Supporting information for

\title{
Iron-Catalyzed Highly Enantioselective Hydrogenation of
}

\author{
Alkenes \\ Peng Lu, ${ }^{\dagger}$ Xiang Ren, ${ }^{\dagger}$ Haofeng Xu, Dongpo Lu, Yufeng Sun and Zhan Lu* \\ Department of Chemistry, Zhejiang University, 310058 Hangzhou, China
}

I. General Information..........................................................

II. Procedures for Preparation of Ligands and Metal Complexes.................S2

III. Procedures for Synthesis of Alkenes..................................S10

IV. Iron-Catalyzed Highly Enantioselective Hydrogenation of Alkenes ..............S23

V. Mechanistic Studies....................................................S43

VI. References..........................................................S56

VII. NMR Spectra.....................................................S58

VIII. HPLC and GC Spectra.............................................S281

\section{General Information}

Toluene was distilled from sodium benzophenone ketyl prior to use. $\mathrm{NaBHEt}_{3}(1.0 \mathrm{M}$ in THF) was purchased from Aldrich and used as received. $\mathrm{FeCl}_{2}(99.5 \%)$ and $\mathrm{FeBr}_{2}$ (99.5\%) were purchased from Alfa Aesar and used as received. The other commercial available chemicals were used as received. NMR spectra were recorded on a Bruker400 instrument or Oxford instrument. ${ }^{1} \mathrm{H}$ NMR chemical shifts were referenced to tetramethylsilane signal $(0 \mathrm{ppm}),{ }^{13} \mathrm{C}$ NMR chemical shifts were referenced to the solvent resonance $\left(77.00 \mathrm{ppm}, \mathrm{CDCl}_{3}\right),{ }^{19} \mathrm{~F}$ NMR chemical shifts were referenced to the solvent resonance. The following abbreviations (or combinations thereof) were used to explain multiplicities: $\mathrm{s}=$ singlet, $\mathrm{d}=$ doublet, $\mathrm{t}=$ triplet, $\mathrm{m}=$ multiplet, $\mathrm{br}=$ broad, $\mathrm{q}=$ quadruplet, $\mathrm{PE}=$ petroleum ether, $\mathrm{EA}=$ ethyl acetate. IR spectra were recorded on 
a Perkin-Elmer Spectrum One FTIR spectrometer with diamond ATR accessory. HPLC analyses were performed on a Shimadzu SPD-20A and GC analyses were performed on Shimadzu GC-2014. High-resolution mass spectra (HRMS) were recorded on Waters XEVOG2-STOF or GCT Premier. X-ray diffraction data was obtained on Gemini A Ultra. Optical rotation data were obtained on PerkinElmer Model 341 Polarimeter. The paramagnetic moment was tested by SQUID on MPMS-XL-5. The data of ESI-HRMS was tested by Agilent 6545 Quadruploe TOF MS.

\section{Procedures for Preparation of Ligands and Metal Complexes}

S1 ${ }^{\text {la }}$ and OIP ligand ${ }^{1 \mathrm{~b}}$ was prepared according to the previously reported procedures.<smiles>C/C(=N\c1c(C)cccc1C)c1ccc2cccc(Br)c2n1</smiles>

(E)-1-(8-bromoquinolin-2-yl)- $N$-(2,6-dimethylphenyl)ethan-1-imine

(S2a). Prepared according to a previously reported procedure ${ }^{2}$ using S1 as starting material, $4.5437 \mathrm{~g}$ (67\% yield). IR (neat): $3065,1645,1595$, 1493, 1468, 1441, $1365 \mathrm{~cm}^{-1} ;{ }^{1} \mathrm{H}$ NMR: (400.0 MHz, $\left.\mathrm{CDCl}_{3}\right) \delta 8.60(\mathrm{~d}$, $J=8.4 \mathrm{~Hz}, 1 \mathrm{H}), 8.23(\mathrm{~d}, J=8.4 \mathrm{~Hz}, 1 \mathrm{H}), 8.09(\mathrm{~d}, J=7.6 \mathrm{~Hz}, 1 \mathrm{H}), 7.85(\mathrm{~d}, J=8.0 \mathrm{~Hz}, 1 \mathrm{H}), 7.45$ $(\mathrm{dd}, J=7.6,8.0 \mathrm{~Hz}, 1 \mathrm{H}), 7.10(\mathrm{~d}, J=7.6 \mathrm{~Hz}, 2 \mathrm{H}), 6.96(\mathrm{dd}, J=7.6,7.6 \mathrm{~Hz}, 1 \mathrm{H}), 2.40(\mathrm{~s}, 3 \mathrm{H}), 2.05$ (s, 6H); ${ }^{13} \mathrm{C}$ NMR: $\left(100.6 \mathrm{MHz}, \mathrm{CDCl}_{3}\right) \delta 167.6,156.5,148.8,144.3,136.7,133.2,130.0,127.9$, $127.8,127.4,125.9,125.2,123.2,119.4,17.9,16.3$; HRMS (ESI) calculated for $\left[\mathrm{C}_{19} \mathrm{H}_{18} \mathrm{BrN}_{2}\right]^{+}$ $\left(\mathrm{M}+\mathrm{H}^{+}\right)$, requires $\mathrm{m} / \mathrm{z} 365.0653$, found $\mathrm{m} / \mathrm{z} 365.0663$.<smiles>C/C(=N\c1c(C(C)C)cccc1C(C)C)c1ccc2cccc(Br)c2n1</smiles>

(E)-1-(8-bromoquinolin-2-yl)- $N$-(2,6-diisopropylphenyl)ethan-1imine (S2b). Prepared according to a previously reported procedure ${ }^{2}$ using S1 as starting material, $7.3078 \mathrm{~g}$ (90\% yield). IR (neat): 2959, 2924, 1696, 1643, 1493, 1462, $1362 \mathrm{~cm}^{-1} ;{ }^{1} \mathrm{H}$ NMR: $\left(400.0 \mathrm{MHz}, \mathrm{CDCl}_{3}\right) \delta$ $8.60(\mathrm{~d}, J=8.8 \mathrm{~Hz}, 1 \mathrm{H}), 8.24(\mathrm{~d}, J=8.4 \mathrm{~Hz}, 1 \mathrm{H}), 8.10(\mathrm{~d}, J=7.2 \mathrm{~Hz}, 1 \mathrm{H}), 7.86(\mathrm{~d}, J=8.0 \mathrm{~Hz}$, 1H), $7.46(\mathrm{dd}, J=8.0,7.6 \mathrm{~Hz}, 1 \mathrm{H}), 7.22-7.17(\mathrm{~m}, 2 \mathrm{H}), 7.15-7.10(\mathrm{~m}, 1 \mathrm{H}), 2.80-2.72(\mathrm{~m}, 2 \mathrm{H}), 2.43$ (s, $3 \mathrm{H}), 1.17$ (d, $J=3.2 \mathrm{~Hz}, 6 \mathrm{H}), 1.15(\mathrm{~d}, J=3.2 \mathrm{~Hz}, 6 \mathrm{H}) ;{ }^{13} \mathrm{C}$ NMR: $\left(100.6 \mathrm{MHz}, \mathrm{CDCl}_{3}\right) \delta 167.3$, 156.5, 146.5, 144.3, 136.6, 135.5, 133.1, 130.0, 127.8, 127.4, 125.9, 123.7, 123.0, 119.4, 28.3, 23.2, 22.8, 16.9; HRMS (ESI) calculated for $\left[\mathrm{C}_{23} \mathrm{H}_{26} \mathrm{BrN}_{2}\right]^{+}\left(\mathrm{M}+\mathrm{H}^{+}\right)$, requires $\mathrm{m} / \mathrm{z} 409.1279$, found $\mathrm{m} / \mathrm{z}$ 409.1290. 
<smiles>COc1cc(C)c(/C=N\c2ccc3cccc(Br)c3n2)c(C=Pc2ccccc2)c1</smiles>

(E)-1-(8-bromoquinolin-2-yl)- $N$-(2,6-dibenzhydryl-4methoxyphenyl)ethan-1-imine (S2c). Prepared according to a previously reported procedure ${ }^{2}$ using $\mathbf{S 1}$ as starting material, $7.7023 \mathrm{~g}$ (82\% yield). IR (neat): 2972, 2902, 1645, 1452, 1407, 1059, $895 \mathrm{~cm}^{-1}$; ${ }^{1} \mathrm{H}$ NMR: $\left(400.0 \mathrm{MHz}, \mathrm{CDCl}_{3}\right) \delta 8.12(\mathrm{q}, \mathrm{J}=16.4,8.0 \mathrm{~Hz}, 2 \mathrm{H}), 8.04(\mathrm{~d}, \mathrm{~J}=8.0 \mathrm{~Hz}$, 1H), $7.78(\mathrm{~d}, \mathrm{~J}=8.0 \mathrm{~Hz}, 1 \mathrm{H}), 7.39(\mathrm{t}, \mathrm{J}=6.4 \mathrm{~Hz}, 1 \mathrm{H}), 7.29-7.11(\mathrm{~m}, 12 \mathrm{H}), 7.08-6.93(\mathrm{~m}, 8 \mathrm{H}), 6.48$ (s, 2H), $5.30(\mathrm{~s}, 2 \mathrm{H}), 3.56(\mathrm{~s}, 3 \mathrm{H}), 1.37$ (s, 3H); $\left.{ }^{13} \mathrm{C} \mathrm{NMR:} \mathrm{(100.6} \mathrm{MHz,} \mathrm{CDCl}_{3}\right) \delta 170.9,156.4$, $155.1,144.2,143.2,142.2,142.1,136.3,133.4,133.0,129.9,129.7,129.4,128.4,128.0,127.6$, $127.3,126.3,126.1,126.0,119.5,113.7,55.1,52.2,16.7$; HRMS (ESI) calculated for $\left[\mathrm{C}_{44} \mathrm{H}_{36} \mathrm{BrN}_{2} \mathrm{O}\right]^{+}\left(\mathrm{M}+\mathrm{H}^{+}\right)$, requires $\mathrm{m} / \mathrm{z}$ 678.2011, found $\mathrm{m} / \mathrm{z}$ 687.2005.<smiles>C/C(=N\c1c(C)cccc1C)c1ccc2cccc(C3=NC(C(C)(C)C)CO3)c2n1</smiles>

(S,E)-1-(8-(4-(tert-butyl)-4,5-dihydrooxazol-2-yl)quinolin-2-yl)- $N$ (2,6-dimethylphenyl)ethan-1-imine (La). Prepared according to a previously reported procedure, ${ }^{3}$ using $1.4134 \mathrm{~g}(4.0 \mathrm{mmol})$ of $(E)-1$ (8-bromoquinolin-2-yl)- $N$-(2,6-dimethylphenyl)ethan-1-imine, 0.7224 $\mathrm{g}(5.4 \mathrm{mmol})$ of (S)-4-(tert-butyl)-4,5-dihydrooxazole, ${ }^{4} 0.0454 \mathrm{~g}(0.12 \mathrm{mmol})$ of $\mathrm{Pd}(\mathrm{OAc})_{2}, 0.0932$ $\mathrm{g}(0.22 \mathrm{mmol})$ of dppe, $0.6423 \mathrm{~g}(8.0 \mathrm{mmol})$ of $t \mathrm{BuOLi}$ and $24 \mathrm{~mL}$ of 1,4-dioxane. The mixture was placed in an oil bath and stirring at $110{ }^{\circ} \mathrm{C}$ for $36 \mathrm{~h}$. The resulting solution was purified by flash column chromatography using PE/EA (10:1) as the eluent to give $1.0945 \mathrm{~g}$ (2.7 mmol, 68\% yield) of the title compound as a yellow oil. Optical Rotation: $[\alpha]^{20}{ }_{\mathrm{D}}=-54.8\left(\mathrm{c} 0.94, \mathrm{CHCl}_{3}\right)$. IR (neat): 2970, 2904, 1644, 1600, 1455, 1401, $1054 \mathrm{~cm}^{-1}$; ${ }^{1} \mathrm{H}$ NMR: $\left(400.0 \mathrm{MHz}, \mathrm{CDCl}_{3}\right) \delta 8.58(\mathrm{~d}, J=8.8$ $\mathrm{Hz}, 1 \mathrm{H}), 8.24(\mathrm{~d}, J=8.8 \mathrm{~Hz}, 1 \mathrm{H}), 8.09(\mathrm{dd}, J=1.2,7.2 \mathrm{~Hz}, 1 \mathrm{H}), 7.96(\mathrm{dd}, J=1.6,8.4 \mathrm{~Hz}, 1 \mathrm{H})$, 7.61 (dd, $J=7.8,8.0 \mathrm{~Hz}, 1 \mathrm{H}), 4.60-4.52(\mathrm{~m}, 1 \mathrm{H}), 4.30-4.20(\mathrm{~m}, 2 \mathrm{H}), 2.81-2.69$ (m, 1H), 2.34 (s, $3 \mathrm{H}), 2.02-1.92(\mathrm{~m}, 1 \mathrm{H}), 1.18-1.12(\mathrm{~m}, 12 \mathrm{H}), 1.11(\mathrm{~d}, J=6.8 \mathrm{~Hz}, 3 \mathrm{H}), 1.03(\mathrm{~d}, J=6.8 \mathrm{~Hz}, 3 \mathrm{H}) ;{ }^{13} \mathrm{C}$ NMR: $\left(100.6 \mathrm{MHz}, \mathrm{CDCl}_{3}\right) \delta 167.4,163.7,156.3,146.6,145.2,136.3,135.6,135.5,131.1,130.3$, $129.0,128.8,126.6,123.6,123.0,122.9,118.9,73.2,70.6,32.8,28.2,23.2,22.8,18.9,18.3,16.9$; HRMS (ESI) calculated for $\left[\mathrm{C}_{26} \mathrm{H}_{30} \mathrm{~N}_{3} \mathrm{O}\right]^{+}\left(\mathrm{M}+\mathrm{H}^{+}\right)$, requires $\mathrm{m} / \mathrm{z} 400.2389$, found $\mathrm{m} / \mathrm{z} 400.2395$. 
<smiles>C/C(=N\c1c(C(C)C)cccc1C(C)C)c1ccc2cccc(C3=NC(C(C)C)CO3)c2n1</smiles>

\section{(S,E)-1-(8-(4-(tert-butyl)-4,5-dihydrooxazol-2-yl)quinolin-2-yl)- $N$ -}

(2,6-diisopropylphenyl)ethan-1-imine (Lb). Prepared according to a previously reported procedure, ${ }^{3}$ using $2.7287 \mathrm{~g}(6.7 \mathrm{mmol})$ of $(E)-1-$ (8-bromoquinolin-2-yl)- $N$-(2,6-diisopropylphenyl)ethan-1-imine, $1.3135 \mathrm{~g}$ (9.0 mmol) of (S)-4-(tert-butyl)-4,5-dihydrooxazole, ${ }^{4} 0.0694 \mathrm{~g}(0.34 \mathrm{mmol})$ of $\mathrm{Pd}(\mathrm{OAc})_{2}$, $0.1478 \mathrm{~g}(0.38 \mathrm{mmol})$ of dppe, $1.0873 \mathrm{~g}(5.0 \mathrm{mmol})$ of $t \mathrm{BuOLi}$ and $40 \mathrm{~mL}$ of 1,4-dioxane. The mixture was placed in an oil bath and stirring at $110^{\circ} \mathrm{C}$ for $40 \mathrm{~h}$. The resulting solution was purified by flash column chromatography using PE/EA (5:1) as the eluent to give $2.8562 \mathrm{~g} \mathrm{(6.3} \mathrm{mmol,} \mathrm{94 \%}$ yield) of the title compound as a yellow oil. Optical Rotation: $[\alpha]^{20}{ }_{\mathrm{D}}=-57.8\left(\mathrm{c} 1.05, \mathrm{CHCl}_{3}\right)$. IR (neat): 2970, 2903, 1647, 1400, 1254, $1060 \mathrm{~cm}^{-1} ;{ }^{1} \mathrm{H}$ NMR: $\left(400.0 \mathrm{MHz}, \mathrm{CDCl}_{3}\right) \delta 8.58(\mathrm{~d}, J=8.8$ $\mathrm{Hz}, 1 \mathrm{H}), 8.24(\mathrm{~d}, J=8.8 \mathrm{~Hz}, 1 \mathrm{H}), 8.09(\mathrm{dd}, J=1.2,7.2 \mathrm{~Hz}, 1 \mathrm{H}), 7.96(\mathrm{dd}, J=1.6,8.4 \mathrm{~Hz}, 1 \mathrm{H})$, $7.61(\mathrm{dd}, J=7.8,8.0 \mathrm{~Hz}, 1 \mathrm{H}), 7.25-7.11(\mathrm{~m}, 3 \mathrm{H}), 4.50(\mathrm{t}, J=1.6 \mathrm{~Hz}, 1 \mathrm{H}), 4.36(\mathrm{t}, J=8.4 \mathrm{~Hz}, 1 \mathrm{H})$, $4.21(\mathrm{t}, J=8.4 \mathrm{~Hz}, 1 \mathrm{H}), 2.81-2.69(\mathrm{~m}, 2 \mathrm{H}), 2.34(\mathrm{~s}, 3 \mathrm{H}), 1.18-1.12(\mathrm{~m}, 12 \mathrm{H}), 1.05(\mathrm{~s}, 9 \mathrm{H}) ;{ }^{13} \mathrm{C}$ NMR: $\left(100.6 \mathrm{MHz}, \mathrm{CDCl}_{3}\right) \delta$ 167.5, 163.7, 156.3, 146.6, 145.2, 136.3, 135.6, 135.5, 131.2, 130.2, $129.1,128.8,126.6,123.6,123.0,122.9,119.0,76.9,69.1,33.8,28.2,26.0,23.2,23.1,22.8,17.1$; HRMS (ESI) calculated for $\left[\mathrm{C}_{30} \mathrm{H}_{38} \mathrm{~N}_{3} \mathrm{O}\right]^{+}\left(\mathrm{M}+\mathrm{H}^{+}\right)$, requires $\mathrm{m} / \mathrm{z} 456.3015$, found $\mathrm{m} / \mathrm{z} 456.3021$.<smiles>COc1cc(C)c(N=C(C)c2ccc3cccc(C4=NC(C(c5ccccc5)c5ccccc5)CO4)c3n2)c(C=Cc2ccccc2)c1</smiles>

(S,E)-1-(8-(4-(tert-butyl)-4,5-dihydrooxazol-2-yl)quinolin-2yl)- $N$-(2,6-dibenzhydryl-4-methoxyphenyl)ethan-1-imine

(Lc). Prepared according to a previously reported procedure, ${ }^{3}$ using $1.7194 \mathrm{~g}(2.5 \mathrm{mmol})$ of $(E)-1$-(8-bromoquinolin-2-yl)- $N$ (2,6-dibenzhydryl-4-methoxyphenyl)ethan-1-imine, $0.4739 \mathrm{~g}(3.4 \mathrm{mmol})$ of $(S)-4$-(tert-butyl)4,5-dihydrooxazole, ${ }^{4} 0.0285 \mathrm{~g}(0.13 \mathrm{mmol})$ of $\mathrm{Pd}(\mathrm{OAc})_{2}, 0.0561 \mathrm{~g}(0.14 \mathrm{mmol})$ of dppe, $0.4004 \mathrm{~g}$ $(5.0 \mathrm{mmol})$ of $t \mathrm{BuOLi}$ and $15 \mathrm{~mL}$ of 1,4-dioxane. The mixture was placed in an oil bath and stirring at $110{ }^{\circ} \mathrm{C}$ for $40 \mathrm{~h}$. The resulting solution was purified by flash column chromatography using PE/EA $(5: 1)$ as the eluent to give $1.2294 \mathrm{~g}(1.68 \mathrm{mmol}, 84 \%$ yield $)$ of the title compound as a yellow oil. Optical Rotation: $[\alpha]^{20}{ }_{\mathrm{D}}=-52.3$ (c 0.37, $\mathrm{CHCl}_{3}$ ). IR (neat): 2972, 2920, 1655, 1498, 1361, $1161 \mathrm{~cm}^{-}$ '; ${ }^{1} \mathrm{H}$ NMR: $\left(399.9 \mathrm{MHz}, \mathrm{CDCl}_{3}\right) \delta 8.59(\mathrm{~d}, J=8.8 \mathrm{~Hz}, 1 \mathrm{H}), 8.25(\mathrm{~d}, J=8.8 \mathrm{~Hz}, 1 \mathrm{H}), 8.07(\mathrm{dd}, J$ $=1.2,7.2 \mathrm{~Hz}, 1 \mathrm{H}), 7.98(\mathrm{dd}, J=1.2,8.0 \mathrm{~Hz}, 1 \mathrm{H}), 7.65-7.59(\mathrm{~m}, 1 \mathrm{H}), 7.35-7.27(\mathrm{~m}, 4 \mathrm{H}), 7.25-7.20$ (m, 1H), 7.19-7.16 (m, 2H), 7.14-7.08 (m, 1H), 4.78-4.69 (m, 1H), $4.51(\mathrm{dd}, J=8.8,9.2 \mathrm{~Hz}, 1 \mathrm{H})$, 
$4.29(\mathrm{dd}, J=7.6,8.0 \mathrm{~Hz}, 1 \mathrm{H}), 3.35(\mathrm{dd}, J=5.2,13.6 \mathrm{~Hz}, 1 \mathrm{H}), 2.88(\mathrm{dd}, J=8.8,14.0 \mathrm{~Hz}, 1 \mathrm{H}), 2.81-$ $2.70(\mathrm{~m}, 2 \mathrm{H}), 2.34$ (s, 3H), 1.18-1.09 (m, 12H); ${ }^{13} \mathrm{C}$ NMR: (100.6 MHz, $\left.\mathrm{CDCl}_{3}\right)$ 8170.6, 164.2, 155.9, 155.0, 145.2, 143.25, 143.24, 142.32, 142.30, 142.2, 135.8, 133.4, 133.2, 130.9, 130.1, 129.8, $129.7,129.43,129.40,128.6,128.4,128.3,128.03,128.00,126.5,126.2,126.1,119.1,113.7,76.6$, 69.2, 55.1, 52.15, 52.13, 33.8, 26.1, 16.6; HRMS (ESI) calculated for $\left[\mathrm{C}_{51} \mathrm{H}_{48} \mathrm{~N}_{3} \mathrm{O}_{2}\right]^{+}\left(\mathrm{M}+\mathrm{H}^{+}\right)$, requires $\mathrm{m} / \mathrm{z} 734.3747$, found $\mathrm{m} / \mathrm{z} 734.3744$.<smiles>C/C(=N\c1c(C(C)C)cccc1C(C)C)c1ccc2cccc(C3=NC(Cc4ccccc4)CO3)c2n1</smiles>
(S,E)-1-(8-(4-benzyl-4,5-dihydrooxazol-2-yl)quinolin-2-yl)- $N$-(2,6diisopropylphenyl)ethan-1-imine (Ld). Prepared according to a previously reported procedure, ${ }^{3}$ using $1.0213 \mathrm{~g}(2.5 \mathrm{mmol})$ of $(E)-1-$ (8-bromoquinolin-2-yl)-N-(2,6-diisopropylphenyl)ethan-1-imine, $0.5498 \mathrm{~g}(3.4 \mathrm{mmol})$ of $(S)$-4-benzyl-4,5-dihydrooxazole ${ }^{4}{ }^{0} 0.0283 \mathrm{~g}(0.13 \mathrm{mmol})$ of $\mathrm{Pd}(\mathrm{OAc})_{2}$, $0.0564 \mathrm{~g}(0.14 \mathrm{mmol})$ of dppe, $0.4021 \mathrm{~g}(5.0 \mathrm{mmol})$ of $t \mathrm{BuOLi}$ and $15 \mathrm{~mL}$ of 1,4-dioxane. The mixture was placed in an oil bath and stirring at $110{ }^{\circ} \mathrm{C}$ for $40 \mathrm{~h}$. The resulting solution was purified by flash column chromatography using PE/EA (5:1) as the eluent to give $1.0227 \mathrm{~g} \mathrm{(2.1} \mathrm{mmol,} \mathrm{84 \%}$ yield) of the title compound as a yellow oil. Optical Rotation: $[\alpha]^{20} \mathrm{D}=-13.5\left(\mathrm{c} 1.05, \mathrm{CHCl}_{3}\right)$. IR (neat): 2962, 2924, 1645, 1497, 1362, $1061 \mathrm{~cm}^{-1} ;{ }^{1} \mathrm{H}$ NMR: $\left(399.9 \mathrm{MHz}, \mathrm{CDCl}_{3}\right) \delta 8.59(\mathrm{~d}, J=8.8$ $\mathrm{Hz}, 1 \mathrm{H}), 8.25(\mathrm{~d}, J=8.8 \mathrm{~Hz}, 1 \mathrm{H}), 8.07$ (dd, $J=1.2,7.2 \mathrm{~Hz}, 1 \mathrm{H}), 7.98(\mathrm{dd}, J=1.2,8.0 \mathrm{~Hz}, 1 \mathrm{H})$, 7.65-7.59 (m, 1H), 7.35-7.27 (m, 4H), 7.25-7.20 (m, 1H), 7.19-7.16 (m, 2H), 7.14-7.08 (m, 1H), 4.78-4.69 (m, 1H), $4.51(\mathrm{dd}, J=8.8,9.2 \mathrm{~Hz}, 1 \mathrm{H}), 4.29(\mathrm{dd}, J=7.6,8.0 \mathrm{~Hz}, 1 \mathrm{H}), 3.35(\mathrm{dd}, J=5.2$, $13.6 \mathrm{~Hz}, 1 \mathrm{H}), 2.88$ (dd, $J=8.8,14.0 \mathrm{~Hz}, 1 \mathrm{H}), 2.81-2.70$ (m, 2H), 2.34 (s, 3H), 1.18-1.09 (m, 12H); ${ }^{13} \mathrm{C}$ NMR: $\left(100.6 \mathrm{MHz}, \mathrm{CDCl}_{3}\right) \delta 167.2,164.4,156.2,146.5,145.2,138.0,136.3,135.5,131.0$, $130.4,129.2,128.8,128.7,128.6,126.7,126.5,123.6,122.9,119.0,72.3,68.1,41.9,28.2,23.2$, 22.8, 16.8; HRMS (ESI) calculated for $\left[\mathrm{C}_{33} \mathrm{H}_{36} \mathrm{~N}_{3} \mathrm{O}\right]^{+}\left(\mathrm{M}+\mathrm{H}^{+}\right)$, requires $\mathrm{m} / \mathrm{z} 490.2858$, found $\mathrm{m} / \mathrm{z}$ 490.2859 .<smiles>C/C(=N\c1c(C(C)C)cccc1C(C)C)c1ccc2cccc(C3=NC(C(C)C)CO3)c2n1</smiles>

(S,E)-N-(2,6-diisopropylphenyl)-1-(8-(4-isopropyl-4,5dihydrooxazol-2-yl)quinolin-2-yl)ethan-1-imine (Le). Prepared according to a previously reported procedure, ${ }^{3}$ using $1.0213 \mathrm{~g}(2.5 \mathrm{mmol})$ of (E)-1-(8-bromoquinolin-2-yl)- $N$-(2,6-diisopropylphenyl)ethan-1imine, $0.6069 \mathrm{~g}(3.8 \mathrm{mmol})$ of $(S)$-4-isopropyl-4,5-dihydrooxazole, ${ }^{4} 0.0279 \mathrm{~g}(0.13 \mathrm{mmol})$ of 
$\mathrm{Pd}(\mathrm{OAc})_{2}, 0.0568 \mathrm{~g}(0.14 \mathrm{mmol})$ of dppe, $0.4063 \mathrm{~g}(5.0 \mathrm{mmol})$ of $t \mathrm{BuOLi}$ and $15 \mathrm{~mL}$ of 1,4-dioxane. The mixture was placed in an oil bath and stirring at $110{ }^{\circ} \mathrm{C}$ for $40 \mathrm{~h}$. The resulting solution was purified by flash column chromatography using PE/EA (5:1) as the eluent to give $0.8456 \mathrm{~g} \mathrm{(1.9}$ mmol, $76 \%$ yield) of the title compound as a yellow oil. Optical Rotation: $[\alpha]^{20}{ }_{\mathrm{D}}=-55.1(\mathrm{c} 1.03$, $\mathrm{CHCl}_{3}$ ). IR (neat): 2960, 2926, 1645, 1414, 1362, $765 \mathrm{~cm}^{-1} ;{ }^{1} \mathrm{H}$ NMR: $\left(400.0 \mathrm{MHz}, \mathrm{CDCl}_{3}\right) \delta 8.58$ $(\mathrm{d}, J=8.8 \mathrm{~Hz}, 1 \mathrm{H}), 8.24(\mathrm{~d}, J=8.8 \mathrm{~Hz}, 1 \mathrm{H}), 8.09(\mathrm{~d}, J=8.0 \mathrm{~Hz}, 1 \mathrm{H}), 7.96(\mathrm{~d}, J=7.2 \mathrm{~Hz}, 1 \mathrm{H})$, $7.61(\mathrm{t}, J=7.2 \mathrm{~Hz}, 1 \mathrm{H}), 7.21-7.15(\mathrm{~m}, 2 \mathrm{H}), 7.14-7.08(\mathrm{~m}, 1 \mathrm{H}), 4.61-4.52(\mathrm{~m}, 1 \mathrm{H}), 4.30-4.21(\mathrm{~m}$, 2H), 2.82-2.70 (m, 2H), $2.34(\mathrm{~s}, 3 \mathrm{H}), 2.03-1.91(\mathrm{~m}, 1 \mathrm{H}), 1.18-1.13(\mathrm{~m}, 12 \mathrm{H}), 1.11(\mathrm{~d}, J=6.8 \mathrm{~Hz}$, 3H), $1.03(\mathrm{~d}, J=6.8 \mathrm{~Hz}, 3 \mathrm{H}) ;{ }^{13} \mathrm{C}$ NMR: $\left(100.6 \mathrm{MHz}, \mathrm{CDCl}_{3}\right) \delta 167.4,163.7,156.3,146.6,145.2$, $136.3,135.6,135.5,131.1,130.3,129.0,128.8,126.6,123.6,123.0,122.9,118.9,73.2,70.6,32.8$, 28.2, 23.2, 22.8, 18.9, 18.3, 16.9; HRMS (ESI) calculated for $\left[\mathrm{C}_{29} \mathrm{H}_{36} \mathrm{~N}_{3} \mathrm{O}\right]^{+}\left(\mathrm{M}+\mathrm{H}^{+}\right)$, requires $\mathrm{m} / \mathrm{z}$ 442.2858, found $\mathrm{m} / \mathrm{z} 442.2862$.

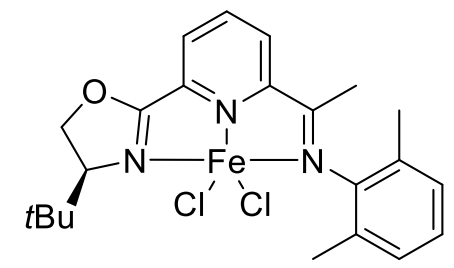

(S)-OIP· $\mathrm{FeCl}_{2}$. A $50 \mathrm{~mL}$ Schleck flask was charged with $0.1722 \mathrm{~g}$ (1.40 mmol) of $\mathrm{FeCl}_{2}, 3 \mathrm{~mL}$ THF and a solution of $0.5452 \mathrm{~g}(1.56$ mmol) of (S)-OIP in $4 \mathrm{~mL}$ THF under atmosphere of nitrogen. The mixture was stirred at room temperature for $12 \mathrm{~h}$. The solvent was removed in vacuo. The resulting mixture was washed with ether and filtered under air. The cake was washed with ether and dried in vacuo to afford $0.5864 \mathrm{~g}(1.23 \mathrm{mmol}, 91 \%$ yield $)$ of the title compound as a brown powder.<smiles></smiles>

(S)-La $\cdot \mathrm{FeCl}_{2}$. A $50 \mathrm{~mL}$ Schleck flask was charged with $0.0603 \mathrm{~g}(0.48$ mmol) of $\mathrm{FeCl}_{2}, 4 \mathrm{~mL}$ THF and a solution of $0.2002 \mathrm{~g}(0.53 \mathrm{mmol})$ of (S)-La in $3 \mathrm{~mL}$ THF under atmosphere of nitrogen. The mixture was stirred at room temperature for $17 \mathrm{~h}$. The solvent was removed in vacuo. The resulting mixture was washed with ether and filtered under air. The cake was washed with ether and dried in vacuo to afford $0.2090 \mathrm{~g}(0.21 \mathrm{mmol}, 79 \%$ yield $)$ of the title compound as a brown powder. Anal. Calcd for $\mathrm{C}_{26} \mathrm{H}_{29} \mathrm{Cl}_{2} \mathrm{FeN}_{3} \mathrm{O}+2 \mathrm{H}_{2} \mathrm{O}: \mathrm{C}, 55.54 ; \mathrm{H}, 5.92 ; \mathrm{N}, 7.47$; Found: $\mathrm{C}, 55.30 ; \mathrm{H}$, 5.54; $\mathrm{N}, 7.40 .{ }^{1} \mathrm{H} \mathrm{NMR}\left(400.0 \mathrm{MHz}, \mathrm{CDCl}_{3}\right.$, all peaks appear as singlets due to their broadness) $\delta$, $11.71(1 \mathrm{H}) .8 .70(3 \mathrm{H}), 7.30(4 \mathrm{H}), 2.16-1.22(13 \mathrm{H}),-0.69(13 \mathrm{H}),-2.61(2 \mathrm{H})$. 
<smiles></smiles>

(S)-Lb $\cdot \mathrm{FeCl}_{2}$. A $50 \mathrm{~mL}$ Schleck flask was charged with $0.0481 \mathrm{~g}(0.38$ mmol $)$ of $\mathrm{FeCl}_{2}, 4 \mathrm{~mL}$ THF and a solution of $0.1835 \mathrm{~g}(0.40 \mathrm{mmol})$ of $(\boldsymbol{S})$-Lb in $3 \mathrm{~mL}$ THF under atmosphere of nitrogen. The mixture was stirred at room temperature for $17 \mathrm{~h}$. The solvent was removed in vacuo. The resulting mixture was washed with ether and filtered under air. The cake was washed with ether and dried in vacuo to afford $0.1628 \mathrm{~g}(0.33 \mathrm{mmol}, 90 \%$ yield $)$ of the title compound as a brown powder. Anal. Calcd for $\mathrm{C}_{30} \mathrm{H}_{37} \mathrm{Cl}_{2} \mathrm{FeN}_{3} \mathrm{O}+1 / 7 \mathrm{H}_{2} \mathrm{O}: \mathrm{C}, 61.60 ; \mathrm{H}, 6.43 ; \mathrm{N}, 7.18$; Found: $\mathrm{C}, 61.61$; $\mathrm{H}, 6.37 ; \mathrm{N}, 7.16 .{ }^{1} \mathrm{H} \mathrm{NMR}\left(400.0 \mathrm{MHz}, \mathrm{CDCl}_{3}\right.$, all peaks appear as singlets due to their broadness) $\delta, 12.36(1 \mathrm{H}), 9.58(3 \mathrm{H}), 7.20(1 \mathrm{H}), 4.77(3 \mathrm{H}), 2.11(1 \mathrm{H}), 0.52(6 \mathrm{H}),-0.31(1 \mathrm{H}),-2.41(1 \mathrm{H}),-1.94$ $(4 \mathrm{H}),-3.06(11 \mathrm{H})$.

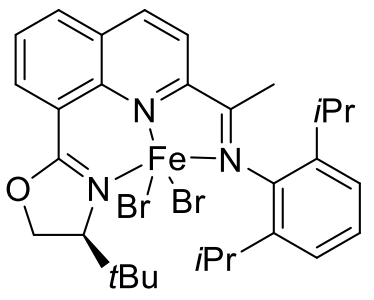

$(\boldsymbol{S})-\mathbf{L b} \cdot \mathrm{FeBr}_{2}$. A $50 \mathrm{~mL}$ Schleck flask was charged with $0.0820 \mathrm{~g}(0.38$ $\mathrm{mmol})$ of $\mathrm{FeBr}_{2}, 4 \mathrm{~mL}$ THF and a solution of $0.1845 \mathrm{~g}(0.40 \mathrm{mmol})$ of (S)-Lb in $3 \mathrm{~mL}$ THF under atmosphere of nitrogen. The mixture was stirred at room temperature for $17 \mathrm{~h}$. The solvent was removed in vacuo. The resulting mixture was washed with ether and filtered under air. The cake was washed with ether and dried in vacuo to afford $0.2200 \mathrm{~g}(0.33 \mathrm{mmol}, 90 \%$ yield $)$ of the title compound as a brown powder. Anal. Calcd for $\mathrm{C}_{30} \mathrm{H}_{37} \mathrm{Br}_{2} \mathrm{FeN}_{3} \mathrm{O}+1 / 3 \mathrm{H}_{2} \mathrm{O}$ : C, 53.20; H, 5.61; N, 6.20; Found: C, 53.21; $\mathrm{H}, 5.59$; $\mathrm{N}, 6.04$. CCDC Number 2011869. ${ }^{1} \mathrm{H}$ NMR $\left(400.0 \mathrm{MHz}, \mathrm{CDCl}_{3}\right.$, all peaks appear as singlets due to their broadness) $\delta 14.05(2 \mathrm{H}), 11.28(1 \mathrm{H}), 7.24(2 \mathrm{H}), 3.76(4 \mathrm{H}), 1.27(10 \mathrm{H}),-0.52$ $(8 \mathrm{H}),-1.91(3 \mathrm{H})$. Based on the measurement of SQUID, the iron catalyst is paramagnetic and the paramagnetic moment is $4.5 \mu_{\mathrm{B}}$.

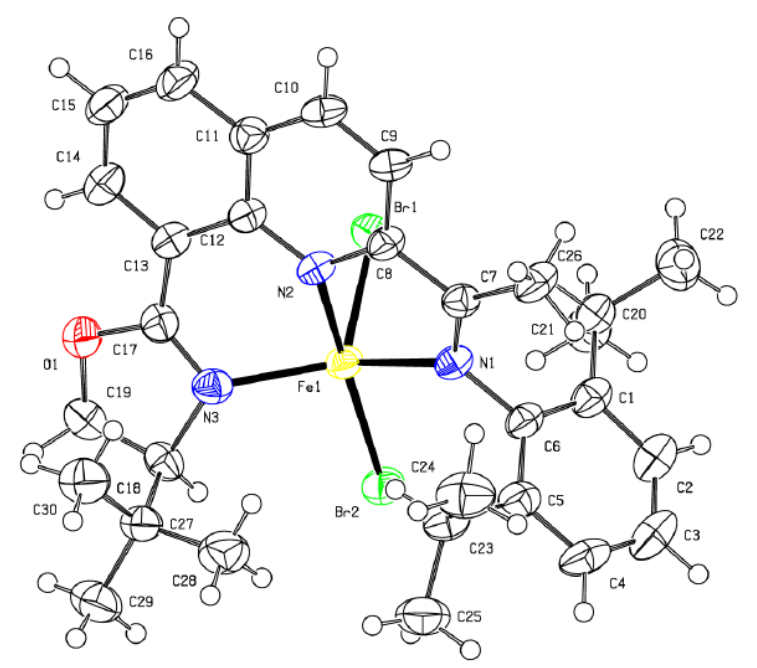


Selected Bond Length for $(\boldsymbol{S})-\mathbf{L b} \cdot \mathrm{FeBr}_{2}$ complex

\begin{tabular}{|l|l|}
\hline Selected Bond Length & Distance $(\AA)$ \\
\hline Fe1-N1 & $2.134(6)$ \\
\hline Fe1-N2 & $2.166(6)$ \\
\hline Fe1-N3 & $2.110(6)$ \\
\hline Fe1-Br1 & $2.5234(15)$ \\
\hline Fe1-Br2 & $2.4319(14)$ \\
\hline
\end{tabular}

Selected Bond Angles for $(\boldsymbol{S})-\mathbf{L b} \cdot \mathrm{FeBr}_{2}$ complex

\begin{tabular}{|l|l|}
\hline Selected Bond Angles & $($ deg $)$ \\
\hline N2- Fe1-N3 & $83.1(2)$ \\
\hline N2- Fe1-N1 & $75.4(2)$ \\
\hline N2- Fe1-Br2 & $168.47(16)$ \\
\hline N2- Fe1- Br1 & $83.43(16)$ \\
\hline N3- Fe1-N1 & $139.0(2)$ \\
\hline N3- Fe1- Br2 & $97.96(16)$ \\
\hline N3- Fe1- Br1 & $110.64(18)$ \\
\hline N1- Fe1- Br2 & $96.78(15)$ \\
\hline N1- Fe1- Br1 & $101.24(17)$ \\
\hline $\mathrm{Br} 2-\mathrm{Fe} 1-\mathrm{Br} 1$ & $106.70(5)$ \\
\hline
\end{tabular}

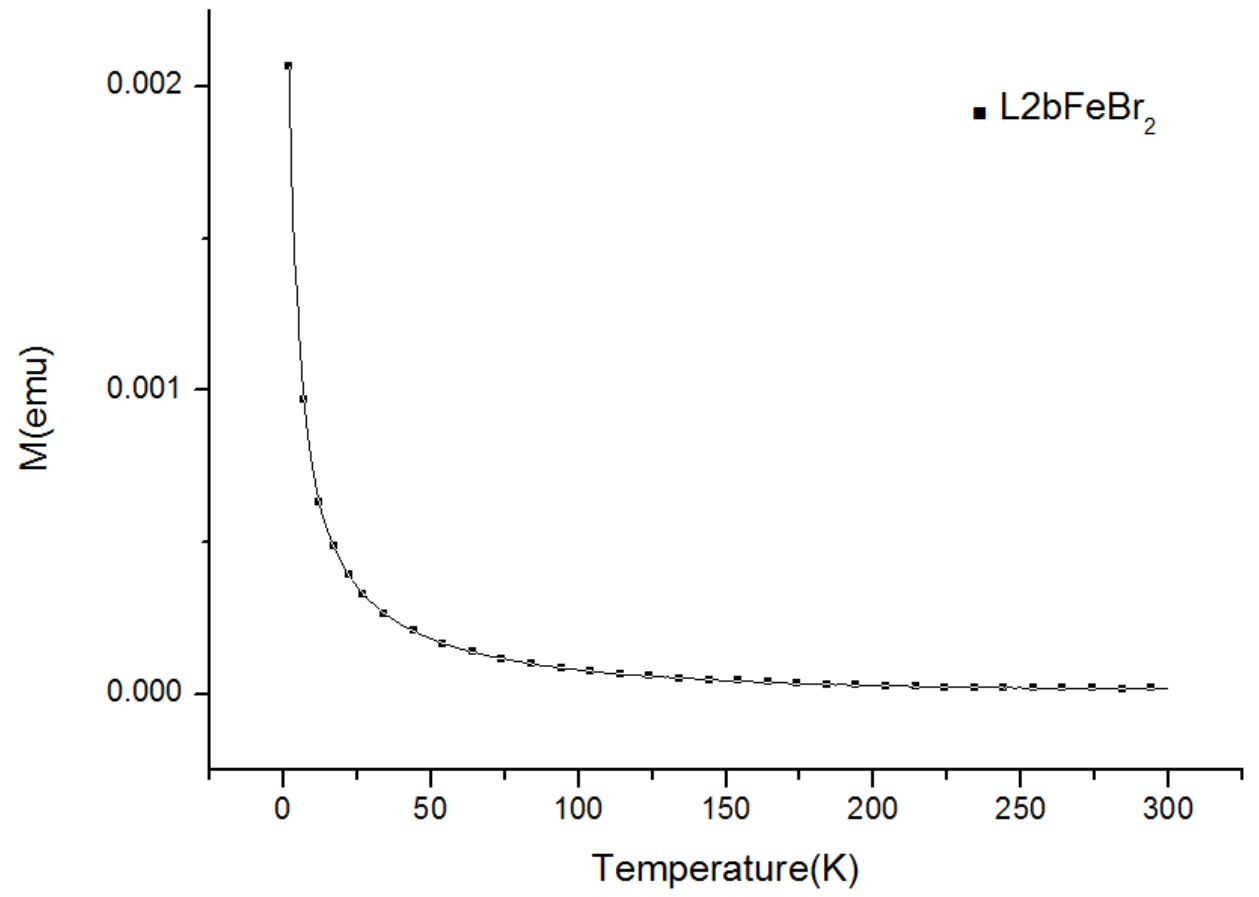

The M-T curve of the iron catalyst $\mathbf{L b} \cdot \mathrm{FeCl}_{2}$ 


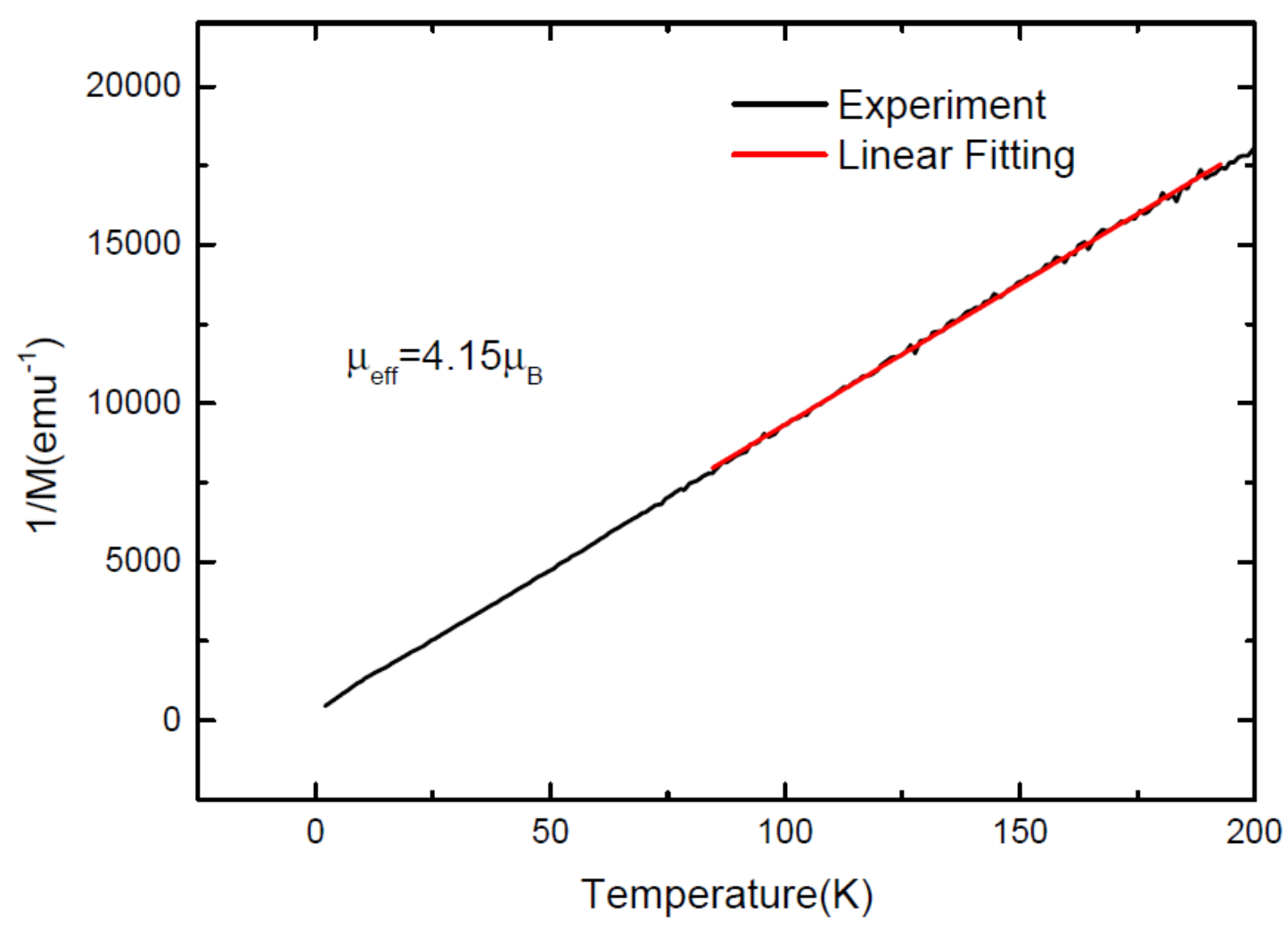

The magnetic moment of iron catalyst $\mathbf{L b} \cdot \mathrm{FeCl}_{2}$

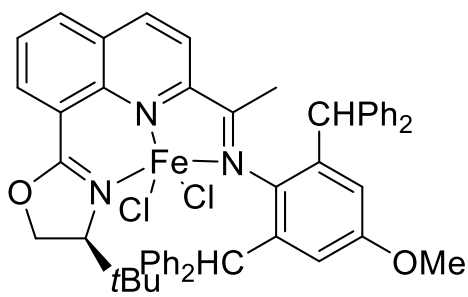

(S)-Lc $\cdot \mathrm{FeCl}_{2}$. A $50 \mathrm{~mL}$ Schleck flask was charged with $0.0253 \mathrm{~g}$ $(0.20 \mathrm{mmol})$ of $\mathrm{FeCl}_{2}, 3 \mathrm{~mL}$ THF and a solution of $0.1471 \mathrm{~g}(0.20$ mmol) of (S)-Le in $3 \mathrm{~mL}$ THF under atmosphere of nitrogen. The mixture was stirred at room temperature for $5 \mathrm{~h}$. The solvent was removed in vacuo. The resulting mixture was washed with ether and filtered under air. The cake was washed with ether and dried in vacuo to afford $0.1548 \mathrm{~g}(0.18 \mathrm{mmol}, 90 \%$ yield $)$ of the title compound as a brown powder. Anal. Calcd for $\mathrm{C}_{51} \mathrm{H}_{47} \mathrm{Cl}_{2} \mathrm{FeN}_{3} \mathrm{O}_{2}+1.5 \mathrm{H}_{2} \mathrm{O}$ : C, 69.00; $\mathrm{H}, 5.68 ; \mathrm{N}$, 4.73; Found: C, 69.05; H, 5.60; N, 4.77.

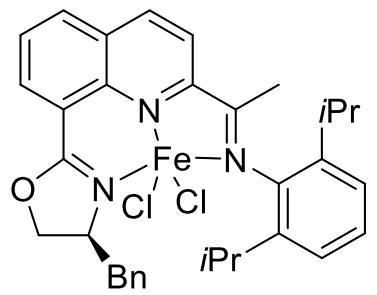

(S)-Ld $\cdot \mathrm{FeCl}_{2}$. A $50 \mathrm{~mL}$ Schleck flask was charged with $0.0761 \mathrm{~g}(0.60$ $\mathrm{mmol})$ of $\mathrm{FeCl}_{2}, 4 \mathrm{~mL}$ THF and a solution of $0.3080 \mathrm{~g}(0.63 \mathrm{mmol})$ of $(\boldsymbol{S})$-Ld in $3 \mathrm{~mL}$ THF under atmosphere of nitrogen. The mixture was stirred at room temperature for $9 \mathrm{~h}$. The solvent was removed in vacuo. The resulting mixture was washed with ether and filtered under air. The cake was washed with ether and dried in vacuo to afford $0.3340 \mathrm{~g}(0.54 \mathrm{mmol}, 90 \%$ yield $)$ of the title compound as a brown powder. Anal. Calcd for $\mathrm{C}_{33} \mathrm{H}_{35} \mathrm{Cl}_{2} \mathrm{FeN}_{3} \mathrm{O}+3 / 4 \mathrm{H}_{2} \mathrm{O}: \mathrm{C}, 62.92 ; \mathrm{H}, 5.84 ; \mathrm{N}, 6.67$; Found: $\mathrm{C}, 62.90$; 
$\mathrm{H}, 5.68 ; \mathrm{N}, 6.61 .{ }^{1} \mathrm{H} \mathrm{NMR}\left(400.0 \mathrm{MHz}, \mathrm{CDCl}_{3}\right.$, all peaks appear as singlets due to their broadness) $\delta, 19.26(1 \mathrm{H}), 13.50(1 \mathrm{H}), 8.10(1 \mathrm{H}), 7.01(6 \mathrm{H}), 6.28(3 \mathrm{H}), 3.47(2 \mathrm{H}), 2.52(3 \mathrm{H}), 1.26(11 \mathrm{H}),-2.21$ $(1 \mathrm{H}),-3.10(3 \mathrm{H})$.

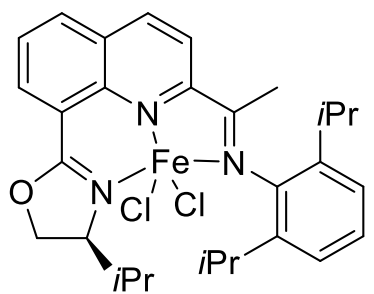

(S)-Le $\cdot \mathrm{FeCl}_{2}$. A $50 \mathrm{~mL}$ Schleck flask was charged with $0.0625 \mathrm{~g}(0.50$ mmol) of $\mathrm{FeCl}_{2}, 4 \mathrm{~mL}$ THF and a solution of $0.2212 \mathrm{~g}(0.50 \mathrm{mmol})$ of $(\boldsymbol{S})$-Le in $3 \mathrm{~mL}$ THF under atmosphere of nitrogen. The mixture was stirred at room temperature for $17 \mathrm{~h}$. The solvent was removed in vacuo.

The resulting mixture was washed with ether and filtered under air. The cake was washed with ether and dried in vacuo to afford $0.2530 \mathrm{~g}(0.45 \mathrm{mmol}, 90 \%$ yield $)$ of the title compound as a brown powder. Anal. Calcd for $\mathrm{C}_{29} \mathrm{H}_{35} \mathrm{Cl}_{2} \mathrm{FeN}_{3} \mathrm{O}+1 / 4 \mathrm{H}_{2} \mathrm{O}: \mathrm{C}, 60.80 ; \mathrm{H}, 6.25 ; \mathrm{N}, 7.34$; Found: $\mathrm{C}, 60.78$; $\mathrm{H}, 6.28 ; \mathrm{N}, 7.09 .{ }^{1} \mathrm{H} \mathrm{NMR}\left(400.0 \mathrm{MHz}, \mathrm{CDCl}_{3}\right.$, all peaks appear as singlets due to their broadness) $\delta, 18.9(1 \mathrm{H}) .13 .0(1 \mathrm{H}), 7.30(3 \mathrm{H}), 7.19(3 \mathrm{H}), 3.74(1.0 \mathrm{H}), 2.91(4 \mathrm{H}), 1.81-0.51(12 \mathrm{H}),-2.37(1 \mathrm{H})$, $-3.61(6 \mathrm{H})$.

\section{Procedures for Synthesis of Alkenes}

\section{Method A:}

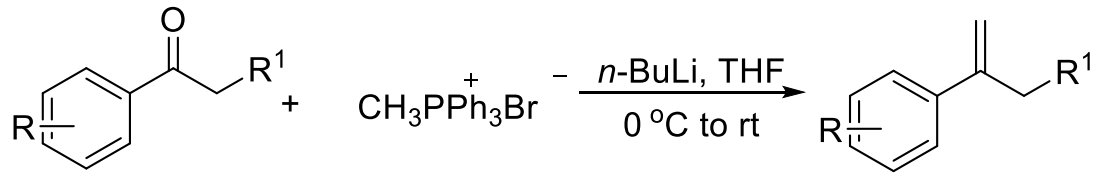

\section{Method B:}

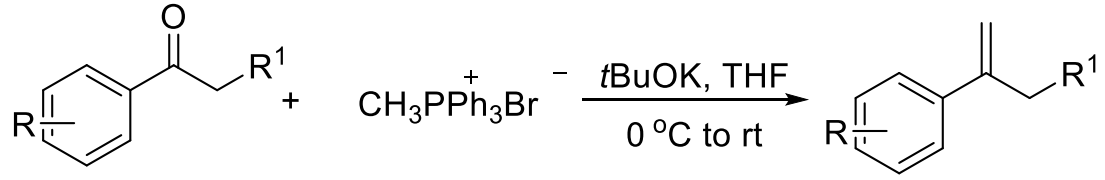

\section{Method C:}

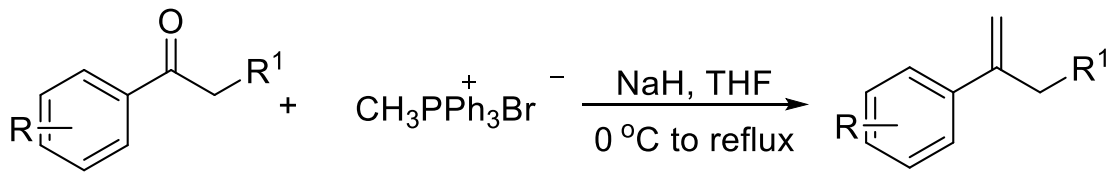

Method A: To a flame dried round bottom flask, charged with a magnetic stir bar and the corresponding methyltriphenylphosphonium bromide (1.2-1.5 equiv) was added anhydrous THF $(0.25 \mathrm{M})$, the flask was then cooled to $0{ }^{\circ} \mathrm{C}$ in an ice bath with stirring. Then, $n$-BuLi $(2.4 \mathrm{M}$ solution in hexanes, $1.20-1.50$ equiv) was added slowly over 10-15 min under stirring. After the resulting 
orange mixture was maintained at $0{ }^{\circ} \mathrm{C}$ for $0.5-1.0 \mathrm{~h}$. A solution of the aryl ketone derivative $(1.0$ equiv) in dry THF $(1.0 \mathrm{M})$ was added dropwise over $10-15$ min at $0{ }^{\circ} \mathrm{C}$. The mixture was warmed gradually to room temperature and allowed to stir overnight. The mixture was quenched with saturated aqueous ammonium chloride solution. The aqueous layer was separated and extracted with ether ( 3 times). The organic layers were combined, dried over anhydrous $\mathrm{Na}_{2} \mathrm{SO}_{4}$, filtered and concentrated in vacuo. The crude material was subsequently purified via column chromatography on silica gel to afford the desired compound as a 1,1-disubstituted alkene.

Method B: To a flame dried round bottom flask, charged with a magnetic stir bar and the corresponding methyltriphenylphosphonium bromide (1.2-1.5 equiv) was added anhydrous THF $(0.25 \mathrm{M})$, the flask was then cooled to $0{ }^{\circ} \mathrm{C}$ in an ice bath with stirring. Potassium $t$-butoxide $(1.2-1.5$ equiv), dissolved in dry THF (1 M), was then introduced dropwise via syringe. The resulting solution was stirred at $0{ }^{\circ} \mathrm{C}$ for $0.5-1.0 \mathrm{~h}$, a solution of the aryl ketone derivative (1.0 equiv) in dry THF $(1.0 \mathrm{M})$ was added dropwise over $10-15 \mathrm{~min}$ at $0{ }^{\circ} \mathrm{C}$. The mixture was warmed gradually to room temperature and allowed to stir overnight. After this time TLC analysis showed the formation of a principal product. The mixture was quenched with saturated aqueous ammonium chloride solution. The aqueous layer was separated and extracted with ether (3 times). The organic layers were combined, dried over anhydrous $\mathrm{Na}_{2} \mathrm{SO}_{4}$, filtered and concentrated in vacuo. The crude material was subsequently purified via column chromatography on silica gel to afford the desired compound as a 1,1-disubstituted alkene.

Method C: To a flame dried round bottom flask, charged with a magnetic stir bar and the corresponding methyltriphenylphosphonium bromide (1.2-1.5 equiv) was added anhydrous THF $(0.25 \mathrm{M})$, the flask was then cooled to $0{ }^{\circ} \mathrm{C}$ in an ice bath with stirring. $\mathrm{NaH}(60 \%$ dispersion in mineral oil, $1.2-1.5$ equiv) was then introduced slowly. The resulting solution was stirred at $0{ }^{\circ} \mathrm{C}$ for $0.5-1.0 \mathrm{~h}$, a solution of the aryl ketone derivative (1.0 equiv) in dry THF (1.0 M) was added dropwise over $10-15 \min$ at $0{ }^{\circ} \mathrm{C}$. The mixture was warmed gradually to RT and refluxed in oil bath for overnight. The mixture was quenched with saturated aqueous ammonium chloride solution. The aqueous layer was separated and extracted with ether (3 times). The organic layers were combined, dried over anhydrous $\mathrm{Na}_{2} \mathrm{SO}_{4}$, filtered and concentrated in vacuo. The crude material was subsequently purified via column chromatography on silica gel to afford the desired compound as 
a 1,1-disubstituted alkene.

$\mathbf{1 a},{ }^{5} \mathbf{1 d},{ }^{6} 1 \mathbf{e},{ }^{7} \mathbf{1 h},{ }^{7} 1 \mathbf{i},{ }^{7} \mathbf{1 j},{ }^{7} 1 \mathbf{k},{ }^{10} 1 \mathbf{1},{ }^{7} \mathbf{1} \mathbf{m},{ }^{7} 1 \mathbf{a c}^{11}$ were prepared according to the previously reported procedures.<smiles>C=C(CC)c1ccc(OCc2ccccc2)cc1</smiles>

1-(benzyloxy)-4-(but-1-en-2-yl)benzene (1b). According to method $\mathbf{C}$, the reaction afforded $0.8568 \mathrm{~g}$ (18\% yield) of $\mathbf{1 b}$ as a colorless oil. IR (neat): 2925, 2903, 1606, 1511, 1458, $1378 \mathrm{~cm}^{-1} ;{ }^{1} \mathrm{H}$

NMR: (399.9 MHz, $\left.\mathrm{CDCl}_{3}\right) \delta$ 7.47-7.28 (m, 7H), $6.93(\mathrm{~d}, J=8.8 \mathrm{~Hz}, 2 \mathrm{H}), 5.21(\mathrm{~s}, 1 \mathrm{H}), 5.07(\mathrm{~s}$, 2H), 4.98 (s, 1H), 2.49 (q, $J=14.4,7.2 \mathrm{~Hz}, 2 \mathrm{H}), 1.10$ (t, $J=7.6 \mathrm{~Hz}, 3 \mathrm{H}) ;{ }^{13} \mathrm{C}$ NMR: (100.0 MHz, $\left.\mathrm{CDCl}_{3}\right) \delta 158.2,149.3,137.0,134.2,133.8,128.6,127.5,127.1,114.5,109.5,70.0,28.0,13.0$; HRMS (EI) calculated for $\left[\mathrm{C}_{17} \mathrm{H}_{18} \mathrm{O}\right]^{+}$requires $\mathrm{m} / \mathrm{z} 238.1358$, found $\mathrm{m} / \mathrm{z} 238.1358$.<smiles>C=C(CC)c1ccc(OC(C)(C)C)cc1</smiles>

(4-(but-1-en-2-yl)phenoxy)(tert-butyl)dimethylsilane (1c). According to method $\mathbf{C}$, the reaction afforded $2.7676 \mathrm{~g}$ ( $81 \%$ yield) of $\mathbf{1 c}$ as a colorless oil. IR (neat): 2962, 2861, 1604, 1509, 1468, $1260 \mathrm{~cm}^{-1} ;{ }^{1} \mathrm{H}$ NMR: (400.0 MHz, $\left.\mathrm{CDCl}_{3}\right) \delta 7.29(\mathrm{~d}, J=8.8 \mathrm{~Hz}, 2 \mathrm{H}), 6.79(\mathrm{~d}, J=8.8 \mathrm{~Hz}, 2 \mathrm{H}), 5.21(\mathrm{~s}, 1 \mathrm{H}), 4.97$ (s, 1H), 2.52-2.44 (m, 2H), 1.10 (t, $J=7.2 \mathrm{~Hz}, 3 \mathrm{H}), 0.98$ (s, 9H), 0.20 (s, 6H); ${ }^{13} \mathrm{C}$ NMR: $(100.0$ $\left.\mathrm{MHz}, \mathrm{CDCl}_{3}\right) \delta 155.1,149.4,134.4,126.9,119.7,109.3,28.0,25.7,18.2,13.0,-4.4$; HRMS (EI) calculated for $\left[\mathrm{C}_{16} \mathrm{H}_{26} \mathrm{OSi}\right]^{+}$requires $\mathrm{m} / \mathrm{z} 262.1753$, found $\mathrm{m} / \mathrm{z} 262.1755$.<smiles>C=C(CC)c1ccc(SC)cc1</smiles>

(4-(but-1-en-2-yl)phenyl)(methyl)sulfane (1f). According to method C, the reaction afforded $1.7655 \mathrm{~g}(83 \%$ yield) of $\mathbf{1 f}$ as a colorless oil. IR (neat): 2969, 2921, 1625, 1595, 1494, $1436 \mathrm{~cm}^{-1},{ }^{1} \mathrm{H}$ NMR: (400.0 MHz, $\mathrm{CDCl}_{3}$ ) $\delta 7.35(\mathrm{~d}, J=8.4 \mathrm{~Hz}, 2 \mathrm{H}), 7.22(\mathrm{~d}, J=8.4 \mathrm{~Hz}, 2 \mathrm{H}), 5.26(\mathrm{~s}, 1 \mathrm{H}), 5.03(\mathrm{~s}, 1 \mathrm{H}), 2.54-2.42(\mathrm{~m}, 5 \mathrm{H})$, $1.10(\mathrm{~m}, J=7.6 \mathrm{~Hz}, 3 \mathrm{H}) ;{ }^{13} \mathrm{C} \mathrm{NMR}:\left(100.0 \mathrm{MHz}, \mathrm{CDCl}_{3}\right) \delta 149.2,138.3,137.3,126.5,126.4,110.5$, 27.9, 15.9, 12.9; HRMS (EI) calculated for $\left[\mathrm{C}_{11} \mathrm{H}_{14} \mathrm{~S}\right]^{+}$requires $\mathrm{m} / \mathrm{z}$ 178.0816, found m/z 178.0814.

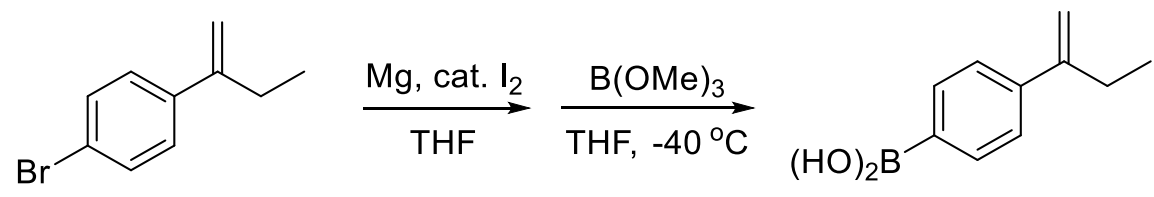<smiles>C=C(CC)c1ccc(B(O)O)cc1</smiles> 


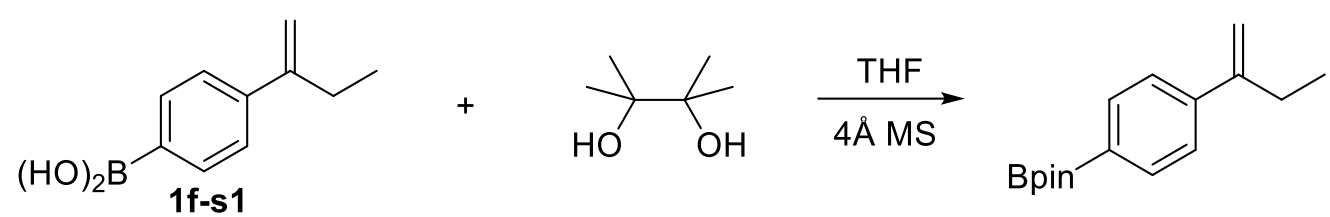<smiles>C=C(CC)c1ccc(Cc2ccccc2)cc1</smiles>

2-(4-(but-1-en-2-yl)phenyl)-4,4,5,5-tetramethyl-1,3,2-dioxaborolane (1g). According to the previous method, ${ }^{9}$ the reaction afforded $1.5239 \mathrm{~g}$ (49\% yield) of $\mathbf{1 g}$ as a colorless oil. IR (neat): 2977, 1609, 1514, 1398, $1359 \mathrm{~cm}^{-1} ;{ }^{1} \mathrm{H}$ NMR: $\left(400.0 \mathrm{MHz}, \mathrm{CDCl}_{3}\right) \delta 7.77(\mathrm{~d}, J=8.0 \mathrm{~Hz}, 2 \mathrm{H}), 7.42(\mathrm{~d}, J=7.6 \mathrm{~Hz}, 2 \mathrm{H}), 5.31$ (s, 1H), 5.09 (s, 1H), 2.52 (q, $J=15.2,7.6 \mathrm{~Hz}, 2 \mathrm{H}), 1.34(\mathrm{~s}, 12 \mathrm{H}), 1.09$ (t, $J=7.6 \mathrm{~Hz}, 3 \mathrm{H}) ;{ }^{13} \mathrm{C}$ NMR: (100.0 MHz, $\left.\mathrm{CDCl}_{3}\right) \delta 150.0,144.3,134.8,125.3,111.6,83.7,27.9,24.8,12.9$; HRMS (EI) calculated for $\left[\mathrm{C}_{16} \mathrm{H}_{23} \mathrm{O}_{2} \mathrm{~B}\right]^{+}$requires $\mathrm{m} / \mathrm{z} 258.1791$, found $\mathrm{m} / \mathrm{z} 258.1789$.<smiles>C=C(CC)c1cccc(OC(C)(C)C)c1</smiles>

According to method $\mathbf{C}$, the reaction afforded $1.9532 \mathrm{~g}$ ( $74 \%$ yield) of $1 \mathbf{m}$ as a colorless oil. IR (neat): 2961, 2859, 1598, 1576, 1484, $1429 \mathrm{~cm}^{-}$ ${ }^{1}$; ${ }^{1} \mathrm{H}$ NMR: $\left(400.0 \mathrm{MHz}, \mathrm{CDCl}_{3}\right) \delta 7.17(\mathrm{t}, J=8.0 \mathrm{~Hz}, 1 \mathrm{H}), 7.01(\mathrm{~d}, J=7.2 \mathrm{~Hz}, 1 \mathrm{H}), 6.88(\mathrm{~s}, 1 \mathrm{H})$, 6.77-6.72 (m, 1H), $5.25(\mathrm{~s}, 1 \mathrm{H}), 5.03(\mathrm{~s}, 1 \mathrm{H}), 2.48(\mathrm{q}, J=14.4,6.8 \mathrm{~Hz}, 2 \mathrm{H}), 1.10(\mathrm{t}, J=7.6 \mathrm{~Hz}$, 3H), 0.99 (s, 9H), 0.20 (s, 6H); ${ }^{13} \mathrm{C}$ NMR: (100.6 MHz, $\left.\mathrm{CDCl}_{3}\right) \delta$ 155.5, 149.8, 143.0, 129.0, 119.1, $118.9,117.9,110.9,28.1,25.7,18.2,12.9,-4.4$; HRMS (EI) calculated for $\left[\mathrm{C}_{16} \mathrm{H}_{26} \mathrm{OSi}\right]^{+}$requires $\mathrm{m} / \mathrm{z} 262.1753$, found $\mathrm{m} / \mathrm{z} 262.1752$.<smiles>C=C(CC)c1ccccc1OC</smiles>

1-(but-1-en-2-yl)-2-methoxybenzene (1n). According to method C, the reaction afforded $1.9458 \mathrm{~g}$ (57\% yield) of $\mathbf{1} \mathbf{n}$ as a colorless oil. IR (neat): 2967, 1597, 1490, 1461, 1435, $1242 \mathrm{~cm}^{-1} ;{ }^{1} \mathrm{H}$ NMR: (400.0 MHz, $\left.\mathrm{CDCl}_{3}\right) \delta$ 7.28-7.21 (m, $\left.1 \mathrm{H}\right), 7.13$ (d, $J=6.0 \mathrm{~Hz}, 1 \mathrm{H}), 6.95-6.84(\mathrm{~m}, 2 \mathrm{H}), 5.14$ (s, 1H), 4.99 (s, 1H), 3.81 (s, 3H), 2.47 (q, $J=14.8$, $7.6 \mathrm{~Hz}, 2 \mathrm{H}), 1.00(\mathrm{t}, J=7.6 \mathrm{~Hz}, 3 \mathrm{H}) ;{ }^{13} \mathrm{C}$ NMR: $\left(100.6 \mathrm{MHz}, \mathrm{CDCl}_{3}\right) \delta 156.5,150.7,132.4,130.1$, 128.2, 120.4, 112.7, 110.6, 55.4, 29.1, 12.7; HRMS (EI) calculated for $\left[\mathrm{C}_{11} \mathrm{H}_{14} \mathrm{O}\right]^{+}$requires $\mathrm{m} / \mathrm{z}$ 162.1045 , found $\mathrm{m} / \mathrm{z} 162.1045$.<smiles>C=C(CC)c1ccc2cc(OC)ccc2c1</smiles>

2-(but-1-en-2-yl)-6-methoxynaphthalene (10). According to method C, the reaction afforded $4.2115 \mathrm{~g}$ (99\% yield) of $\mathbf{1 0}$ as a colorless oil. IR (neat): 2962, 1627, 1600, 1485, 1459, $1391 \mathrm{~cm}^{-1} ;{ }^{1} \mathrm{H}$ NMR: (400.0 MHz, $\left.\mathrm{CDCl}_{3}\right)$ 8 7.79-7.54 (m, 4H), 7.16-7.10 (m, 2H), $5.39(\mathrm{~s}, 1 \mathrm{H}), 5.12(\mathrm{~s}, 1 \mathrm{H}), 3.92$ (s, 3H), $2.62(\mathrm{q}, J=14.0,7.6 \mathrm{~Hz}, 2 \mathrm{H}), 1.15(\mathrm{t}, J=7.6 \mathrm{~Hz}, 3 \mathrm{H}) ;{ }^{13} \mathrm{C} \mathrm{NMR}:\left(100.0 \mathrm{MHz}, \mathrm{CDCl}_{3}\right) \delta$ 
157.6, 149.7, 136.4, 133.9, 129.6, 128.8, 126.6, 125.0, 124.3, 118.8, 110.7, 105.5, 55.2, 28.0, 13.1; HRMS (EI) calculated for $\left[\mathrm{C}_{15} \mathrm{H}_{16} \mathrm{O}\right]^{+}$requires $\mathrm{m} / \mathrm{z} 212.1201$, found $\mathrm{m} / \mathrm{z} 212.1199$.<smiles>C=C(CC)c1ccc2c(ccn2C)c1</smiles>

5-(but-1-en-2-yl)-1-methyl-1H-indole (1p). According to method C, the reaction afforded $0.5059 \mathrm{~g}$ ( $23 \%$ yield) of $\mathbf{1 p}$ as a colorless oil. IR (neat): 3076, 2968, 1622, 1514, 1489, $1369 \mathrm{~cm}^{-1} ;{ }^{1} \mathrm{H}$ NMR: $\left(400.0 \mathrm{MHz}, \mathrm{CDCl}_{3}\right) \delta$ $7.66(\mathrm{~s}, 1 \mathrm{H}), 7.37-7.23(\mathrm{~m}, 2 \mathrm{H}), 7.02(\mathrm{~d}, J=3.2 \mathrm{~Hz}, 1 \mathrm{H}), 6.46(\mathrm{~d}, J=2.8 \mathrm{~Hz}, 1 \mathrm{H}), 5.26(\mathrm{~s}, 1 \mathrm{H})$, $5.01(\mathrm{~s}, 1 \mathrm{H}), 3.77(\mathrm{~s}, 3 \mathrm{H}), 2.60(\mathrm{q}, J=14.8,7.6 \mathrm{~Hz}, 2 \mathrm{H}), 1.23(\mathrm{t}, J=14.8,7.2 \mathrm{~Hz}, 3 \mathrm{H}) ;{ }^{13} \mathrm{C}$ NMR: $\left(100.0 \mathrm{MHz}, \mathrm{CDCl}_{3}\right) \delta 151.0,136.2,132.9,129.1,128.4,120.3,118.2,109.3,108.8,101.2,32.8$, 28.7, 13.2; HRMS (EI) calculated for $\left[\mathrm{C}_{13} \mathrm{H}_{15} \mathrm{~N}\right]^{+}$requires $\mathrm{m} / \mathrm{z}$ 185.1204, found $\mathrm{m} / \mathrm{z} 185.1203$.<smiles>C=C(CC)c1ccc(OC)nc1</smiles>

5-(but-1-en-2-yl)-2-methoxypyridine (1q). According to method $\mathrm{C}$, the reaction afforded $3.5827 \mathrm{~g}$ ( $74 \%$ yield) of 1q as a colorless oil. IR (neat): 2970, 1601, 1495, 1370, 1287, $1027 \mathrm{~cm}^{-1}$; ${ }^{1} \mathrm{H}$ NMR: (400.0 MHz, $\mathrm{CDCl}_{3}$ ) $\delta 8.22(\mathrm{~d}, J=2.8 \mathrm{~Hz}, 1 \mathrm{H}), 7.63(\mathrm{dd}, J=8.4,2.4 \mathrm{~Hz}, 1 \mathrm{H}), 6.71(\mathrm{~d}, J=8.4 \mathrm{~Hz}, 1 \mathrm{H}), 5.22(\mathrm{~s}, 1 \mathrm{H})$, 5.03 (s, 1H), 3.94 (s, 3H), 2.47 (q, $J=15.2,8.0 \mathrm{~Hz}, 2 \mathrm{H}), 1.10(\mathrm{t}, J=7.2 \mathrm{~Hz}, 3 \mathrm{H}) ;{ }^{13} \mathrm{C}$ NMR: $(100.0$ $\left.\mathrm{MHz}, \mathrm{CDCl}_{3}\right) \delta 163.5,146.6,144.1,136.4,130.1,110.5,110.2,53.4,27.9,12.8$; HRMS (EI) calculated for $\left[\mathrm{C}_{10} \mathrm{H}_{13} \mathrm{NO}\right]^{+}$requires $\mathrm{m} / \mathrm{z}$ 163.0997, found $\mathrm{m} / \mathrm{z} 163.0997$.<smiles>C=C(C(=C)C(C)(C)C)c1ccc(OC)cc1</smiles>

1-methoxy-4-(pent-1-en-2-yl)benzene (1r). According to method C, the reaction afforded $2.2054 \mathrm{~g}$ (85\% yield) of $1 \mathrm{r}$ as a colorless oil. IR (neat): 2958, 1609, 1512, 1460, 1285, $1248 \mathrm{~cm}^{-1}$; ${ }^{1} \mathrm{H} \mathrm{NMR}:\left(399.9 \mathrm{MHz}, \mathrm{CDCl}_{3}\right.$ ) $\delta 7.35(\mathrm{~d}, J=8.8 \mathrm{~Hz}, 2 \mathrm{H}), 6.86(\mathrm{~d}, J=8.4 \mathrm{~Hz}, 2 \mathrm{H}), 5.20(\mathrm{~s}, 1 \mathrm{H}), 4.97(\mathrm{~s}, 1 \mathrm{H}), 3.81(\mathrm{~s}, 3 \mathrm{H}), 2.45(\mathrm{t}$, $J=7.6 \mathrm{~Hz}, 2 \mathrm{H}), 1.53-1.40(\mathrm{~m}, 2 \mathrm{H}), 0.91(\mathrm{t}, 3 \mathrm{H}) ;{ }^{13} \mathrm{C} \mathrm{NMR}:\left(100.0 \mathrm{MHz}, \mathrm{CDCl}_{3}\right) \delta 158.9,147.7$, 133.8, 127.1, 113.5, 110.6, 55.2, 37.5, 21.4, 13.7; HRMS (EI) calculated for $\left[\mathrm{C}_{12} \mathrm{H}_{16} \mathrm{O}\right]^{+}$requires $\mathrm{m} / \mathrm{z}$ 176.1201, found m/z 176.1203.<smiles>C=C(c1ccc(OC)cc1)C(C)(C)C</smiles>

1-(hex-1-en-2-yl)-4-methoxybenzene (1s). According to method C, the reaction afforded $1.8003 \mathrm{~g}$ (71\% yield) of $1 \mathrm{~s}$ as a colorless oil. IR (neat): 2957, 1609, 1512, 1461, 1284, $1248 \mathrm{~cm}^{-1}$; ${ }^{1} \mathrm{H}$ NMR: (399.9 MHz, $\mathrm{CDCl}_{3}$ ) $\delta 7.35(\mathrm{~d}, J=8.8 \mathrm{~Hz}, 2 \mathrm{H}), 6.86(\mathrm{~d}, J=8.4 \mathrm{~Hz}, 2 \mathrm{H}), 5.19(\mathrm{~s}, 1 \mathrm{H}), 4.97(\mathrm{~s}, 1 \mathrm{H}), 3.81(\mathrm{~s}, 3 \mathrm{H}), 2.47$ (s, 2H), 1.48-1.28 (m, 4H), 0.89 (t, $J=7.6 \mathrm{~Hz}, 3 \mathrm{H}) ;{ }^{13} \mathrm{C} \mathrm{NMR}:\left(100.0 \mathrm{MHz}, \mathrm{CDCl}_{3}\right) \delta 158.9,148.0$, 133.8, 127.1, 113.5, 110.5, 55.2, 35.1, 30.5, 22.4, 13.9; HRMS (EI) calculated for $\left[\mathrm{C}_{13} \mathrm{H}_{18} \mathrm{O}\right]^{+}$ 
requires $\mathrm{m} / \mathrm{z}$ 190.1358, found $\mathrm{m} / \mathrm{z} 190.1357$.<smiles>C=C(c1ccccc1)C(C)(C)C(C)(C)C</smiles>

hept-1-en-2-ylbenzene (1t). According to method C, the reaction afforded 0.86 $\mathrm{g}(37 \%$ yield) of $\mathbf{1 t}$ as a colorless oil. IR (neat): 2958, 2925, 1627, 1525, 1463, $1378 \mathrm{~cm}^{-1}$; ${ }^{1} \mathrm{H}$ NMR: $\left(400.0 \mathrm{MHz}, \mathrm{CDCl}_{3}\right) \delta$ 7.43-7.23 (m, 5H), $5.25(\mathrm{~s}, 1 \mathrm{H}), 5.05$ (s, 1H), $2.49(\mathrm{t}, J=7.2 \mathrm{~Hz}, 2 \mathrm{H}), 1.50-1.23(\mathrm{~m}, 6 \mathrm{H}), 0.92-0.83(\mathrm{~m}, 3 \mathrm{H}) ;{ }^{13} \mathrm{C}$ NMR: (100.6 MHz, $\left.\mathrm{CDCl}_{3}\right) \delta 148.8,141.5,128.2,127.2,126.1,112.0,35.3,31.6,27.9,22.5,14.0$; HRMS (EI) calculated for $\left[\mathrm{C}_{13} \mathrm{H}_{18}\right]^{+}$requires m/z 174.1409, found m/z 174.1408.<smiles>C=C(c1ccc(OC)cc1)C(C)(C)C</smiles>

1-(hept-1-en-2-yl)-4-methoxybenzene (1u). According to method C, the reaction afforded $1.3359 \mathrm{~g}$ (58\% yield) of $1 \mathbf{u}$ as a colorless oil. IR (neat): 2932, 1609, 1512, 1462, 1285, $1248 \mathrm{~cm}^{-1} ;{ }^{1} \mathrm{H}$ NMR: $\left(400.0 \mathrm{MHz}, \mathrm{CDCl}_{3}\right)$ $\delta 7.35(\mathrm{~d}, J=8.4 \mathrm{~Hz}, 2 \mathrm{H}), 6.86(\mathrm{~d}, J=8.8 \mathrm{~Hz}, 2 \mathrm{H}), 5.19(\mathrm{~s}, 1 \mathrm{H}), 4.96(\mathrm{~s}, 1 \mathrm{H}), 3.81(\mathrm{~s}, 3 \mathrm{H}), 2.46(\mathrm{t}$, $J=7.6 \mathrm{~Hz}, 2 \mathrm{H}), 1.50-1.39(\mathrm{~m}, 2 \mathrm{H}), 1.36-1.24(\mathrm{~m}, 4 \mathrm{H}), 0.87(\mathrm{t}, J=6.8 \mathrm{~Hz}, 3 \mathrm{H}) ;{ }^{13} \mathrm{C}$ NMR: $(100.0$ $\left.\mathrm{MHz}, \mathrm{CDCl}_{3}\right) \delta 158.9,148.0,133.8,127.1,113.5,110.4,55.2,35.4,31.6,28.0,22.5,14.1 ;$ HRMS (EI) calculated for $\left[\mathrm{C}_{12} \mathrm{H}_{20} \mathrm{O}\right]^{+}$requires $\mathrm{m} / \mathrm{z} 204.1514$, found $\mathrm{m} / \mathrm{z} 204.1515$.<smiles>C=C(c1ccc(OC)cc1)C(C)(C)C</smiles>

1-methoxy-4-(oct-1-en-2-yl)benzene (1v). According to method C, the reaction afforded $1.7770 \mathrm{~g}(58 \%$ yield $)$ of $\mathbf{1 v}$ as a colorless oil. IR (neat): 2929, 1609, 1512, 1462, 1285, $1248 \mathrm{~cm}^{-1} ;{ }^{1} \mathrm{H}$ NMR: $\left(400.0 \mathrm{MHz}, \mathrm{CDCl}_{3}\right)$ $\delta 7.35(\mathrm{~d}, J=8.8 \mathrm{~Hz}, 2 \mathrm{H}), 6.86(\mathrm{~d}, J=8.8 \mathrm{~Hz}, 2 \mathrm{H}), 5.19(\mathrm{~s}, 1 \mathrm{H}), 4.96(\mathrm{~s}, 1 \mathrm{H}), 3.81(\mathrm{~s}, 3 \mathrm{H}), 2.46(\mathrm{t}$, $J=7.6 \mathrm{~Hz}, 2 \mathrm{H}), 1.49-1.38(\mathrm{~m}, 2 \mathrm{H}), 1.37-1.21(\mathrm{~m}, 6 \mathrm{H}), 0.87(\mathrm{t}, J=6.4 \mathrm{~Hz}, 3 \mathrm{H}) ;{ }^{13} \mathrm{C}$ NMR: $(100.0$ $\left.\mathrm{MHz}, \mathrm{CDCl}_{3}\right) \delta 158.9,148.0,133.8,127.1,113.5,110.4,55.2,35.4,31.7,29.0,28.3,22.6,14.1$; HRMS (EI) calculated for $\left[\mathrm{C}_{15} \mathrm{H}_{22} \mathrm{O}\right]^{+}$requires $\mathrm{m} / \mathrm{z} 218.1671$, found $\mathrm{m} / \mathrm{z} 218.1672$.<smiles>C=C(c1ccc(OC(C)(C)C)cc1)C(C)(C)C</smiles>

tert-butyldimethyl(4-(oct-1-en-2-yl)phenoxy)silane (1w). According to method $\mathbf{C}$, the reaction afforded $3.3288 \mathrm{~g}$ ( $75 \%$ yield) of $\mathbf{1 w}$ as a colorless oil. IR (neat): 2928, 2857, 1604, 1509, 1465, $1255 \mathrm{~cm}^{-1} ;{ }^{1} \mathrm{H}$ NMR: $\left(400.0 \mathrm{MHz}, \mathrm{CDCl}_{3}\right) \delta 7.28(\mathrm{~d}, J=8.8 \mathrm{~Hz}, 2 \mathrm{H}), 6.78(\mathrm{~d}, J=8.8 \mathrm{~Hz}, 2 \mathrm{H}), 5.19(\mathrm{~s}, 1 \mathrm{H}), 4.96$ (s, 1H), $2.45(\mathrm{t}, J=6.8 \mathrm{~Hz}, 2 \mathrm{H}), 1.49-1.39(\mathrm{~m}, 2 \mathrm{H}), 1.37-1.22(\mathrm{~m}, 6 \mathrm{H}), 0.98(\mathrm{~s}, 9 \mathrm{H}), 0.87(\mathrm{t}, J=6.8$ $\mathrm{Hz}, 3 \mathrm{H}), 0.20(\mathrm{~s}, 6 \mathrm{H}) ;{ }^{13} \mathrm{C} \mathrm{NMR}:\left(100.0 \mathrm{MHz}, \mathrm{CDCl}_{3}\right) \delta 155.0,148.1,134.4,127.0,119.7,110.4$, 35.4, 31.7, 29.0, 28.3, 25.7, 22.6, 18.2, 14.1, -4.4; HRMS (EI) calculated for $\left[\mathrm{C}_{20} \mathrm{H}_{34} \mathrm{SiO}\right]^{+}$requires $\mathrm{m} / \mathrm{z} 318.2379$, found $\mathrm{m} / \mathrm{z} 318.2378$. 
<smiles>C=C(CC(C)C)c1ccc(OC)cc1</smiles>

methoxy-4-(4-methylpent-1-en-2-yl)benzene (1x). According to method $\mathbf{C}$, the reaction afforded $2.4066 \mathrm{~g}(66 \%$ yield $)$ of $\mathbf{1 x}$ as a colorless oil. IR (neat): 2954, 2867, 1608, 1511, $1462 \mathrm{~cm}^{-1} ;{ }^{1} \mathrm{H}$ NMR: $\left(400.0 \mathrm{MHz}, \mathrm{CDCl}_{3}\right) \delta 7.33(\mathrm{~d}, J=8.4 \mathrm{~Hz}, 2 \mathrm{H}), 6.85(\mathrm{~d}, J=8.8 \mathrm{~Hz}, 2 \mathrm{H}), 5.19(\mathrm{~s}, 1 \mathrm{H}), 4.94(\mathrm{~s}, 1 \mathrm{H})$, $3.81(\mathrm{~s}, 3 \mathrm{H}), 2.35(\mathrm{~d}, J=7.2 \mathrm{~Hz}, 2 \mathrm{H}), 1.74-1.60(\mathrm{~m}, 1 \mathrm{H}), 0.87(\mathrm{~d}, J=6.4 \mathrm{~Hz}, 6 \mathrm{H}) ;{ }^{13} \mathrm{C}$ NMR: $(100.0$ $\left.\mathrm{MHz}, \mathrm{CDCl}_{3}\right) \delta 158.9,147.1,133.8,127.3,113.5,111.9,55.2,45.2,26.4,22.4 ; \mathrm{HRMS}$ (EI) calculated for $\left[\mathrm{C}_{13} \mathrm{H}_{18} \mathrm{O}\right]^{+}$requires m/z 190.1358, found $\mathrm{m} / \mathrm{z} 190.1358$.<smiles>C=C(CCc1ccccc1)c1ccccc1</smiles>

but-3-ene-1,3-diyldibenzene (1y). According to method $B$, the reaction afforded $1.3054 \mathrm{~g}$ ( $66 \%$ yield) of $\mathbf{1 y}$ as a colorless oil. IR (neat): 2925, 2861, 1628, 1601, 1494, $1453 \mathrm{~cm}^{-1}$; ${ }^{1} \mathrm{H}$ NMR: (400.0 MHz, $\left.\mathrm{CDCl}_{3}\right)$ $\delta$ 7.50-7.12 (m, 10H), $5.29(\mathrm{~s}, 1 \mathrm{H}), 5.06(\mathrm{~s}, 1 \mathrm{H}), 2.87-2.71(\mathrm{~m}, 4 \mathrm{H}) ;{ }^{13} \mathrm{C} \mathrm{NMR}:\left(100.0 \mathrm{MHz}, \mathrm{CDCl}_{3}\right)$ $\delta 147.8,141.9,141.0,128.4,128.3,128.3,127.4,126.1,125.8,112.7,37.3,34.7$; HRMS (EI) calculated for $\left[\mathrm{C}_{16} \mathrm{H}_{16}\right]^{+}$requires $\mathrm{m} / \mathrm{z}$ 208.1252, found $\mathrm{m} / \mathrm{z} 208.1254$.<smiles>C=C(C)N(C)C</smiles>

$N, N$-dimethyl-4-(p-tolyl)pent-4-en-1-amine (1z). According to method $\mathrm{C}$, the reaction afforded $0.5846 \mathrm{~g}$ ( $57 \%$ yield) of $1 \mathrm{z}$ as a colorless oil. IR (neat): 2942, 2859, 1626, 1513, $1459 \mathrm{~cm}^{-1} ;{ }^{1} \mathrm{H}$ NMR: (400.0 MHz, $\left.\mathrm{CDCl}_{3}\right) \delta 7.31(\mathrm{~d}, J=8.0 \mathrm{~Hz}, 2 \mathrm{H}), 7.12(\mathrm{~d}, J=8.0 \mathrm{~Hz}, 2 \mathrm{H}), 5.25(\mathrm{~s}, 1 \mathrm{H}), 5.03(\mathrm{~s}, 1 \mathrm{H}), 2.50(\mathrm{t}, J=$ 7.6 Hz, 2H), $2.34(\mathrm{~s}, 3 \mathrm{H}), 2.28(\mathrm{t}, J=7.6 \mathrm{~Hz}, 2 \mathrm{H}), 2.19(\mathrm{~s}, 6 \mathrm{H}), 1.66-1.57(\mathrm{~m}, 2 \mathrm{H}),{ }^{13} \mathrm{C}$ NMR: $(100.0$ $\left.\mathrm{MHz}, \mathrm{CDCl}_{3}\right) \delta 148.0,138.2,136.9,128.8,125.9,111.4,59.3,45.4,33.0,26.3,21.0 ;$ HRMS (EI) calculated for $\left[\mathrm{C}_{14} \mathrm{H}_{21} \mathrm{~N}\right]^{+}$requires $\mathrm{m} / \mathrm{z}$ 203.1674, found $\mathrm{m} / \mathrm{z} 2203.1675$.<smiles>C=C(CCC1OCCO1)c1ccc(C)cc1</smiles>

2-(3-(p-tolyl)but-3-en-1-yl)-1,3-dioxolane (1aa). According to method B, the reaction afforded $2.4344 \mathrm{~g}$ (89\% yield) of $\mathbf{1 a a}$ as a colorless oil. IR (neat): 2956, 2924, 2878, 1626, 1513, $1456 \mathrm{~cm}^{-1} ;{ }^{1} \mathrm{H}$ NMR: $\left(400.0 \mathrm{MHz}, \mathrm{CDCl}_{3}\right) \delta 7.32(\mathrm{~d}, J=8.0 \mathrm{~Hz}, 2 \mathrm{H}), 7.13(\mathrm{~d}, J=7.6 \mathrm{~Hz}, 2 \mathrm{H}), 5.27(\mathrm{~s}, 1 \mathrm{H}), 5.05$ (s, 1H), $4.92(\mathrm{t}, J=4.8 \mathrm{~Hz}, 1 \mathrm{H}), 4.03-3.81(\mathrm{~m}, 4 \mathrm{H}), 2.62(\mathrm{t}, J=8.0 \mathrm{~Hz}, 2 \mathrm{H}), 2.34(\mathrm{~s}, 3 \mathrm{H}), 1.87-1.79$ (m, 2H); ${ }^{13} \mathrm{C}$ NMR: $\left(100.0 \mathrm{MHz}, \mathrm{CDCl}_{3}\right) \delta$ 147.3, 137.9, 137.1, 128.9, 125.9, 111.5, 104.1, 64.9, 32.5, 29.4, 21.0; HRMS (EI) calculated for $\left[\mathrm{C}_{14} \mathrm{H}_{18} \mathrm{O}_{2}\right]^{+}$requires $\mathrm{m} / \mathrm{z} 218.1307$, found $\mathrm{m} / \mathrm{z} 218.1306$. 
<smiles>C=C(CCC1OCCO1)c1ccc(C)c(OC)c1</smiles>

(1ab). According to method B, the reaction afforded $1.9860 \mathrm{~g}(71 \%$ yield) of 1ab as a colorless oil. IR (neat): 2954, 2924, 1610, 1573, 1509, $1463 \mathrm{~cm}^{-1}$; ${ }^{1} \mathrm{H}$ NMR: $\left(400.0 \mathrm{MHz}, \mathrm{CDCl}_{3}\right) \delta 7.08(\mathrm{~d}, J=7.6 \mathrm{~Hz}, 1 \mathrm{H}), 6.92(\mathrm{~d}, J=8.0 \mathrm{~Hz}$, 1H), $6.88(\mathrm{~s}, 1 \mathrm{H}), 5.28(\mathrm{~s}, 1 \mathrm{H}), 5.06(\mathrm{~s}, 1 \mathrm{H}), 4.92(\mathrm{t}, J=4.4 \mathrm{~Hz}, 1 \mathrm{H}), 4.01-3.81(\mathrm{~m}, 7 \mathrm{H}), 2.62(\mathrm{t}, J$ $=8.0 \mathrm{~Hz}, 2 \mathrm{H}), 2.21(\mathrm{~s}, 3 \mathrm{H}), 1.88-1.80(\mathrm{~m}, 2 \mathrm{H}) ;{ }^{13} \mathrm{C} \mathrm{NMR}:\left(100.0 \mathrm{MHz}, \mathrm{CDCl}_{3}\right) \delta$ 157.5, 147.7, $139.8,130.3,125.9,117.9,111.6,107.8,104.0,64.8,55.2,32.6,29.5,15.9$; HRMS (EI) calculated for $\left[\mathrm{C}_{15} \mathrm{H}_{20} \mathrm{O}_{3}\right]^{+}$requires $\mathrm{m} / \mathrm{z} 248.1412$, found $\mathrm{m} / \mathrm{z} 248.1411$.<smiles>N#Cc1ccc(F)cc1</smiles>

$\mathrm{RO}$<smiles>CCC(=O)c1ccc(C)cc1</smiles>

s2

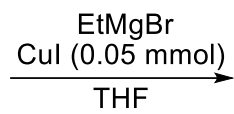

1<smiles>CCC(=O)c1ccc(Oc2ccccc2)cc1</smiles>

\section{4-(((1R,2S,5R)-2-isopropyl-5-methylcyclohexyl)oxy)benzonitrile}

(1ad-s1). According to the previous method, ${ }^{12}$ the reaction afforded $2.3114 \mathrm{~g}(90 \%$ yield) of $\mathbf{1 a d}-\mathbf{s} 1$ as a white solid. IR (neat): 2955, 2927, 2869, 2224, 1604, $1504 \mathrm{~cm}^{-1}$; ${ }^{1} \mathrm{H}$ NMR: (400.0 MHz, $\left.\mathrm{CDCl}_{3}\right) \delta 7.56(\mathrm{~d}$, $J=8.8 \mathrm{~Hz}, 2 \mathrm{H}), 6.93(\mathrm{~d}, J=8.8 \mathrm{~Hz}, 2 \mathrm{H}), 4.15-4.07(\mathrm{~m}, 1 \mathrm{H}), 2.15-2.06(\mathrm{~m}, 2 \mathrm{H}), 1.78-1.70(\mathrm{~m}, 2 \mathrm{H})$, 1.59-1.42 (m, 2H), 1.17-0.99 (m, 2H), 0.99-0.88 (m, 7H), $0.74(\mathrm{~d}, J=7.2 \mathrm{~Hz}, 3 \mathrm{H}) ;{ }^{13} \mathrm{C}$ NMR: $(100.0$ $\left.\mathrm{MHz}, \mathrm{CDCl}_{3}\right) \delta 161.8,134.0,119.4,115.8,103.2,77.8,47.8,39.8,34.3,31.3,26.1,23.7,22.0,20.6$, 16.6; HRMS(ESI) calculated for $\left[\mathrm{C}_{17} \mathrm{H}_{24} \mathrm{NO}\right]^{+}\left(\mathrm{M}+\mathrm{H}^{+}\right)$, requires $\mathrm{m} / \mathrm{z} 258.1858$, found $\mathrm{m} / \mathrm{z} 258.1854$.<smiles>C=C(CC)c1ccc(O[C@H]2C[C@H](C)CCC2C(C)C)cc1</smiles>

1-(but-1-en-2-yl)-4-(((1S,2R,5S)-2-isopropyl-5methylcyclohexyl)oxy)benzene (1ad). According to method $\mathbf{B}$, the reaction afforded $1.0589 \mathrm{~g}(60 \%$ yield $)$ of $\mathbf{1} \mathbf{a d}$ as a colorless oil. IR (neat): 2955, 2869, 1606, 1508, $1278 \mathrm{~cm}^{-1}$; ${ }^{1} \mathrm{H}$ NMR: $(400.0 \mathrm{MHz}$, $\left.\mathrm{CDCl}_{3}\right) \delta 7.34(\mathrm{~d}, J=8.8 \mathrm{~Hz}, 2 \mathrm{H}), 6.85(\mathrm{~d}, J=8.8 \mathrm{~Hz}, 2 \mathrm{H}), 5.20(\mathrm{~s}, 1 \mathrm{H}), 4.96(\mathrm{~s}, 1 \mathrm{H}), 4.03(\mathrm{dt}, J=$ 10.6, $4.4 \mathrm{~Hz}, 1 \mathrm{H}), 2.48$ (q, $J=14.8,7.2 \mathrm{~Hz}, 2 \mathrm{H}), 2.26-2.13(\mathrm{~m}, 2 \mathrm{H}), 1.76-1.68(\mathrm{~m}, 2 \mathrm{H}), 1.54-1.39$ (m, 2H), 1.14-0.87 (m, 12H), $0.77(\mathrm{~d}, J=6.8 \mathrm{~Hz}, 3 \mathrm{H}) ;{ }^{13} \mathrm{C} \mathrm{NMR}:\left(100.0 \mathrm{MHz}, \mathrm{CDCl}_{3}\right) \delta 157.8$, 149.3, 133.6, 127.0, 115.4, 109.1, 48.0, 40.3, 34.5, 31.4, 28.0, 26.0, 23.7, 22.1, 20.7, 16.6, 13.0; 
HRMS (EI) calculated for $\left[\mathrm{C}_{20} \mathrm{H}_{30} \mathrm{O}\right]^{+}$requires $\mathrm{m} / \mathrm{z} 286.2297$, found $\mathrm{m} / \mathrm{z} 286.2299$.<smiles>CC1(C)C2CCC1(C)C(Oc1ccc(C#N)cc1)C2</smiles>

\section{4-(((2R,4R)-4,7,7-trimethylbicyclo[2.2.1]heptan-2-}

yl)oxy)benzonitrile (1ae-s1). According to the previous method, ${ }^{12}$ the reaction afforded $3.5678 \mathrm{~g}$ (70\% yield) of 1ae-s1 as a white solid. IR (neat): 2952, 2225, 1656, 1605, $1507 \mathrm{~cm}^{-1}$; ${ }^{1} \mathrm{H}$ NMR: $\left(400.0 \mathrm{MHz}, \mathrm{CDCl}_{3}\right) \delta 7.55(\mathrm{~d}, J=8.8 \mathrm{~Hz}$, 2H), $6.87(\mathrm{~d}, J=9.2 \mathrm{~Hz}, 2 \mathrm{H}), 4.39-4.33(\mathrm{~m}, 1 \mathrm{H}), 2.44-2.34(\mathrm{~m}, 1 \mathrm{H}), 2.23-2.13(\mathrm{~m}, 1 \mathrm{H}), 1.84-1.71$ (m, 2H), 1.43-1.32 (m, 1H), 1.29-1.20 (m, 1H), $1.07(\mathrm{dd}, J=13.6,3.6 \mathrm{~Hz}, 1 \mathrm{H}), 0.97-0.90(\mathrm{~m}, 9 \mathrm{H})$; ${ }^{13} \mathrm{C}$ NMR: $\left(100.0 \mathrm{MHz}, \mathrm{CDCl}_{3}\right) \delta 162.4,133.9,119.4,116.0,103.2,83.5,49.5,47.6,45.0,36.5$, 27.8, 26.7, 19.6, 18.9, 13.7; HRMS (EI) calculated for $\left[\mathrm{C}_{17} \mathrm{H}_{21} \mathrm{NO}\right]^{+}$requires $\mathrm{m} / \mathrm{z} 255.1623$, found $\mathrm{m} / \mathrm{z} 255.1625$.<smiles>C=C(CC)c1ccc(OC2CC3(C)CCC2C3(C)C)cc1</smiles>

$(1 R, 3 R)-3-(4-(b u t-1-e n-2-y l) p h e n o x y)-1,7,7-$ trimethylbicyclo[2.2.1]heptane (1ae). According to the method B, the reaction afforded $1.3537 \mathrm{~g}$ (79\% yield) of 1ae as a colorless oil. IR (neat): 2950, 2876, 1606, 1571, $1509 \mathrm{~cm}^{-1}$; ${ }^{1} \mathrm{H}$ NMR: $\left(400.0 \mathrm{MHz}, \mathrm{CDCl}_{3}\right) \delta 7.33(\mathrm{~d}, J=8.8 \mathrm{~Hz}$, 2H), $6.79(\mathrm{~d}, J=8.8 \mathrm{~Hz}, 2 \mathrm{H}), 5.19(\mathrm{~s}, 1 \mathrm{H}), 4.95(\mathrm{~s}, 1 \mathrm{H}), 4.35-4.29(\mathrm{~m}, 1 \mathrm{H}), 2.48(\mathrm{q}, J=15.2,6.8$ $\mathrm{Hz}, 2 \mathrm{H}), 2.42-2.32$ (m, 1H), 2.28-2.19 (m, 1H), 1.81-1.70 (m, 2H), 1.39-1.20 (m, 2H), 1.16-1.07 (m, 4H), 0.96-0.89 (m, 9H); ${ }^{13} \mathrm{C}$ NMR: (100.0 MHz, $\left.\mathrm{CDCl}_{3}\right) \delta 158.5,149.3,133.3,126.9,115.0,109.1$, 82.7, 49.5, 47.5, 45.2, 36.8, 28.1, 27.9, 26.8, 19.7, 18.9, 13.7, 13.0; HRMS (EI) calculated for $\left[\mathrm{C}_{20} \mathrm{H}_{28} \mathrm{O}\right]^{+}$requires $\mathrm{m} / \mathrm{z} 284.2140$, found $\mathrm{m} / \mathrm{z} 284.2142$.<smiles>CC(C)=CCC/C(C)=C/COc1ccc(C#N)cc1</smiles>

(E)-4-((3,7-dimethylocta-2,6-dien-1yl)oxy)benzonitrile (1af-s1). According to the previous method, ${ }^{12}$ the reaction afforded $4.7858 \mathrm{~g}$ (93\% yield) of $\mathbf{1 a f}-\mathbf{s} 1$ as a white solid. IR (neat): 2920, 2851, 2225, 1739, 1605, $1508 \mathrm{~cm}^{-1} ;{ }^{1} \mathrm{H}$ NMR: (400.0 MHz, $\left.\mathrm{CDCl}_{3}\right) \delta$ 7.58-7.54 (m, 2H), 6.99-6.91 (m, 2H), 5.49-5.30 (m, 1H), 5.12-5.03 (m, 1H), $4.59(\mathrm{~d}, J=6.8 \mathrm{~Hz}, 2 \mathrm{H}), 2.19-2.07(\mathrm{~m}, 4 \mathrm{H}), 1.74$ (s, 3H), 1.67 (s, 3H), 1.63-1.56 (m, 3H); ${ }^{13} \mathrm{C}$ NMR: (100.0 MHz, $\left.\mathrm{CDCl}_{3}\right) \delta$ 162.2, 142.3, 133.9, 131.9, 123.6, 119.3, 118.4, 115.4, 103.7, 65.2, 39.5, 26.2, 25.7, 17.7, 16.7; HRMS (EI) calculated for $\left[\mathrm{C}_{17} \mathrm{H}_{21} \mathrm{NO}\right]^{+}$requires $\mathrm{m} / \mathrm{z} 255.1623$, found $\mathrm{m} / \mathrm{z} 255.1623$.<smiles>C=C(CC)c1ccc(OC/C=C(\C)CCC=C(C)C)cc1</smiles>

(E)-1-(but-1-en-2-yl)-4-((3,7-dimethylocta-2,6dien-1-yl)oxy)benzene (1af). According to the 
method B, the reaction afforded $0.7075 \mathrm{~g}$ (55\% yield) of 1 af as a colorless oil. IR (neat): 2969 , 2916, 1670, 1607, 1572, $1510 \mathrm{~cm}^{-1}$; ${ }^{1} \mathrm{H}$ NMR: $\left(400.0 \mathrm{MHz}, \mathrm{CDCl}_{3}\right) \delta 7.35(\mathrm{~d}, J=8.8 \mathrm{~Hz}, 2 \mathrm{H}), 6.87$ $(\mathrm{d}, J=8.8 \mathrm{~Hz}, 2 \mathrm{H}), 5.50(\mathrm{t}, J=6.4 \mathrm{~Hz}, 1 \mathrm{H}), 5.21(\mathrm{~s}, 1 \mathrm{H}), 5.10(\mathrm{t}, J=5.6 \mathrm{~Hz}, 1 \mathrm{H}), 4.97(\mathrm{~s}, 1 \mathrm{H})$, $4.54(\mathrm{~d}, J=6.4 \mathrm{~Hz}, 2 \mathrm{H}), 2.48(\mathrm{q}, J=14.8,7.2 \mathrm{~Hz}, 2 \mathrm{H}), 2.18-2.04(\mathrm{~m}, 4 \mathrm{H}), 1.73(\mathrm{~s}, 3 \mathrm{H}), 1.68(\mathrm{~s}$, 3H), $1.61(\mathrm{~s}, 3 \mathrm{H}), 1.10(\mathrm{t}, J=7.6 \mathrm{~Hz}, 3 \mathrm{H}) ;{ }^{13} \mathrm{C} \mathrm{NMR}:\left(100.0 \mathrm{MHz}, \mathrm{CDCl}_{3}\right) \delta 158.3,149.3,141.1$, $133.8,131.8,127.0,123.8,119.5,114.3,109.3,64.8,39.5,28.0,26.3,25.7,17.7,16.6,13.0$; HRMS (EI) calculated for $\left[\mathrm{C}_{20} \mathrm{H}_{28} \mathrm{O}\right]^{+}$requires $\mathrm{m} / \mathrm{z} 284.2140$, found $\mathrm{m} / \mathrm{z} 284.2142$.

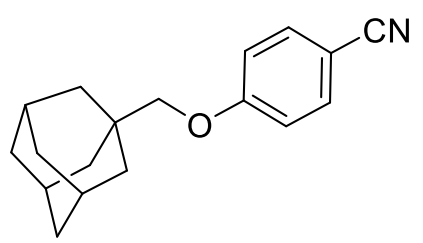

4-((adamantan-1-yl)methoxy)benzonitrile (1ag-s1). According to the previous method, ${ }^{12}$ the reaction afforded $4.5292 \mathrm{~g}$ (85\% yield) of 1ag-s1 as a white solid. IR (neat): 2906, 2851 1607, 2225, $1606 \mathrm{~cm}^{-}$ 1; ${ }^{1} \mathrm{H}$ NMR: (400.0 MHz, $\left.\mathrm{CDCl}_{3}\right) \delta 7.57(\mathrm{~d}, J=9.2 \mathrm{~Hz}, 2 \mathrm{H}), 6.94(\mathrm{~d}$, $J=9.2 \mathrm{~Hz}, 2 \mathrm{H}), 3.53(\mathrm{~s}, 2 \mathrm{H}), 2.03(\mathrm{~s}, 3 \mathrm{H}), 1.82-1.60(\mathrm{~m}, 12 \mathrm{H}) ;{ }^{13} \mathrm{C} \mathrm{NMR}:\left(100.0 \mathrm{MHz}, \mathrm{CDCl}_{3}\right) \delta$ $162.9,133.9,119.4,115.2,103.4,78.5,39.3,37.0,33.7,28.1$; HRMS (ESI) calculated for $\left[\mathrm{C}_{18} \mathrm{H}_{21} \mathrm{NONa}\right]^{+}\left(\mathrm{M}+\mathrm{Na}^{+}\right)$, requires $\mathrm{m} / \mathrm{z} 290.1515$, found $\mathrm{m} / \mathrm{z} 290.1515$.

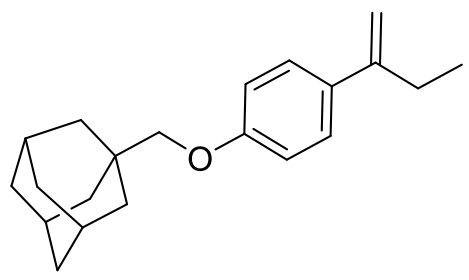

According to the method B, the reaction afforded $1.1010 \mathrm{~g}(53 \%$ yield) of 1ag as a colorless oil. IR (neat): 2901, 2849, 1607, 1572, $1460 \mathrm{~cm}^{-1} ;{ }^{1} \mathrm{H}$ NMR: $\left(400.0 \mathrm{MHz}, \mathrm{CDCl}_{3}\right) \delta 7.34(\mathrm{~d}, J=8.8 \mathrm{~Hz}$, 2H), $6.85(\mathrm{~d}, J=8.8 \mathrm{~Hz}, 2 \mathrm{H}), 5.19(\mathrm{~s}, 1 \mathrm{H}), 4.96(\mathrm{~s}, 1 \mathrm{H}), 3.50(\mathrm{~s}, 2 \mathrm{H}), 2.48$ (q, $J=14.8,7.2 \mathrm{~Hz}, 2 \mathrm{H})$, $2.02(\mathrm{~s}, 3 \mathrm{H}), 1.80-1.63(\mathrm{~m}, 12 \mathrm{H}), 1.09(\mathrm{t}, J=6.8 \mathrm{~Hz}, 3 \mathrm{H}) ;{ }^{13} \mathrm{C} \mathrm{NMR}:\left(100.0 \mathrm{MHz}, \mathrm{CDCl}_{3}\right) \delta 159.1$, 149.4, 133.5, 126.9, 114.1, 109.1, 78.3, 39.5, 37.1, 33.8, 28.2, 28.1, 13.0; HRMS (EI) calculated for $\left[\mathrm{C}_{21} \mathrm{H}_{28} \mathrm{O}\right]^{+}$requires $\mathrm{m} / \mathrm{z} 296.2140$, found $\mathrm{m} / \mathrm{z} 296.2139$.

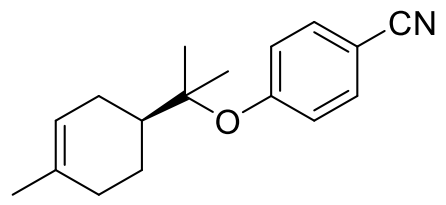

(S)-4-((2-(4-methylcyclohex-3-en-1-yl)propan-2yl)oxy)benzonitrile (1ah-1). According to the previous method, ${ }^{12}$ the reaction afforded $1.7000 \mathrm{~g}$ ( $33 \%$ yield) of $\mathbf{1 a h}-\mathbf{s} \mathbf{1}$ as a colorless oil. IR (neat): 2926, 2225, 1602, 1568, 1502, $1442 \mathrm{~cm}^{-1}$; ${ }^{1} \mathrm{H}$ NMR: $\left(399.9 \mathrm{MHz}, \mathrm{CDCl}_{3}\right) \delta 7.55$ (d, $J=8.4 \mathrm{~Hz}, 2 \mathrm{H}), 7.02(\mathrm{~d}, J=8.8 \mathrm{~Hz}, 2 \mathrm{H}), 5.40(\mathrm{t}, J=3.2 \mathrm{~Hz}, 1 \mathrm{H}), 2.14-1.80(\mathrm{~m}, 6 \mathrm{H}), 1.67(\mathrm{~s}, 3 \mathrm{H})$, 1.46-1.34 (m, 1H), $1.32(\mathrm{~s}, 6 \mathrm{H}) ;{ }^{13} \mathrm{C}$ NMR: (100.0 MHz, $\left.\mathrm{CDCl}_{3}\right) \delta 160.0,134.0,133.3,122.8,120.3$, $119.1,105.3,84.6,43.9,30.8,26.7,24.4,24.0,23.6,23.3$; HRMS (ESI) calculated for 
$\left[\mathrm{C}_{17} \mathrm{H}_{21} \mathrm{NONa}\right]^{+}\left(\mathrm{M}+\mathrm{Na}^{+}\right)$, requires $\mathrm{m} / \mathrm{z} 278.1515$, found $\mathrm{m} / \mathrm{z} 278.1514$.

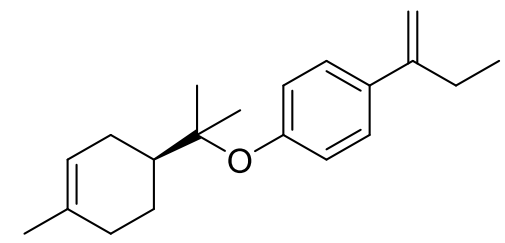

(S)-1-(but-1-en-2-yl)-4-((2-(4-methylcyclohex-3-en-1yl)propan-2-yl)oxy)benzene (1ah). According to the method $\mathbf{B}$, the reaction afforded $0.2445 \mathrm{~g}$ ( $22 \%$ yield) of $\mathbf{1 a h}$ as a colorless oil. IR (neat): 2972, 2888, 1603, 1507, 1439, $1237 \mathrm{~cm}^{-}$

1; ${ }^{1} \mathrm{H}$ NMR: (400.0 MHz, $\left.\mathrm{CDCl}_{3}\right) \delta 7.31(\mathrm{~d}, J=8.4 \mathrm{~Hz}, 2 \mathrm{H}), 6.92(\mathrm{~d}, J=8.4 \mathrm{~Hz}, 2 \mathrm{H}), 5.41(\mathrm{t}, J=$ $1.2 \mathrm{~Hz}, 1 \mathrm{H}), 5.24(\mathrm{~s}, 1 \mathrm{H}), 5.00(\mathrm{~s}, 1 \mathrm{H}), 2.49$ (q, $J=14.8,7.6 \mathrm{~Hz}, 2 \mathrm{H}), 2.18-1.78(\mathrm{~m}, 6 \mathrm{H}), 1.67(\mathrm{~s}$, 3H), 1.46-1.33 (m, 1H), $1.23(\mathrm{~d}, J=3.2 \mathrm{~Hz}, 6 \mathrm{H}), 1.10(\mathrm{t}, J=7.2 \mathrm{~Hz}, 3 \mathrm{H}) ;{ }^{13} \mathrm{C}$ NMR: $(100.0 \mathrm{MHz}$, $\left.\mathrm{CDCl}_{3}\right) \delta 154.8,149.3,136.1,134.1,126.3,123.7,120.7,109.8,82.8,43.8,31.0,28.0,27.1,24.5$, 24.1, 23.4, 23.3, 13.0; HRMS (EI) calculated for $\left[\mathrm{C}_{20} \mathrm{H}_{28} \mathrm{O}\right]^{+}$requires $\mathrm{m} / \mathrm{z} 284.2140$, found $\mathrm{m} / \mathrm{z}$ 284.2140<smiles>C=C(CCc1ccc(OC)cc1)C(C)C</smiles>
of alkyl alkene 1ai as a colorless oil. ${ }^{1} \mathrm{H}$ NMR: $\left(400.0 \mathrm{MHz}, \mathrm{CDCl}_{3}\right)$ $\delta 7.12(\mathrm{~d}, \mathrm{~J}=8.4 \mathrm{~Hz}, 2 \mathrm{H}), 6.83(\mathrm{~d}, \mathrm{~J}=8.4 \mathrm{~Hz}, 2 \mathrm{H}), 4.79(\mathrm{~s}, 1 \mathrm{H}), 4.73(\mathrm{~s}, 1 \mathrm{H}), 3.79(\mathrm{~s}, 3 \mathrm{H}), 2.70$ (t, $\left.\mathrm{J}=8.0 \mathrm{~Hz}, 2 \mathrm{H}), 2.34-2.21(\mathrm{~m}, 3 \mathrm{H}), 1.04(\mathrm{~d}, 6 \mathrm{H}) ;{ }^{13} \mathrm{C} \mathrm{NMR:} \mathrm{(100.0} \mathrm{MHz,} \mathrm{CDCl}_{3}\right) \delta 157.7,155.5$, $134.5,129.2,113.7,106.5,55.2,36.4,34.0,33.7,21.8$; HRMS (EI) calculated for $\left[\mathrm{C}_{14} \mathrm{H}_{20} \mathrm{O}\right]^{+}$ requires $\mathrm{m} / \mathrm{z} 204.1514$, found $\mathrm{m} / \mathrm{z} 204.1515$.<smiles>C=C(CCC=C(C)C)c1ccc(C)c(OC)c1</smiles>

\section{2-methoxy-1-methyl-4-(6-methylhepta-1,5-dien-2-yl)benzene}

(1aj). According to method A, the reaction afforded $1.1748 \mathrm{~g}(81 \%$ yield) of 1aj as a colorless oil. IR (neat): 2924, 1611, 1573, 1508, 1462, $1407 \mathrm{~cm}^{-1}$; ${ }^{1} \mathrm{H}$ NMR: $\left(400.0 \mathrm{MHz}, \mathrm{CDCl}_{3}\right) \delta 7.08(\mathrm{~d}, J=7.6 \mathrm{~Hz}, 1 \mathrm{H}), 6.91(\mathrm{~d}, J=7.6 \mathrm{~Hz}$, 1H), $6.87(\mathrm{~s}, 1 \mathrm{H}), 5.26(\mathrm{~s}, 1 \mathrm{H}), 5.16(\mathrm{t}, J=6.8 \mathrm{~Hz}, 1 \mathrm{H}), 5.03(\mathrm{~s}, 1 \mathrm{H}), 3.85(\mathrm{~s}, 3 \mathrm{H}), 2.50(\mathrm{t}, J=7.6$ $\mathrm{Hz}, 2 \mathrm{H}), 2.21$ (s, 3H), 2.19-2.10 (m, 2H), 1.69 (s, 3H), 1.56 (s, 3H); ${ }^{13} \mathrm{C} \mathrm{NMR}:\left(100.0 \mathrm{MHz}, \mathrm{CDCl}_{3}\right)$ $\delta 157.5,148.5,140.3,131.8,130.3,125.8,123.9,118.0,111.5,107.9,55.2,35.6,27.0,25.7,17.7$, 15.9; HRMS (EI) calculated for $\left[\mathrm{C}_{16} \mathrm{H}_{22} \mathrm{O}\right]^{+}$requires $\mathrm{m} / \mathrm{z} 230.1671$, found $\mathrm{m} / \mathrm{z} 230.1669$.

$n-\mathrm{C}_{18} \mathrm{H}_{37} \mathrm{SiH}_{3}$ octadecylsilane. A three-necked flask equipped with a reflux condenser and a magnetic stir bar was flame-dried under reduced pressure, and then cooled under a $\mathrm{N}_{2}$ atmosphere. $\mathrm{LiAlH}_{4}\left(1.25 \mathrm{~g}, 33.0 \mathrm{mmol}, 1.1\right.$ equiv) and $\mathrm{Et}_{2} \mathrm{O}(50 \mathrm{~mL})$ was added successively under $0{ }^{\circ} \mathrm{C}$, then 
followed by $n-\mathrm{C}_{18} \mathrm{H}_{37} \mathrm{Si}(\mathrm{OMe})_{3}(11.25 \mathrm{~g}, 30.0 \mathrm{mmol}, 1.0$ equiv) dropwise over $15 \mathrm{~min}$. The resulting mixture was refluxed for overnight. After the reaction was completed, the resulting suspension was filtered through celite and distilled under reduced pressure to provide $n-\mathrm{C}_{18} \mathrm{H}_{37} \mathrm{SiH}_{3}$ as a colorless oil with $86 \%$ yield. IR (neat): 2921, 2852, 2149, 1464, $924 \mathrm{~cm}^{-1} ;{ }^{1} \mathrm{H}$ NMR: $\left(400.0 \mathrm{MHz}, \mathrm{CDCl}_{3}\right) \delta$ $3.48(\mathrm{t}, J=4.0 \mathrm{~Hz}, 3 \mathrm{H}), 1.47-1.37(\mathrm{~m}, 2 \mathrm{H}), 1.36-1.21(\mathrm{~m}, 30 \mathrm{H}), 0.88(\mathrm{t}, J=6.4 \mathrm{~Hz}, 3 \mathrm{H}), 0.78-0.70$

(m, 2H); $\left.{ }^{13} \mathrm{C} \mathrm{NMR:} \mathrm{(100.0} \mathrm{MHz,} \mathrm{CDCl}_{3}\right) \delta$ 67.9, 32.6, 32.0, 29.8, 29.7, 29.6, 29.4, 29.3, 26.4, 25.6, 22.7, 14.1, 5.9; HRMS (EI) calculated for $\left[\mathrm{C}_{18} \mathrm{H}_{40} \mathrm{Si}^{+}\right.$requires $\mathrm{m} / \mathrm{z} 284.2899$, found $\mathrm{m} / \mathrm{z} 284.2900$. $\boldsymbol{n}-\mathrm{C}_{18} \mathrm{H}_{37} \mathrm{SiD}_{3}$ octadecylsilane- $\boldsymbol{d}_{3}$. Using $\mathrm{LiAlD}_{4}$ instead of $\mathrm{LiAlH}_{4}$, provide $n-\mathrm{C}_{18} \mathrm{H}_{37} \mathrm{SiD}_{3}$ as a colorless oil with 99\% deuterium incorporation (4.39 g, 76\% yield). IR (neat): 2924, 2854, 1570, 1463, $1376 \mathrm{~cm}^{-1}$; ${ }^{1} \mathrm{H}$ NMR: (400.0 MHz, $\left.\mathrm{CDCl}_{3}\right) \delta$ 1.47-1.37 (m, 2H), 1.36-1.21 (m, 30H), $0.88(\mathrm{t}$, $J=6.4 \mathrm{~Hz}, 3 \mathrm{H}), 0.78-0.70(\mathrm{~m}, 2 \mathrm{H}) ;{ }^{13} \mathrm{C} \mathrm{NMR}:\left(100.0 \mathrm{MHz}, \mathrm{CDCl}_{3}\right) \delta 32.5,31.9,29.7,29.7,29.5$, 29.4, 29.3, 26.3, 22.7, 14.1, 5.7. HRMS (EI) calculated for $\left[\mathrm{C}_{18} \mathrm{H}_{37} \mathrm{D}_{3} \mathrm{Si}\right]^{+}$requires $\mathrm{m} / \mathrm{z} 287.3088$, found $\mathrm{m} / \mathrm{z} 287.3090$.<smiles></smiles>

1-(but-1-en-2-yl-3,3-d2)-4-methoxybenzene (d1-1a). According to the previous method A, the reaction afforded $2.1188 \mathrm{~g}$ ( $90 \%$ yield) of $\boldsymbol{d 1}$-1a as a colorless oil. IR (neat): 2963, 1609, 1511, 1461, $1285 \mathrm{~cm}^{-1}$; ${ }^{1} \mathrm{H}$ NMR: (399.9 MHz, $\left.\mathrm{CDCl}_{3}\right) \delta 7.36(\mathrm{~d}, J=8.4 \mathrm{~Hz}, 2 \mathrm{H}), 6.86(\mathrm{~d}, J=8.4 \mathrm{~Hz}, 2 \mathrm{H}), 5.21(\mathrm{~s}, 1 \mathrm{H})$, 4.98 (s, 1H), 1.08 (s, 3H). ${ }^{13} \mathrm{C}$ NMR: (100.0 MHz, $\left.\mathrm{CDCl}_{3}\right) \delta 158.9,149.1,133.7,127.0,113.5,109.3$, 55.1, 27.7-26.9 (m, 1C), 12.8; HRMS (EI) calculated for $\left[\mathrm{C}_{11} \mathrm{H}_{12} \mathrm{D}_{2} \mathrm{O}\right]^{+}$requires $\mathrm{m} / \mathrm{z}$ 164.1170, found $\mathrm{m} / \mathrm{z} 164.1168$.<smiles>COc1ccc(C(=C([O-])[O-])C(C)C([O-])[O-])cc1</smiles>
1-(but-1-en-2-yl-1,1-d2)-4-methoxybenzene (d2-1a). According to the previous method $\mathbf{B}$, using $\mathrm{Ph}_{3} \mathrm{PCD}_{3} \mathrm{Br}(92 \% \mathrm{D})$ as Yelide reagent, the $\mathrm{Ph}_{3} \mathrm{PCD}_{3} \mathrm{Br}$ was synthesized according to the previous method. ${ }^{13}$ The reaction afforded $0.8823 \mathrm{~g}$ (68\% yield) of $\mathbf{d 2}-\mathbf{1 a}$ as a colorless oil, the D-incorporation was determined by ${ }^{1} \mathrm{H}$ NMR. ${ }^{1} \mathrm{H}$ NMR: (399.9 MHz, $\left.\mathrm{CDCl}_{3}\right) \delta 7.36(\mathrm{~d}, J=8.4$ $\mathrm{Hz}, 2 \mathrm{H}), 6.86(\mathrm{~d}, J=8.4 \mathrm{~Hz}, 2 \mathrm{H}), 5.21(\mathrm{~s}, 0.21 \mathrm{H}), 4.98(\mathrm{~s}, 0.21 \mathrm{H}), 2.51-2.46(\mathrm{~m}, 1.85 \mathrm{H}), 1.10(\mathrm{t}, J$ $=7.6 \mathrm{~Hz}, 3 \mathrm{H})$. HRMS (EI) calculated for $\left[\mathrm{C}_{11} \mathrm{H}_{12} \mathrm{D}_{2} \mathrm{O}\right]^{+}$requires $\mathrm{m} / \mathrm{z} 164.1170$, found $\mathrm{m} / \mathrm{z} 164.1170$. 
<smiles>COc1ccc(Br)cc1</smiles>

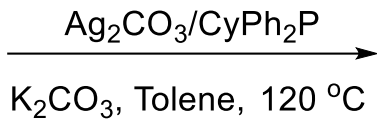

$(0.87)$<smiles>COc1c([18O])c([18O])c(Br)c([18O])c1[18O]</smiles>

According to the previous literature, ${ }^{14}$ 4-bromoanisole $(2.81 \mathrm{~g}, 15$ mmol) was added to a vigorously stirred solution of silver carbonate (0.5530 g, $3.0 \mathrm{mmol})$, cyclohexyldiphenylphosphine (2.10 g, 7.5 mmol), potassium carbonate $(2.10 \mathrm{~g}, 15 \mathrm{mmol})$ and $\mathrm{D}_{2} \mathrm{O}(12.0 \mathrm{~g}, 600$ Then the reaction was quenched with saturated $\mathrm{NH}_{4} \mathrm{Cl}$ solution. The product was extracted with dichloromethane $(3 \times 40 \mathrm{~mL})$. The combined organic layer was washed with brine and dried over $\mathrm{Na}_{2} \mathrm{SO}_{4}$. After removal of solvents under vacuum, the crude product was purified via column chromatography. Isolated yield: (2.67 g, 87\%), yellow oil. ${ }^{1} \mathrm{H}$ NMR: $\left(400.1 \mathrm{MHz}, \mathrm{CDCl}_{3}\right) \delta 7.38(\mathrm{~s}$, $0.42 \mathrm{H}), 6.79(\mathrm{~s}, 0.26 \mathrm{H}), 3.78(\mathrm{~s}, 3 \mathrm{H})$.

$(0.87)$

$$
\text { (0.80)D }
$$<smiles>COc1c([18O])c([18O])c([18O])c(Br)c1Br</smiles>

$(0.87)$<smiles>CCC(=O)c1c([18OH])c([18OH])c(O[18O])c([18OH])c1[18OH]</smiles>

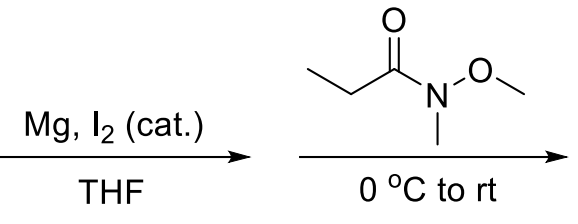

$(0.87)$<smiles>CCC(=O)c1c([18O])c([18OH])c([18OH])c([18OH])c1[18OH]</smiles>

According to the previous method, In an over-dried, $\mathrm{N}_{2}$ purged round flask, the Grignard solution was prepared from magnesium turnings $(14.2 \mathrm{mmol})$ reacting with $\mathrm{D}$-p-methoxylbromobenzene (14.2 mmol) in anhydrous THF $(10 \mathrm{~mL})$. N-propionamide $(1.88 \mathrm{~g}$, $14.2 \mathrm{mmol})$ was added to a $100-\mathrm{mL}$ round-bottom flask, and THF $(10 \mathrm{~mL})$ was then added. After cooling the solution to $0{ }^{\circ} \mathrm{C}$, D-p-methoxylphenylmagnesium bromide was added dropwise over 10 min. The solution was allowed to warm to room temperature and stirred overnight. After completion of reaction as determined by TLC, the reaction was cooled to $0{ }^{\circ} \mathrm{C}$ and quenched with saturated $\mathrm{NH}_{4} \mathrm{Cl}(10 \mathrm{~mL})$. Upon separation of the two layers, the aqueous layer was extracted with $\mathrm{EA}(3 \mathrm{x}$ $20 \mathrm{~mL}$ ). The combined organic layers were then dried over $\mathrm{Na}_{2} \mathrm{SO}_{4}$, filtered, and concentrated under reduced pressure. The resulting crude oil was then purified by column chromatography. Isolated yield: $\left(1.3609\right.$ g, 57\%), colorless oil. ${ }^{1} \mathrm{H}$ NMR: $\left(400.0 \mathrm{MHz}, \mathrm{CDCl}_{3}\right) \delta 7.96(\mathrm{~s}, 0.40 \mathrm{H}), 6.93(\mathrm{~s}$, 
$0.25 \mathrm{H}), 3.87(\mathrm{~s}, 3.0 \mathrm{H}), 3.01-2.90(\mathrm{~m}, 2 \mathrm{H}), 1.25-1.16(\mathrm{~m}, 3 \mathrm{H})$.

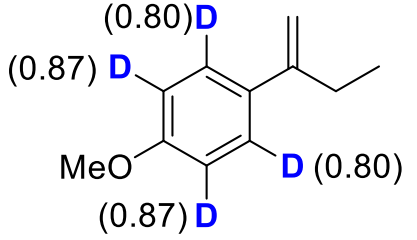

According to the previous method $\mathbf{B}$, the reaction afforded 0.5128 g (39\% yield) of $\boldsymbol{d} \mathbf{3}-\mathbf{1 a}$ as a colorless oil. ${ }^{1} \mathrm{H}$ NMR: $(400.0 \mathrm{MHz}$, $\left.\mathrm{CDCl}_{3}\right) \delta 7.37(\mathrm{~s}, 0.40 \mathrm{H}), 6.87(\mathrm{~s}, 0.26 \mathrm{H}), 5.21(\mathrm{~s}, 1 \mathrm{H}), 4.97(\mathrm{~s}, 1 \mathrm{H})$, $3.81(\mathrm{~s}, 3 \mathrm{H}), 2.49$ (q, J = 14.8, 7.2 Hz, 2H), 1.10 (t, J = 7.2 Hz, 3H).

\section{Iron-Catalyzed Highly Enantioselective Hydrogenation of alkenes}

\section{Table S1: control experiments ${ }^{a}$}

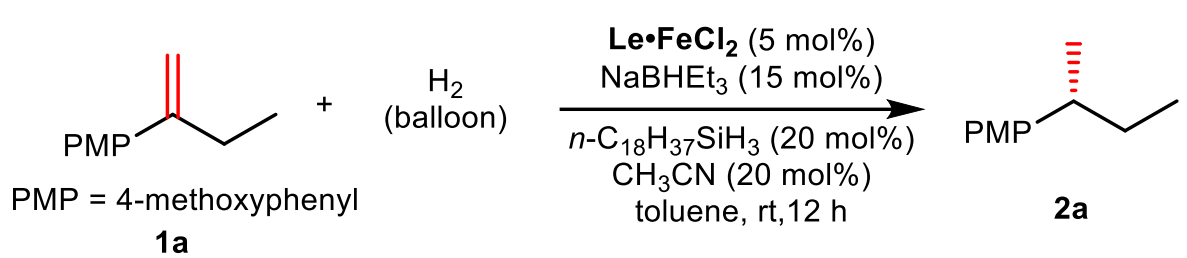

\begin{tabular}{|c|c|c|c|c|c|}
\hline entry & $\begin{array}{c}\text { Variation from } \\
\text { standard conditions } \\
\end{array}$ & $\begin{array}{l}\text { yield of } \\
\mathbf{2 a}(\%)^{b}\end{array}$ & $\begin{array}{c}\text { recovery of } \\
\mathbf{1 a}(\%) \\
\end{array}$ & $\begin{array}{c}\text { isom. (3a) } \\
(\%) \\
\end{array}$ & $\begin{array}{c}e e \text { of } \mathbf{2 a} \\
(\%)^{b}\end{array}$ \\
\hline 1 & none & $>99$ & $\mathbf{0}$ & $\mathbf{0}$ & 99 \\
\hline 2 & no $\mathrm{CH}_{3} \mathrm{CN}$ & 65 & 0 & 35 & 96 \\
\hline 3 & no $[\mathrm{Si}-\mathrm{H}]$ & 0 & $>99$ & 0 & / \\
\hline 4 & $\begin{array}{c}\text { no } \mathrm{CH}_{3} \mathrm{CN} \text { and } \\
{[\mathrm{Si}-\mathrm{H}]}\end{array}$ & 27 & 46 & 27 & 99 \\
\hline 5 & no $\mathrm{H}_{2}$ & 10 & 89 & 1 & 91 \\
\hline $6^{c}$ & no $\mathrm{H}_{2}$ & 13 & 59 & $28+0$ & 89 \\
\hline $7^{c}$ & $10 \mathrm{~mol} \%[\mathrm{Si}-\mathrm{H}]$ & 84 & 16 & 0 & 99 \\
\hline 8 & $\begin{array}{l}\text { 3-methoxybenzonitrile } \\
\text { instead of } \mathrm{CH}_{3} \mathrm{CN}\end{array}$ & $>99$ & 0 & 0 & 98 \\
\hline $9^{d}$ & $\begin{array}{c}\text { no } \mathrm{CH}_{3} \mathrm{CN} \text { and } \\
{[\mathrm{Si}-\mathrm{H}]}\end{array}$ & 55 & 16 & 29 & 98 \\
\hline
\end{tabular}

${ }^{a}$ Standard conditions: 1 (0.5 mmol), $\mathrm{H}_{2}$ (balloon), $n-\mathrm{C}_{18} \mathrm{H}_{37} \mathrm{SiH}_{3}(0.10 \mathrm{mmol}), \mathrm{CH}_{3} \mathrm{CN}$ (0.10 mmol), Le $\cdot \mathrm{FeCl}_{2}$ $(0.025 \mathrm{mmol}, 5 \mathrm{~mol} \%)$ and $\mathrm{NaBHEt}_{3}(0.075 \mathrm{mmol})$ in toluene $(0.5 \mathrm{M})$ under rt for $12 \mathrm{~h} .{ }^{b}$ Conversions and recovery were determined by ${ }^{1} \mathrm{H}$ NMR using TMSPh as an internal standard; $e e$ values were determined by GC using chiral column; ${ }^{c}$ the reaction time is extended to $42 \mathrm{~h},{ }^{d}$ using MeLi instead of NaBHEt 3 as an activator of precatalyst.

$$
\mathrm{R}^{1} \mathrm{R}^{2}+\underset{\text { (balloon) }}{\mathrm{H}_{2}} \stackrel{\begin{array}{c}
\mathrm{Le} \cdot \mathrm{FeCl}_{2}(5 \mathrm{~mol} \%) \\
\mathrm{NaBHEt}_{3}(15 \mathrm{~mol} \%)
\end{array}}{\begin{array}{c}
\mathrm{n}-\mathrm{C}_{18} \mathrm{H}_{37} \mathrm{SiH}_{3}(20 \mathrm{~mol} \%) \\
\mathrm{CH}_{3} \mathrm{CN}(20 \mathrm{~mol} \%) \\
\text { toluene, } \mathrm{rt}, 12 \mathrm{~h}
\end{array}} \mathrm{R}^{1{ }^{1}} \mathrm{R}^{2}
$$

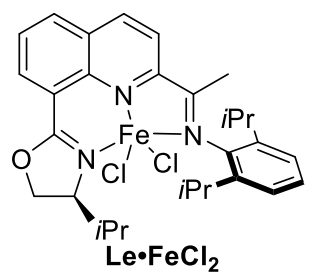

General procedure for asymmetric hydrogenation of 1,1-disubstituted alkenes: A 25 $\mathrm{mL}$ flame-dried Schleck flask was cooled at room temperature under nitrogen, charged 
with $\mathbf{L e} \cdot \mathbf{F e C l}_{2}(0.025 \mathrm{mmol})$, alkene $(0.50 \mathrm{mmol})$, hydrosilane $(0.10 \mathrm{mmol})$, the system was purged one time, The acetonitrile $(0.10 \mathrm{mmol})$ and dry toluene $(1 \mathrm{~mL})$ were added successively, then $\mathrm{NaBHEt}_{3}(1 \mathrm{M}$ in THF) $(75 \mu \mathrm{L}, 0.075 \mathrm{mmol})$ was injected slowly, the reaction was stirred $5 \mathrm{~min}$, a balloon with hydrogen was added and the system was purged three times. The mixture was stirred for 12 hours in room temp. The reaction was quenched by PE. The mixture was filtered through a pad of silica gel and washed with PE $(50 \mathrm{~mL})$. The filtrate was concentrated without further purification to afford the corresponding product.

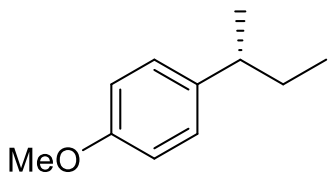

(R)-1-(sec-butyl)-4-methoxybenzene (2a). ${ }^{15}$ Prepared according to the general procedure: using $\mathbf{L e} \bullet \mathrm{FeCl}_{2}(0.0149 \mathrm{~g}, 0.025 \mathrm{mmol}), \mathbf{1 a}(81 \mu \mathrm{L}$, $1.03 \mathrm{~g} / \mathrm{mL}, 0.50 \mathrm{mmol}), n-\mathrm{C}_{18} \mathrm{H}_{37} \mathrm{SiH}_{3}(32 \mu \mathrm{L}, 0.8906 \mathrm{~g} / \mathrm{mL}, 0.10 \mathrm{mmol})$, $\mathrm{CH}_{3} \mathrm{CN}(6.4 \mu \mathrm{L}, 0.6406 \mathrm{~g} / \mathrm{mL}, 0.10 \mathrm{mmol}), 1 \mathrm{~mL}$ of toluene, NaBHEt $3(75 \mu \mathrm{L}, 0.075 \mathrm{mmol})$. After 12 hours, the reaction afforded $\mathbf{2 a}$ with $>99 \%$ conversion (97\% yield) as a colorless oil. Optical Rotation: $[\alpha]^{20} \mathrm{D}=-23.2\left(\mathrm{c} 1.11, \mathrm{CHCl}_{3}\right)\left\{\mathrm{Lit}^{15}[\alpha]^{23} \mathrm{D}=+27.0\left(\mathrm{c} 1.0, \mathrm{CHCl}_{3}\right), 94 \%\right.$ ee $\} .99 \%$ ee, determined by GC, GC conditions: Chiralcel B-DM, $100 \mathrm{kPa} \mathrm{N} 2\left(60{ }^{\circ} \mathrm{C}-30 \mathrm{~min}-1{ }^{\circ} \mathrm{C} / \mathrm{min}-110\right.$ ${ }^{\circ} \mathrm{C}-5 \mathrm{~min}$ ), tr 67.9 (minor), 68.5 (major). ${ }^{1} \mathrm{H} \mathrm{NMR}:\left(400.0 \mathrm{MHz}, \mathrm{CDCl}_{3}\right) \delta 7.10(\mathrm{~d}, J=8.4 \mathrm{~Hz}, 2 \mathrm{H})$, $6.84(\mathrm{~d}, J=8.4 \mathrm{~Hz}, 2 \mathrm{H}), 3.79(\mathrm{~s}, 3 \mathrm{H}), 2.60-2.49(\mathrm{~m}, 1 \mathrm{H}), 1.62-1.48(\mathrm{~m}, 2 \mathrm{H}), 1.21(\mathrm{~d}, J=7.2 \mathrm{~Hz}$, $3 \mathrm{H}), 0.81(\mathrm{t}, J=7.6 \mathrm{~Hz}, 3 \mathrm{H})$. All the spectroscopic data were in agreement with the reported ones. ${ }^{14}$<smiles>CC[C@H](C)c1ccc(OCc2ccccc2)cc1</smiles>
(R)-1-(sec-butyl)-4-methoxybenzene (2b). Prepared according to the general procedure: using $\mathbf{L e} \cdot \mathrm{FeCl}_{2}(0.0142 \mathrm{~g}, 0.025 \mathrm{mmol}), \mathbf{1 b}(0.1176 \mathrm{~g}$, $0.50 \mathrm{mmol}), n-\mathrm{C}_{18} \mathrm{H}_{37} \mathrm{SiH}_{3}(32 \mu \mathrm{L}, 0.8906 \mathrm{~g} / \mathrm{mL}, 0.10 \mathrm{mmol}), \mathrm{CH}_{3} \mathrm{CN}(6.4$ $\mu \mathrm{L}, 0.6406 \mathrm{~g} / \mathrm{mL}, 0.10 \mathrm{mmol}), 1 \mathrm{~mL}$ of toluene, NaBHEt3 $(75 \mu \mathrm{L}, 0.075 \mathrm{mmol})$. After 12 hours, the reaction afforded $\mathbf{2 b}$ with $>99 \%$ conversion ( $93 \%$ yield) as a colorless oil. Optical Rotation: $[\alpha]^{20} \mathrm{D}$ $=-8.6\left(\mathrm{c} 1.35, \mathrm{CHCl}_{3}\right) .98 \%$ ee, determined by HPLC, HPLC conditions: Chiralcel OJ-H, $n-$ hexane $/ i-\mathrm{PrOH}=99 / 1,1.0 \mathrm{~mL} / \mathrm{min}, \mathrm{n}=220 \mathrm{~nm}, \operatorname{tr} 9.8$ (major), 10.3 (minor). IR (neat): 2961, 2925, 1510, $1488 \mathrm{~cm}^{-1}$; ${ }^{1} \mathrm{H}$ NMR: (400.0 MHz, $\left.\mathrm{CDCl}_{3}\right) \delta$ 7.46-7.30 (m, 5H), $7.10(\mathrm{~d}, J=8.8 \mathrm{~Hz}, 2 \mathrm{H}), 6.91$ $(\mathrm{d}, J=8.4 \mathrm{~Hz}, 2 \mathrm{H}), 5.04(\mathrm{~s}, 2 \mathrm{H}), 2.59-2.51(\mathrm{~m}, 1 \mathrm{H}), 1.63-1.51(\mathrm{~m}, 2 \mathrm{H}), 1.21(\mathrm{~d}, J=6.8 \mathrm{~Hz}, 3 \mathrm{H})$, $0.82(\mathrm{t}, J=7.2 \mathrm{~Hz}, 3 \mathrm{H}) ;{ }^{13} \mathrm{C} \mathrm{NMR}:\left(100.0 \mathrm{MHz}, \mathrm{CDCl}_{3}\right) \delta 156.9,140.1,137.3,128.5,127.9,127.8$, 127.5, 114.5, 70.0, 40.8, 31.3, 22.0, 12.2; HRMS (EI) calculated for $\left[\mathrm{C}_{17} \mathrm{H}_{20} \mathrm{O}\right]^{+}$requires $\mathrm{m} / \mathrm{z}$ 
240.1514, found $\mathrm{m} / \mathrm{z} 240.1516$.<smiles>CC[C@H](C)c1ccc(O[AsH3])cc1</smiles>

(R)-tert-butyl(4-(sec-butyl)phenoxy)dimethylsilane (2c). Prepared according to the general procedure: using $\mathbf{L e} \bullet \mathrm{FeCl}_{2}(0.0148 \mathrm{~g}, 0.025$ mmol), 1c (0.1325 g, $0.50 \mathrm{mmol}), n-\mathrm{C}_{18} \mathrm{H}_{37} \mathrm{SiH}_{3}(32 \mu \mathrm{L}, 0.8906 \mathrm{~g} / \mathrm{mL}$, $0.10 \mathrm{mmol}), \mathrm{CH}_{3} \mathrm{CN}(6.4 \mu \mathrm{L}, 0.6406 \mathrm{~g} / \mathrm{mL}, 0.10 \mathrm{mmol}), 1 \mathrm{~mL}$ of toluene, NaBHEt3 $(75 \mu \mathrm{L}, 0.075$ mmol). After 12 hours, the reaction afforded $\mathbf{2 c}$ with $>99 \%$ conversion ( $99 \%$ yield) as a colorless oil. Optical Rotation: $[\alpha]^{20}{ }_{\mathrm{D}}=-13.7\left(\mathrm{c} 1.52, \mathrm{CHCl}_{3}\right) .96 .5 \%$ ee, determined by HPLC after conversion to the corresponding phenol according to previously reported procedures, ${ }^{16}$ HPLC conditions: Chiralcel OJ-H, $n$-hexane $/ i-\mathrm{PrOH}=99 / 1,1.0 \mathrm{~mL} / \mathrm{min}, \mathrm{n}=220 \mathrm{~nm}$, tr 14.3 (minor ), 15.2 (major). IR (neat): 2959, 2931, 1608, 1510, $1257 \mathrm{~cm}^{-1} ;{ }^{1} \mathrm{H}$ NMR: $\left(400.0 \mathrm{MHz}, \mathrm{CDCl}_{3}\right) \delta 7.01(\mathrm{~d}, J$ $=8.0 \mathrm{~Hz}, 2 \mathrm{H}), 6.75(\mathrm{~d}, J=8.4 \mathrm{~Hz}, 2 \mathrm{H}), 2.58-2.47(\mathrm{~m}, 1 \mathrm{H}), 1.59-1.50(\mathrm{~m}, 2 \mathrm{H}), 1.20(\mathrm{~d}, J=6.8 \mathrm{~Hz}$, 3H), $0.98(\mathrm{~s}, 9 \mathrm{H}), 0.80(\mathrm{t}, J=7.2 \mathrm{~Hz}, 3 \mathrm{H}), 0.18(\mathrm{~s}, 6 \mathrm{H}) ;{ }^{13} \mathrm{C} \mathrm{NMR:}\left(100.0 \mathrm{MHz}, \mathrm{CDCl}_{3}\right) \delta 153.5$, $140.3,127.8,119.6,40.9,31.4,25.7,22.0,18.2,12.2,-4.4$; HRMS (EI) calculated for $\left[\mathrm{C}_{16} \mathrm{H}_{28} \mathrm{OSi}\right]^{+}$ requires $\mathrm{m} / \mathrm{z} 264.1909$, found $\mathrm{m} / \mathrm{z} 264.1906$.<smiles>CC[C@H](C)c1ccc(O)cc1</smiles>

(R)-4-(sec-butyl)phenol (2c-1). Be converted by the $2 \mathrm{c}$ according to previously reported procedures, ${ }^{16}$ IR (neat): $3370,2961,2927,1706,1514$ cm ${ }^{-1}$; ${ }^{1} \mathrm{H}$ NMR: $\left(400.0 \mathrm{MHz}, \mathrm{CDCl}_{3}\right) \delta 7.04(\mathrm{~d}, J=8.4 \mathrm{~Hz}, 2 \mathrm{H}), 6.76(\mathrm{~d}, J$ $=8.4 \mathrm{~Hz}, 2 \mathrm{H}), 4.93(\mathrm{~s}, 1 \mathrm{H}), 2.58-2.48(\mathrm{~m}, 1 \mathrm{H}), 1.59-1.49(\mathrm{~m}, 2 \mathrm{H}), 1.20(\mathrm{~d}, J=6.4 \mathrm{~Hz}, 3 \mathrm{H}), 0.80(\mathrm{t}$, $J=7.2 \mathrm{~Hz}, 3 \mathrm{H}) ;{ }^{13} \mathrm{C} \mathrm{NMR}:\left(100.0 \mathrm{MHz}, \mathrm{CDCl}_{3}\right) \delta 153.3,140.0,128.0,115.0,40.8,31.3,22.0,12.2$; HRMS (EI) calculated for $\left[\mathrm{C}_{10} \mathrm{H}_{14} \mathrm{O}\right]^{+}$requires m/z 150.1045, found m/z 150.1046 .<smiles>CCC(C)c1ccc(C)cc1</smiles>

(R)-1-(sec-butyl)-4-methylbenzene (2d). ${ }^{15}$ Prepared according to the general procedure: using $\mathbf{L e} \cdot \mathrm{FeCl}_{2}(0.0131 \mathrm{~g}, 0.025 \mathrm{mmol})$, 1d $(0.0731 \mathrm{~g}$, $0.50 \mathrm{mmol}), n-\mathrm{C}_{18} \mathrm{H}_{37} \mathrm{SiH}_{3}(32 \mu \mathrm{L}, 0.8906 \mathrm{~g} / \mathrm{mL}, 0.10 \mathrm{mmol}), \mathrm{CH}_{3} \mathrm{CN}(6.4$ $\mu \mathrm{L}, 0.6406 \mathrm{~g} / \mathrm{mL}, 0.10 \mathrm{mmol}), 1 \mathrm{~mL}$ of toluene, NaBHEt3 $(75 \mu \mathrm{L}, 0.075 \mathrm{mmol})$. After 12 hours, the reaction afforded $\mathbf{2 d}$ with $>99 \%$ conversion ( $95 \%$ yield) as a colorless oil. Optical Rotation: $[\alpha]^{20}{ }_{D}$ $=+3.5\left(\mathrm{c} 0.39, \mathrm{CHCl}_{3}\right) .\left\{\mathrm{Lit}^{15}\left[\alpha{ }^{23} \mathrm{D}=+25\left(\mathrm{c} 2.0, \mathrm{CHCl}_{3}\right) .88 \% e e\right\} .97 .3 \% e e\right.$, determined by GC, GC conditions: Chiralcel B-DM, $100 \mathrm{kPa} \mathrm{N} 2\left(50{ }^{\circ} \mathrm{C}-5 \mathrm{~min}-1{ }^{\circ} \mathrm{C} / \mathrm{min}-160{ }^{\circ} \mathrm{C}-5 \mathrm{~min}\right)$, tr 50.7 (minor), 51.5 (major). ${ }^{1} \mathrm{H}$ NMR: (400.0 MHz, $\left.\mathrm{CDCl}_{3}\right) \delta$ 7.11-7.08 (m, 4H), 2.60-2.50 (m, 1H), 2.32 (s, 3H), 1.62-1.51 (m, 2H), $1.22(\mathrm{~d}, J=6.8 \mathrm{~Hz}, 3 \mathrm{H}), 0.82(\mathrm{t}, J=7.2 \mathrm{~Hz}, 3 \mathrm{H})$. All the spectroscopic 
data were in agreement with the reported ones. ${ }^{14}$<smiles>CCC(C)c1ccc(N(C)C)cc1</smiles>

(R)-4-(sec-butyl)- $N, N$-dimethylaniline (2e). Prepared according to the general procedure: using $\mathbf{L e} \bullet \mathrm{FeCl}_{2}(0.0149 \mathrm{~g}, 0.025 \mathrm{mmol}), \mathbf{1 e}(0.0870 \mathrm{~g}$, $0.50 \mathrm{mmol}), n-\mathrm{C}_{18} \mathrm{H}_{37} \mathrm{SiH}_{3}(32 \mu \mathrm{L}, 0.8906 \mathrm{~g} / \mathrm{mL}, 0.10 \mathrm{mmol}), \mathrm{CH}_{3} \mathrm{CN}$ (6.4 $\mu \mathrm{L}, 0.6406 \mathrm{~g} / \mathrm{mL}, 0.10 \mathrm{mmol}), 1 \mathrm{~mL}$ of toluene, NaBHEt3 $(75 \mu \mathrm{L}, 0.075 \mathrm{mmol})$. After 12 hours, the reaction afforded $\mathbf{2 e}$ with $>99 \%$ conversion ( $94 \%$ yield) as a colorless oil. Optical Rotation: $[\alpha]^{20}$ $\mathrm{D}=-22.5\left(\mathrm{c} 1.46, \mathrm{CHCl}_{3}\right) .97 .9 \%$ ee, determined by HPLC, HPLC conditions: Chiralcel OJ-H, $n-$ hexane $/ \mathrm{i}-\mathrm{PrOH}=99 / 1,1.0 \mathrm{~mL} / \mathrm{min}, \mathrm{n}=220 \mathrm{~nm}, \operatorname{tr} 12.2$ (major), 14.0 (minor). IR (neat): 2959, 2925, 1616, 1567, 1485, $1269 \mathrm{~cm}^{-1}$; ${ }^{1} \mathrm{H}$ NMR: $\left(400.0 \mathrm{MHz}, \mathrm{CDCl}_{3}\right) \delta 7.06(\mathrm{~d}, J=7.6 \mathrm{~Hz}, 2 \mathrm{H}), 6.71(\mathrm{~d}, J$ $=7.6 \mathrm{~Hz}, 2 \mathrm{H}), 2.91(\mathrm{~s}, 6 \mathrm{H}), 2.52-2.47(\mathrm{~m}, 1 \mathrm{H}), 1.58-1.50(\mathrm{~m}, 2 \mathrm{H}), 1.20(\mathrm{~d}, J=7.2 \mathrm{~Hz}, 3 \mathrm{H}), 0.82(\mathrm{t}$, $J=7.2 \mathrm{~Hz}, 3 \mathrm{H}) ;{ }^{13} \mathrm{C} \mathrm{NMR}:\left(100.0 \mathrm{MHz}, \mathrm{CDCl}_{3}\right) \delta 149.0,136.0,127.6,112.9,40.9,40.6,31.3,22.0$, 12.3; HRMS (EI) calculated for $\left[\mathrm{C}_{12} \mathrm{H}_{19} \mathrm{~N}\right]^{+}$requires m/z 177.1517, found $\mathrm{m} / \mathrm{z}$ 177.1519.<smiles>CC[C@H](C)c1ccc(SC)cc1</smiles>

(R)-(4-(sec-butyl)phenyl)(methyl)sulfane (2f). Prepared according to the general procedure: using $\mathbf{L e} \cdot \mathrm{FeCl}_{2}(0.0142 \mathrm{~g}, 0.025 \mathrm{mmol}), \mathbf{1 f}(0.0888$ g, $0.50 \mathrm{mmol}), n-\mathrm{C}_{18} \mathrm{H}_{37} \mathrm{SiH}_{3}(32 \mu \mathrm{L}, 0.8906 \mathrm{~g} / \mathrm{mL}, 0.10 \mathrm{mmol}), \mathrm{CH}_{3} \mathrm{CN}$ ( $6.4 \mu \mathrm{L}, 0.6406 \mathrm{~g} / \mathrm{mL}, 0.10 \mathrm{mmol}), 1 \mathrm{~mL}$ of toluene, NaBHEt3 $(75 \mu \mathrm{L}, 0.075 \mathrm{mmol})$. After 12 hours, the reaction afforded $\mathbf{2 f}$ with $>99 \%$ conversion ( $72 \%$ yield) as a colorless oil. Optical Rotation: $[\alpha]^{20}$ $\mathrm{D}=-18.1\left(\mathrm{c} 1.03, \mathrm{CHCl}_{3}\right) .95 .1 \%$ ee, determined by GC, GC conditions: Chiralcel B-DM, $100 \mathrm{kPa}$ $\mathrm{N}_{2}\left(60{ }^{\circ} \mathrm{C}-40 \mathrm{~min}-1{ }^{\circ} \mathrm{C} / \mathrm{min}-175^{\circ} \mathrm{C}-5 \mathrm{~min}\right)$, tr 104.4 (minor), 104.9 (major). IR (neat): 2960 , 2923, 1561, 1494, 1458, $1144 \mathrm{~cm}^{-1} ;{ }^{1} \mathrm{H}$ NMR: $\left(400.0 \mathrm{MHz}, \mathrm{CDCl}_{3}\right) \delta 7.21(\mathrm{~d}, J=8.0 \mathrm{~Hz}, 2 \mathrm{H}), 8.3$ $(\mathrm{d}, J=8.0 \mathrm{~Hz}, 2 \mathrm{H}), 2.61-2.50(\mathrm{~m}, 1 \mathrm{H}), 2.47(\mathrm{~s}, 3 \mathrm{H}), 1.62-1.55(\mathrm{~m}, 2 \mathrm{H}), 1.21(\mathrm{~d}, J=6.8 \mathrm{~Hz}, 3 \mathrm{H})$, $0.81(\mathrm{t}, J=7.6 \mathrm{~Hz}, 3 \mathrm{H}) ;{ }^{13} \mathrm{C}$ NMR: $\left(100.0 \mathrm{MHz}, \mathrm{CDCl}_{3}\right) \delta 144.9,135.0,127.6,127.1,41.2,31.1$, 21.8, 16.3, 12.2; HRMS (EI) calculated for $\left[\mathrm{C}_{11} \mathrm{H}_{16} \mathrm{~S}\right]^{+}$requires m/z 180.0973, found $\mathrm{m} / \mathrm{z} 180.0974$.<smiles>CC[C@H](C)c1ccc(Cc2ccccc2)cc1</smiles>

(R)-2-(4-(sec-butyl)phenyl)-4,4,5,5-tetramethyl-1,3,2-dioxaborolane (2g). Prepared according to the general procedure: using $\mathbf{L e} \cdot \mathrm{FeCl}_{2}(0.0148$ g, $0.025 \mathrm{mmol}), 1 \mathrm{~g}(0.0660 \mathrm{~g}, 0.50 \mathrm{mmol}),{ }^{-} \mathrm{C}_{18} \mathrm{H}_{37} \mathrm{SiH}_{3}(32 \mu \mathrm{L}, 0.8906$ $\mathrm{g} / \mathrm{mL}, 0.10 \mathrm{mmol}), \mathrm{CH}_{3} \mathrm{CN}(6.4 \mu \mathrm{L}, 0.6406 \mathrm{~g} / \mathrm{mL}, 0.10 \mathrm{mmol}), 1 \mathrm{~mL}$ of toluene, NaBHEt3 $(75 \mu \mathrm{L}$, $0.075 \mathrm{mmol})$. After 12 hours, the reaction afforded $2 \mathrm{~g}$ with $>99 \%$ conversion $(95 \%$ yield) as a colorless oil. Optical Rotation: $[\alpha]^{20} \mathrm{D}=-2.1$ (c 1.18, $\left.\mathrm{CHCl}_{3}\right) .94 .4 \%$ ee, determined by GC, GC 
conditions: Chiralcel B-DM, $100 \mathrm{kPa} \mathrm{N} 2\left(110{ }^{\circ} \mathrm{C}-200 \mathrm{~min}-1{ }^{\circ} \mathrm{C} / \mathrm{min}-180{ }^{\circ} \mathrm{C}-5 \mathrm{~min}\right), \operatorname{tr} 211.2$ (minor), 214.6 (major). IR (neat): 2960, 2855, 1611, 1401, 1362, $1145 \mathrm{~cm}^{-1}$; ${ }^{1} \mathrm{H}$ NMR: (400.0 MHz, $\left.\mathrm{CDCl}_{3}\right) \delta 7.75(\mathrm{~d}, J=8.0 \mathrm{~Hz}, 2 \mathrm{H}), 7.19(\mathrm{~d}, J=8.0 \mathrm{~Hz}, 2 \mathrm{H}), 2.65-2.55(\mathrm{~m}, 1 \mathrm{H}), 1.65-1.55(\mathrm{~m}, 2 \mathrm{H})$, $1.33(\mathrm{~s}, 12 \mathrm{H}), 1.23(\mathrm{~d}, J=6.8 \mathrm{~Hz}, 3 \mathrm{H}), 0.81(\mathrm{t}, J=7.6 \mathrm{~Hz}, 3 \mathrm{H}) ;{ }^{13} \mathrm{C} \mathrm{NMR:}\left(100.0 \mathrm{MHz}, \mathrm{CDCl}_{3}\right) \delta$ 151.1, 134.8, 126.5, 83.6, 41.9, 31.0, 24.8, 21.7, 12.2; HRMS (EI) calculated for $\left[\mathrm{C}_{16} \mathrm{H}_{25} \mathrm{O}_{2} \mathrm{~B}\right]^{+}$ requires m/z 260.1948, found m/z 260.1949. CCDC number: 2055703. The X-Ray of $\mathbf{2 g}$ is below.

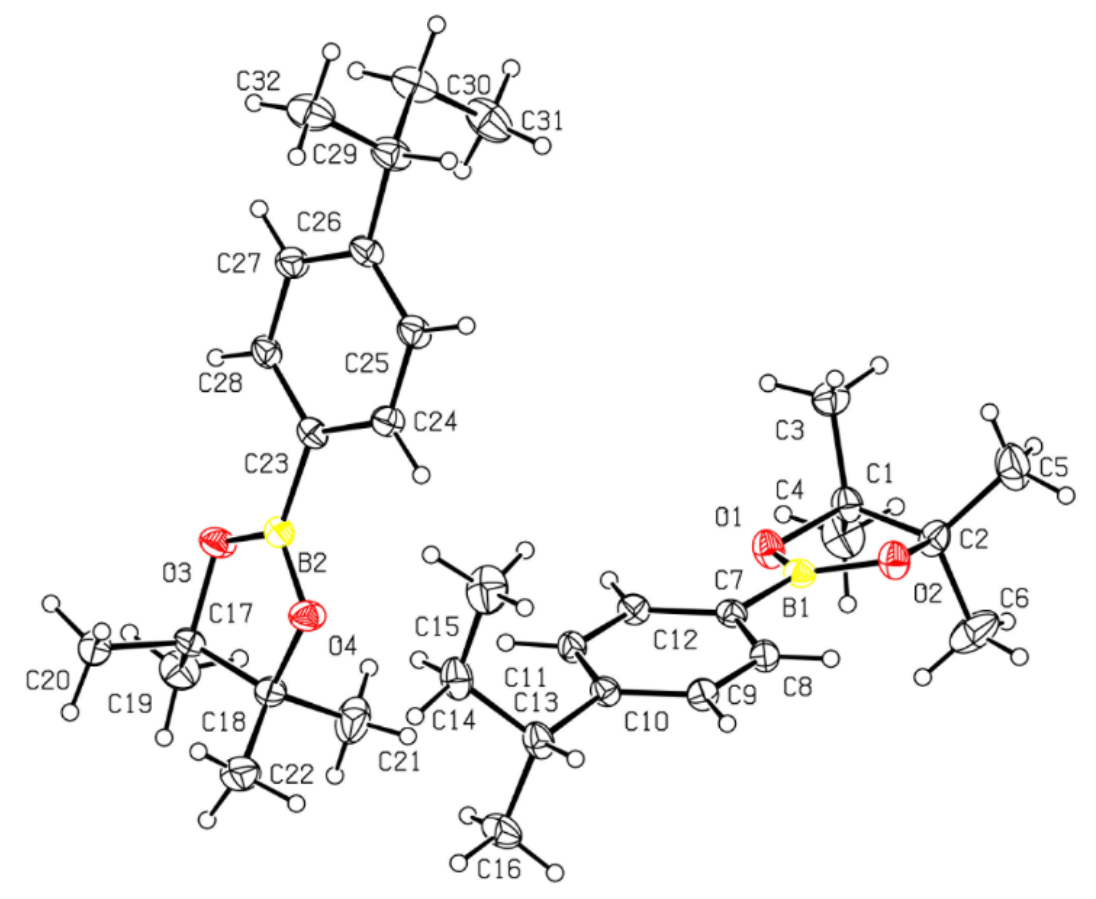<smiles>CC[C@H](C)c1ccc2c(c1)OCO2</smiles>

(R)-5-(sec-butyl)benzo[d][1,3]dioxole (2h). Prepared according to the general procedure: using $\mathbf{L e} \cdot \mathrm{FeCl}_{2}(0.0146 \mathrm{~g}, 0.025 \mathrm{mmol}), \mathbf{1 h}(0.0879 \mathrm{~g}$, $0.50 \mathrm{mmol}), n-\mathrm{C}_{18} \mathrm{H}_{37} \mathrm{SiH}_{3}(32 \mu \mathrm{L}, 0.8906 \mathrm{~g} / \mathrm{mL}, 0.10 \mathrm{mmol}), \mathrm{CH}_{3} \mathrm{CN}(6.4$ $\mu \mathrm{L}, 0.6406 \mathrm{~g} / \mathrm{mL}, 0.10 \mathrm{mmol}), 1 \mathrm{~mL}$ of toluene, NaBHEt3 $(75 \mu \mathrm{L}, 0.075 \mathrm{mmol})$. After 12 hours, the reaction afforded $\mathbf{2 h}$ with $>99 \%$ conversion ( $98 \%$ yield) as a colorless oil. Optical Rotation: $[\alpha]^{20} \mathrm{D}$ $=-12.8\left(\mathrm{c} 0.30, \mathrm{CHCl}_{3}\right) .98 .6 \%$ ee, determined by HPLC after conversion to the corresponding phenol according to previously reported procedures, ${ }^{17}$ HPLC conditions: Chiralcel OJ-H, $n$ hexane $/ i-\mathrm{PrOH}=98 / 2,0.8 \mathrm{~mL} / \mathrm{min}, \mathrm{n}=220 \mathrm{~nm}, \operatorname{tr} 84.6$ (minor), 86.5 (major). IR (neat): 2961, 2927, 1487, 1440, $1249 \mathrm{~cm}^{-1}$; ${ }^{1} \mathrm{H}$ NMR: $\left(400.0 \mathrm{MHz}, \mathrm{CDCl}_{3}\right) \delta 6.73(\mathrm{~d}, J=8.0 \mathrm{~Hz}, 1 \mathrm{H}), 6.68(\mathrm{~s}, 1 \mathrm{H}), 6.62$ $(\mathrm{d}, J=8.0 \mathrm{~Hz}, 1 \mathrm{H}), 5.92(\mathrm{~s}, 2 \mathrm{H}), 2.55-2.48(\mathrm{~m}, 1 \mathrm{H}), 1.64-1.48(\mathrm{~m}, 2 \mathrm{H}), 1.19(\mathrm{~d}, J=6.8 \mathrm{~Hz}, 3 \mathrm{H})$, $0.81(\mathrm{t}, J=7.2 \mathrm{~Hz}, 3 \mathrm{H}) ;{ }^{13} \mathrm{C}$ NMR: $\left(100.0 \mathrm{MHz}, \mathrm{CDCl}_{3}\right) \delta 147.5,145.4,141.7,119.9,107.9,107.2$, 100.7, 41.5, 31.3, 22.1, 12.2; HRMS (EI) calculated for $\left[\mathrm{C}_{11} \mathrm{H}_{14} \mathrm{O}_{2}\right]^{+}$requires $\mathrm{m} / \mathrm{z}$ 178.0994, found 
$\mathrm{m} / \mathrm{z} 178.0992$.<smiles>CC[C@H](C)c1ccc(O)c(O)c1</smiles>

(R)-4-(sec-butyl)benzene-1,2-diol (2h-1). Be converted by the $\mathbf{2 h}$ according to previously reported procedures ${ }^{17}$ as a colorless oil; IR (neat): 3650, 3551, 2962, 2871, $1261 \mathrm{~cm}^{-1}$; ${ }^{1} \mathrm{H}$ NMR: $\left(400.0 \mathrm{MHz}, \mathrm{CDCl}_{3}\right) \delta 6.78$ $(\mathrm{d}, J=8.0 \mathrm{~Hz}, 1 \mathrm{H}), 6.71(\mathrm{~d}, J=2.0 \mathrm{~Hz}, 1 \mathrm{H}), 6.62(\mathrm{~d}, J=8.4 \mathrm{~Hz}, 1 \mathrm{H}), 5.15(\mathrm{~s}, 1 \mathrm{H}), 5.06(\mathrm{~s}, 1 \mathrm{H})$, 2.54-2.41 (m, 1H), 1.57-1.49 (m, 2H), $1.18(\mathrm{~d}, J=6.8 \mathrm{~Hz}, 3 \mathrm{H}), 0.80(\mathrm{t}, J=7.2 \mathrm{~Hz}, 3 \mathrm{H}) ;{ }^{13} \mathrm{C}$ NMR: $\left(100.0 \mathrm{MHz}, \mathrm{CDCl}_{3}\right) \delta 143.3,141.2,141.1,119.5,115.2,114.0,41.0,31.2,22.0,12.2 ; \mathrm{HRMS}$ (EI) calculated for $\left[\mathrm{C}_{11} \mathrm{H}_{14} \mathrm{O}_{2}\right]^{+}$requires $\mathrm{m} / \mathrm{z} 166.0994$, found $\mathrm{m} / \mathrm{z} 166.0993$.<smiles>CC[C@H](C)c1ccc(F)cc1</smiles>

(R)-1-(sec-butyl)-4-fluorobenzene (2i). ${ }^{18}$ Prepared according to the general procedure: using $\mathbf{L e} \cdot \mathrm{FeCl}_{2}(0.0143 \mathrm{~g}, 0.025 \mathrm{mmol}), \mathbf{1 i}(0.0749 \mathrm{~g}, 0.50 \mathrm{mmol})$, $n-\mathrm{C}_{18} \mathrm{H}_{37} \mathrm{SiH}_{3}(32 \mu \mathrm{L}, 0.8906 \mathrm{~g} / \mathrm{mL}, 0.10 \mathrm{mmol}), \mathrm{CH}_{3} \mathrm{CN}(6.4 \mu \mathrm{L}, 0.6406$ $\mathrm{g} / \mathrm{mL}, 0.10 \mathrm{mmol}), 1 \mathrm{~mL}$ of toluene, NaBHEt3 $(75 \mu \mathrm{L}, 0.075 \mathrm{mmol})$. After 12 hours, the reaction afforded $2 \mathbf{i}$ with $91 \%$ hydrogenated conversion ( $84 \%$ yield, purification by $m$-CPBA, removal of olefin) as a colorless oil. Optical Rotation: $[\alpha]^{20} \mathrm{D}=-12.7\left(\mathrm{c} 1.25, \mathrm{CHCl}_{3}\right) \cdot\left\{\mathrm{Lit}^{18}{ }^{1}[\alpha]^{25} \mathrm{D}=+22.45\right.$ (c $0.44, \mathrm{CHCl}_{3}$ ), 96\% ee $.92 .1 \%$ ee, determined by GC, GC conditions: Chiralcel B-DM, $100 \mathrm{kPa}$ $\mathrm{N}_{2}\left(60{ }^{\circ} \mathrm{C}-30 \mathrm{~min}-2{ }^{\circ} \mathrm{C} / \mathrm{min}-180{ }^{\circ} \mathrm{C}-10 \mathrm{~min}\right.$, tr 26.1 (minor), 27.2 (major). ${ }^{1} \mathrm{H}$ NMR: (400.0 $\left.\mathrm{MHz}, \mathrm{CDCl}_{3}\right) \delta$ 7.17-7.08 (m, 2H), 7.00-6.90 (m, 2H), 2.63-2.53 (m, 1H), 1.61-1.51 (m, 2H), 1.21 $(\mathrm{d}, J=6.8 \mathrm{~Hz}, 3 \mathrm{H}), 0.80(\mathrm{t}, J=7.2 \mathrm{~Hz}, 3 \mathrm{H}) .{ }^{19} \mathrm{~F} \mathrm{NMR:}\left(367 \mathrm{MHz}, \mathrm{CDCl}_{3}\right) \delta-118.1$. All the spectroscopic data were in agreement with the reported ones. ${ }^{18}$<smiles>CC[C@H](C)c1ccc(Cl)cc1</smiles>

(R)-1-(sec-butyl)-4-chlorobenzene (2j). Prepared according to the general procedure: using $\mathbf{L e} \cdot \mathrm{FeCl}_{2}(0.0149 \mathrm{~g}, 0.025 \mathrm{mmol}), \mathbf{1 j}(0.0409 \mathrm{~g}, 0.25 \mathrm{mmol})$, $n-\mathrm{C}_{18} \mathrm{H}_{37} \mathrm{SiH}_{3}(32 \mu \mathrm{L}, 0.8906 \mathrm{~g} / \mathrm{mL}, 0.10 \mathrm{mmol}), \mathrm{CH}_{3} \mathrm{CN}(6.4 \mu \mathrm{L}, 0.6406$ $\mathrm{g} / \mathrm{mL}, 0.10 \mathrm{mmol}), 1 \mathrm{~mL}$ of toluene, NaBHEt3 $(75 \mu \mathrm{L}, 0.075 \mathrm{mmol})$. After 12 hours, the reaction afforded $\mathbf{2} \mathbf{j}$ with $>99 \%$ conversion ( $96 \%$ yield) as a colorless oil. Optical Rotation: $[\alpha]^{20}{ }_{D}=-3.2$ (c 1.37, $\left.\mathrm{CHCl}_{3}\right) .94 .3 \%$ ee, determined by GC, GC conditions: Chiralcel B-DM, $100 \mathrm{kPa} \mathrm{N} \mathrm{N}_{2}\left(60^{\circ} \mathrm{C}-\right.$ $90 \mathrm{~min}-2{ }^{\circ} \mathrm{C} / \mathrm{min}-180^{\circ} \mathrm{C}-10 \mathrm{~min}$, tr 102.2 (minor), 104.0 (major). IR (neat): 2958, 2925, 2855, 1493, 1461, $1378 \mathrm{~cm}^{-1}$; ${ }^{1} \mathrm{H}$ NMR: (400.0 MHz, $\left.\mathrm{CDCl}_{3}\right) \delta 7.25$ (d, $\left.J=8.4 \mathrm{~Hz}, 2 \mathrm{H}\right), 7.10$ (d, $J=8.4$ $\mathrm{Hz}, 2 \mathrm{H}), 2.62-2.50(\mathrm{~m}, 1 \mathrm{H}), 1.62-1.52(\mathrm{~m}, 2 \mathrm{H}), 1.21(\mathrm{~d}, J=6.8 \mathrm{~Hz}, 3 \mathrm{H}), 0.80(\mathrm{t}, J=7.2 \mathrm{~Hz}, 3 \mathrm{H})$; ${ }^{13} \mathrm{C}$ NMR: $\left(100.0 \mathrm{MHz}, \mathrm{CDCl}_{3}\right) \delta$ 146.1, 131.3, 128.4, 128.3, 41.1, 31.1, 21.8, 12.1; HRMS (EI) 
calculated for $\left[\mathrm{C}_{10} \mathrm{H}_{13} \mathrm{Cl}\right]^{+}$requires $\mathrm{m} / \mathrm{z} 168.0706$, found $\mathrm{m} / \mathrm{z} 168.0706$.<smiles>CC[C@H](C)c1ccc(Br)cc1</smiles>

(R)-1-bromo-4-(sec-butyl)benzene (2k). ${ }^{18}$ Prepared according to the general procedure: using $\mathbf{L e} \cdot \mathrm{FeCl}_{2}(0.0149 \mathrm{~g}, 0.025 \mathrm{mmol}), \mathbf{1 k}(0.0520 \mathrm{~g}$, $0.25 \mathrm{mmol}), n-\mathrm{C}_{18} \mathrm{H}_{37} \mathrm{SiH}_{3}(32 \mu \mathrm{L}, 0.8906 \mathrm{~g} / \mathrm{mL}, 0.10 \mathrm{mmol}), \mathrm{CH}_{3} \mathrm{CN}(6.4$ $\mu \mathrm{L}, 0.6406 \mathrm{~g} / \mathrm{mL}, 0.10 \mathrm{mmol}), 1 \mathrm{~mL}$ of toluene, NaBHEt3 $(75 \mu \mathrm{L}, 0.075 \mathrm{mmol})$. After 12 hours, the reaction afforded $\mathbf{2 k}$ with $>99 \%$ conversion ( $95 \%$ yield) as a colorless oil. Optical Rotation: $[\alpha]^{20} \mathrm{D}$ $=-7.9\left(\mathrm{c} 1.02, \mathrm{CHCl}_{3}\right) .\left\{\mathrm{Lit}^{18}[\alpha]^{25} \mathrm{D}=+22.05\left(\mathrm{c} 0.45, \mathrm{CHCl}_{3}\right), 94 \% e e\right\} .93 .8 \% e e$, determined by $\mathrm{GC}$, GC conditions: Chiralcel B-DM, $100 \mathrm{kPa} \mathrm{N}\left(70{ }^{\circ} \mathrm{C}-90 \min -3{ }^{\circ} \mathrm{C} / \mathrm{min}-175{ }^{\circ} \mathrm{C}-5 \mathrm{~min}\right), \mathrm{tr}_{\mathrm{r}}$ 103.3 (minor), 104.3 (major). ${ }^{1} \mathrm{H}$ NMR: $\left(400.0 \mathrm{MHz}, \mathrm{CDCl}_{3}\right) \delta 7.40(\mathrm{~d}, J=8.4 \mathrm{~Hz}, 2 \mathrm{H}), 7.05(\mathrm{~d}, J$ $=8.4 \mathrm{~Hz}, 2 \mathrm{H}), 2.61-2.52(\mathrm{~m}, 1 \mathrm{H}), 1.61-1.52(\mathrm{~m}, 2 \mathrm{H}), 1.21(\mathrm{~d}, J=6.8 \mathrm{~Hz}, 3 \mathrm{H}), 0.80(\mathrm{t}, J=7.2 \mathrm{~Hz}$, $3 \mathrm{H})$. All the spectroscopic data were in agreement with the reported ones. ${ }^{18}$<smiles>CC[C@H](C)c1cccc(OC)c1</smiles>

(R)-1-(sec-butyl)-3-methoxybenzene (2l). Prepared according to the general procedure: using $\mathbf{L e} \cdot \mathrm{FeCl}_{2}(0.0150 \mathrm{~g}, 0.025 \mathrm{mmol}), \mathbf{1 l}$ (0.0807 g, $0.50 \mathrm{mmol}), n-\mathrm{C}_{18} \mathrm{H}_{37} \mathrm{SiH}_{3}(32 \mu \mathrm{L}, 0.8906 \mathrm{~g} / \mathrm{mL}, 0.10 \mathrm{mmol}), \mathrm{CH}_{3} \mathrm{CN}(6.4$ $\mu \mathrm{L}, 0.6406 \mathrm{~g} / \mathrm{mL}, 0.10 \mathrm{mmol}), 1 \mathrm{~mL}$ of toluene, NaBHEt $3(75 \mu \mathrm{L}, 0.075 \mathrm{mmol})$. After 12 hours, the reaction afforded $\mathbf{2 l}$ with $>99 \%$ conversion $\left(96 \%\right.$ yield) as a colorless oil. Optical Rotation: $[\alpha]^{20} \mathrm{D}$ $=-21.4\left(\mathrm{c} 0.97, \mathrm{CHCl}_{3}\right) .95 .4 \%$ ee, determined by HPLC after conversion to the corresponding phenol according to previously reported procedures. ${ }^{17}$ HPLC conditions: Chiralcel OJ-H x 2, $n$ hexane $/ i-\mathrm{PrOH}=90 / 10,1.0 \mathrm{~mL} / \mathrm{min}, \mathrm{n}=220 \mathrm{~nm}, \operatorname{tr} 13.0$ (major), 13.7 (minor). IR (neat): 2960, 2922, 1604, 1487, 1458, $1261 \mathrm{~cm}^{-1}$; ${ }^{1} \mathrm{H}$ NMR: $\left(400.0 \mathrm{MHz}, \mathrm{CDCl}_{3}\right) \delta 7.21(\mathrm{t}, J=7.8 \mathrm{~Hz}, 1 \mathrm{H}), 6.78-$ $6.71(\mathrm{~m}, 3 \mathrm{H}), 3.80(\mathrm{~s}, 3 \mathrm{H}), 2.61-2.53(\mathrm{~m}, 1 \mathrm{H}), 1.65-1.00(\mathrm{~m}, 2 \mathrm{H}), 1.23(\mathrm{~d}, J=6.8 \mathrm{~Hz}, 3 \mathrm{H}), 0.83(\mathrm{t}$, $J=7.2 \mathrm{~Hz}, 3 \mathrm{H}) ;{ }^{13} \mathrm{C}$ NMR: $\left(100.0 \mathrm{MHz}, \mathrm{CDCl}_{3}\right) \delta 159.6,149.5,129.1,119.5,113.0,110.7,55.1$, 41.7, 31.1, 21.8, 12.2; HRMS (EI) calculated for $\left[\mathrm{C}_{11} \mathrm{H}_{16} \mathrm{O}\right]^{+}$requires $\mathrm{m} / \mathrm{z} 164.1201$, found $\mathrm{m} / \mathrm{z}$ 164.1203.<smiles>CC[C@H](C)c1cccc(O)c1</smiles>

(R)-3-(sec-butyl)phenol (2l-1) Be converted by the $2 \mathrm{l}$ according to previously reported procedures, ${ }^{17}$ IR (neat): 3330, 2961, 2926, 1592, 1456, $1170 \mathrm{~cm}^{-1} ;{ }^{1} \mathrm{H}$ NMR: $\left(400.0 \mathrm{MHz}, \mathrm{CDCl}_{3}\right) \delta 7.15(\mathrm{t}, J=7.8 \mathrm{~Hz}, 1 \mathrm{H}), 6.77-$ $6.62(\mathrm{~m}, 3 \mathrm{H}), 4.66(\mathrm{~s}, 1 \mathrm{H}), 2.59-2.51(\mathrm{~m}, 1 \mathrm{H}), 1.61-1.52(\mathrm{~m}, 2 \mathrm{H}), 1.21(\mathrm{~d}, J=7.6 \mathrm{~Hz}, 3 \mathrm{H}), 0.82(\mathrm{t}$, $J=7.2 \mathrm{~Hz}, 3 \mathrm{H}) ;{ }^{13} \mathrm{C} \mathrm{NMR}:\left(100.0 \mathrm{MHz}, \mathrm{CDCl}_{3}\right) \delta 155.4,149.8,129.3,119.7,113.9,112.6,41.6$, 
31.0, 21.7, 12.2; HRMS (EI) calculated for $\left[\mathrm{C}_{10} \mathrm{H}_{14} \mathrm{O}\right]^{+}$requires $\mathrm{m} / \mathrm{z} 150.1045$, found $\mathrm{m} / \mathrm{z} 150.1046$.<smiles>CC[C@H](C)c1cccc(OC(C)(C)C)c1</smiles>

(R)-tert-butyl(3-(sec-butyl)phenoxy)dimethylsilane (2m). Prepared according to the general procedure: using $\mathbf{L e} \cdot \mathrm{FeCl}_{2}(0.0146 \mathrm{~g}, 0.025$ mmol), $1 \mathbf{m}(0.1309 \mathrm{~g}, 0.50 \mathrm{mmol}),{ }-\mathrm{C}_{18} \mathrm{H}_{37} \mathrm{SiH}_{3}(32 \mu \mathrm{L}, 0.8906 \mathrm{~g} / \mathrm{mL}$, $0.10 \mathrm{mmol}), \mathrm{CH}_{3} \mathrm{CN}(6.4 \mu \mathrm{L}, 0.6406 \mathrm{~g} / \mathrm{mL}, 0.10 \mathrm{mmol}), 1 \mathrm{~mL}$ of toluene, NaBHEt3 $(75 \mu \mathrm{L}, 0.075$ mmol). After 12 hours, the reaction afforded $\mathbf{2 m}$ with $>99 \%$ conversion ( $96 \%$ yield) as a colorless oil. Optical Rotation: $[\alpha]^{20}{ }_{\mathrm{D}}=-15.8\left(\mathrm{c} 0.84, \mathrm{CHCl}_{3}\right) .95 .1 \%$ ee, determined by HPLC after conversion to the corresponding phenol according to previously reported procedures, ${ }^{16}$ HPLC conditions: Chiralcel OJ-H x 2, n-hexane $/ i-\mathrm{PrOH}=90 / 10,1.0 \mathrm{~mL} / \mathrm{min}, \mathrm{n}=220 \mathrm{~nm}, \operatorname{tr} 12.5$ (major), 13.0 (minor). IR (neat): 2959, 2859, 1602, 1583, 1483, $1277 \mathrm{~cm}^{-1}$; ${ }^{1} \mathrm{H}$ NMR: $\left(400.0 \mathrm{MHz}, \mathrm{CDCl}_{3}\right)$ $\delta$ 7.16-7.06 (m, 1H), $6.77(\mathrm{~d}, J=7.6 \mathrm{~Hz}, 1 \mathrm{H}), 6.68-6.46(\mathrm{~m}, 1 \mathrm{H}), 2.60-2.48(\mathrm{~m}, 1 \mathrm{H}), 1.61-1.52(\mathrm{~m}$, 2H), $1.21(\mathrm{~d}, J=6.8 \mathrm{~Hz}, 3 \mathrm{H}), 0.98(\mathrm{~s}, 9 \mathrm{H}), 0.81$ (t, $J=7.2 \mathrm{~Hz}, 3 \mathrm{H}), 0.19(\mathrm{~s}, 6 \mathrm{H}) ;{ }^{13} \mathrm{C}$ NMR: $(100.0$ $\left.\mathrm{MHz}, \mathrm{CDCl}_{3}\right) \delta 155.6,149.3,129.0,120.2,118.8,117.4,41.6,31.1,25.7,21.8,18.2,12.2,-4.4$; HRMS (EI) calculated for $\left[\mathrm{C}_{16} \mathrm{H}_{28} \mathrm{OSi}\right]^{+}$requires $\mathrm{m} / \mathrm{z} 264.1909$, found $\mathrm{m} / \mathrm{z} 264.1909$.

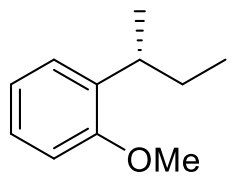

(R)-1-(sec-butyl)-2-methoxybenzene (2n). Prepared according to the general procedure: using Le• $\mathrm{FeCl}_{2}(0.0149 \mathrm{~g}, 0.025 \mathrm{mmol}), \mathbf{1 n}(0.0447 \mathrm{~g}, 0.25 \mathrm{mmol}), n$ $\mathrm{C}_{18} \mathrm{H}_{37} \mathrm{SiH}_{3}(16 \mu \mathrm{L}, 0.8906 \mathrm{~g} / \mathrm{mL}, 0.05 \mathrm{mmol}), \mathrm{CH}_{3} \mathrm{CN}(6.4 \mu \mathrm{L}, 0.6406 \mathrm{~g} / \mathrm{mL}$, $0.10 \mathrm{mmol}), 1 \mathrm{~mL}$ of toluene, NaBHEt3 $(75 \mu \mathrm{L}, 0.075 \mathrm{mmol})$. After 12 hours, the reaction afforded 2n with $93 \%$ conversion ( $86 \%$ yield, purification by $m$-CPBA, removal of olefin) as a colorless oil. Optical Rotation: $[\alpha]^{20}{ }_{\mathrm{D}}=-1.4\left(\mathrm{c} 0.51, \mathrm{CHCl}_{3}\right) .77 .4 \% e e$, determined by HPLC after conversion to the corresponding phenol according to previously reported procedures, ${ }^{17}$ HPLC conditions: Chiralcel OJ-H, $n$-hexane $/ i-\mathrm{PrOH}=90 / 10,1.0 \mathrm{~mL} / \mathrm{min}, \mathrm{n}=220 \mathrm{~nm}$, tr 7.8 (major), 9.0 (minor). IR (neat): 2921, 2853, 1511, 1464, 1380, $1246 \mathrm{~cm}^{-1}$; ${ }^{1} \mathrm{H}$ NMR: (400.0 MHz, $\left.\mathrm{CDCl}_{3}\right) \delta$ 7.19-7.12 (m, 2H), 6.95-6.82 (m, 2H), $3.81(\mathrm{~s}, 3 \mathrm{H}), 3.16-3.04(\mathrm{~m}, 1 \mathrm{H}), 1.70-1.54(\mathrm{~m}, 2 \mathrm{H}), 1.19(\mathrm{~d}, J=6.8 \mathrm{~Hz}$, 3H), $0.84(\mathrm{t}, J=6.8 \mathrm{~Hz}, 3 \mathrm{H}) ;{ }^{13} \mathrm{C} \mathrm{NMR}:\left(100.6 \mathrm{MHz}, \mathrm{CDCl}_{3}\right) \delta 157.1,135.9,126.7,126.4,120.5$, 110.4, 55.3, 33.4, 29.8, 20.4, 12.1; HRMS (EI) calculated for $\left[\mathrm{C}_{11} \mathrm{H}_{16} \mathrm{O}\right]^{+}$requires $\mathrm{m} / \mathrm{z}$ 164.1201, found $\mathrm{m} / \mathrm{z} 164.1202$. 
<smiles>CCC(C)c1ccc2cc(OC)ccc2c1</smiles>

(R)-2-(sec-butyl)-6-methoxynaphthalene

(20). ${ }^{19} \quad$ Prepared according to the general procedure: using $\mathbf{L e} \cdot \mathrm{FeCl}_{2}(0.0175 \mathrm{~g}, 0.030$ mmol), $10(0.1060$ g, $0.50 \mathrm{mmol}), n-\mathrm{C}_{18} \mathrm{H}_{37} \mathrm{SiH}_{3}(32 \mu \mathrm{L}, 0.8906$ $\mathrm{g} / \mathrm{mL}, 0.10 \mathrm{mmol}), \mathrm{CH}_{3} \mathrm{CN}(6.4 \mu \mathrm{L}, 0.6406 \mathrm{~g} / \mathrm{mL}, 0.10 \mathrm{mmol}), 1 \mathrm{~mL}$ of toluene, NaBHEt3 $(90 \mu \mathrm{L}$, $0.090 \mathrm{mmol}$ ). After 12 hours, the reaction afforded $2 \mathrm{o}$ with $91 \%$ conversion ( $84 \%$ yield, purification by $m$-CPBA, removal of olefin) as a colorless oil. Optical Rotation: $[\alpha]^{20}{ }_{\mathrm{D}}=-24.4\left(\mathrm{c} 0.44, \mathrm{CHCl}_{3}\right)$. $\left\{\right.$ Lit. $\left.^{18}[\alpha]^{23} \mathrm{D}=-26.0\left(\mathrm{c} 0.97, \mathrm{CHCl}_{3}\right), 85 \% e e\right\}, 96.1 \% e e$, determined by HPLC, HPLC conditions: Chiralcel OJ-H , $n$-hexane $/ i$-PrOH $=99.5 / 0.5,1.0 \mathrm{~mL} / \mathrm{min}, \mathrm{n}=220 \mathrm{~nm}, \operatorname{tr} 16.9$ (minor), 17.9 (major). ${ }^{1} \mathrm{H}$ NMR: (400.0 MHz, $\left.\mathrm{CDCl}_{3}\right) \delta$ 7.72-7.68 (m, 2H), $7.53(\mathrm{~s}, 1 \mathrm{H}), 7.31(\mathrm{dd}, J=8.8,1.6 \mathrm{~Hz}, 1 \mathrm{H})$, 7.18-7.09 (m, 2H), $3.90(\mathrm{~s}, 3 \mathrm{H}), 2.78-2.66(\mathrm{~m}, 1 \mathrm{H}), 1.73-1.61(\mathrm{~m}, 2 \mathrm{H}), 1.31(\mathrm{~d}, J=6.8 \mathrm{~Hz}, 3 \mathrm{H})$, $0.84(\mathrm{t}, J=7.6 \mathrm{~Hz}, 3 \mathrm{H}) .{ }^{13} \mathrm{C} \mathrm{NMR}:\left(100.0 \mathrm{MHz}, \mathrm{CDCl}_{3}\right) \delta 157.1,142.8,133.1,129.1,129.0,126.7$, $126.3,125.0,118.5,105.6,55.2,41.6,31.1,21.9,12.3$. All the spectroscopic data were in agreement with the reported ones. ${ }^{19}$

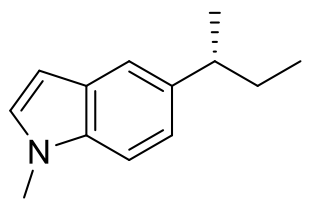

(R)-5-(sec-butyl)-1-methyl-1H-indole (2p). Prepared according to the general procedure: using $\mathbf{L e} \cdot \mathrm{FeCl}_{2}(0.0166 \mathrm{~g}, 0.030 \mathrm{mmol}), \mathbf{1 p}(0.0922 \mathrm{~g}$, $0.50 \mathrm{mmol}), n-\mathrm{C}_{18} \mathrm{H}_{37} \mathrm{SiH}_{3}(32 \mu \mathrm{L}, 0.8906 \mathrm{~g} / \mathrm{mL}, 0.10 \mathrm{mmol}), \mathrm{CH}_{3} \mathrm{CN}(6.4$ $\mu \mathrm{L}, 0.6406 \mathrm{~g} / \mathrm{mL}, 0.10 \mathrm{mmol}), 1 \mathrm{~mL}$ of toluene, NaBHEt3 $(90 \mu \mathrm{L}, 0.090 \mathrm{mmol})$. After 12 hours, the reaction afforded $\mathbf{2 p}$ with $>99 \%$ conversion ( $94 \%$ yield) as a colorless oil. Optical Rotation: $[\alpha]^{20} \mathrm{D}$ $=-16.0\left(\mathrm{c} 1.11, \mathrm{CHCl}_{3}\right) .97 .9 \%$ ee, determined by HPLC, HPLC conditions: Chiralcel OJ-H x 2, $n-$ hexane $/ i-\operatorname{PrOH}=95 / 5,1.0 \mathrm{~mL} / \mathrm{min}, \mathrm{n}=220 \mathrm{~nm}, \operatorname{tr} 41.4$ (major), 45.4 (minor). IR (neat): 2958, 2919, 1513, 1490 1450, $1352 \mathrm{~cm}^{-1} ;{ }^{1} \mathrm{H}$ NMR: (400.0 MHz, $\left.\mathrm{CDCl}_{3}\right) \delta$ 7.42 (s, 1H), 7.28-7.23 (m, 1H), 7.07 $(\mathrm{d}, J=7.6 \mathrm{~Hz}, 1 \mathrm{H}), 7.01(\mathrm{~d}, J=2.8 \mathrm{~Hz}, 1 \mathrm{H}), 6.42(\mathrm{~d}, J=2.8 \mathrm{~Hz}, 1 \mathrm{H}), 3.77(\mathrm{~s}, 3 \mathrm{H}), 2.74-2.63(\mathrm{~m}$, 1H), 1.71-1.59 (m, 2H), $1.29(\mathrm{~d}, J=6.8 \mathrm{~Hz}, 3 \mathrm{H}), 0.83(\mathrm{t}, J=7.2 \mathrm{~Hz}, 3 \mathrm{H}) ;{ }^{13} \mathrm{C}$ NMR: $(100.0 \mathrm{MHz}$, $\left.\mathrm{CDCl}_{3}\right) \delta 138.6,135.4,128.7,128.5,121.1,118.6,108.8,100.5,41.7,32.7,31.6,22.6,12.4$; HRMS (EI) calculated for $\left[\mathrm{C}_{13} \mathrm{H}_{17} \mathrm{~N}\right]^{+}$requires $\mathrm{m} / \mathrm{z}$ 187.1361, found $\mathrm{m} / \mathrm{z} 187.1362$.

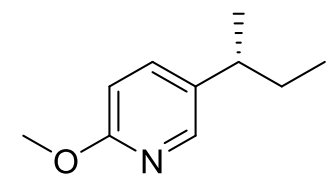

(R)-5-(sec-butyl)-2-methoxypyridine (2q). Prepared according to the general procedure: using $\mathbf{L e} \cdot \mathrm{FeCl}_{2}(0.0149 \mathrm{~g}, 0.025 \mathrm{mmol}), \mathbf{1 q}(0.0409 \mathrm{~g}$, $0.25 \mathrm{mmol}), n-\mathrm{C}_{18} \mathrm{H}_{37} \mathrm{SiH}_{3}(32 \mu \mathrm{L}, 0.8906 \mathrm{~g} / \mathrm{mL}, 0.10 \mathrm{mmol}), \mathrm{CH}_{3} \mathrm{CN}(6.4$ $\mu \mathrm{L}, 0.6406 \mathrm{~g} / \mathrm{mL}, 0.10 \mathrm{mmol}), 1 \mathrm{~mL}$ of toluene, $\operatorname{NaBHEt}_{3}(75 \mu \mathrm{L}, 0.075 \mathrm{mmol})$. After 12 hours, the 
reaction afforded $2 \mathbf{q}$ with $99 \%$ conversion (95\% yield) as a colorless oil. Optical Rotation: $[\alpha]^{20} \mathrm{D}=$ -15.3 (c $\left.0.88, \mathrm{CHCl}_{3}\right) .70 \%$ ee, determined by GC, GC conditions: Chiralcel B-DM, $100 \mathrm{kPa} \mathrm{N}_{2}(60$ ${ }^{\circ} \mathrm{C}-30 \mathrm{~min}-2{ }^{\circ} \mathrm{C} / \min -180^{\circ} \mathrm{C}-5 \mathrm{~min}, \operatorname{tr} 55.3$ (minor), 55.8 (major). IR (neat): 2958, 2925, 2855, 1653, 1494, $1285 \mathrm{~cm}^{-1}$; ${ }^{1} \mathrm{H}$ NMR: $\left(400.0 \mathrm{MHz}, \mathrm{CDCl}_{3}\right) \delta 7.96(\mathrm{~s}, 1 \mathrm{H}), 7.40(\mathrm{~d}, J=6.4 \mathrm{~Hz}, 1 \mathrm{H})$, $6.69(\mathrm{~d}, J=8.4 \mathrm{~Hz}, 1 \mathrm{H}), 3.92(\mathrm{~s}, 3 \mathrm{H}), 2.64-2.52(\mathrm{~m}, 1 \mathrm{H}), 1.65-1.42(\mathrm{~m}, 2 \mathrm{H}), 1.22(\mathrm{~d}, J=6.8 \mathrm{~Hz}$, $3 \mathrm{H}), 0.82(\mathrm{t}, J=7.6 \mathrm{~Hz}, 3 \mathrm{H}) ;{ }^{13} \mathrm{C} \mathrm{NMR}:\left(100.0 \mathrm{MHz}, \mathrm{CDCl}_{3}\right) \delta 162.7,145.2,137.2,135.2,110.5$, 53.2, 38.3, 31.0, 21.7, 12.1; HRMS (EI) calculated for $\left[\mathrm{C}_{10} \mathrm{H}_{15} \mathrm{NO}\right]^{+}$requires $\mathrm{m} / \mathrm{z} 165.1154$, found $\mathrm{m} / \mathrm{z} 165.1154$.<smiles>COc1ccc(C(C)C(C)(C)C)cc1</smiles>

(R)-1-methoxy-4-(pentan-2-yl)benzene (2r). Prepared according to the general procedure: using $\mathbf{L e} \cdot \mathrm{FeCl}_{2}(0.0147 \mathrm{~g}, 0.025 \mathrm{mmol}), \mathbf{1 r}(0.0887 \mathrm{~g}$, $0.50 \mathrm{mmol}), n-\mathrm{C}_{18} \mathrm{H}_{37} \mathrm{SiH}_{3}(32 \mu \mathrm{L}, 0.8906 \mathrm{~g} / \mathrm{mL}, 0.10 \mathrm{mmol}), \mathrm{CH}_{3} \mathrm{CN}(6.4$ $\mu \mathrm{L}, 0.6406 \mathrm{~g} / \mathrm{mL}, 0.10 \mathrm{mmol}), 1 \mathrm{~mL}$ of toluene, NaBHEt3 $(75 \mu \mathrm{L}, 0.075 \mathrm{mmol})$. After 12 hours, the reaction afforded $\mathbf{2 r}$ with $>99 \%$ conversion (95\% yield) as a colorless oil. Optical Rotation: $[\alpha]^{20} \mathrm{D}$ $=-7.2\left(\mathrm{c} 0.96, \mathrm{CHCl}_{3}\right) .98 .4 \%$ ee, determined by GC, GC conditions: Chiralcel B-DM, $100 \mathrm{kPa} \mathrm{N}_{2}$ $\left(60{ }^{\circ} \mathrm{C}-30 \mathrm{~min}-2{ }^{\circ} \mathrm{C} / \mathrm{min}-180{ }^{\circ} \mathrm{C}-5 \mathrm{~min}\right.$ ), tr 60.7 (minor), 60.9 (major). IR (neat): 2957, 2928 , 2870, 1613, 1513, $1247 \mathrm{~cm}^{-1}$; ${ }^{1} \mathrm{H}$ NMR: $\left(400.0 \mathrm{MHz}, \mathrm{CDCl}_{3}\right) \delta 7.10(\mathrm{~d}, J=7.6 \mathrm{~Hz}, 2 \mathrm{H}), 6.83(\mathrm{~d}, J$ $=7.6 \mathrm{~Hz}, 2 \mathrm{H}), 3.78(\mathrm{~s}, 3 \mathrm{H}), 2.70-2.58(\mathrm{~m}, 1 \mathrm{H}), 1.59-1.45(\mathrm{~m}, 2 \mathrm{H}), 1.35-1.16(\mathrm{~m}, 5 \mathrm{H}), 0.86(\mathrm{t}, J=$ 7.6 Hz, 3H); ${ }^{13} \mathrm{C}$ NMR: (100.0 MHz, $\left.\mathrm{CDCl}_{3}\right) \delta 157.6,140.0,127.8,113.6,55.2,40.9,38.8,22.5$, 20.8, 14.1; HRMS (EI) calculated for $\left[\mathrm{C}_{12} \mathrm{H}_{18} \mathrm{O}\right]^{+}$requires $\mathrm{m} / \mathrm{z}$ 178.1358, found $\mathrm{m} / \mathrm{z} 178.1358$.<smiles>COc1ccc(C(C)C(C)(C)C)cc1</smiles>

(R)-1-(hexan-2-yl)-4-methoxybenzene (2s). Prepared according to the general procedure: using $\mathbf{L e} \cdot \mathrm{FeCl}_{2}(0.0167 \mathrm{~g}, 0.025 \mathrm{mmol}), \mathbf{1 s}(0.0950 \mathrm{~g}$, $0.50 \mathrm{mmol}), n-\mathrm{C}_{18} \mathrm{H}_{37} \mathrm{SiH}_{3}(32 \mu \mathrm{L}, 0.8906 \mathrm{~g} / \mathrm{mL}, 0.10 \mathrm{mmol}), \mathrm{CH}_{3} \mathrm{CN}(6.4$ $\mu \mathrm{L}, 0.6406 \mathrm{~g} / \mathrm{mL}, 0.10 \mathrm{mmol}), 1 \mathrm{~mL}$ of toluene, NaBHEt3 $(75 \mu \mathrm{L}, 0.075 \mathrm{mmol})$. After 12 hours, the reaction afforded $\mathbf{2 s}$ with $>99 \%$ conversion (97\% yield) as a colorless oil. Optical Rotation: $[\alpha]^{20} \mathrm{D}$ $=-27.4\left(\mathrm{c} 0.89, \mathrm{CHCl}_{3}\right) .93 .9 \%$ ee, determined by HPLC after conversion to the corresponding phenol according to previously reported procedures, ${ }^{17}$ HPLC conditions: Chiralcel OJ-H, $n$ hexane $/ i-\mathrm{PrOH}=97 / 3,0.8 \mathrm{~mL} / \mathrm{min}, \mathrm{n}=220 \mathrm{~nm}, \operatorname{tr} 23.5$ (minor), 25.1 (major). IR (neat): 2957, 2927 , 1611, 1512, 1461, $1247 \mathrm{~cm}^{-1}$; ${ }^{1} \mathrm{H}$ NMR: $\left(400.0 \mathrm{MHz}, \mathrm{CDCl}_{3}\right) \delta 7.10$ (d, $\left.J=8.8 \mathrm{~Hz}, 2 \mathrm{H}\right), 6.83(\mathrm{~d}, J$ $=8.8 \mathrm{~Hz}, 2 \mathrm{H}), 3.79(\mathrm{~s}, 3 \mathrm{H}), 2.67-2.57(\mathrm{~s}, 1 \mathrm{H}), 1.56-1.48(\mathrm{~m}, 2 \mathrm{H}), 1.34-1.07(\mathrm{~m}, 7 \mathrm{H}), 0.85(\mathrm{t}, J=$ 
$6.8 \mathrm{~Hz}, 3 \mathrm{H}) ;{ }^{13} \mathrm{C} \mathrm{NMR}:\left(100.0 \mathrm{MHz}, \mathrm{CDCl}_{3}\right) \delta 157.6,140.1,127.8,113.6,55.2,39.0,38.3,29.9$, 22.8, 22.5, 14.0; HRMS (EI) calculated for $\left[\mathrm{C}_{13} \mathrm{H}_{20} \mathrm{O}\right]^{+}$requires $\mathrm{m} / \mathrm{z} 192.1514$, found $\mathrm{m} / \mathrm{z} 192.1514$. (1) (R)-4-(hexan-2-yl)phenol (2s-1). Be converted by the $2 \mathrm{~s}$ according to previously reported procedures, ${ }^{17}$ IR (neat): 3331, 2957, 2924, 1611 1513, $1451 \mathrm{~cm}^{-1} ;{ }^{1} \mathrm{H}$ NMR: $\left(400.0 \mathrm{MHz}, \mathrm{CDCl}_{3}\right) \delta 7.04(\mathrm{~d}, J=8.4 \mathrm{~Hz}, 2 \mathrm{H}), 6.76$ $(\mathrm{d}, J=8.4 \mathrm{~Hz}, 2 \mathrm{H}), 4.61(\mathrm{~s}, 1 \mathrm{H}), 2.66-2.55(\mathrm{~m}, 1 \mathrm{H}), 1.56-1.47(\mathrm{~m}, 2 \mathrm{H}), 1.32-1.11(\mathrm{~m}, 7 \mathrm{H}), 0.84(\mathrm{t}$, $J=7.2 \mathrm{~Hz}, 3 \mathrm{H}) ;{ }^{13} \mathrm{C} \mathrm{NMR}:\left(100.0 \mathrm{MHz}, \mathrm{CDCl}_{3}\right) \delta 153.4,140.3,128.0,115.0,39.0,38.3,31.6,29.9$, 22.5, 14.0; HRMS (EI) calculated for $\left[\mathrm{C}_{12} \mathrm{H}_{18} \mathrm{O}\right]^{+}$requires $\mathrm{m} / \mathrm{z} 178.1358$, found $\mathrm{m} / \mathrm{z} 178.1356$.<smiles>CC(C)C(C)C(C)C(C)C</smiles>

(R)-heptan-2-ylbenzene (2t). Prepared according to the general procedure: using $\mathbf{L e} \cdot \mathrm{FeCl}_{2}(0.0165 \mathrm{~g}, 0.025 \mathrm{mmol}), \mathbf{1 t}(0.0860 \mathrm{~g}, 0.50 \mathrm{mmol}), n-\mathrm{C}_{18} \mathrm{H}_{37} \mathrm{SiH}_{3}$ (32 $\mu \mathrm{L}, 0.8906 \mathrm{~g} / \mathrm{mL}, 0.10 \mathrm{mmol}), \mathrm{CH}_{3} \mathrm{CN}(6.4 \mu \mathrm{L}, 0.6406 \mathrm{~g} / \mathrm{mL}, 0.10 \mathrm{mmol}), 1$ $\mathrm{mL}$ of toluene, NaBHEt3 $(75 \mu \mathrm{L}, 0.075 \mathrm{mmol})$. After 12 hours, the reaction afforded $\mathbf{2 t}$ with $96 \%$ conversion (91\% yield, purification by $m$-CPBA, removal of olefin) as a colorless oil. Optical Rotation: $[\alpha]^{20}{ }_{\mathrm{D}}=-7.0\left(\mathrm{c} 1.25, \mathrm{CHCl}_{3}\right) .99 \%$ ee, determined by GC, GC conditions: Chiralcel B$\mathrm{DM}, 100 \mathrm{kPa} \mathrm{N} 2\left(80{ }^{\circ} \mathrm{C}-100 \mathrm{~min}-1{ }^{\circ} \mathrm{C} / \mathrm{min}-150{ }^{\circ} \mathrm{C}-5 \mathrm{~min}\right), \operatorname{tr} 45.4$ (minor), 45.9 (major). IR (neat): 2956, 2865, 1727, 1563, $1497 \mathrm{~cm}^{-1}$; ${ }^{1} \mathrm{H}$ NMR: (400.0 MHz, $\left.\mathrm{CDCl}_{3}\right) \delta$ 7.31-7.25 (m, 2H), 7.20-7.12 (m, 3H), 2.72-2.61 (m, 1H), 1.63-1.48 (m, 2H), 1.30-1.18 (m, 9H), $0.84(\mathrm{t}, J=6.4 \mathrm{~Hz}$, 3H); $\left.{ }^{13} \mathrm{C} \mathrm{NMR:} \mathrm{(100.0} \mathrm{MHz,} \mathrm{CDCl}_{3}\right) \delta 148.0,128.2,127.0,125.7,39.9,38.4,31.9,27.4,22.6,22.3$, 14.1; HRMS (EI) calculated for $\left[\mathrm{C}_{13} \mathrm{H}_{20}\right]^{+}$requires $\mathrm{m} / \mathrm{z} 176.1565$, found $\mathrm{m} / \mathrm{z} 176.1564$.<smiles>COc1ccc(C(C)C(C)(C)C)cc1</smiles>

(R)-1-(heptan-2-yl)-4-methoxybenzene (2u). Prepared according to the general procedure: using $\mathbf{L e} \cdot \mathrm{FeCl}_{2}(0.0147 \mathrm{~g}, 0.025 \mathrm{mmol}), \mathbf{1} \mathbf{u}(0.1021 \mathrm{~g}$, $0.50 \mathrm{mmol}), n-\mathrm{C}_{18} \mathrm{H}_{37} \mathrm{SiH}_{3}(32 \mu \mathrm{L}, 0.8906 \mathrm{~g} / \mathrm{mL}, 0.10 \mathrm{mmol}), \mathrm{CH}_{3} \mathrm{CN}(6.4$ $\mu \mathrm{L}, 0.6406 \mathrm{~g} / \mathrm{mL}, 0.10 \mathrm{mmol}), 1 \mathrm{~mL}$ of toluene, NaBHEt3 $(75 \mu \mathrm{L}, 0.075 \mathrm{mmol})$. After 12 hours, the reaction afforded $\mathbf{2} \mathbf{u}$ with $>99 \%$ conversion ( $98 \%$ yield) as a colorless oil. Optical Rotation: $[\alpha]^{20} \mathrm{D}$ $=-26.7\left(\mathrm{c} 0.75, \mathrm{CHCl}_{3}\right) .94 .8 \%$ ee, determined by HPLC after conversion to the corresponding phenol according to previously reported procedures, ${ }^{17}$ HPLC conditions: Chiralcel OJ-H, $n$ hexane $/ i-\mathrm{PrOH}=97 / 3,0.8 \mathrm{~mL} / \mathrm{min}, \mathrm{n}=220 \mathrm{~nm}, \operatorname{tr} 18.2$ (minor), 21.0 (major). IR (neat): 2923, 2854, 1612, 1512, 1461, $1375 \mathrm{~cm}^{-1}$; ${ }^{1} \mathrm{H}$ NMR: $\left(400.0 \mathrm{MHz}, \mathrm{CDCl}_{3}\right) \delta 7.09$ (d, $\left.J=8.8 \mathrm{~Hz}, 2 \mathrm{H}\right), 6.83$ (d, $J$ $=8.4 \mathrm{~Hz}, 2 \mathrm{H}), 3.79(\mathrm{~s}, 3 \mathrm{H}), 2.67-2.57(\mathrm{~m}, 1 \mathrm{H}), 1.55-1.47(\mathrm{~m}, 2 \mathrm{H}), 1.30-1.10(\mathrm{~m}, 9 \mathrm{H}), 0.85(\mathrm{t}, J=$ 
$6.4 \mathrm{~Hz}, 3 \mathrm{H}) ;{ }^{13} \mathrm{C}$ NMR: $\left(100.0 \mathrm{MHz}, \mathrm{CDCl}_{3}\right) \delta$ 157.6, 140.1, 127.7, 113.6, 55.1, 39.0, 38.6, 31.9, 27.4, 22.6, 22.5, 14.1; HRMS (EI) calculated for $\left[\mathrm{C}_{14} \mathrm{H}_{22} \mathrm{O}\right]^{+}$requires $\mathrm{m} / \mathrm{z} 206.1671$, found $\mathrm{m} / \mathrm{z}$ 206.1672 .<smiles>CC(C)C(C)(C)c1ccc(O)cc1</smiles>

(R)-4-(heptan-2-yl)phenol (2u-1). Be converted by the $\mathbf{2 u}$ according to previously reported procedures $;{ }^{17}$ IR (neat): 3382, 2958, 2926, 1612 1513, $1449 \mathrm{~cm}^{-1}$; ${ }^{1} \mathrm{H}$ NMR: $\left(400.0 \mathrm{MHz}, \mathrm{CDCl}_{3}\right) \delta 7.04(\mathrm{~d}, J=8.4 \mathrm{~Hz}, 2 \mathrm{H}), 6.75$ $(\mathrm{d}, J=8.4 \mathrm{~Hz}, 2 \mathrm{H}), 4.58(\mathrm{~s}, 1 \mathrm{H}), 2.66-2.56(\mathrm{~m}, 1 \mathrm{H}), 1.54-1.45(\mathrm{~m}, 2 \mathrm{H}), 1.29-1.65(\mathrm{~m}, 9 \mathrm{H}), 0.85(\mathrm{t}$, $J=6.8 \mathrm{~Hz}, 3 \mathrm{H}) ;{ }^{13} \mathrm{C} \mathrm{NMR:}\left(100.0 \mathrm{MHz}, \mathrm{CDCl}_{3}\right) \delta 153.4,140.3,128.0,115.0,39.1,38.6,31.9,27.3$, 22.6, 22.5, 14.1; HRMS (EI) calculated for $\left[\mathrm{C}_{13} \mathrm{H}_{20} \mathrm{O}\right]^{+}$requires $\mathrm{m} / \mathrm{z} 192.1514$, found $\mathrm{m} / \mathrm{z} 192.1515$.<smiles>COc1ccc(C(C)C(C)(C)C)cc1</smiles>

(R)-1-methoxy-4-(octan-2-yl)benzene (2v). Prepared according to the general procedure: using $\mathbf{L e} \cdot \mathrm{FeCl}_{2}(0.0147 \mathrm{~g}, 0.025 \mathrm{mmol}), \mathbf{1 v}(0.1101 \mathrm{~g}$, $0.50 \mathrm{mmol}), n-\mathrm{C}_{18} \mathrm{H}_{37} \mathrm{SiH}_{3}(32 \mu \mathrm{L}, 0.8906 \mathrm{~g} / \mathrm{mL}, 0.10 \mathrm{mmol}), \mathrm{CH}_{3} \mathrm{CN}(6.4$ $\mu \mathrm{L}, 0.6406 \mathrm{~g} / \mathrm{mL}, 0.10 \mathrm{mmol}), 1 \mathrm{~mL}$ of toluene, NaBHEt3 $(75 \mu \mathrm{L}, 0.075 \mathrm{mmol})$. After 12 hours, the reaction afforded $2 \mathbf{v}$ with $>99 \%$ conversion ( $98 \%$ yield) as a colorless oil. Optical Rotation: $[\alpha]^{20} \mathrm{D}$ $=-23.4\left(\mathrm{c} 1.20, \mathrm{CHCl}_{3}\right) .90 .8 \%$ ee, determined by HPLC after conversion to the corresponding phenol according to previously reported procedures, ${ }^{17}$ HPLC conditions: Chiralcel OJ-H, $n$ hexane $/ i-\mathrm{PrOH}=97 / 3,0.8 \mathrm{~mL} / \mathrm{min}, \mathrm{n}=220 \mathrm{~nm}$, tr 14.8 (minor), 16.1 (major). IR (neat): 2955, 2854, 1612 1512, 1461, $1375 \mathrm{~cm}^{-1}$; ${ }^{1} \mathrm{H}$ NMR: $\left(400.0 \mathrm{MHz}, \mathrm{CDCl}_{3}\right) \delta 7.09(\mathrm{~d}, J=8.8 \mathrm{~Hz}, 2 \mathrm{H}), 6.83(\mathrm{~d}, J$ $=8.4 \mathrm{~Hz}, 2 \mathrm{H}), 3.79(\mathrm{~s}, 3 \mathrm{H}), 2.67-2.57(\mathrm{~m}, 1 \mathrm{H}), 1.56-1.48(\mathrm{~m}, 2 \mathrm{H}), 1.30-1.10(\mathrm{~m}, 11 \mathrm{H}), 0.85(\mathrm{t}, J=$ $6.4 \mathrm{~Hz}, 3 \mathrm{H}) ;{ }^{13} \mathrm{C} \mathrm{NMR}:\left(100.0 \mathrm{MHz}, \mathrm{CDCl}_{3}\right) \delta 157.6,140.1,127.7,113.6,55.1,39.1,38.6,31.8$, 29.4, 27.7, 22.6, 22.5, 14.1; HRMS (EI) calculated for $\left[\mathrm{C}_{15} \mathrm{H}_{24} \mathrm{O}\right]^{+}$requires $\mathrm{m} / \mathrm{z} 220.1827$, found $\mathrm{m} / \mathrm{z} 220.1826$.<smiles>CC(C)(C)C(C)(C)c1ccc(O)cc1</smiles>

(R)-4-(octan-2-yl)phenol (2v-1). Be converted by the $2 \mathrm{v}$ according to previously reported procedures; ${ }^{17}$ IR (neat): 3374, 2958, 2957, 1613, 1513, $1460 \mathrm{~cm}^{-1} ;{ }^{1} \mathrm{H}$ NMR: $\left(400.0 \mathrm{MHz}, \mathrm{CDCl}_{3}\right) \delta 7.04(\mathrm{~d}, J=8.4 \mathrm{~Hz}, 2 \mathrm{H}), 6.75$ (d, $J=8.8 \mathrm{~Hz}, 2 \mathrm{H}), 4.58(\mathrm{~s}, 1 \mathrm{H}), 2.66-2.55(\mathrm{~m}, 1 \mathrm{H}), 1.55-1.64(\mathrm{~m}, 2 \mathrm{H}), 1.29-1.57(\mathrm{~m}, 11 \mathrm{H}), 0.85$ (t, $J=6.4 \mathrm{~Hz}, 3 \mathrm{H}) ;{ }^{13} \mathrm{C} \mathrm{NMR}:\left(100.0 \mathrm{MHz}, \mathrm{CDCl}_{3}\right) \delta 153.4,140.3,128.0,115.0,39.0,38.6,31.8$, 29.4, 27.6, 22.6, 22.5, 14.1; HRMS (EI) calculated for $\left[\mathrm{C}_{14} \mathrm{H}_{22} \mathrm{O}\right]^{+}$requires $\mathrm{m} / \mathrm{z} 206.1671$, found $\mathrm{m} / \mathrm{z} 206.1673$. 
<smiles>CC(C)(C)Oc1ccc(C(C)(C)C(C)(C)C)cc1</smiles>

(R)-tert-butyldimethyl(4-(octan-2-yl)phenoxy)silane (2w). Prepared according to the general procedure: using $\mathbf{L e} \bullet \mathrm{FeCl}_{2}(0.0149 \mathrm{~g}, 0.025$ mmol), 1 w (0.1311 g, $0.50 \mathrm{mmol}), n-\mathrm{C}_{18} \mathrm{H}_{37} \mathrm{SiH}_{3}(32 \mu \mathrm{L}, 0.8906 \mathrm{~g} / \mathrm{mL}$, mmol). After 12 hours, the reaction afforded $\mathbf{2 w}$ with $>99 \%$ conversion ( $98 \%$ yield) as a colorless oil. Optical Rotation: $[\alpha]^{20}{ }_{\mathrm{D}}=-15.0\left(\mathrm{c} 0.90, \mathrm{CHCl}_{3}\right) .95 .9 \%$ ee, determined by HPLC after conversion to the corresponding phenol according to previously reported procedures, ${ }^{16}$ HPLC conditions: Chiralcel OJ-H, $n$-hexane $/ i-\mathrm{PrOH}=97 / 3,0.8 \mathrm{~mL} / \mathrm{min}, \mathrm{n}=220 \mathrm{~nm}$, tr 14.7 (minor), 15.7 (major). IR (neat): 2923, 2854, 1607, 1510, $1463 \mathrm{~cm}^{-1}$; ${ }^{1} \mathrm{H}$ NMR: $\left(399.9 \mathrm{MHz}, \mathrm{CDCl}_{3}\right) \delta 7.01(\mathrm{~d}, J$ $=8.4 \mathrm{~Hz}, 2 \mathrm{H}), 6.74(\mathrm{~d}, J=8.8 \mathrm{~Hz}, 2 \mathrm{H}), 2.65-2.54(\mathrm{~m}, 1 \mathrm{H}), 1.55-1.45(\mathrm{~m}, 2 \mathrm{H}), 1.29-1.16(\mathrm{~m}, 11 \mathrm{H})$, $0.98(\mathrm{~s}, 9 \mathrm{H}), 0.85$ (t, $\left.J=6.8 \mathrm{~Hz}, 3 \mathrm{H}), 0.18(\mathrm{~s}, 6 \mathrm{H}) ;{ }^{13} \mathrm{C} \mathrm{NMR:} \mathrm{(100.0} \mathrm{MHz,} \mathrm{CDCl}_{3}\right) \delta 153.4,140.6$, 127.7, 119.6, 39.1, 38.7, 31.8, 29.4, 27.7, 25.7, 22.6, 22.4, 18.2, 14.1, -4.4; HRMS (EI) calculated for $\left[\mathrm{C}_{20} \mathrm{H}_{36} \mathrm{OSi}\right]^{+}$requires $\mathrm{m} / \mathrm{z} 320.2535$, found $\mathrm{m} / \mathrm{z} 320.2533$.<smiles>COc1ccc([C@@H](C)CC(C)C)cc1</smiles>

(R)-1-methoxy-4-(4-methylpentan-2-yl)benzene $\quad(2 x) . \quad$ Prepared according to the general procedure: using $\mathbf{L e} \bullet \mathrm{FeCl}_{2}(0.0147 \mathrm{~g}, 0.025$ mmol), 1 x (0.0950 g, $0.50 \mathrm{mmol}), n-\mathrm{C}_{18} \mathrm{H}_{37} \mathrm{SiH}_{3}(32 \mu \mathrm{L}, 0.8906 \mathrm{~g} / \mathrm{mL}$, $0.10 \mathrm{mmol}), \mathrm{CH}_{3} \mathrm{CN}(6.4 \mu \mathrm{L}, 0.6406 \mathrm{~g} / \mathrm{mL}, 0.10 \mathrm{mmol}), 1 \mathrm{~mL}$ of toluene, NaBHEt3 $(75 \mu \mathrm{L}, 0.075$ mmol). After 12 hours, the reaction afforded $\mathbf{2} \mathbf{x}$ with $>99 \%$ conversion (98\% yield) as a colorless oil. Optical Rotation: $[\alpha]^{20} \mathrm{D}=-3.5$ (c $\left.0.90, \mathrm{CHCl}_{3}\right) .97 .9 \%$ ee, determined by $\mathrm{GC}, \mathrm{GC}$ conditions: Chiralcel B-DM, $100 \mathrm{kPa} \mathrm{N} 2\left(60{ }^{\circ} \mathrm{C}-30 \mathrm{~min}-1{ }^{\circ} \mathrm{C} / \mathrm{min}-130{ }^{\circ} \mathrm{C}-5 \mathrm{~min}\right)$, tr 82.1 (minor), 83.1 (major). IR (neat): 2921, 2853, 1612, 1512, $1462 \mathrm{~cm}^{-1}$; ${ }^{1} \mathrm{H}$ NMR: $\left(400.0 \mathrm{MHz}, \mathrm{CDCl}_{3}\right) \delta 7.10$ (d, $J$ $=8.8 \mathrm{~Hz}, 2 \mathrm{H}), 6.83(\mathrm{~d}, J=8.8 \mathrm{~Hz}, 2 \mathrm{H}), 3.79(\mathrm{~s}, 3 \mathrm{H}), 2.79-2.68(\mathrm{~m}, 1 \mathrm{H}), 1.52-1.30(\mathrm{~m}, 3 \mathrm{H}), 1.18$ $(\mathrm{d}, J=6.8 \mathrm{~Hz}, 3 \mathrm{H}), 0.89-0.80(\mathrm{~m}, 6 \mathrm{H}) ;{ }^{13} \mathrm{C} \mathrm{NMR}:\left(100.0 \mathrm{MHz}, \mathrm{CDCl}_{3}\right) \delta 157.6,140.1,127.8,113.6$, 55.2, 48.0, 36.6, 25.5, 23.0, 22.3; HRMS (EI) calculated for $\left[\mathrm{C}_{13} \mathrm{H}_{20} \mathrm{O}\right]^{+}$requires $\mathrm{m} / \mathrm{z} 192.1514$, found $\mathrm{m} / \mathrm{z} 192.1513$.<smiles>C[C@H](CCc1ccccc1)c1ccccc1</smiles>

(R)-butane-1,3-diyldibenzene (2y). Prepared according to the general procedure: using $\mathbf{L e} \cdot \mathrm{FeCl}_{2}(0.0171 \mathrm{~g}, 0.030 \mathrm{mmol}), \mathbf{1 y}(0.1043 \mathrm{~g}, 0.50$ mmol), $n-\mathrm{C}_{18} \mathrm{H}_{37} \mathrm{SiH}_{3}(38 \mu \mathrm{L}, 0.8906 \mathrm{~g} / \mathrm{mL}, 0.12 \mathrm{mmol}), \mathrm{CH}_{3} \mathrm{CN}(7.8$ $\mu \mathrm{L}, 0.6406 \mathrm{~g} / \mathrm{mL}, 0.12 \mathrm{mmol}), 1 \mathrm{~mL}$ of toluene, NaBHEt3 $(90 \mu \mathrm{L}, 0.090 \mathrm{mmol})$. After 12 hours, the 
reaction afforded $2 \mathbf{y}$ with $91 \%$ conversion ( $88 \%$ yield, purification by $m$-CPBA, removal of olefin) as a colorless oil. Optical Rotation: $[\alpha]^{20}{ }_{\mathrm{D}}=-1.8\left(\mathrm{c} 1.01, \mathrm{CHCl}_{3}\right) .88 \%$ ee, determined by HPLC, HPLC conditions: Chiralcel OJ-H x 2, $n$-hexane $/ i-\mathrm{PrOH}=99.5 / 0.5,1.0 \mathrm{~mL} / \mathrm{min}, \mathrm{n}=254 \mathrm{~nm}, \operatorname{tr} 23.8$ (major), 26.3 (minor). IR (neat): 2922, 2853 1598, 1461, $1377 \mathrm{~cm}^{-1},{ }^{1} \mathrm{H}$ NMR: (400.0 MHz, $\mathrm{CDCl}_{3}$ ) $\delta$ 7.36-7.10 (m, 10H), 2.79-2.65 (m, 1H), 2.60-2.43 (m, 2H), 2.00-1.80 (m, 2H), $1.28(\mathrm{~d}, J=7.2 \mathrm{~Hz}$, 3H); ${ }^{13} \mathrm{C}$ NMR: (100.0 MHz, $\left.\mathrm{CDCl}_{3}\right) \delta 147.3,142.5,128.4,128.3,128.2,127.1,125.9,125.6,40.0$, 39.5, 33.9, 22.5. HRMS (EI) calculated for $\left[\mathrm{C}_{16} \mathrm{H}_{18}\right]^{+}$requires $\mathrm{m} / \mathrm{z} 210.1409$, found $\mathrm{m} / \mathrm{z} 210.1409$.<smiles>Cc1ccc(C(C)C(C)N(C)C)cc1</smiles>

$(R)-N, N$-dimethyl-4-( $p$-tolyl)pentan-1-amine (2z). Prepared according to the general procedure: using $\mathbf{L e} \cdot \mathrm{FeCl}_{2}(0.0165 \mathrm{~g}, 0.025 \mathrm{mmol}), \mathbf{1 z}(0.1020$ g, $0.50 \mathrm{mmol}), n-\mathrm{C}_{18} \mathrm{H}_{37} \mathrm{SiH}_{3}(32 \mu \mathrm{L}, 0.8906 \mathrm{~g} / \mathrm{mL}, 0.10 \mathrm{mmol}), \mathrm{CH}_{3} \mathrm{CN}$ ( $6.4 \mu \mathrm{L}, 0.6406 \mathrm{~g} / \mathrm{mL}, 0.10 \mathrm{mmol}), 1 \mathrm{~mL}$ of toluene, NaBHEt 3 ( $75 \mu \mathrm{L}, 0.075 \mathrm{mmol})$. After 12 hours, the reaction afforded $\mathbf{2 z}$ with $>99 \%$ conversion (96\% yield) as a colorless oil. Optical Rotation: $[\alpha]^{20}$ $\mathrm{D}=-17.4\left(\mathrm{c} 0.92, \mathrm{CHCl}_{3}\right) .96 .6 \%$ ee, determined by HPLC, HPLC conditions: Chiralcel OJ-H x 2, $n$-hexane $/ i-\mathrm{PrOH}=95 / 5,1.0 \mathrm{~mL} / \mathrm{min}, \mathrm{n}=220 \mathrm{~nm}$, tr 9.5 (minor), 10.0 (major). IR (neat): 2941, 2858, 2763, 1651, 1515, $1374 \mathrm{~cm}^{-1},{ }^{1} \mathrm{H}$ NMR: (400.0 MHz, $\left.\mathrm{CDCl}_{3}\right) \delta$ 7.12-7.04 (m, 4H), 2.70-2.59 (m, 1H), $2.31(\mathrm{~s}, 3 \mathrm{H}), 2.25-2.14(\mathrm{~m}, 8 \mathrm{H}), 1.61-1.52(\mathrm{~m}, 2 \mathrm{H}), 1.47-1.28(\mathrm{~m}, 2 \mathrm{H}), 1.23(\mathrm{~d}, J=6.8 \mathrm{~Hz}$ 3H); ${ }^{13} \mathrm{C}$ NMR: $\left(100.0 \mathrm{MHz}, \mathrm{CDCl}_{3}\right) \delta$ 144.4, 135.2, 128.9, 126.8, 59.8, 45.4, 39.5, 36.1, 25.8, 22.4, 20.9; HRMS (EI) calculated for $\left[\mathrm{C}_{14} \mathrm{H}_{23} \mathrm{~N}\right]^{+}$requires $\mathrm{m} / \mathrm{z}$ 205.1830, found $\mathrm{m} / \mathrm{z} 205.1832$.

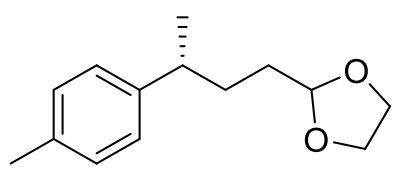

(R)-2-(3-(p-tolyl)butyl)-1,3-dioxolane (2aa). Prepared according to the general procedure: using $\mathbf{L e} \cdot \mathrm{FeCl}_{2}(0.0141 \mathrm{~g}, 0.025 \mathrm{mmol})$, 1aa (0.1080 g, $0.50 \mathrm{mmol}), n-\mathrm{C}_{18} \mathrm{H}_{37} \mathrm{SiH}_{3}(32 \mu \mathrm{L}, 0.8906 \mathrm{~g} / \mathrm{mL}, 0.10$ $\mathrm{mmol}), \mathrm{CH}_{3} \mathrm{CN}(6.4 \mu \mathrm{L}, 0.6406 \mathrm{~g} / \mathrm{mL}, 0.10 \mathrm{mmol}), 1 \mathrm{~mL}$ of toluene, NaBHEt3 $(75 \mu \mathrm{L}, 0.075 \mathrm{mmol})$. After 12 hours, the reaction afforded 2 aa with $>99 \%$ conversion (94\% yield) as a colorless oil. Optical Rotation: $[\alpha]^{20}{ }_{\mathrm{D}}=-3.8\left(\mathrm{c} 0.58, \mathrm{CHCl}_{3}\right) .96 .1 \%$ ee, determined by HPLC, HPLC conditions: Chiralcel AD-H, $n$-hexane $/ i$-PrOH $=99.5 / 0.5,0.5 \mathrm{~mL} / \mathrm{min}, \mathrm{n}=220 \mathrm{~nm}, \operatorname{tr} 15.9$ (minor), 16.9 (major). IR (neat): 2957, 2925, 1740, 1515, 1458, $1143 \mathrm{~cm}^{-1}$; ${ }^{1} \mathrm{H}$ NMR: $\left(400.0 \mathrm{MHz}, \mathrm{CDCl}_{3}\right) \delta$ 7.12-7.04 (m, 4H), $4.81(\mathrm{t}, J=4.8 \mathrm{~Hz}, 1 \mathrm{H}), 3.98-3.80(\mathrm{~m}, 4 \mathrm{H}), 2.73-2.62(\mathrm{~m}, 1 \mathrm{H}), 2.31(\mathrm{~s}, 3 \mathrm{H}), 1.73-1.47(\mathrm{~m}, 4 \mathrm{H})$, $1.24(\mathrm{~d}, J=6.8 \mathrm{~Hz}, 3 \mathrm{H}) ;{ }^{13} \mathrm{C} \mathrm{NMR}:\left(100.0 \mathrm{MHz}, \mathrm{CDCl}_{3}\right) \delta 144.0,135.3,129.0,126.8,104.6,64.8$, 39.4, 32.4, 32.1, 22.4, 20.9; HRMS (EI) calculated for $\left[\mathrm{C}_{14} \mathrm{H}_{20} \mathrm{O}_{2}\right]^{+}$requires $\mathrm{m} / \mathrm{z} 220.1463$, found 
$\mathrm{m} / \mathrm{z} 220.1461$.<smiles>COc1cc([C@@H](C)CCC2OCCO2)ccc1C</smiles>

(R)-2-(3-(3-methoxy-4-methylphenyl)butyl)-1,3-dioxolane (2ab).

Prepared according to the general procedure: using $\mathbf{L e} \cdot \mathrm{FeCl}_{2}(0.0142$ g, $0.025 \mathrm{mmol}), \mathbf{a b}(0.1240 \mathrm{~g}, 0.50 \mathrm{mmol}), n-\mathrm{C}_{18} \mathrm{H}_{37} \mathrm{SiH}_{3}(32 \mu \mathrm{L}$, $0.8906 \mathrm{~g} / \mathrm{mL}, 0.10 \mathrm{mmol}), \mathrm{CH}_{3} \mathrm{CN}(6.4 \mu \mathrm{L}, 0.6406 \mathrm{~g} / \mathrm{mL}, 0.10 \mathrm{mmol}), 1 \mathrm{~mL}$ of toluene, NaBHEt 3 (75 $\mu \mathrm{L}, 0.075 \mathrm{mmol}$ ). After 12 hours, the reaction afforded $2 \mathbf{a b}$ with $>99 \%$ conversion ( $94 \%$ yield) as a colorless oil. Optical Rotation: $[\alpha]^{20}{ }_{\mathrm{D}}=-11.4\left(\mathrm{c} 1.98, \mathrm{CHCl}_{3}\right) .97 .2 \% e e$, determined by HPLC, HPLC conditions: Chiralcel AD-H x 2, $n$-hexane $/ i-\mathrm{PrOH}=99 / 1,1.0 \mathrm{~mL} / \mathrm{min}, \mathrm{n}=220 \mathrm{~nm}$, tr 12.4 (minor), 13.2 (major). IR (neat): 2954, 2926, 1611, 1582, 1462, $1256 \mathrm{~cm}^{-1}$; ${ }^{1} \mathrm{H}$ NMR: (400.0 MHz, $\left.\mathrm{CDCl}_{3}\right) \delta 7.03(\mathrm{~d}, J=7.6 \mathrm{~Hz}, 1 \mathrm{H}), 6.71-6.61(\mathrm{~m}, 2 \mathrm{H}), 4.81(\mathrm{t}, J=4.8 \mathrm{~Hz}, 1 \mathrm{H}), 4.01-3.79(\mathrm{~m}, 7 \mathrm{H})$, 2.73-2.59 (m, 1H), 2.17 (s, 3H), 1.73-1.49 (m, 4H), 1.25 (d, $J=7.2 \mathrm{~Hz}, 3 \mathrm{H}) ;{ }^{13} \mathrm{C}$ NMR: (100.0 MHz, $\left.\mathrm{CDCl}_{3}\right) \delta 157.6,146.1,130.4,124.0,118.6,108.8,104.6,64.8,55.2,39.9,32.4,32.1,22.4,15.8 ;$ HRMS (EI) calculated for $\left[\mathrm{C}_{15} \mathrm{H}_{22} \mathrm{O}_{3}\right]^{+}$requires $\mathrm{m} / \mathrm{z} 250.1569$, found $\mathrm{m} / \mathrm{z} 250.1569$.<smiles>C[C@H]1CCCCc2ccccc21</smiles>

$\begin{array}{llll}\text { (R)-5-methyl-6,7,8,9-tetrahydro-5H-benzo[7]annulene } \quad \text { (2ac). }{ }^{20} \quad \text { Prepared } & \end{array}$ according to the general procedure: using $\mathbf{L e} \bullet \mathrm{FeCl}_{2}(0.0140 \mathrm{~g}, 0.025 \mathrm{mmol}), \mathbf{1 a c}$ (0.0397 g, $0.25 \mathrm{mmol}), n-\mathrm{C}_{18} \mathrm{H}_{37} \mathrm{SiH}_{3}(32 \mu \mathrm{L}, 0.8906 \mathrm{~g} / \mathrm{mL}, 0.10 \mathrm{mmol}), \mathrm{CH}_{3} \mathrm{CN}$ (6.4 $\mu \mathrm{L}, 0.6406 \mathrm{~g} / \mathrm{mL}, 0.10 \mathrm{mmol}), 1 \mathrm{~mL}$ of toluene, NaBHEt3 $(75 \mu \mathrm{L}, 0.075 \mathrm{mmol})$. After 12 hours, the reaction afforded 2 ac with $>99 \%$ conversion (94\% yield) as a colorless oil. Optical Rotation: $[\alpha]^{20}{ }_{\mathrm{D}}=+10.6\left(\mathrm{c} 0.50, \mathrm{CHCl}_{3}\right) . \operatorname{Ref}^{20}\left\{[\alpha]^{23} \mathrm{D}=+10.0^{\circ}\left(\mathrm{c} 5, \mathrm{CHCl}_{3}\right)\right\} .90 .5 \%$ ee, determined by GC, GC conditions: Chiralcel B-DM, $100 \mathrm{kPa} \mathrm{N}_{2}\left(60^{\circ} \mathrm{C}-30 \mathrm{~min}-2{ }^{\circ} \mathrm{C} / \mathrm{min}-180^{\circ} \mathrm{C}-5 \mathrm{~min}\right)$, $\mathrm{tr}$ 61.4 (major), 63.4 (minor). ${ }^{1} \mathrm{H}$ NMR: (400.0 MHz, $\mathrm{CDCl}_{3}$ ) $\delta$ 7.22-7.04 (m, 4H), 3.10-2.99 (m, 1H), 2.93-2.83 (m, 1H), 2.83-2.74 (m, 1H), 1.98-1.86 (m, 1H), 1.86-1.68 (m, 3H), 1.51-1.30 (m, 5H); ${ }^{13} \mathrm{C}$ NMR: (100.0 MHz, $\left.\mathrm{CDCl}_{3}\right) \delta 146.5,142.8,129.2,126.0,125.6,125.2,37.7,36.1,36.0,30.1$, 27.8, 20.4. All the spectroscopic data were in agreement with the reported ones. ${ }^{20}$

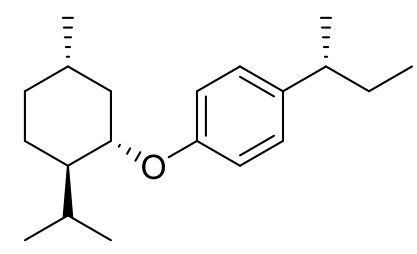

1-((R)-sec-butyl)-4-(((1S,2R,5S)-2-isopropyl-5methylcyclohexyl)oxy)benzene (2ad). Prepared according to the general procedure: using $\mathbf{L e} \bullet \mathrm{FeCl}_{2}(0.0166 \mathrm{~g}, 0.025 \mathrm{mmol})$, $\mathbf{1 a d}$ (0.1429 g, $0.50 \mathrm{mmol}), n-\mathrm{C}_{18} \mathrm{H}_{37} \mathrm{SiH}_{3}(32 \mu \mathrm{L}, 0.8906 \mathrm{~g} / \mathrm{mL}, 0.10$ mmol), $\mathrm{CH}_{3} \mathrm{CN}(6.4 \mu \mathrm{L}, 0.6406 \mathrm{~g} / \mathrm{mL}, 0.10 \mathrm{mmol}), 1 \mathrm{~mL}$ of toluene, NaBHEt3 $(75 \mu \mathrm{L}, 0.075 \mathrm{mmol})$. 
After 12 hours, the reaction afforded 2ad with $>99 \%$ conversion ( $99 \%$ yield) as a colorless oil. Optical Rotation: $[\alpha]^{20}{ }_{\mathrm{D}}=-97.6\left(\mathrm{c} 0.69, \mathrm{CHCl}_{3}\right) .96 .9 \%$ ee, determined by HPLC after conversion to the corresponding phenol according to previously reported procedures, ${ }^{17}$ HPLC conditions: Chiralcel OJ-H, $n$-hexane $/ i-\mathrm{PrOH}=95 / 5,1.0 \mathrm{~mL} / \mathrm{min}, \mathrm{n}=220 \mathrm{~nm}$, tr 14.0 (minor), 15.1 (major). IR (neat): 2957, 2926, 2871, 1611, 1509, $1457 \mathrm{~cm}^{-1} ;{ }^{1} \mathrm{H}$ NMR: (400.0 MHz, $\left.\mathrm{CDCl}_{3}\right) \delta 7.06(\mathrm{~d}, J=8.4$ $\mathrm{Hz}, 2 \mathrm{H}), 6.82(\mathrm{~d}, J=8.8 \mathrm{~Hz}, 2 \mathrm{H}), 4.04-3.94(\mathrm{~m}, 1 \mathrm{H}), 2.58-2.47(\mathrm{~m}, 1 \mathrm{H}), 2.28-2.12(\mathrm{~m}, 2 \mathrm{H}), 1.75-$ $1.67(\mathrm{~m}, 2 \mathrm{H}), 1.61-1.39(\mathrm{~m}, 5 \mathrm{H}), 1.21(\mathrm{~d}, J=6.8 \mathrm{~Hz}, 3 \mathrm{H}), 1.03-0.87(\mathrm{~m}, 8 \mathrm{H}), 0.85-0.78(\mathrm{~m}, 6 \mathrm{H})$; ${ }^{13}$ C NMR: (100.0 MHz, $\left.\mathrm{CDCl}_{3}\right) \delta 156.4,139.6,127.8,115.7,48.1,40.8,40.4,34.6,31.4,31.3,26.0$, 23.7, 22.1, 21.9, 20.8, 16.5, 12.3; HRMS (EI) calculated for $\left[\mathrm{C}_{20} \mathrm{H}_{32} \mathrm{O}\right]^{+}$requires $\mathrm{m} / \mathrm{z} 288.2453$, found $\mathrm{m} / \mathrm{z} 288.2453$.

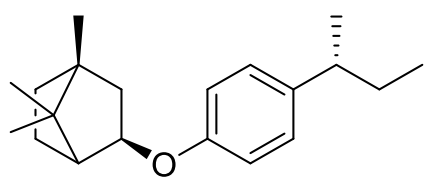

$(1 R, 3 R)-3-(4-((R)-s e c-b u t y l) p h e n o x y)-1,7,7-$ trimethylbicyclo[2.2.1]heptane (2ae). Prepared according to the general procedure: using $\mathbf{L e} \cdot \mathrm{FeCl}_{2}(0.0165 \mathrm{~g}, 0.025 \mathrm{mmol})$, 1ae (0.1400 g, $0.50 \mathrm{mmol}), n-\mathrm{C}_{18} \mathrm{H}_{37} \mathrm{SiH}_{3}(32 \mu \mathrm{L}, 0.8906 \mathrm{~g} / \mathrm{mL}, 0.10 \mathrm{mmol}), \mathrm{CH}_{3} \mathrm{CN}(6.4 \mu \mathrm{L}, 0.6406$ $\mathrm{g} / \mathrm{mL}, 0.10 \mathrm{mmol}), 1 \mathrm{~mL}$ of toluene, NaBHEt3 $(75 \mu \mathrm{L}, 0.075 \mathrm{mmol})$. After 12 hours, the reaction afforded 2ae with $>99 \%$ conversion (100\% yield) as a colorless oil. Optical Rotation: $[\alpha]^{20}{ }_{D}=-88.3$ (c $0.79, \mathrm{CHCl}_{3}$ ). $96 \%$ ee, determined by HPLC after conversion to the corresponding phenol according to previously reported procedures, ${ }^{17} \mathrm{HPLC}$ conditions: Chiralcel OJ-H, $n$-hexane $/ i$-PrOH $=95 / 5,1.0 \mathrm{~mL} / \mathrm{min}, \mathrm{n}=220 \mathrm{~nm}$, tr 14.3 (minor), 15.3 (major). IR (neat): 2955, 2875, 1610, 1510, 1455, $1245 \mathrm{~cm}^{-1}$; ${ }^{1} \mathrm{H}$ NMR: $\left(400.0 \mathrm{MHz}, \mathrm{CDCl}_{3}\right) \delta 7.06(\mathrm{~d}, J=8.8 \mathrm{~Hz}, 2 \mathrm{H}), 6.76(\mathrm{~d}, J=8.8 \mathrm{~Hz}$, 2H), 4.32-4.25 (m, 1H), 2.58-2.47 (m, 1H), 2.40-2.30 (m, 1H), 2.29-2.18 (m, 1H), 1.81-1.69 (m, 2H), 1.61-1.50 (m, 2H), 1.23-1.37 (m, 2H), $1.20(\mathrm{~d}, J=7.2 \mathrm{~Hz}, 3 \mathrm{H}) 1.13(\mathrm{dd}, J=13.2,, 3.2 \mathrm{~Hz}$, 1H), 0.97-0.88 (m, 9H), $\left.0.81(\mathrm{t}, J=7.2 \mathrm{~Hz}, 3 \mathrm{H}) ;{ }^{13} \mathrm{C} \mathrm{NMR:} \mathrm{(100.0} \mathrm{MHz,} \mathrm{CDCl}_{3}\right) \delta 157.2,139.2$, $127.7,115.1,82.7,49.5,47.5,45.2,40.8,36.9,31.3,27.9,26.8,22.0,19.7,19.0,13.8,12.3$. HRMS (EI) calculated for $\left[\mathrm{C}_{20} \mathrm{H}_{30} \mathrm{O}\right]^{+}$requires $\mathrm{m} / \mathrm{z} 286.2297$, found $\mathrm{m} / \mathrm{z} 286.2297$.

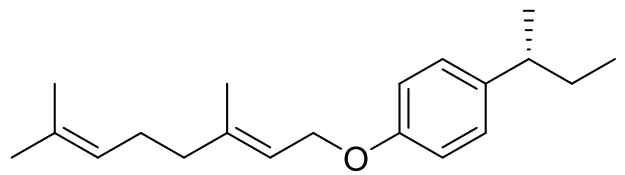

(R,E)-1-(sec-butyl)-4-((3,7-dimethylocta-2,6-dien1-yl)oxy)benzene (2af). Prepared according to the general procedure: using $\mathbf{L e} \cdot \mathrm{FeCl}_{2}(0.0162 \mathrm{~g}, 0.025$ mmol), 1af (0.1430 g, $0.50 \mathrm{mmol}), n-\mathrm{C}_{18} \mathrm{H}_{37} \mathrm{SiH}_{3}(32 \mu \mathrm{L}, 0.8906 \mathrm{~g} / \mathrm{mL}, 0.10 \mathrm{mmol}), \mathrm{CH}_{3} \mathrm{CN}(6.4$ 
$\mu \mathrm{L}, 0.6406 \mathrm{~g} / \mathrm{mL}, 0.10 \mathrm{mmol}), 1 \mathrm{~mL}$ of toluene, NaBHEt3 $(75 \mu \mathrm{L}, 0.075 \mathrm{mmol})$. After 12 hours, the reaction afforded 2af with $>99 \%$ conversion ( $92 \%$ yield) as a colorless oil. Optical Rotation: $[\alpha]^{20}$ $\mathrm{D}=-13.1\left(\mathrm{c} 0.78, \mathrm{CHCl}_{3}\right) .97 .2 \%$ ee, determined by HPLC after conversion to the corresponding phenol according to previously reported procedures, ${ }^{17}$ HPLC conditions: Chiralcel OJ-H, $n$ hexane $/ i-\mathrm{PrOH}=95 / 5,1.0 \mathrm{~mL} / \mathrm{min}, \mathrm{n}=220 \mathrm{~nm}, \operatorname{tr} 14.5$ (minor), 16.1 (major). IR (neat): 2963, 2922, 1671, 1611, 1511, $1455 \mathrm{~cm}^{-1}$; ${ }^{1} \mathrm{H}$ NMR: (400.0 MHz, $\left.\mathrm{CDCl}_{3}\right) \delta 7.08$ (d, $\left.J=8.8 \mathrm{~Hz}, 2 \mathrm{H}\right), 6.85$ (d, $J$ $=8.4 \mathrm{~Hz}, 2 \mathrm{H}), 5.50(\mathrm{t}, J=6.8 \mathrm{~Hz}, 1 \mathrm{H}), 5.10(\mathrm{t}, J=5.2 \mathrm{~Hz}, 1 \mathrm{H}), 4.51(\mathrm{~d}, J=6.8 \mathrm{~Hz}, 2 \mathrm{H}), 2.59-2.48$ (m, 1H), 2.18-2.04 (m, 4H), 1.73(s, 3H), 1.68 (s, 3H), $1.58(\mathrm{~s}, 3 \mathrm{H}), 1.57-1.51(\mathrm{~m}, 2 \mathrm{H}), 1.21(\mathrm{~d}, J=$ $6.8 \mathrm{~Hz}, 3 \mathrm{H}), 0.81(\mathrm{t}, J=7.6 \mathrm{~Hz}, 3 \mathrm{H}) ;{ }^{13} \mathrm{C} \mathrm{NMR}:\left(100.0 \mathrm{MHz}, \mathrm{CDCl}_{3}\right) \delta 157.0,140.8,139.6,131.7$, $127.8,123.8,119.8,114.4,64.8,40.8,39.5,31.3,26.3,25.6,22.0,17.6,16.6,12.2$. HRMS (EI) calculated for $\left[\mathrm{C}_{20} \mathrm{H}_{30} \mathrm{O}\right]^{+}$requires $\mathrm{m} / \mathrm{z} 286.2297$, found $\mathrm{m} / \mathrm{z} 286.2298$.

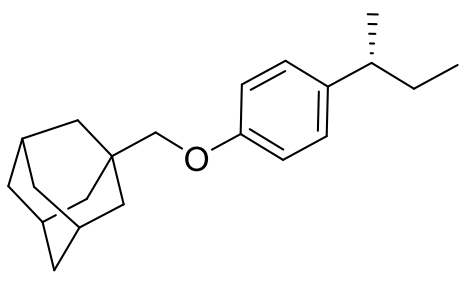

(1s,3s)-1-((4-((R)-sec-butyl)phenoxy)methyl)adamantine (2ag). Prepared according to the general procedure: using Le• $\mathrm{FeCl}_{2}(0.0160 \mathrm{~g}, 0.025 \mathrm{mmol}), \mathbf{1 a g}(0.1405 \mathrm{~g}, 0.50 \mathrm{mmol}), n$ $\mathrm{C}_{18} \mathrm{H}_{37} \mathrm{SiH}_{3}(32 \mu \mathrm{L}, 0.8906 \mathrm{~g} / \mathrm{mL}, 0.10 \mathrm{mmol}), \mathrm{CH}_{3} \mathrm{CN}(6.4 \mu \mathrm{L}$, $0.6406 \mathrm{~g} / \mathrm{mL}, 0.10 \mathrm{mmol}), 1 \mathrm{~mL}$ of toluene, NaBHEt3 $(75 \mu \mathrm{L}, 0.075 \mathrm{mmol})$. After 12 hours, the reaction afforded 2 ag with $>99 \%$ conversion ( $94 \%$ yield) as a colorless oil. Optical Rotation: $[\alpha]^{20}$ $\mathrm{D}=-16.8\left(\mathrm{c} 0.83, \mathrm{CHCl}_{3}\right) .95 .1 \%$ ee, determined by HPLC after conversion to the corresponding phenol according to previously reported procedures, ${ }^{17}$ HPLC conditions: Chiralcel OJ-H, $n$ hexane $/ i-\mathrm{PrOH}=95 / 5,1.0 \mathrm{~mL} / \mathrm{min}, \mathrm{n}=220 \mathrm{~nm}, \operatorname{tr} 14.3$ (minor), 15.2 (major). IR (neat): 2961, 2901, 2848, 1611, 1511, $1457 \mathrm{~cm}^{-1}$; ${ }^{1} \mathrm{H}$ NMR: $\left(400.0 \mathrm{MHz}, \mathrm{CDCl}_{3}\right) \delta 7.07(\mathrm{~d}, J=8.4 \mathrm{~Hz}, 2 \mathrm{H}), 6.82(\mathrm{~d}, J$ $=8.8 \mathrm{~Hz}, 2 \mathrm{H}), 3.47(\mathrm{~s}, 2 \mathrm{H}), 2.58-2.48(\mathrm{~m}, 1 \mathrm{H}), 2.01(\mathrm{~s}, 3 \mathrm{H}), 1.80-1.66(\mathrm{~m}, 6 \mathrm{H}), 1.65(\mathrm{~d}, J=3.2 \mathrm{~Hz}$, $6 \mathrm{H}), 1.60-1.50(\mathrm{~m}, 2 \mathrm{H}), 1.20(\mathrm{~d}, J=6.8 \mathrm{~Hz}, 3 \mathrm{H}), 0.80(\mathrm{t}, J=7.2 \mathrm{~Hz}, 3 \mathrm{H}) ;{ }^{13} \mathrm{C}$ NMR: $(100.0 \mathrm{MHz}$, $\left.\mathrm{CDCl}_{3}\right) \delta 157.8,139.3,127.7,114.2,78.2,40.8,39.5,37.2,33.8,31.3,28.2,22.1,12.2$; HRMS (EI) calculated for $\left[\mathrm{C}_{21} \mathrm{H}_{30} \mathrm{O}\right]^{+}$requires $\mathrm{m} / \mathrm{z} 298.2297$, found $\mathrm{m} / \mathrm{z} 298.2295$.

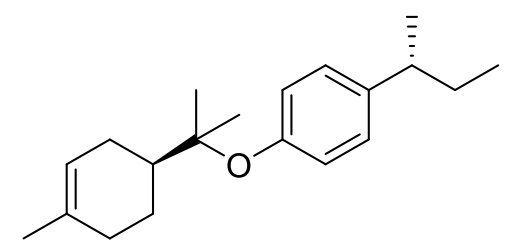

1-((R)-sec-butyl)-4-((2-((S)-4-methylcyclohex-3-en-1yl)propan-2-yl)oxy)benzene (2ah). Prepared according to the general procedure: using $\mathbf{L e} \cdot \mathrm{FeCl}_{2}(0.0149 \mathrm{~g}, 0.025 \mathrm{mmol}), \mathbf{1 a h}$ (0.1400 g, $0.50 \mathrm{mmol}), n-\mathrm{C}_{18} \mathrm{H}_{37} \mathrm{SiH}_{3}(32 \mu \mathrm{L}, 0.8906 \mathrm{~g} / \mathrm{mL}$, 
$0.10 \mathrm{mmol}), \mathrm{CH}_{3} \mathrm{CN}(6.4 \mu \mathrm{L}, 0.6406 \mathrm{~g} / \mathrm{mL}, 0.10 \mathrm{mmol}), 1 \mathrm{~mL}$ of toluene, NaBHEt3 $(75 \mu \mathrm{L}, 0.075$ mmol). After 12 hours, the reaction afforded 2ah with $>99 \%$ conversion ( $96 \%$ yield) as a colorless oil. Optical Rotation: $[\alpha]^{20} \mathrm{D}=-30.2\left(\mathrm{c} 0.76, \mathrm{CHCl}_{3}\right) .95 .5 \%$ ee, determined by HPLC after conversion to the corresponding phenol according to previously reported procedures, ${ }^{17}$ HPLC conditions: Chiralcel OJ-H, $n$-hexane $/ i-\mathrm{PrOH}=95 / 5,1.0 \mathrm{~mL} / \mathrm{min}, \mathrm{n}=220 \mathrm{~nm}$, tr 14.5 (minor), 15.4 (major). IR (neat): 2961, 2924, 1607, 1576, 1506, $1458 \mathrm{~cm}^{-1} ;{ }^{1} \mathrm{H}$ NMR: $\left(400.0 \mathrm{MHz}, \mathrm{CDCl}_{3}\right) \delta 7.04$ $(\mathrm{d}, J=8.4 \mathrm{~Hz}, 2 \mathrm{H}), 6.87(\mathrm{~d}, J=8.0 \mathrm{~Hz}, 2 \mathrm{H}), 5.41(\mathrm{~d}, J=2.8 \mathrm{~Hz}, 1 \mathrm{H}), 2.60-2.49(\mathrm{~m}, 1 \mathrm{H}), 2.18-.75$ (m, 6H), $1.67(\mathrm{~s}, 3 \mathrm{H}), 1.60-1.50(\mathrm{~m}, 2 \mathrm{H}), 1.48-1.30(\mathrm{~m}, 1 \mathrm{H}), 1.23-1.67(\mathrm{~m}, 9 \mathrm{H}), 0.80(\mathrm{t}, J=7.6 \mathrm{~Hz}$ 3H); ${ }^{13} \mathrm{C}$ NMR: $\left(100.0 \mathrm{MHz}, \mathrm{CDCl}_{3}\right) \delta$ 153.1, 142.2, 134.0, 127.2, 123.9, 120.7, 82.2, 43.8, 40.9, 31.3, 31.0, 27.1, 24.4, 24.1, 23.4, 23.2, 21.8, 12.2; HRMS (EI) calculated for $\left[\mathrm{C}_{20} \mathrm{H}_{30} \mathrm{O}\right]^{+}$requires $\mathrm{m} / \mathrm{z} 286.2297$, found $\mathrm{m} / \mathrm{z} 286.2296$.

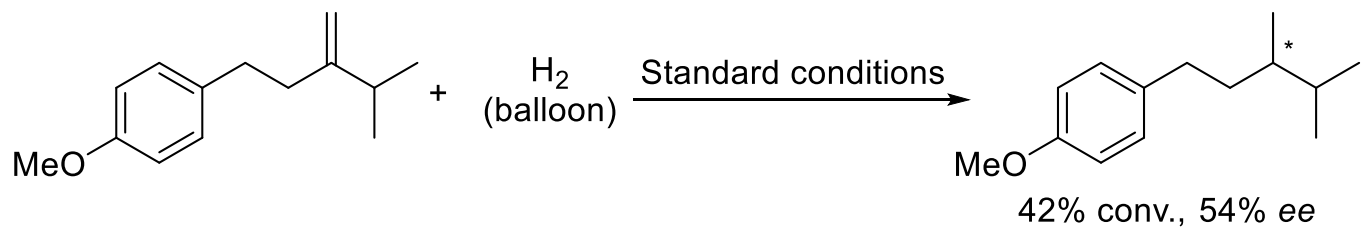<smiles>COc1ccc(CCC(C)C(C)C)cc1</smiles>
(R)-1-(3,4-dimethylpentyl)-4-methoxybenzene (2ai). Prepared according to the general procedure: using $\mathbf{L e} \cdot \mathrm{FeCl}_{2}(0.0141 \mathrm{~g}, 0.025$ mmol), 1-methoxy-4-(4-methyl-3-methylenepentyl)benzene (0.1000 g, $0.50 \mathrm{mmol}), n-\mathrm{C}_{18} \mathrm{H}_{37} \mathrm{SiH}_{3}(32 \mu \mathrm{L}, 0.8906 \mathrm{~g} / \mathrm{mL}, 0.10 \mathrm{mmol}), \mathrm{CH}_{3} \mathrm{CN}(6.4 \mu \mathrm{L}, 0.6406$ $\mathrm{g} / \mathrm{mL}, 0.10 \mathrm{mmol}), 1 \mathrm{~mL}$ of toluene, NaBHEt3 $(75 \mu \mathrm{L}, 0.075 \mathrm{mmol})$. After 12 hours, the reaction afforded chiral alkane 2 ai product with $42 \%$ conversion ( $40 \%$ yield) as a colorless oil. Optical Rotation: $[\alpha]^{20}{ }_{\mathrm{D}}=-19.9\left(\mathrm{c} 1.05, \mathrm{CHCl}_{3}\right) .54 \%$ ee, determined by HPLC, HPLC conditions: Chiralcel OB-H, $n$-hexane $/ i-\mathrm{PrOH}=99.5 / 0.5,0.5 \mathrm{~mL} / \mathrm{min}, \mathrm{n}=220 \mathrm{~nm}$, tr 9.7 (major), 10.8 (minor). ${ }^{1} \mathrm{H}$ NMR: $\left(400.0 \mathrm{MHz}, \mathrm{CDCl}_{3}\right) \delta 7.10(\mathrm{~d}, \mathrm{~J}=8.0 \mathrm{~Hz}, 2 \mathrm{H}), 6.83(\mathrm{~d}, \mathrm{~J}=8.4 \mathrm{~Hz}, 2 \mathrm{H}), 3.79(\mathrm{~s}, 3 \mathrm{H}), 2.66-2.56(\mathrm{~m}$, 1H), 2.51-2.41 (m, 1H), 1.68-1.58 (m, 2H), 1.43-1.28 (m, 2H), 0.93-0.77 (m, 9H); ${ }^{13} \mathrm{C}$ NMR: $(100.0$ $\left.\mathrm{MHz}, \mathrm{CDCl}_{3}\right) \delta 157.5,135.3,129.2,113.6,55.2,38.1,36.4,33.0,31.9,20.1,17.9,15.2 ; \mathrm{HRMS}(\mathrm{EI})$ calculated for $\left[\mathrm{C}_{14} \mathrm{H}_{22} \mathrm{O}\right]^{+}$requires $\mathrm{m} / \mathrm{z} 206.1671$, found $\mathrm{m} / \mathrm{z} 206.1673$.<smiles>COc1cc([C@@H](C)CCC=C(C)C)ccc1C</smiles>

(R)-2-methoxy-1-methyl-4-(6-methylhept-5-en-2-yl)benzene

(2aj). Prepared according to the general procedure: using $\mathbf{L b} \cdot \mathrm{FeCl}_{2}(0.0141 \mathrm{~g}, 0.025 \mathrm{mmol}), \mathbf{1 a j}(0.1150 \mathrm{~g}, 0.50 \mathrm{mmol}), n-$ 
$\mathrm{C}_{18} \mathrm{H}_{37} \mathrm{SiH}_{3}(32 \mu \mathrm{L}, 0.8906 \mathrm{~g} / \mathrm{mL}, 0.10 \mathrm{mmol}), \mathrm{CH}_{3} \mathrm{CN}(6.4 \mu \mathrm{L}, 0.6406 \mathrm{~g} / \mathrm{mL}, 0.10 \mathrm{mmol}), 1 \mathrm{~mL}$ of toluene, NaBHEt3 $(75 \mu \mathrm{L}, 0.075 \mathrm{mmol}$ ). After 12 hours, the reaction afforded 2aj with $>99 \%$ conversion ( $95 \%$ yield) as a colorless oil. Optical Rotation: $[\alpha]^{20}{ }_{\mathrm{D}}=-32.5\left(\mathrm{c} 1.75, \mathrm{CHCl}_{3}\right) .93 \%$ ee, determined by HPLC after conversion to the corresponding phenol according to previously reported procedures, ${ }^{17} \mathrm{HPLC}$ conditions: Chiralcel OJ-H, $n$-hexane $/ i$-PrOH $=95 / 5,0.8 \mathrm{~mL} / \mathrm{min}, \mathrm{n}=220 \mathrm{~nm}$, tr 7.5 (minor), 9.9 (major). IR (neat): 2958, 2923, 2855, 1613, 1583, $1512 \mathrm{~cm}^{-1} ;{ }^{1} \mathrm{H}$ NMR: (400.0 $\left.\mathrm{MHz}, \mathrm{CDCl}_{3}\right) \delta 7.04(\mathrm{~d}, J=7.2 \mathrm{~Hz}, 1 \mathrm{H}), 6.69(\mathrm{~d}, J=7.6 \mathrm{~Hz}, 1 \mathrm{H}), 6.65(\mathrm{~s}, 1 \mathrm{H}), 5.10(\mathrm{t}, J=6.4 \mathrm{~Hz}$, $1 \mathrm{H}), 3.83(\mathrm{~s}, 3 \mathrm{H}), 2.71-2.59(\mathrm{~m}, 1 \mathrm{H}), 2.18(\mathrm{~s}, 3 \mathrm{H}), 1.96-1.83(\mathrm{~m}, 2 \mathrm{H}), 1.68(\mathrm{~s}, 3 \mathrm{H}), 1.65-1.55(\mathrm{~m}$, 2H), $1.53(\mathrm{~s}, 3 \mathrm{H}), 1.22(\mathrm{~d}, J=7.2 \mathrm{~Hz}, 3 \mathrm{H}) ;{ }^{13} \mathrm{C} \mathrm{NMR}:\left(100.0 \mathrm{MHz}, \mathrm{CDCl}_{3}\right) \delta$ 157.5, 146.7, 131.4, $130.3,124.5,123.8,118.6,108.8,55.2,39.5,38.4,26.2,25.7,22.5,17.7,15.8$; HRMS (EI) calculated for $\left[\mathrm{C}_{16} \mathrm{H}_{24} \mathrm{O}\right]^{+}$requires $\mathrm{m} / \mathrm{z} 232.1827$, found $\mathrm{m} / \mathrm{z} 232.1826$.

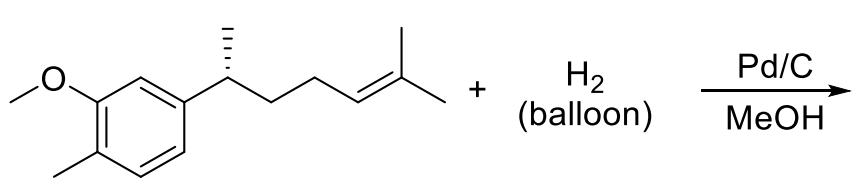

2aj<smiles>COc1cc([C@@H](C)CCCC(C)C)ccc1C</smiles>

(R)-2-methoxy-1-methyl-4-(6-methylheptan-2-yl)benzene (2aj-

1). According to the previous method, got $\mathbf{2 a j - 1}$ as a colorless oil. IR (neat): 2955, 2926, 1869, 1612, 1584, $1512 \mathrm{~cm}^{-1} ;{ }^{1} \mathrm{H}$ NMR: $\left(400.0 \mathrm{MHz}, \mathrm{CDCl}_{3}\right) \delta 7.04(\mathrm{~d}, J=7.6 \mathrm{~Hz}, 1 \mathrm{H}), 6.69$ (d, $\left.J=7.6 \mathrm{~Hz}, 1 \mathrm{H}\right), 6.65(\mathrm{~s}, 1 \mathrm{H}), 3.83(\mathrm{~s}, 3 \mathrm{H})$, 2.69-2.58 (m, 1H), $2.18(\mathrm{~s}, 3 \mathrm{H}), 1.55-1.42(\mathrm{~m}, 2 \mathrm{H}), 1.32-1.10(\mathrm{~m}, 7 \mathrm{H}), 0.87-0.78(\mathrm{~m}, 6 \mathrm{H}) ;{ }^{13} \mathrm{C} \mathrm{NMR}$ : $\left(100.0 \mathrm{MHz}, \mathrm{CDCl}_{3}\right) \delta 157.5,147.0,130.3,123.7,118.5,108.8,55.2,40.0,39.0,38.7,27.8,25.5$, 22.7, 22.6, 22.4, 15.8; HRMS (EI) calculated for $\left[\mathrm{C}_{16} \mathrm{H}_{26} \mathrm{O}\right]^{+}$requires $\mathrm{m} / \mathrm{z} 234.1984$, found $\mathrm{m} / \mathrm{z}$ 234.1983.<smiles>Cc1ccc(CCCCC(C)C)cc1O</smiles>

(R)-2-methyl-5-(6-methylheptan-2-yl)phenol $\quad(2 \mathrm{aj}-2) . \quad$ Be converted by the $\mathbf{2 a j}-\mathbf{1}$ according to previously reported procedures ${ }^{17}$ IR (neat): 3442, 2956, 2926, 1621, 1576, $1505 \mathrm{~cm}^{-1}$; ${ }^{1} \mathrm{H}$ NMR: (400.0 MHz, $\left.\mathrm{CDCl}_{3}\right) \delta 7.03(\mathrm{~d}, J=7.6 \mathrm{~Hz}, 1 \mathrm{H}), 6.68(\mathrm{~d}, J=7.6 \mathrm{~Hz}, 1 \mathrm{H}), 6.61(\mathrm{~s}, 1 \mathrm{H})$, $4.61(\mathrm{~s}, 1 \mathrm{H}), 2.65-2.54(\mathrm{~m}, 1 \mathrm{H}), 2.22(\mathrm{~s}, 3 \mathrm{H}), 1.55-1.40(\mathrm{~m}, 3 \mathrm{H}), 1.29-1.08(\mathrm{~m}, 7 \mathrm{H}), 0.86-0.79(\mathrm{~m}$, 6H); ${ }^{13} \mathrm{C}$ NMR: $\left(100.0 \mathrm{MHz}, \mathrm{CDCl}_{3}\right) \delta 153.5,147.5,130.7,120.7,119.4,113.4,39.5,39.0,38.6$, 27.8, 25.4, 22.7, 22.6, 22.3, 15.3; HRMS (EI) calculated for $\left[\mathrm{C}_{15} \mathrm{H}_{24} \mathrm{O}\right]^{+}$requires $\mathrm{m} / \mathrm{z} 220.1827$, 
found $\mathrm{m} / \mathrm{z} 220.1829$.

\section{Gram-scale experiment:}

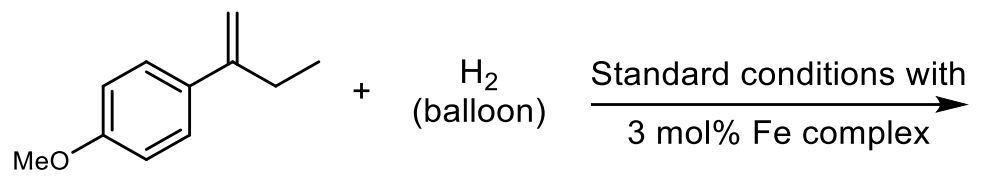<smiles>CC[C@H](C)c1ccc(OC)cc1</smiles>

Prepared according to the general procedure: using $\mathbf{L e} \bullet \mathrm{FeCl}_{2}(0.1184 \mathrm{~g}, 0.21 \mathrm{mmol}), \mathbf{1 a}(1.1037 \mathrm{~g}$, $7.0 \mathrm{mmol}), n-\mathrm{C}_{18} \mathrm{H}_{37} \mathrm{SiH}_{3}(270 \mu \mathrm{L}, 0.8906 \mathrm{~g} / \mathrm{mL}, 0.84 \mathrm{mmol}), \mathrm{CH}_{3} \mathrm{CN}(54 \mu \mathrm{L}, 0.6406 \mathrm{~g} / \mathrm{mL}, 0.84$ mmol), $3 \mathrm{~mL}$ of toluene, NaBHEt $3(630 \mu \mathrm{L}, 0.63 \mathrm{mmol})$. After 22 hours, the reaction afforded $2 \mathrm{a}$ with $>99 \%$ conversion ( $98 \%$ yield, $1.0925 \mathrm{~g}$ ) as a colorless oil. $98.2 \% e e$, determined by GC, GC conditions: Chiralcel B-DM, $100 \mathrm{kPa} \mathrm{N} 2\left(60{ }^{\circ} \mathrm{C}-30 \mathrm{~min}-1{ }^{\circ} \mathrm{C} / \mathrm{min}-110{ }^{\circ} \mathrm{C}-5 \mathrm{~min}\right), \operatorname{tr} 67.5$ (minor), 67.9 (major).

The chiral alkanes 2aa and $\mathbf{2 a b}$ could be further transformed through known procedures to deliver bioactive natural compounds (+)-erogorgiaene ${ }^{21}$ and (+)-mutisianthol (Figure S1). ${ }^{22}$

a)<smiles>CCO[C@H]1CC[C@H](C(C)CCC=C(C)C)c2cc(C)ccc2[C@H]1C(C)CCC=O</smiles>

2aa: $96 \%$ ee<smiles>COc1cc([C@@H](C)CCC2OCCO2)ccc1C</smiles>

2ab: $97 \%$ ee
$88 \%$ yield $96 \%$ ee

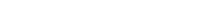

(+)-Erogorgiaene<smiles>Cc1cc2c(cc1O)[C@H](C)C[C@H]2CC(C)C</smiles>

(+)-Mutisianthol

Figure S1. Synthetic applications of hydrogenation products<smiles>Cc1ccc([C@@H](C)CCCC2OCCO2)cc1</smiles><smiles>Cc1ccc([C@@H](C)CCC=O)cc1</smiles>

(R)-4-(p-tolyl)pentanal $\quad$ (4aa). ${ }^{23} \quad$ To $\quad(R)-2-(3-(p$-tolyl)butyl)-1,3dioxolane (2aa) (0.0404 g, 1 equiv) was added a solution of $\mathrm{AcOH}$ (6 $\mathrm{ml})$ and $\mathrm{H}_{2} \mathrm{O}(2 \mathrm{ml})$. The reaction mixture was stirred at $60{ }^{\circ} \mathrm{C}$ for $5 \mathrm{~h}$ and then was cooled down to room temperature. Adding the sodium hydroxide solution to adjust $\mathrm{pH}$ to 7 , then exacted with ethyl acetate $(3 \times 10 \mathrm{~mL})$, the solvent was removed in vacuo, providing the 
crude residue. The crude material was purified by column chromatography (PE/EA (30/1)) to give 4aa $(0.0286 \mathrm{~g}, 88 \%$ yield $)$ as a colorless oil. Optical Rotation: $[\alpha]^{20} \mathrm{D}=-33.9\left(\mathrm{c} 0.91, \mathrm{CHCl}_{3}\right) .96 \%$ $e e$, determined by HPLC, HPLC conditions: Chiralcel OJ-H, $n$-hexane $/ i-\mathrm{PrOH}=99 / 1,1.0 \mathrm{~mL} / \mathrm{min}$, $\mathrm{n}=220 \mathrm{~nm}$, tr 12.1 (minor), 14.1 (major). ${ }^{1} \mathrm{H}$ NMR: $\left(400.0 \mathrm{MHz}, \mathrm{CDCl}_{3}\right) \delta 9.68(\mathrm{t}, J=1.6 \mathrm{~Hz}, 1 \mathrm{H})$, 7.14-7.03 (m, 4H), 2.75-2.63 (m, 1H), 2.37-2.26 (m, 5H), 1.97-1.80 (m, 2H), $1.26(\mathrm{~d}, J=7.2 \mathrm{~Hz}$, 3H); ${ }^{13} \mathrm{C}$ NMR: $\left(100.0 \mathrm{MHz}, \mathrm{CDCl}_{3}\right) \delta 202.4,142.9,135.7,129.1,126.8,42.1,38.8,30.3,22.3$, 20.9. All the spectroscopic data were in agreement with the reported ones. ${ }^{23}$<smiles>COc1cc([C@@H](C)CCC2OCCO2)ccc1C</smiles><smiles>COc1cc2c(cc1C)C=CC[C@H]2C</smiles>

(R)-7-methoxy-1,6-dimethyl-1,2-dihydronaphthalene (4ab). ${ }^{24}$ To $(R)-2-$ (3-(3-methoxy-4-methylphenyl)butyl)-1,3-dioxolane (2ab) (0.0655 g, 1 equiv) was added a solution of $\mathrm{HCl}(6 \mathrm{M}, 20 \mathrm{~mL})$. The reaction mixture was stirred at room temperature for $19 \mathrm{~h}$. Then washed with DCM $(3 \times 10 \mathrm{~mL})$, The solvent was removed in vacuo, providing the crude residue. The crude material was purified by column chromatography (PE) to give 4ab (0.0463 g, 94\% yield) as a colorless oil. $97.7 \%$ ee, determined by $\mathrm{GC}, \mathrm{GC}$ conditions: Chiralcel B-DM, $100 \mathrm{kPa} \mathrm{N} 2\left(40{ }^{\circ} \mathrm{C}-90 \mathrm{~min}^{-} 1^{\circ} \mathrm{C} / \mathrm{min}-150{ }^{\circ} \mathrm{C}-5 \mathrm{~min}\right), \operatorname{tr} 183.7$ (major), 186.8 (minor). ${ }^{1} \mathrm{H}$ NMR: $\left(400.0 \mathrm{MHz}, \mathrm{CDCl}_{3}\right) \delta 6.83(\mathrm{~s}, 1 \mathrm{H}), 6.66(\mathrm{~s}, 1 \mathrm{H}), 6.36(\mathrm{~d}, J=7.6$ Hz, 1H), 5.83-5.76 (m, 1H), 3.84 (s, 3H), 2.94-2.83 (m, 1H), 2.49-2.40 (m, 1H), 2.17 (s, 3H), 2.13$2.03(\mathrm{~m}, 1 \mathrm{H}), 1.23(\mathrm{~d}, J=6.8 \mathrm{~Hz}, 3 \mathrm{H}) ;{ }^{13} \mathrm{C}$ NMR: $\left(100.0 \mathrm{MHz}, \mathrm{CDCl}_{3}\right) \delta 156.8,139.4,128.7,126.7$, $125.9,124.2,123.9,108.5,55.4,32.0,31.1,20.3,15.7$. All the spectroscopic data were in agreement with the reported ones. ${ }^{24}$

\section{Mechanistic Studies}

\section{Deuterium labeling experiments:}

In order to figure out the reaction cycle, the $(R)$ and $(S)$-alkyl magnesium bromide $((\boldsymbol{R})$-2a-MgBr and $(\boldsymbol{S})$-2a$\mathbf{M g B r}$ ) have been prepared following the previously reported methods (Figure S2a and S2b). The iron precatalyst $\mathbf{L} 2 \mathbf{e} \cdot \mathbf{F e C l}_{2}$ could be activated by 2 equiv. of MeLi to generate a proposed iron methyl species based on literatures. ${ }^{24}$ The 1 equiv. of $(\boldsymbol{R})-\mathbf{2} \mathbf{a}-\mathbf{M g B r}$ was added to a solution of proposed iron methyl species in which a proposed iron-alkyl species $((\boldsymbol{R})$-2a-Fe $)$ could be obtained. Then the D-hydrosilane was added to the 
system, the D-alkane $\mathbf{d 5 - 2 a}$ was obtained in $43 \%$ yield with $99 \%$ ee and $0.34 \mathrm{D}$-atom on the methyl group, the d3-1a and 3a have also been observed with $43 \%$ recovery and 5\% yield, respectively (Figure S2c). The generation of $\boldsymbol{d 3 - 1 a}$ and 3a demonstrated that the iron-alkyl species $((\boldsymbol{R})-\mathbf{2 a}-\mathbf{F e})$ should be formed and then underwent $\beta$-hydride elimination (alkene insertion and $\beta$-hydride elimination for 3a). In the control experiment of $(\boldsymbol{R})$-2a-MgBr with D-hydrosilane in the absence of the iron complex, no deuterium cooperation was observed on the product which indicated that the $(R)$-alkyl magnesium bromide $(99 \%$ ee $)$ did not react with D-hydrosilane (Figure S2e). Compared to the control experiment, the proposed iron-alkyl species $((\boldsymbol{R})$ 2a-Fe) may react with D-hydrosilane via $\sigma$-bond metathesis to afford $\boldsymbol{d 5 - 2 a}$ and the Fe-Si species (eq. S1). The reaction of $(S)$-alkyl magnesium bromide $((\boldsymbol{S})-\mathbf{2} \mathbf{a}-\mathbf{M g B r},-94 \%$ ee $)$ with a proposed iron methyl species afforded the deuterium alkane $\mathbf{d 6 - 2 a}$ in $73 \%$ yield with $-82 \%$ ee and $0.66 \mathrm{D}$-atom on the methyl group, the $\mathbf{1 a}$ and 3a with 13\% recovery and 2\% yield, respectively (Figure S2d). The decrease of enantioselectivity from $-94 \%$ to $-82 \%$ indicated that the iron alkyl species $((\boldsymbol{S})$-2a-Fe $)$ could be formed and then underwent $\beta$-hydride elimination to give 1a and iron hydride species which could undergo alkene insertion to afford the iron alkyl species $((\boldsymbol{R})$-2a-Fe). This result also supported the formation of iron hydride species and $\sigma$-bond metathesis of the iron alkyl species with D-hydrosilane (eq. S2). 
a)

$$
\text { 1a } \underset{\text { cat. (S)-OIP-CoCl }}{\stackrel{\text { hydroboration }}{\longrightarrow}} \stackrel{\begin{array}{l}
\text { 1) } \mathrm{H}_{2} \mathrm{O}_{2}, \mathrm{KOH} \\
\mathrm{THF}, 0^{\circ} \mathrm{C}
\end{array}}{\stackrel{\begin{array}{l}
\mathrm{THF}^{2} \mathrm{CBr}_{4}, \mathrm{PPh}_{3} \\
\mathrm{THF}, \mathrm{rt}
\end{array}}{\longrightarrow}}
$$

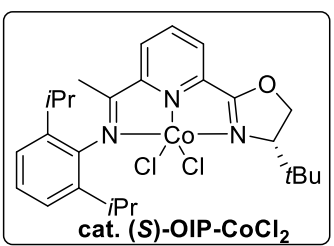

cat. (S)-OIP-CoCl

b)

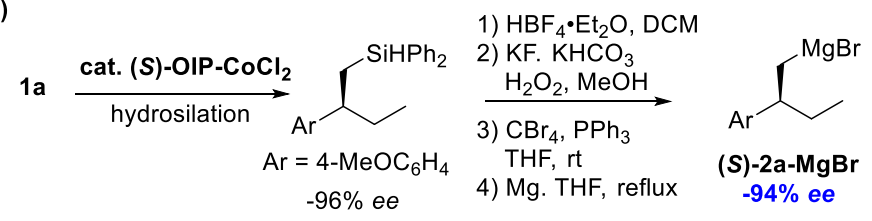

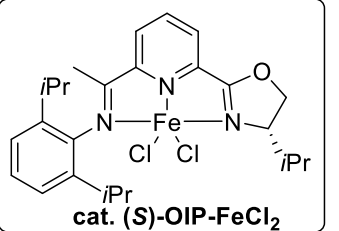

c)

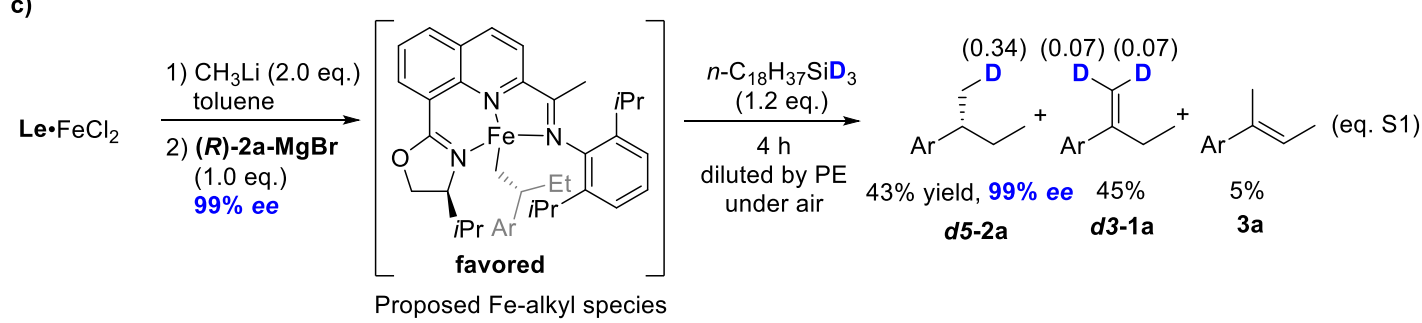

$\begin{array}{ll} & \\ \mathrm{Le} \cdot \mathrm{FeCl}_{2} & \begin{array}{l}\text { 1) } \mathrm{CH}_{3} \mathrm{Li}(2.0 \mathrm{eq} .) \\ \text { toluene }\end{array} \\ & \begin{array}{l}\text { 2) }(\mathrm{S})-2 \mathrm{a}-\mathrm{MgBr} \\ (1.0 \mathrm{eq} .) \\ -94 \% \text { ee }\end{array}\end{array}$

$-94 \%$ ee

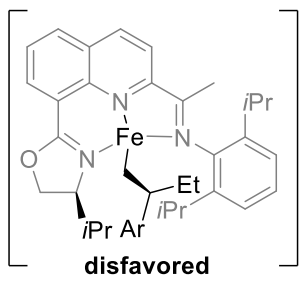

Proposed Fe-alkyl species

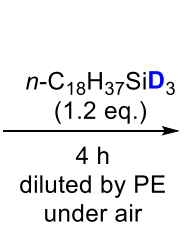

$\overbrace{}^{\mathrm{D}}+$ $73 \%$ yield, $-82 \%$ ee $13 \%$

d6-2a (eq. S2)

e)

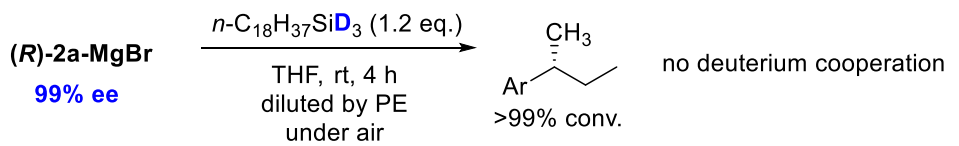

Figure S2. Hydrogenation of $(R)$ and $(S)$-alkyl magnesium bromide $((\boldsymbol{R})-\mathbf{2 a}-\mathbf{M g B r}$ and $(\boldsymbol{S})$-2a-MgBr $)$ with or without the iron complex<smiles>CCC(Cc1ccccc1)c1ccc(OC)cc1</smiles>

(R)-2-(2-(4-methoxyphenyl)butyl)-4,4,5,5-tetramethyl-1,3,2-

dioxaborolane. According to the previous method, ${ }^{26}$ the reaction afford the hydroboration product as a colorless oil (1.0783 g, 72\% yield). IR

(neat): 2977, 2928, 1612, 1512, 1463, $1369 \mathrm{~cm}^{-1} ;{ }^{1} \mathrm{H}$ NMR: $\left(399.9 \mathrm{MHz}, \mathrm{CDCl}_{3}\right) \delta 7.11(\mathrm{~d}, \mathrm{~J}=8.8$ $\mathrm{Hz}, 2 \mathrm{H}), 6.80(\mathrm{~d}, \mathrm{~J}=8.8 \mathrm{~Hz}, 2 \mathrm{H}), 3.77(\mathrm{~s}, 3 \mathrm{H}), 2.74-2.64(\mathrm{~m}, 1 \mathrm{H}), 1.67-1.47(\mathrm{~m}, 2 \mathrm{H}), 1.23-1.18(\mathrm{~m}$, 1H), $1.10(\mathrm{~s}, 12 \mathrm{H}), 1.09-1.01(\mathrm{~m}, 1 \mathrm{H}), 0.76(\mathrm{t}, \mathrm{J}=7.2 \mathrm{~Hz}, 3 \mathrm{H}) ;{ }^{13} \mathrm{C} \mathrm{NMR:}\left(100.6 \mathrm{MHz}, \mathrm{CDCl}_{3}\right) \delta$ 157.6, 139.4, 128.3, 113.3, 82.8, 55.2, 42.4, 32.4, 24.7, 24.6, 12.2; HRMS (EI) calculated for $\left[\mathrm{C}_{17} \mathrm{H}_{27} \mathrm{O}_{3}{ }^{11} \mathrm{~B}\right]^{+}$requires $\mathrm{m} / \mathrm{z} 290.2053$, found $\mathrm{m} / \mathrm{z} 290.2052$. 
<smiles>CC[C@H](CO)c1ccc(OC)cc1</smiles>

(R)-2-(4-methoxyphenyl)butan-1-ol. According to the previous method, ${ }^{27}$ the reaction afford the hydroboration product as a colorless oil (0.6992 g, 97\% yield). IR (neat): 3353, 2960, 2874, 1612, 1583, $1513 \mathrm{~cm}^{-}$

1. ${ }^{1} \mathrm{H}$ NMR: (399.9 MHz, $\left.\mathrm{CDCl}_{3}\right) \delta 7.13(\mathrm{~d}, \mathrm{~J}=8.8 \mathrm{~Hz}, 2 \mathrm{H}), 6.88(\mathrm{~d}, \mathrm{~J}=8.8 \mathrm{~Hz}, 2 \mathrm{H}), 3.80(\mathrm{~s}, 3 \mathrm{H})$, 3.78-3.62 (m, 2H), 2.68-2.59 (m, 1H), 1.79-1.67 (m, 1H), 1.59-1.46 (m, 1H), 1.35-1.27 (s, 1H), 0.83 $(\mathrm{t}, \mathrm{J}=7.6 \mathrm{~Hz}, 3 \mathrm{H}) ;{ }^{13} \mathrm{C} \mathrm{NMR}:\left(100.6 \mathrm{MHz}, \mathrm{CDCl}_{3}\right) \delta 158.3,134.0,128.9,114.0,67.4,55.2,49.6$, 25.0, 11.9; HRMS (EI) calculated for $\left[\mathrm{C}_{11} \mathrm{H}_{16} \mathrm{O}_{2}\right]^{+}$requires $\mathrm{m} / \mathrm{z} 180.1150$, found $\mathrm{m} / \mathrm{z} 180.1150$.<smiles>CC[C@H](CBr)c1ccc(OC)cc1</smiles>
(R)-1-(1-bromobutan-2-yl)-4-methoxybenzene. According to the previous method, ${ }^{27}$ the reaction afford the hydroboration product as a colorless oil (0.7605 g, 85\% yield). IR (neat): 2962, 2835, 1611, 1584, 1513, $1461 \mathrm{~cm}^{-1}$; ${ }^{1} \mathrm{H}$ NMR: (399.9 MHz, $\left.\mathrm{CDCl}_{3}\right) \delta 7.09(\mathrm{~d}, \mathrm{~J}=8.8 \mathrm{~Hz}, 2 \mathrm{H}), 6.87(\mathrm{~d}, \mathrm{~J}=8.4 \mathrm{~Hz}, 2 \mathrm{H})$, $3.80(\mathrm{~s}, 3 \mathrm{H}), 3.54(\mathrm{~d}, \mathrm{~J}=8.0 \mathrm{~Hz}, 2 \mathrm{H}), 2.85-2.74(\mathrm{~m}, 1 \mathrm{H}), 2.02-1.90(\mathrm{~m}, 1 \mathrm{H}), 1.66-1.54(\mathrm{~m}, 1 \mathrm{H})$, $0.81(\mathrm{t}, \mathrm{J}=7.6 \mathrm{~Hz}, 3 \mathrm{H}) ;{ }^{13} \mathrm{C}$ NMR: $\left(100.6 \mathrm{MHz}, \mathrm{CDCl}_{3}\right) \delta 158.4,134.2,128.6,113.8,55.2,48.9$, 39.1, 27.1, 11.9. HRMS (EI) calculated for $\left[\mathrm{C}_{11} \mathrm{H}_{15} \mathrm{OBr}\right]^{+}$requires $\mathrm{m} / \mathrm{z} 242.0305$, found $\mathrm{m} / \mathrm{z}$ 242.0306 .

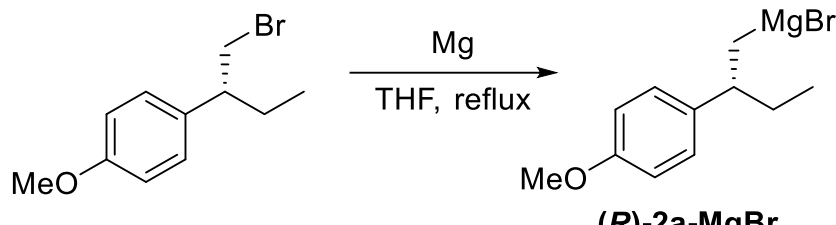

(R)-2a-MgBr

An oven-dried thick walled glass vessel was charged with $0.058 \mathrm{~g}$ (2.4 mmol) magnesium ribbon and a stir bar and heated under vacuum using a heat gun. Upon cooling to room temperature, the flask was charged with $2 \mathrm{~mL}$ anhydrous tetrahydrofuran. To the flask was added $0.4867 \mathrm{~g}(2.0 \mathrm{mmol})$ (R)-1-(1-bromobutan-2-yl)-4-methoxybenzene dropwise. The flask was stirred at $78^{\circ} \mathrm{C}$ for 0.5 hours and stirred at room temperature. The Grignard reagent solution was determined to be $0.313 \mathrm{M}$ according to a literature procedure. ${ }^{28}$ The ee values was tested correspond to the resulting alkanes from the hydrolysis of the alkylmagnesium bromide. $99 \%$ ee, determined by GC, GC conditions: Chiralcel B-DM, $100 \mathrm{kPa} \mathrm{N} 2\left(60{ }^{\circ} \mathrm{C}-30 \mathrm{~min}-1{ }^{\circ} \mathrm{C} / \min -115{ }^{\circ} \mathrm{C}-5 \mathrm{~min}\right), \operatorname{tr} 67.3$ (minor), 67.9 (major). 


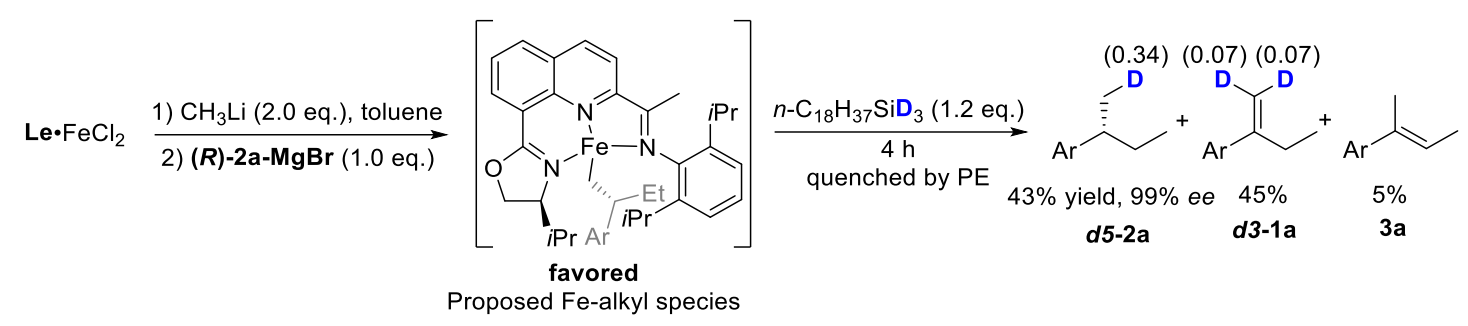

To an oven-dried Shrek tube was added $0.0567 \mathrm{~g}(0.10 \mathrm{mmol}) \mathbf{L 2 e} \cdot \mathrm{FeCl}_{2}$ and $1.0 \mathrm{~mL}$ toluene. To the tube was added 2.0 eq. of $\mathrm{CH}_{3} \mathrm{Li}(1.6 \mathrm{M}$ in THF, $125 \mu \mathrm{L})$ and stirring for $2 \mathrm{~min}$. Then the tube was added $0.35 \mathrm{~mL}$ of $(\boldsymbol{R})-\mathbf{2 a}-\mathbf{M g B r}$ at room temperature and stirring for 2 min., finally the $n$ $\mathrm{C}_{18} \mathrm{H}_{37} \mathrm{SiD}_{3}$ (1.2 eq., $0.0341 \mathrm{~g}$ ) was added and stirring for $4 \mathrm{~h}$. The organics were quched by PE. The D-incorporation was determined by ${ }^{2} \mathrm{H}$ NMR using $\mathrm{CDCl}_{3}$ as the internal standard $(0.0374 \mathrm{~g}$ of

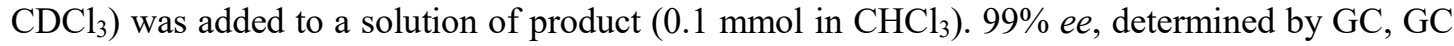
conditions: Chiralcel B-DM, $100 \mathrm{kPa} \mathrm{N} 2\left(60{ }^{\circ} \mathrm{C}-30 \mathrm{~min}-1{ }^{\circ} \mathrm{C} / \mathrm{min}-115{ }^{\circ} \mathrm{C}-5 \mathrm{~min}\right), \operatorname{tr} 67.4$ (minor), 67.9 (major). When the ( $\boldsymbol{R})-\mathbf{2 a}-\mathbf{M g B r}$ reacted with $n-\mathrm{C}_{18} \mathrm{H}_{37} \mathrm{SiD}_{3}$ for $4 \mathrm{~h}$, then quenched by PE, the product was obtained and no deuterium was detected.<smiles>CCC(C[Hg]c1ccccc1)c1ccc(OC)cc1</smiles>

(S)-(2-(4-methoxyphenyl)butyl)diphenylsilane. According to the previous method, ${ }^{29}$ the reaction afford the hydroboration product as a colorless oil (1.0401 g, 54\% yield). ${ }^{1} \mathrm{H}$ NMR: $\left(399.9 \mathrm{MHz}, \mathrm{CDCl}_{3}\right) \delta$ 7.53-7.23 (m, 10H), $6.99(\mathrm{~d}, \mathrm{~J}=8.4 \mathrm{~Hz}, 2 \mathrm{H}), 6.76(\mathrm{~d}, \mathrm{~J}=8.4 \mathrm{~Hz}, 2 \mathrm{H}), 4.63(\mathrm{t}, \mathrm{J}=4.0 \mathrm{~Hz}, 1 \mathrm{H}), 3.78$ (s, 3H), 2.63-2.53 (m, 1H), 1.79-1.67 (m, 1H), 1.50-1.40 (m, 1H), $0.71(\mathrm{t}, \mathrm{J}=7.2 \mathrm{~Hz}, 3 \mathrm{H}) .-96.5 \%$ $e e$, determined by HPLC, HPLC conditions: Chiralcel AD-H, $n$-hexane $/ i-\mathrm{PrOH}=98 / 2,1.0 \mathrm{~mL} / \mathrm{min}$, $\mathrm{n}=220 \mathrm{~nm}, \operatorname{tr} 4.7$ (major), 5.3 (minor). All the spectroscopic data were in agreement with the reported ones. ${ }^{24}$<smiles>CCC(CO)c1ccc(OC)cc1</smiles>

(S)-2-(4-methoxyphenyl)butan-1-ol. Prepared according to a previous reported method. ${ }^{30}$ To $\quad$ a $\quad 50 \quad \mathrm{~mL}$ vial, $\quad(S)-(2-(4-$ methoxyphenyl)butyl)diphenylsilane (1.0401 g, $3.0 \mathrm{mmol})$ and $20 \mathrm{~mL}$ of DCM were added. The solution was stirred at $0{ }^{\circ} \mathrm{C}$. Then $\mathrm{HBF}_{4} \mathrm{Et}_{2} \mathrm{O}(4.38 \mathrm{~g}, 20 \mathrm{mmol}, 40 \% \mathrm{Wt})$ was added. After stirred for 3 hours at ambient temperature, the solvent was removed under reduced pressure. To the residue, $5.0 \mathrm{~mL}$ of THF, $5.0 \mathrm{~mL}$ of $\mathrm{MeOH}, \mathrm{KF}(0.93 \mathrm{~g}, 16 \mathrm{mmol}), \mathrm{KHCO}_{3}(4.0 \mathrm{~g}$, $40 \mathrm{mmol})$ and $\mathrm{H}_{2} \mathrm{O}_{2}(20 \mathrm{~mL}, 30 \% \mathrm{Wt})$ were added in sequence. After stirred at room temperature for $15 \mathrm{~h}$, the mixture was diluted with $\mathrm{H}_{2} \mathrm{O}$, extracted with $\mathrm{Et}_{2} \mathrm{O}$ for three times. The combined 
organic layers were washed with brine, dried over $\mathrm{Na}_{2} \mathrm{SO}_{4}$ and concentrated in vacuo. The crude mixture was purified by flash column chromatography using PE/EtOAc (4/1) as the eluent to give $0.342 \mathrm{~g}(1.9 \mathrm{mmol}, 64 \%$ yield $)$ of the title compound as a colorless oil. ${ }^{1} \mathrm{H}$ NMR: $(399.9 \mathrm{MHz}$, $\left.\mathrm{CDCl}_{3}\right) \delta 7.14(\mathrm{~d}, \mathrm{~J}=8.8 \mathrm{~Hz}, 2 \mathrm{H}), 6.88(\mathrm{~d}, \mathrm{~J}=8.8 \mathrm{~Hz}, 2 \mathrm{H}), 3.80(\mathrm{~s}, 3 \mathrm{H}), 3.79-3.62(\mathrm{~m}, 2 \mathrm{H}), 2.69-$ $2.59(\mathrm{~m}, 1 \mathrm{H}), 1.79-1.68(\mathrm{~m}, 1 \mathrm{H}), 1.59-1.46(\mathrm{~m}, 1 \mathrm{H}), 1.36-1.27(\mathrm{~s}, 1 \mathrm{H}), 0.83(\mathrm{t}, \mathrm{J}=7.6 \mathrm{~Hz}, 3 \mathrm{H})$.

This same procedure was used with another $(S)$-enantioenriched alkylmagnesium bromide reagents.

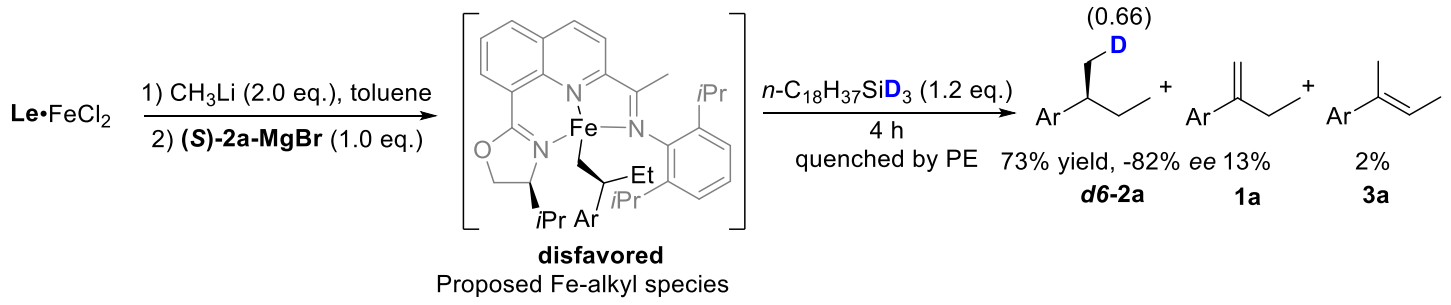

To an oven-dried Shrek tube was added $0.0567 \mathrm{~g}(0.10 \mathrm{mmol})$ of $\mathbf{L 2 e} \cdot \mathrm{FeCl}_{2}$ and $1.0 \mathrm{~mL}$ toluene. To the tube was added 2.0 eq. of $\mathrm{CH}_{3} \mathrm{Li}(1.6 \mathrm{M}$ in THF, $125 \mu \mathrm{L})$ and stirring for $2 \mathrm{~min}$. then the tube was added $0.34 \mathrm{~mL}$ of $(\boldsymbol{S}) \mathbf{- 2} \mathbf{a}-\mathbf{M g B r}$ at room temperature and stirring for $2 \mathrm{~min}$., finally the $n$ $\mathrm{C}_{18} \mathrm{H}_{37} \mathrm{SiD}_{3}$ (1.2 eq., $0.0341 \mathrm{~g}$ ) was added and stirring for $4 \mathrm{~h}$. The mixtures were quenched by PE. The D-incorporation was determined by ${ }^{2} \mathrm{H}$ NMR using $\mathrm{CDCl}_{3}$ as the internal standard $(0.0747 \mathrm{~g}$ of $\left.\mathrm{CDCl}_{3}\right)$ was added to a solution of product $\left(0.0150 \mathrm{~g}\right.$ in $\left.\mathrm{CHCl}_{3}\right) .-82 \%$ ee, determined by HPLC after conversion to the corresponding phenol according to previously reported procedures, ${ }^{17}$ HPLC conditions: Chiralcel OJ-H, $n$-hexane $/ i-\mathrm{PrOH}=95 / 5,1.0 \mathrm{~mL} / \mathrm{min}, \mathrm{n}=220 \mathrm{~nm}$, tr 14.4 (major), 15.8 (minor).

Two control experiments have been carried out. The reaction using $\mathrm{D}_{2}$ was quenched after 4 hours to access $\boldsymbol{d} 7-2 \mathbf{a}$ in $27 \%$ conversion and $73 \%$ recovery of $\mathbf{1 a}$ with a small amount of deuterium atom on the terminal position of alkene (eq. S3). This result did support the possibility of 2,1-insertion in the presence of $\mathrm{MeCN}$, and also demonstrated that $\beta$-hydride elimination of iron intermediate via 2,1-insertion occurred to give the terminal alkene without any trisubstituted alkene.

The reaction using $\mathrm{D}_{2}$ without hydrosilane and $\mathrm{MeCN}$ has been conducted to afford $\boldsymbol{d} \mathbf{8}$-2a in $22 \%$ conversion, d1-3a in $36 \%$ yield, and $42 \%$ recovery of $1 \mathbf{a}$ with a small amount of deuterium atom on the terminal position of alkene (eq. S4). Compared to eq. S3, an observed isomerization product d1-3a in the absence of $\mathrm{MeCN}$ indicated that $\mathrm{MeCN}$ should prevent $\beta$-hydride elimination of iron intermediate via 2,1-insertion to produce the trisubstituted alkene. 
The experiments with $\boldsymbol{d 3 - 1}$ a and 1a has been carried out to afford the product with 0.96 of KIE (eq. S5). This reaction could not rule out the intermediacy of benzyl complexes.

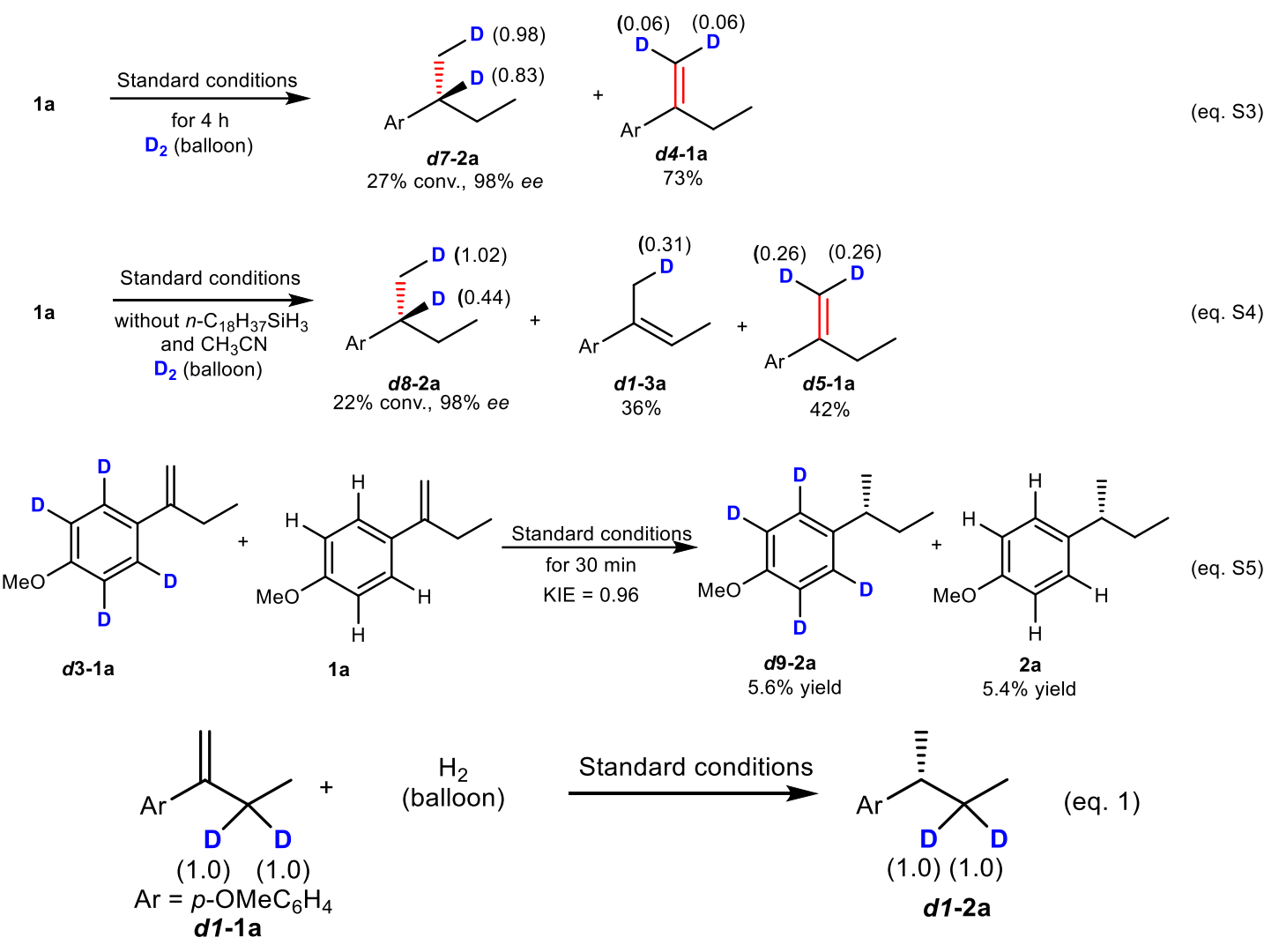
$(\boldsymbol{R})-1-($ butan-2-yl-3,3-d2)-4-methoxybenzene $\quad$ (d1-2a). Prepared
mmol), d1-1a $(0.0871 \mathrm{~g}, 0.50 \mathrm{mmol}), n-\mathrm{C}_{18} \mathrm{H}_{37} \mathrm{SiH}_{3}(32 \mu \mathrm{L}, 0.8906 \mathrm{~g} / \mathrm{mL}$, $0.10 \mathrm{mmol}), \mathrm{CH}_{3} \mathrm{CN}(6.4 \mu \mathrm{L}, 0.6406 \mathrm{~g} / \mathrm{mL}, 0.10 \mathrm{mmol}), 1 \mathrm{~mL}$ of toluene, NaBHEt3 $(75 \mu \mathrm{L}, 0.075$ mmol). After 12 hours, the reaction afforded $\boldsymbol{d 1 - 2 a}$ with $>99 \%$ conversion as a colorless oil. Optical Rotation: $[\alpha]^{20}{ }_{\mathrm{D}}=-6.1\left(\mathrm{c} 1.01, \mathrm{CHCl}_{3}\right) .99 \%$ ee, determined by GC, GC conditions: Chiralcel B$\mathrm{DM}, 100 \mathrm{kPa} \mathrm{N} 2\left(60{ }^{\circ} \mathrm{C}-30 \mathrm{~min}-2{ }^{\circ} \mathrm{C} / \mathrm{min}-110{ }^{\circ} \mathrm{C}-5 \mathrm{~min}\right)$, tr 55.2 (minor), 55.5 (major). IR (neat): 2924, 2853, 1609, 1514, $1464 \mathrm{~cm}^{-1}$; ${ }^{1} \mathrm{H}$ NMR: $\left(399.9 \mathrm{MHz}, \mathrm{CDCl}_{3}\right) \delta 7.09$ (d, $J=8.8 \mathrm{~Hz}$, 2H), $6.84(\mathrm{~d}, J=8.4 \mathrm{~Hz}, 2 \mathrm{H}), 3.79(\mathrm{~s}, 3 \mathrm{H}), 2.53(\mathrm{q}, J=13.6,6.8 \mathrm{~Hz}, 1 \mathrm{H}), 1.20(\mathrm{~d}, J=6.8 \mathrm{~Hz}, 3 \mathrm{H})$, $0.79(\mathrm{~s}, 3 \mathrm{H}) ;{ }^{13} \mathrm{C}$ NMR: $\left(100.0 \mathrm{MHz}, \mathrm{CDCl}_{3}\right) \delta 157.6,139.8,127.8,113.6,55.1,40.6,31.1-22.0(\mathrm{~m}$, 1C), 21.9, 12.0. HRMS (EI) calculated for $\left[\mathrm{C}_{11} \mathrm{H}_{14} \mathrm{D}_{2} \mathrm{O}\right]^{+}$requires $\mathrm{m} / \mathrm{z} 166.1327$, found $\mathrm{m} / \mathrm{z}$ 166.1325 . 


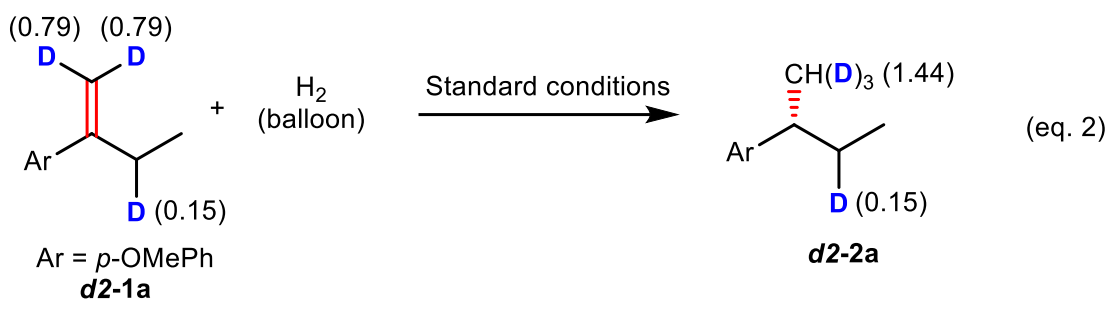

Prepared according to the general procedure: using $\mathbf{L e} \bullet \mathrm{FeCl}_{2}(0.0141 \mathrm{~g}, 0.025 \mathrm{mmol}), \boldsymbol{d} \mathbf{2}-\mathbf{- 1 a}(81$ $\mu \mathrm{L}, 0.50 \mathrm{mmol}), n-\mathrm{C}_{18} \mathrm{H}_{37} \mathrm{SiH}_{3}(0.0285 \mathrm{~g}, 0.10 \mathrm{mmol}), \mathrm{CH}_{3} \mathrm{CN}(6.4 \mu \mathrm{L}, 0.6406 \mathrm{~g} / \mathrm{mL}, 0.10 \mathrm{mmol})$, $1 \mathrm{~mL}$ of toluene, NaBHEt3 $(75 \mu \mathrm{L}, 0.075 \mathrm{mmol})$. After 12 hours, the reaction afforded $\boldsymbol{d} 2-2 \mathrm{a}$ with $>99 \%$ conversion as a colorless oil. The D-incorporation was determined by ${ }^{2} \mathrm{H}$ NMR using $\mathrm{CDCl}_{3}$ as the internal standard $\left(0.0560 \mathrm{~g}\right.$ of $\left.\mathrm{CDCl}_{3}\right)$ was added to a solution of $\boldsymbol{d} \mathbf{2 - 2 a}(0.315 \mathrm{mmol}$ in $\left.\mathrm{CHCl}_{3}\right) .98 .6 \% e e$, determined by GC, GC conditions: Chiralcel B-DM, $100 \mathrm{kPa} \mathrm{N} 2\left(60{ }^{\circ} \mathrm{C}-30\right.$ $\left.\min -1{ }^{\circ} \mathrm{C} / \min -110^{\circ} \mathrm{C}-5 \min \right), \operatorname{tr} 67.1$ (minor), 67.5 (major).
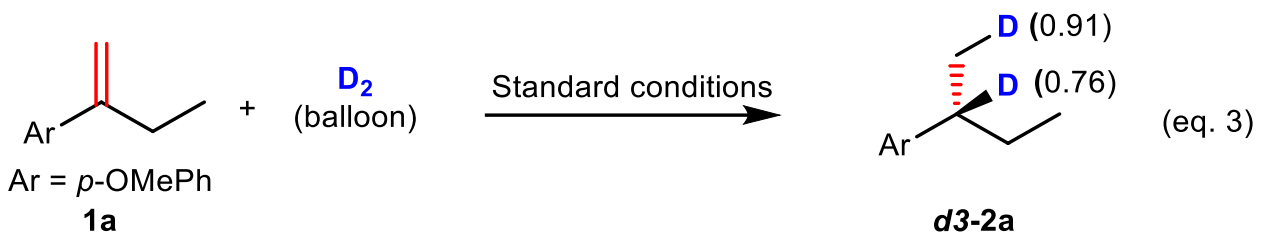

Prepared according to the general procedure: using $\mathbf{L e} \bullet \mathrm{FeCl}_{2}(0.0144 \mathrm{~g}, 0.025 \mathrm{mmol}), \mathbf{1 a}(81 \mu \mathrm{L}$, $0.50 \mathrm{mmol}), n-\mathrm{C}_{18} \mathrm{H}_{37} \mathrm{SiH}_{3}(0.0288 \mathrm{~g}, 0.10 \mathrm{mmol}), \mathrm{CH}_{3} \mathrm{CN}(6.4 \mu \mathrm{L}, 0.6406 \mathrm{~g} / \mathrm{mL}, 0.10 \mathrm{mmol}), 1$ $\mathrm{mL}$ of toluene, NaBHEt3 $(75 \mu \mathrm{L}, 0.075 \mathrm{mmol}$ ). After 12 hours, the reaction afforded $\boldsymbol{d} 3$-2a with $>99 \%$ conversion as a colorless oil. The D-incorporation was determined by ${ }^{2} \mathrm{H} \mathrm{NMR}$ using $\mathrm{CDCl}_{3}$ as the internal standard $\left(0.0264 \mathrm{~g}\right.$ of $\left.\mathrm{CDCl}_{3}\right)$ was added to a solution of $\mathbf{d 3 - 4 a}\left(0.246 \mathrm{mmol}\right.$ in $\left.\mathrm{CHCl}_{3}\right) .99 \%$ $e e$, determined by GC, GC conditions: Chiralcel B-DM, $100 \mathrm{kPa} \mathrm{N} 2\left(60{ }^{\circ} \mathrm{C}-30 \mathrm{~min}-1{ }^{\circ} \mathrm{C} / \mathrm{min}-\right.$ $\left.110^{\circ} \mathrm{C}-5 \mathrm{~min}\right), \operatorname{tr} 66.9$ (minor), 67.2 (major).
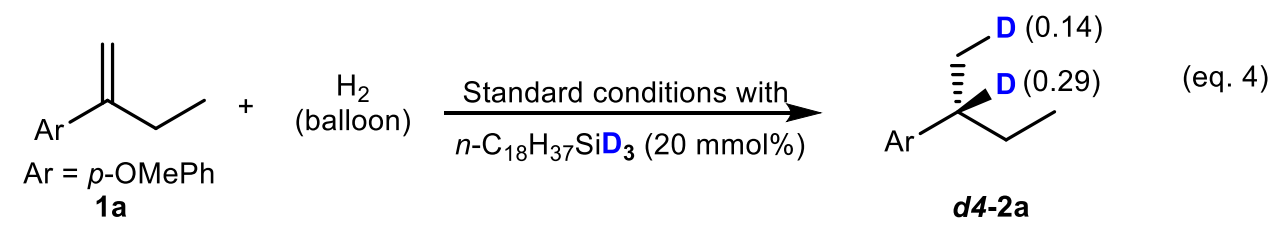

Prepared according to the general procedure: using $\mathbf{L e} \cdot \mathrm{FeCl}_{2}(0.0142 \mathrm{~g}, 0.025 \mathrm{mmol}), \mathbf{1 a}(81 \mu \mathrm{L}$, $0.50 \mathrm{mmol}), n-\mathrm{C}_{18} \mathrm{H}_{37} \mathrm{SiD}_{3}(0.0288 \mathrm{~g}, 0.10 \mathrm{mmol}), \mathrm{CH}_{3} \mathrm{CN}(6.4 \mu \mathrm{L}, 0.6406 \mathrm{~g} / \mathrm{mL}, 0.10 \mathrm{mmol}), 1$ $\mathrm{mL}$ of toluene, NaBHEt $3(75 \mu \mathrm{L}, 0.075 \mathrm{mmol})$. After 12 hours, the reaction afforded $\boldsymbol{d} 4-2 \mathrm{a}$ with $>99 \%$ conversion as a colorless oil. The D-incorporation was determined by ${ }^{2} \mathrm{H} \mathrm{NMR}$ using $\mathrm{CDCl}_{3}$ as the internal standard ( $0.0443 \mathrm{~g}$ of $\mathrm{CDCl}_{3}$ was added to a solution of $\boldsymbol{d} 4-\mathbf{2 a}\left(0.287 \mathrm{mmol}\right.$ in $\left.\mathrm{CHCl}_{3}\right) .98 \%$ 
$e e$, determined by GC, GC conditions: Chiralcel B-DM, $100 \mathrm{kPa} \mathrm{N} 2\left(60{ }^{\circ} \mathrm{C}-30 \mathrm{~min}-1{ }^{\circ} \mathrm{C} / \mathrm{min}-\right.$ $\left.110{ }^{\circ} \mathrm{C}-5 \mathrm{~min}\right), \operatorname{tr} 67.3$ (minor), 67.7 (major).

$$
n-\mathrm{C}_{18} \mathrm{H}_{37} \mathrm{SiD}_{3}+\underset{\text { (balloon) }}{\mathrm{H}_{2}} \stackrel{\text { Standard conditions }}{\longrightarrow} \underset{\substack{n-\mathrm{C}_{18} \mathrm{H}_{37} \mathrm{SiH}(\mathrm{D})_{3} \\ 61 \% \mathrm{D}}}{\text { (eq. 5) }}
$$

Prepared according to the general procedure: using $\mathbf{L e} \cdot \mathrm{FeCl}_{2}(0.0142 \mathrm{~g}, 0.025 \mathrm{mmol}), n-\mathrm{C}_{18} \mathrm{H}_{37} \mathrm{SiD}_{3}$ (0.1430 g, $0.50 \mathrm{mmol}), \mathrm{CH}_{3} \mathrm{CN}(6.4 \mu \mathrm{L}, 0.6406 \mathrm{~g} / \mathrm{mL}, 0.10 \mathrm{mmol}), 1 \mathrm{~mL}$ of toluene, NaBHEt 3 (75 $\mu \mathrm{L}, 0.075 \mathrm{mmol})$. After 12 hours, the reaction afforded $\boldsymbol{n}-\mathbf{C}_{18} \mathbf{H}_{37} \mathbf{S i H}(\mathbf{D})_{3}$ with $39 \%$ of hydrogen atom exchange using TMSPh $(10 \mu \mathrm{L})$ as the internal standard.

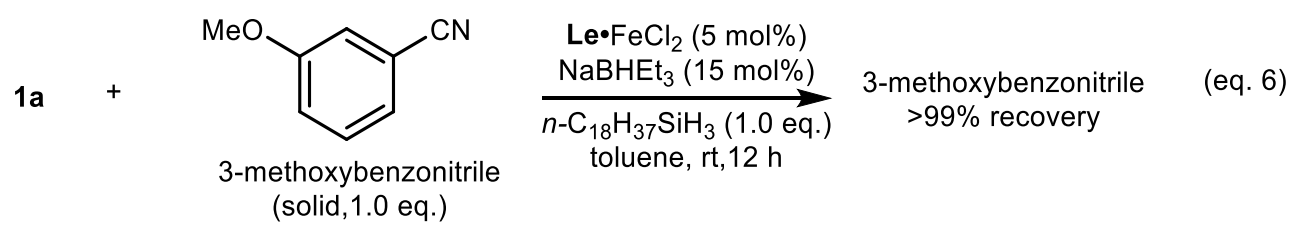

Prepared according to the general procedure: using $\mathbf{L e} \bullet \mathrm{FeCl}_{2}(0.0085 \mathrm{~g}, 0.015 \mathrm{mmol}), \mathbf{1 a}(48 \mu \mathrm{L}$, $0.30 \mathrm{mmol})$, the solid 3-methoxybenzonitrile $(0.0350 \mathrm{~g}, 0.30 \mathrm{mmol}), n-\mathrm{C}_{18} \mathrm{H}_{37} \mathrm{SiH}_{3}(0.0862 \mathrm{~g}, 0.30$ mmol), $1 \mathrm{~mL}$ of toluene, NaBHEt 3 ( $45 \mu \mathrm{L}, 0.045 \mathrm{mmol}$ ). After 12 hours, the reaction afforded $>99 \%$ of recovered 3-methoxybenzonitrile and 1a. The reaction shows that the nitrile couldn't be reduced by hydrosilane.

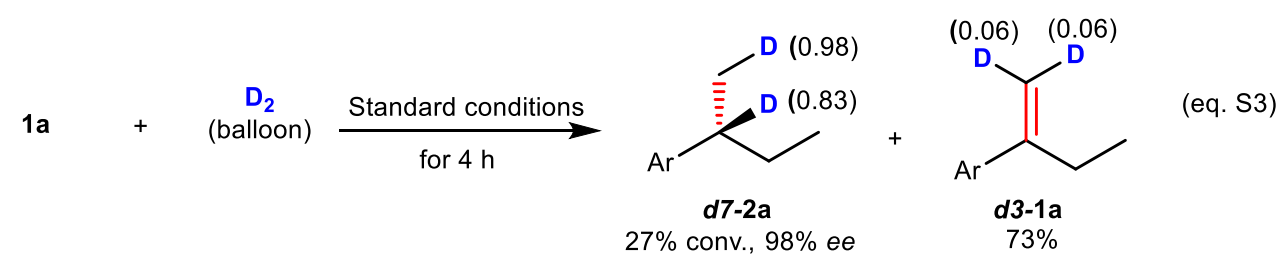

Prepared according to the general procedure: using $\mathbf{L e} \cdot \mathrm{FeCl}_{2}(0.0141 \mathrm{~g}, 0.025 \mathrm{mmol}), \mathbf{1 a}(81 \mu \mathrm{L}$, $0.50 \mathrm{mmol}), n-\mathrm{C}_{18} \mathrm{H}_{37} \mathrm{SiH}_{3}(0.0288 \mathrm{~g}, 0.10 \mathrm{mmol}), \mathrm{CH}_{3} \mathrm{CN}(6.4 \mu \mathrm{L}, 0.6406 \mathrm{~g} / \mathrm{mL}, 0.10 \mathrm{mmol}), 1$ $\mathrm{mL}$ of toluene, NaBHEt3 $(75 \mu \mathrm{L}, 0.075 \mathrm{mmol})$. After 4 hours, the reaction afforded $\mathbf{d 7 - 2 a}$ with $27 \%$ conversion as a colorless oil. The D-incorporation was determined by ${ }^{2} \mathrm{H} \mathrm{NMR}$ using $\mathrm{CDCl}_{3}$ as the internal standard $\left(0.0481 \mathrm{~g}\right.$ of $\left.\mathrm{CDCl}_{3}\right)$ was added to a solution of product $\left(0.0676 \mathrm{~g}\right.$ in $\mathrm{CHCl}_{3}, 0.11$ mmol of $\boldsymbol{d} 7-2 \mathrm{a}, 0.29 \mathrm{mmol}$ of $\boldsymbol{d} 3-\mathbf{1 a}) .98 .3 \% e e$, determined by GC, GC conditions: Chiralcel B$\mathrm{DM}, 100 \mathrm{kPa} \mathrm{N} 2\left(60{ }^{\circ} \mathrm{C}-30 \mathrm{~min}-1{ }^{\circ} \mathrm{C} / \mathrm{min}-110{ }^{\circ} \mathrm{C}-5 \mathrm{~min}\right)$, tr 66.9 (minor), 67.1 (major). 


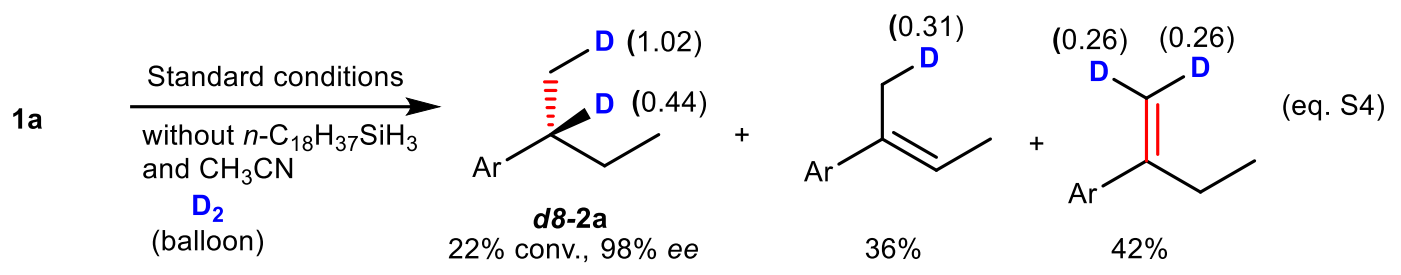

Prepared according to the general procedure: using $\mathbf{L e} \bullet \mathrm{FeCl}_{2}(0.0141 \mathrm{~g}, 0.025 \mathrm{mmol}), \mathbf{1 a}(81 \mu \mathrm{L}$, $0.50 \mathrm{mmol}), 1 \mathrm{~mL}$ of toluene, NaBHEt $3(75 \mu \mathrm{L}, 0.075 \mathrm{mmol})$. After 12 hours, the reaction afforded $\boldsymbol{d 8}$-2a with $>99 \%$ conversion as a colorless oil. The D-incorporation was determined by ${ }^{2} \mathrm{H}$ NMR using $\mathrm{CDCl}_{3}$ as the internal standard $\left(0.0493 \mathrm{~g}\right.$ of $\left.\mathrm{CDCl}_{3}\right)$ was added to a solution of mixtures $\left(0.0680 \mathrm{~g}\right.$ in $\left.\mathrm{CHCl}_{3}\right) .98 .4 \%$ ee, determined by $\mathrm{GC}, \mathrm{GC}$ conditions: Chiralcel B-DM, $100 \mathrm{kPa} \mathrm{N} 2$ $\left(60{ }^{\circ} \mathrm{C}-30 \mathrm{~min}-1{ }^{\circ} \mathrm{C} / \min -110{ }^{\circ} \mathrm{C}-5 \mathrm{~min}\right.$ ), $\operatorname{tr} 66.7$ (minor), 66.9 (major).

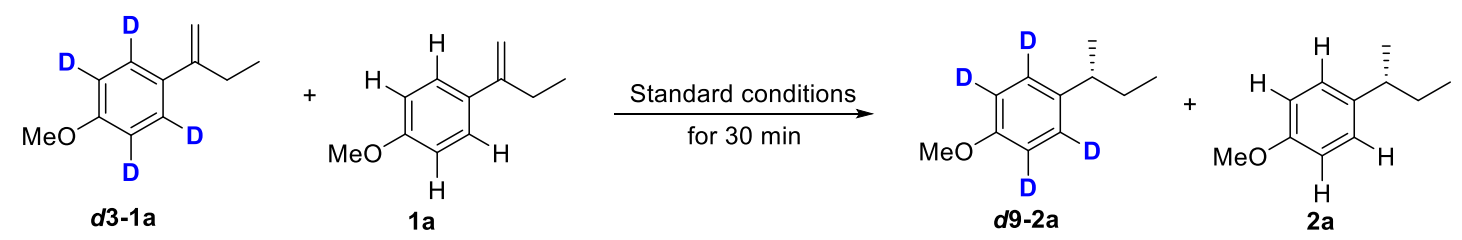

Prepared according to the general procedure: using $\mathbf{L e} \cdot \mathrm{FeCl}_{2}(0.0142 \mathrm{~g}, 0.025 \mathrm{mmol}), \boldsymbol{d 3}-\mathbf{1 a}(48 \mu \mathrm{L}$, $0.313 \mathrm{mmol}), 1 \mathrm{a}(32 \mu \mathrm{L}, 0.187 \mathrm{mmol}), n-\mathrm{C}_{18} \mathrm{H}_{37} \mathrm{SiD}_{3}(0.0288 \mathrm{~g}, 0.10 \mathrm{mmol}), \mathrm{CH}_{3} \mathrm{CN}(6.4 \mu \mathrm{L}$, $0.6406 \mathrm{~g} / \mathrm{mL}, 0.10 \mathrm{mmol}), 1 \mathrm{~mL}$ of toluene, NaBHEt3 $(75 \mu \mathrm{L}, 0.075 \mathrm{mmol})$. After $30 \mathrm{~min}$, the reaction afforded 5.6\% yield of $\mathbf{d} \mathbf{9 - 2 a}$ and $5.4 \%$ yield of $\mathbf{2 a}$ with as a colorless oil. The Dueterium KIE is 0.96 .

\section{A Primary Model to Predict the Stereochemical Outcome}

The possible mechanism of the cobalt-catalyzed hydrogenation of alkenes has already been proposed by Chirik's group (ref 17 in the manuscript) to form a cobalt alkene hydride intermediate. Based on their proposed intermediate, we made a primary model to predict the stereochemical outcome of this reaction. In the proposed intermediate, the hydride group on iron and isopropyl group on oxazoline would sit opposite at paper plane because of steric effect. In Model I, the more steric bulky phenyl ring on the styrene is favor to sit far from isopropyl group on oxazoline. The insertion reaction of the styrene to $\mathrm{Fe}-\mathrm{H}$ bond occurred to form $\mathrm{C}-\mathrm{H}$ bond and $\mathrm{C}-\mathrm{Fe}$ bond which followed by $\sigma$-bond metathesis to construct the $R$-configuration carbon center, it is consistent with the experimental results. In other Models, the phenyl ring is close to isopropyl group on oxazoline or imine, however, it is disfavor due to steric effect. More in-depth mechanistic studies are required and are currently underway. 

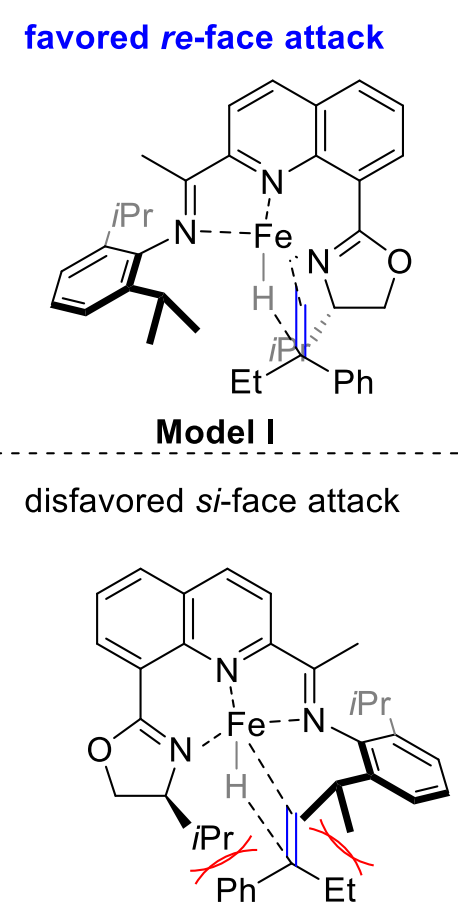

Model III disfavored re-face attack

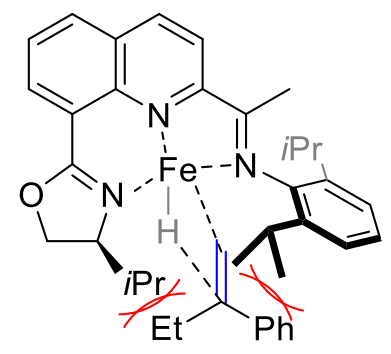

Model II

disfavored si-face attack

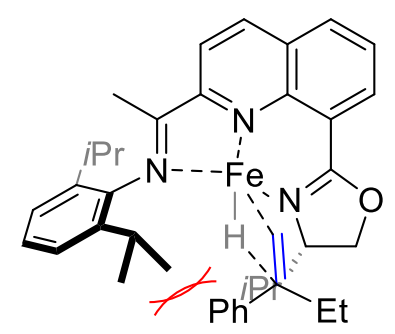

Model IV

Figure S3. A primary model to predict the stereochemical outcome.

The possible mechanism of $N N N$-tridentate ligand-promoted asymmetric cobalt-catalyzed hydrogenation of alkenes has already been proposed by Chirik's group (ref 19 in the manuscript) to form a cobalt alkene hydride intermediate. In our previous studies, we also proposed a $N N N$ tridentate ligand-promoted asymmetric iron or cobalt-catalyzed hydrofunctionalization of 1,1disubstituted alkene via a metal hydride species (ref 7a; 16a, c; 3c; Jianhui Chen, Ligand design for iron and cobalt-catalyzed asymmetric hydrofunctionalization of alkenes [D]. Zhejiang, Zhejiang University, 2017). Here, we proposed that the $N N N$-tridentate OIQ ligand could generate the similar metal hydride intermediate.

The x-ray diffractions of 8 -OIQ $\cdot \mathrm{FeCl}_{2}$ has been compared with that of OIP. $\mathrm{FeCl}_{2}(\mathrm{Org}$. Lett. 2014, 16, 6452-6455) (Fig. S4).

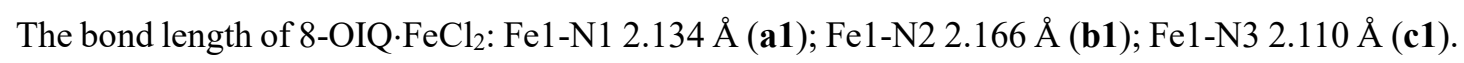

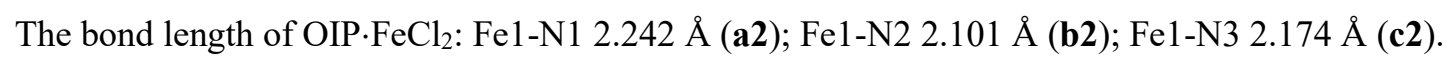
Compared with the side iron-nitrogen bond length (a and $\mathbf{c})$, the $\mathbf{a} 1$ and $\mathbf{c} 1$ in $8-\mathrm{OIQ} \cdot \mathrm{FeCl}_{2}$ are shorter than $\mathbf{a} 2$ and $\mathbf{c 2}$ in OIP. $\mathrm{FeCl}_{2}$ which illustrated that 8-OIQ ligand could provide a narrower space for iron center than OIP ligand. This phenomenon might explain that the 8-OIQ ligand could improve the enantioselectivity in the alkene insertion step. 


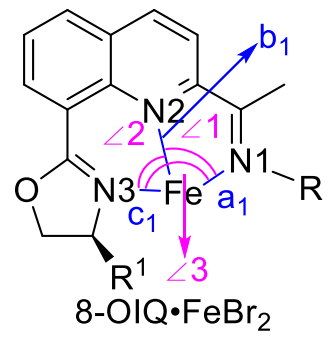

bond length bond angle

$a_{1}=2.134(6) \quad \angle 1=75.4$

$\mathrm{b}_{1}=2.166(6) \quad \angle 2=83.1$

$c_{1}=2.110(6) \quad \angle 3=139.0$

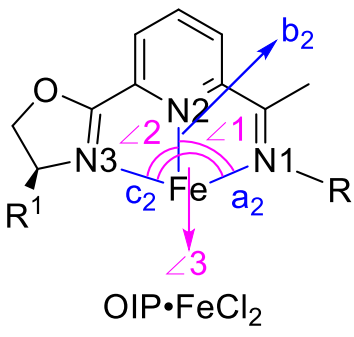

bond length bond angle

$a_{2}=2.2417(58) \angle 1=73.28$

$\mathrm{b}_{2}=2.1005(51) \angle 2=74.25$

$c_{2}=2.1740(53) \angle 3=140.18$

Figure S4. The comparison of steric hindrance with 8-OIQ $\cdot \mathrm{FeBr}_{2}$ and $\mathrm{OIP} \cdot \mathrm{FeCl}_{2}$

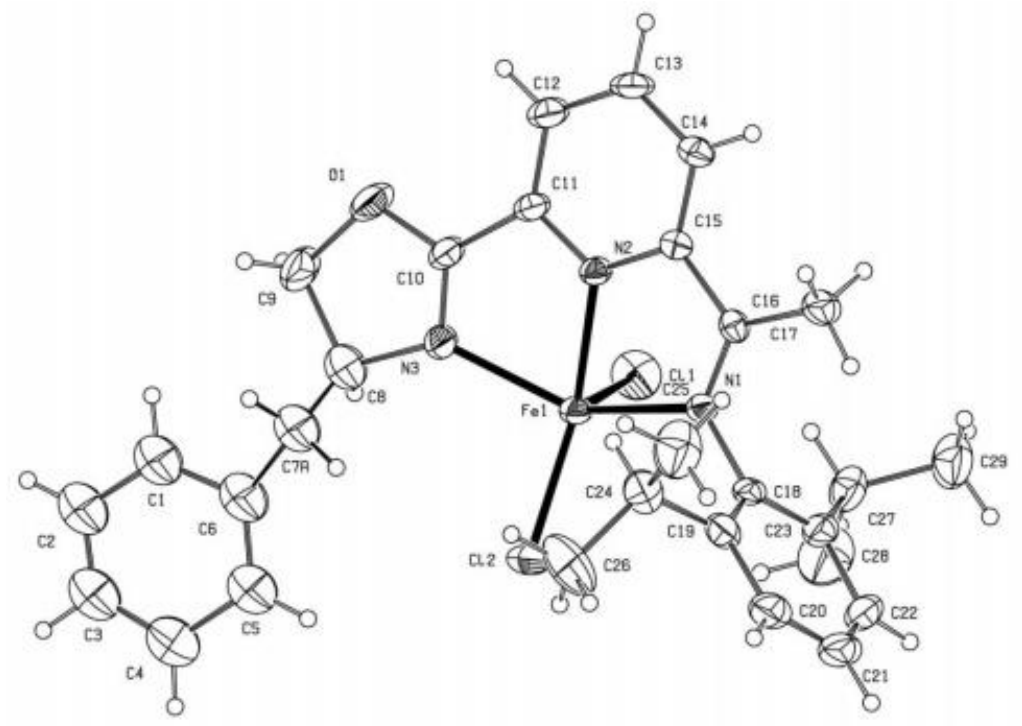

Selected Bond Length for $\mathbf{O I P - F e C l} \mathbf{2}_{\mathbf{2}}$ complex

\begin{tabular}{|l|l|}
\hline Selected Bond Length & Distance $(\AA)$ \\
\hline Fe1-N2 & $2.1005(53)$ \\
\hline Fe1-N3 & $2.1740(58)$ \\
\hline Fe1-N1 & $2.2417(51)$ \\
\hline Fe1-Cl2 & $2.2702(23)$ \\
\hline Fe1-Cl1 & $2.3296(26)$ \\
\hline
\end{tabular}

Selected Bond Angles for OIP-FeCl $\mathbf{~}_{\mathbf{2}}$

\begin{tabular}{|l|l|}
\hline Selected Bond Angles & $(\mathrm{deg})$ \\
\hline $\mathrm{N} 2-\mathrm{Fe} 1-\mathrm{N} 3$ & $74.25(21)$ \\
\hline $\mathrm{N} 2-\mathrm{Fe} 1-\mathrm{N} 1$ & $72.38(20)$ \\
\hline $\mathrm{N} 2-\mathrm{Fe} 1-\mathrm{Cl} 2$ & $150.73(18)$ \\
\hline $\mathrm{N} 2-\mathrm{Fe} 1-\mathrm{Cl} 1$ & $91.37(17)$ \\
\hline $\mathrm{N} 3-\mathrm{Fe} 1-\mathrm{N} 1$ & $140.18(22)$ \\
\hline N3-Fe1-Cl2 & $99.99(18)$ \\
\hline N3-Fe1-Cl1 & $97.91(20)$ \\
\hline $\mathrm{N} 1-\mathrm{Fe} 1-\mathrm{Cl} 2$ & $98.57(16)$ \\
\hline
\end{tabular}




\begin{tabular}{|l|l|}
\hline N1-Fe1-Cl1 & $103.95(16)$ \\
\hline Cl2-Fe1-Cl1 & $117.90(11)$ \\
\hline
\end{tabular}

\section{A proposed mechanism}

Based on the deuterium-labeling experimental results and literatures, a possible mechanism was proposed in Fig. S5. An iron hydride species A was proposed to be generated in the presence of $\mathrm{NaBHEt}_{3}$. To support this hypothesis, a Q-TOF-MS analysis experiment using a mixture of the reaction solution was conducted. Indeed, a signal of $540.2553\left(\mathrm{M}+\mathrm{H}^{+}\right)$is detected (Fig. S6), displaying that iron hydride species A coordinated with acetonitrile might be present. Following olefin coordination and insertion with species $\mathbf{A}$ afforded the 1,2-insertion iron alkyl species $\mathbf{B}$. The iron alkyl species B could go through $\sigma$-bond metathesis with hydrosilane to afford the chiral alkane product and the iron silyl species $\mathbf{C}$ which would react with $\mathrm{H}_{2}$ to regenerate the iron hydride species A via $\sigma$-bond metathesis. The possibility of $\sigma$-bond metathesis of the iron alkyl species $\mathbf{B}$ with hydrogen could not be ruled out. The pathway of the formation of 2,1-insertion species B' was less possible. In the absence of acetonitrile, the 2,1-insertion species B' would be formed and then undergo $\beta$-hydride elimination to afford the isomerization side-product (entry 7, table 1). The role of $\mathrm{MeCN}$ was to prevent the 2,1-insertion and might also be to inhibit $\beta$-hydride elimination of iron intermediate via 2,1-insertion to produce the trisubstituted alkene. A primary possible model to predict the stereochemical outcome of this reaction is shown in Figure S3 in the Supporting Information. More in-depth mechanistic studies are required and are currently underway. 


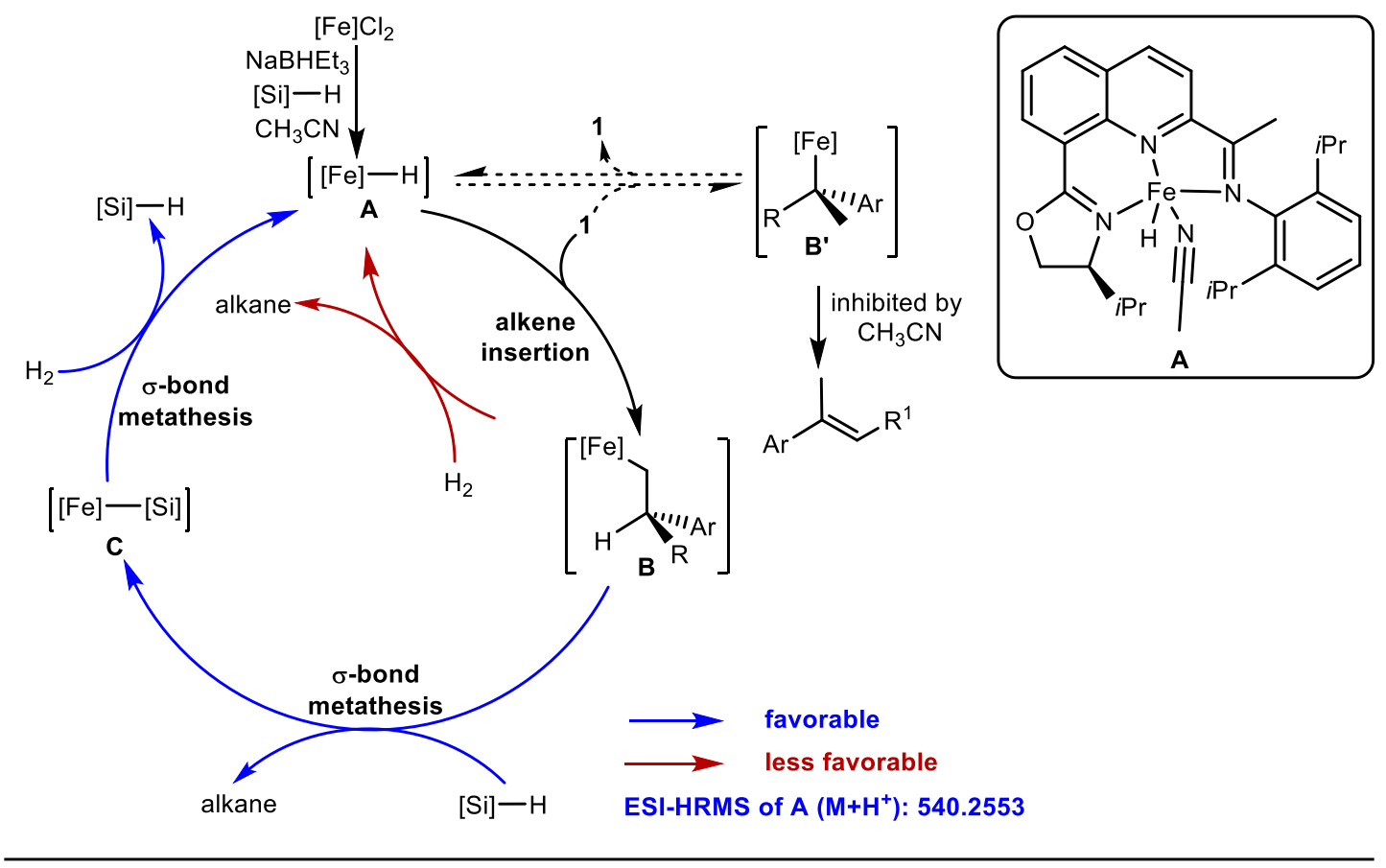

Figure S5. Proposed mechanism

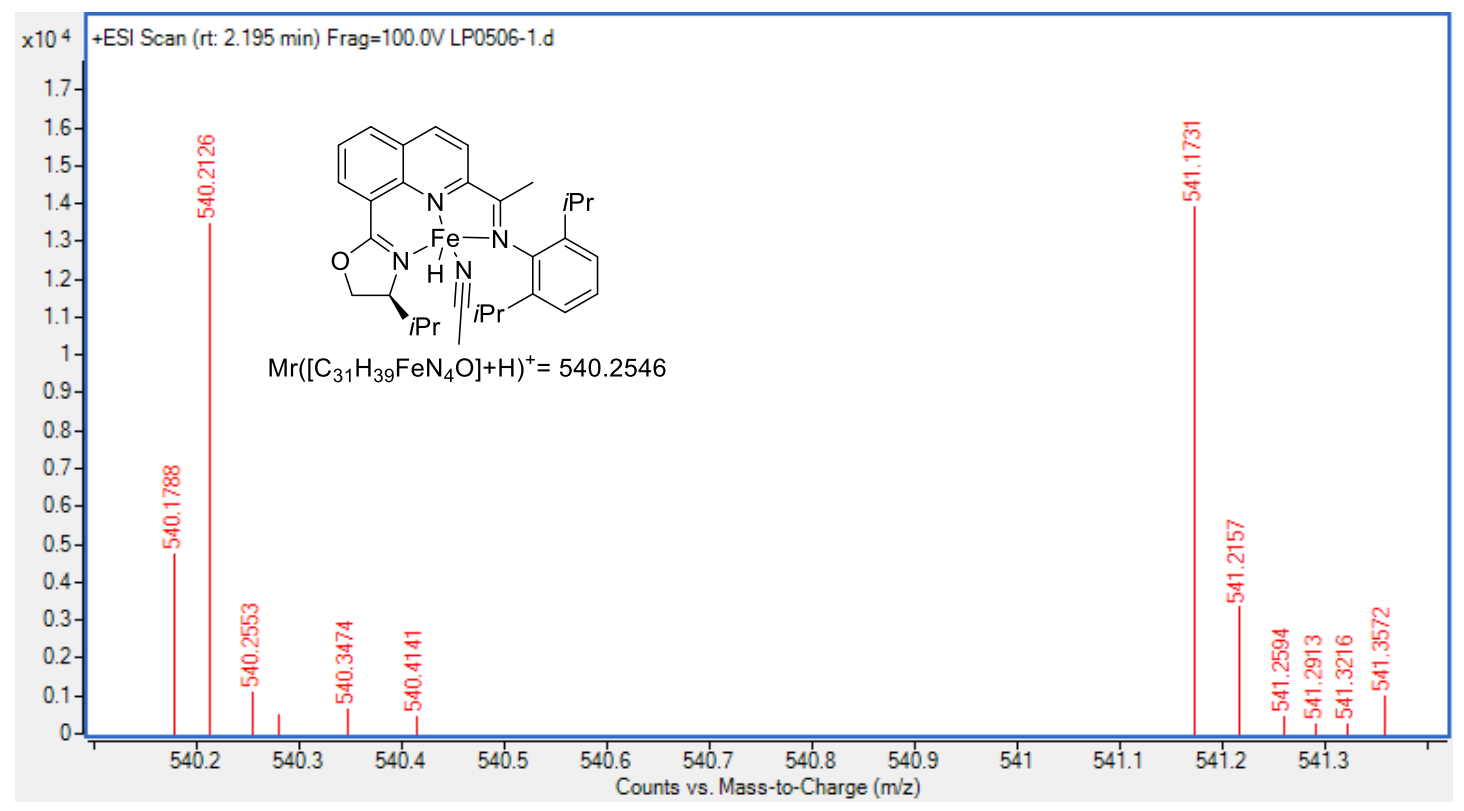

Figure S6. The analysis data of ESI-HRMS for the iron hydride species coordinated with acetonitrile

\section{References}

(1) (a) Nagy, S.; Winslow, L. N.; Mihan, S.; Lukesova, L.; Nifant'ev, E.; Ivchenko, P. V.; Bagrov, V.; US Patent 2012/0016092 2012. (b) Lu, P.; Ji, C.-L.; Lu, Z. Asian J. Org. Chem. 2018, 7, 542-544.

(2) Dai, S.; Sui, X.; Chen, C. Angew. Chem. Int. Ed. 2015, 54, 9948-9953.

(3) (a) Xi, T.; Mei, Y.-C.; Lu, Z. Org. Lett. 2015, 17, 5939-5941; (b) Chen, J.-H.; Cheng, B.; Cao, M.-Y.; Lu, Z. Angew. Chem. Int. Ed. 2015, 54, 4661-4664.

(4) Leonard, W. R.; Romine, J. L.; Meyers, A. I. J. Org. Chem. 1991, 56, 1961-1963. 
(5) Larsen, C. R.; Grotjahn, D. B. J. Am. Chem. Soc. 2012, 134, 10357-10360.

(6) Suzuki, H.; Fuchita, T.; Iwasa, A.; Mishina, T. Synthesis 1978, 12, 905-908.

(7) Zhao, J.; Cheng, B.; Chen, C.; Zhan Lu. Org. Lett. 2020, 22, 837-841.

(8) Wang, Z.-T; Gu, Y.; Ma, M.-Y.; Chen, M. Macromolecules 2020, 53, 3, 956-964.

(9) Iwamoto, T.; Okuzono, C.; Adak, L.; Jinc, M.; Nakamura M. Chem. Commun. 2019, 55, 11281131.

(10) Lim, H.-J.; Smith, C. R.; RajanBabu, T. V. J. Org. Chem. 2009, 74, 4565-4572.

(11) Iwai, R.; Suzuki, S.; Sasaki, S.; Sairi, A. S.; Igawa, K.; Suenobu T.; Morokuma, K.; Konishi, G. Angew. Chem. Int. Ed. 2020, 59, 10566-10573.

(12) Kiel, G. R.; Samkian, A. E.; Nicolay, A.; Witzke, R. J.; Tilley. T. D. J. Am. Chem. Soc. 2018, 140, 2450-2454.

(13) Nguyen, J.; Chong, A.; Lalic, G. Chem. Sci. 2019, 10, 3231-3236.

(14) Hu, G.-Q.; Bai, J.-W.; Li, E.-C.; Liu, K.-H.; Sheng, F.-F.; Zhang, H.-H. Org. Lett. 2021, 23, 1554-1560.

(15) McIntyre, S.; Höermann, E.; Menges, F.; Smidt, S. P.; Pfaltz, A. Adv. Synth. Catal. 2005, 347, 282-288.

(16) Zhang, Y.-M.; Tortorella, M.-D.; Wang, Y.-C.; Liu, J.-Q.; Tu, Z.-C.; Liu, X.-R.; Bai, Y.; Wen, D.-S.; Lu, X.; Lu, Y.-Z.; Talley, J.-J. ACS Med. Chem. Lett. 2014, 5, 1162-1166.

(17) Cao, Y.-Q.; Yang, X.-J.; Du, D.-X.; Xu, X.-T.; Song, F.-R.; Xu, L.-Y. International Joural of Chemistry 2011, 3, 113-117.

(18) K, S.; Wu, H.-B.; Liu, J.-G.; Rabten, W.; Singh, T.; Andersson, P. G. Chem. Sci. 2019, 10, 3649.

(19) Taylor, B. L. H.; Swift, E. C.; Waetzig, J. D.; Jarvo, E. R. J. Am. Chem. Soc. 2011, 133, 389391.

(20) Friedfeld, M. R.; Shevlin, M.; Margulieux, G. W.; Campeau, L.-C.; Chirik, P. J. J. Am. Chem. Soc. 2016, 138, 3314-3324.

(21) (a) Elford, T. G.; Nave, S.; Sonawane, R. P.; Aggarwal, V. K. J. Am. Chem. Soc. 2011, 133, 16798-16801. (b) Wu, L.; Zhong, J.-C.; Liu, S.-K.; Liu, F.-P.; Gao, Z.-D.; Wang, M.; Bian. Q.-H. Tetrahedron: Asymmetry 2016, 27, 78-83.

(22) Bianco, G. G.; Ferraz, H. M. C.; Costa, A. M.; Costa-Lotufo L.V.; Pessoa, C.; Moraes, M. O.; Schrems, M. G.; Pfaltz, A.; Luiz F. Silva, Jr. J. Org. Chem. 2009, 74, 2561-2566.

(23) Song, S.; Zhu, S.-F.; Yang, S.; Li, S.; Zhou, Q.-L. Angew. Chem. Int. Ed. 2012, 51, 2708.

(24) Bianco, G. G.; Ferraz, H. M. C.; Costa, A. M.; Pessoa, C.-L. L. V. C.; Moraes, M. O.; Schrems, M. G.; Pfaltz, A.; Silva, Jr. L. F. J. Org. Chem. 2009, 74, 2561-2566.

(25) (a) Zhang, L.; Zuo, Z.; Wan, X.; Huang, Z. J. Am. Chem. Soc. 2014, 136, 15501-15504. (b) Schaefer, B. A.; Margulieux, G. W.; Small, B. L.; Chirik, P. J. Organometallics 2015, 34, 1307-1320.

(26) Chen, J.; Xi, T.; Ren, X.; Cheng, B.; Guo, J.; Lu, Z. Org. Chem. Front. 2014, 1, 1306-1309.

(27) Krasovskiy, A.; Knochel, P. Synthesis 2006, 890.

(28) Medard, G. Tetrahedron 2014, 70, 186-196.

(29) Jianhui Chen, Ligand design for iron and cobalt-catalyzed asymmetric hydrofunctionalization of alkenes [D]. Zhejiang: Zhejiang university, 2017.

(30) Bergens, S. H.; Noheda, P.; Whelan, J.; Bosnich, B. J. Am. Chem. Soc. 1992, 114, 2121-2128. 
VII. NMR Spectra

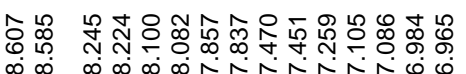

呬

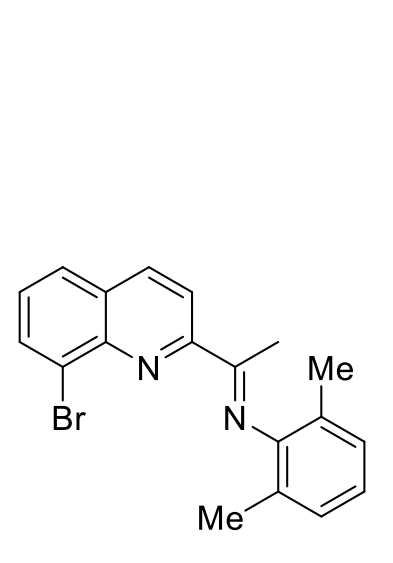

S2a

${ }^{1} \mathrm{H}$ NMR

$\mathrm{CDCl}_{3}$

$400 \mathrm{MHz}$

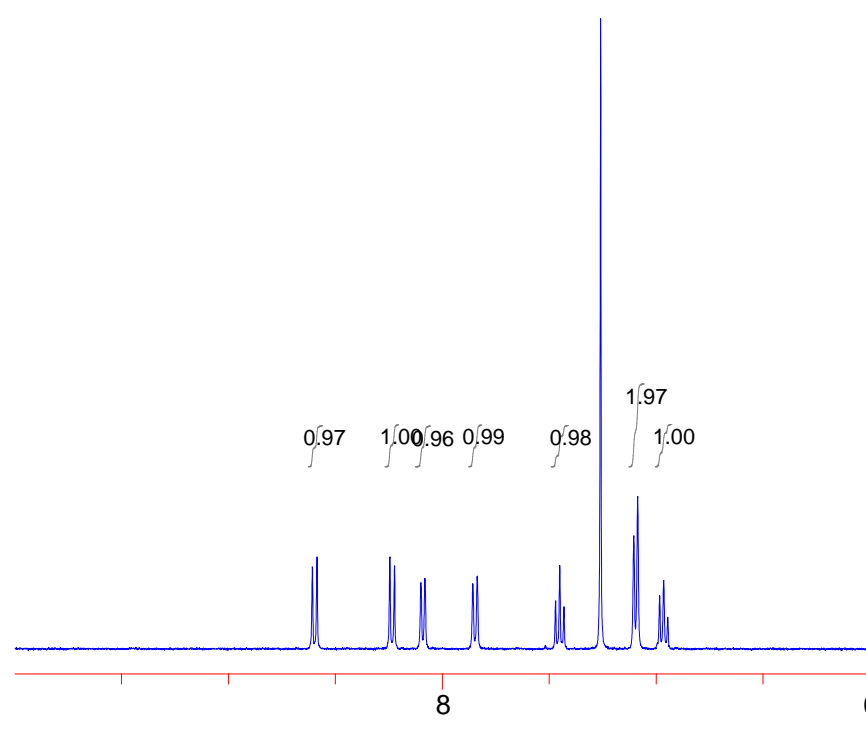

6

2

0 PPM 


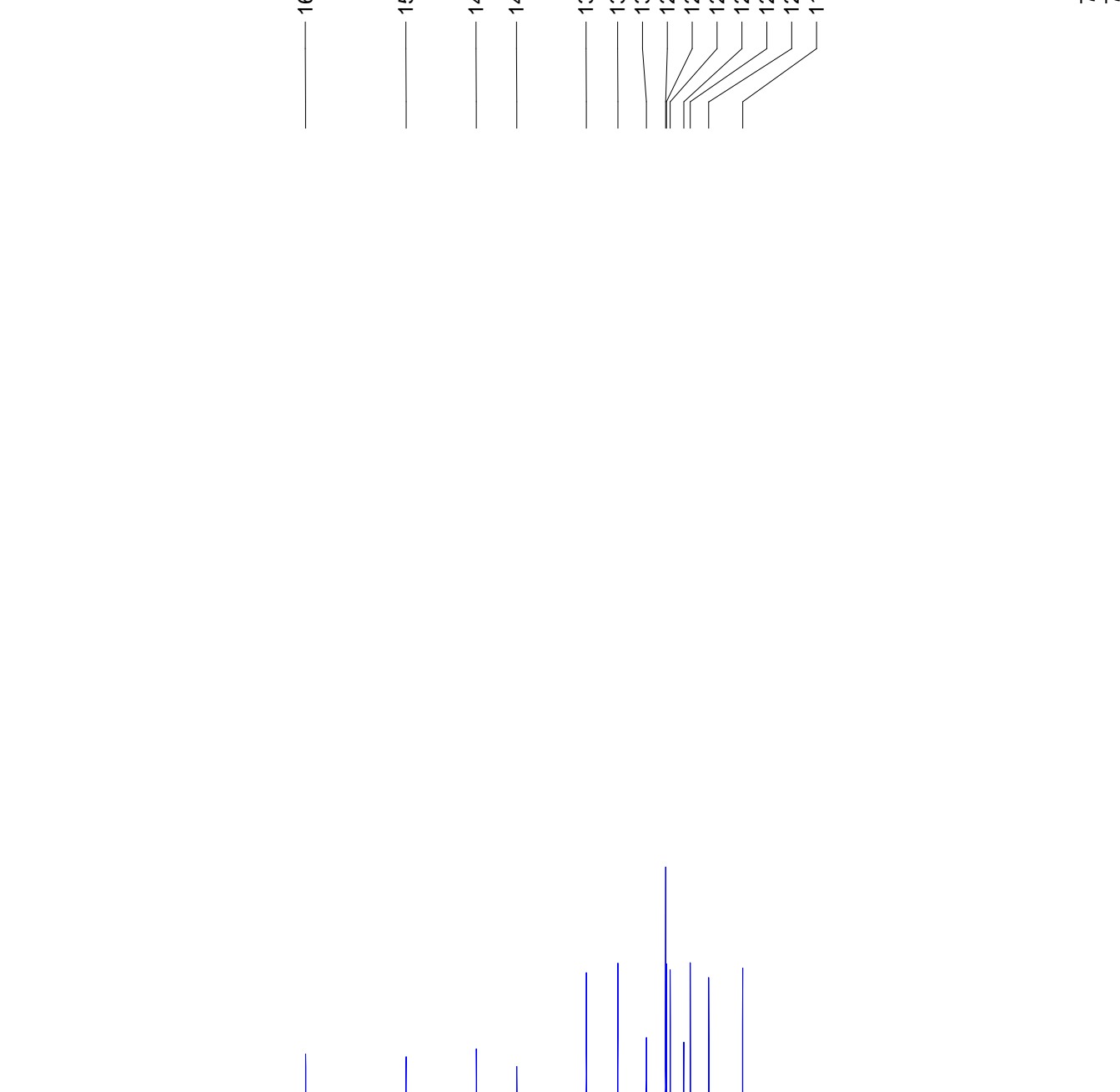

19tw 


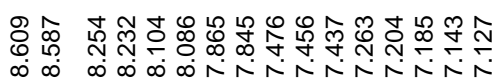

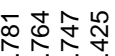

ง

楚

:

I W
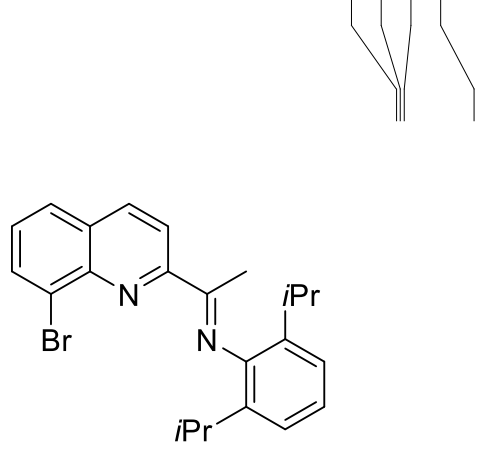

S2b

${ }^{1} \mathrm{H}$ NMR

$\mathrm{CDCl}_{3}$

$400 \mathrm{MHz}$

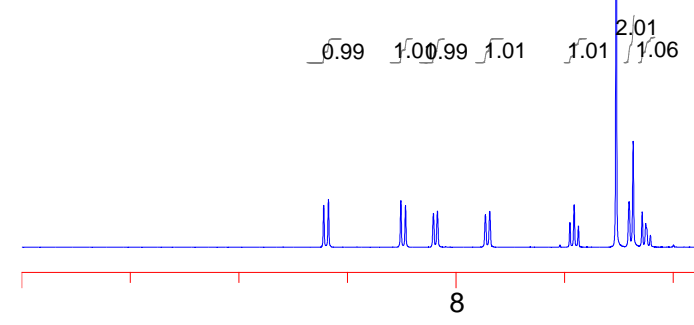

8 

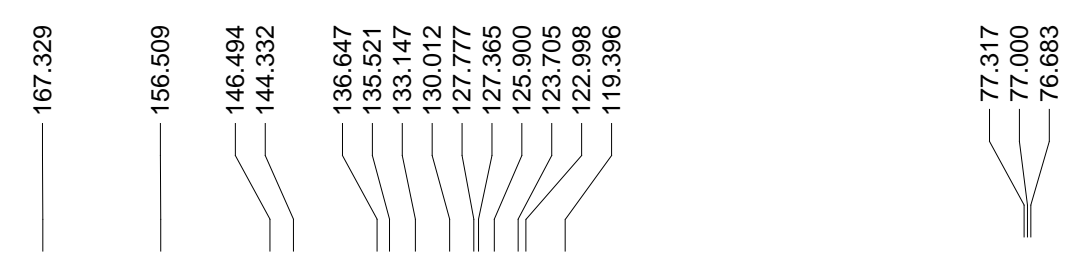

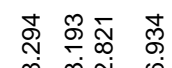

N N N
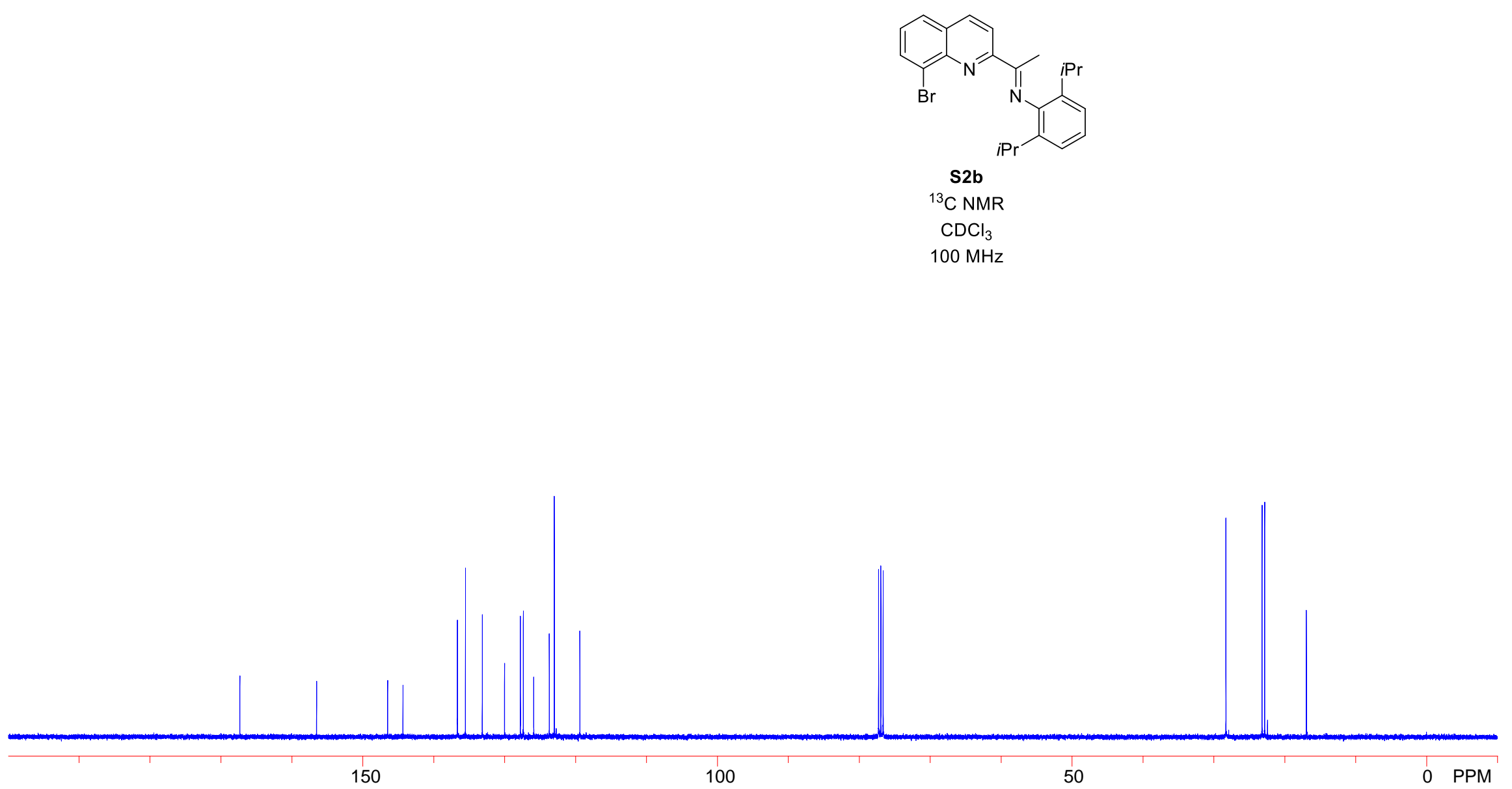


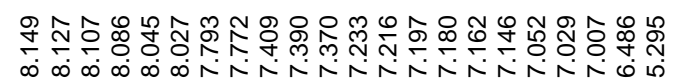
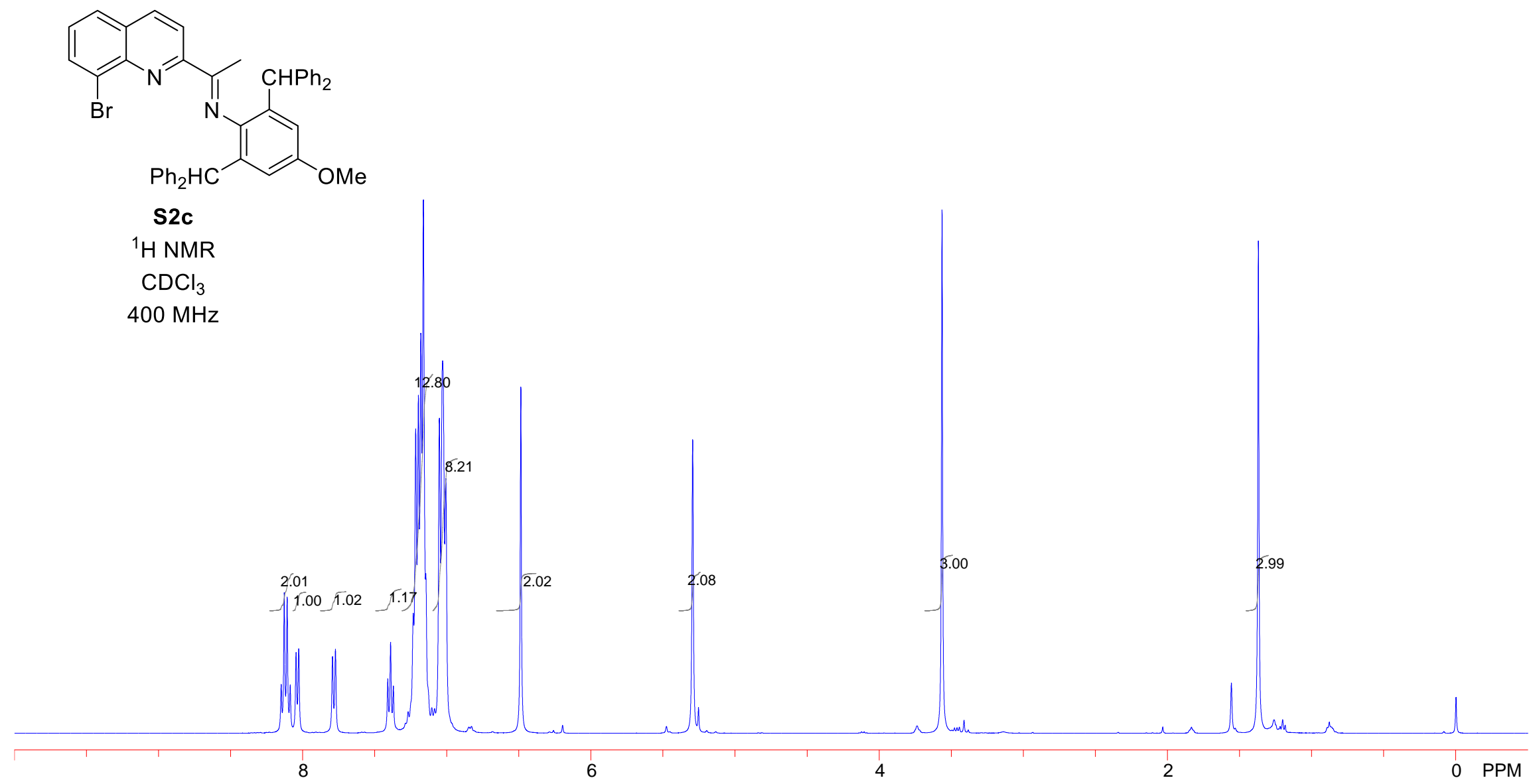


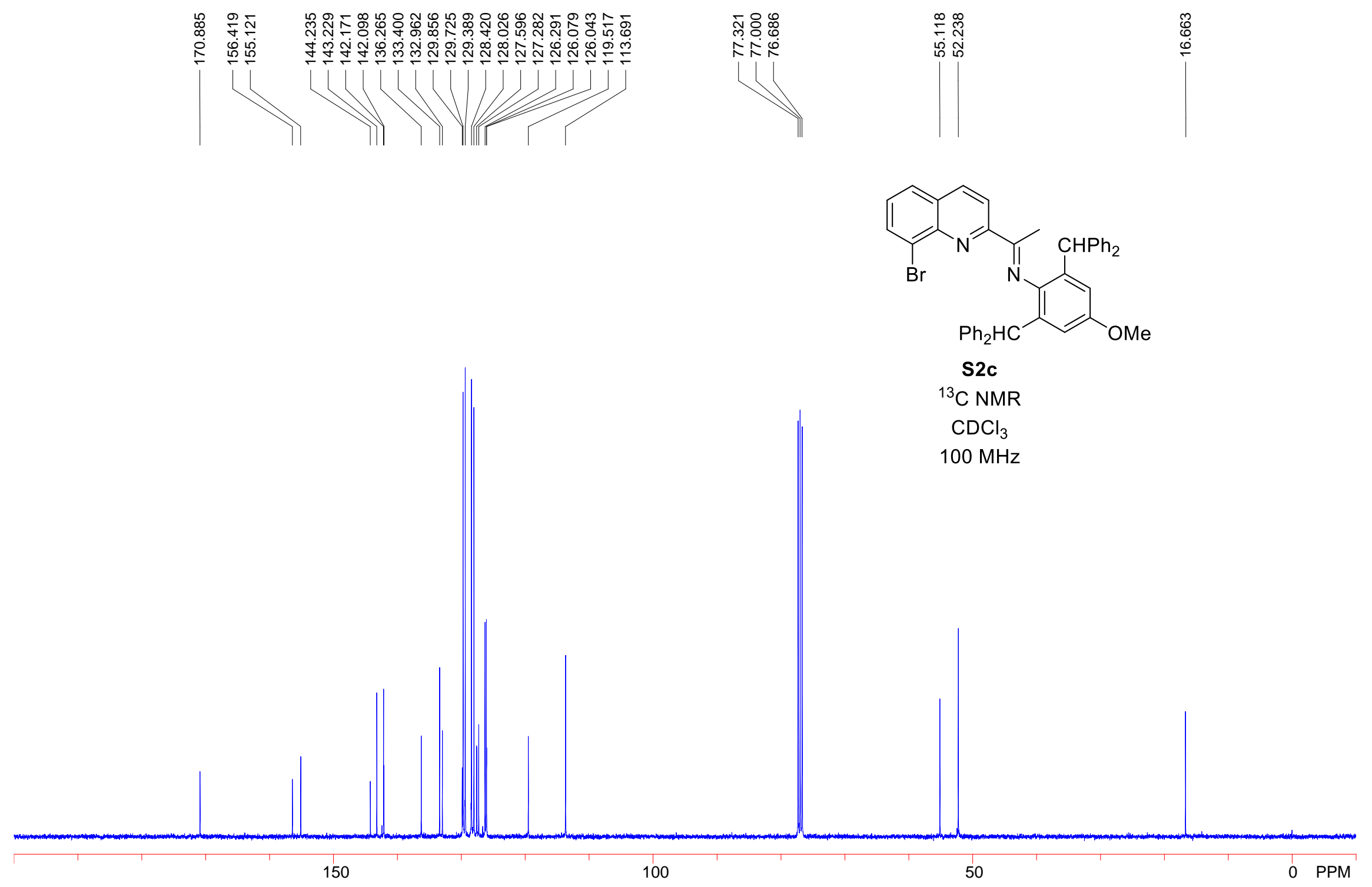




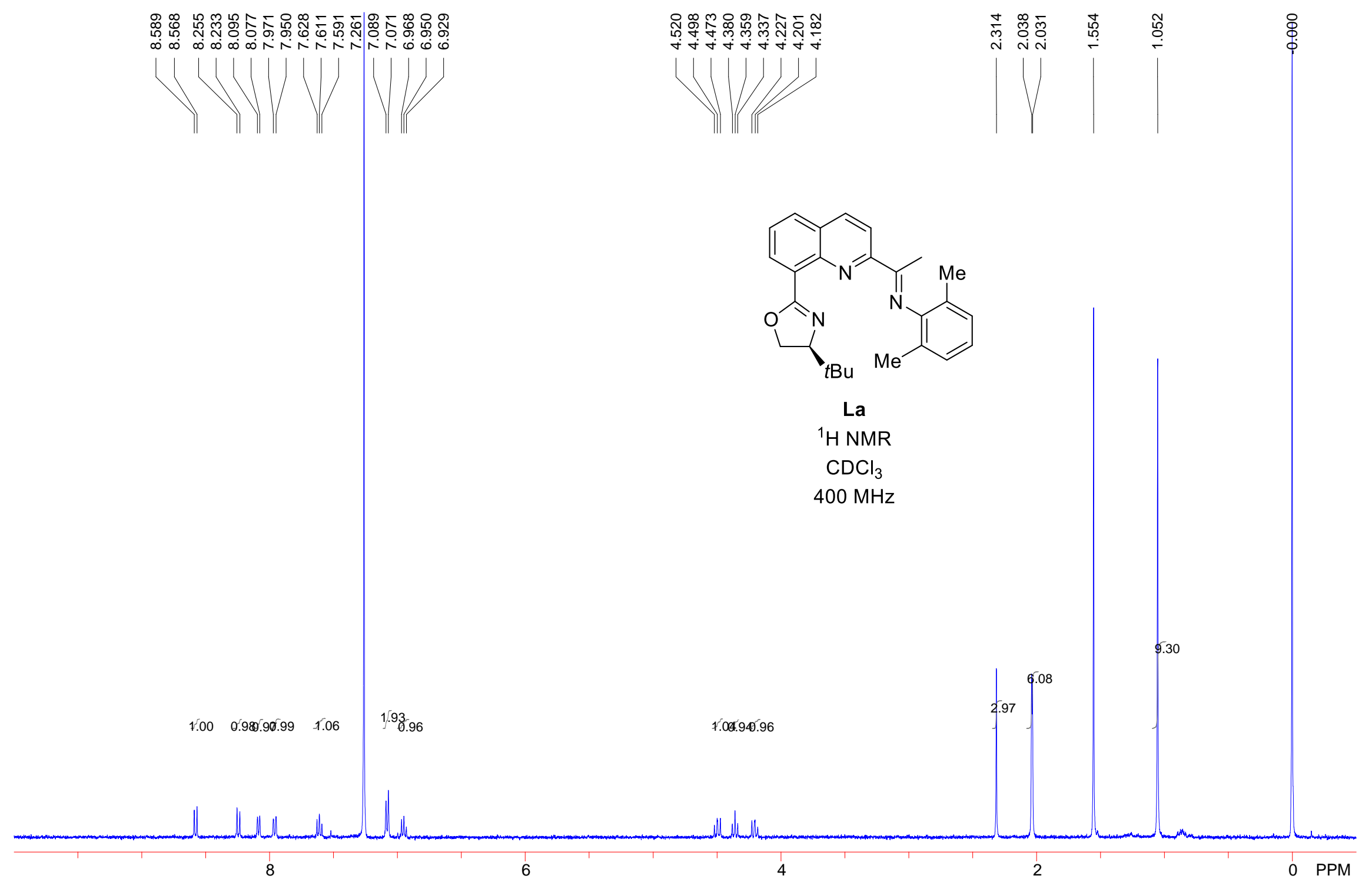




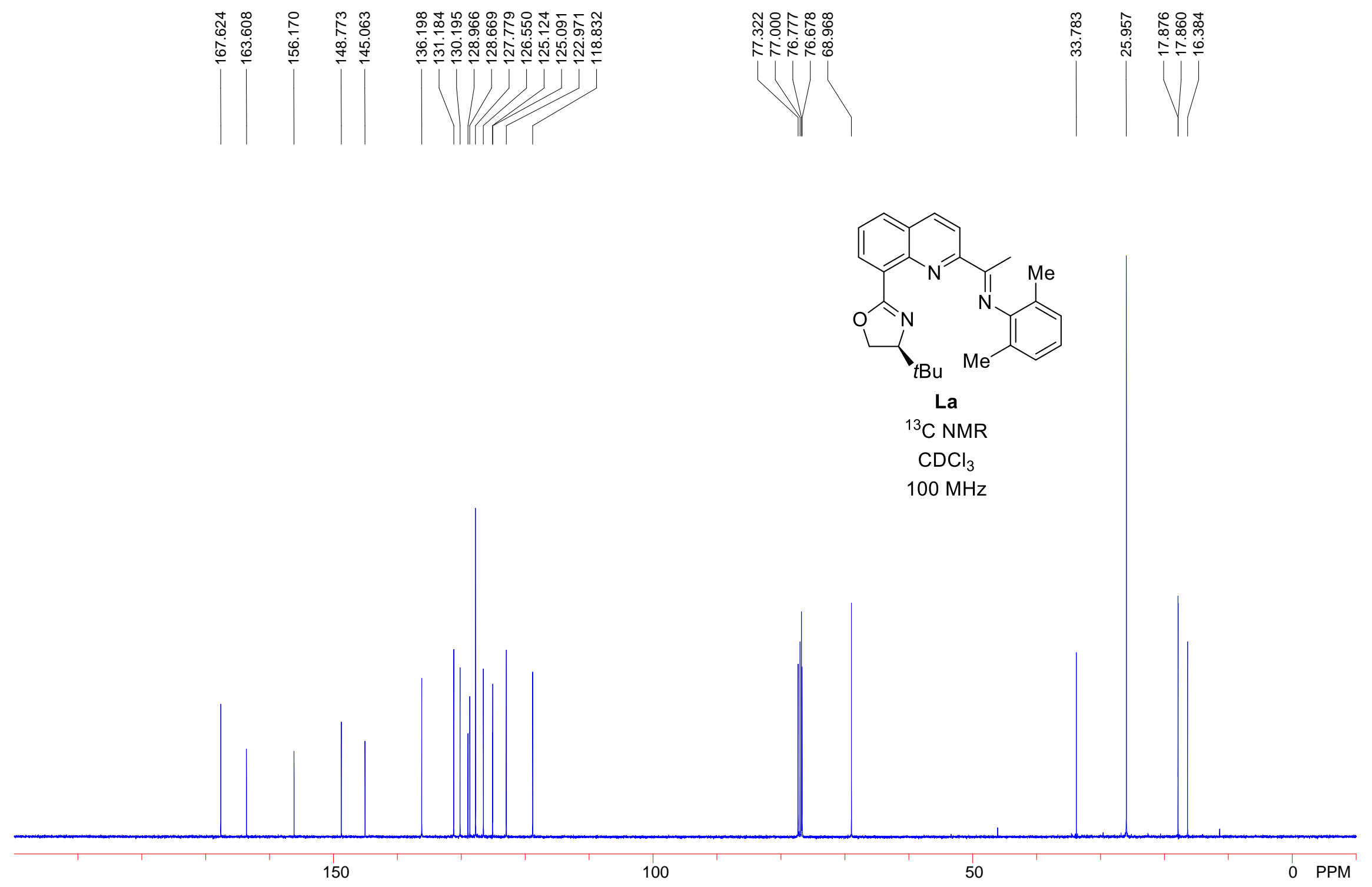




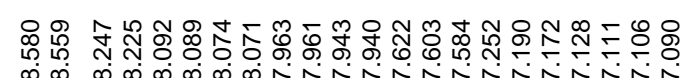

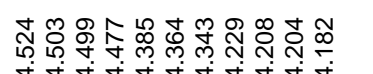

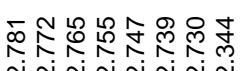

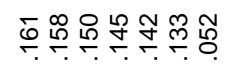

:

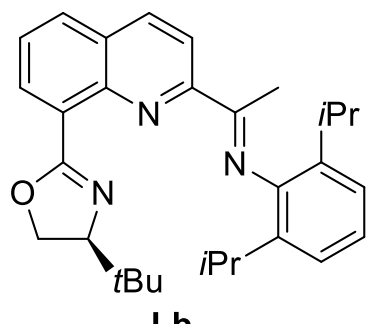

Lb

${ }^{1} \mathrm{H} N M R$

$\mathrm{CDCl}_{3}$

$400 \mathrm{MHz}$

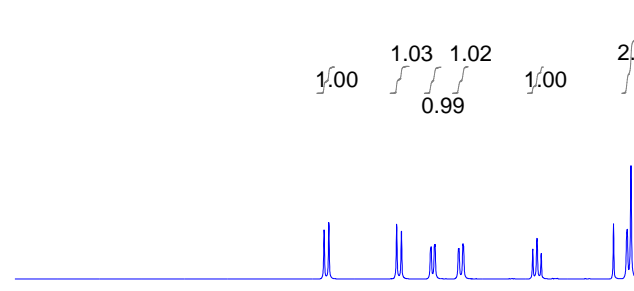

8
2.04

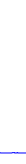

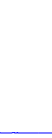

1010302

3.08

H 4

2

0 PPM 

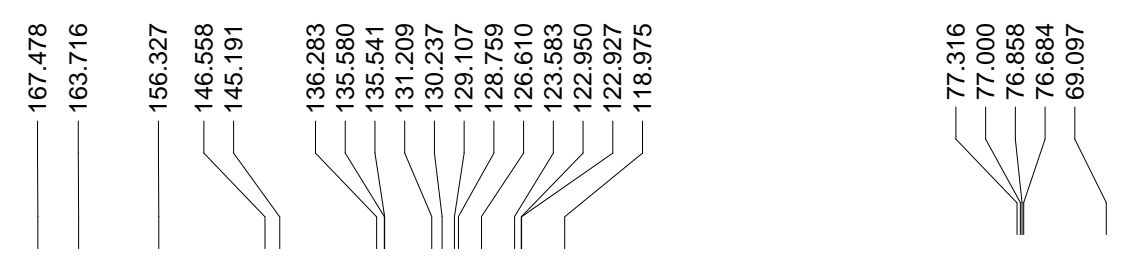

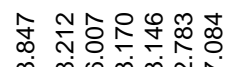

๓ำ ํำ

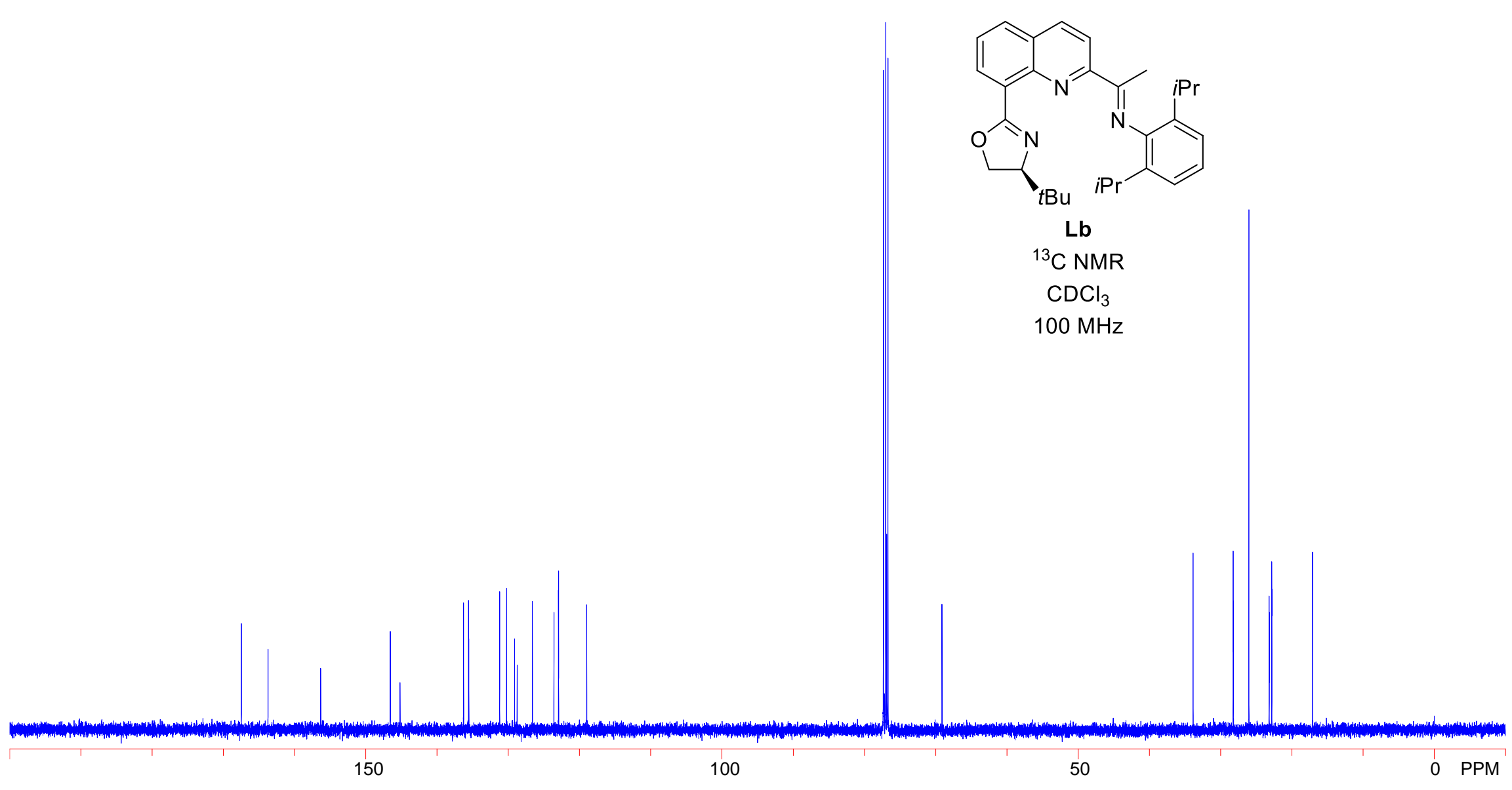




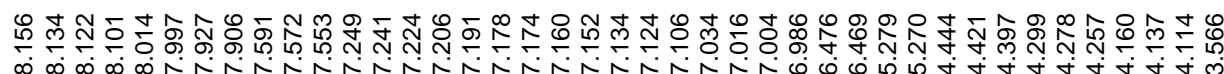

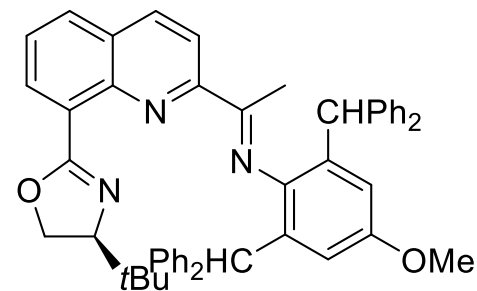

Lc

${ }^{1} \mathrm{H}$ NMR

$\mathrm{CDCl}_{3}$

$400 \mathrm{MHz}$

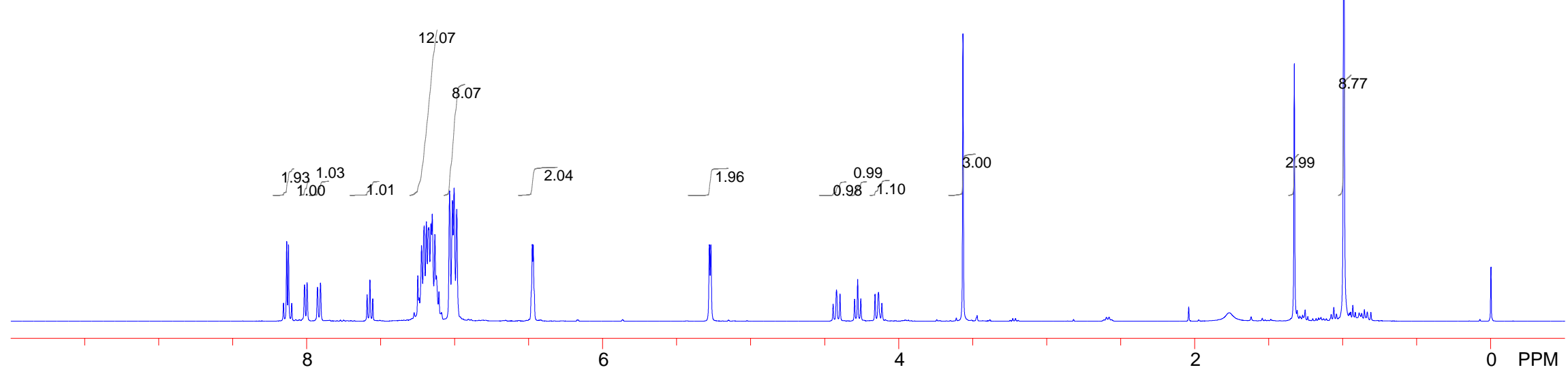




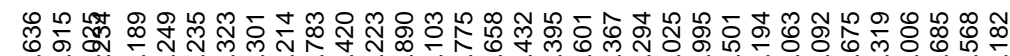

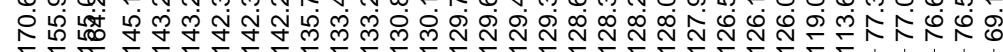

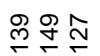

นค่ ถิ่

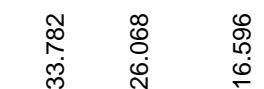
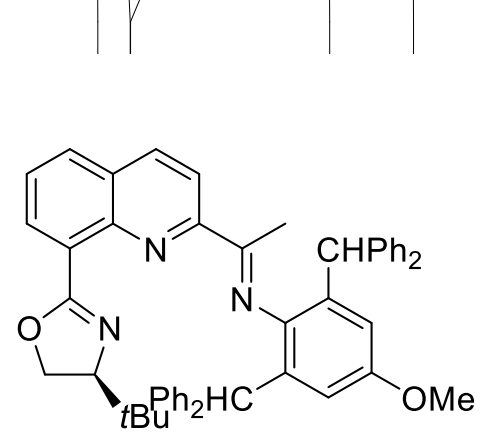

Lc

${ }^{13} \mathrm{C}$ NMR

$\mathrm{CDCl}_{3}$

$100 \mathrm{MHz}$ 


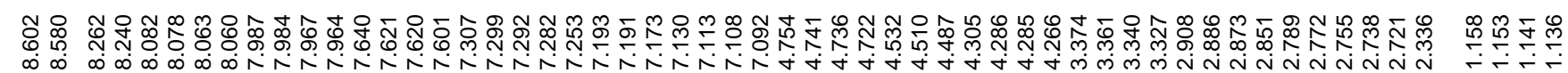
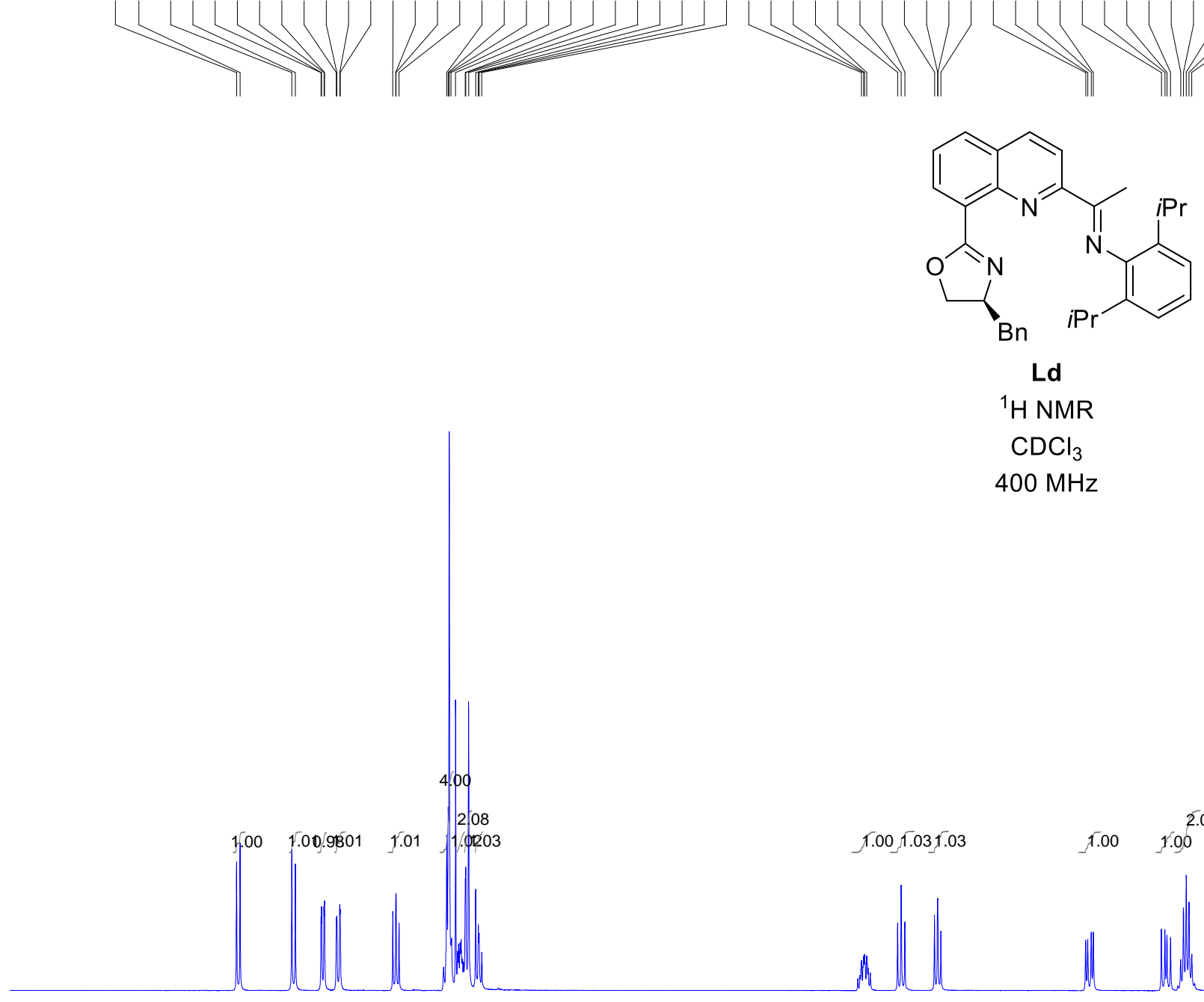

Ld

${ }^{1} \mathrm{H}$ NMR

$\mathrm{CDCl}_{3}$

$400 \mathrm{MHz}$

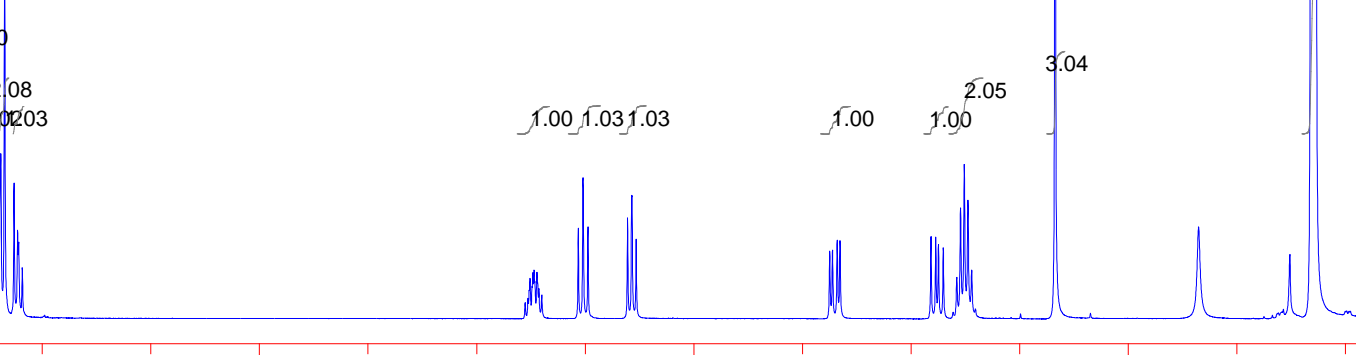




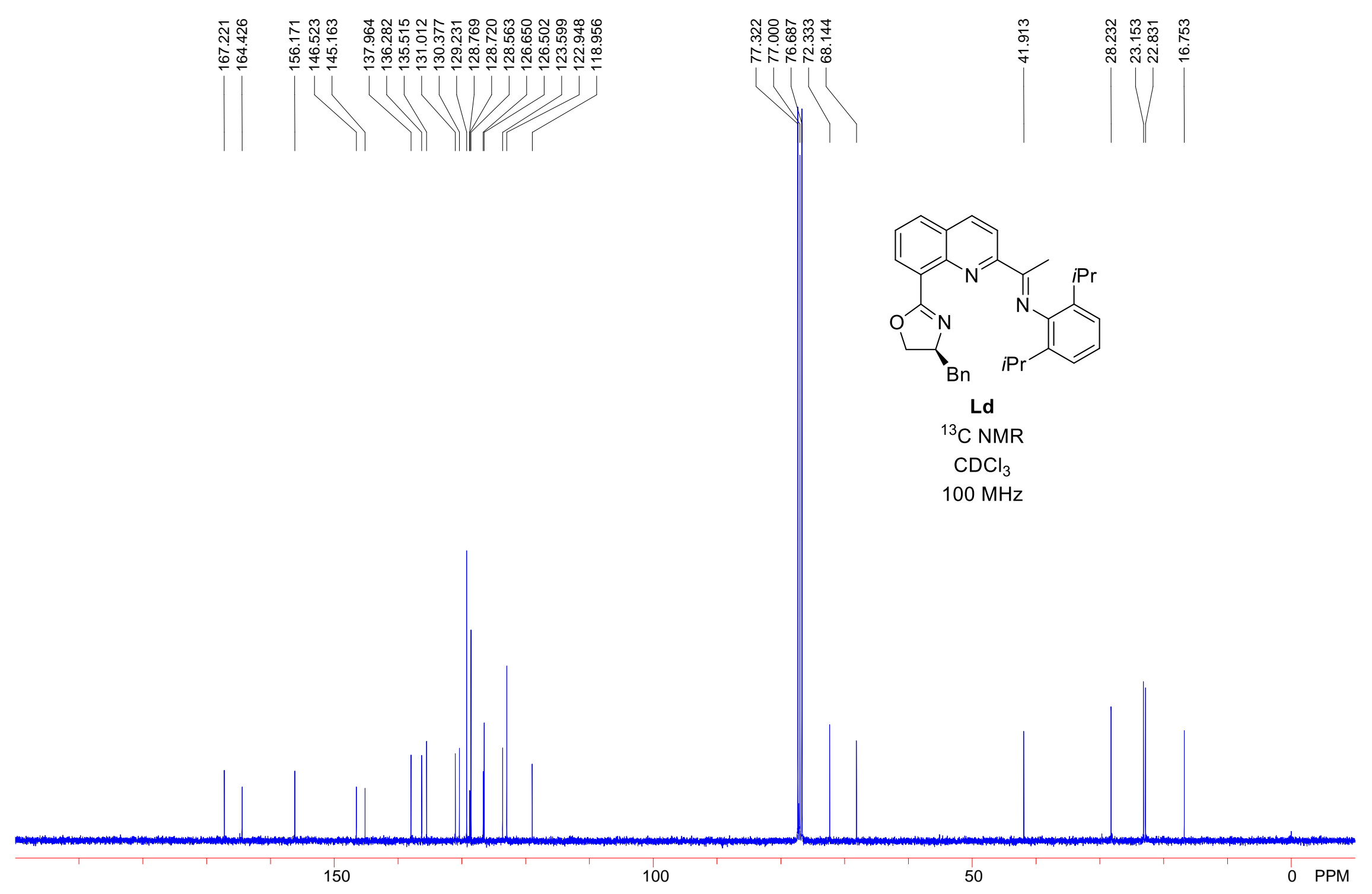




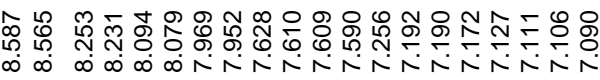

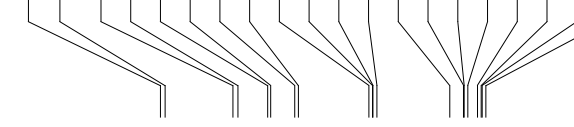

I

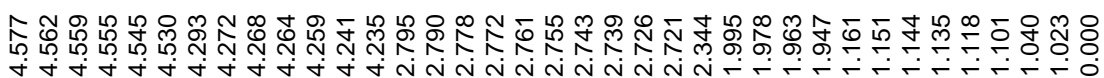

-

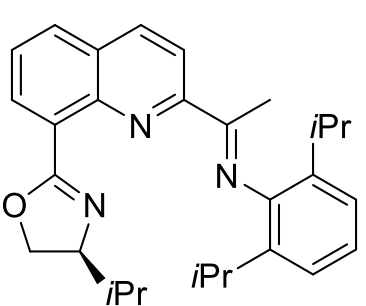

Le

${ }^{1} \mathrm{H}$ NMR

$\mathrm{CDCl}_{3}$

$400 \mathrm{MHz}$

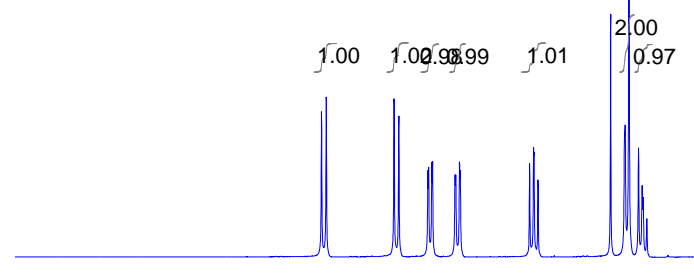

8

1.98

1.01

2.01
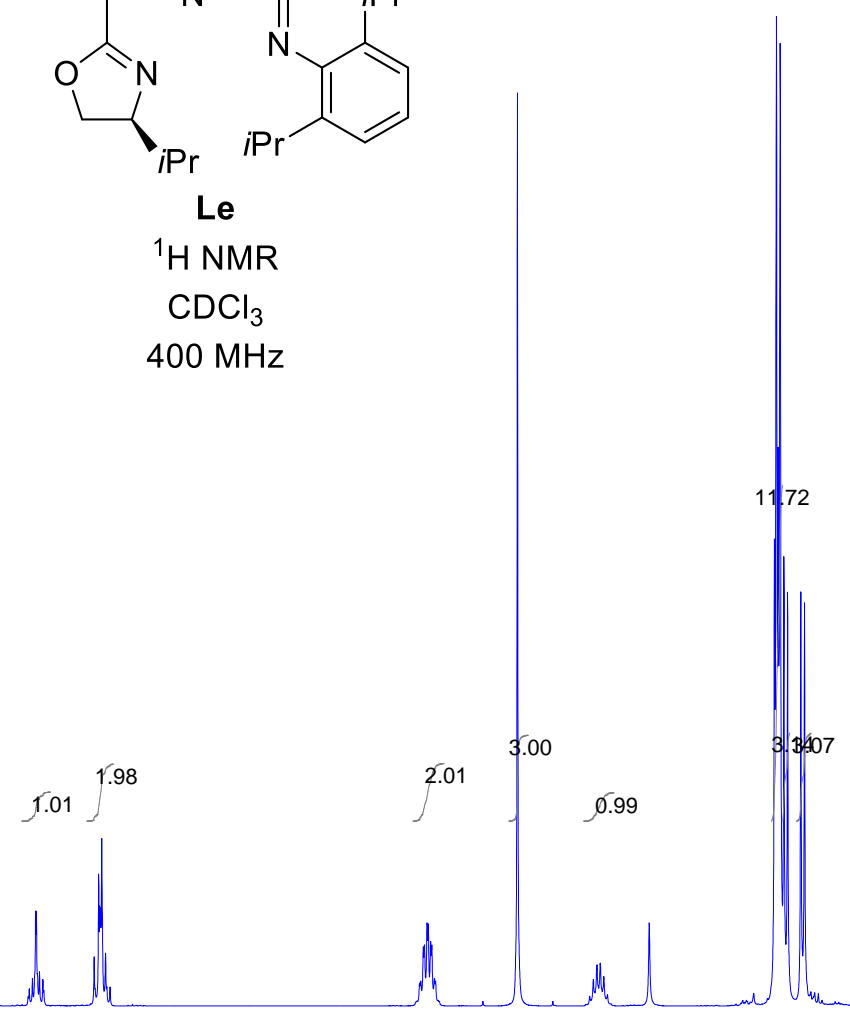

4

0 PPM 


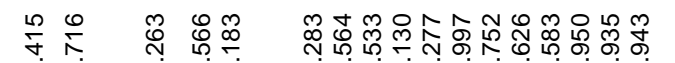

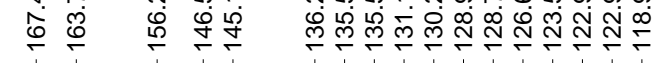

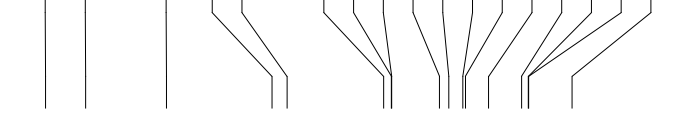

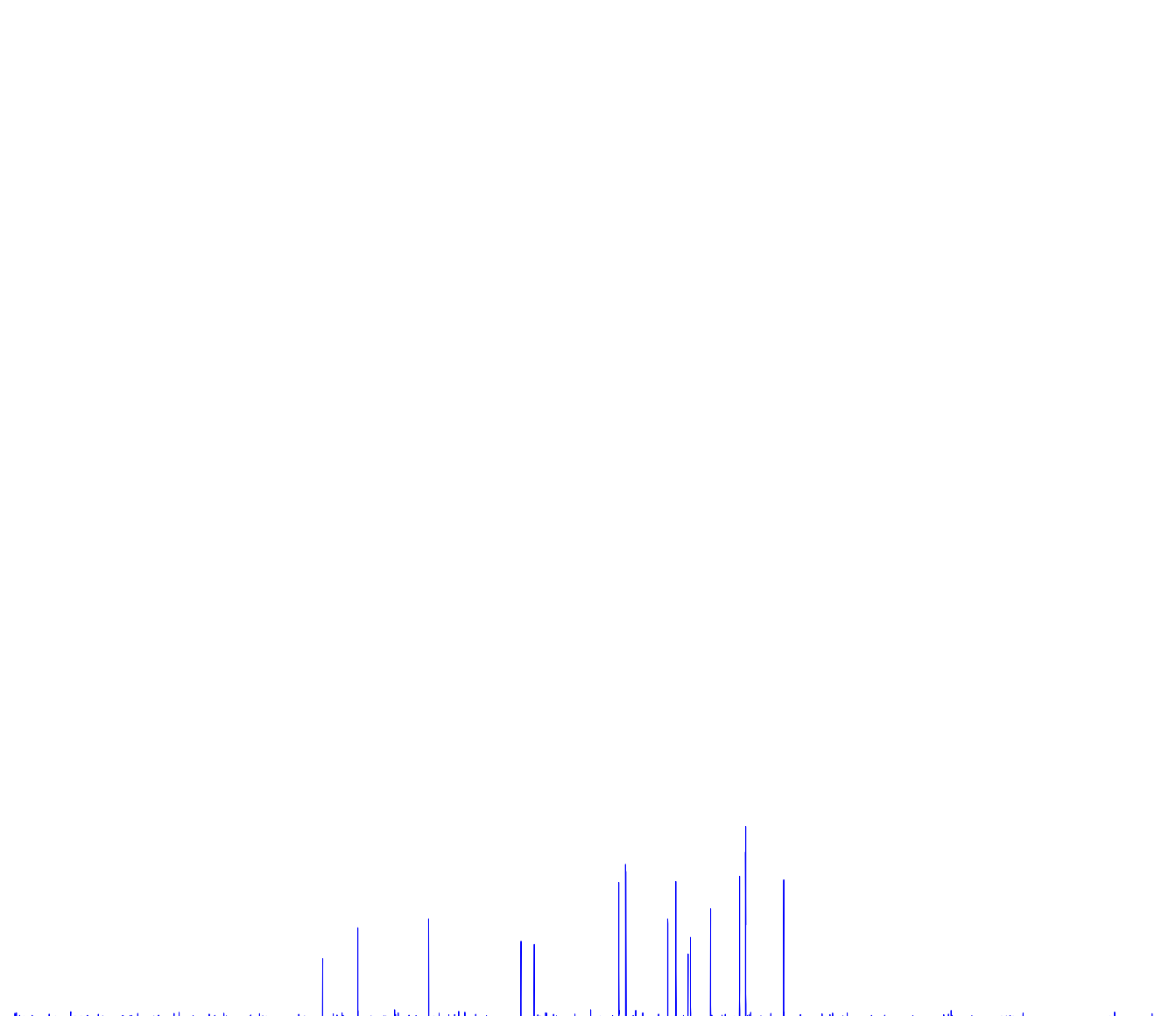

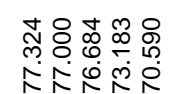

U

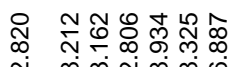

लं

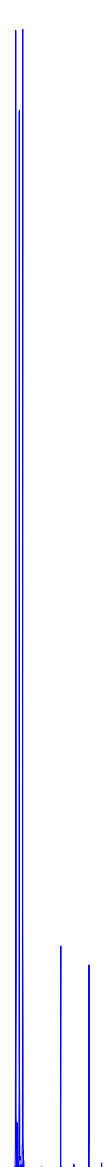

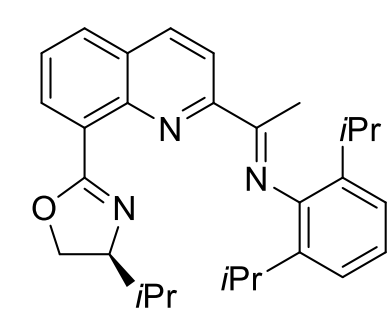

Le

${ }^{13} \mathrm{C} \mathrm{NMR}$

$\mathrm{CDCl}_{3}$

$100 \mathrm{MHz}$ 


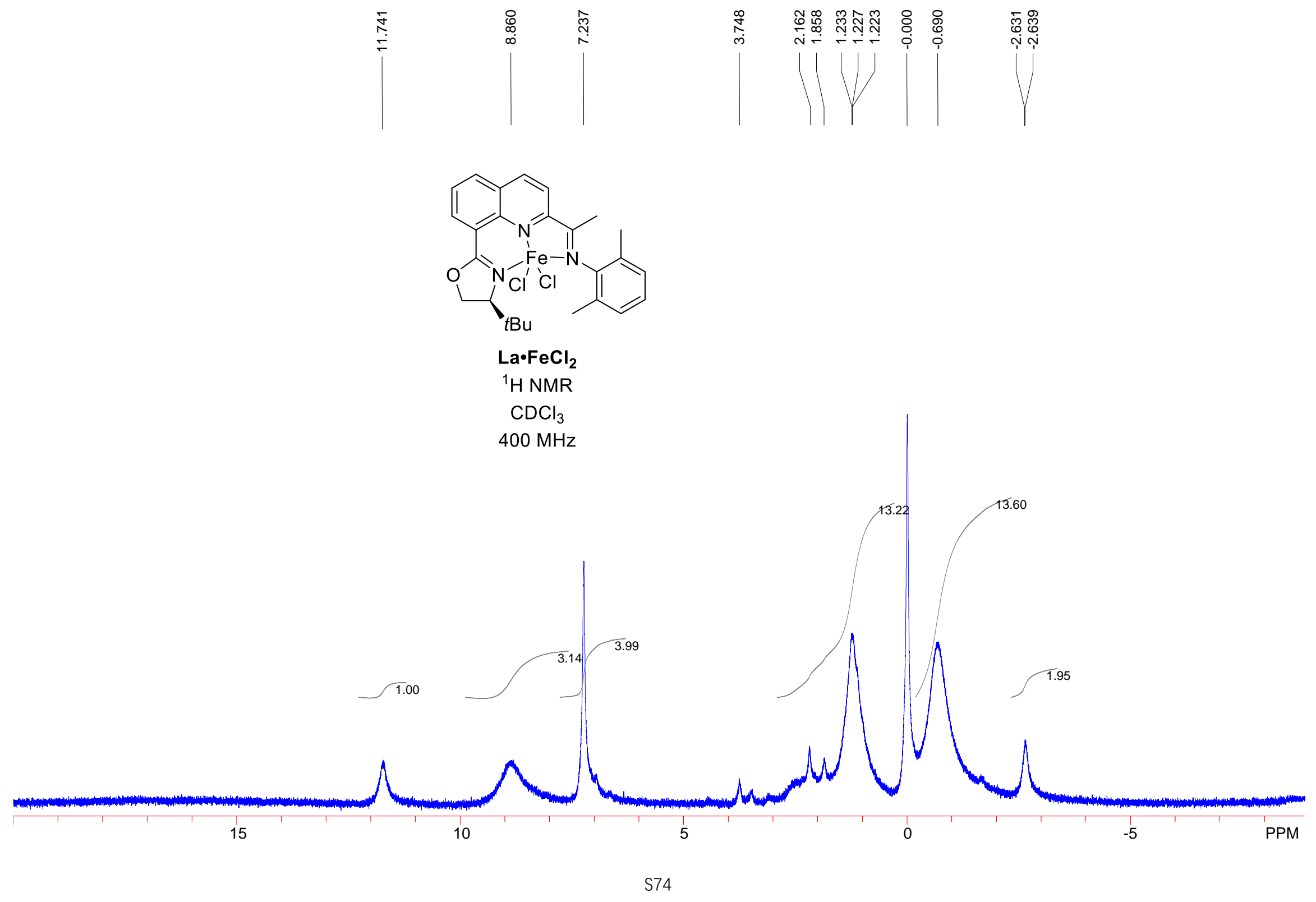




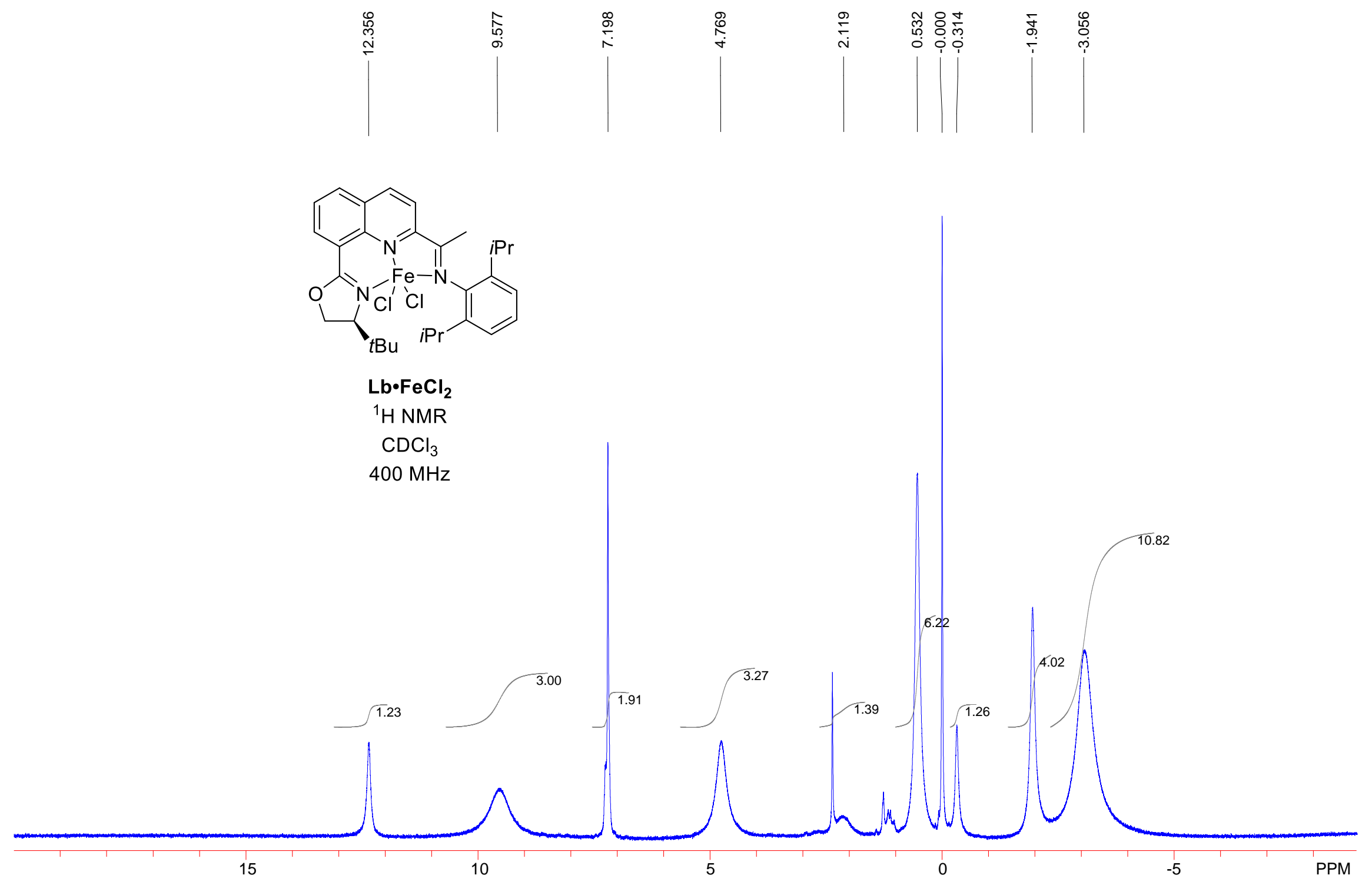




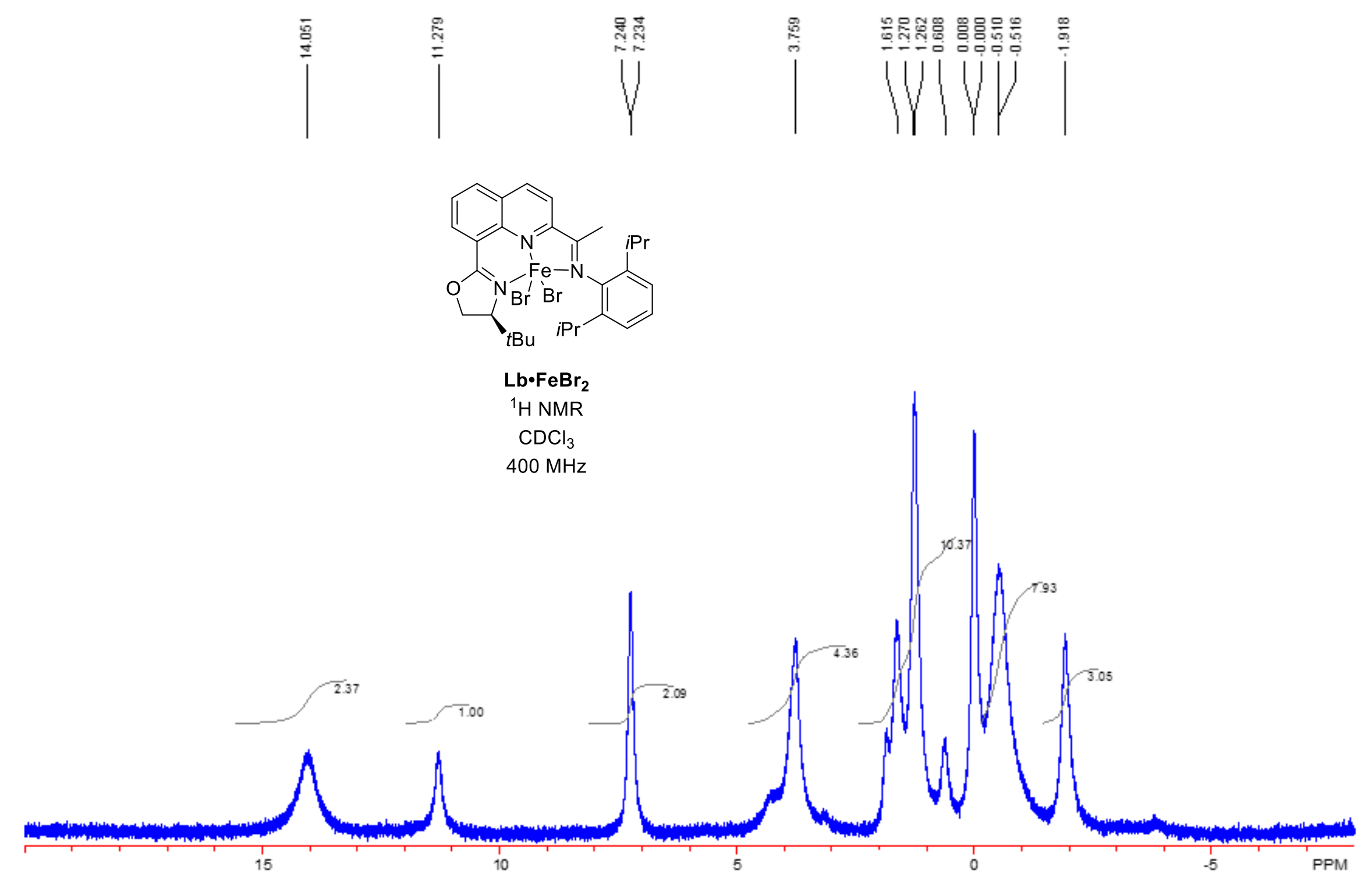




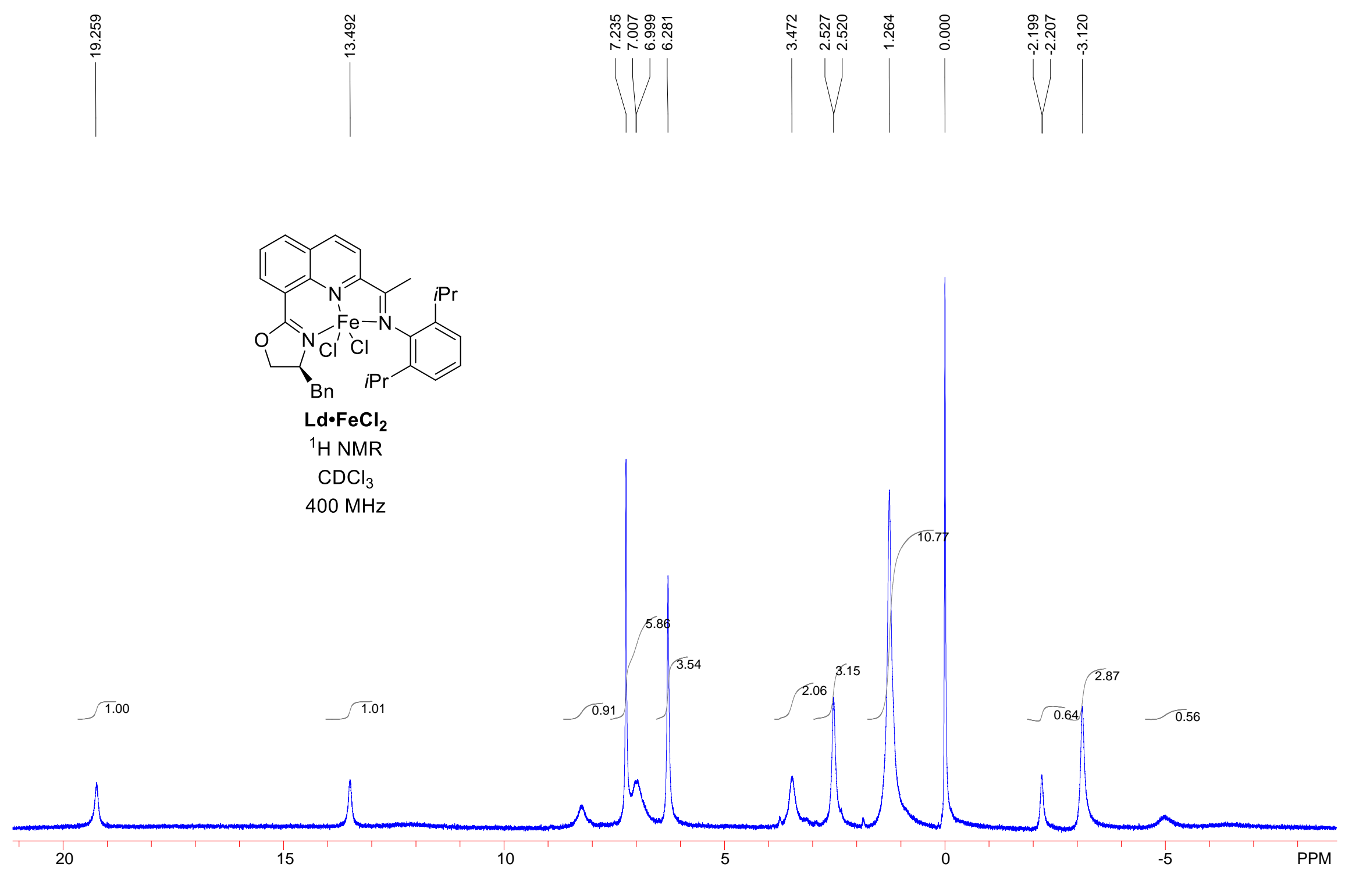




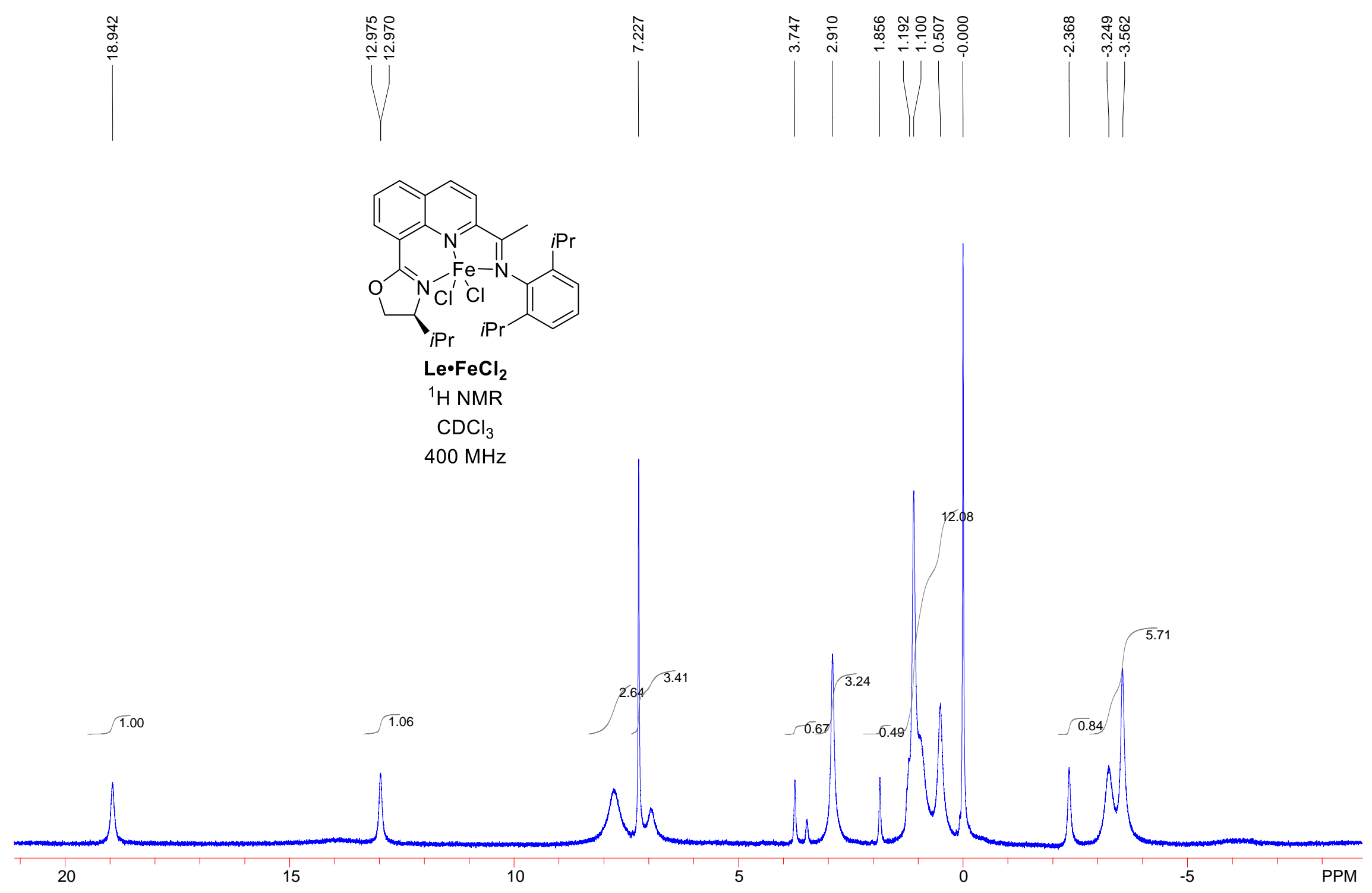




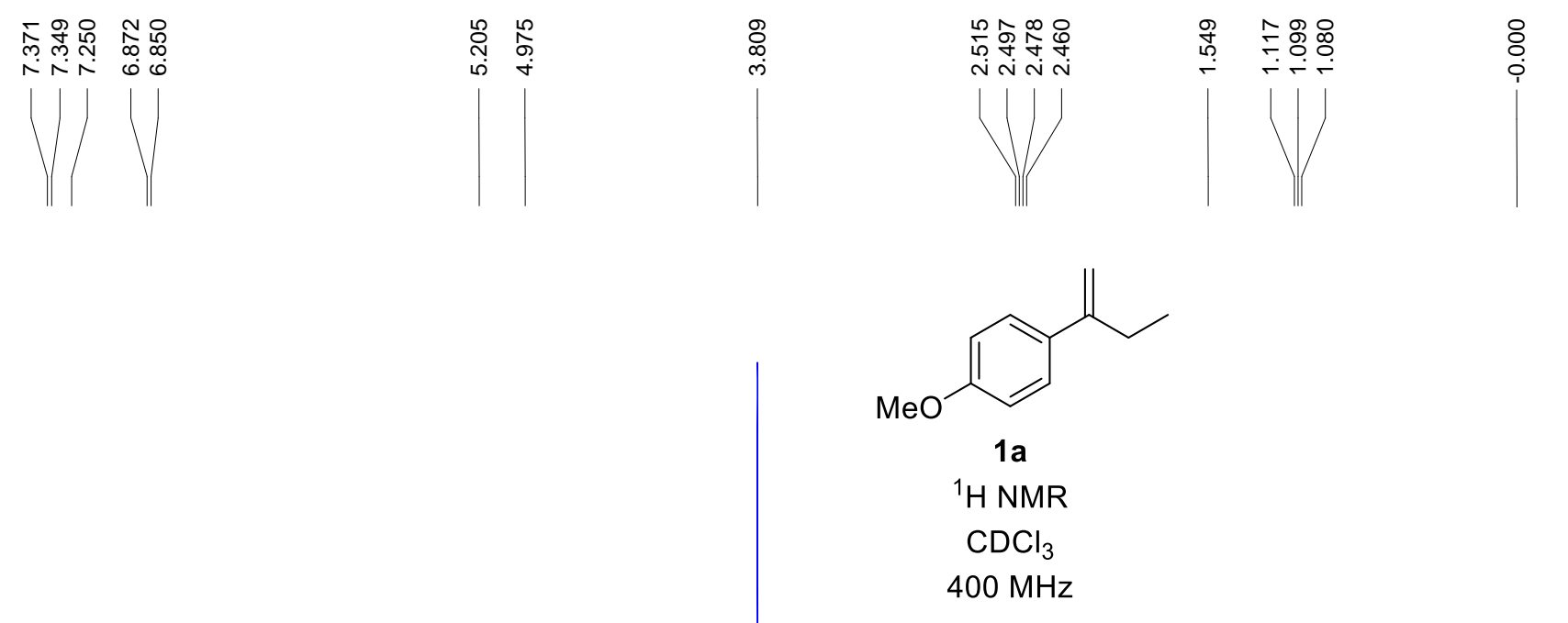

3.02

2.96

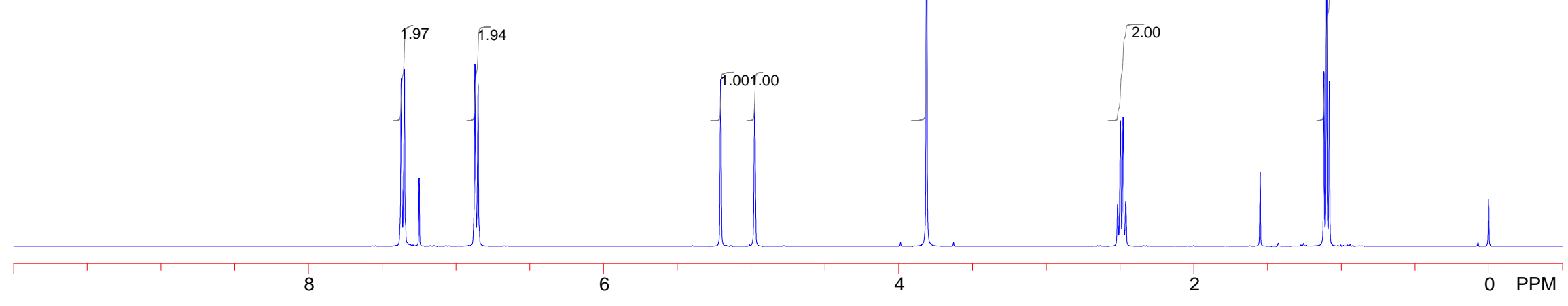




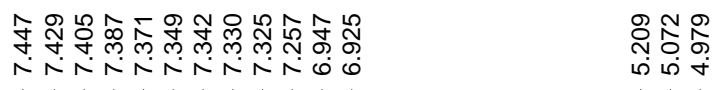
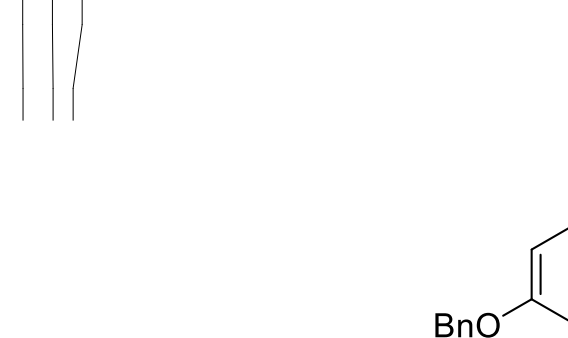

$$
\begin{gathered}
\text { 1b } \\
{ }^{1} \mathrm{H} \mathrm{NMR} \\
\mathrm{CDCl}_{3} \\
400 \mathrm{MHz}
\end{gathered}
$$

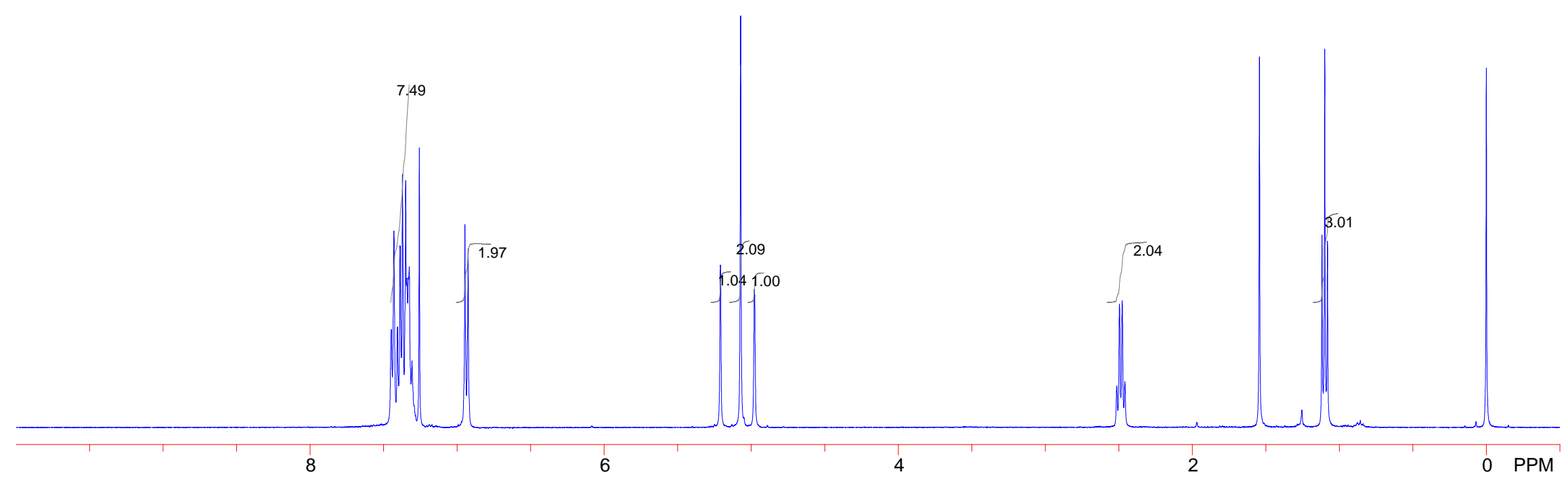




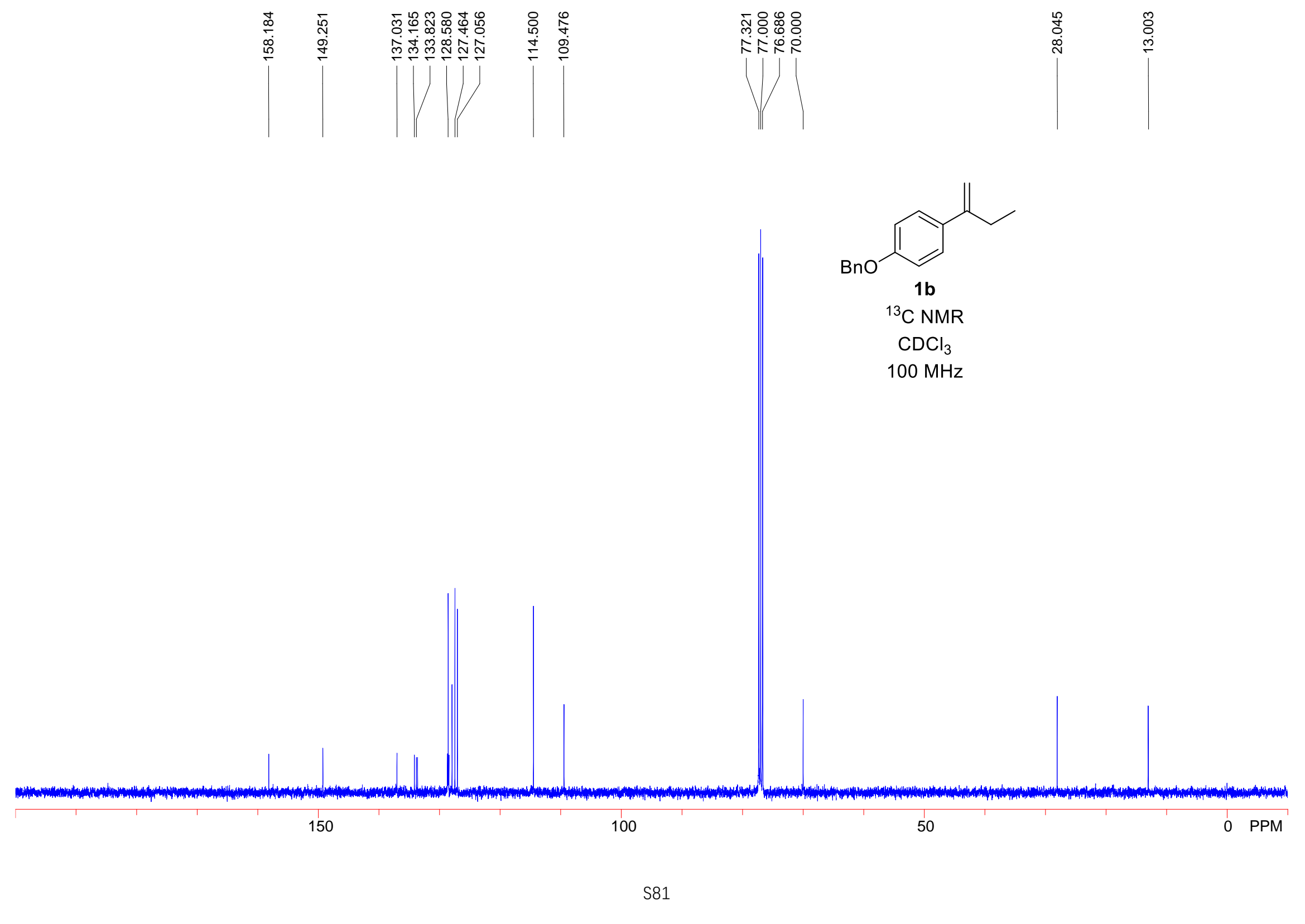



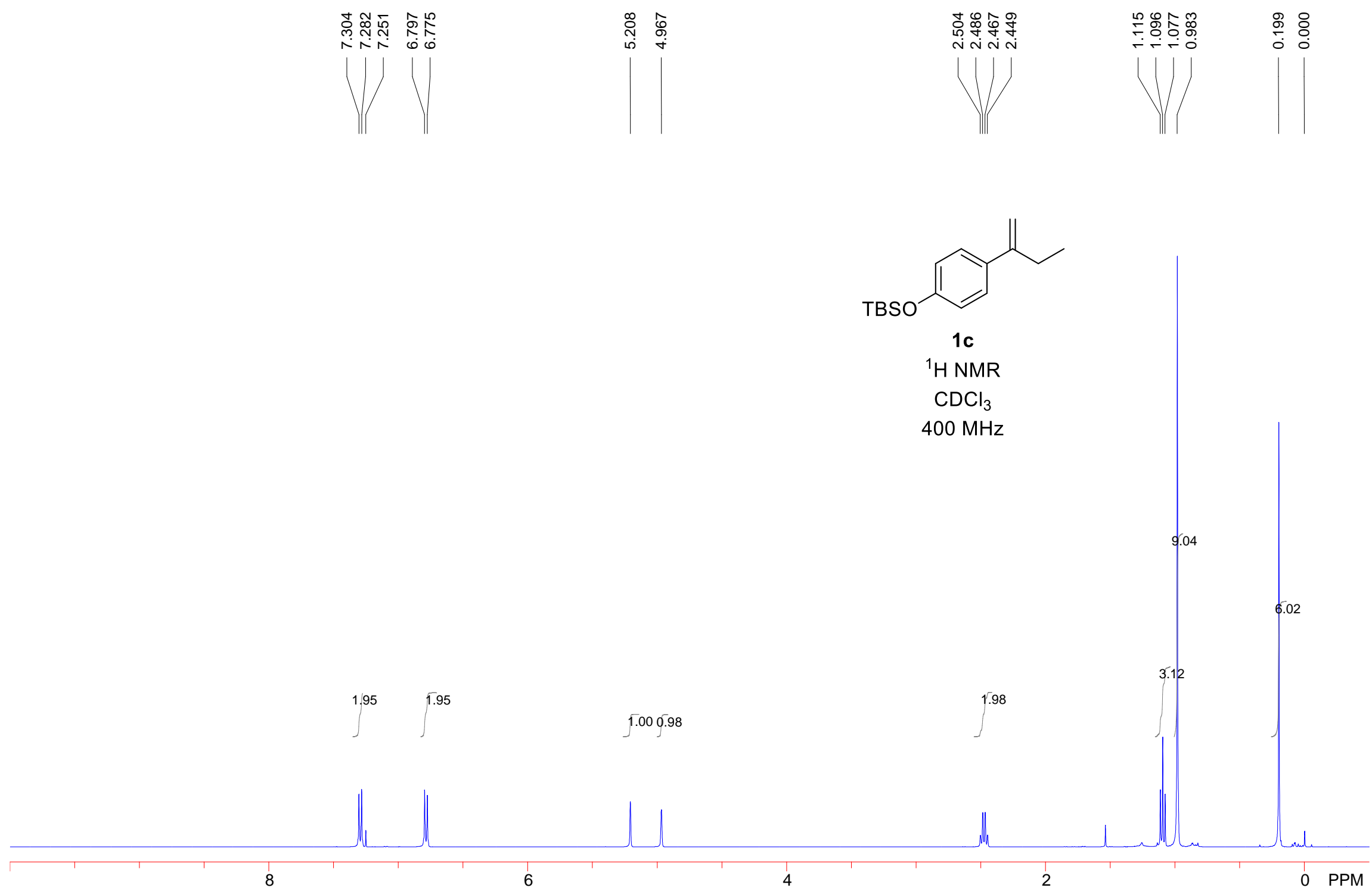

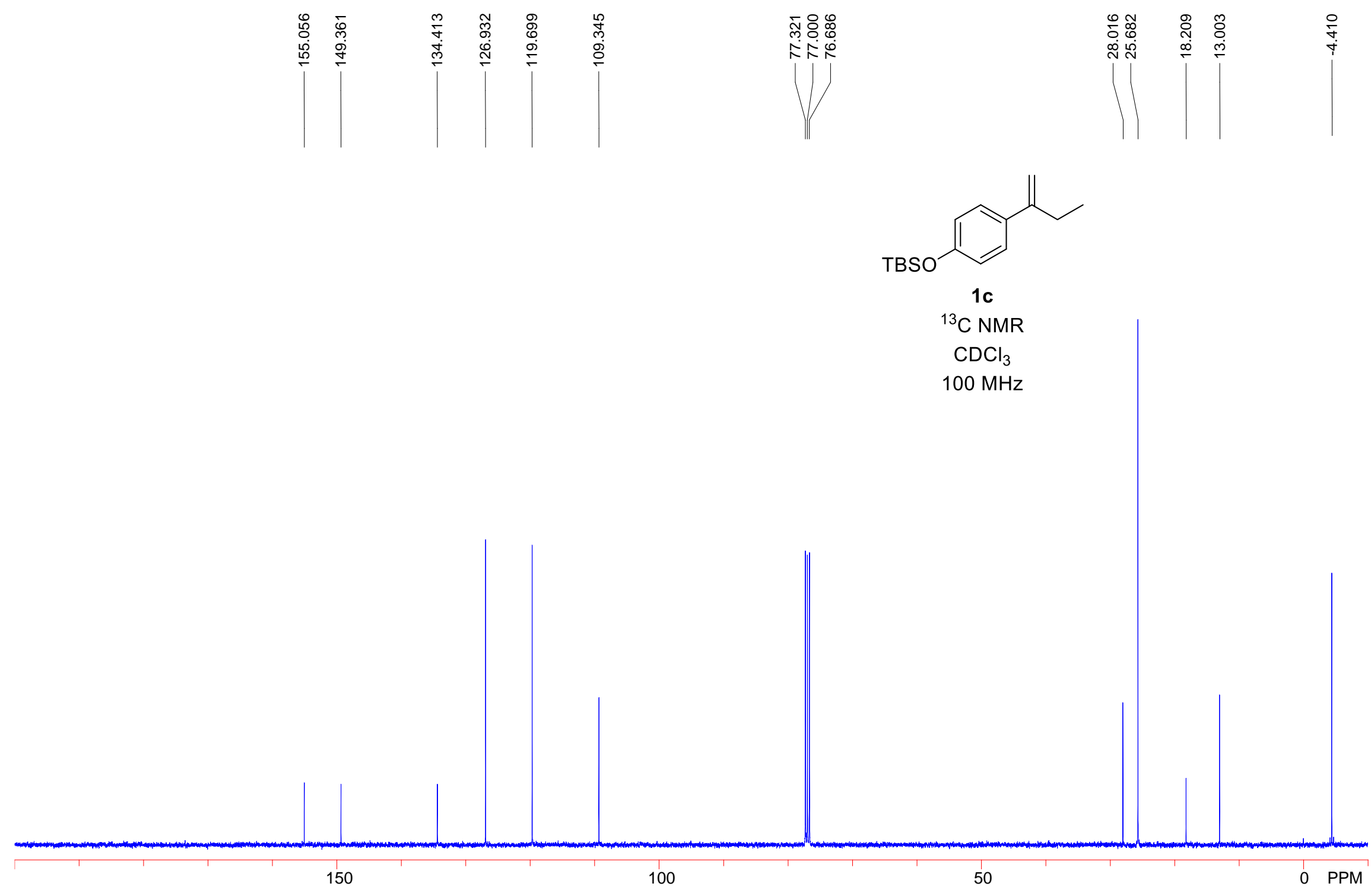


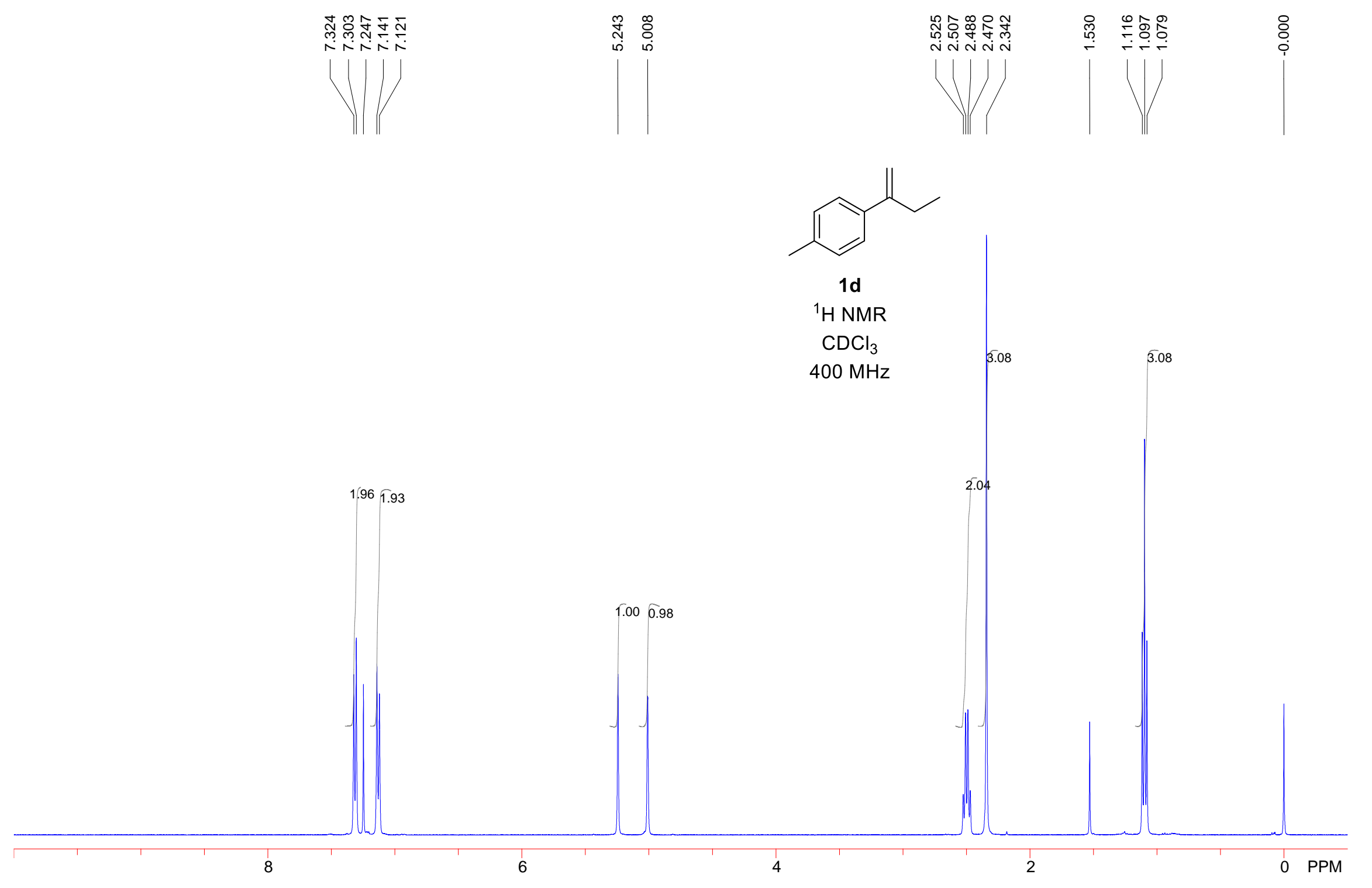




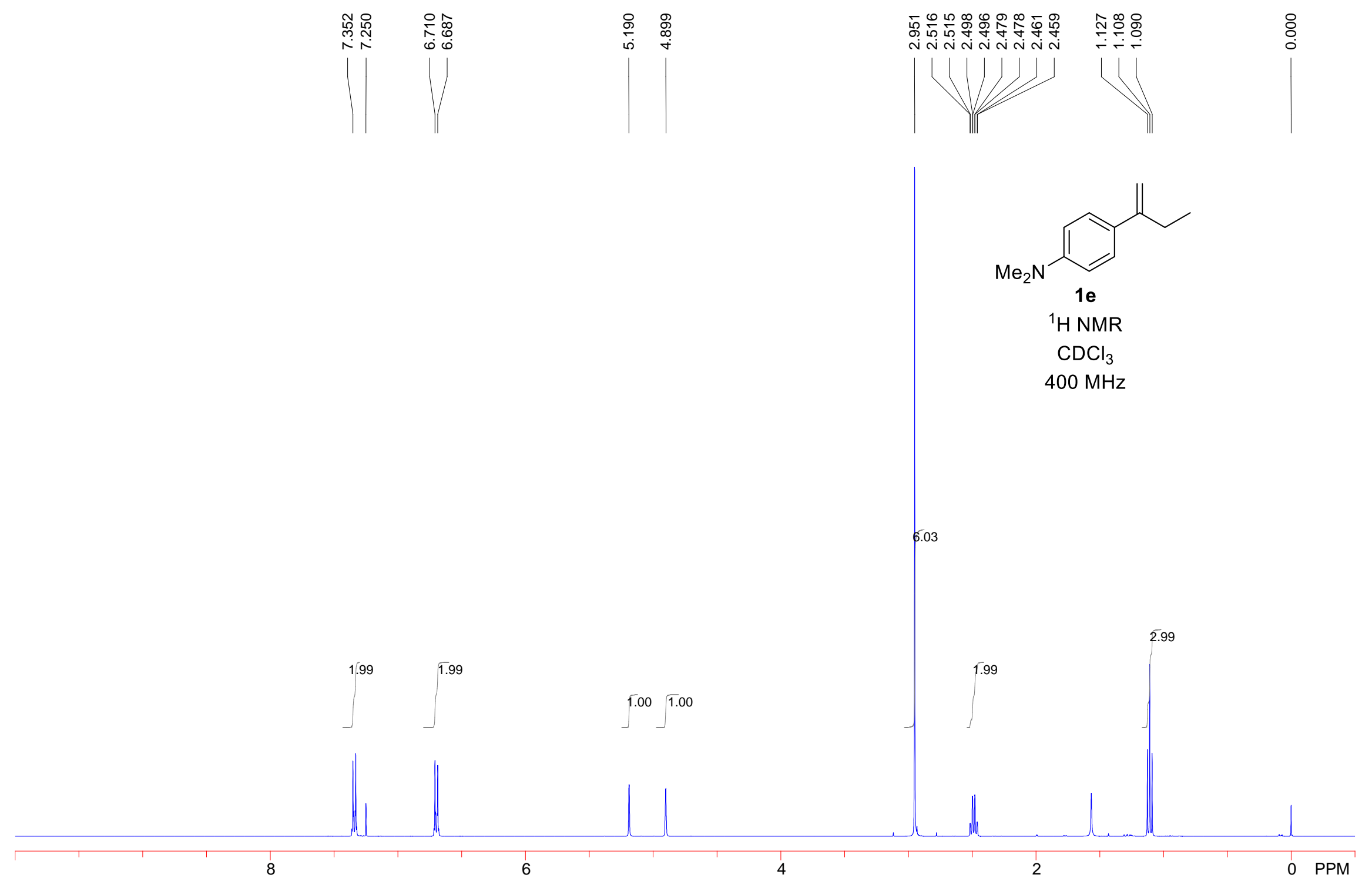




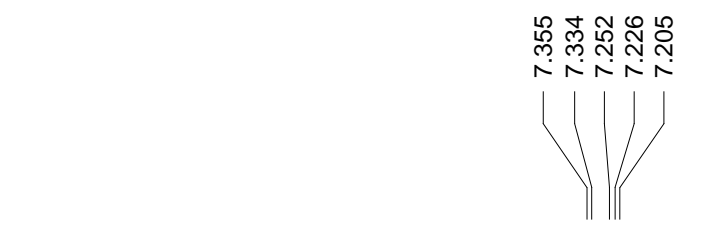

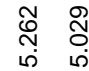

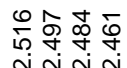

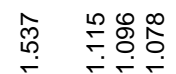

:

U

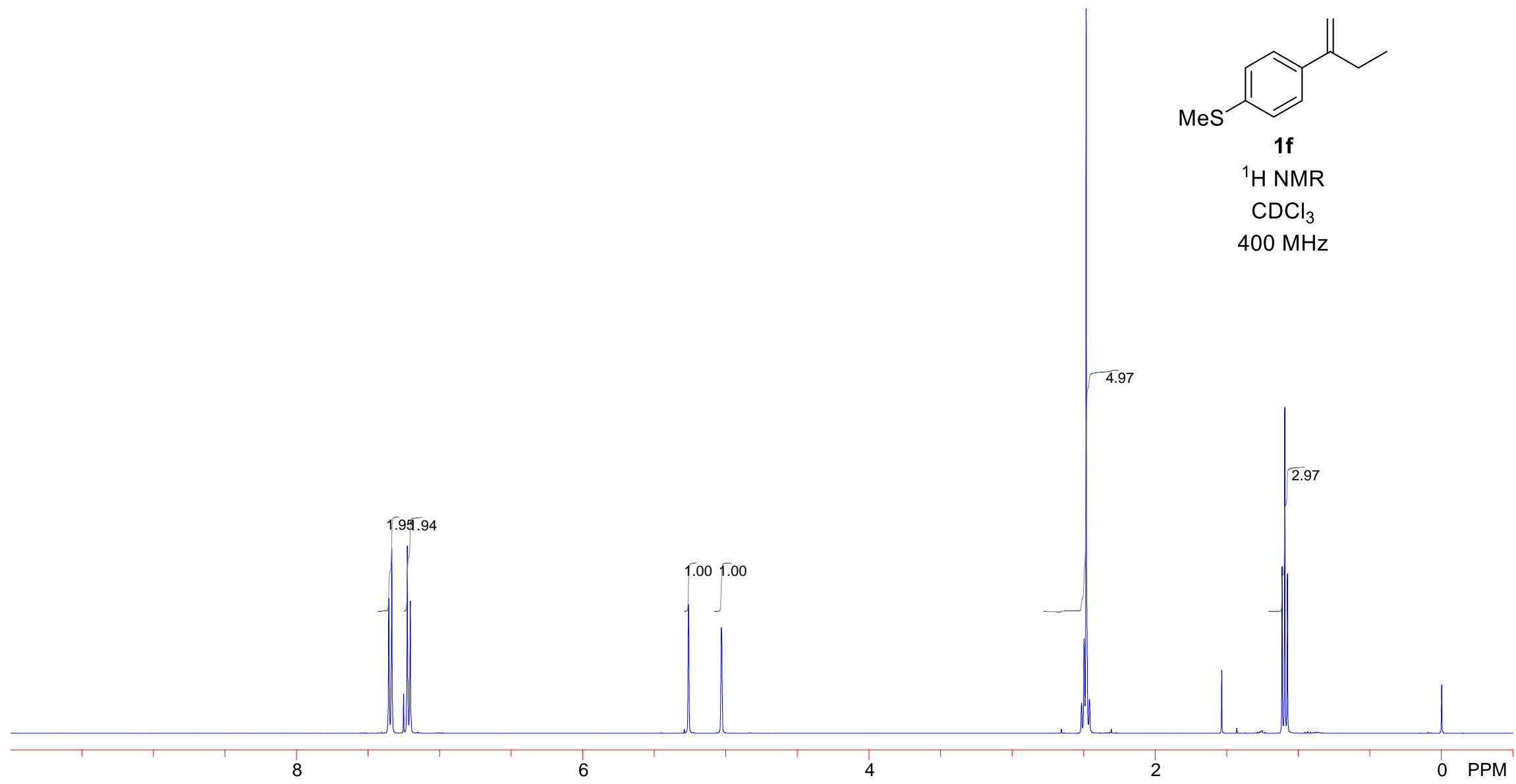




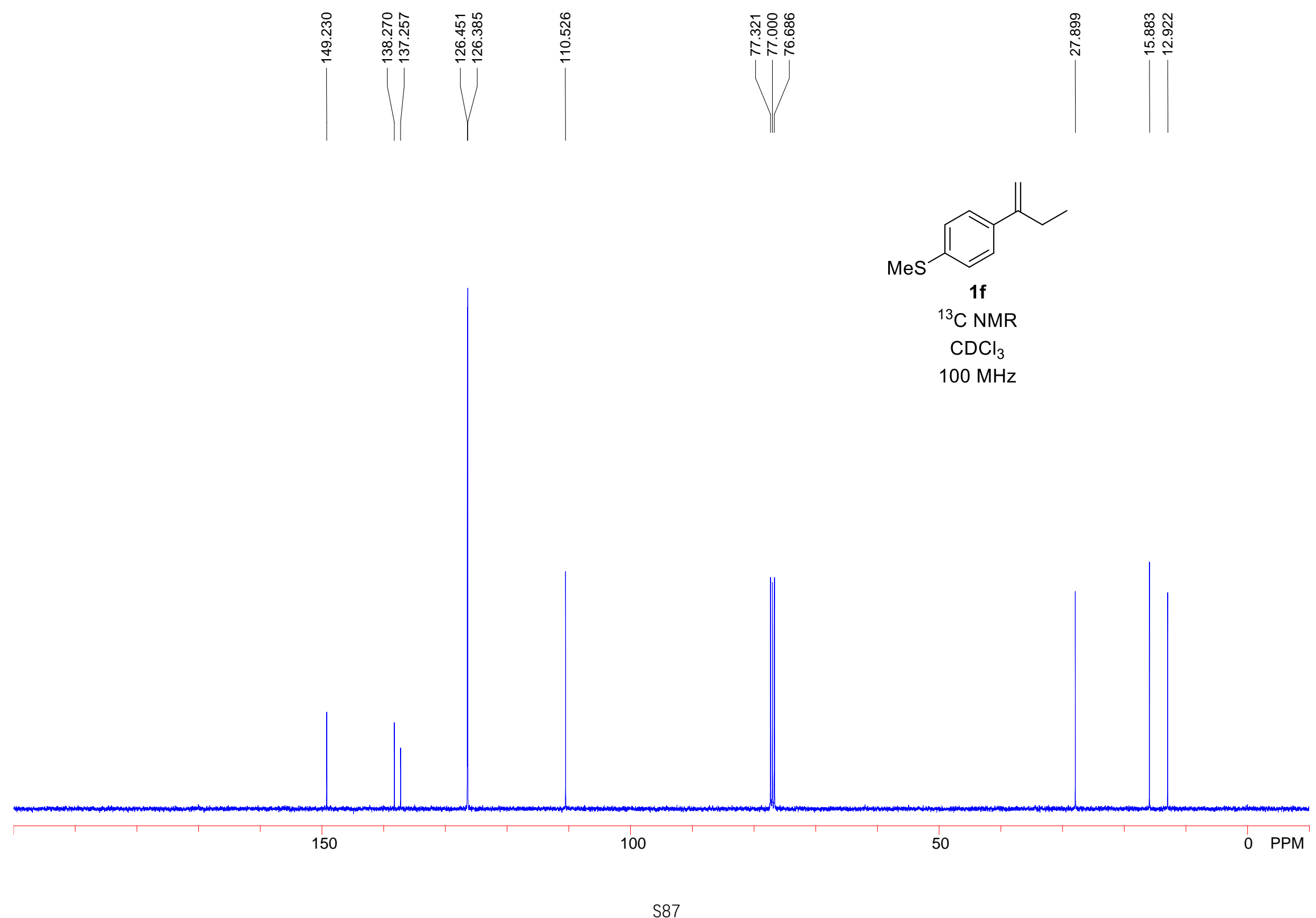




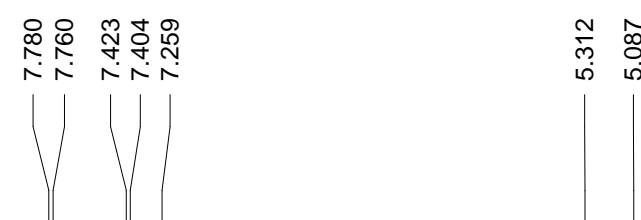

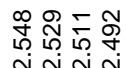

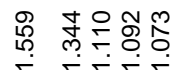

:

i

NaNa

$1 \mathrm{~g}$
${ }^{1} \mathrm{H} \mathrm{NMR}$
$\mathrm{CDCl}_{3}$
$400 \mathrm{MHz}$

$1.98 \quad 1.95$

1.001 .00

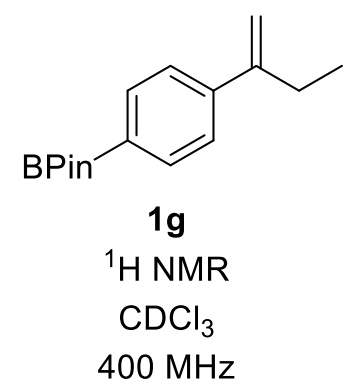

12.47

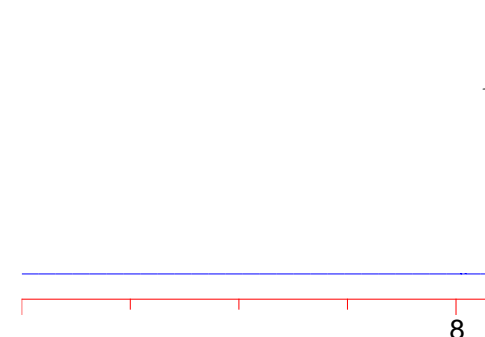




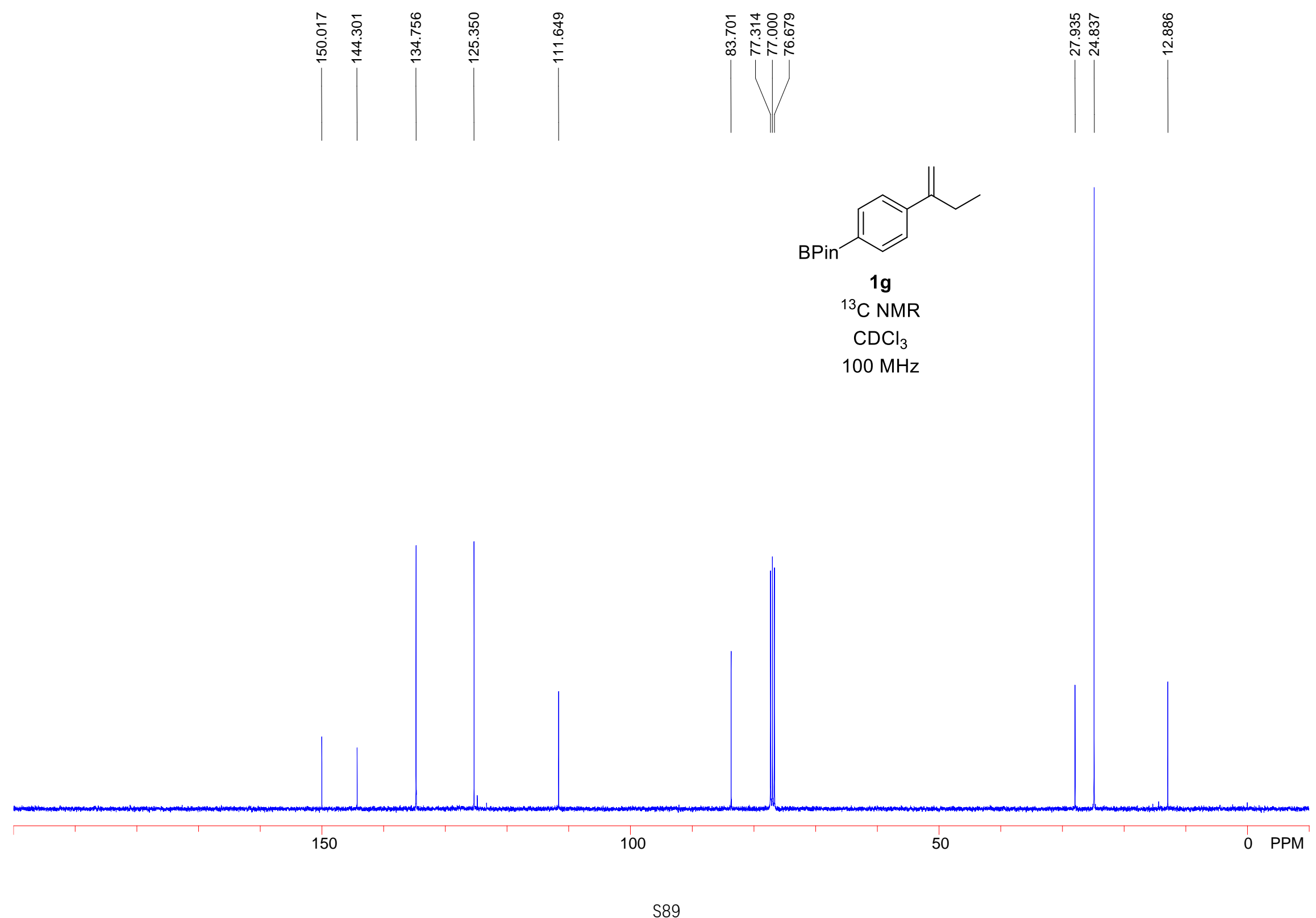




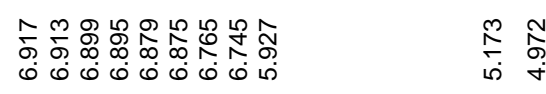

夺造臬举

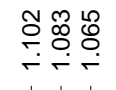

:

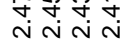

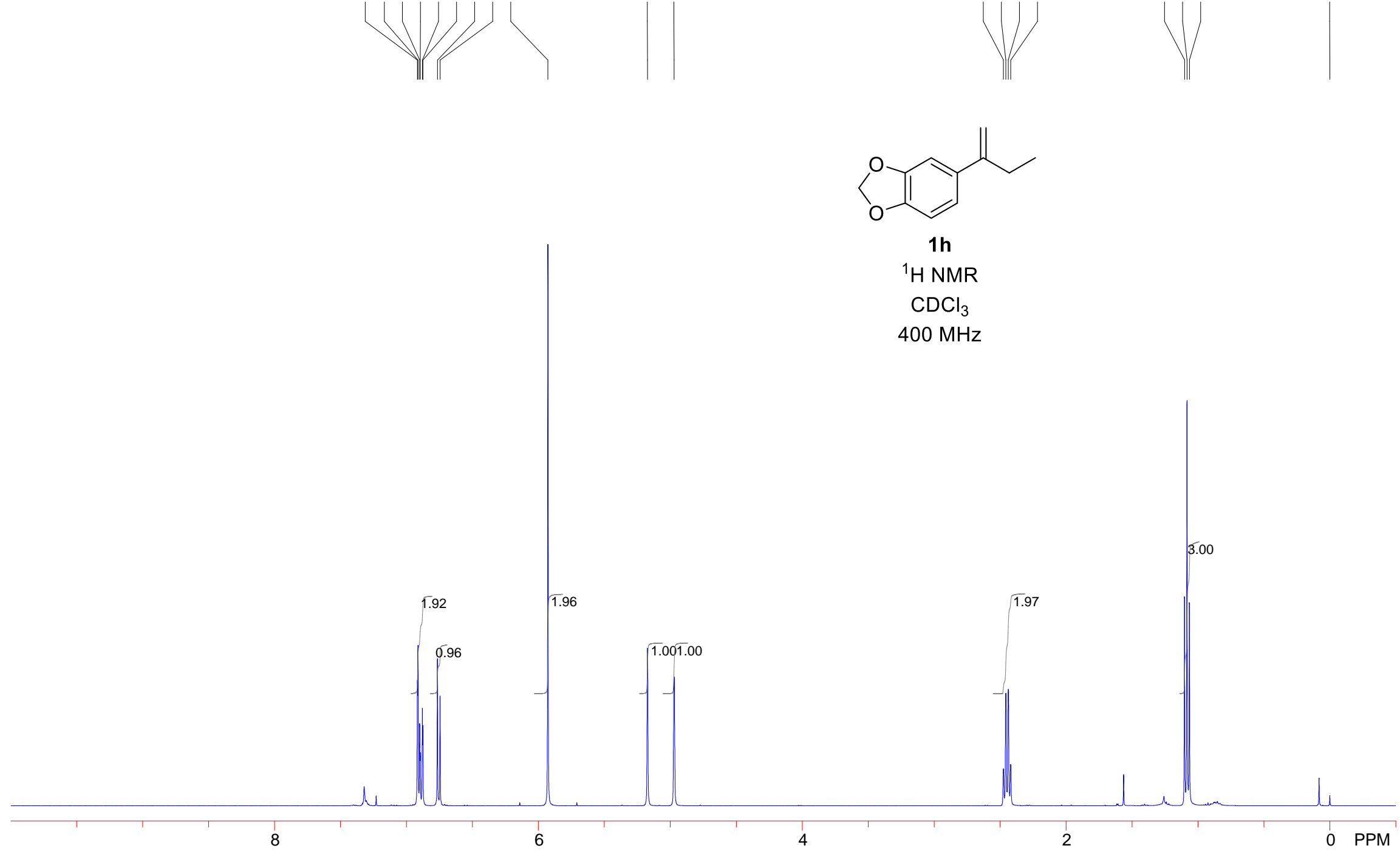




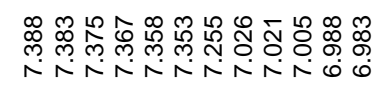
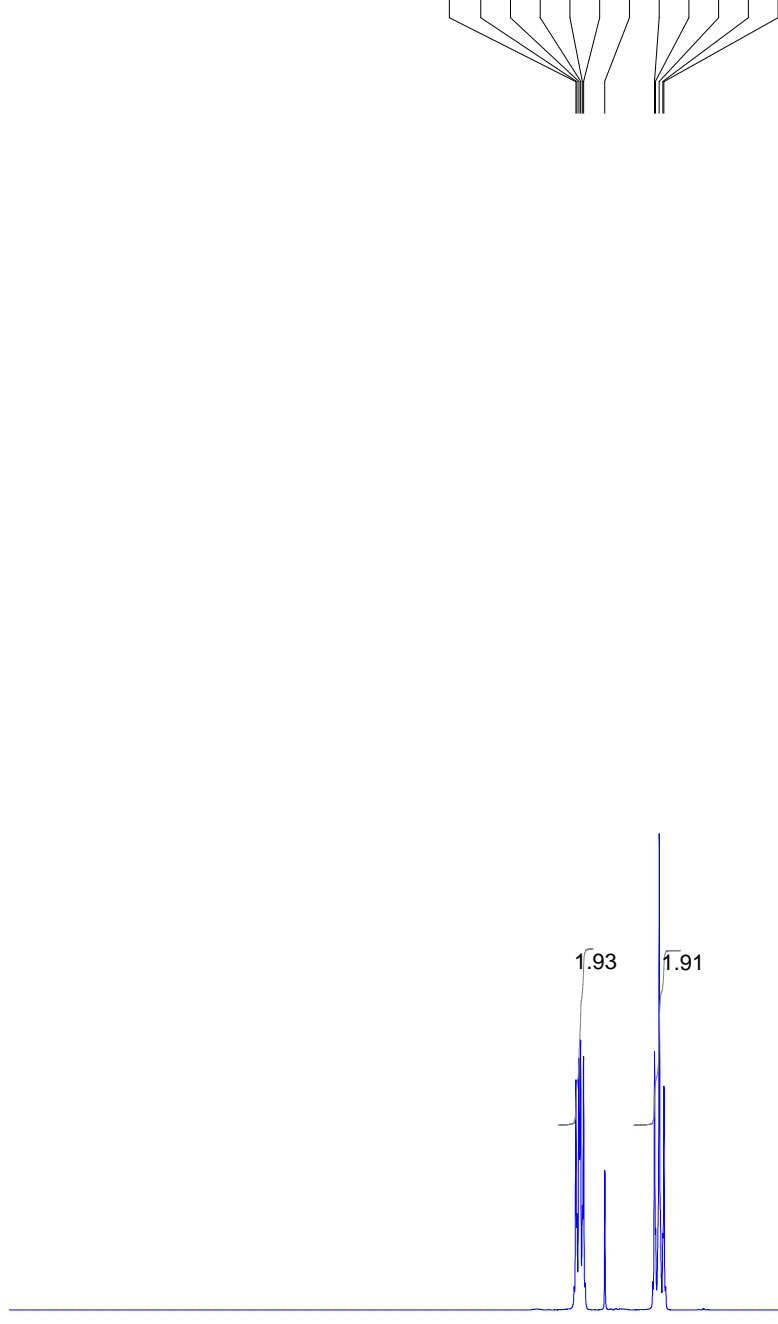

8

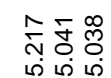

V

$1.93 \quad 1.91$

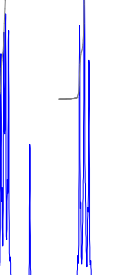

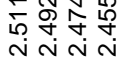

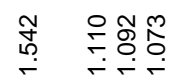

:
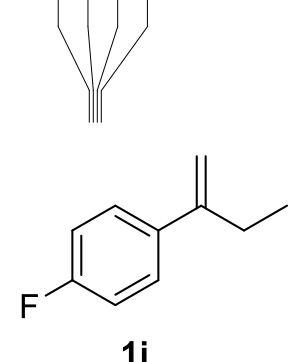

$1 \mathrm{i}$

${ }^{1} \mathrm{H}$ NMR

$\mathrm{CDCl}_{3}$

$400 \mathrm{MHz}$

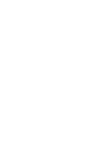

เ 


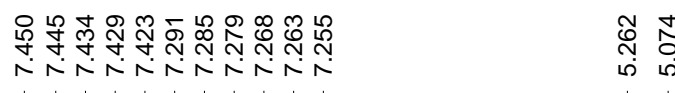

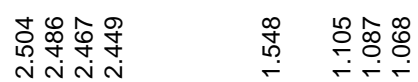

$\stackrel{8}{\circ}$
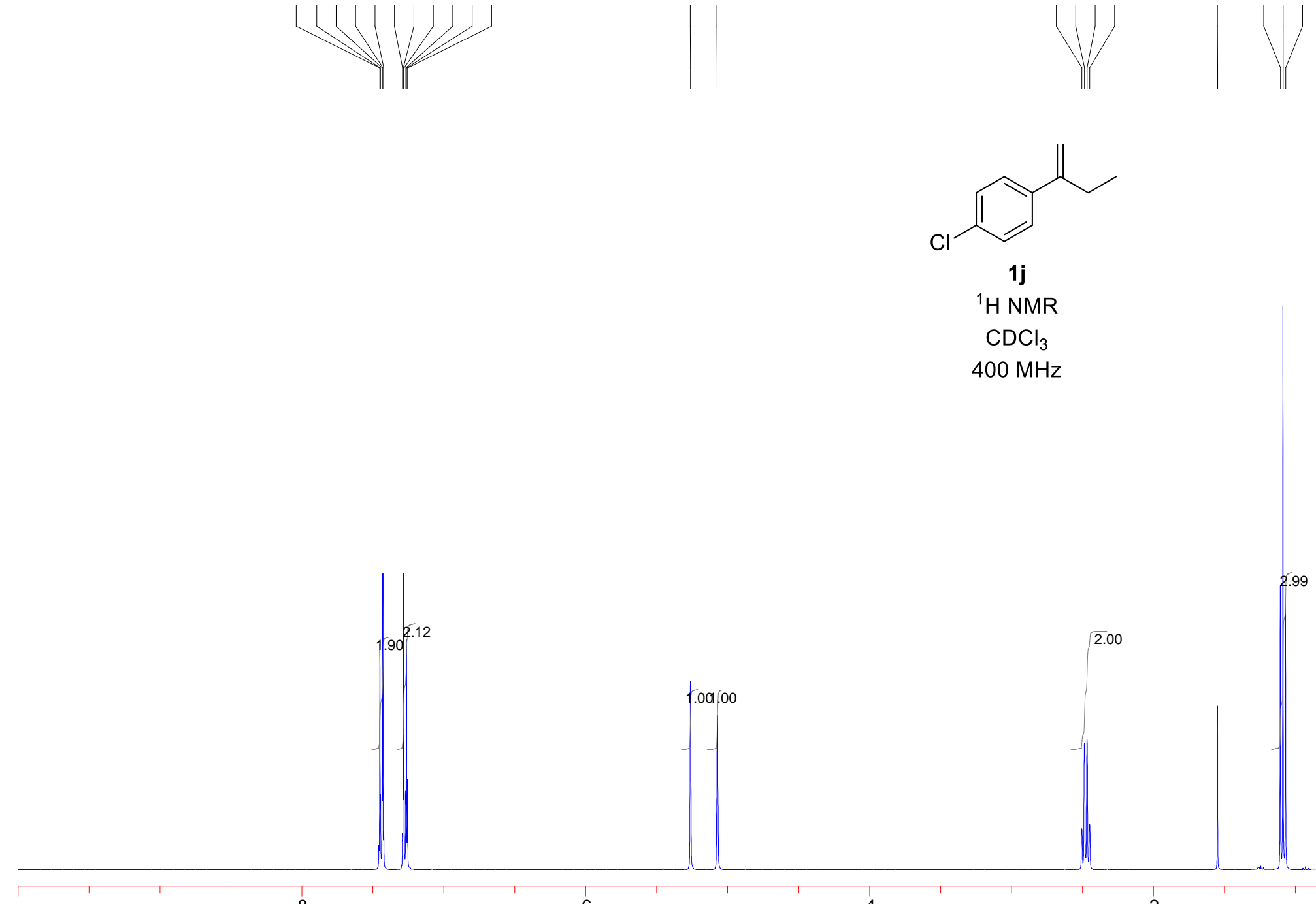


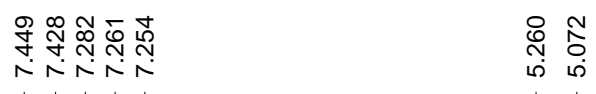

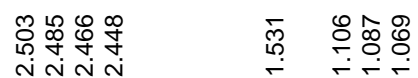

$\stackrel{8}{\circ}$

(H)
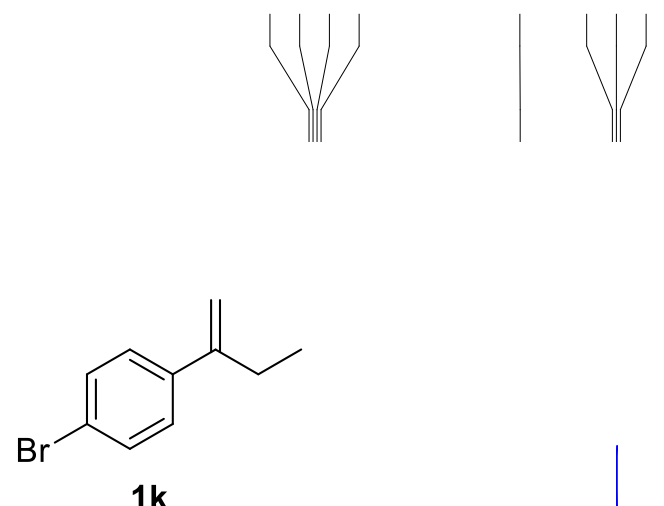

${ }^{1} \mathrm{H}$ NMR

$\mathrm{CDCl}_{3}$

$400 \mathrm{MHz}$

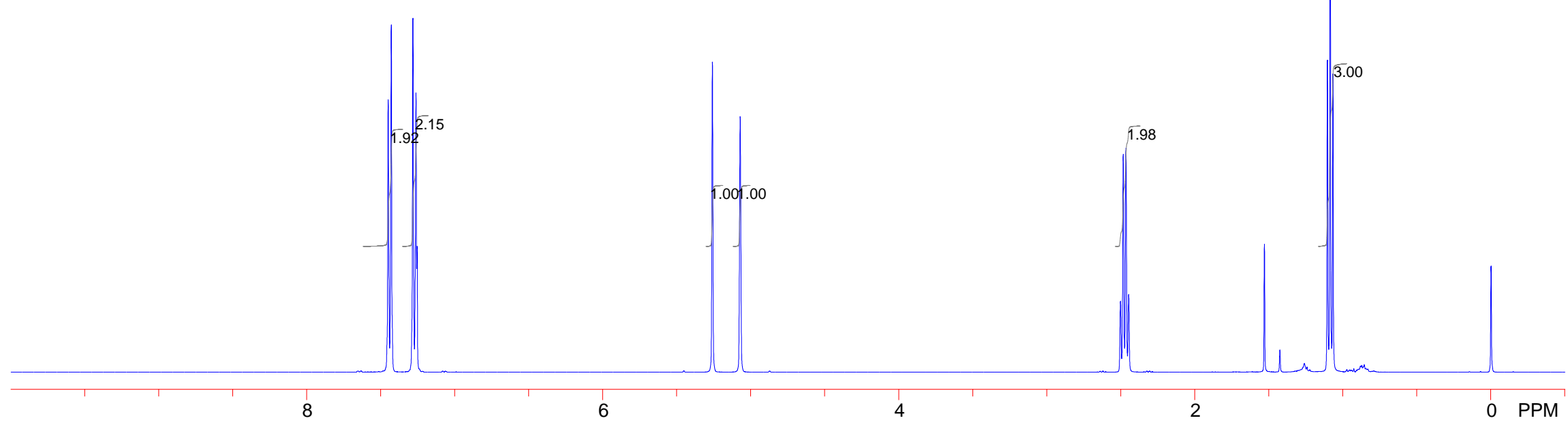




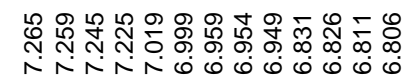

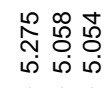

$\underset{\infty}{\infty}$
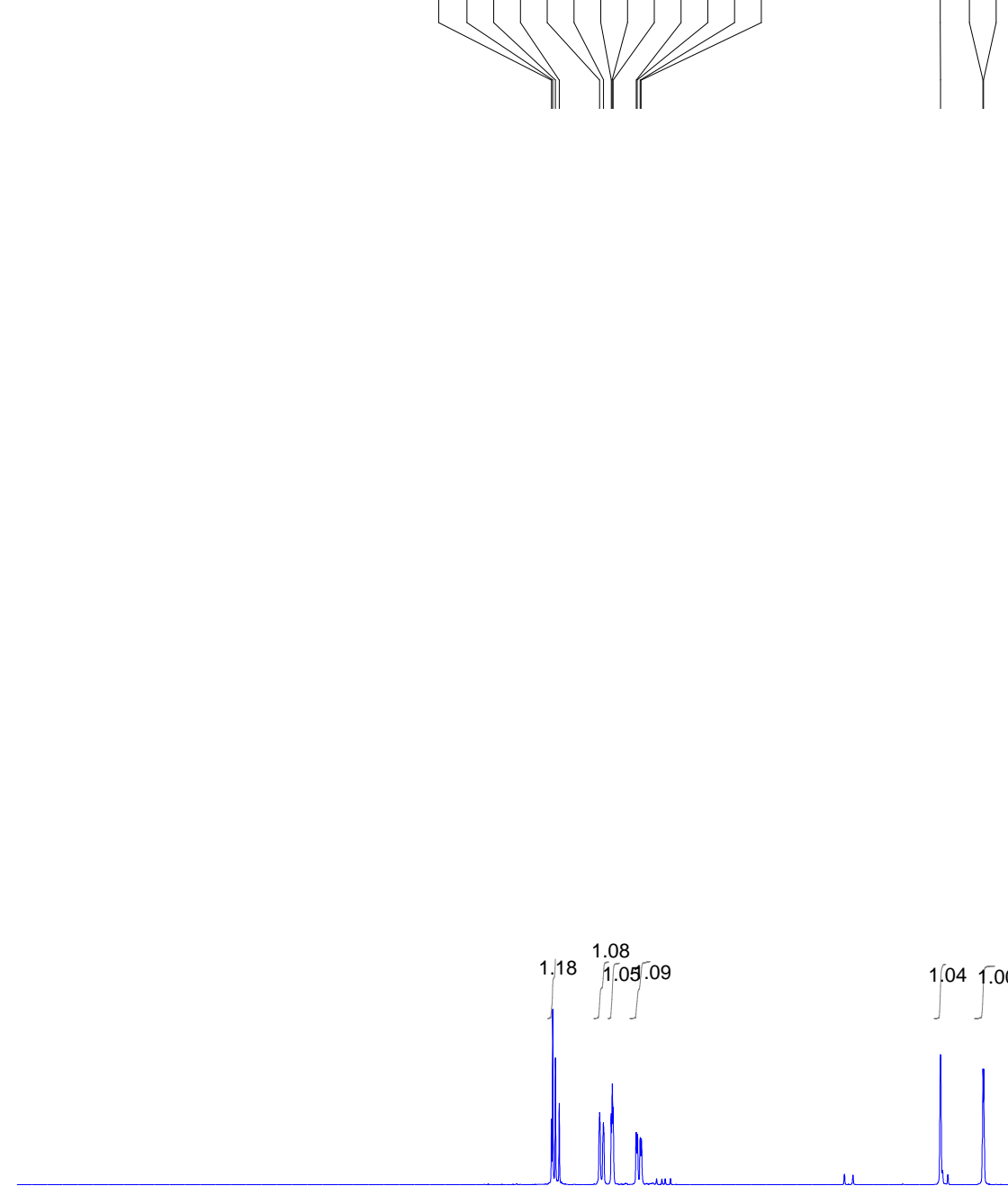

$1.04 \quad 1.00$

$1.18 \stackrel{1.08}{1.05 .09}$

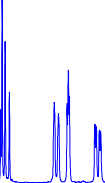

$\mathrm{MeO}$

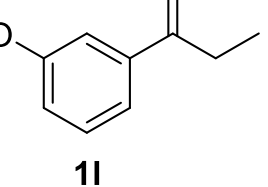

${ }^{1} \mathrm{H}$ NMR

$\mathrm{CDCl}_{3}$

$400 \mathrm{MHz}$ 


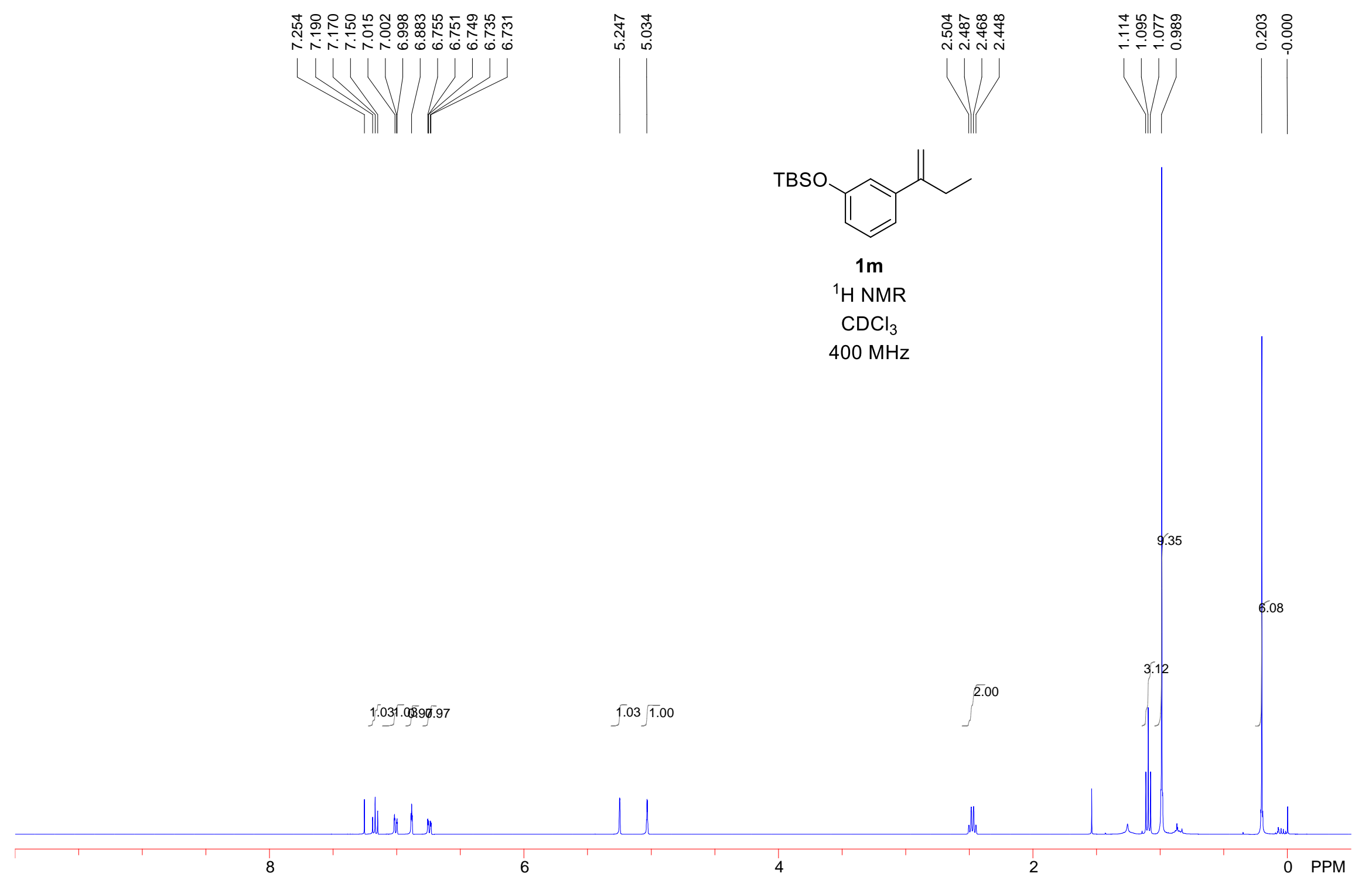




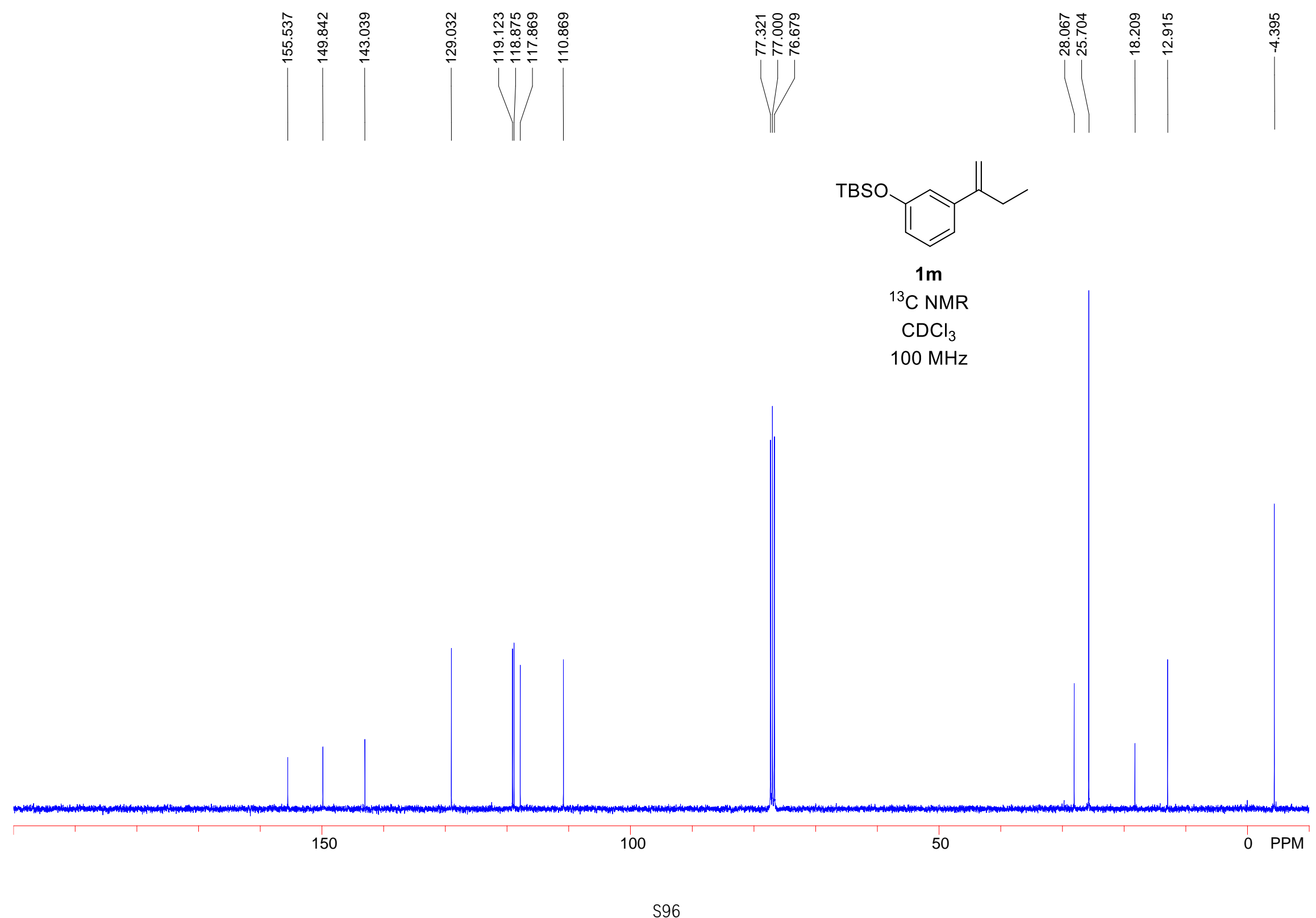




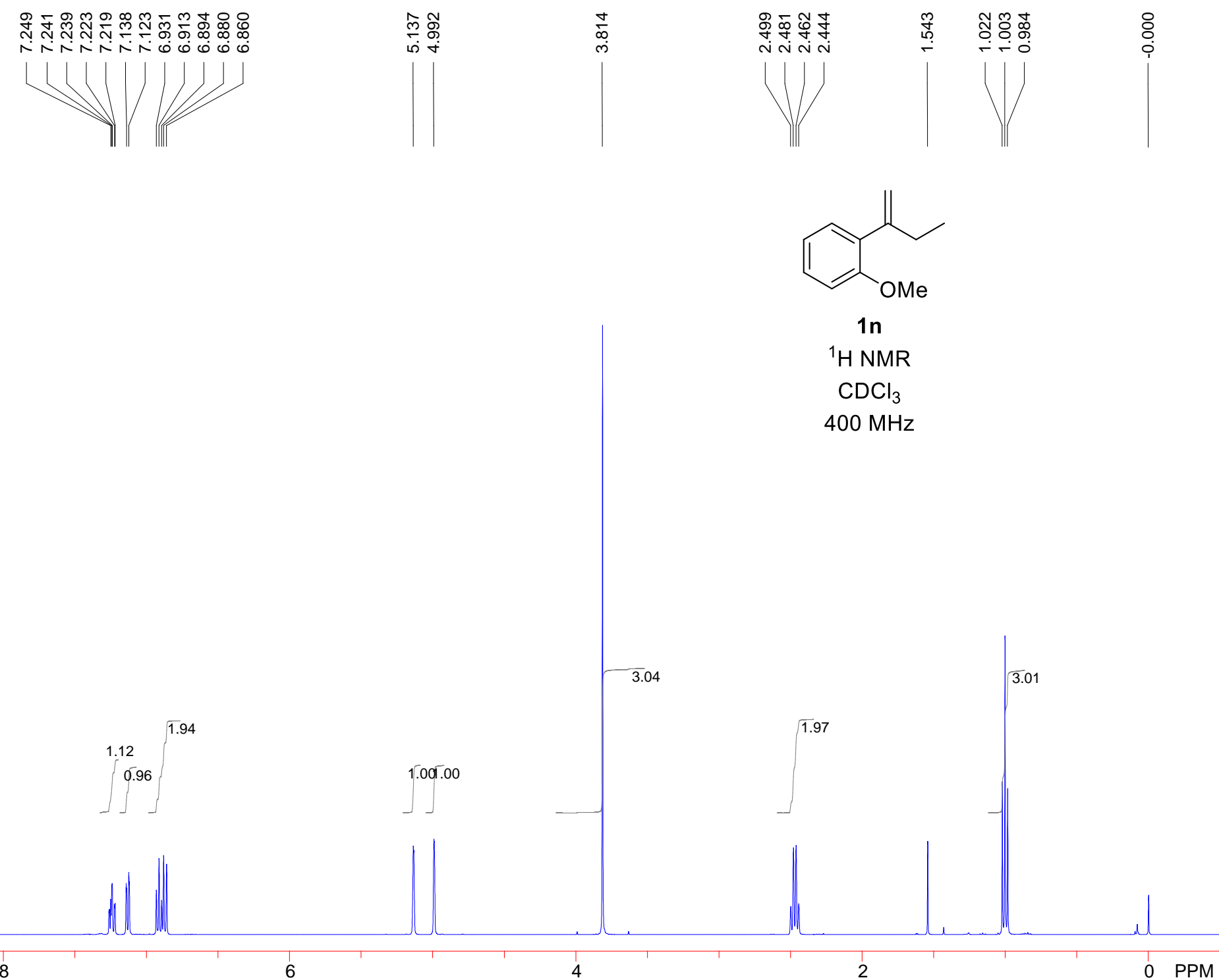




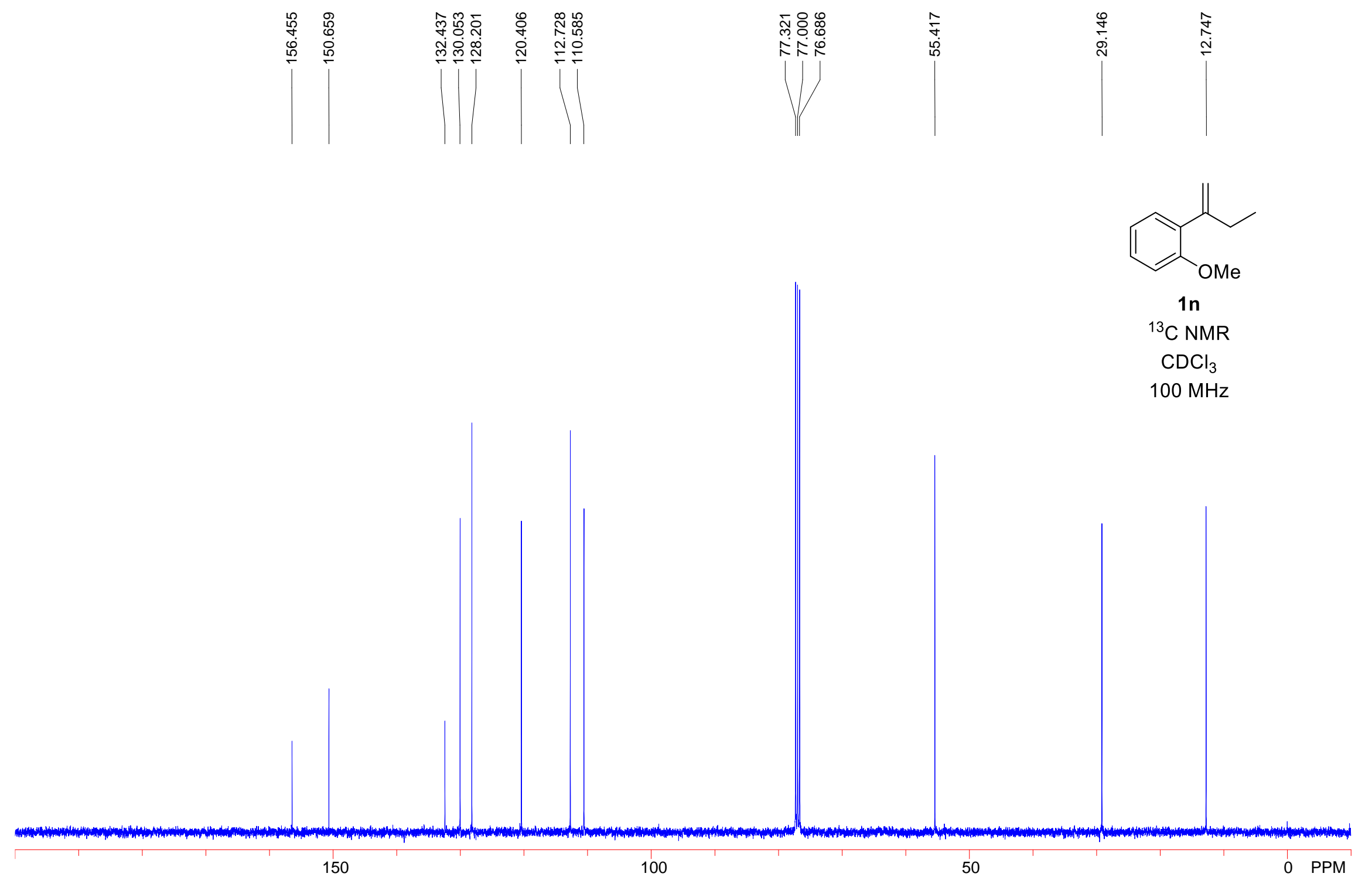




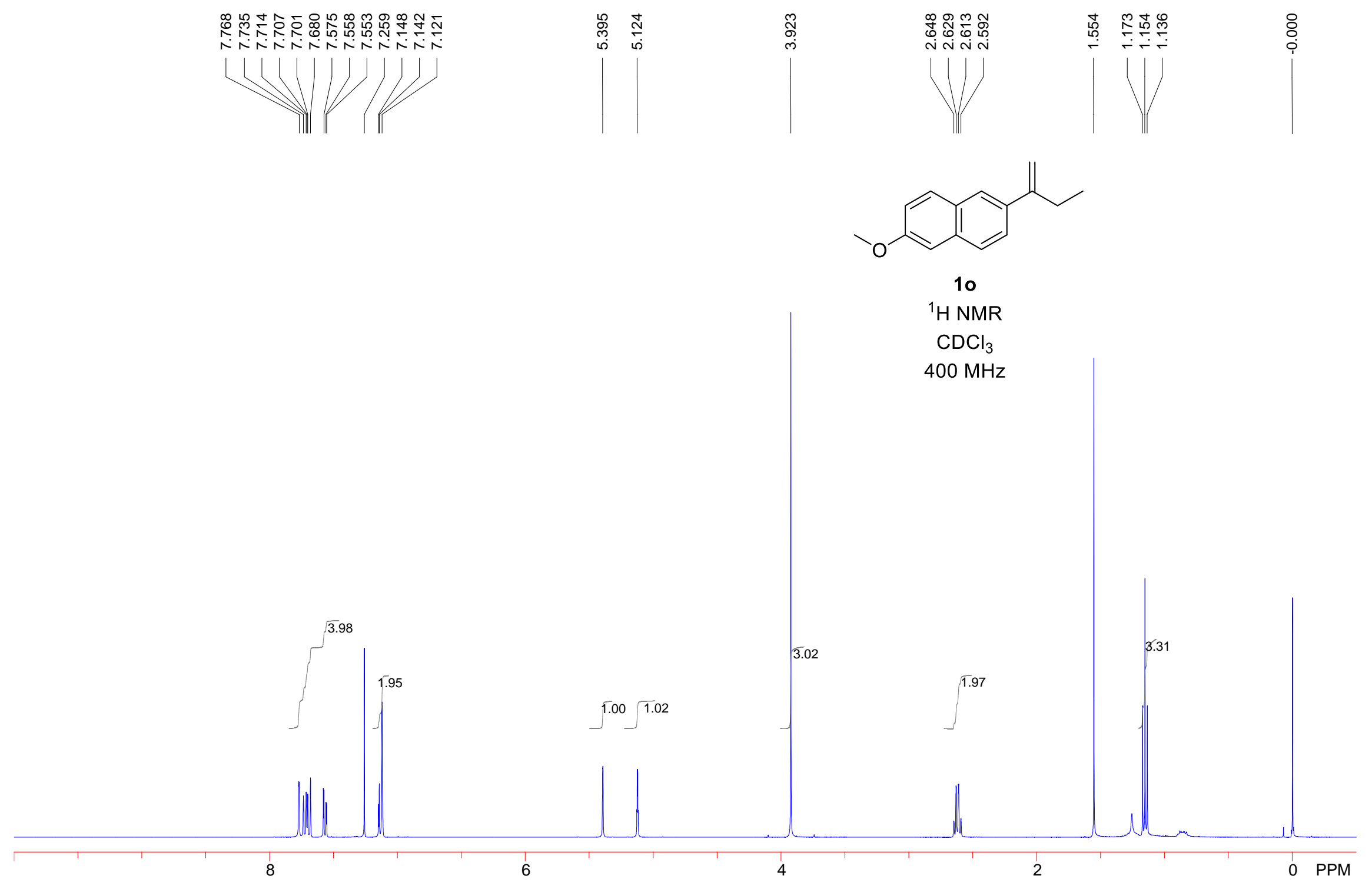




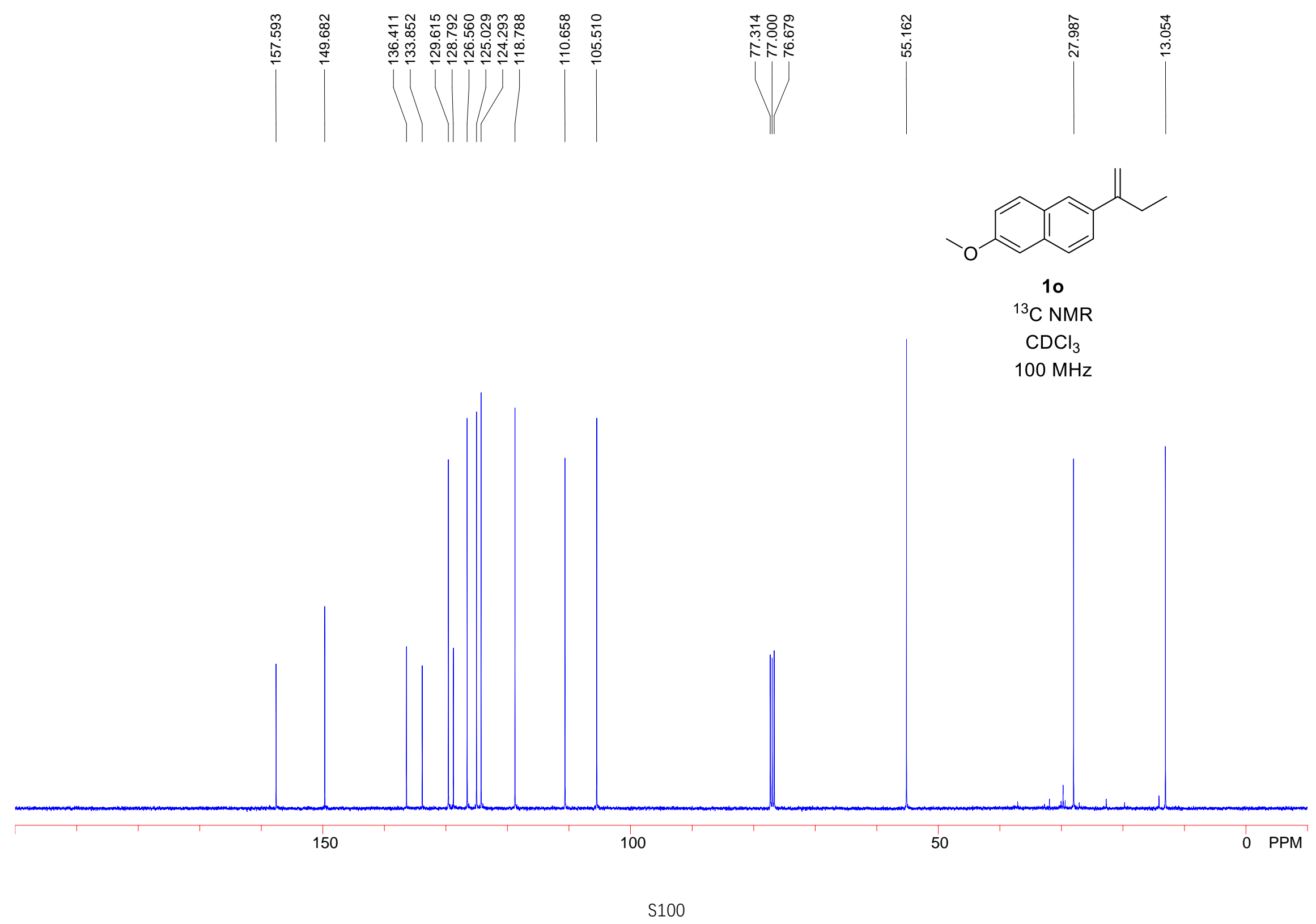




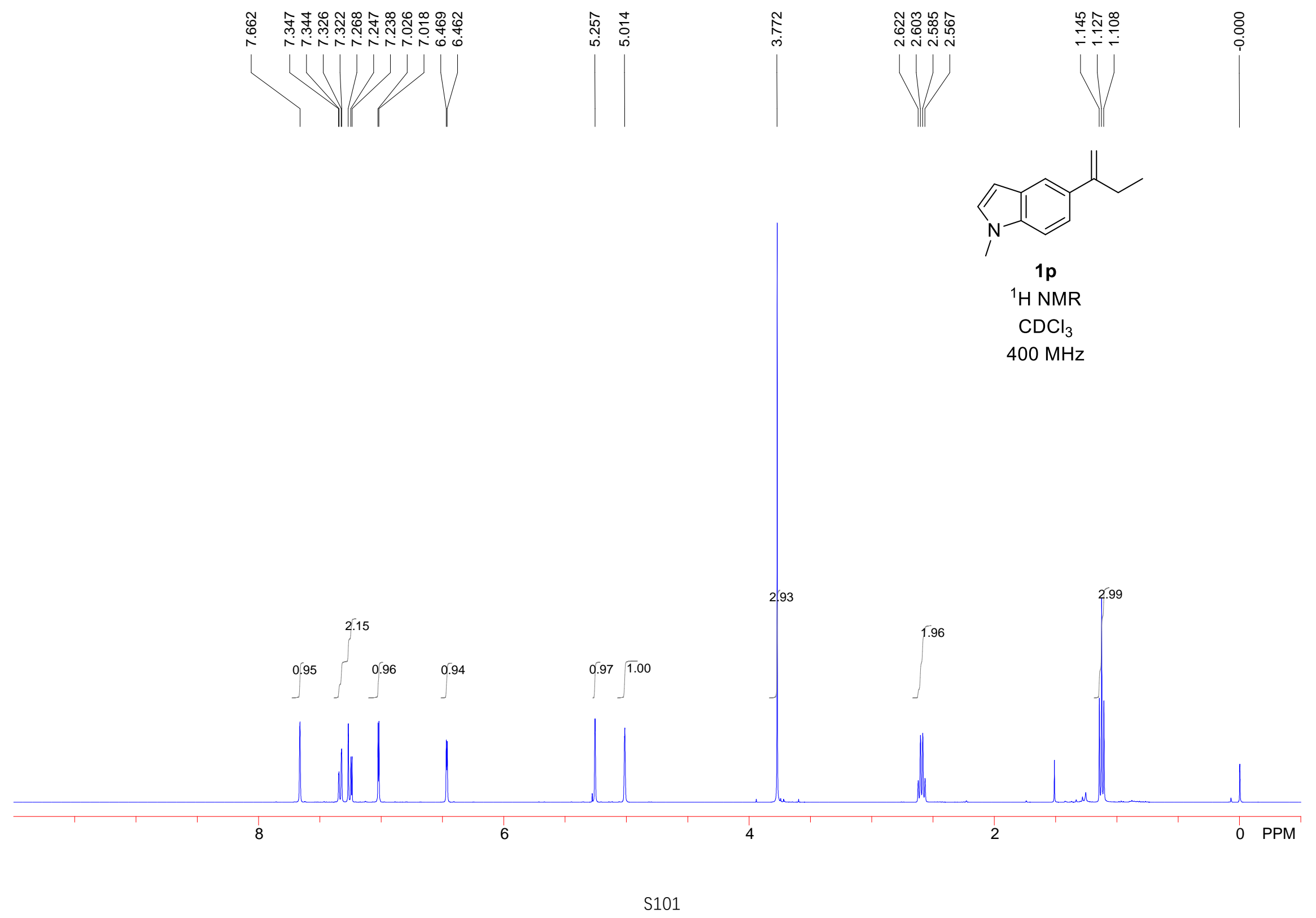




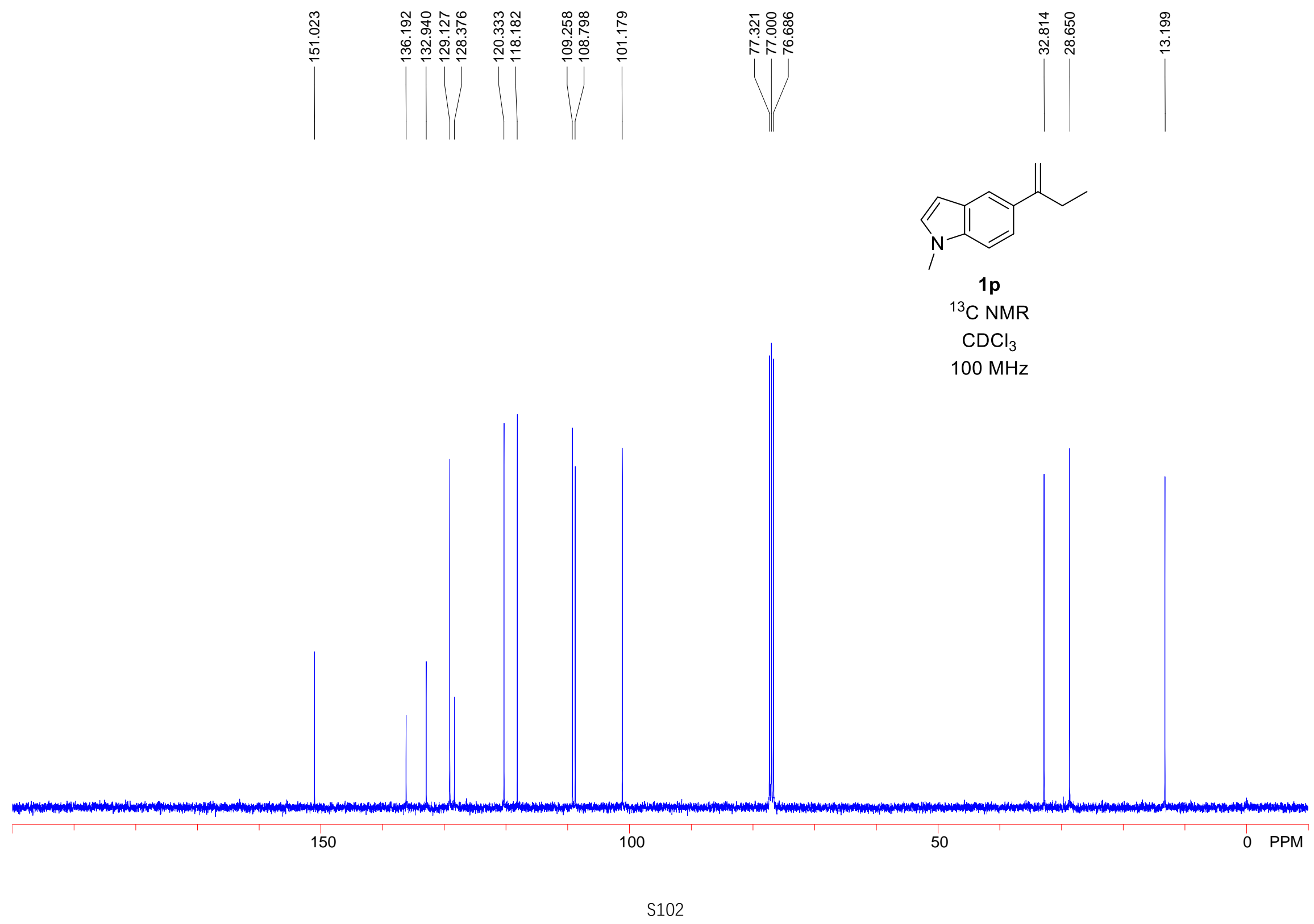




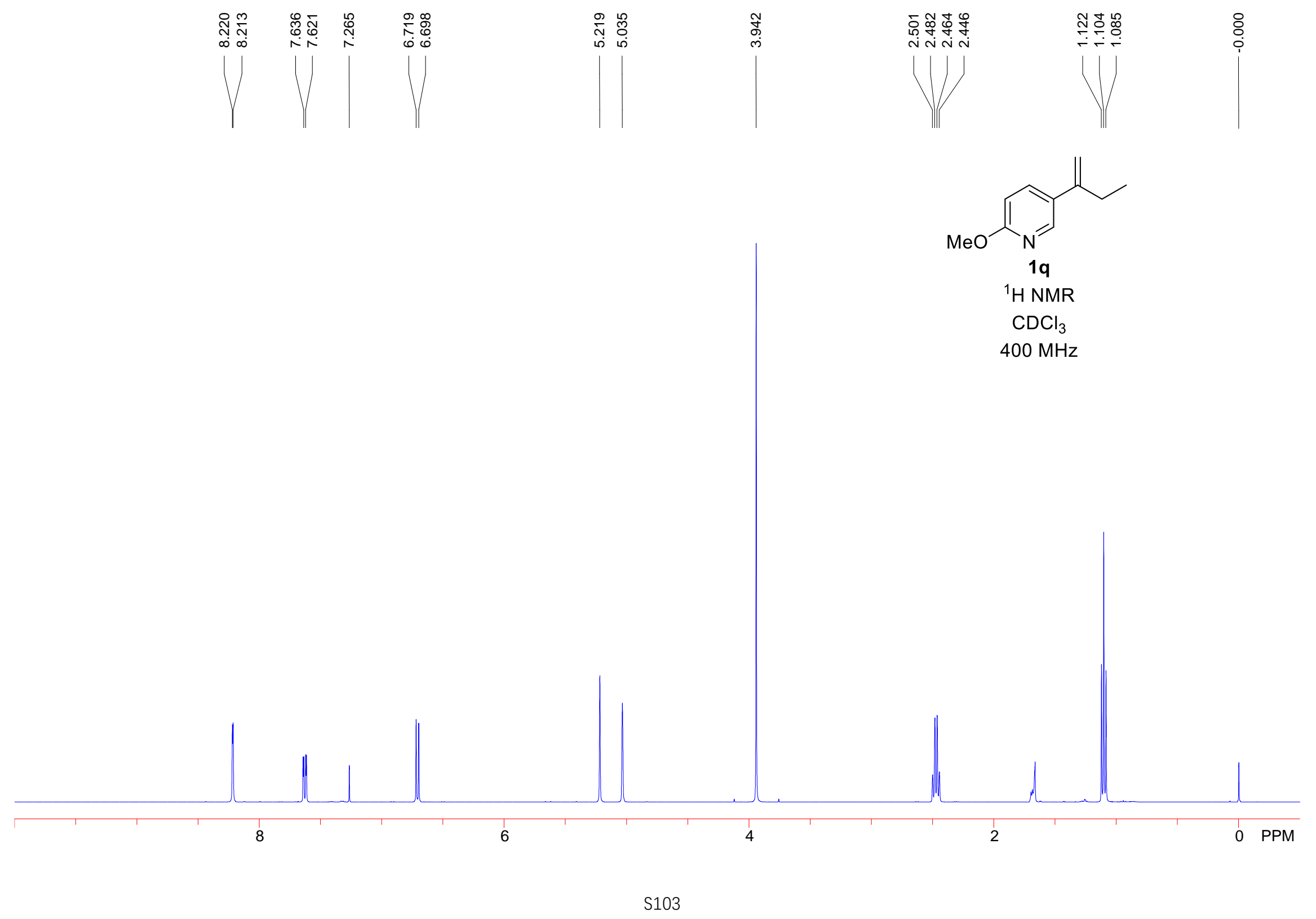




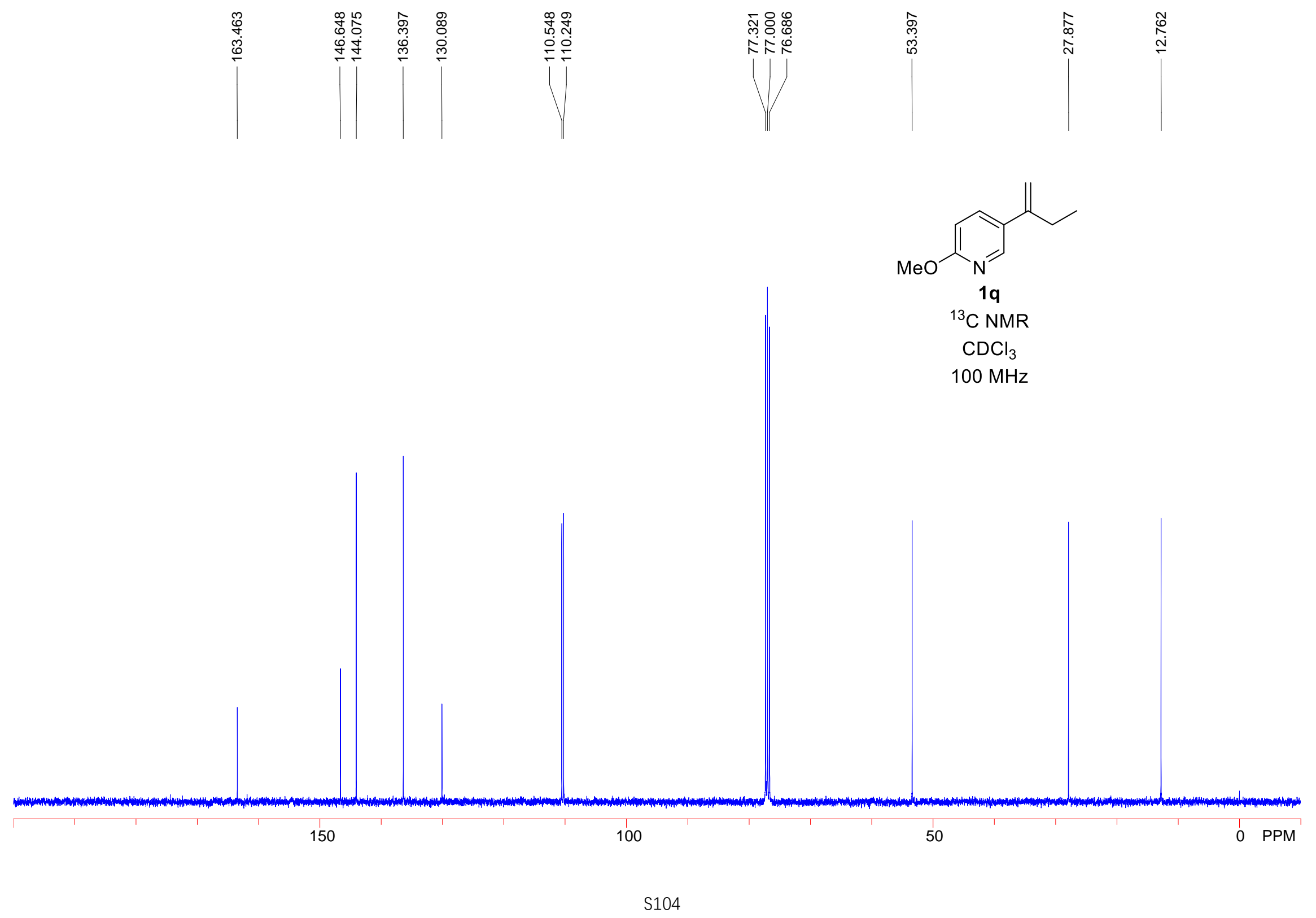




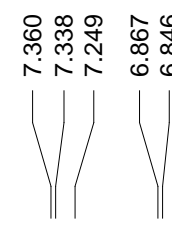

过

ఏ్

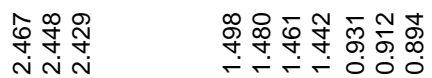

:

V
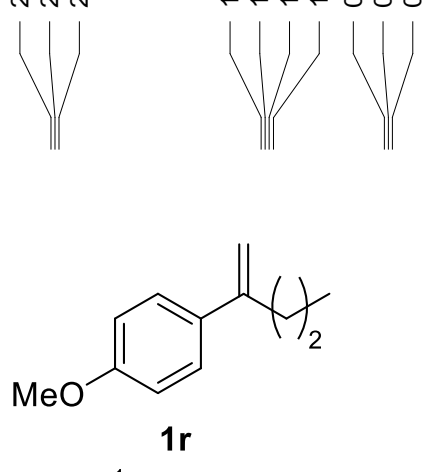

${ }^{1} \mathrm{H}$ NMR

$\mathrm{CDCl}_{3}$

$400 \mathrm{MHz}$

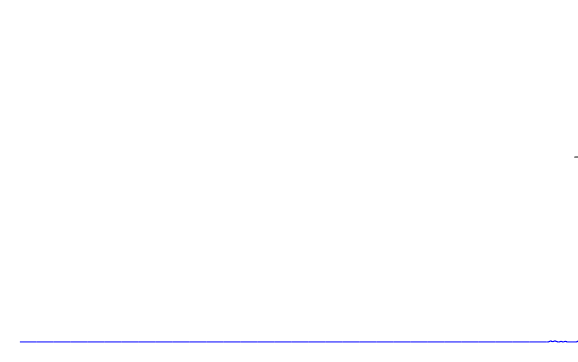

1.94 


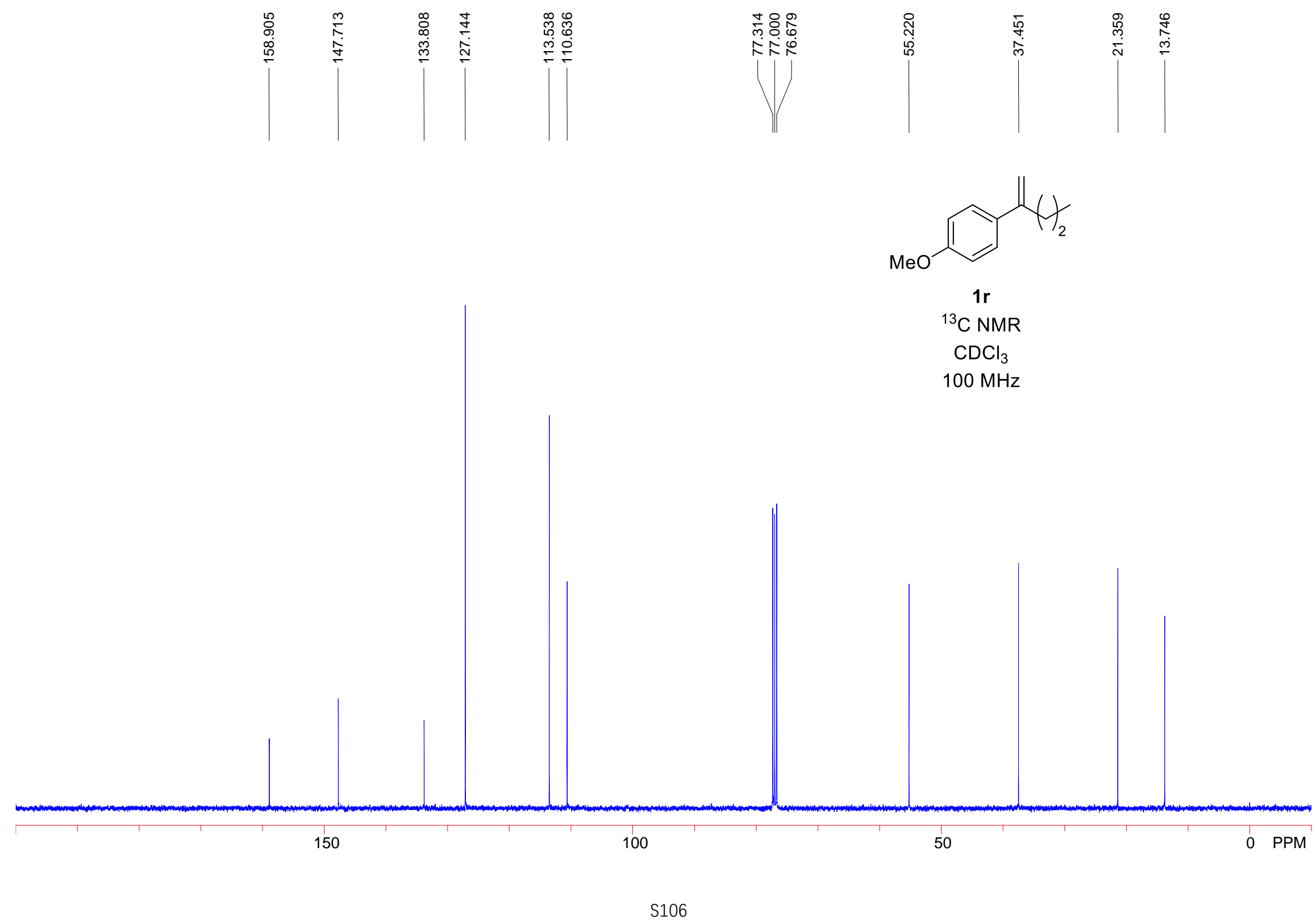




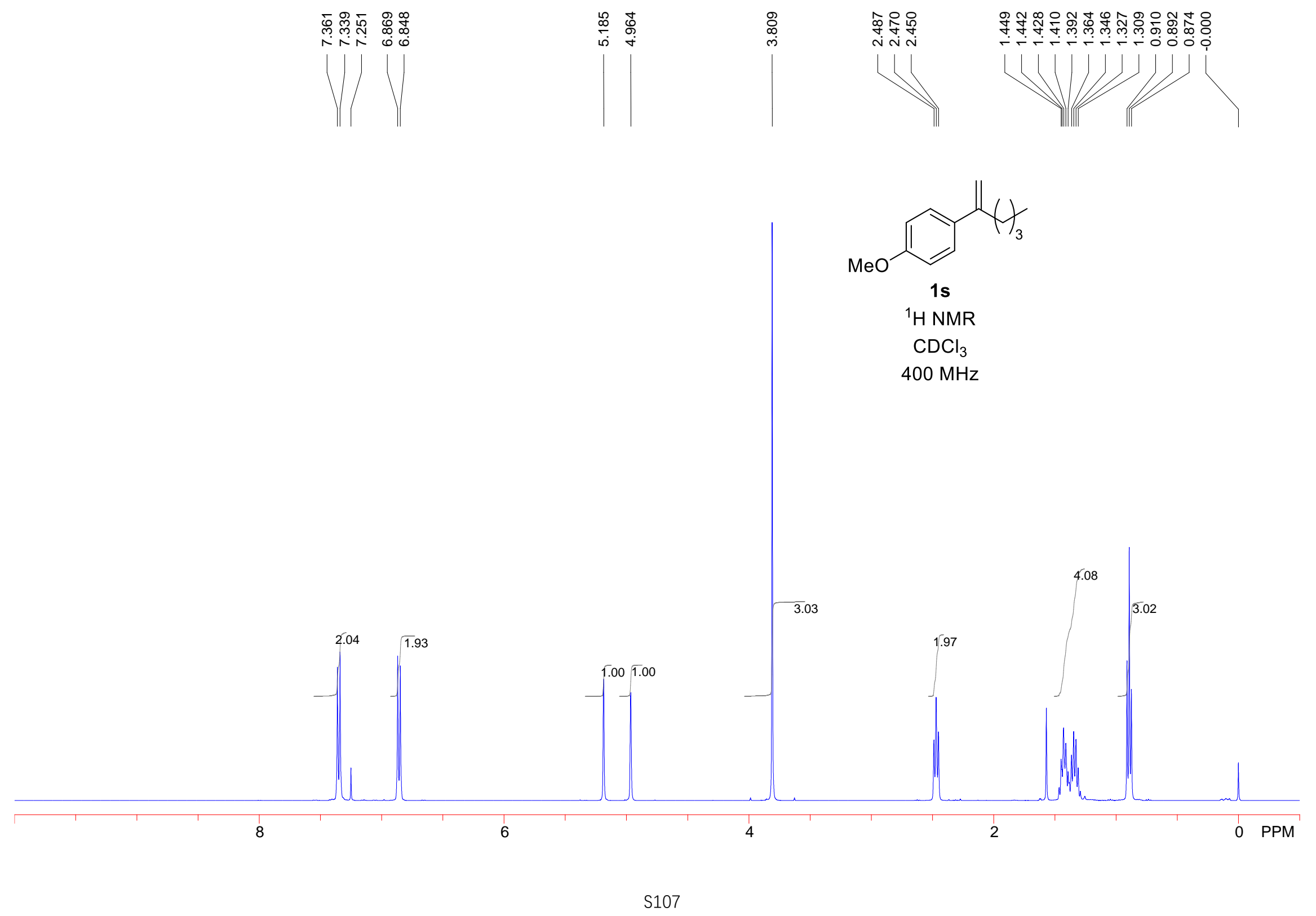




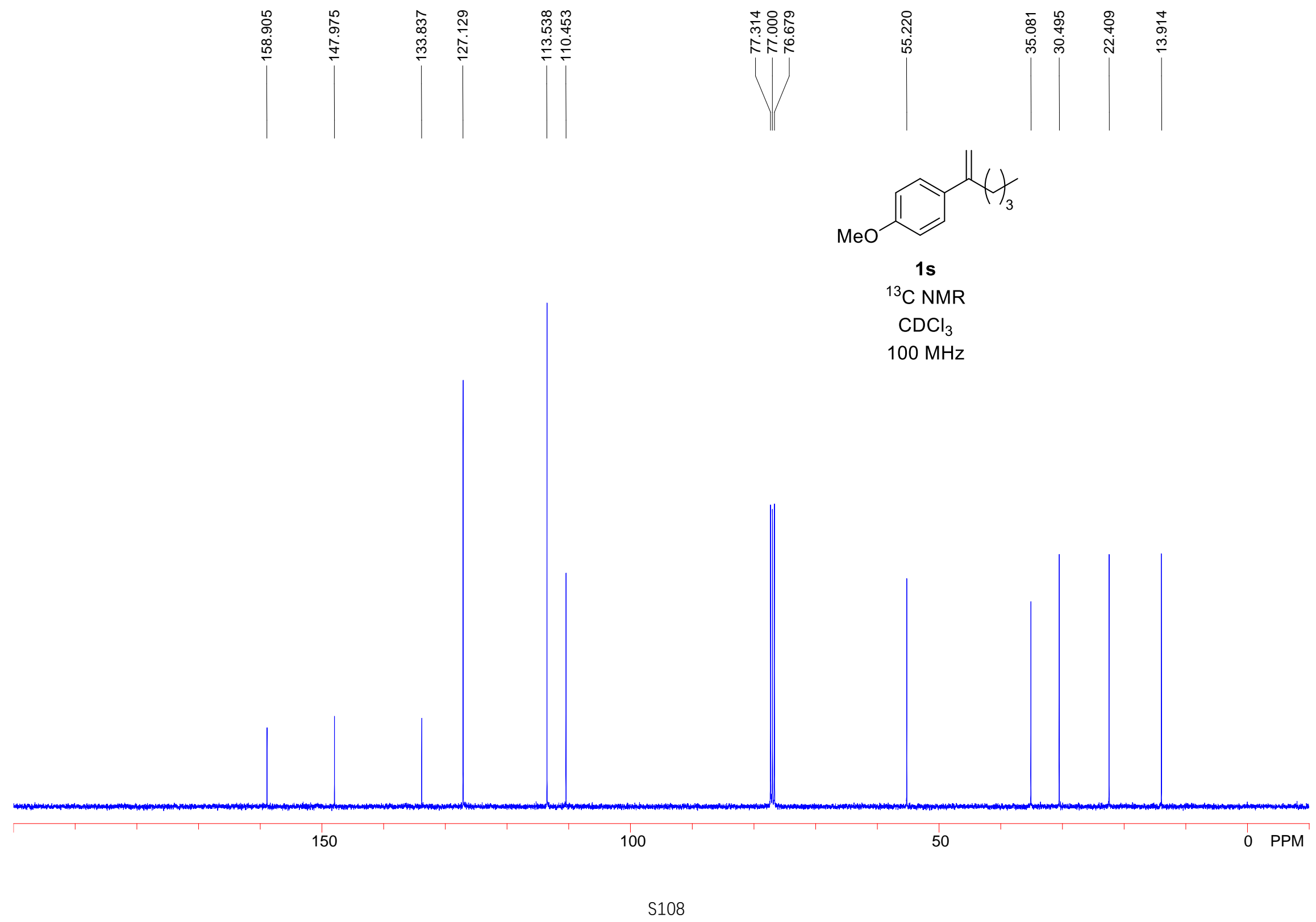




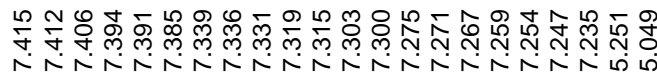

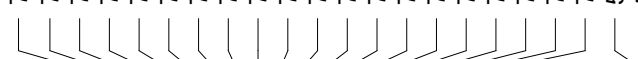

nlí
造哭

กั่ง

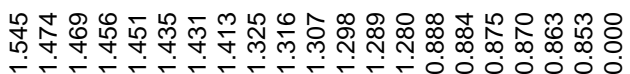

L

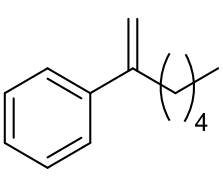

$1 \mathrm{t}$

${ }^{1} \mathrm{H}$ NMR

$\mathrm{CDCl}_{3}$

$400 \mathrm{MHz}$

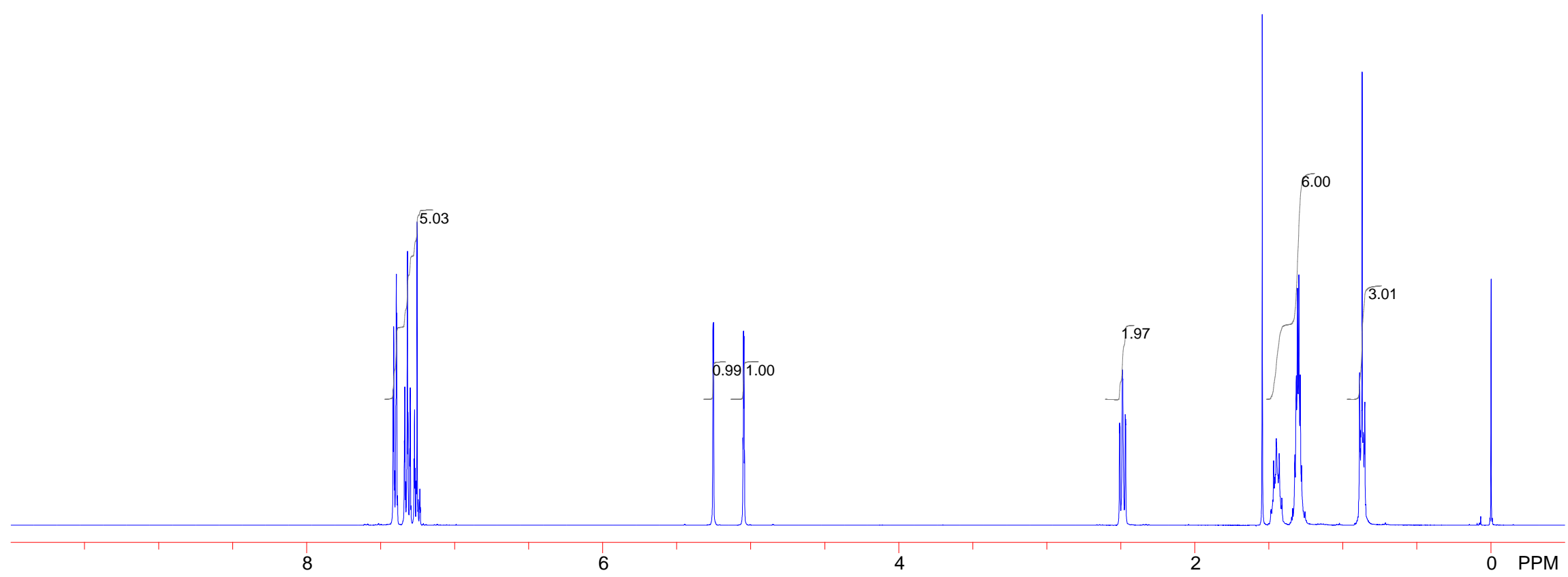




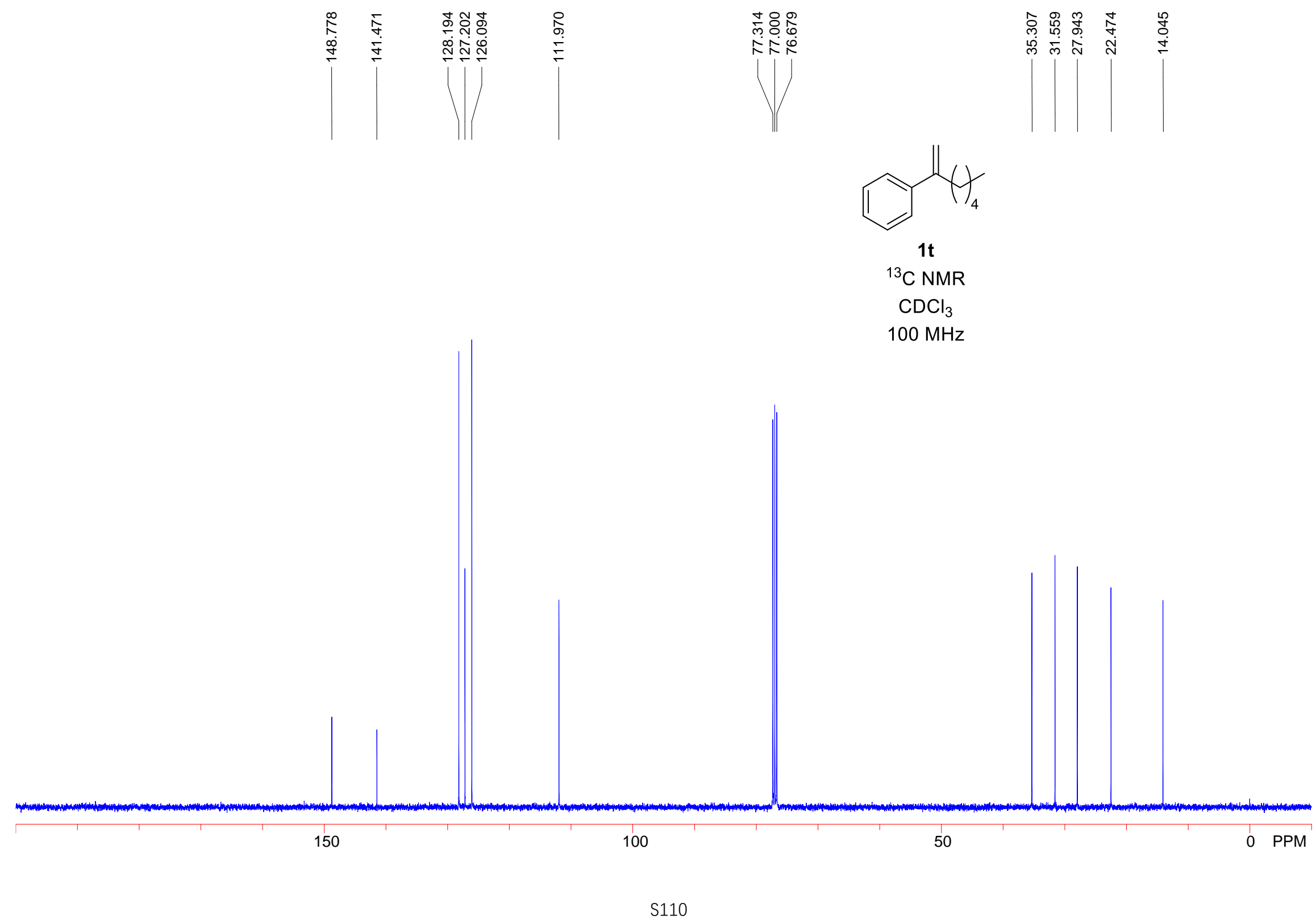




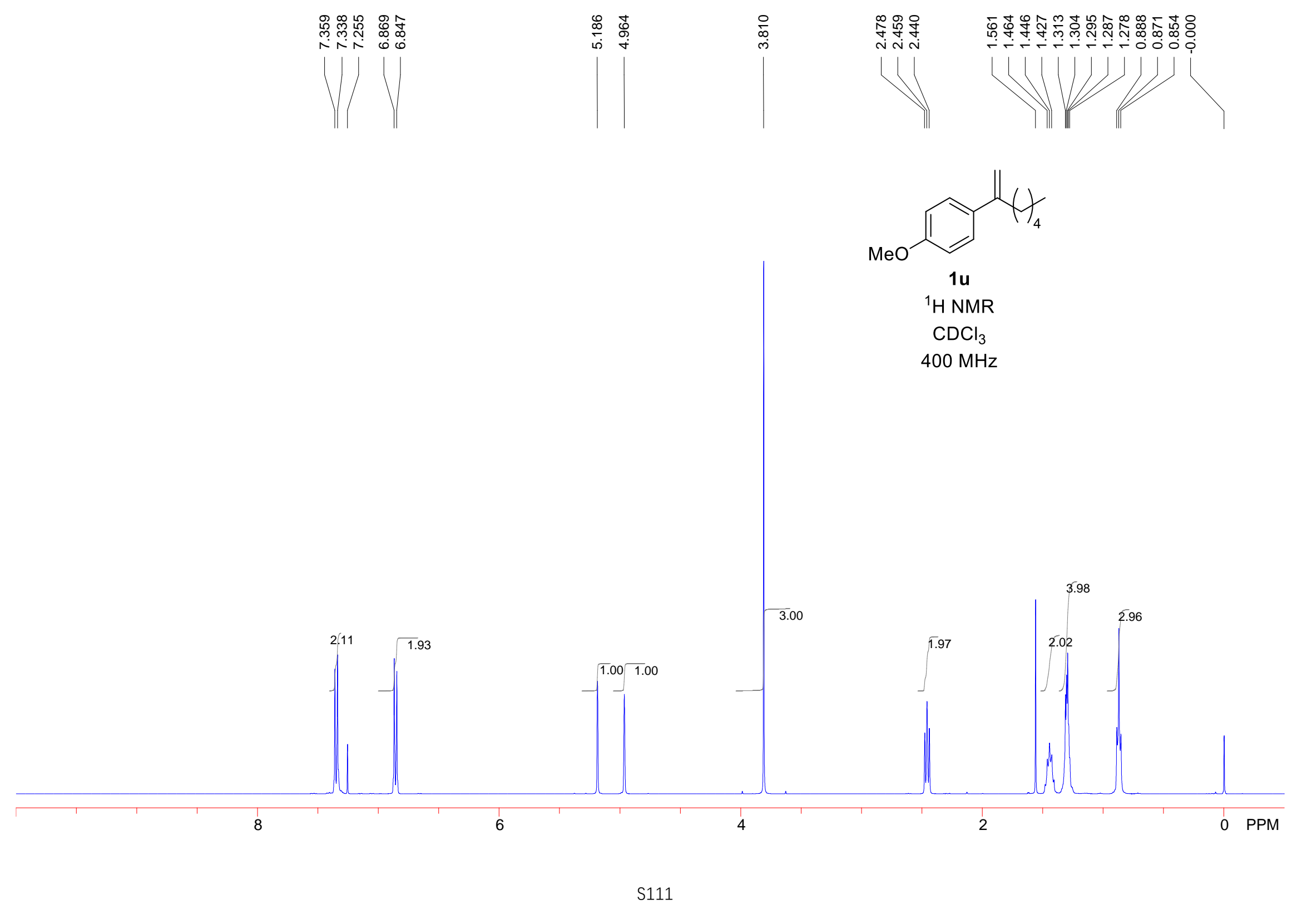



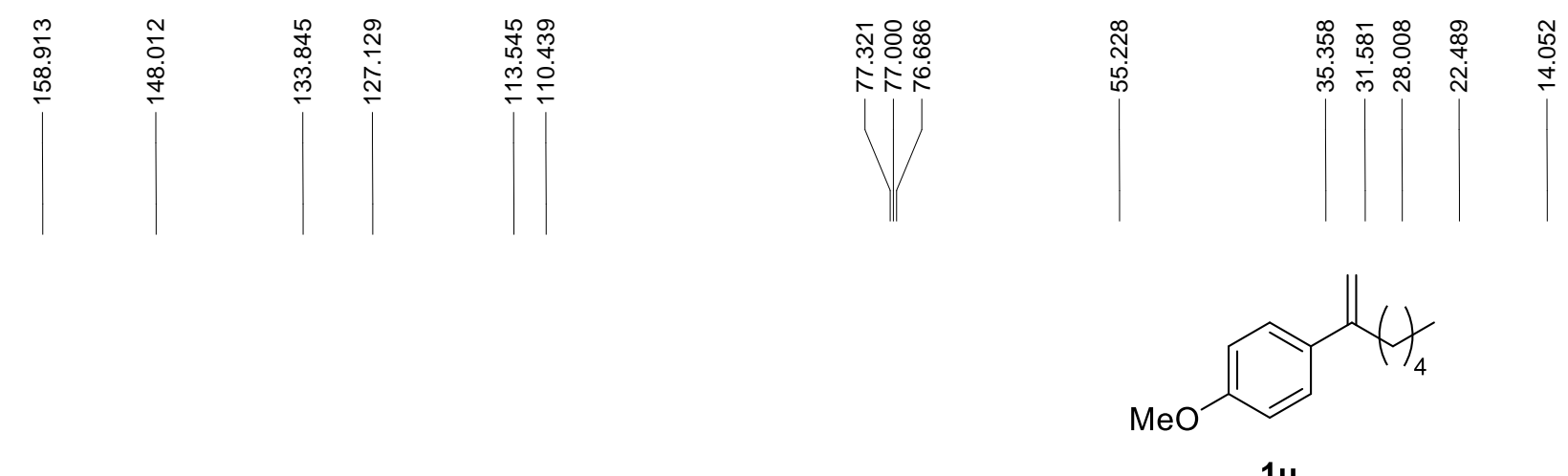

${ }^{13} \mathrm{C} \mathrm{NMR}$
$\mathrm{CDCl}_{3}$
$100 \mathrm{MHz}$

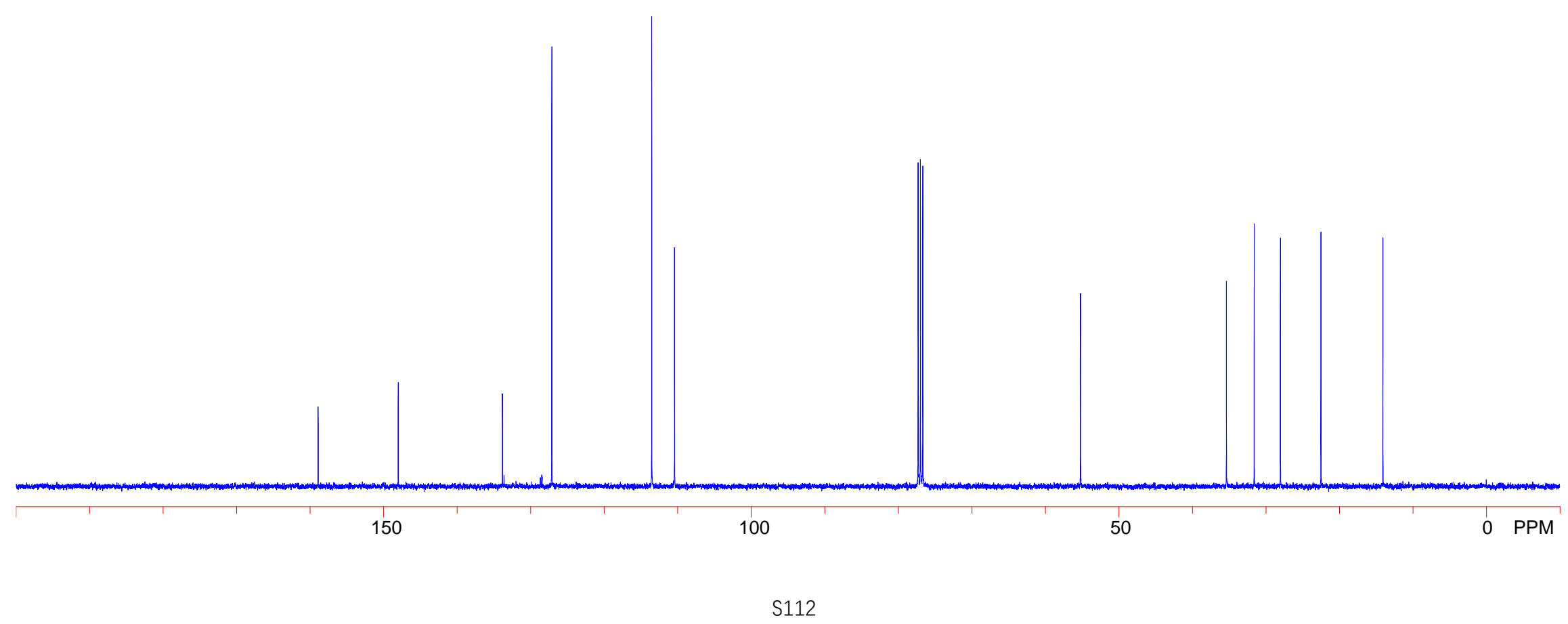




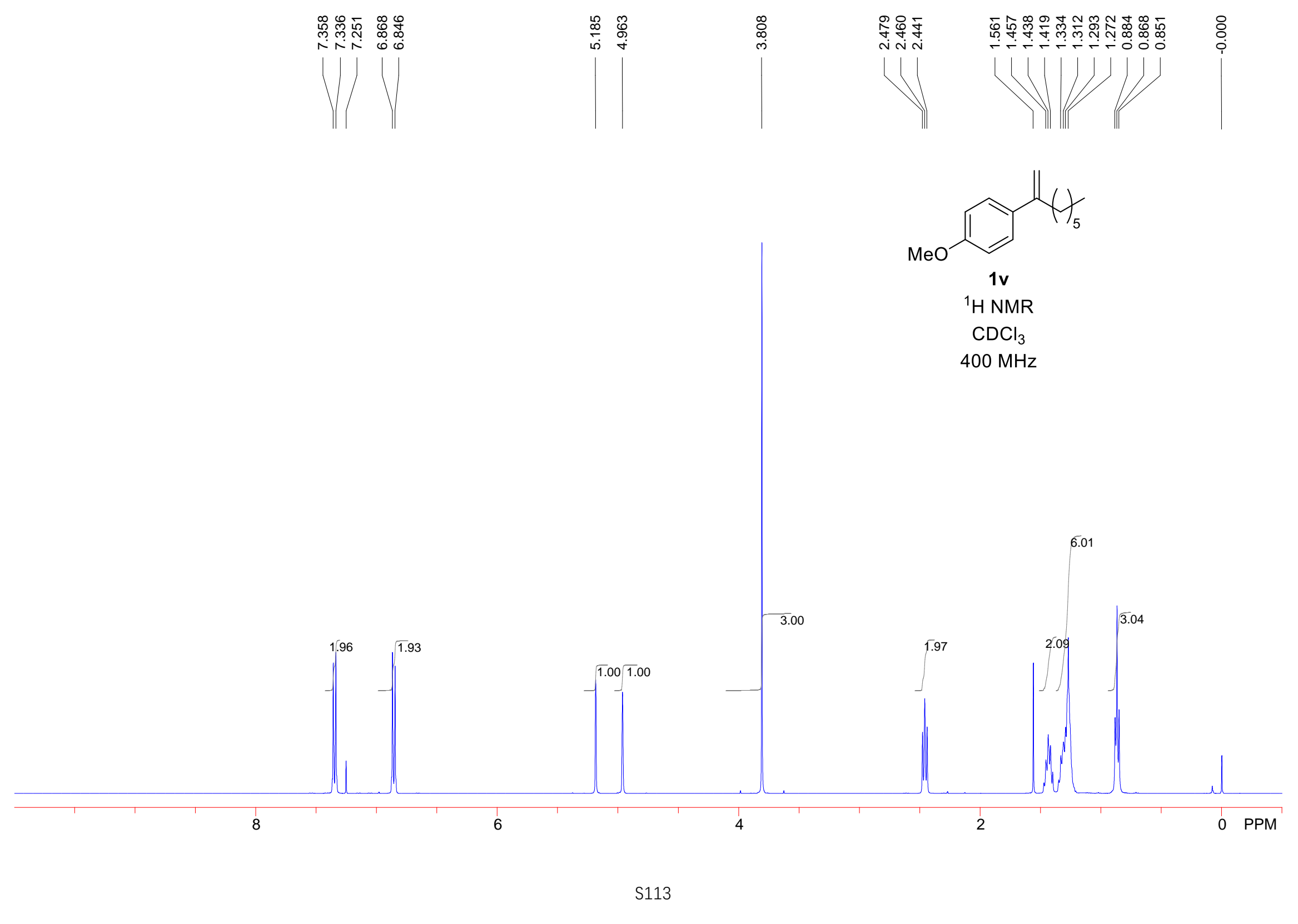




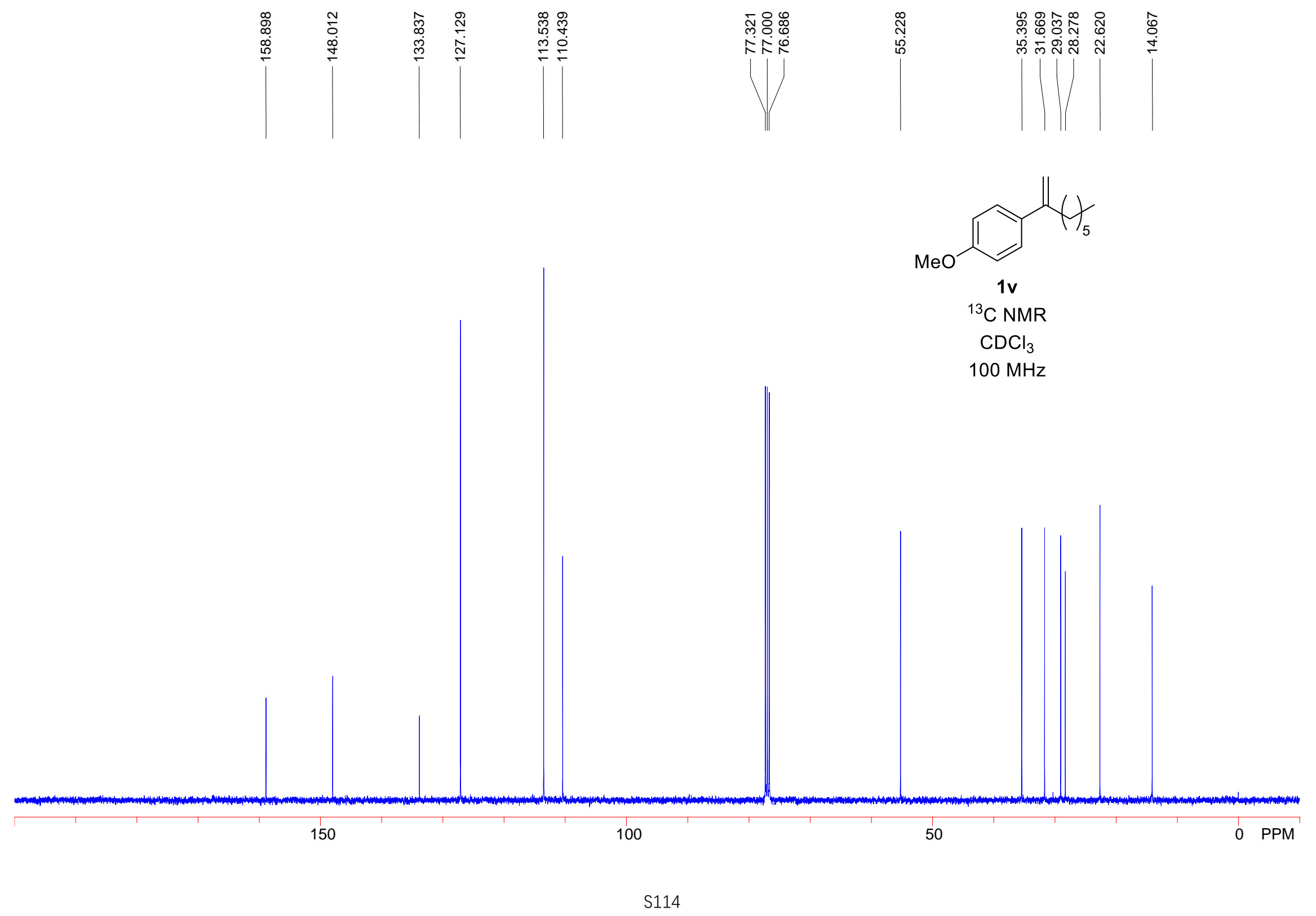




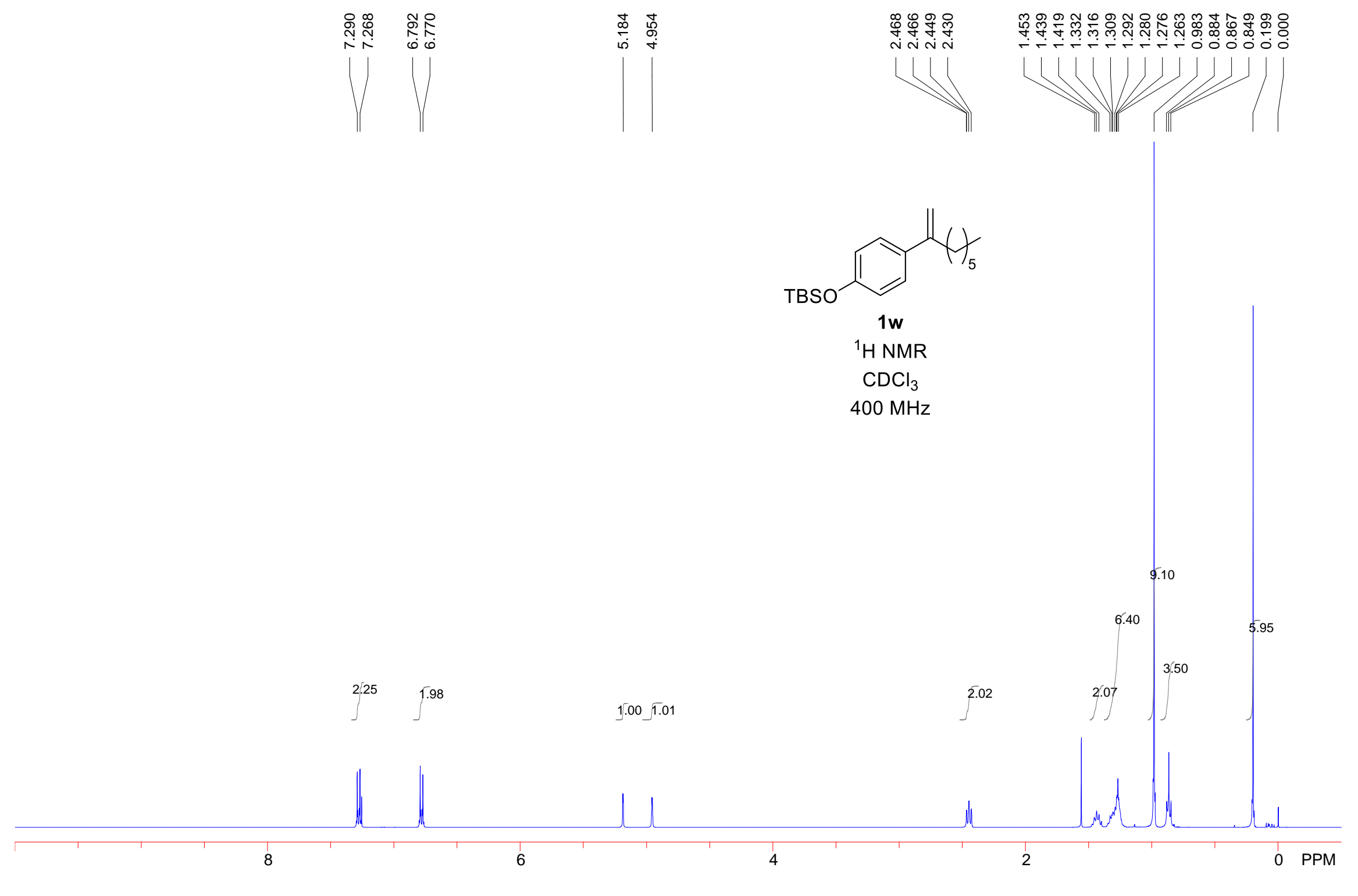




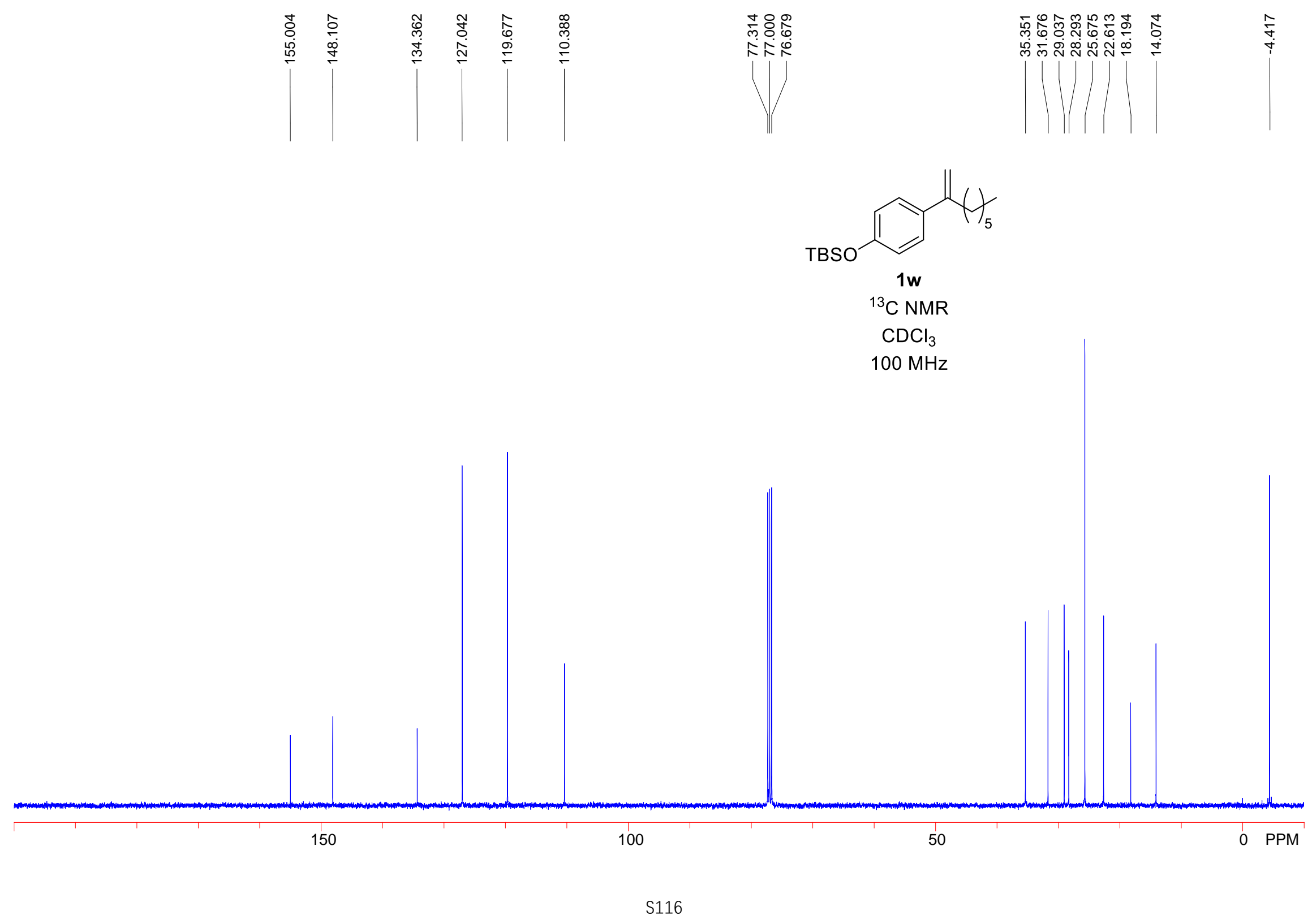




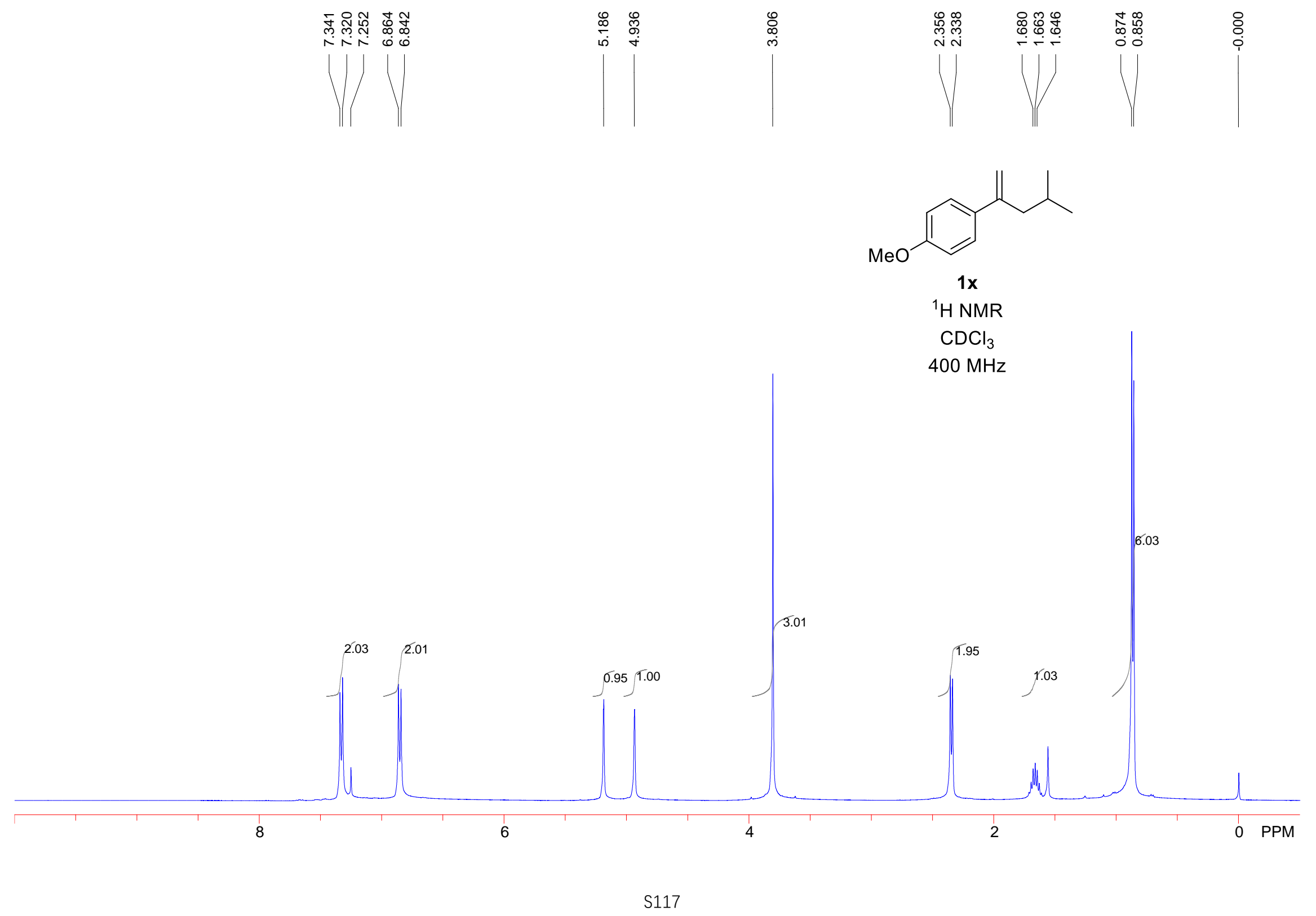




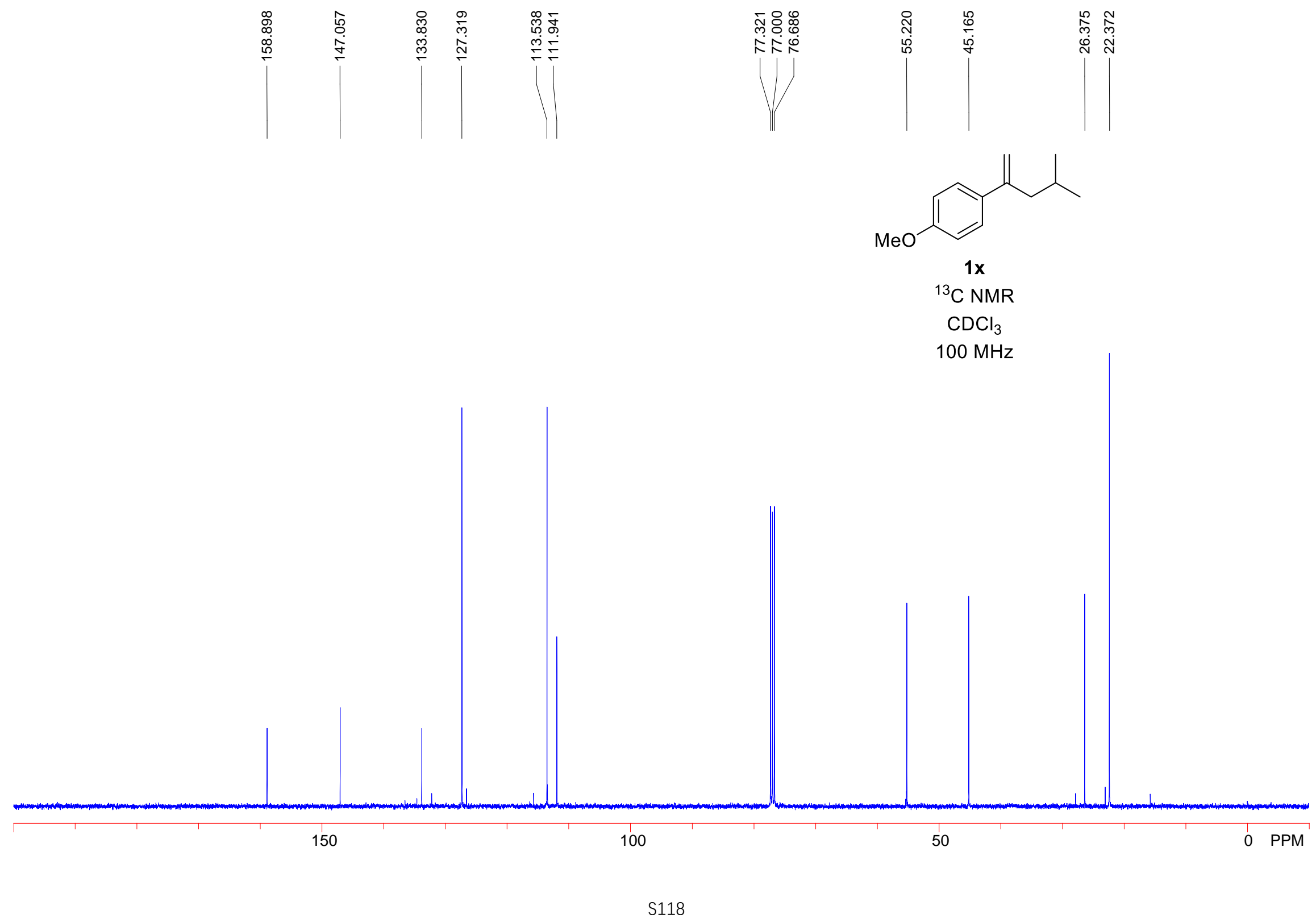




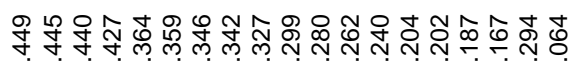

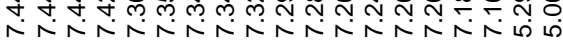

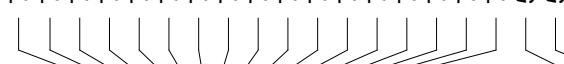
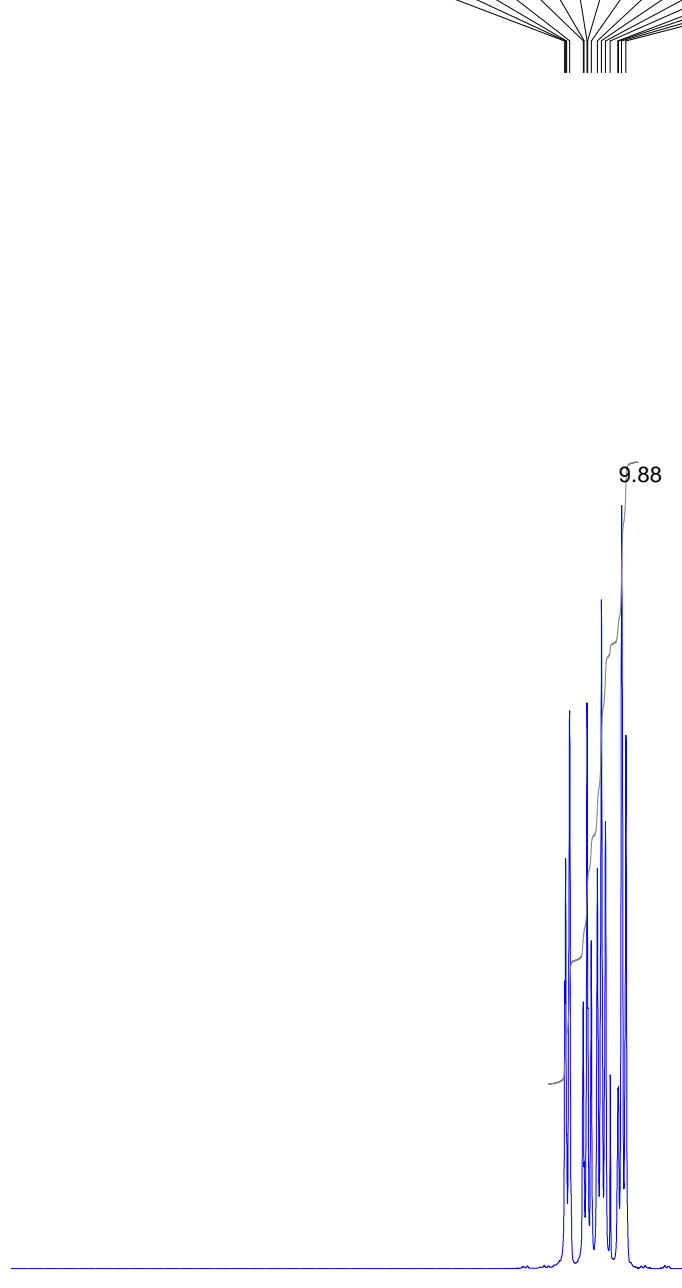

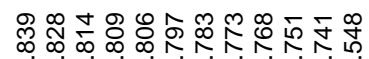

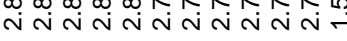

:

L

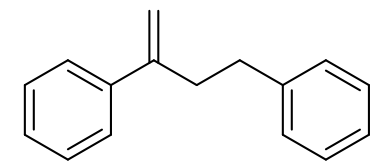

$1 y$

${ }^{1} \mathrm{H}$ NMR

$\mathrm{CDCl}_{3}$

$400 \mathrm{MHz}$

1.011 .00

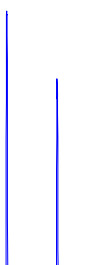




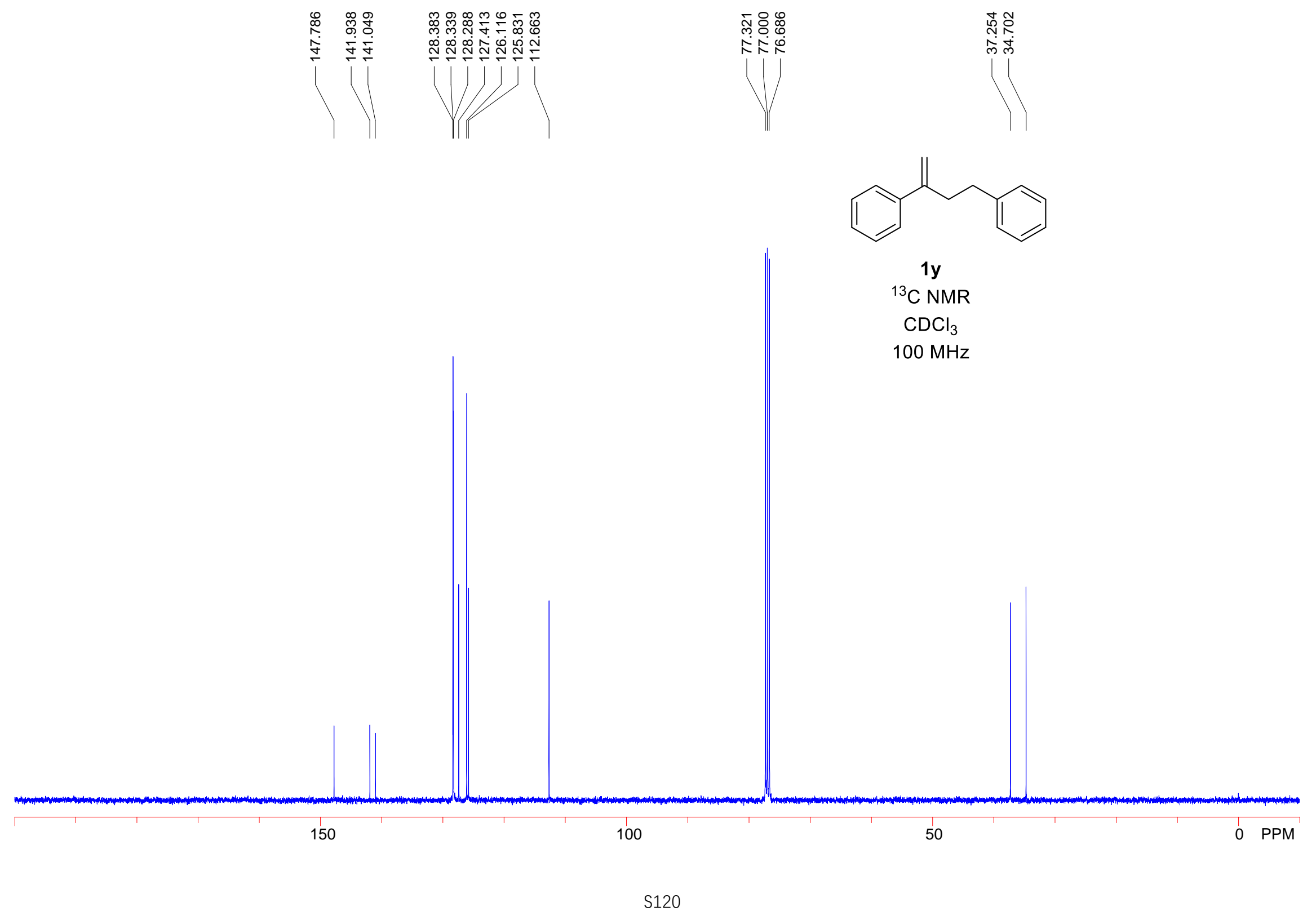




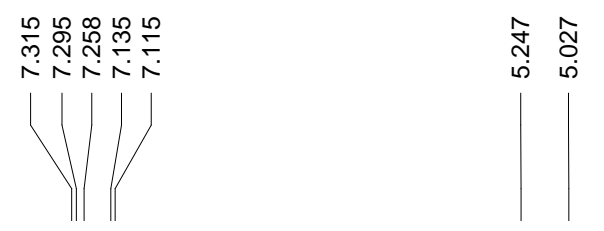

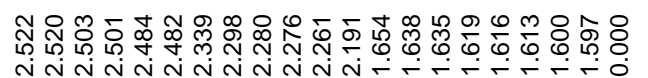

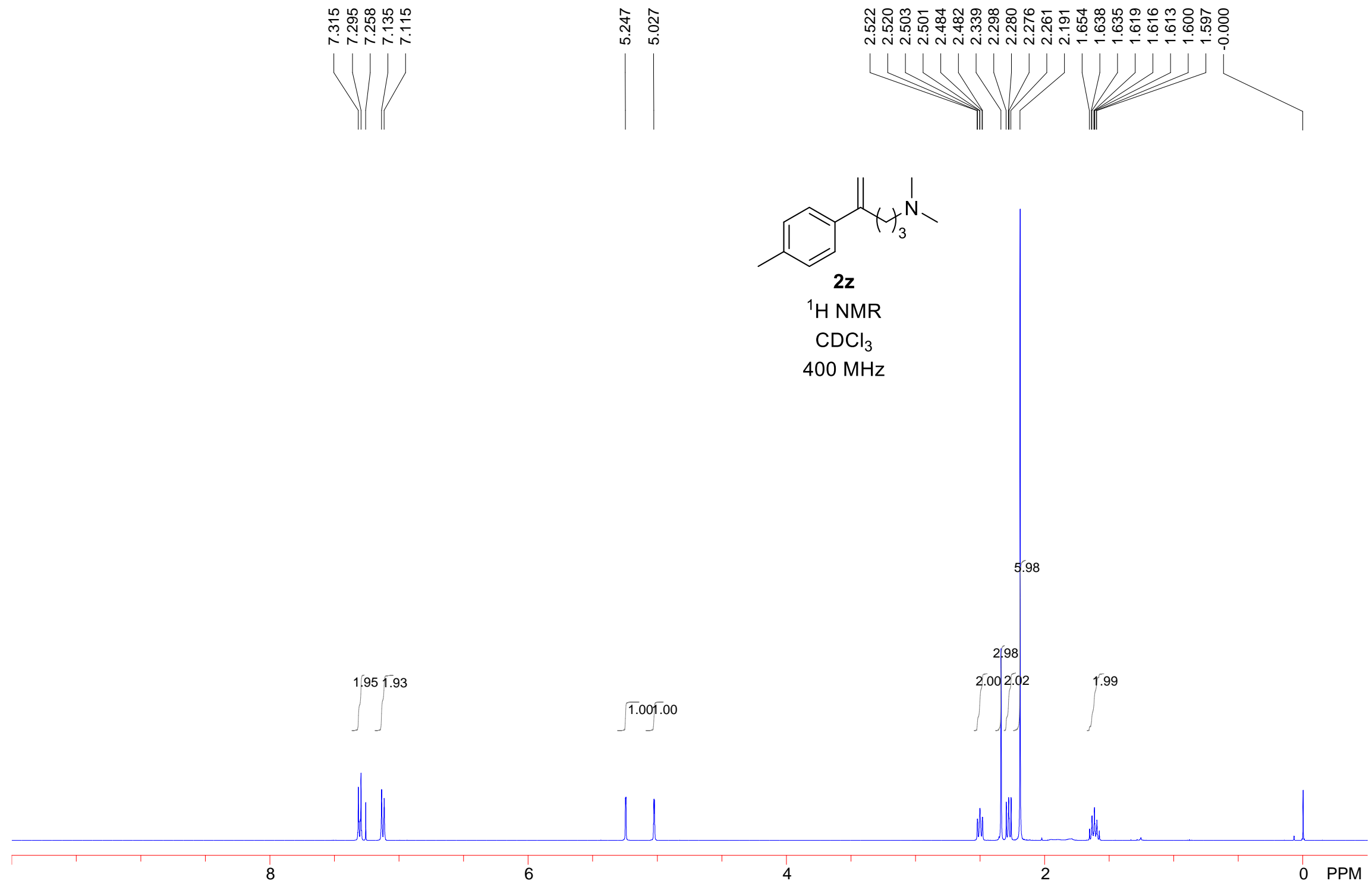




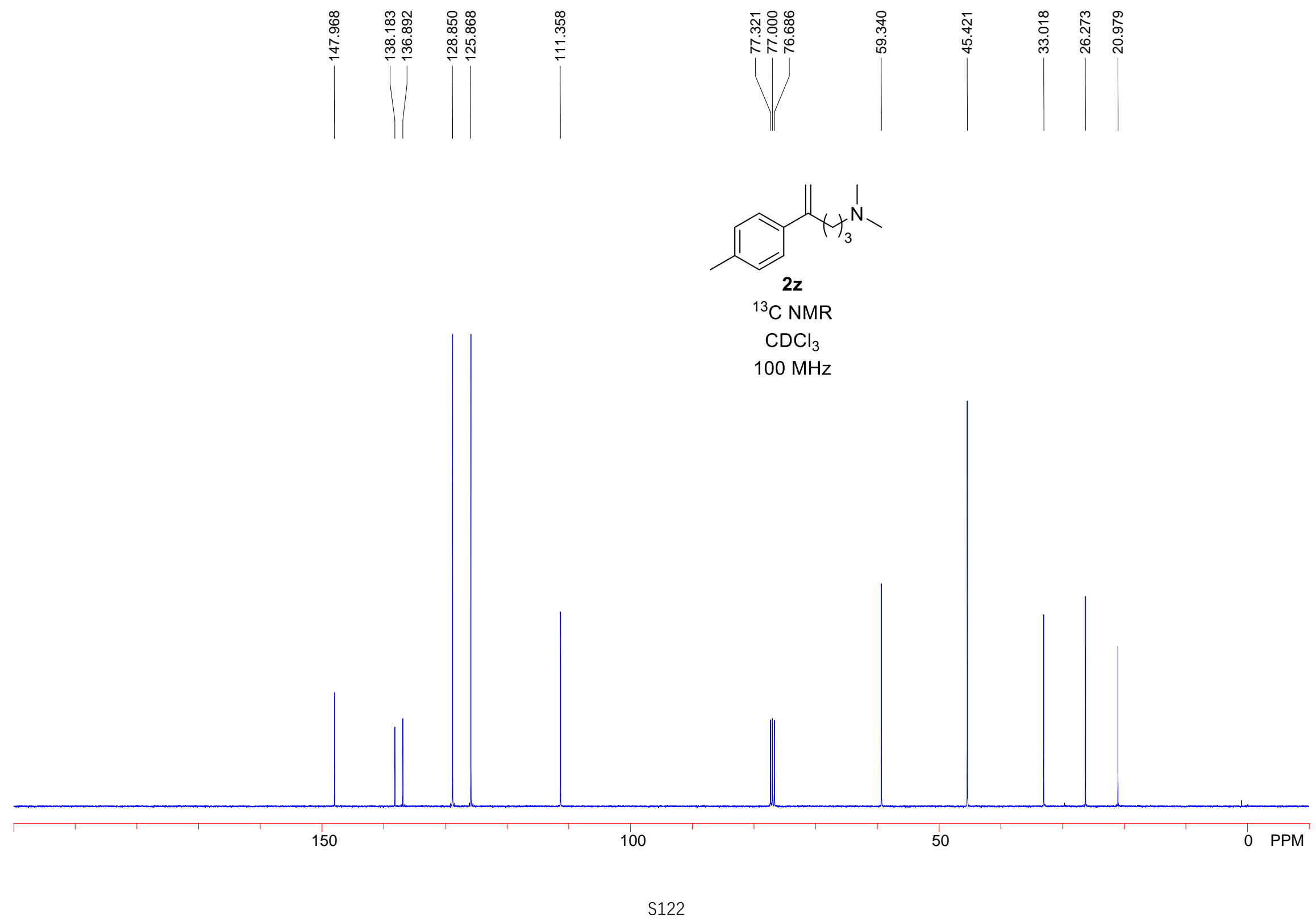




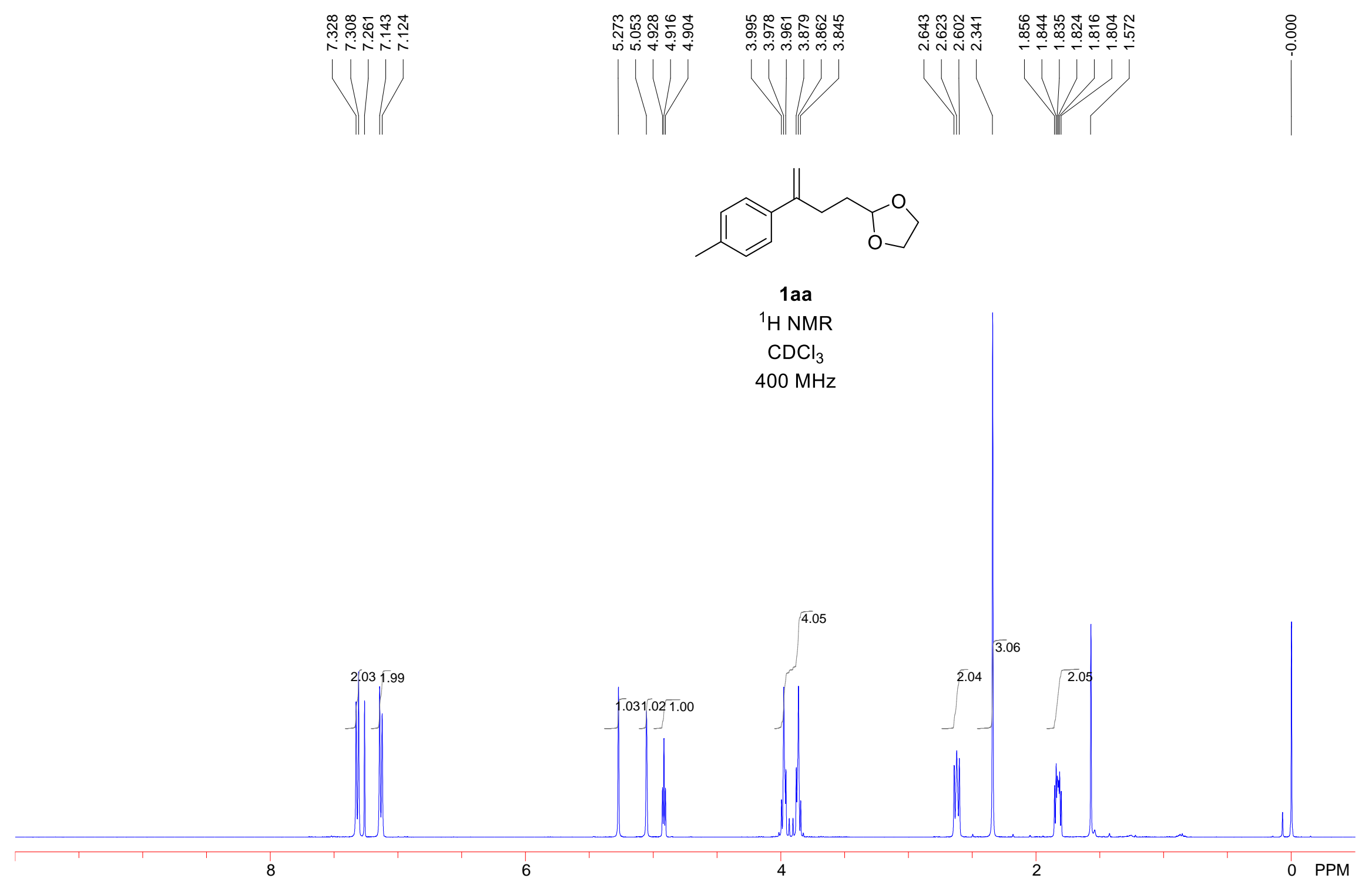




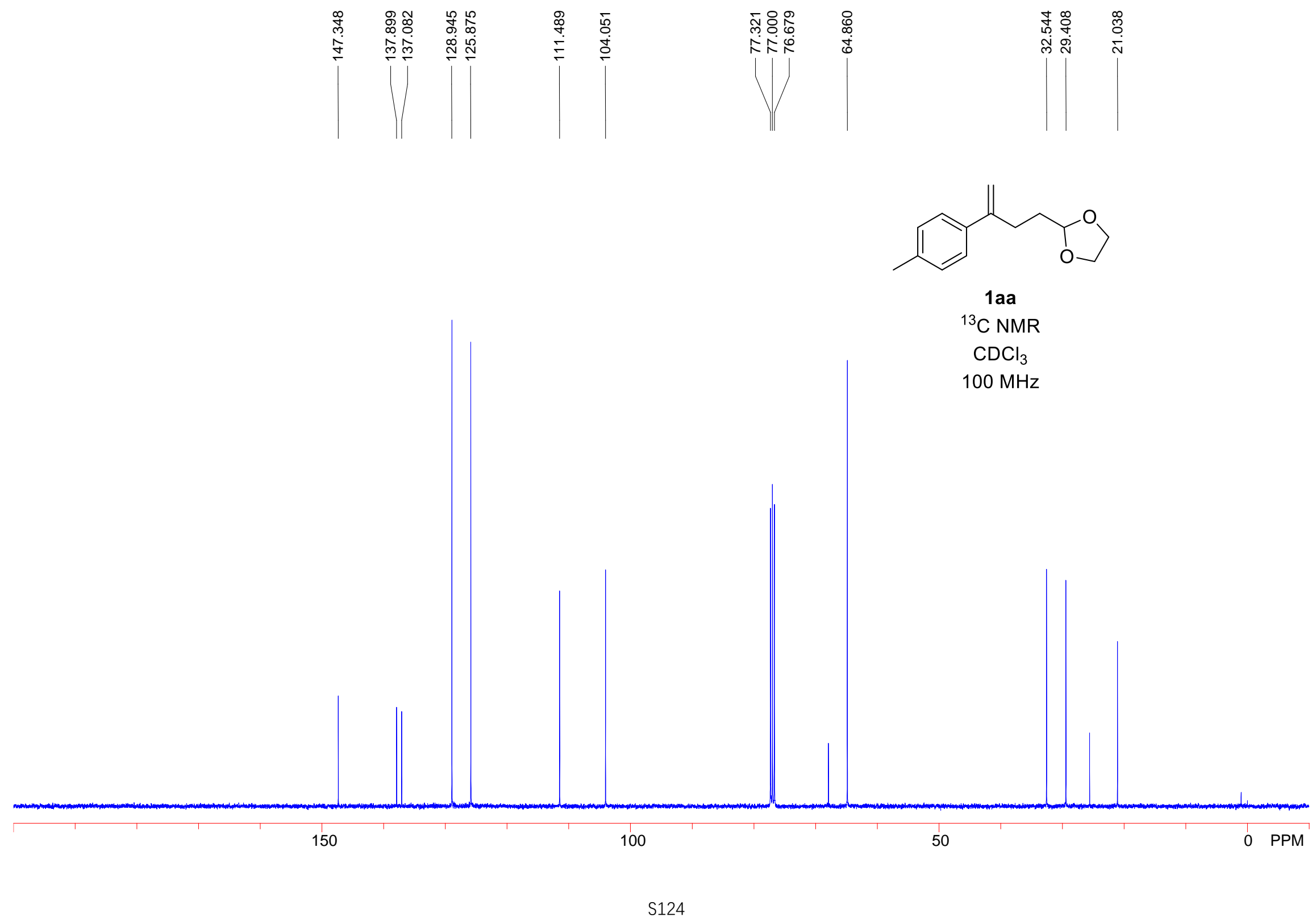




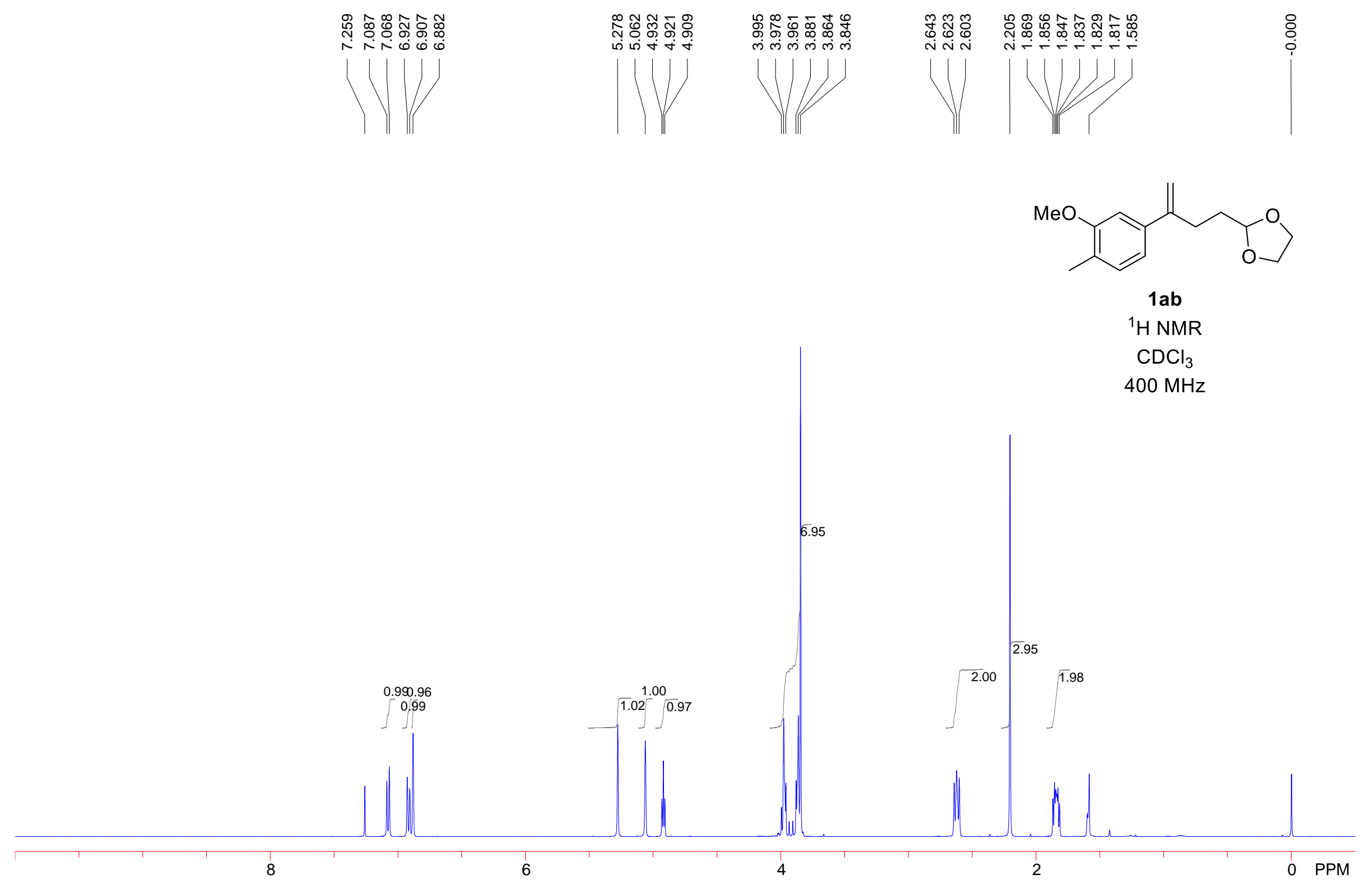




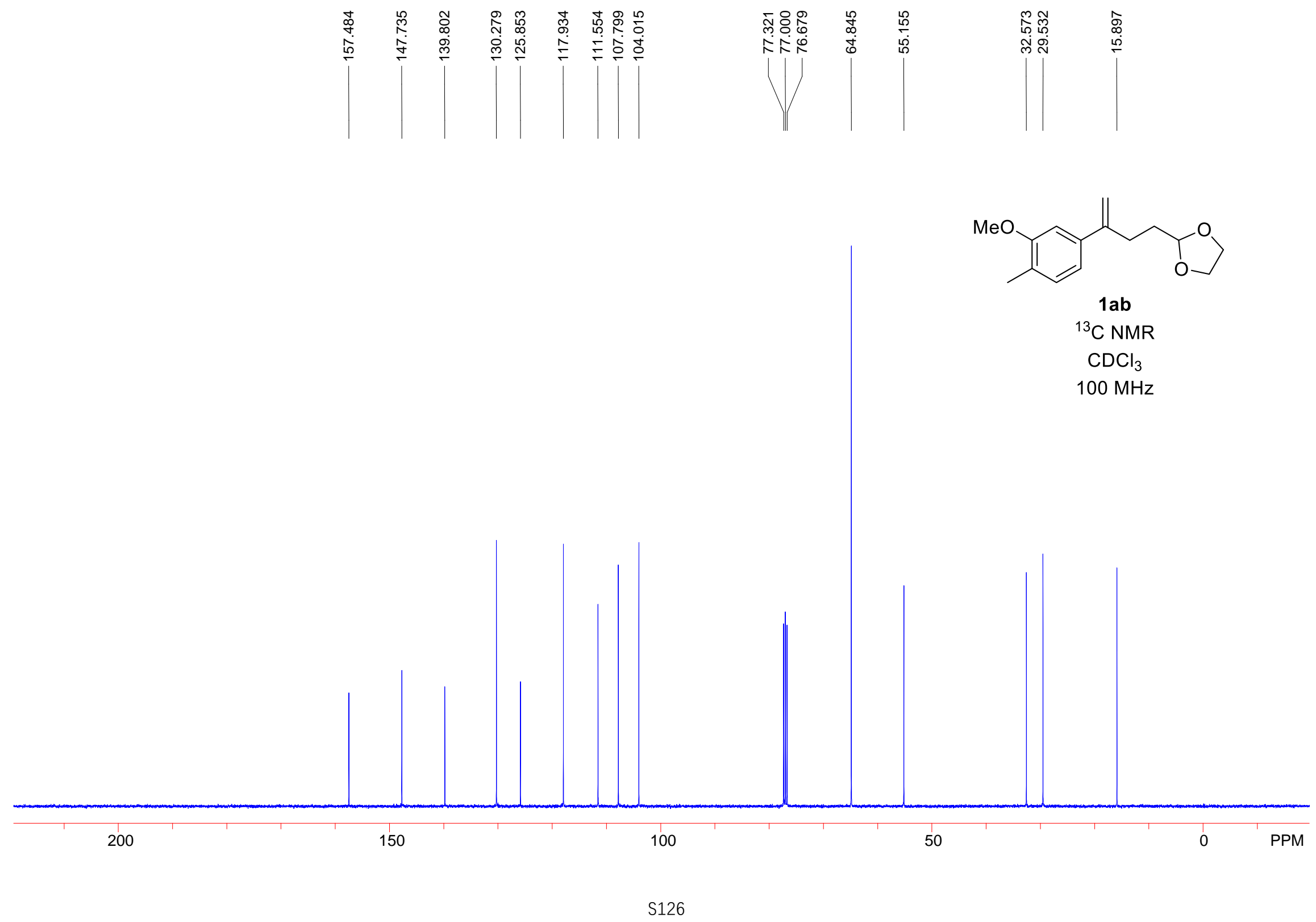




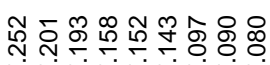

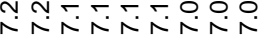

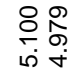

(U)」JJ

MI

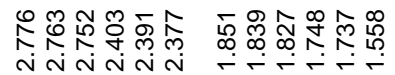
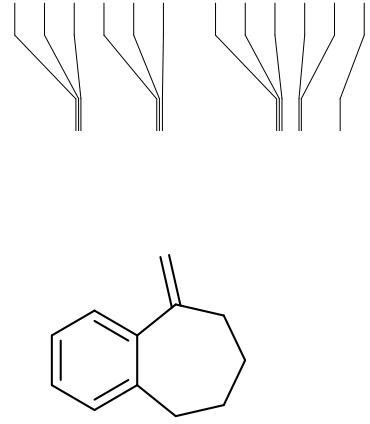

1 ac

${ }^{1} \mathrm{H}$ NMR

$\mathrm{CDCl}_{3}$

$400 \mathrm{MHz}$

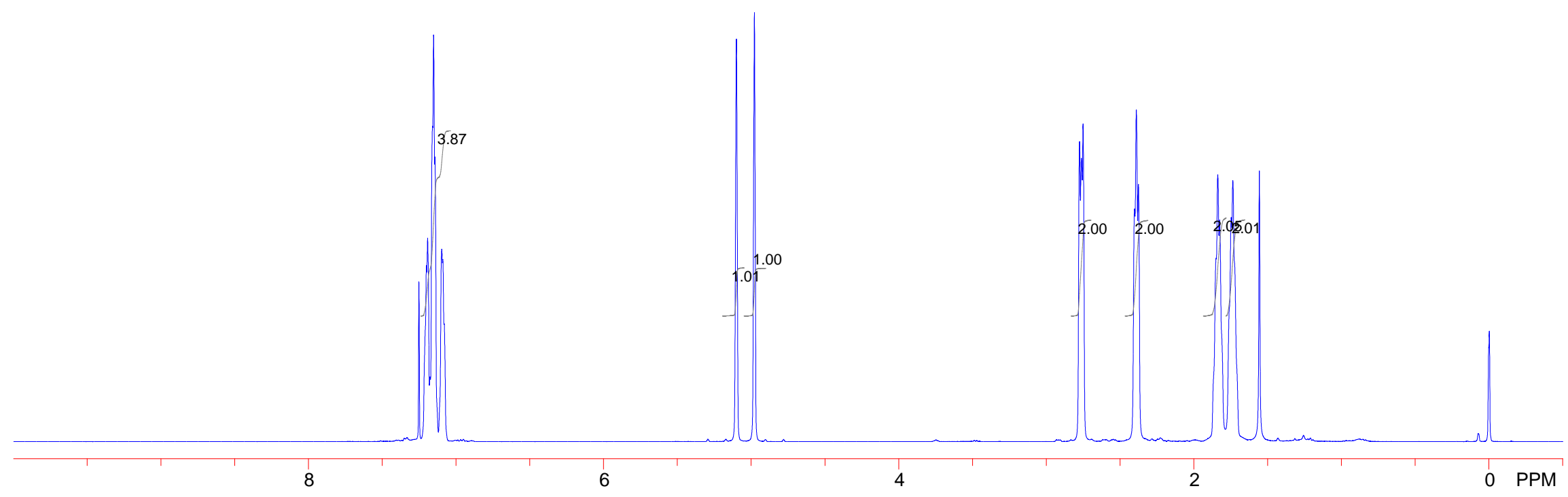




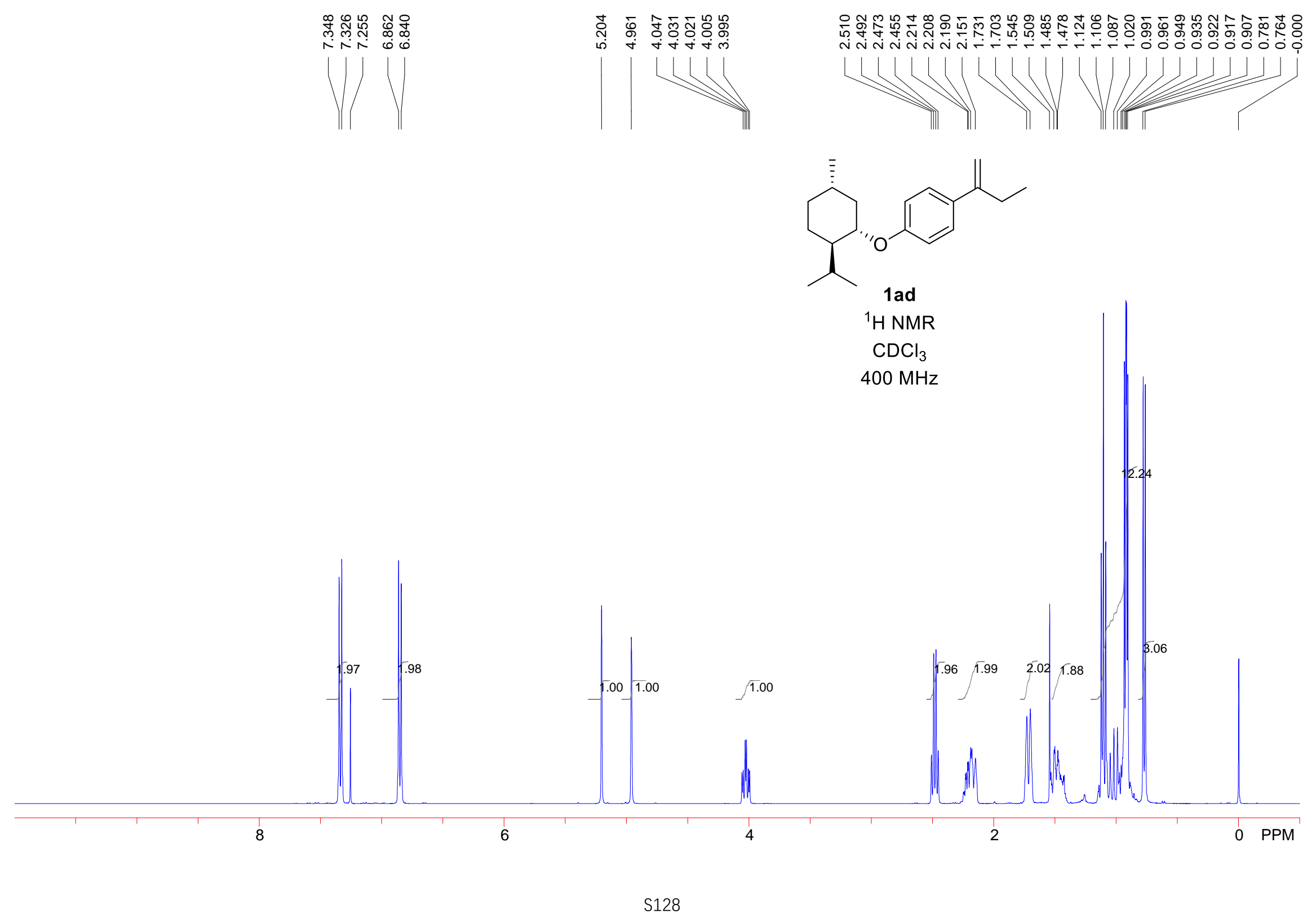




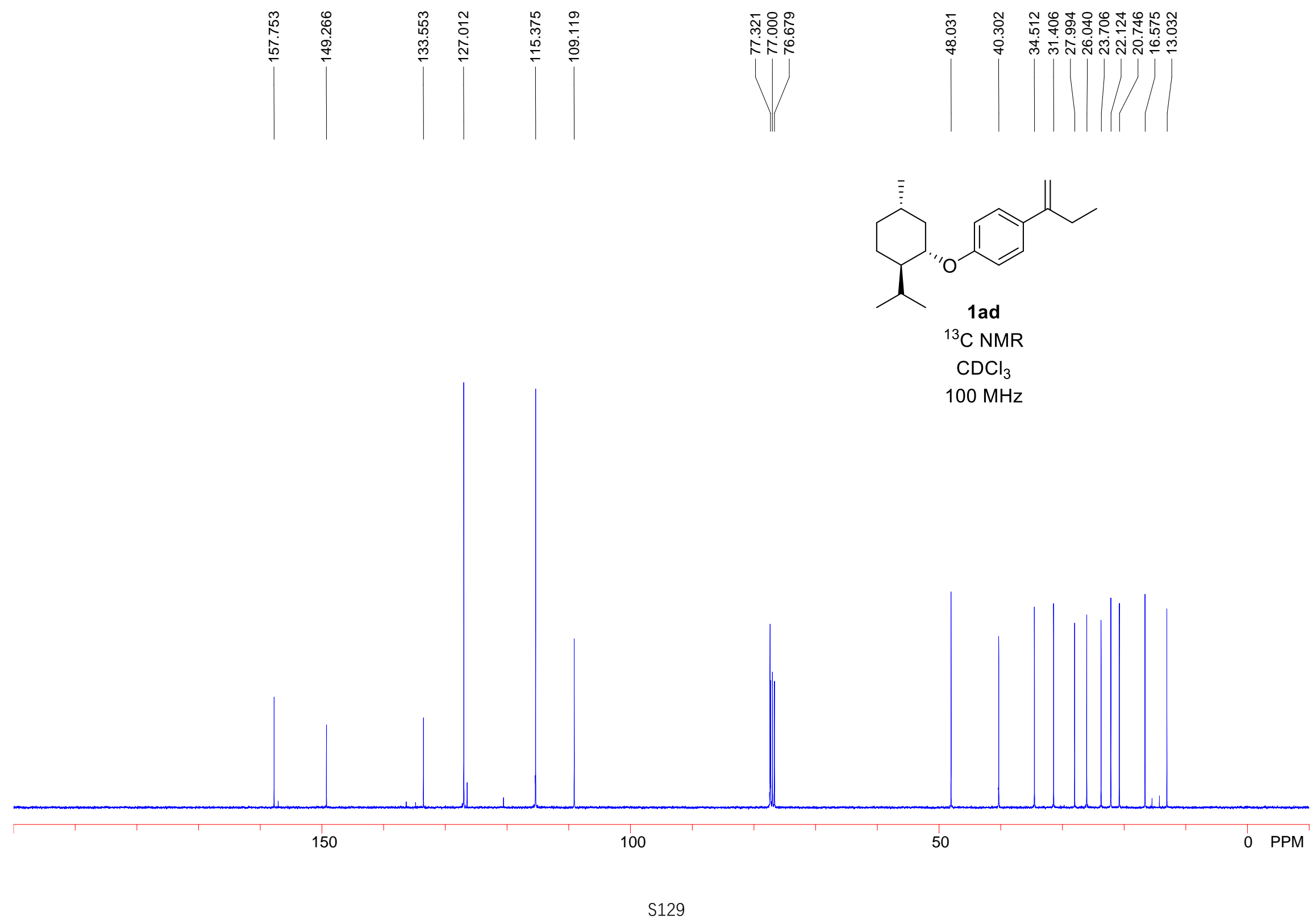




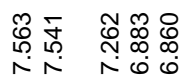

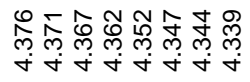

H

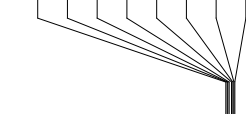

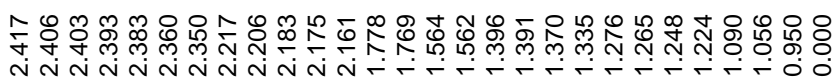
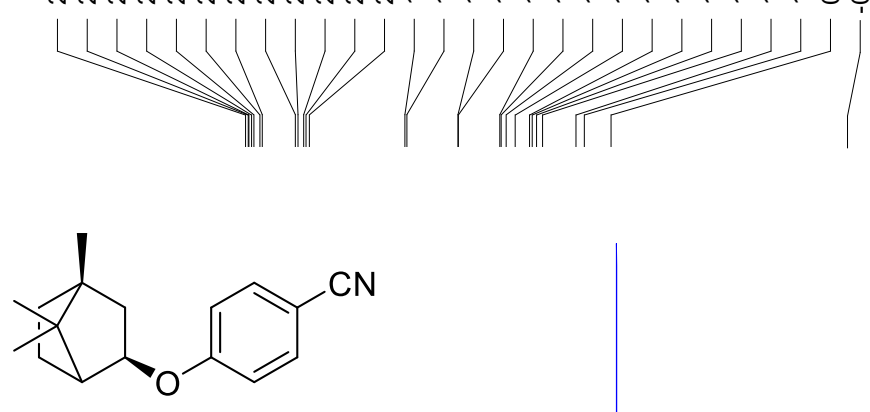

1ae-s1

${ }^{1} \mathrm{H}$ NMR

$\mathrm{CDCl}_{3}$

$400 \mathrm{MHz}$

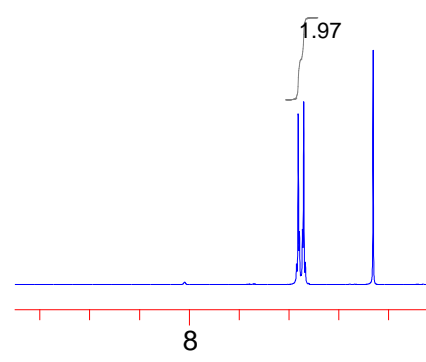

2.02
1.00
1.031 .02

2.10 $1.06 \quad 1.09$

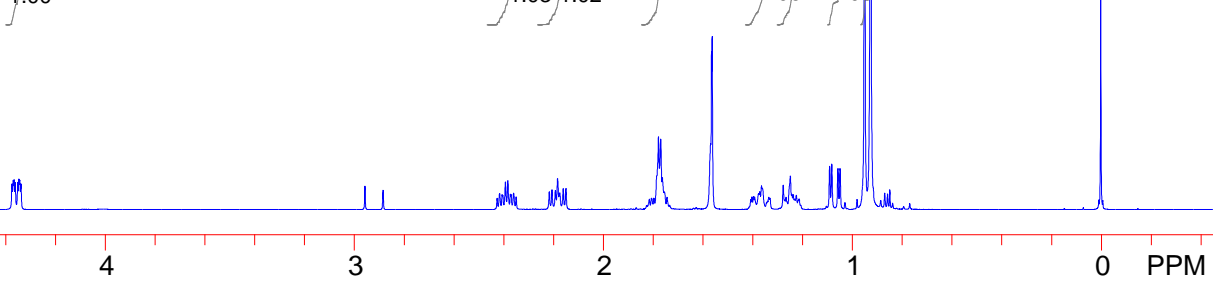




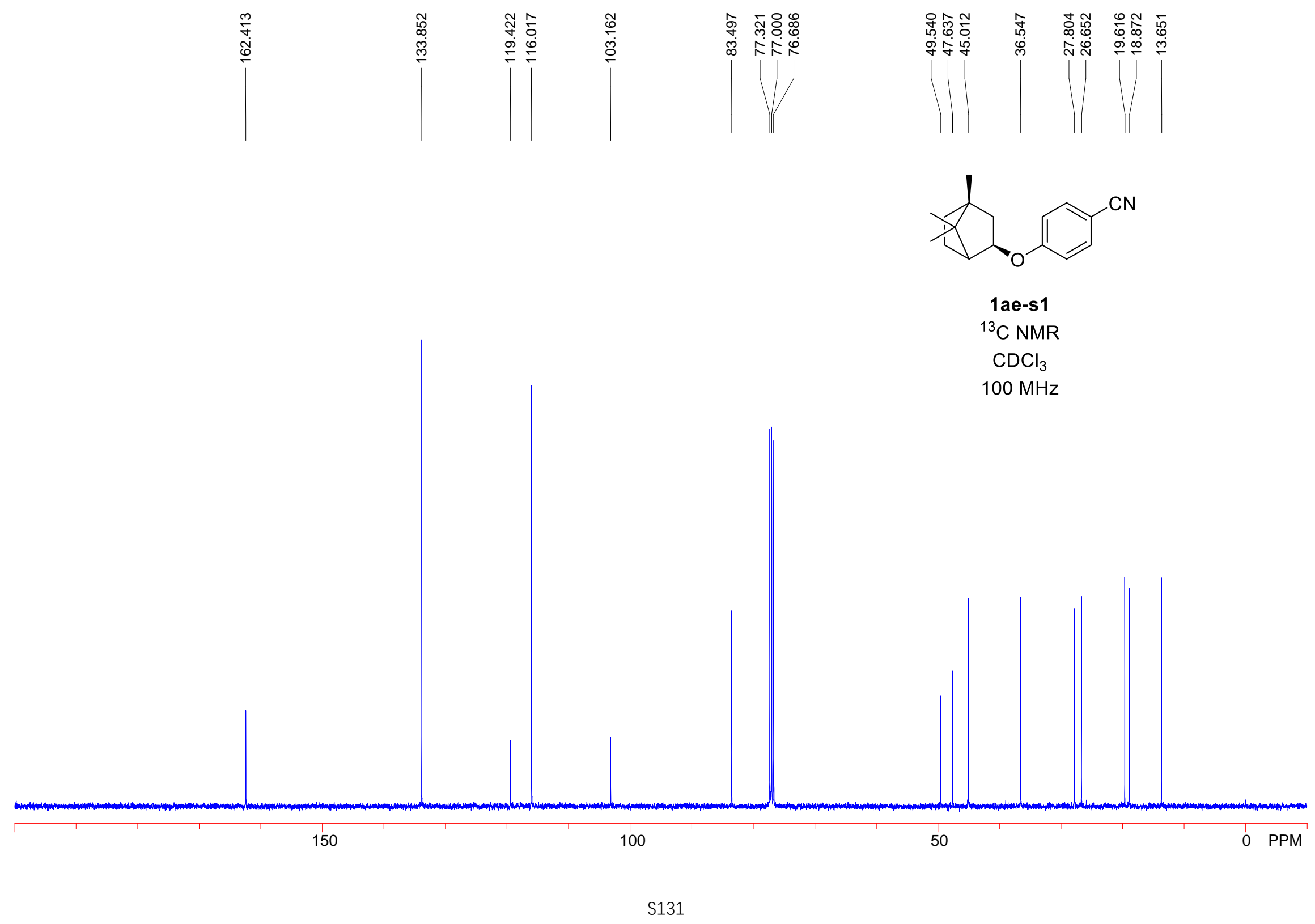




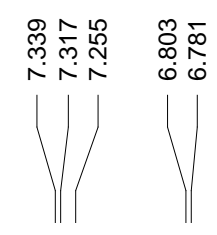

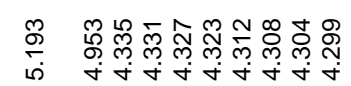

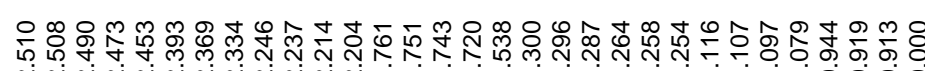
ชัง
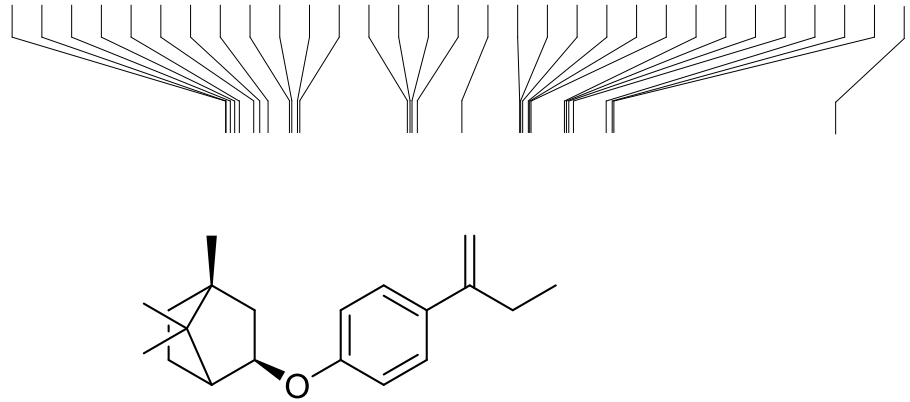

1 ae

${ }^{1} \mathrm{H}$ NMR

$\mathrm{CDCl}_{3}$

$400 \mathrm{MHz}$

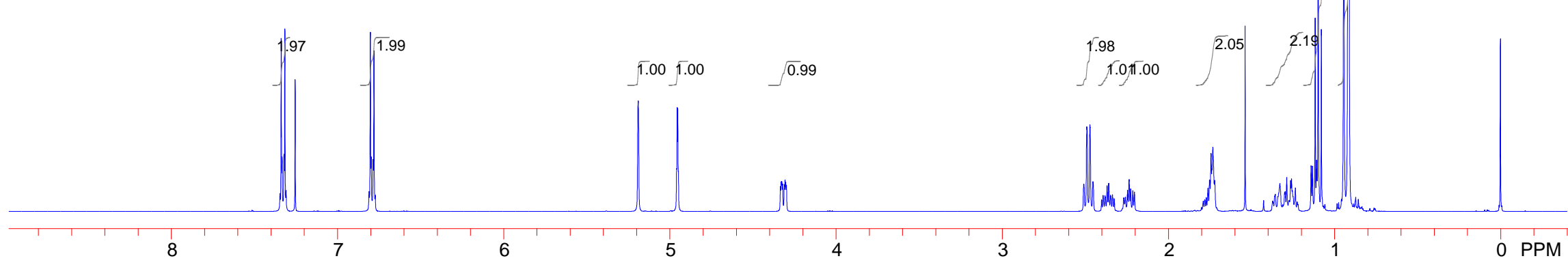




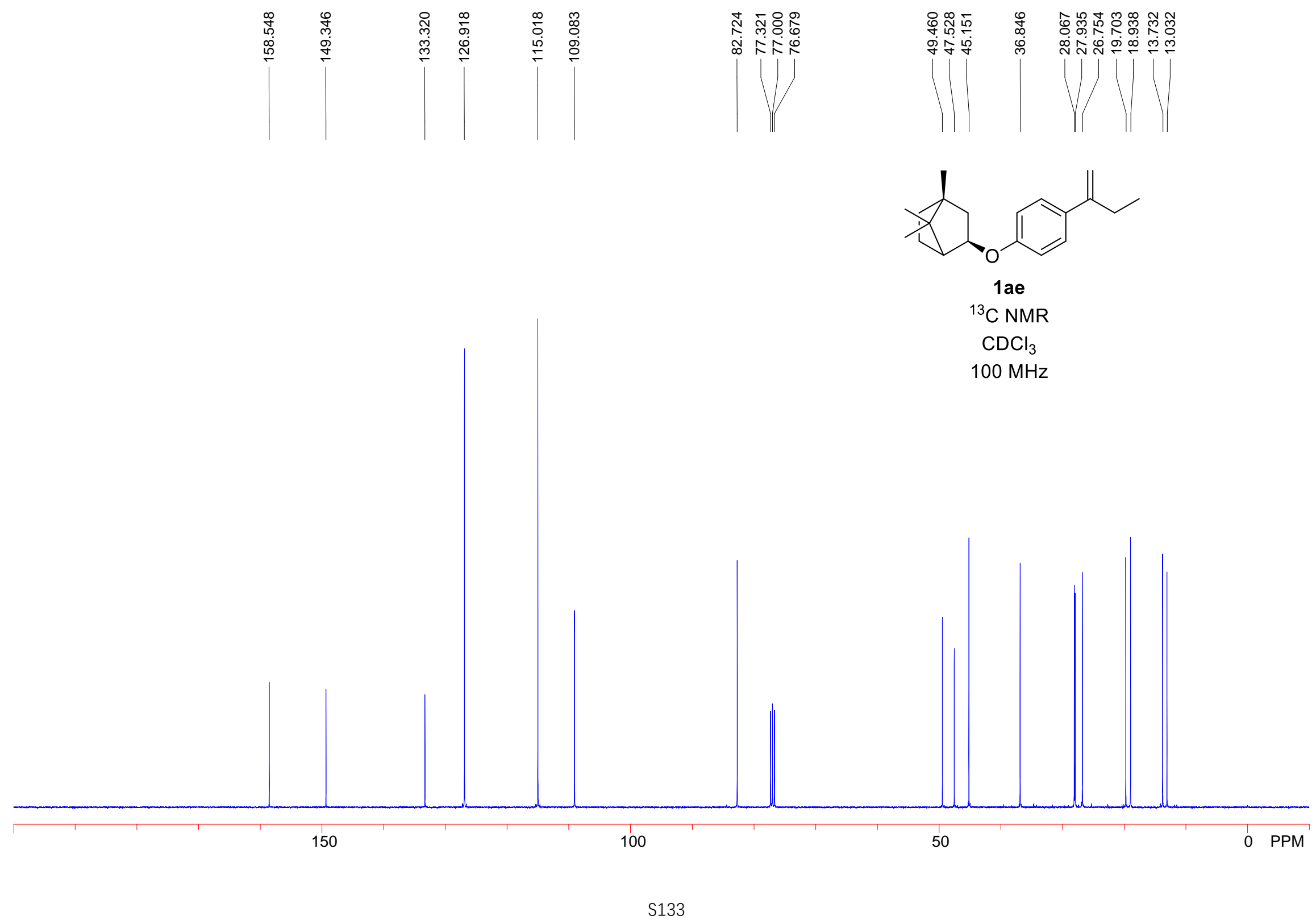


फलแक

ลNลNลN-

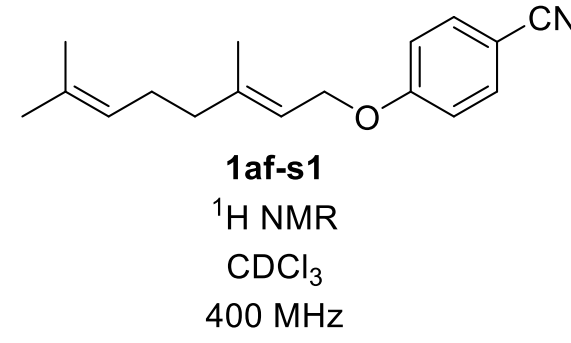

3.92

$400 \mathrm{MHz}$

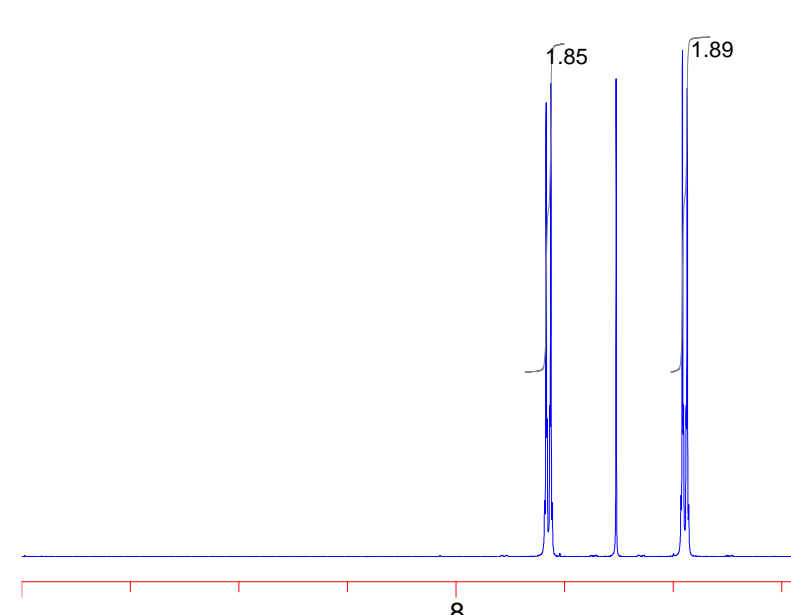

2.03 


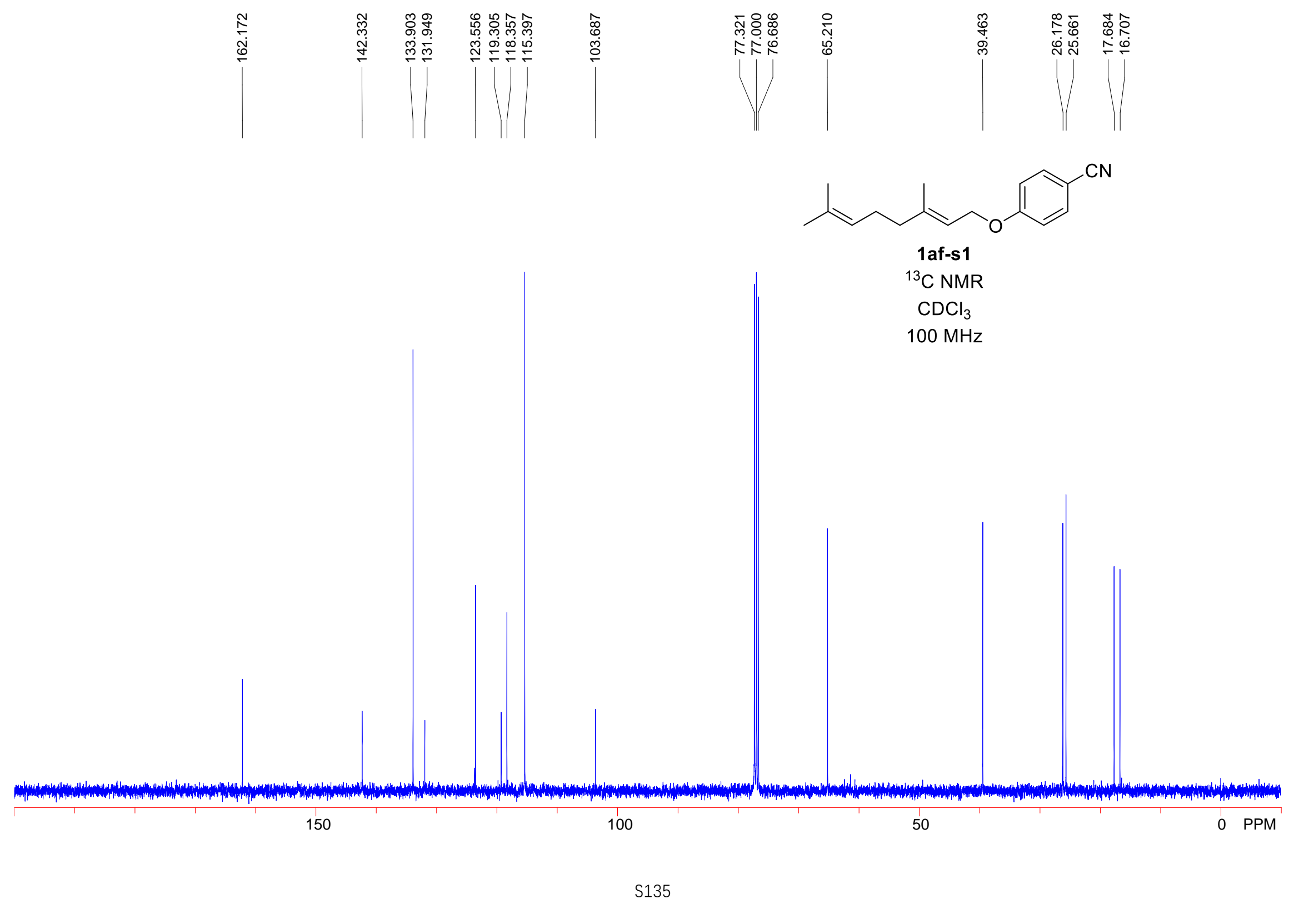




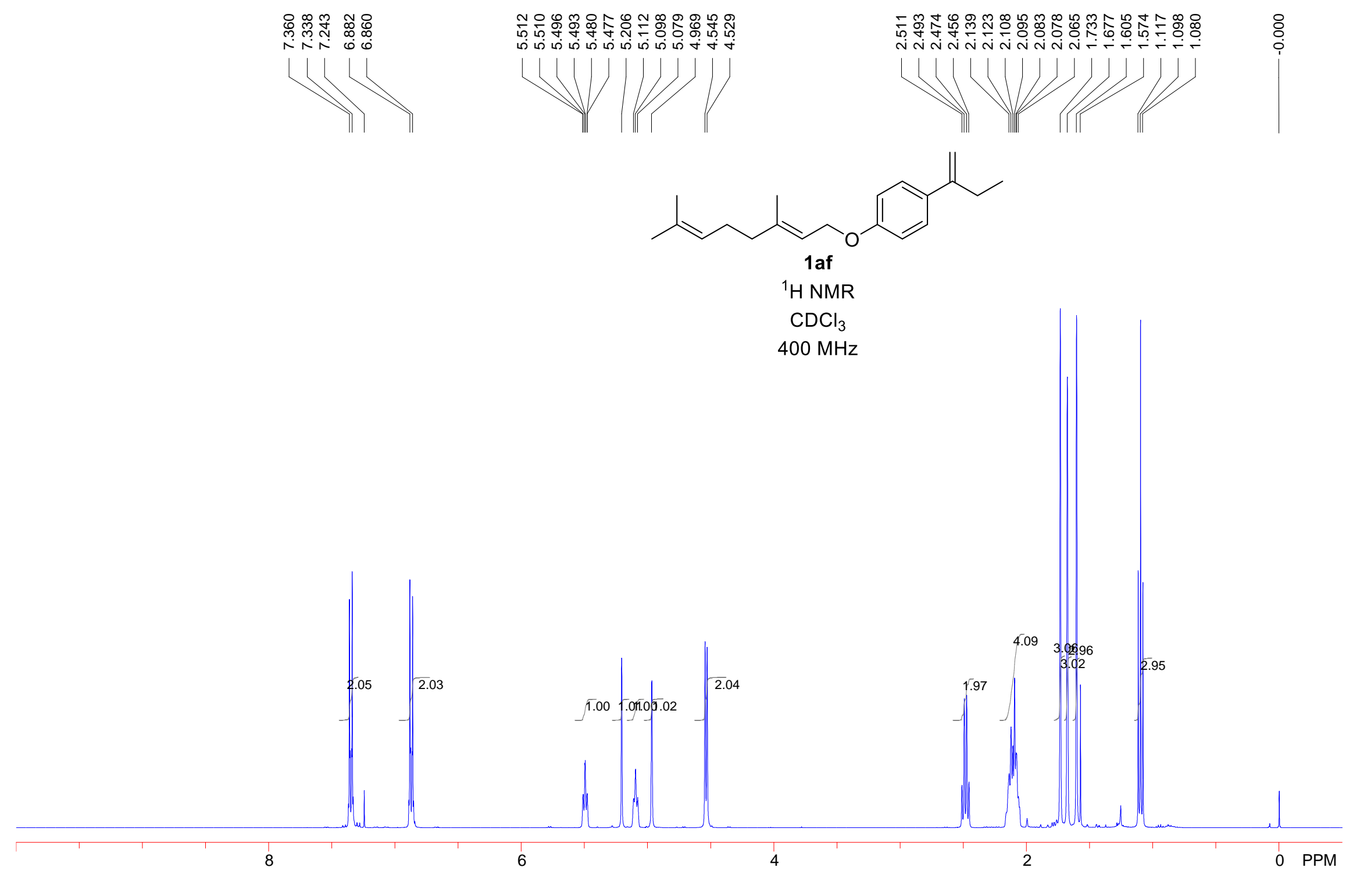




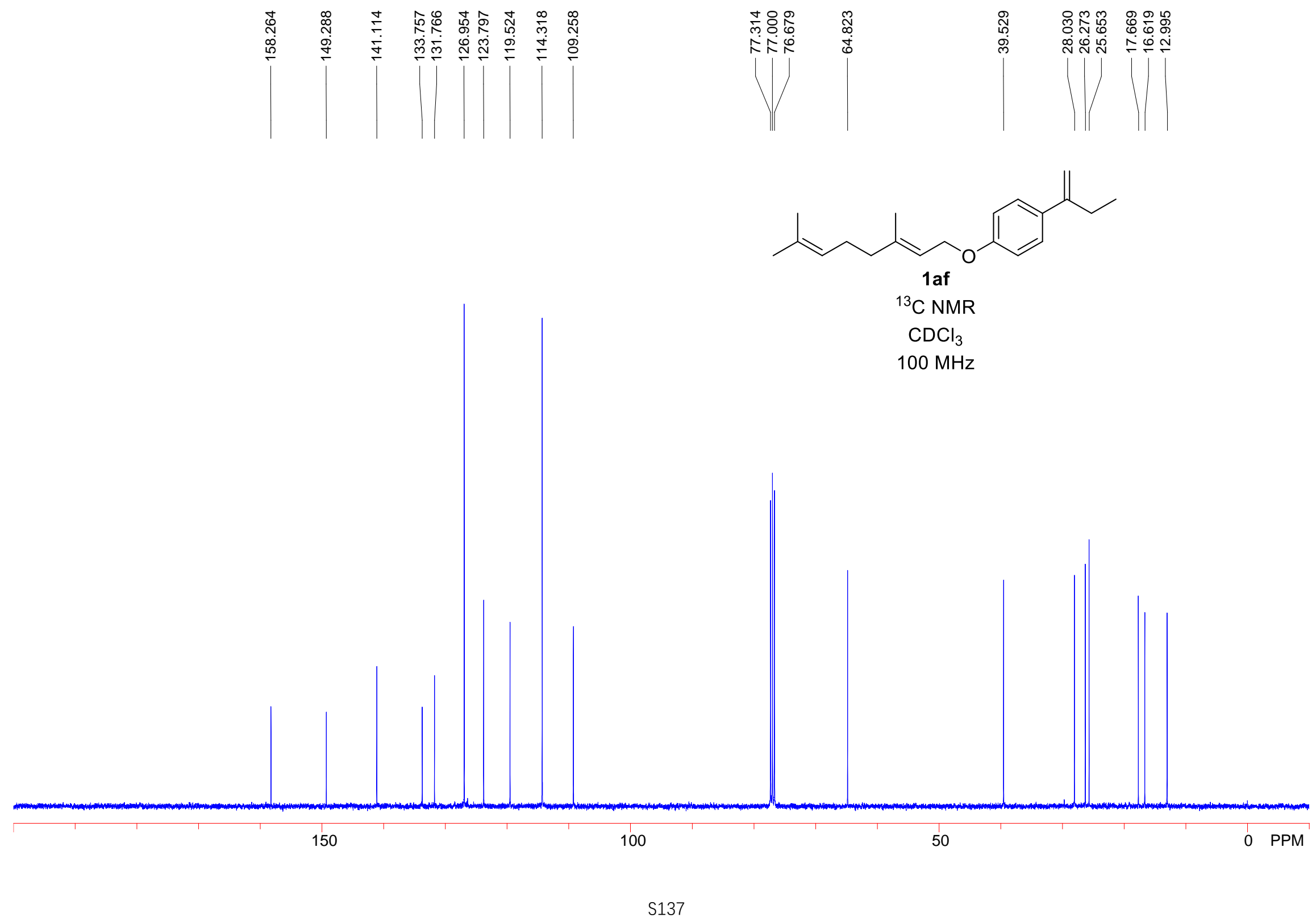




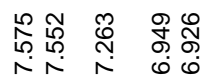

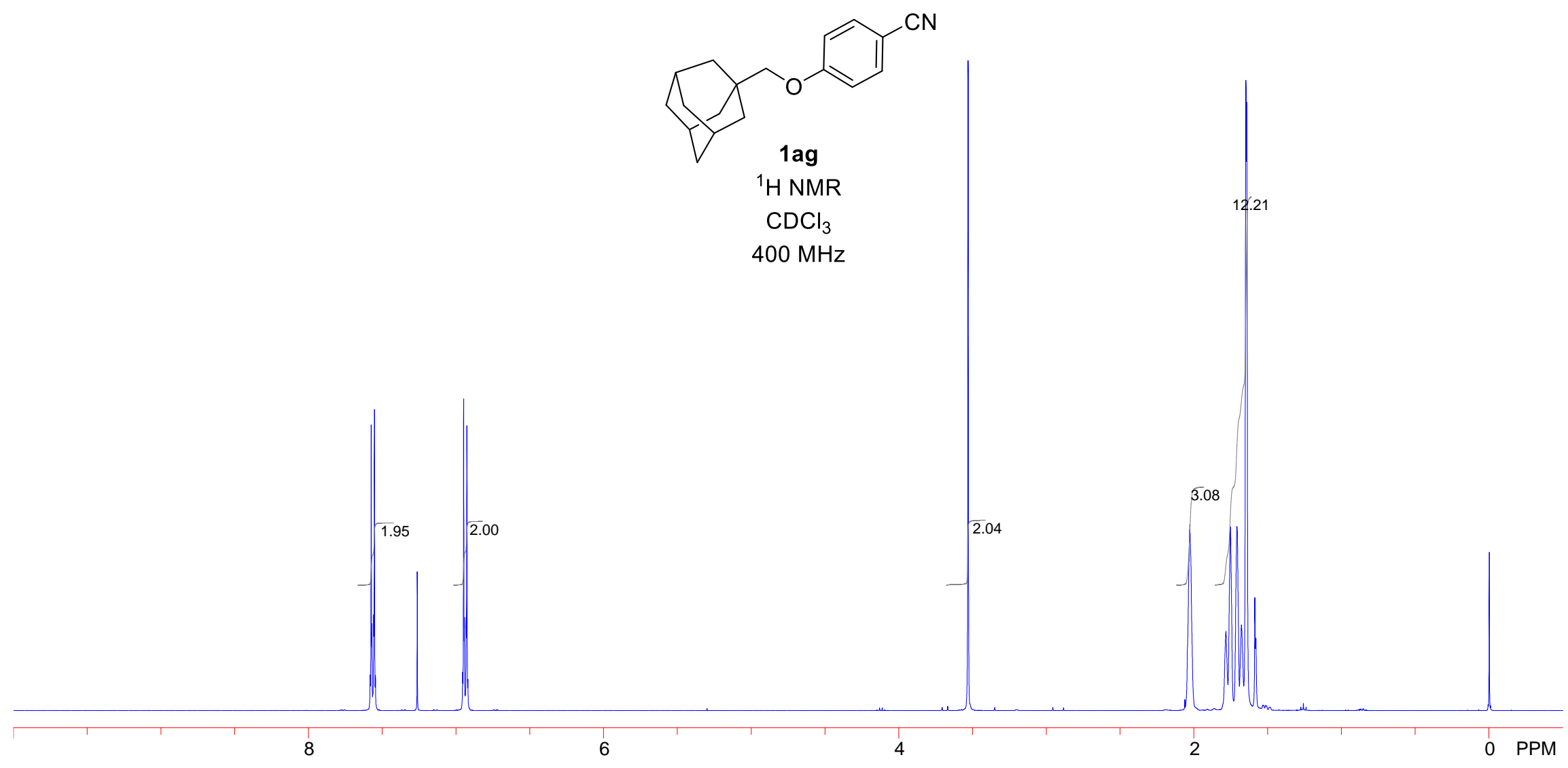




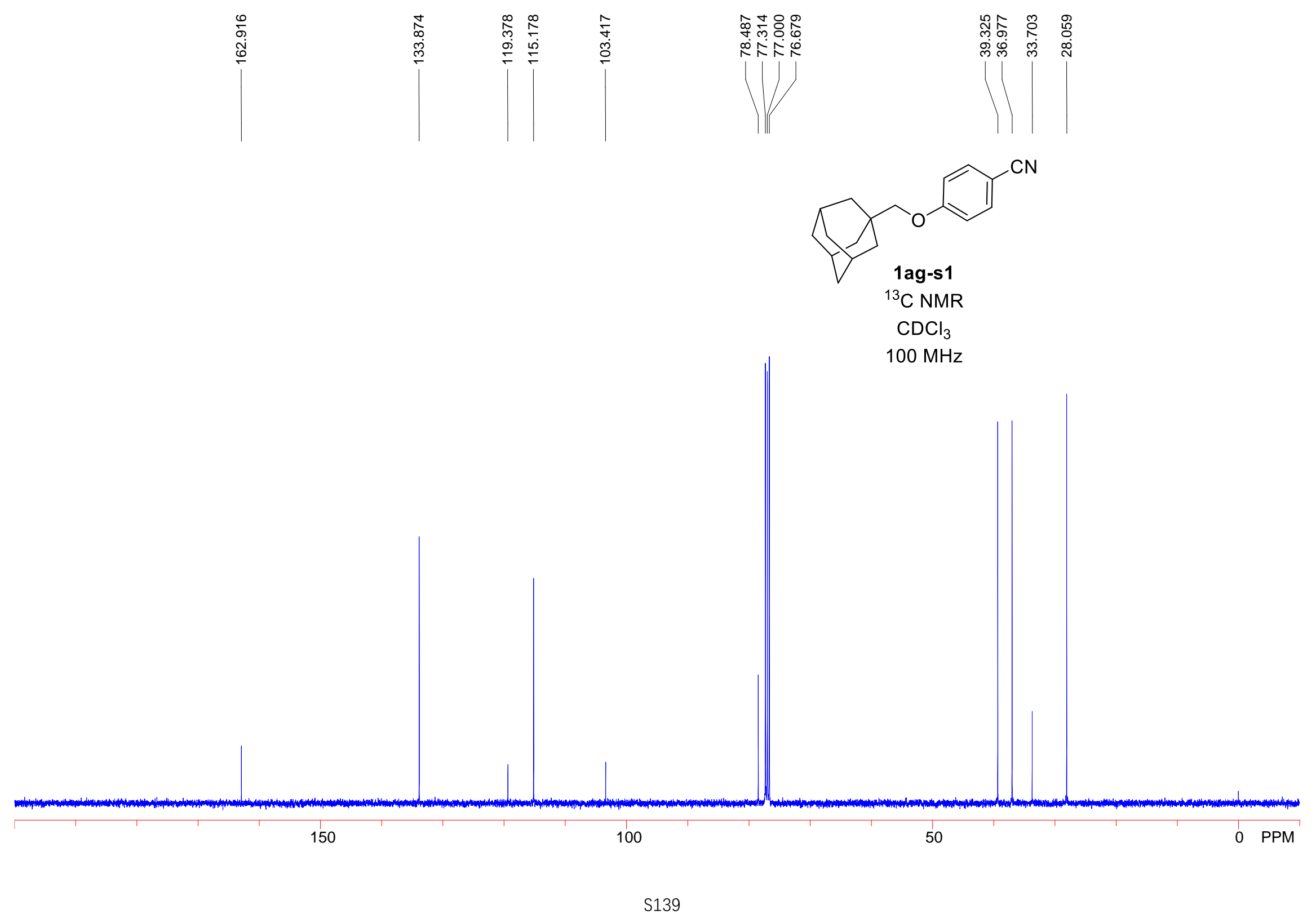




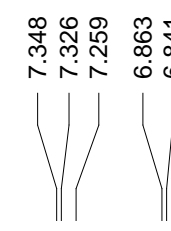

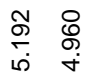

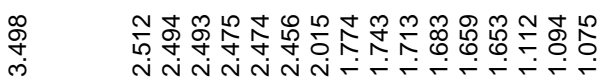

:
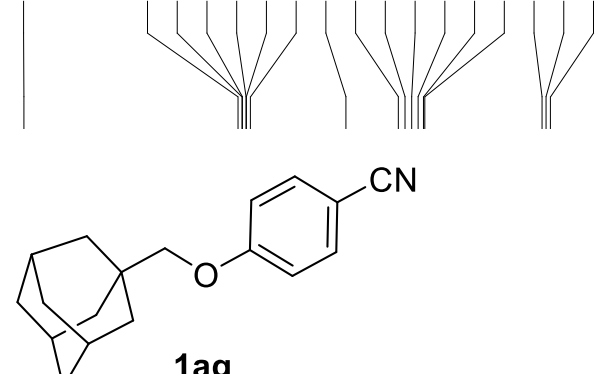

${ }^{1} \mathrm{H}$ NMR

$\mathrm{CDCl}_{3}$

$400 \mathrm{MHz}$

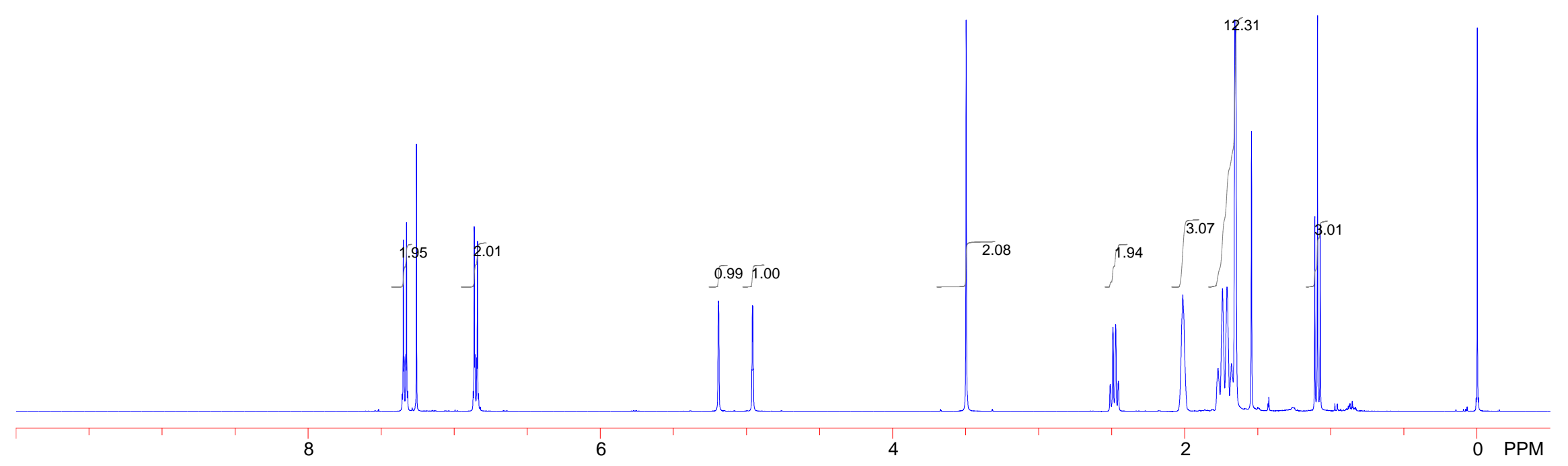




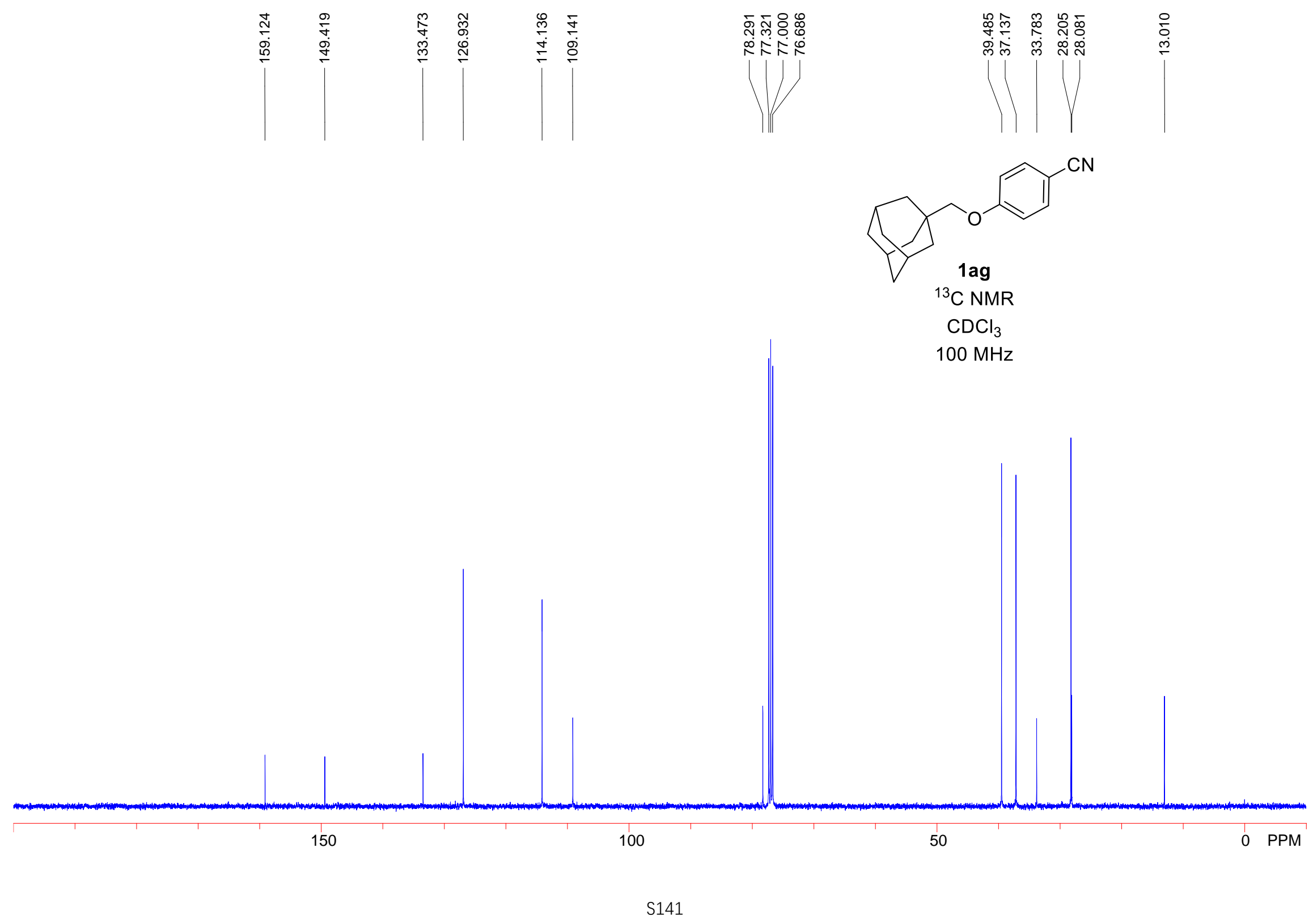




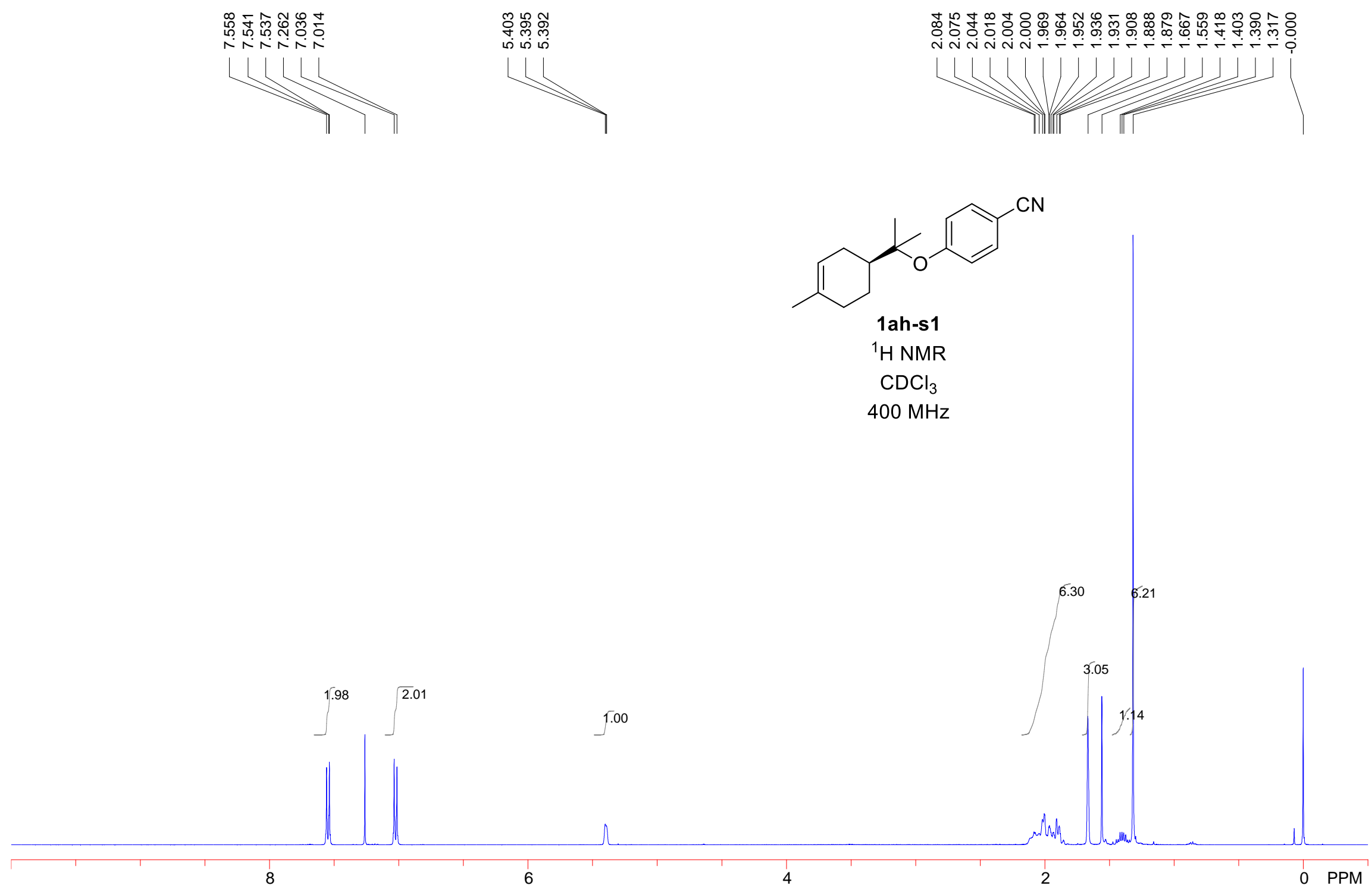

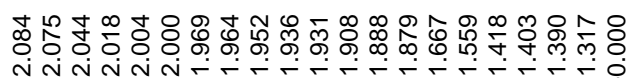




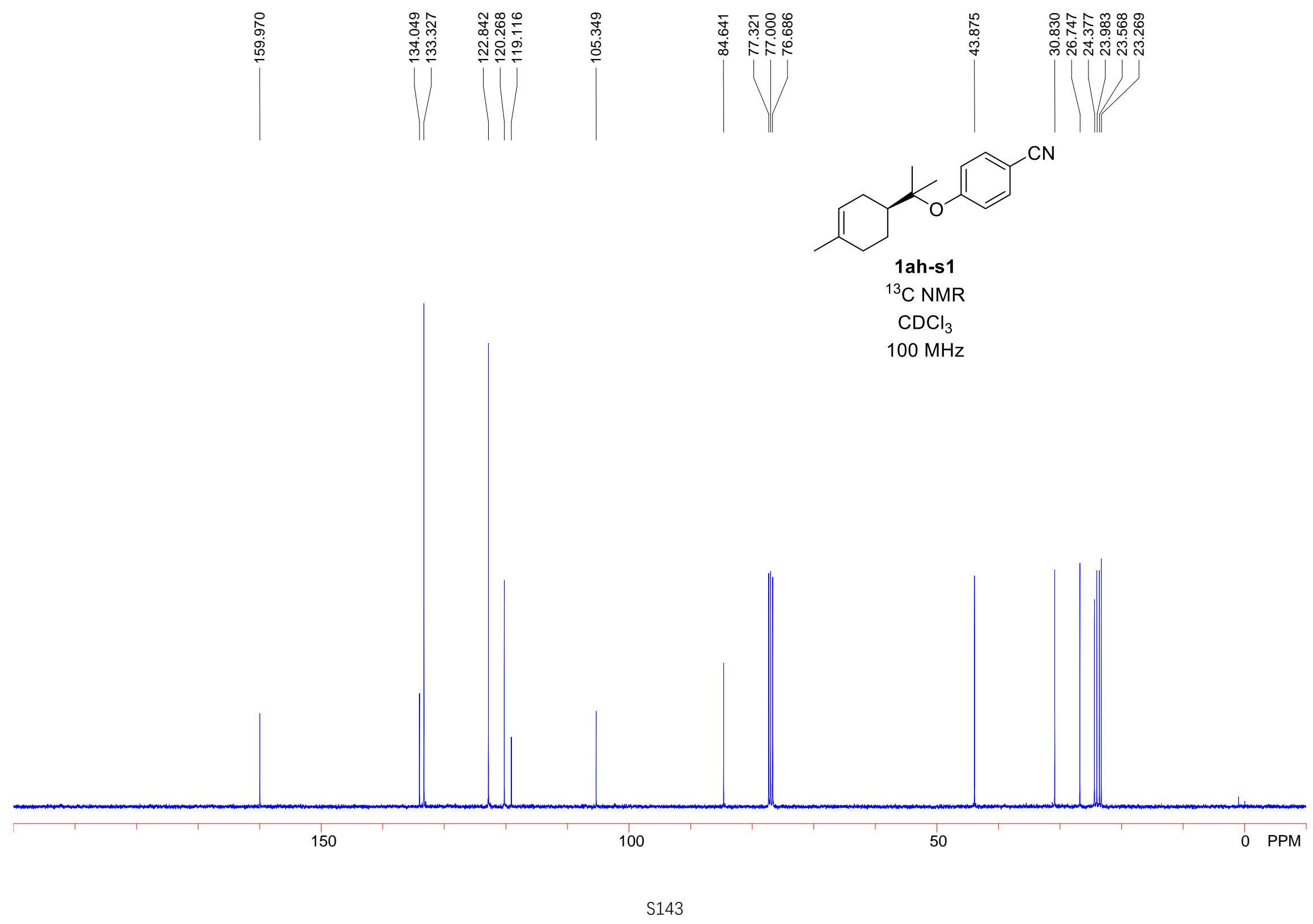




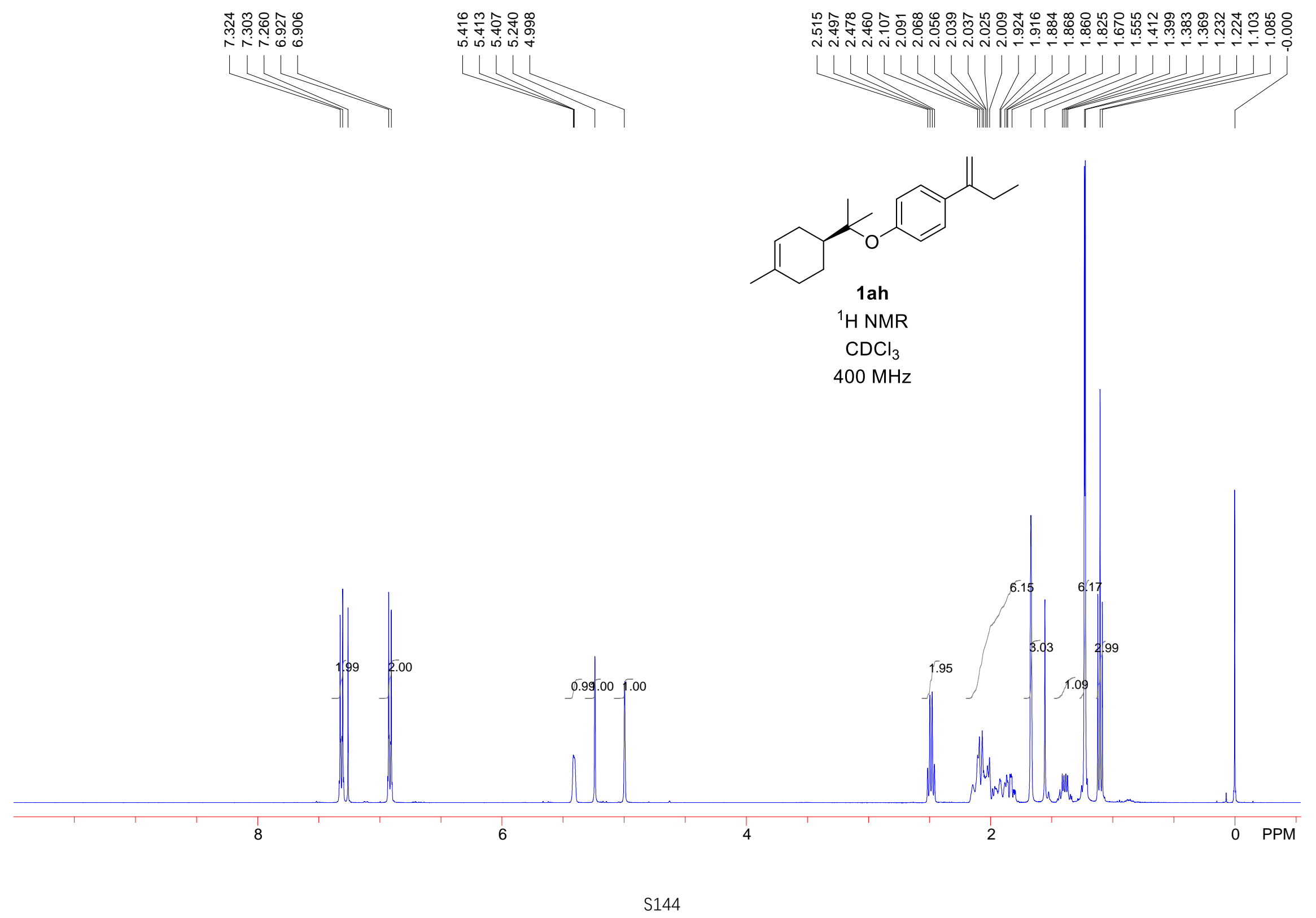




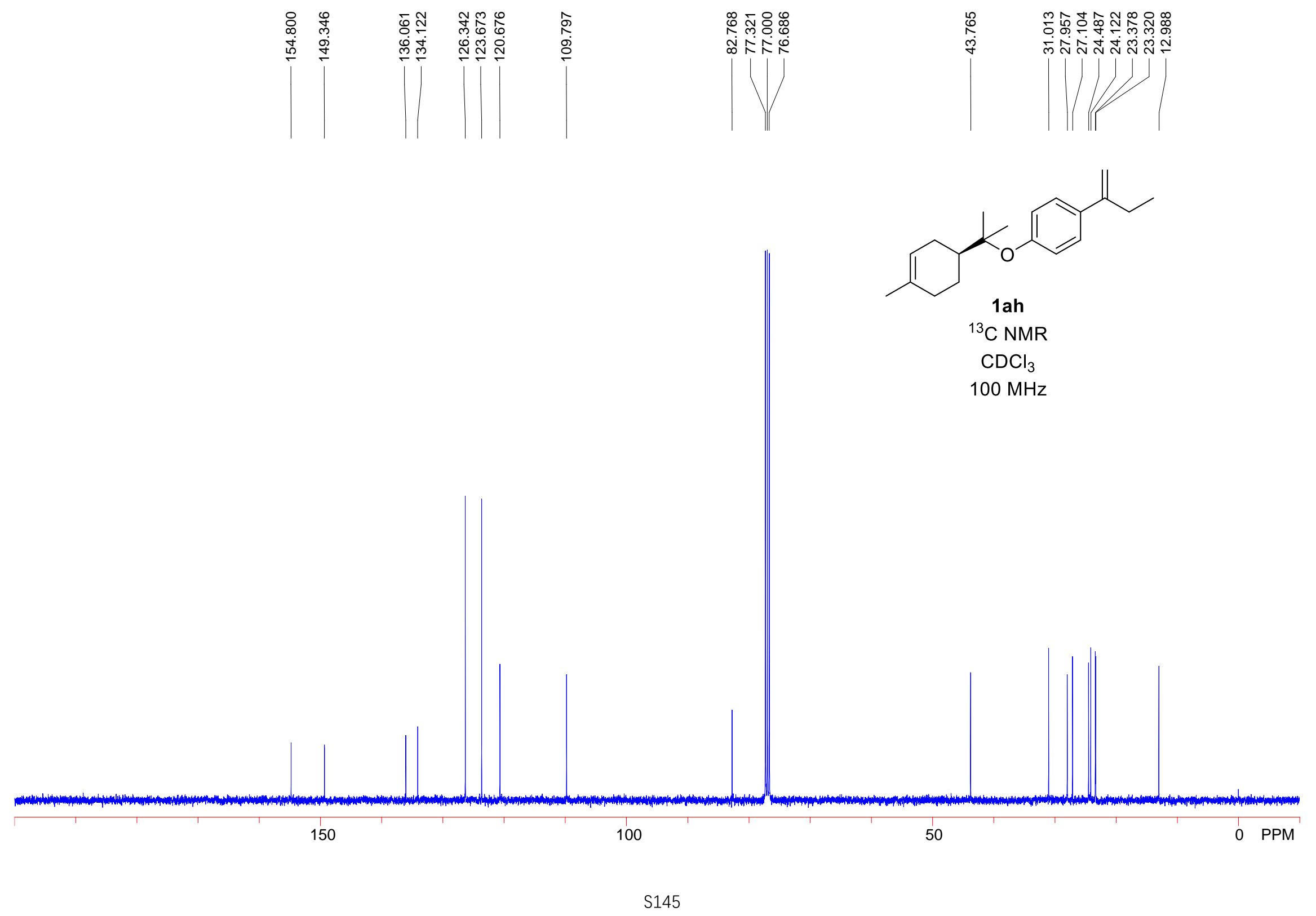




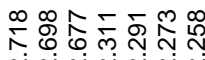

งั่ง ํํำ

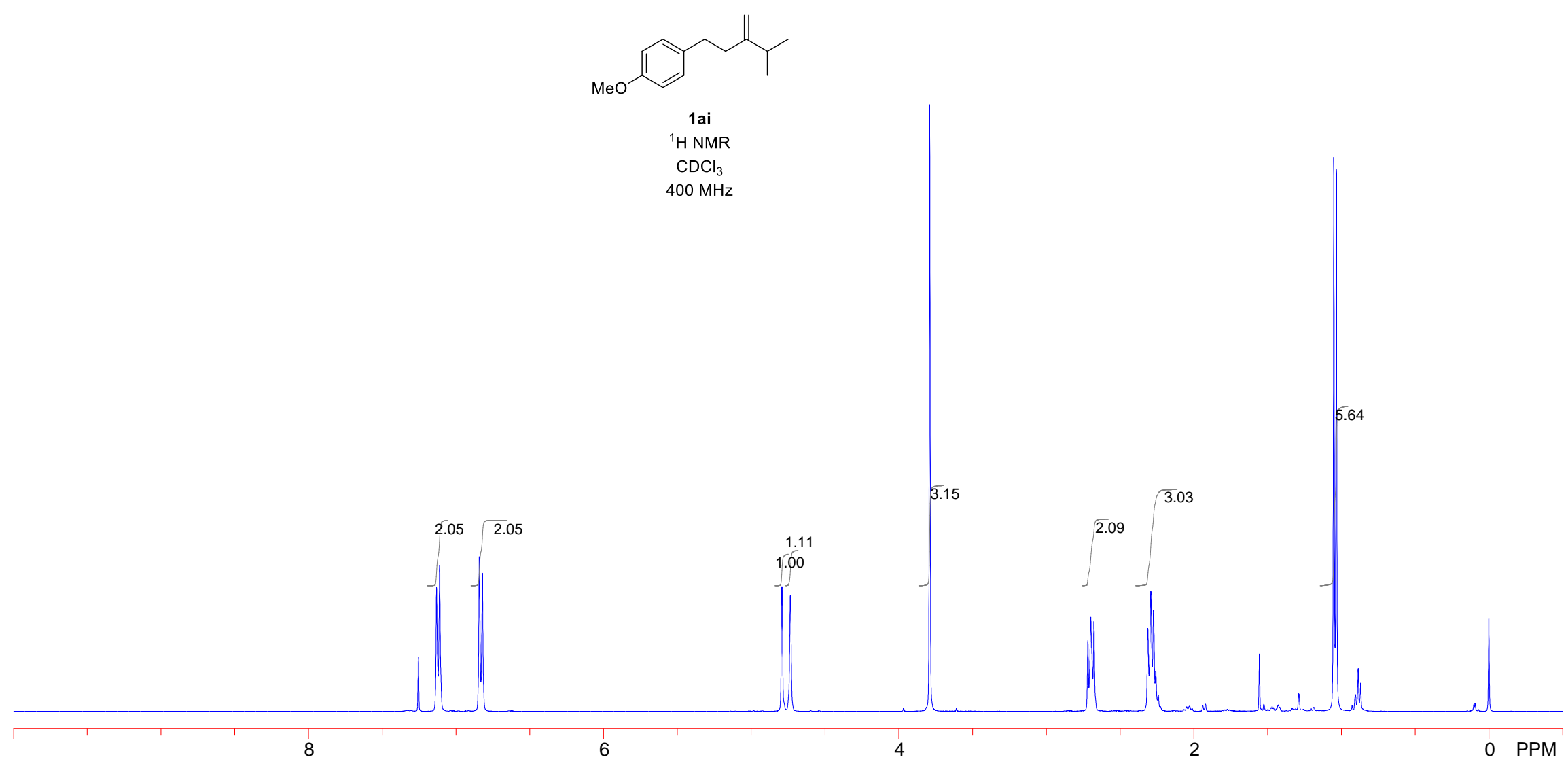




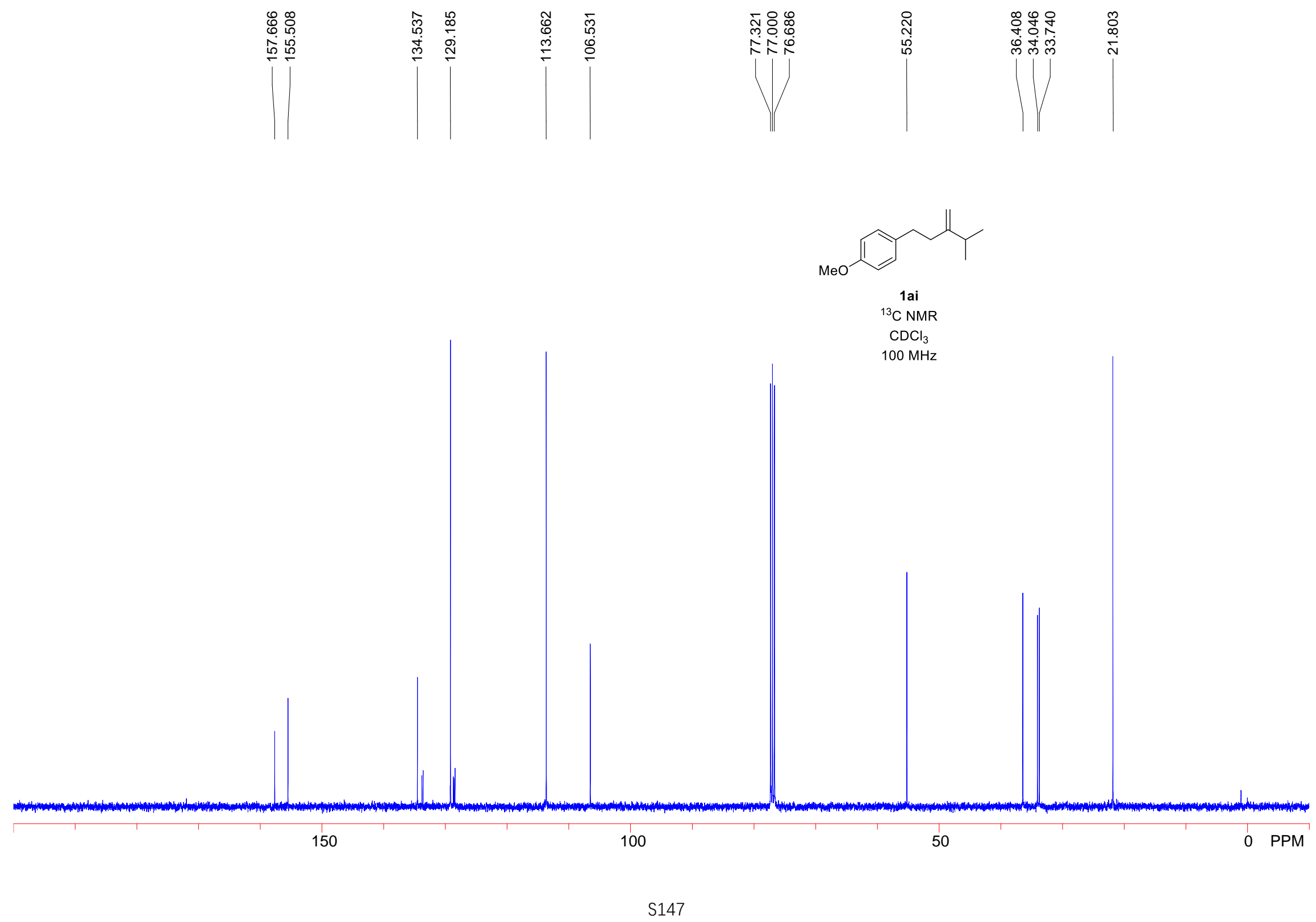




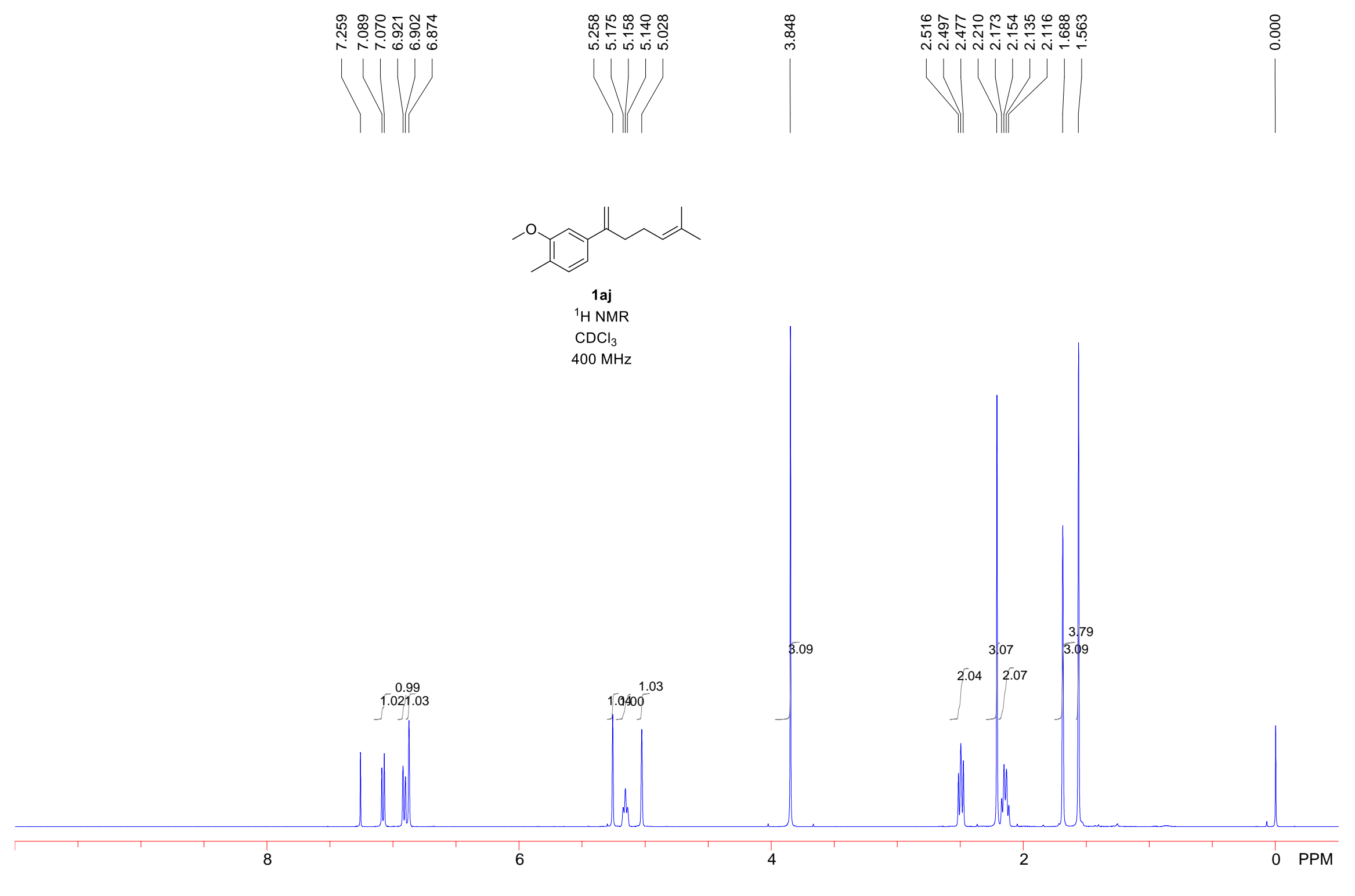




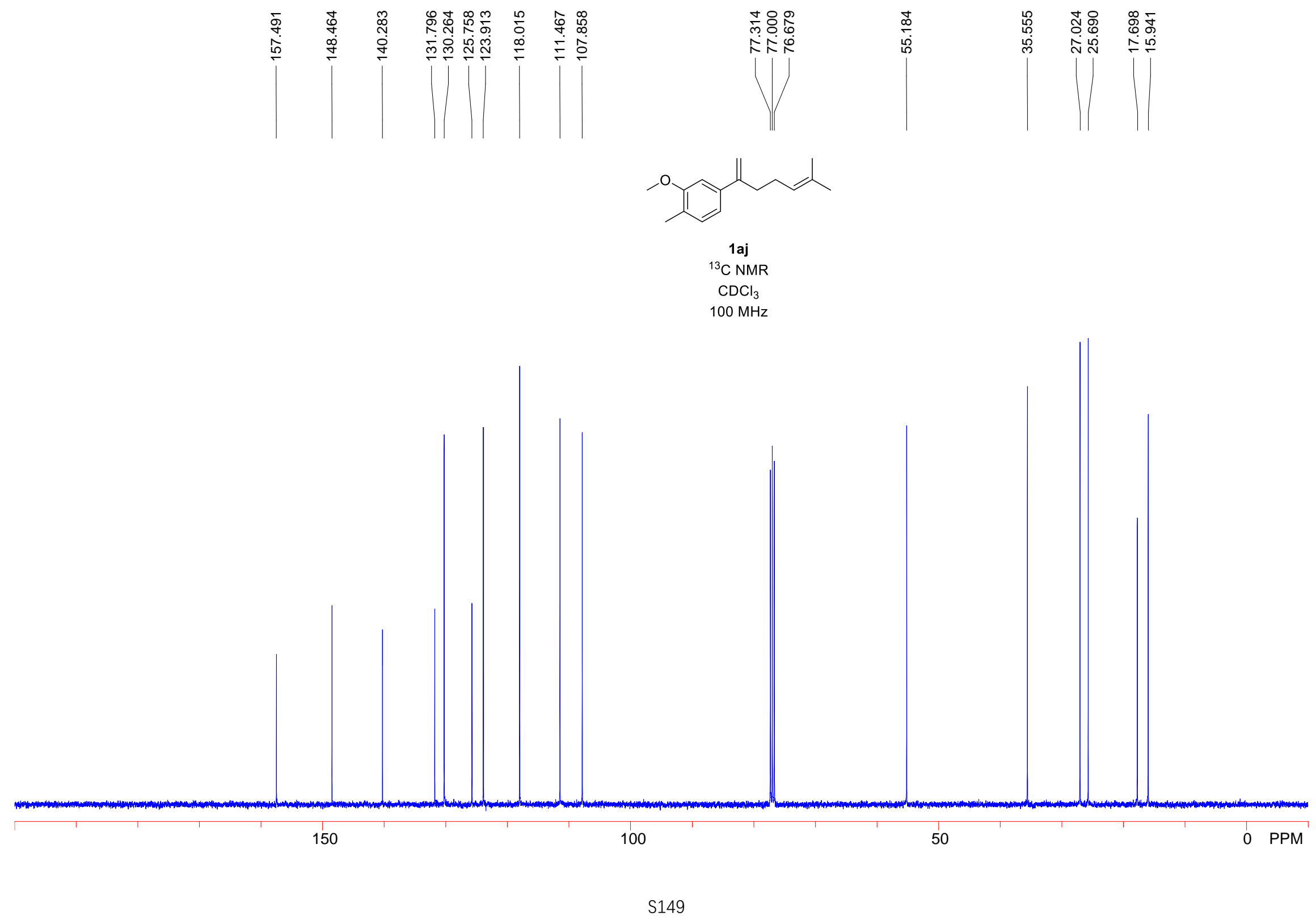



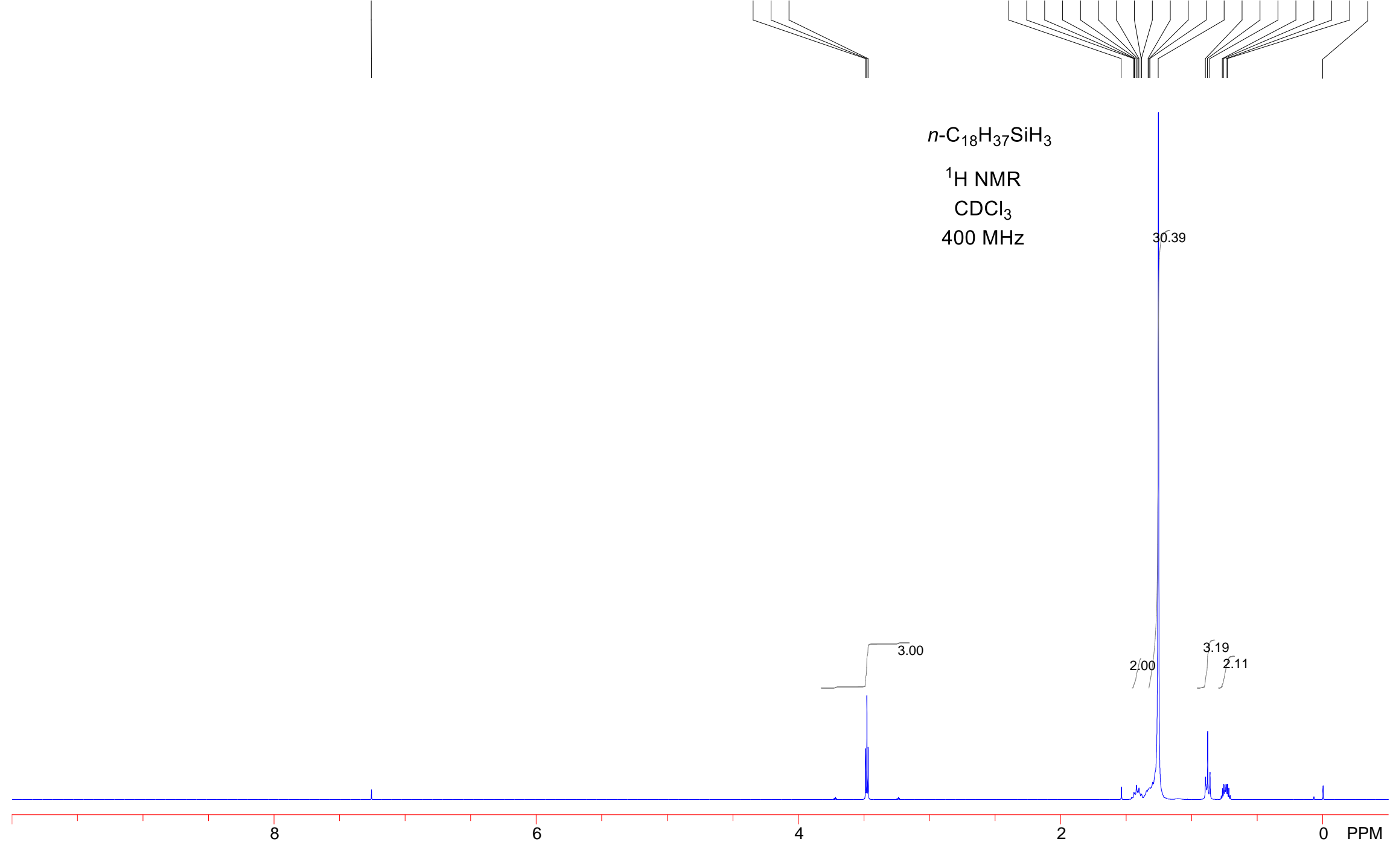


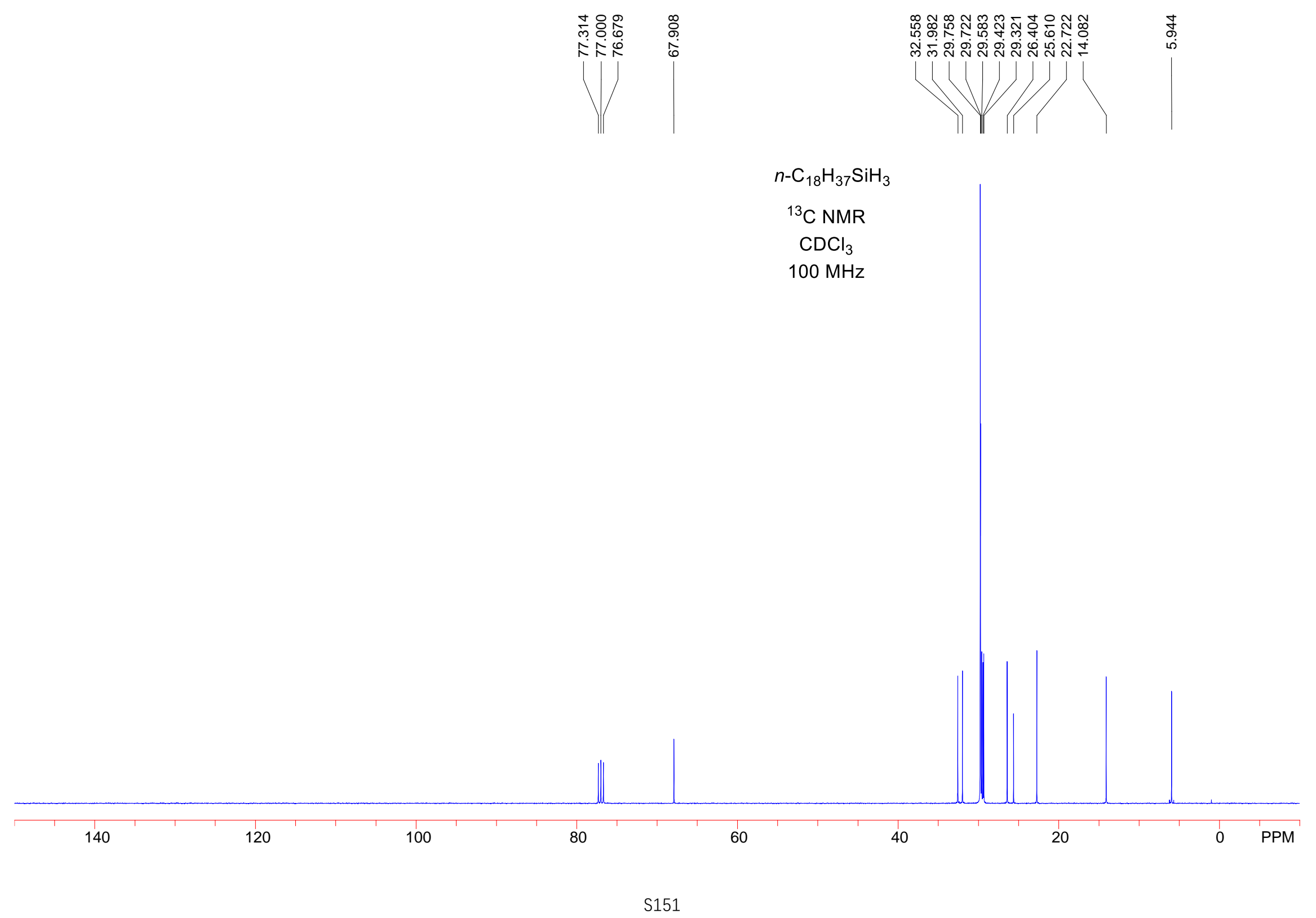




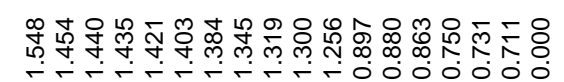

ल ल

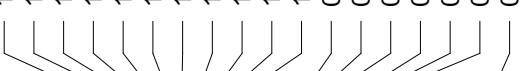

$n-\mathrm{C}_{18} \mathrm{H}_{37} \mathrm{SiD}_{3}$

${ }^{1} \mathrm{H}$ NMR

$\mathrm{CDCl}_{3}$

$400 \mathrm{MHz}$

30.26

2.26

2.00

0.02 


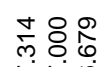

章金
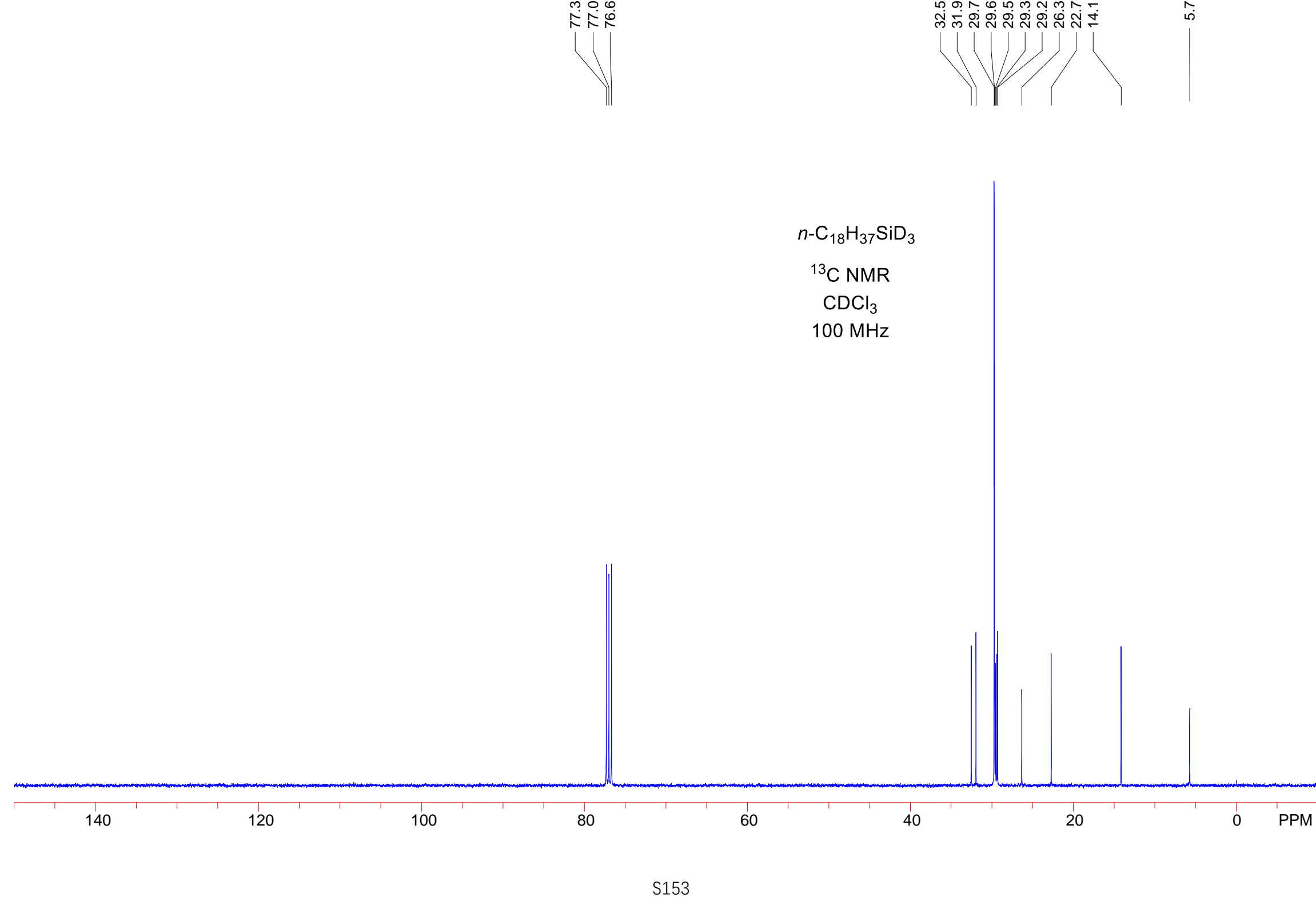

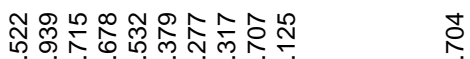

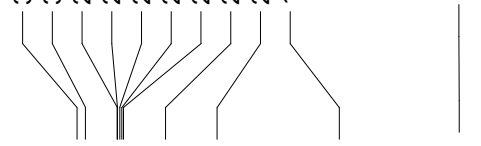

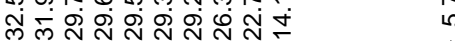



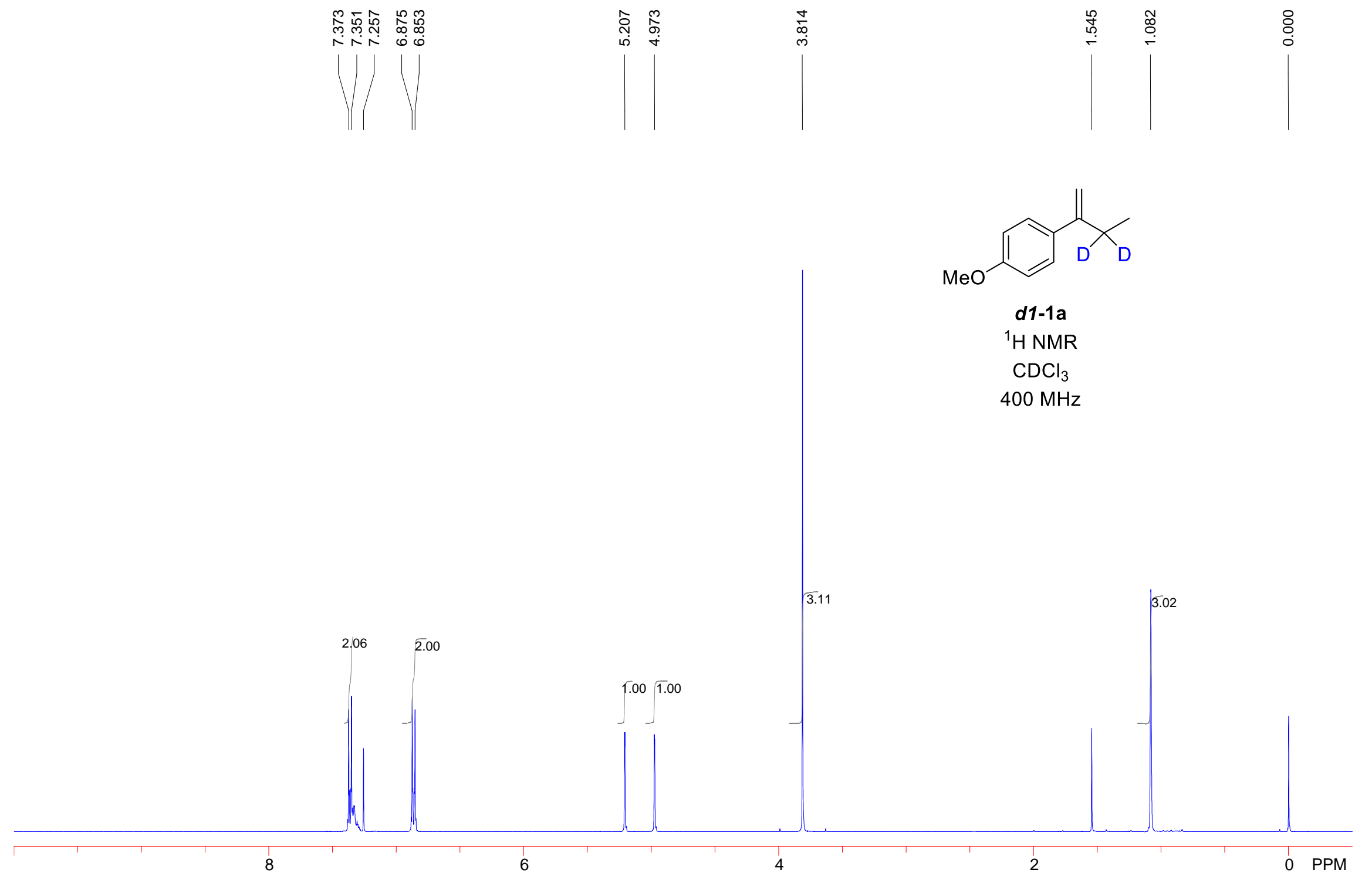


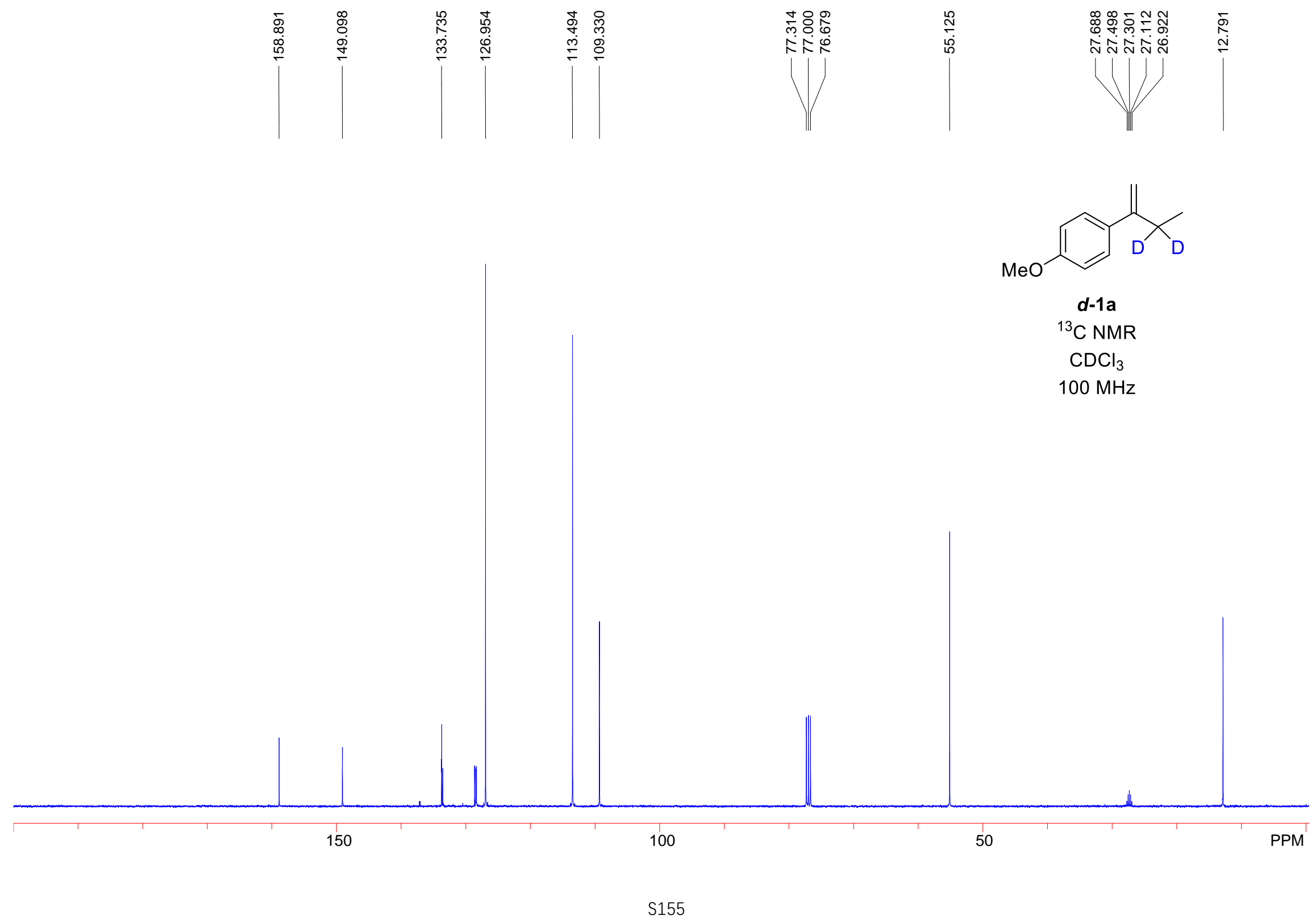




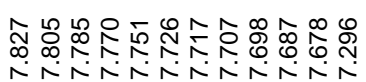

$\mathrm{Ph}_{3} \mathrm{PCD}_{3} \mathrm{Br}$
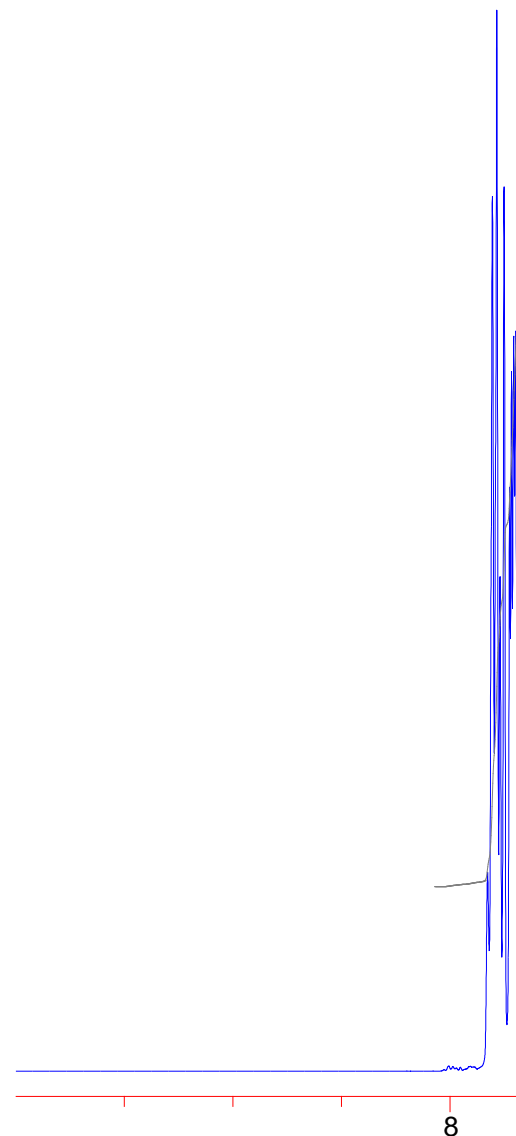

15.00

0.24

\section{${ }^{1} \mathrm{H}$ NMR}

$\mathrm{CDCl}_{3}$

$400 \mathrm{MHz}$ 


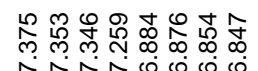

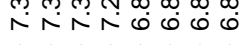

号

$\underset{\substack{\infty \\ \infty}}{\infty}$

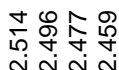

息

웅

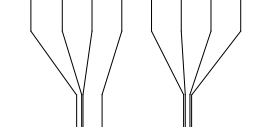

NNN
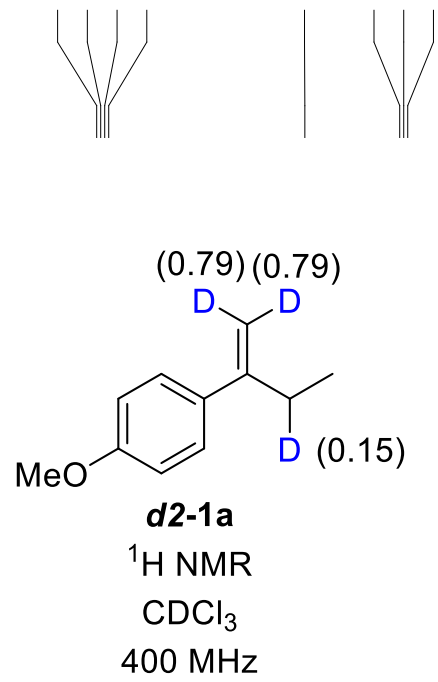

3.06

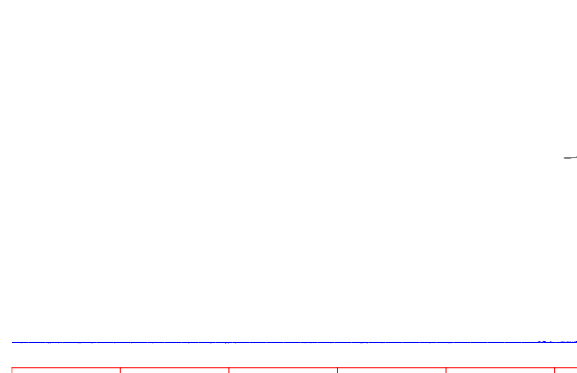

$1.95 \quad 1.97$

0.210 .21 


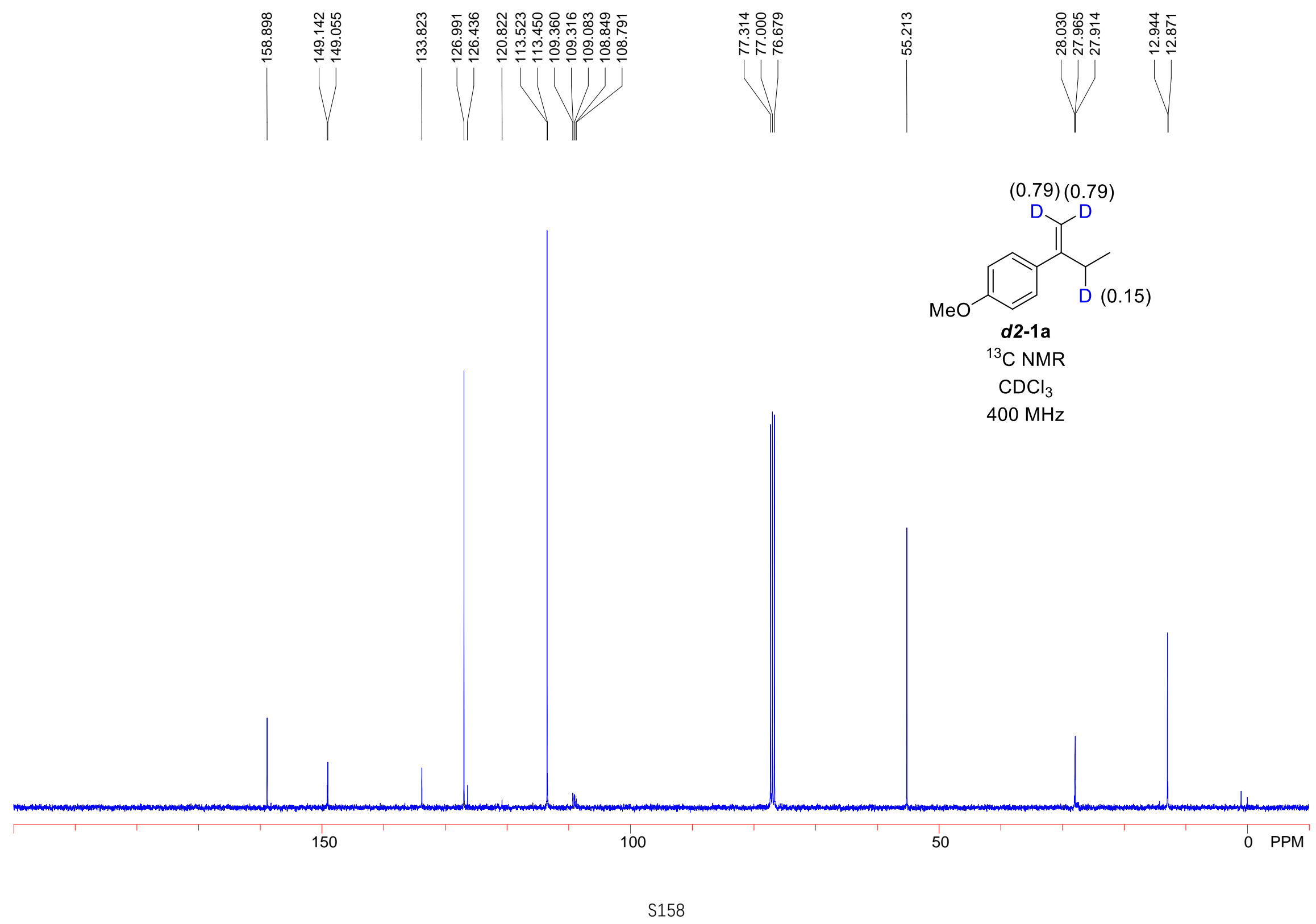




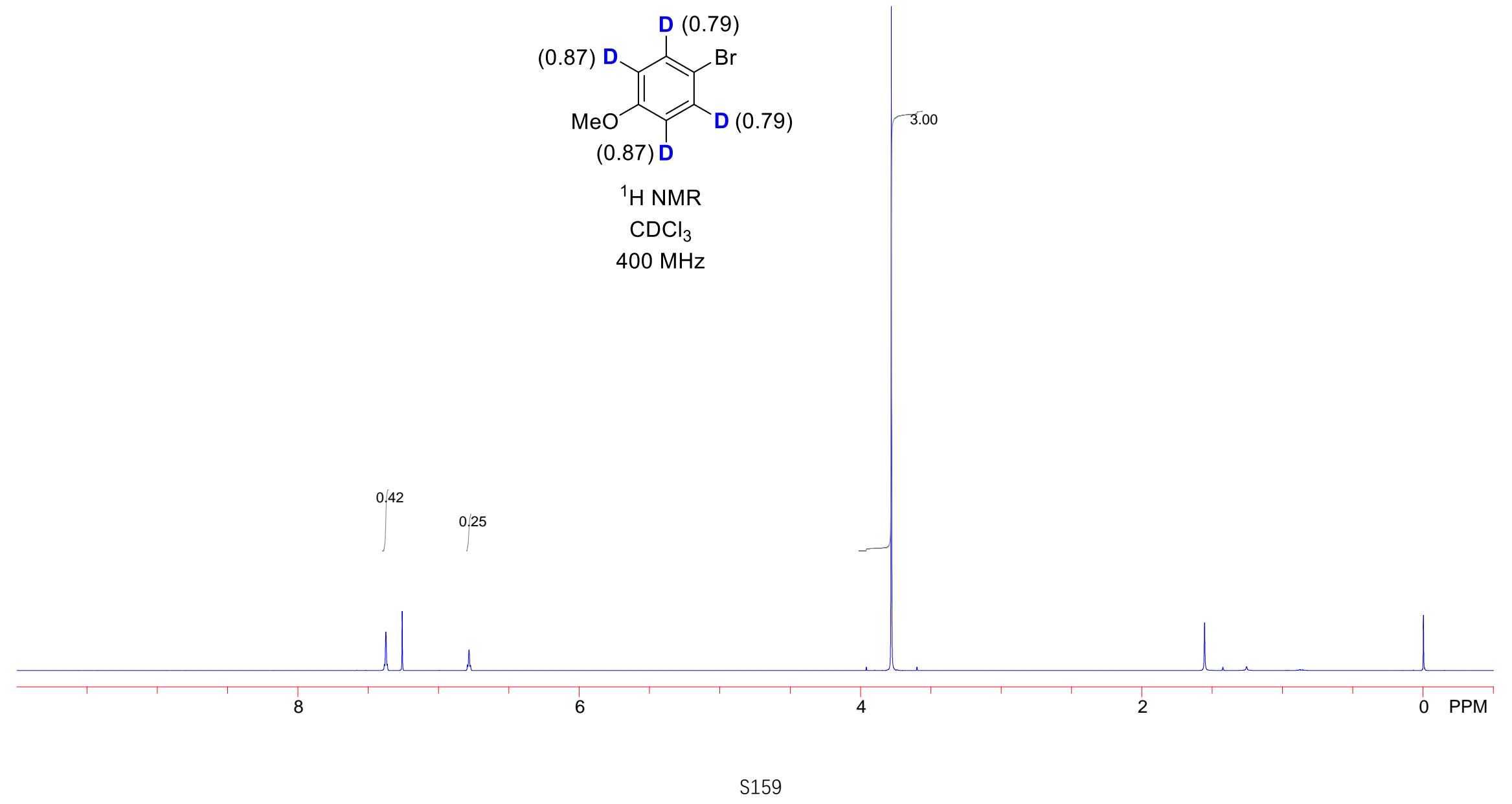




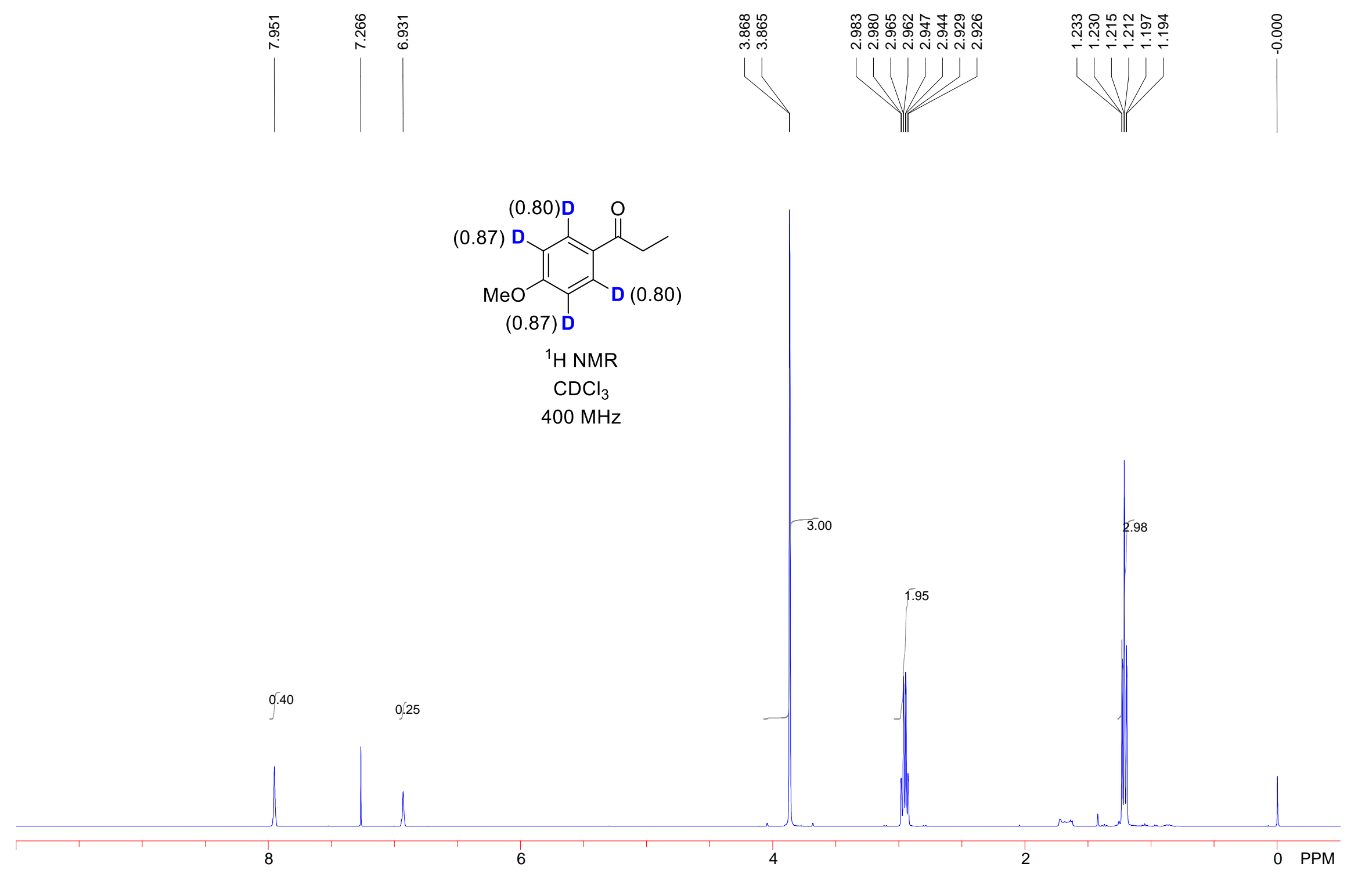




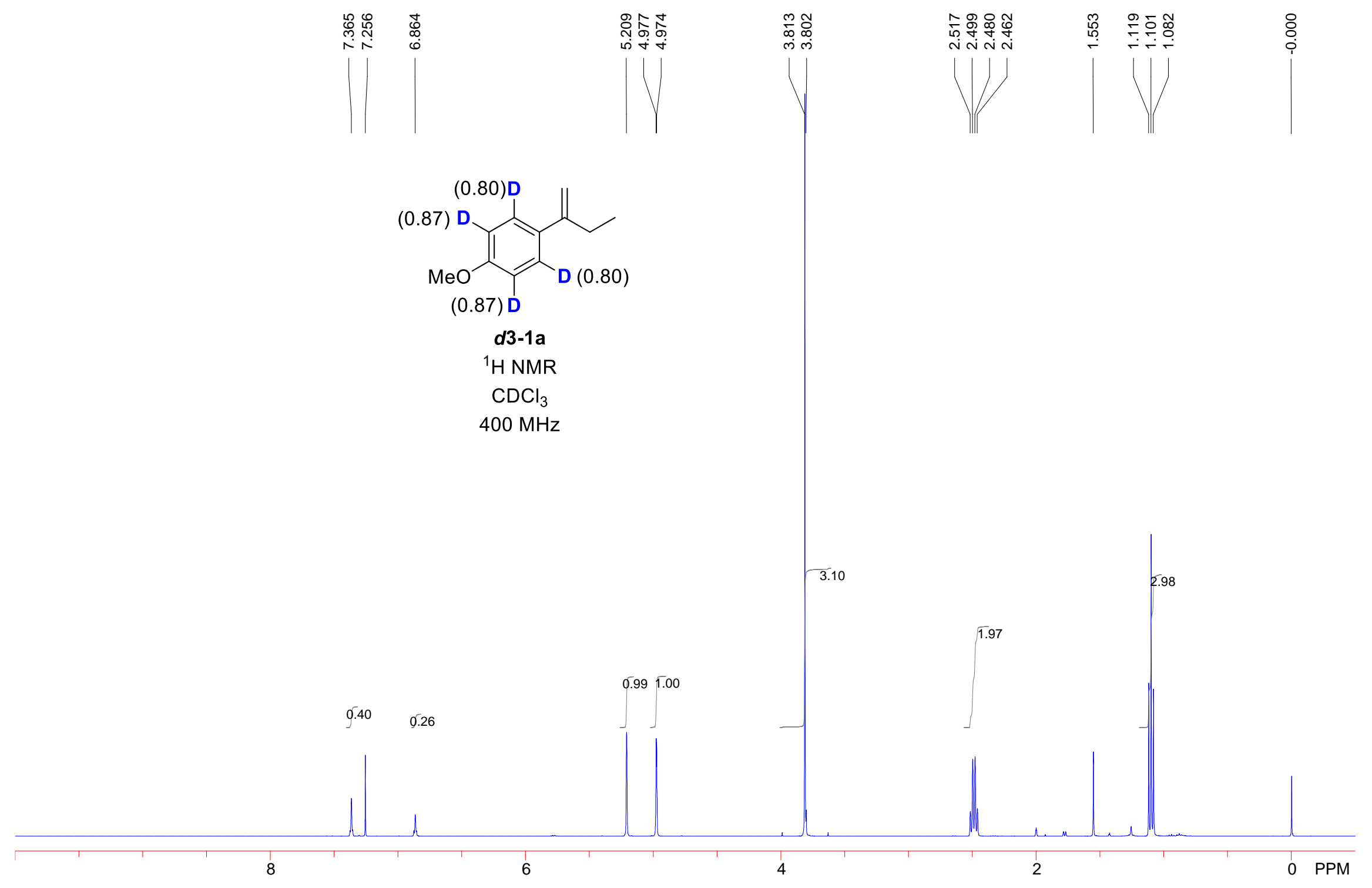




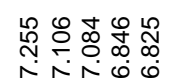

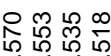

ง ํํำ ํํำ

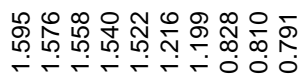

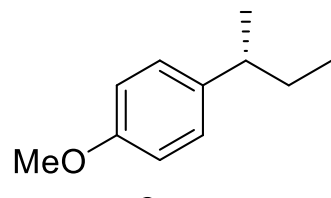

2a

${ }^{1} \mathrm{H}$ NMR

$\mathrm{CDCl}_{3}$

$400 \mathrm{MHz}$

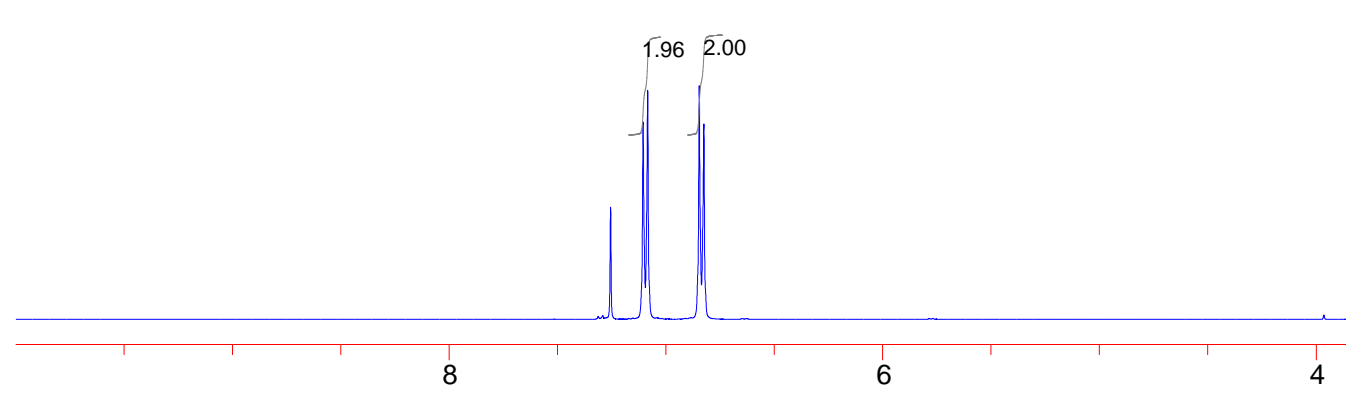

302

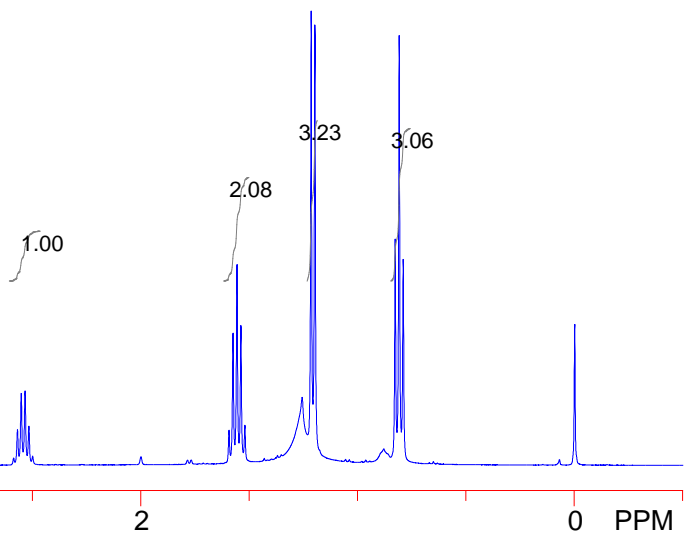




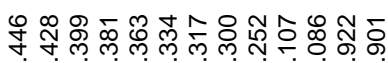

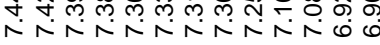

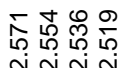

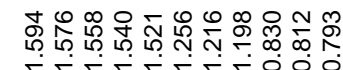

웅

กิن
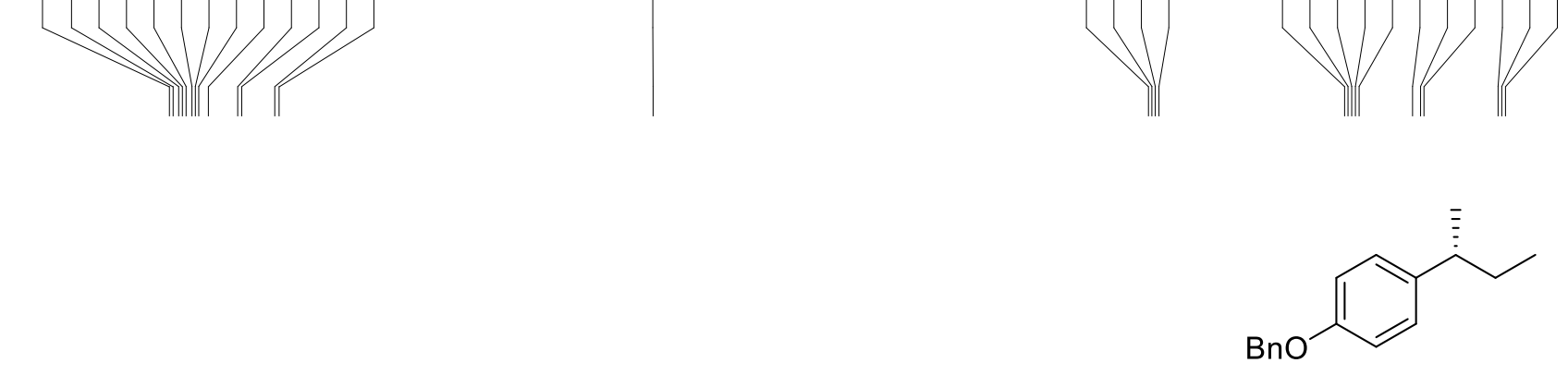

2b

\section{${ }^{1} \mathrm{H}$ NMR}

$\mathrm{CDCl}_{3}$

$400 \mathrm{MHz}$

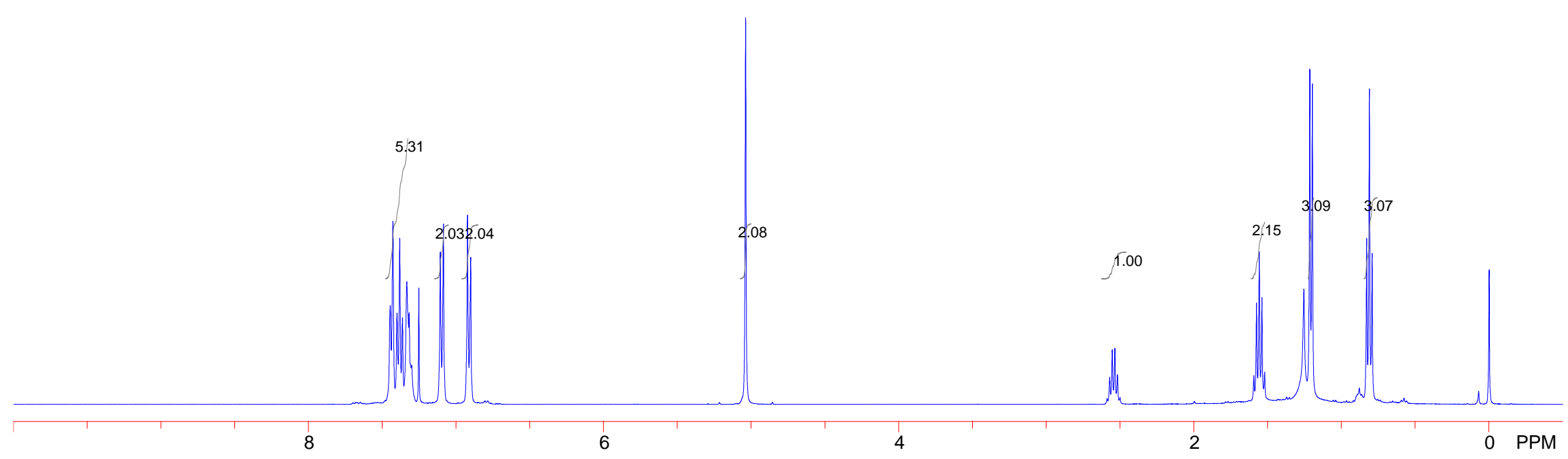




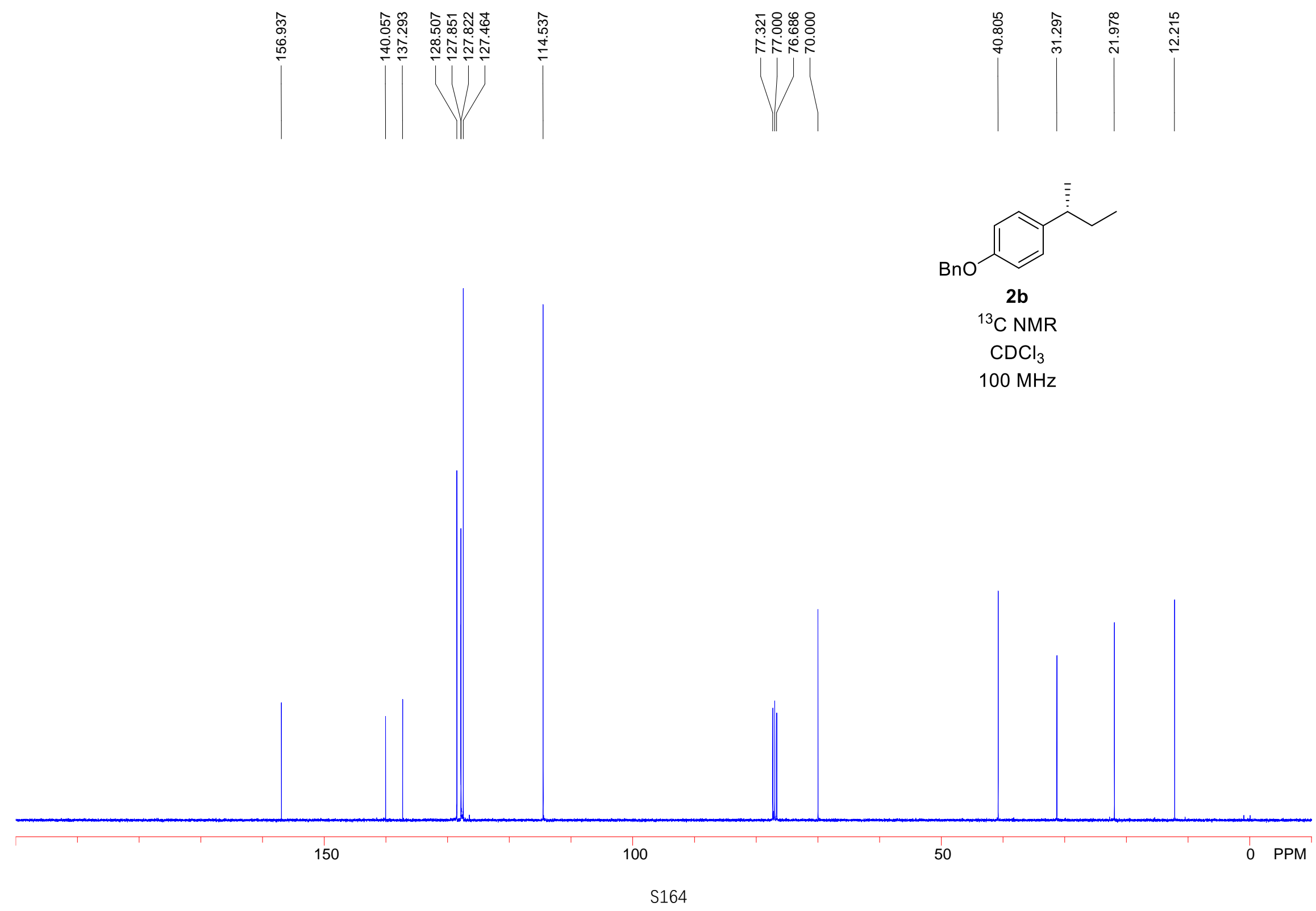




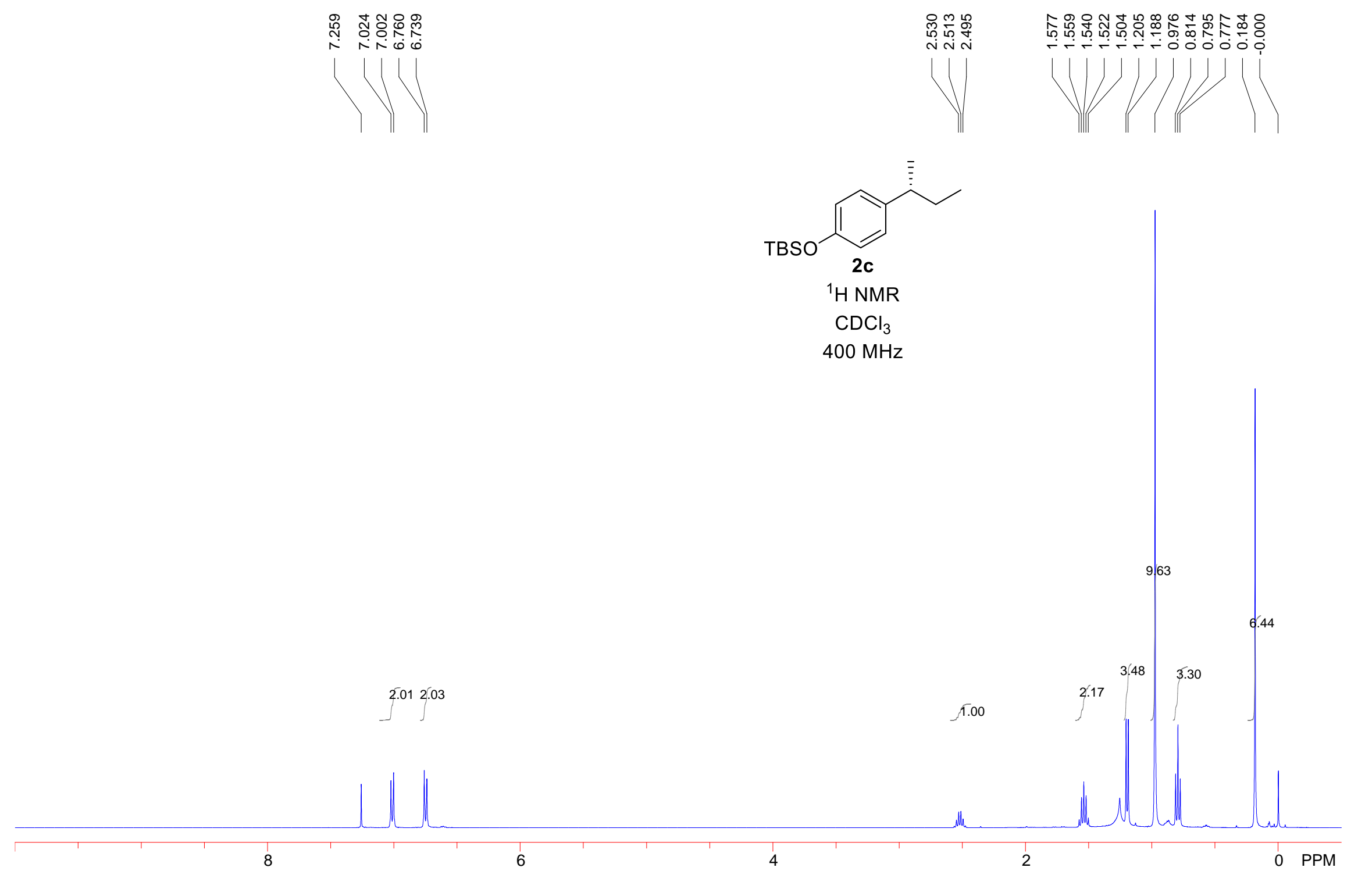




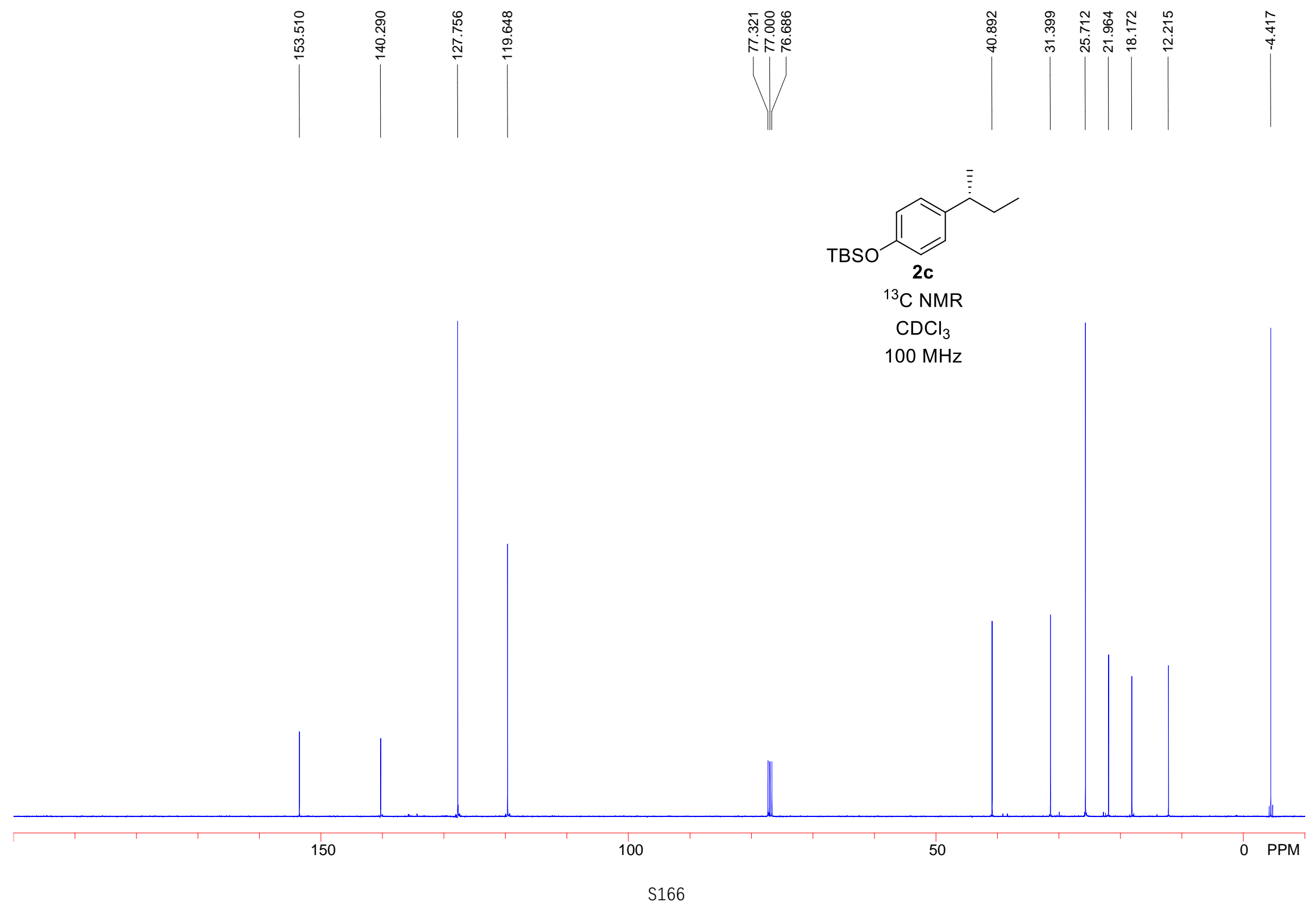




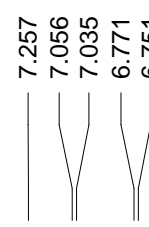

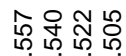

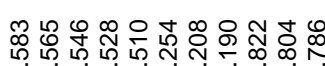

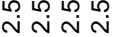

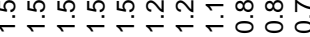

웅
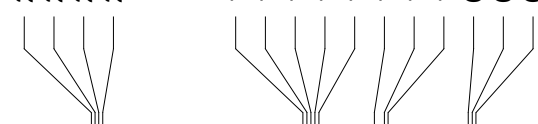

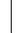

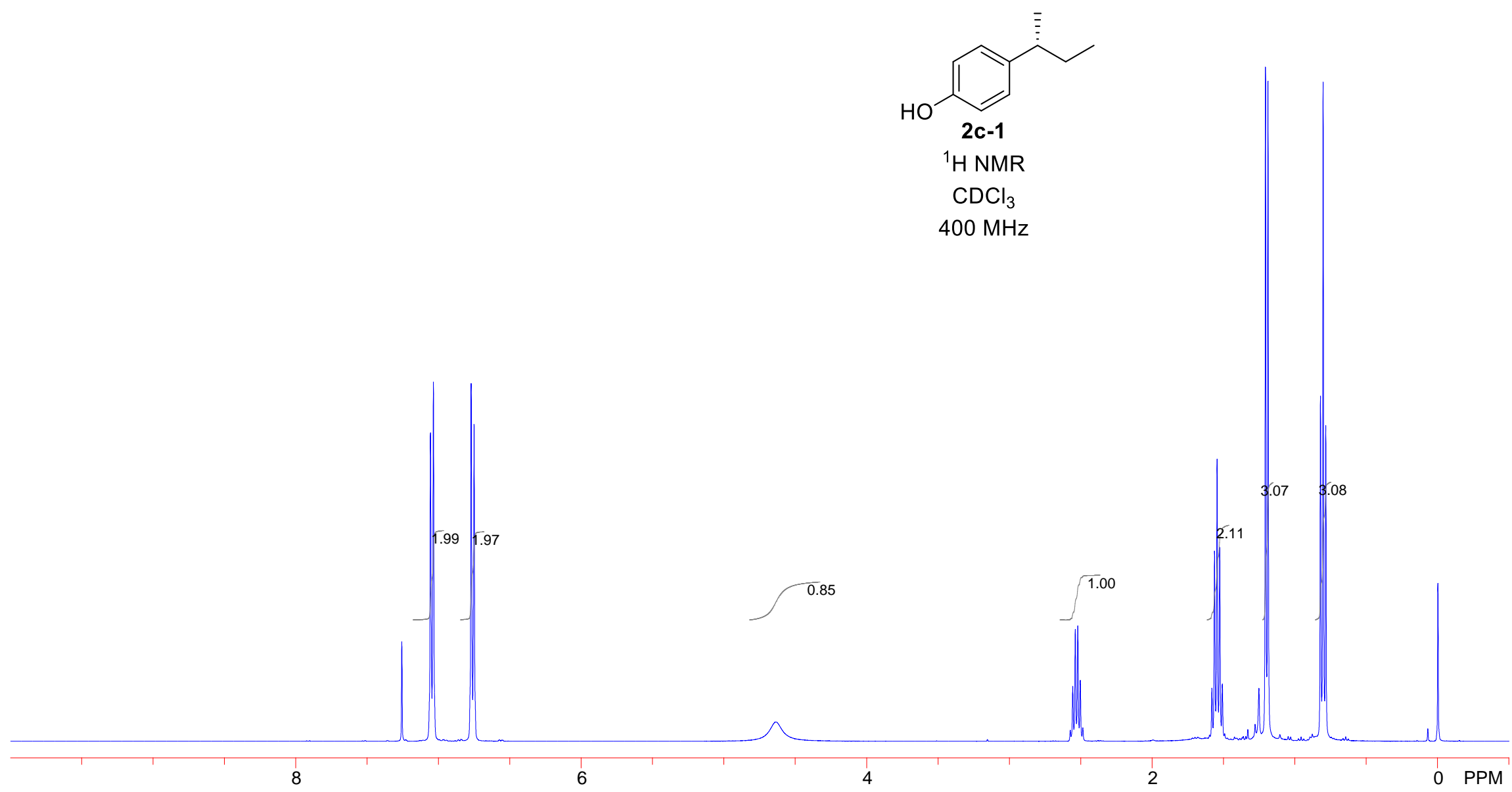




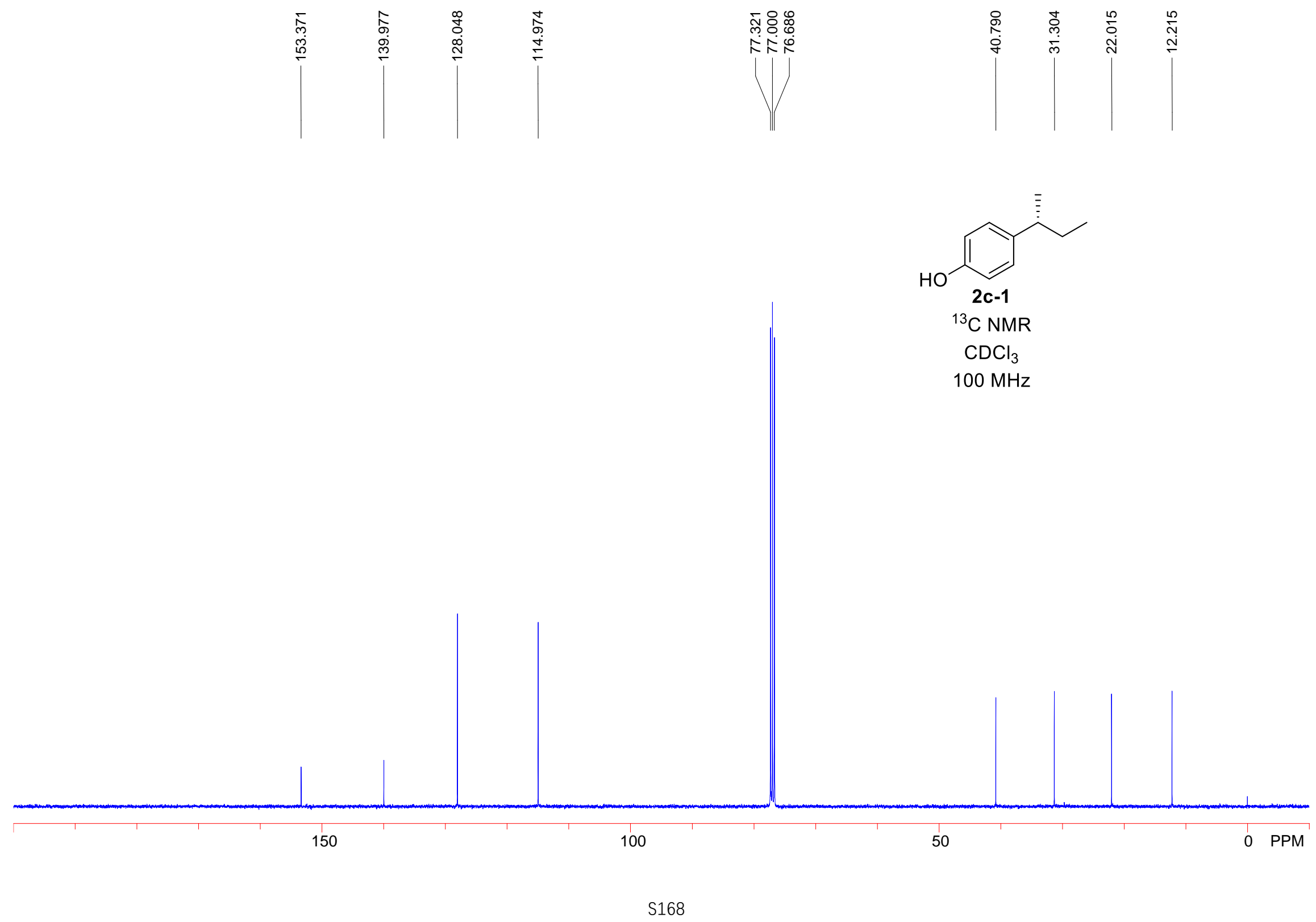




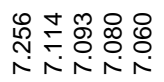

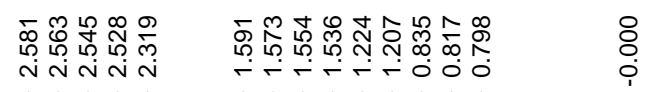

$(1)$
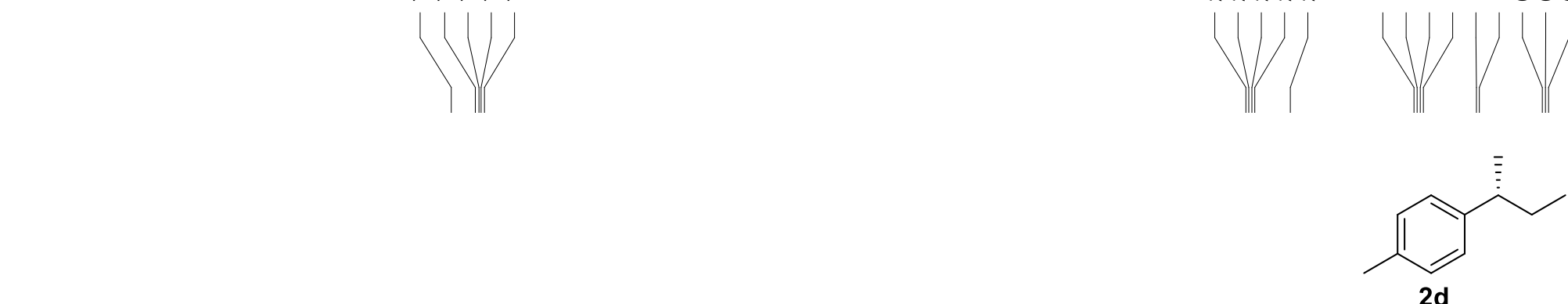

${ }^{1} \mathrm{H}$ NMR

$\mathrm{CDCl}_{3}$

$400 \mathrm{MHz}$

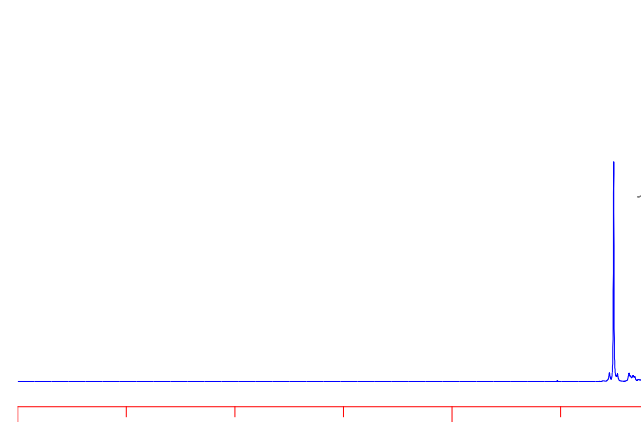

3.95 
壾繁要

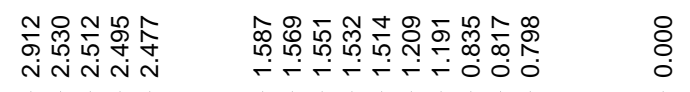

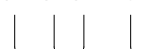

$\mathrm{Me}_{2} \mathrm{~N}$

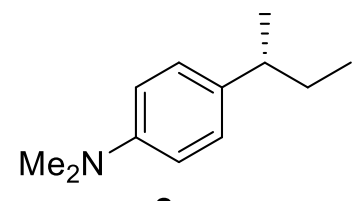

$2 e$

${ }^{1} \mathrm{H}$ NMR

$\mathrm{CDCl}_{3}$

$400 \mathrm{MHz}$

0 PPM 


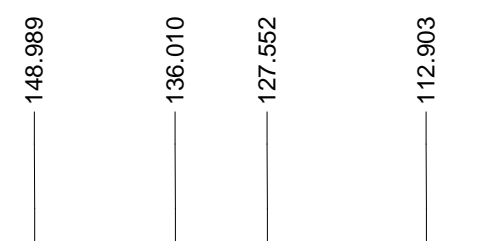

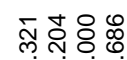

定㝊定

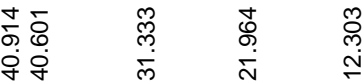

$\sqrt{1}$

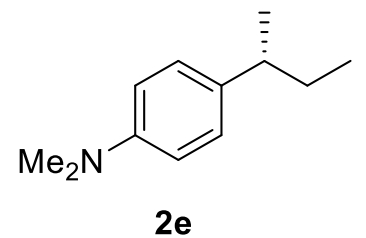

${ }^{13} \mathrm{C}$ NMR

$\mathrm{CDCl}_{3}$

$100 \mathrm{MHz}$

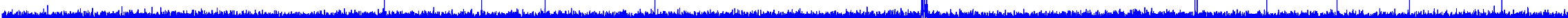




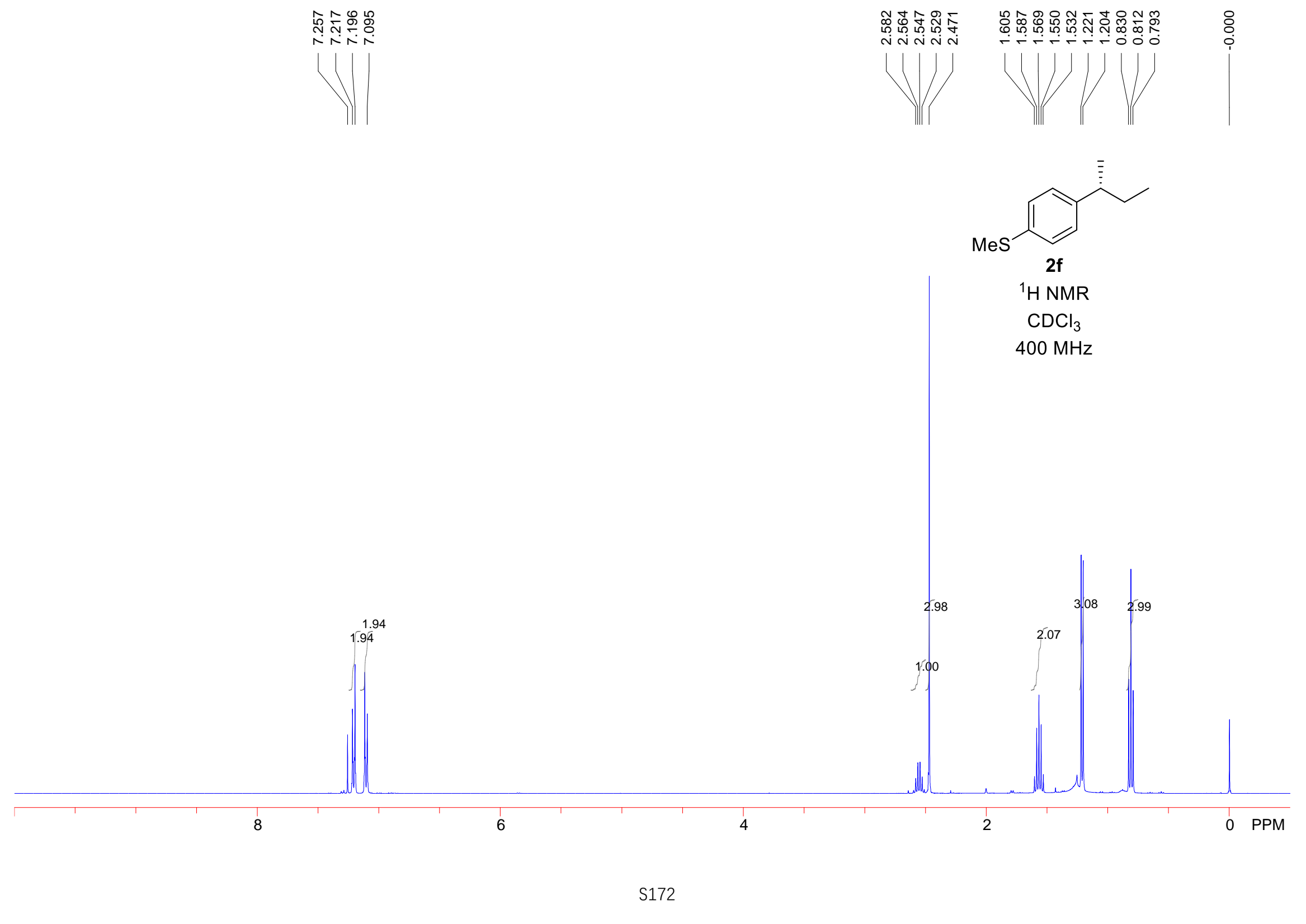




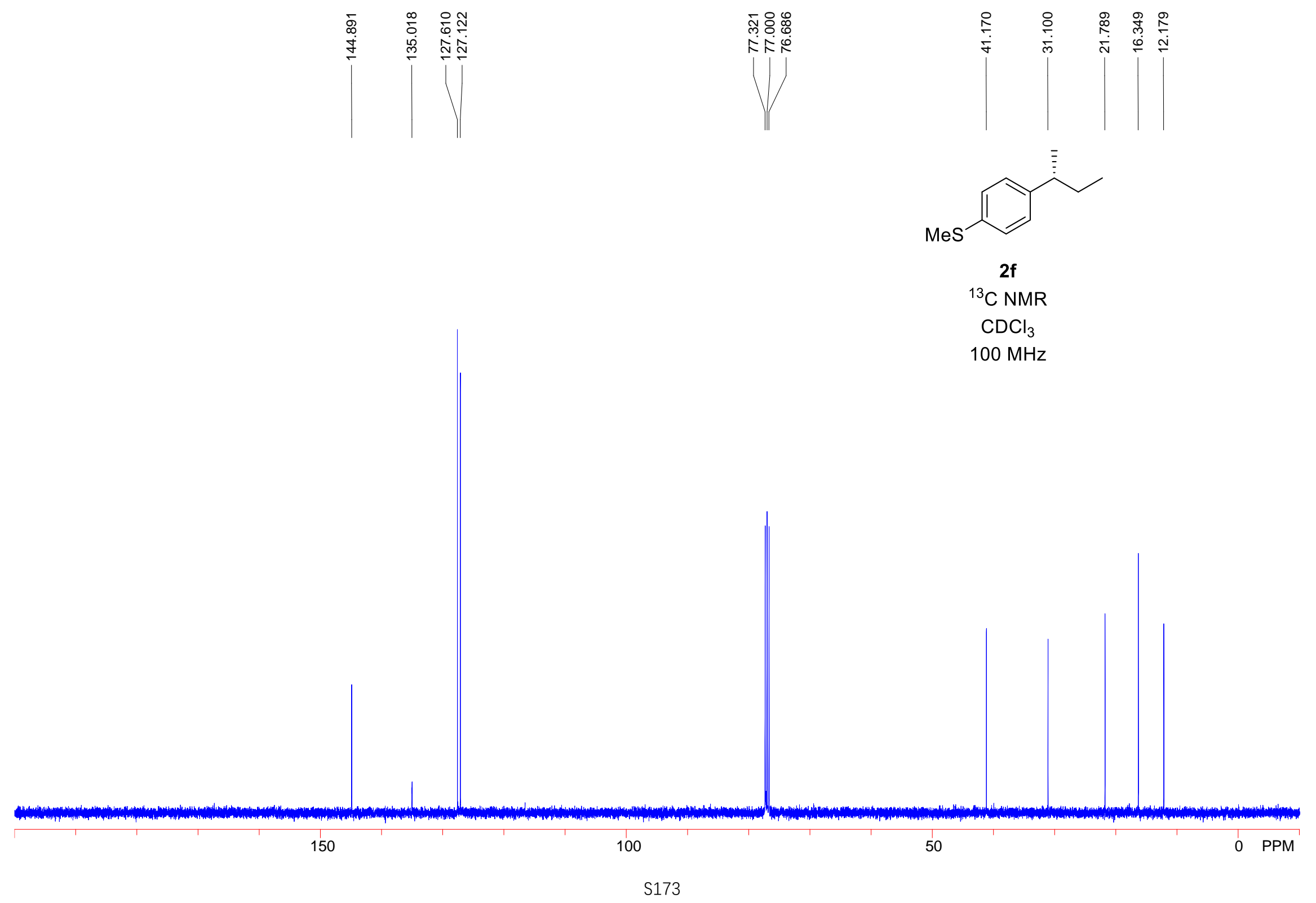




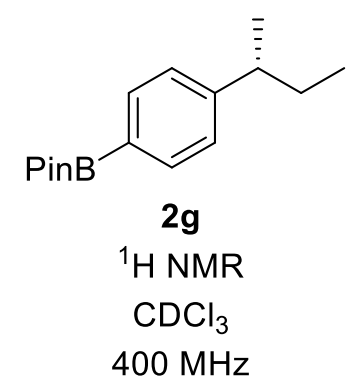

1.00 


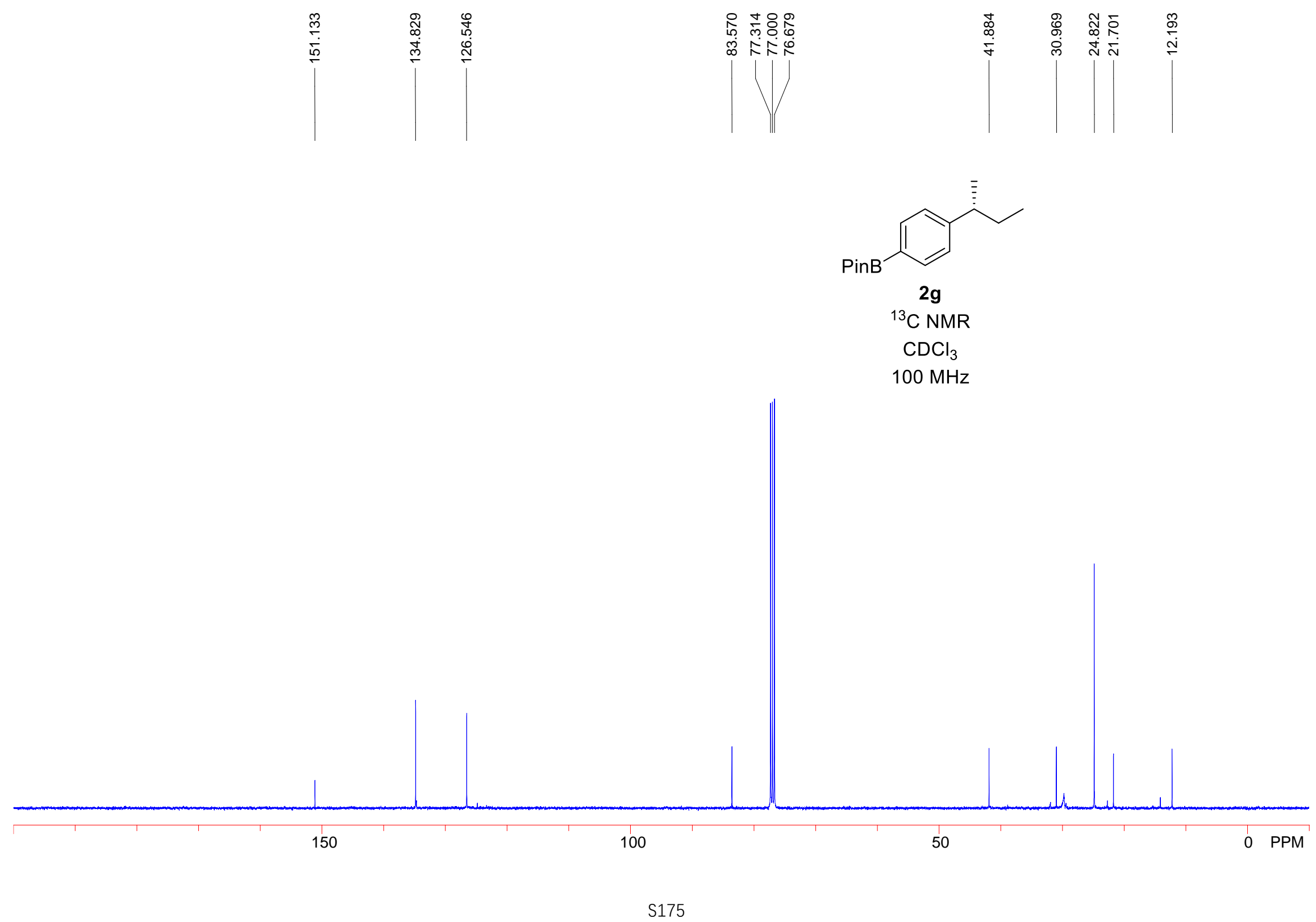




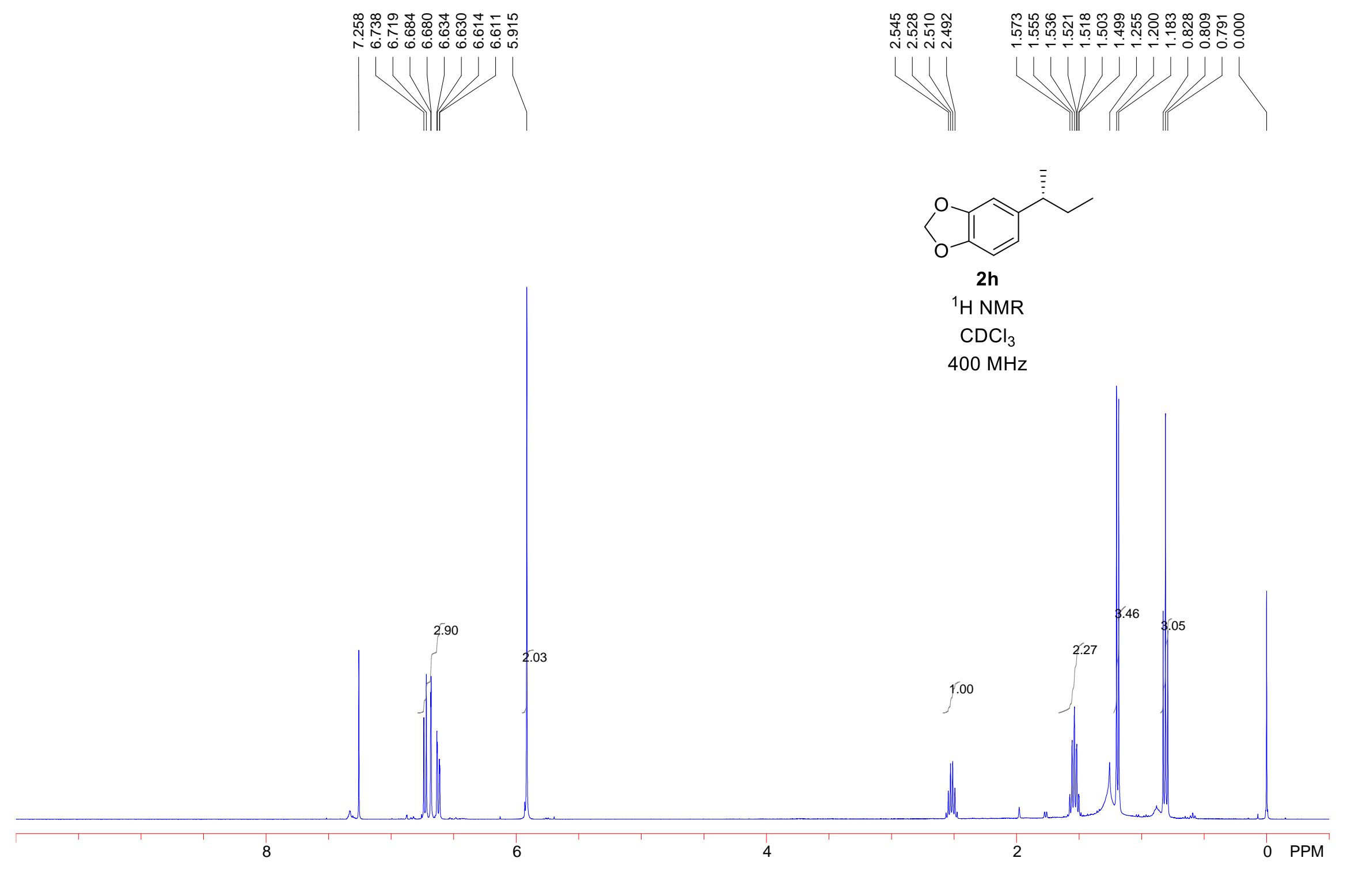




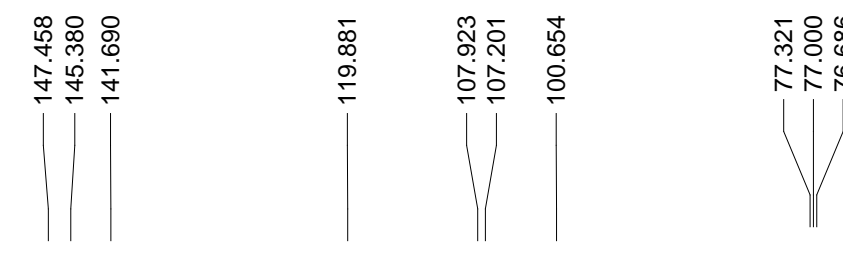

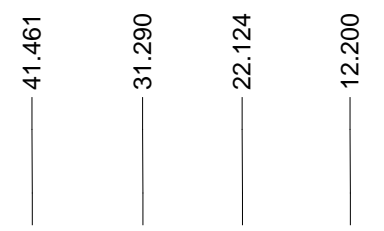

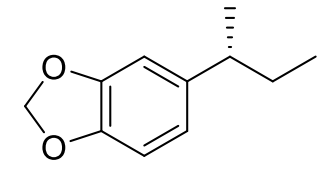

$2 \mathrm{~h}$

${ }^{13} \mathrm{C}$ NMR

$\mathrm{CDCl}_{3}$

$100 \mathrm{MHz}$

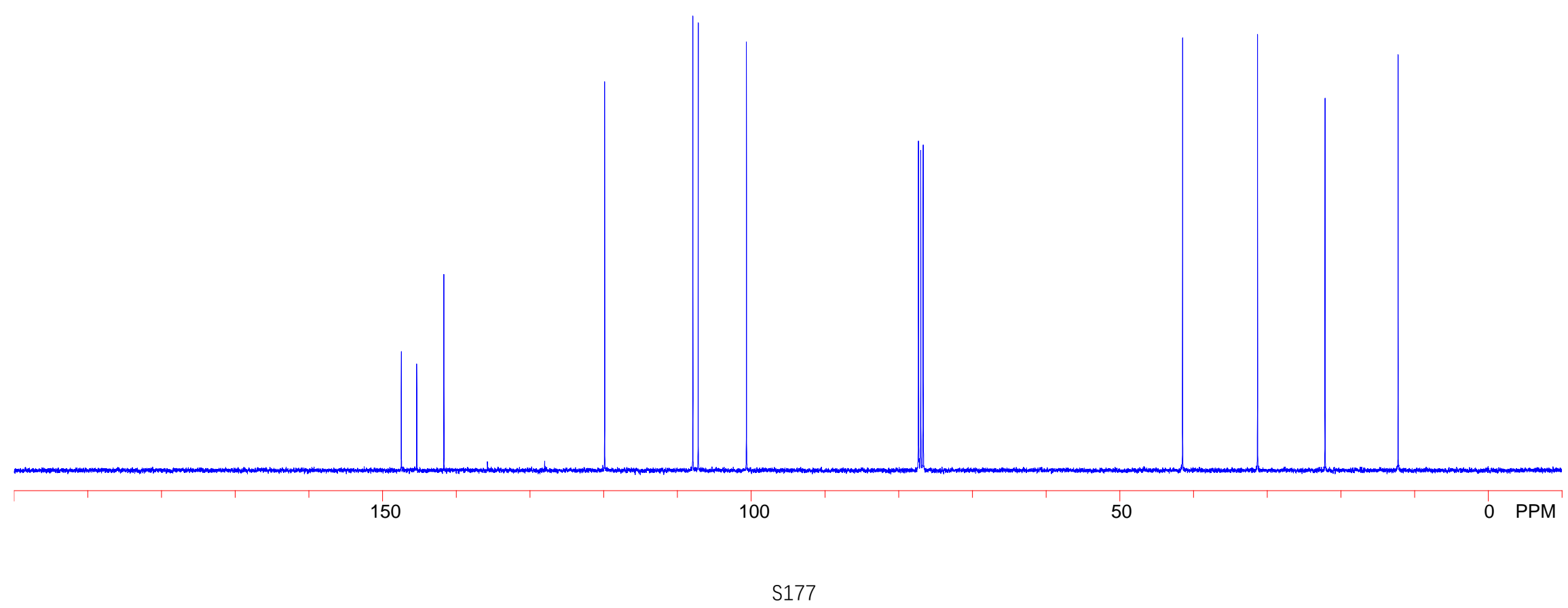



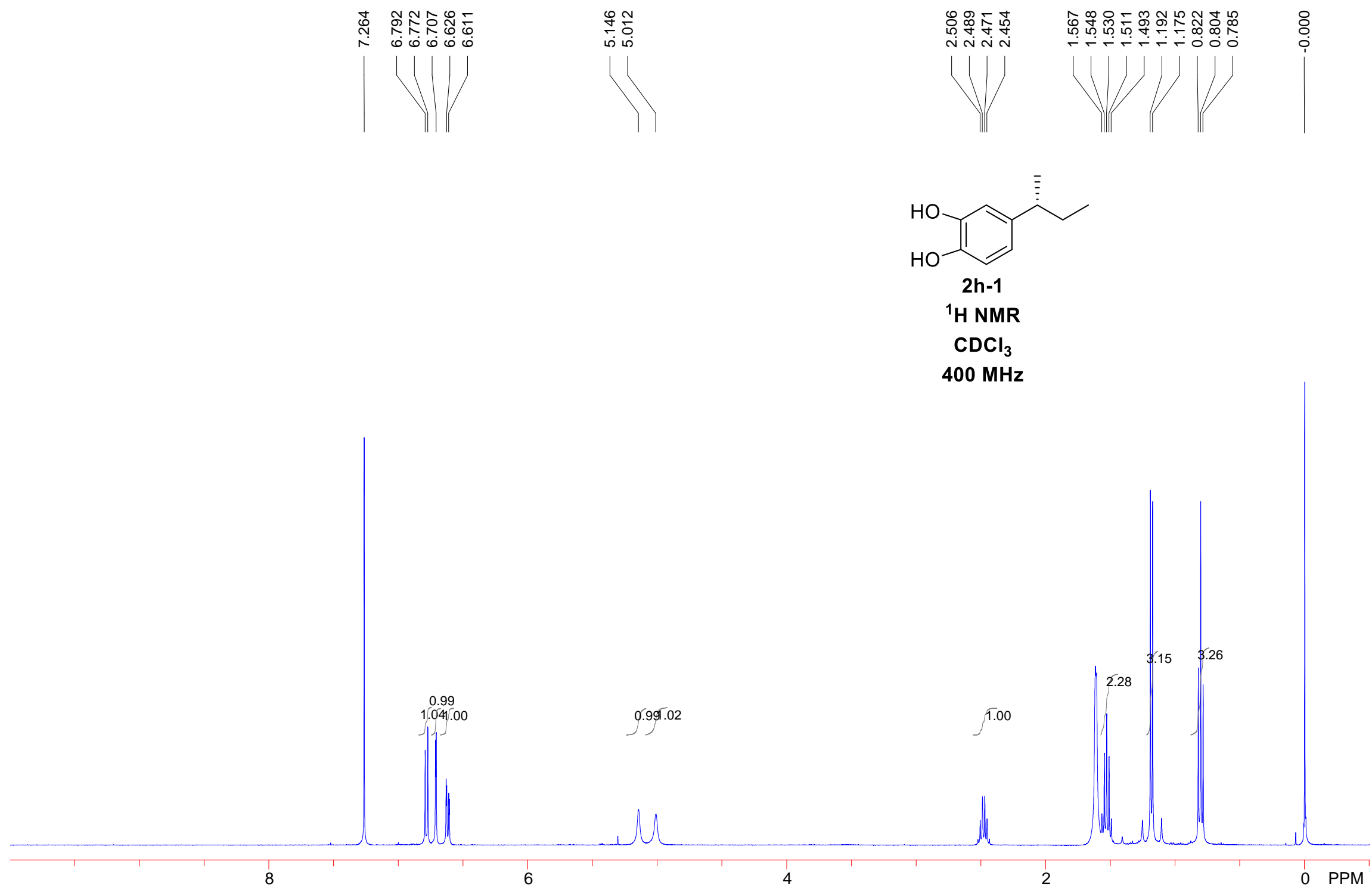


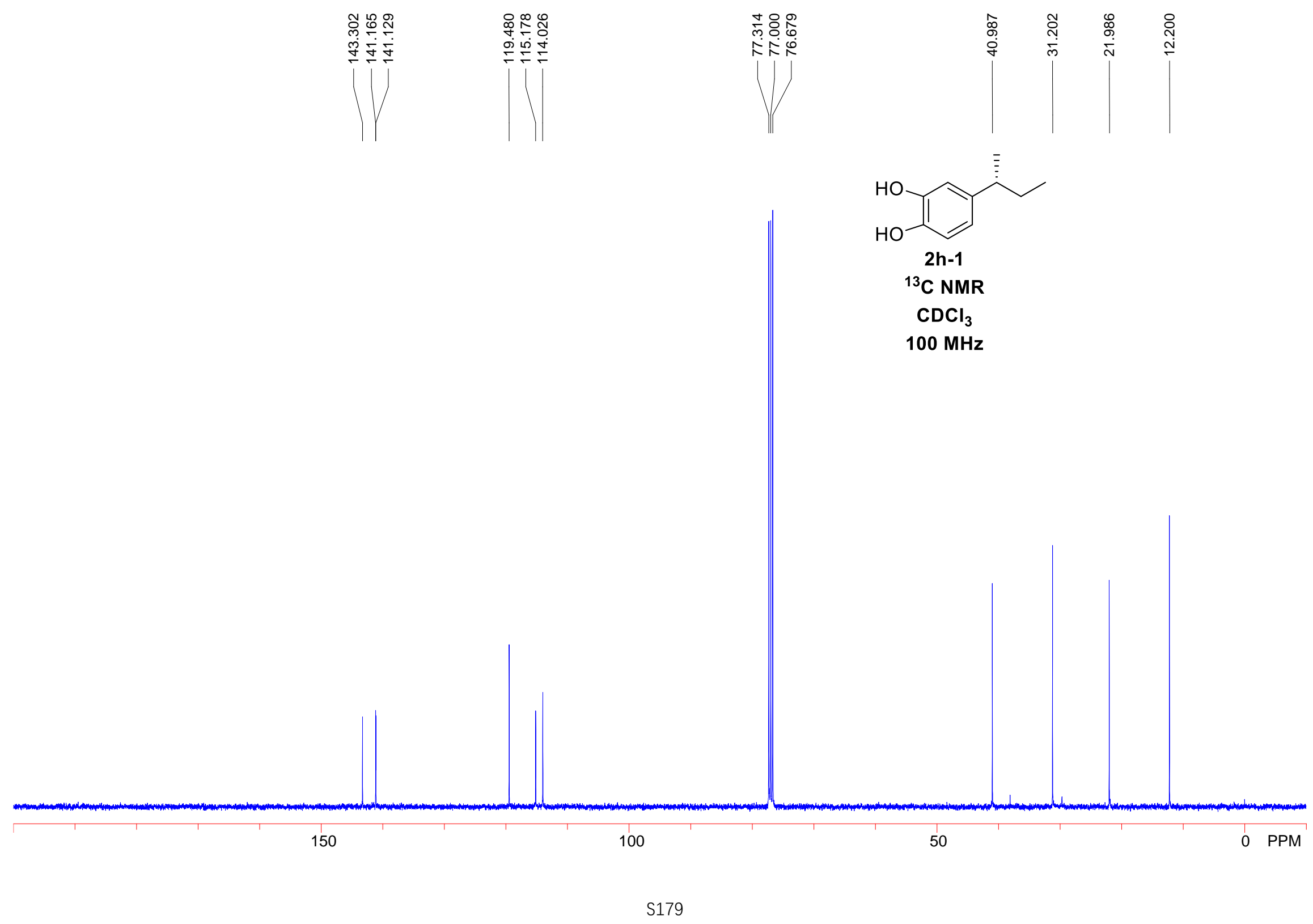




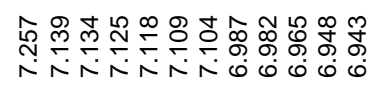

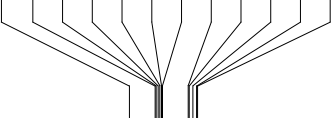

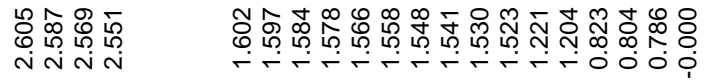



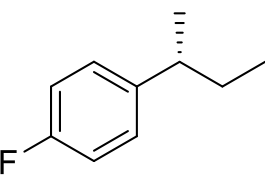

2i

${ }^{1} \mathrm{H}$ NMR

$\mathrm{CDCl}_{3}$

$400 \mathrm{MHz}$

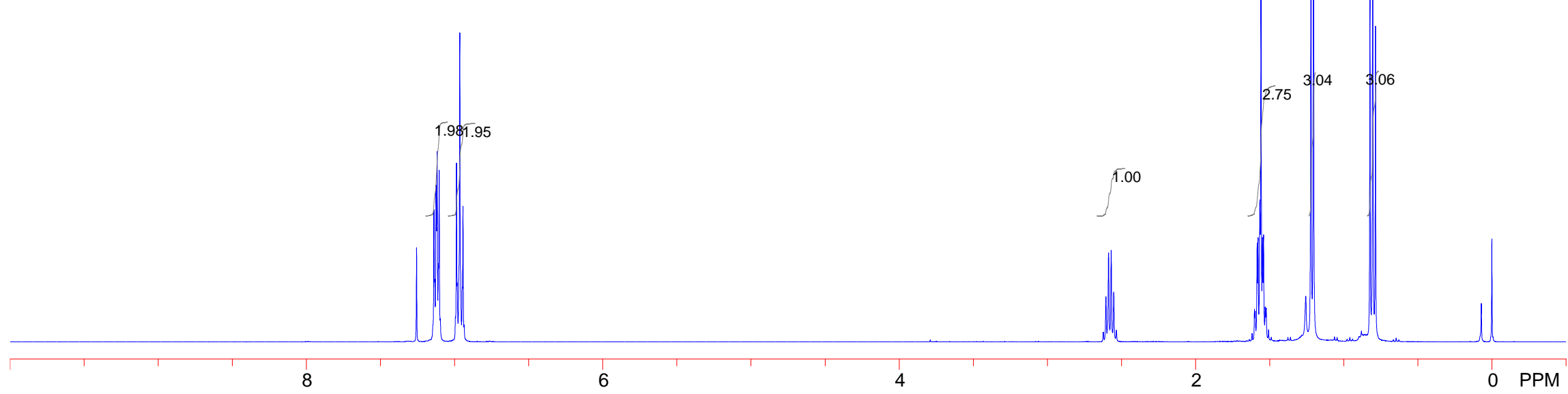




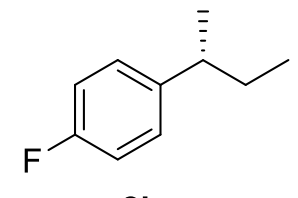

$2 \mathbf{i}$

${ }^{19} \mathrm{~F}$ NMR

$\mathrm{CDCl}_{3}$

$376 \mathrm{MHz}$ 


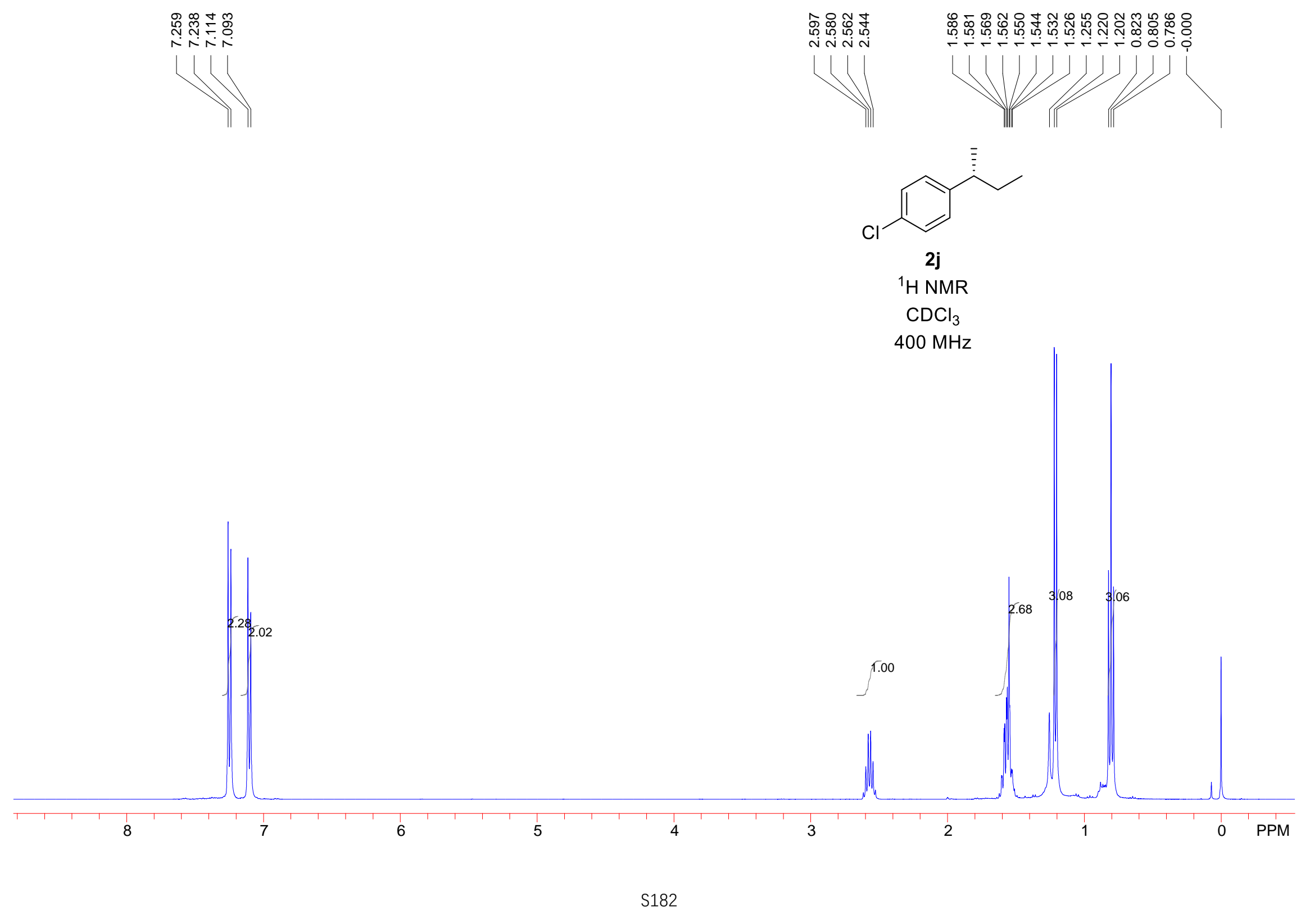




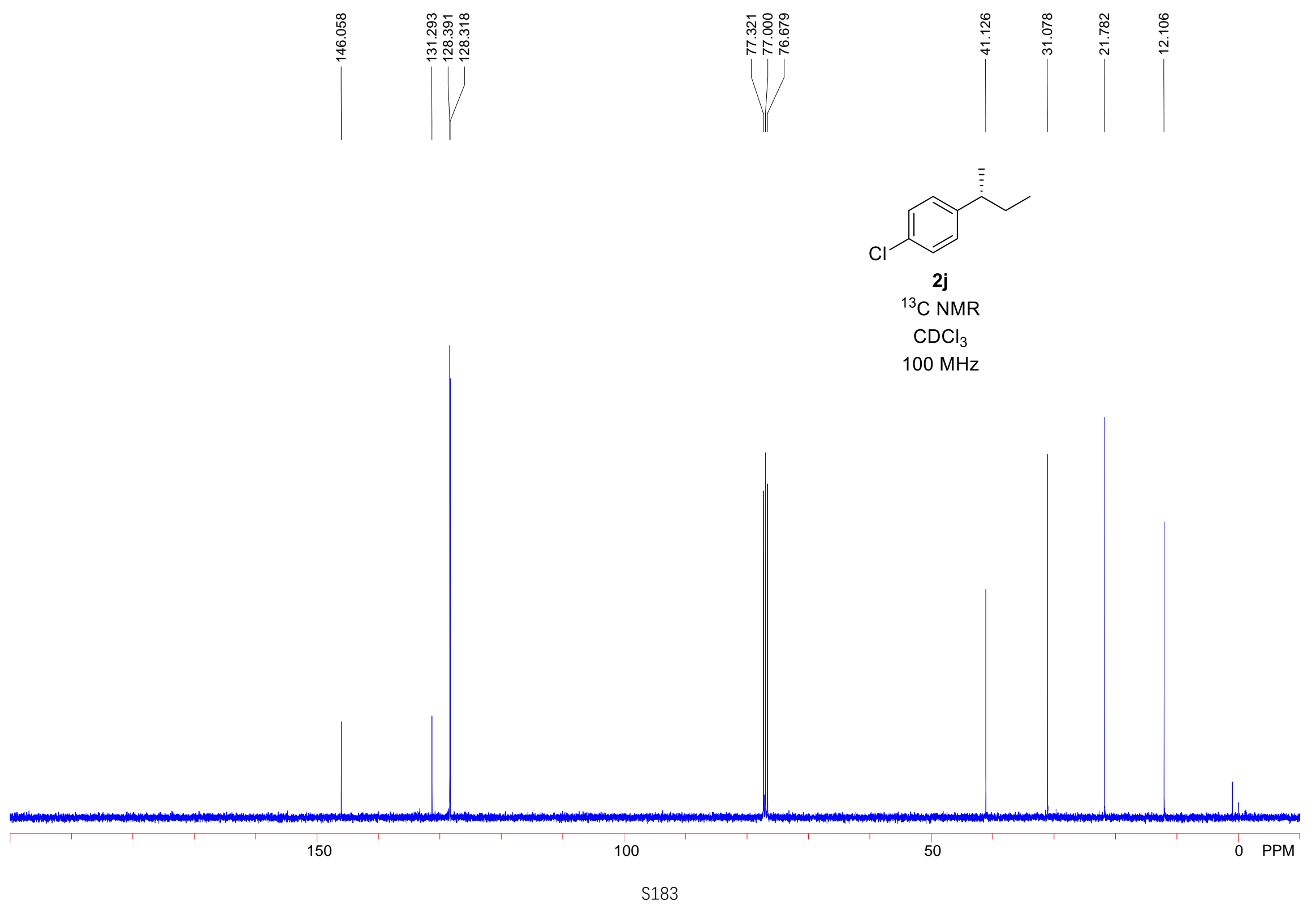




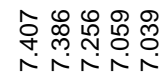

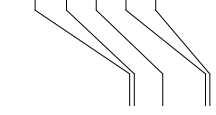

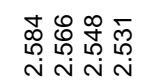

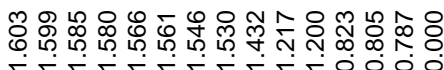
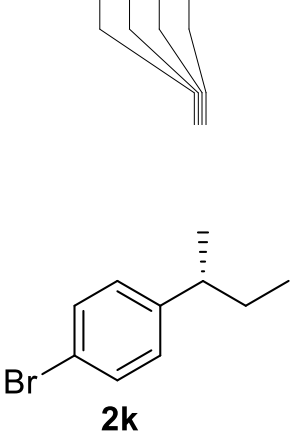

H NMR

$\mathrm{CDCl}_{3}$

$400 \mathrm{MHz}$

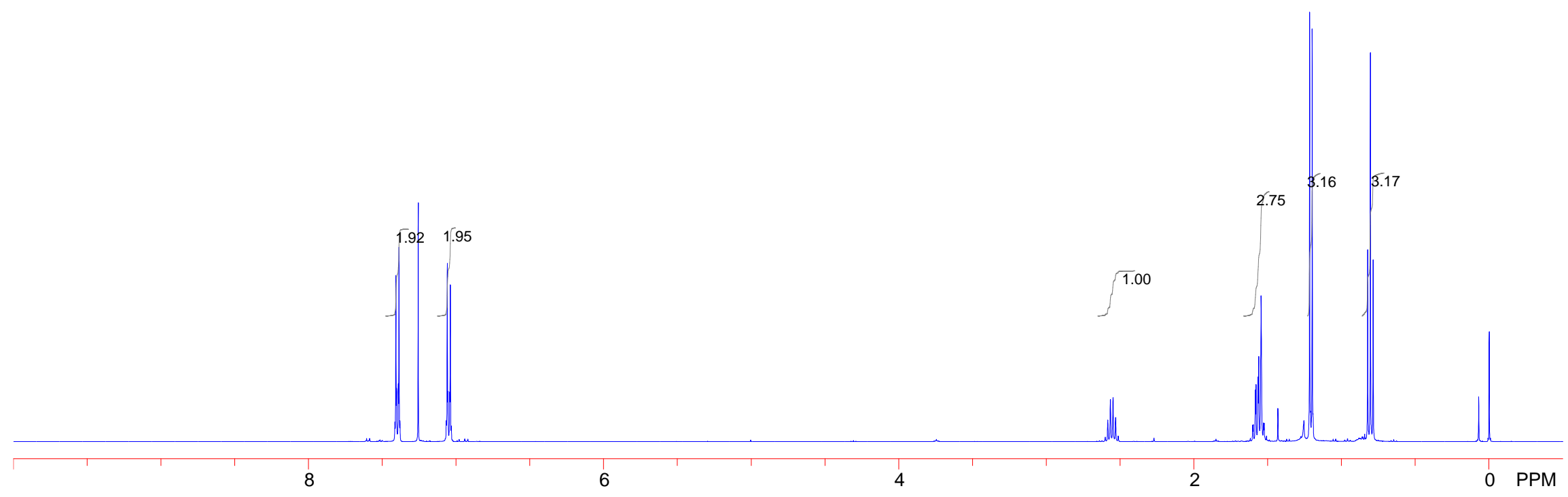




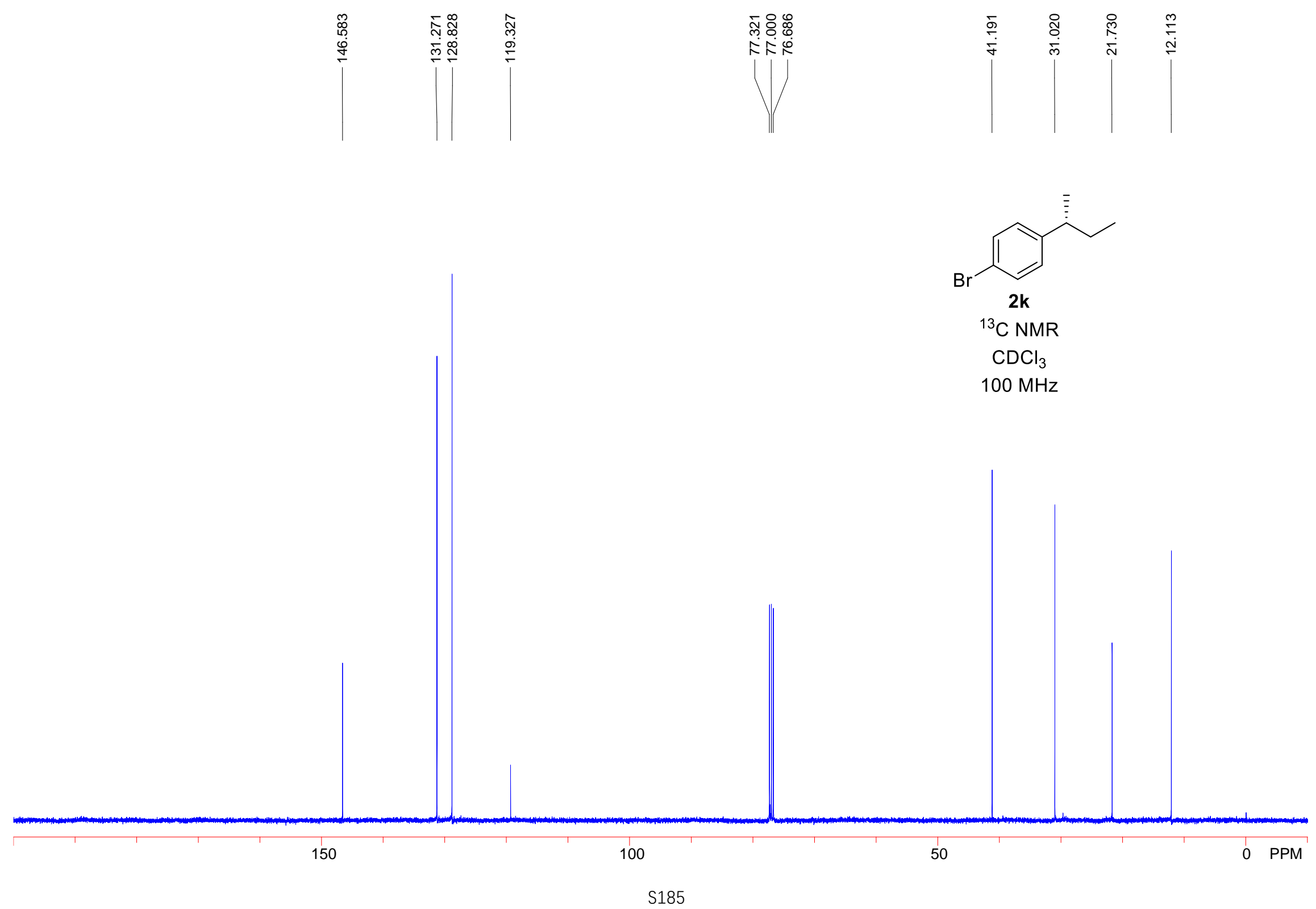




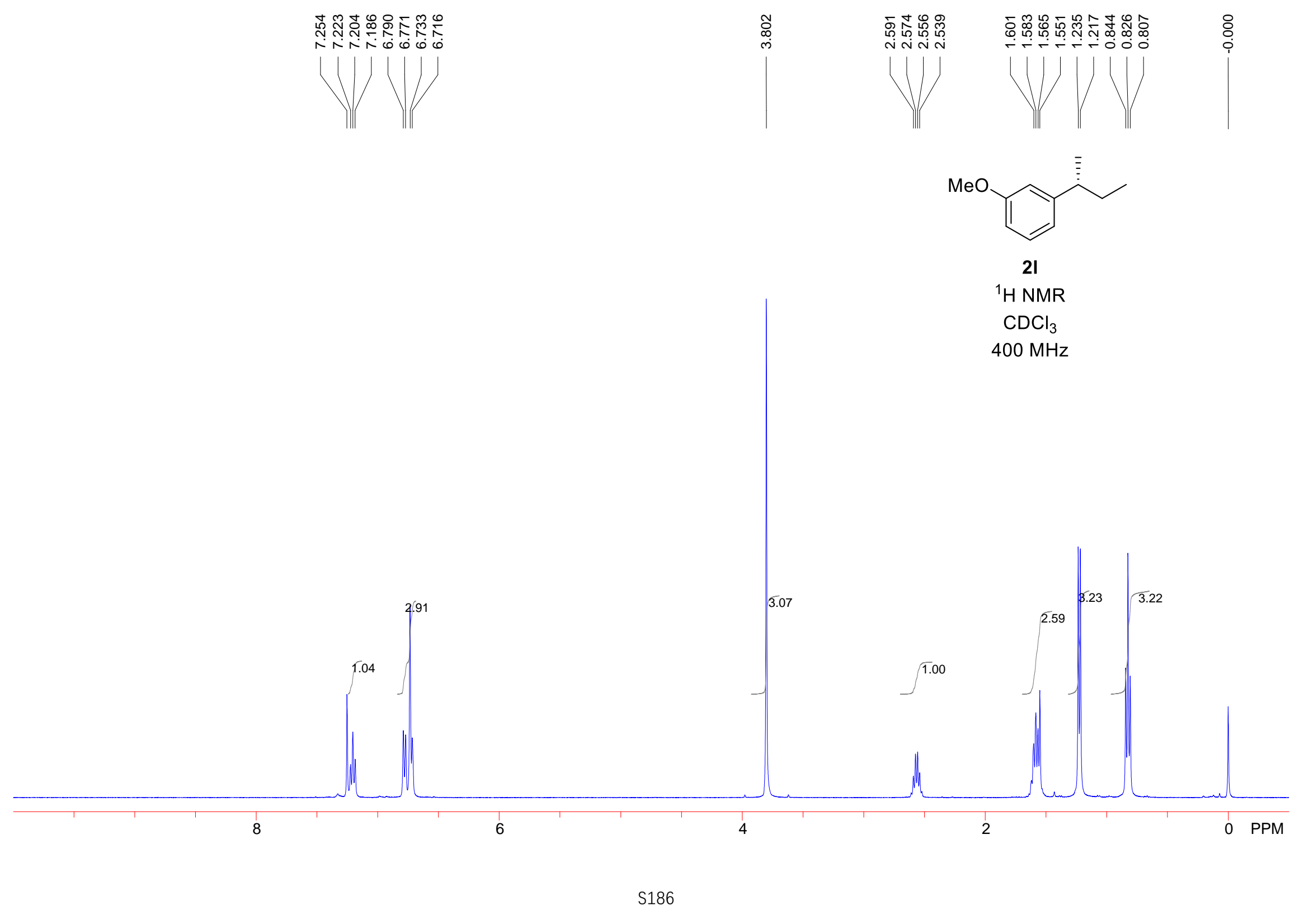



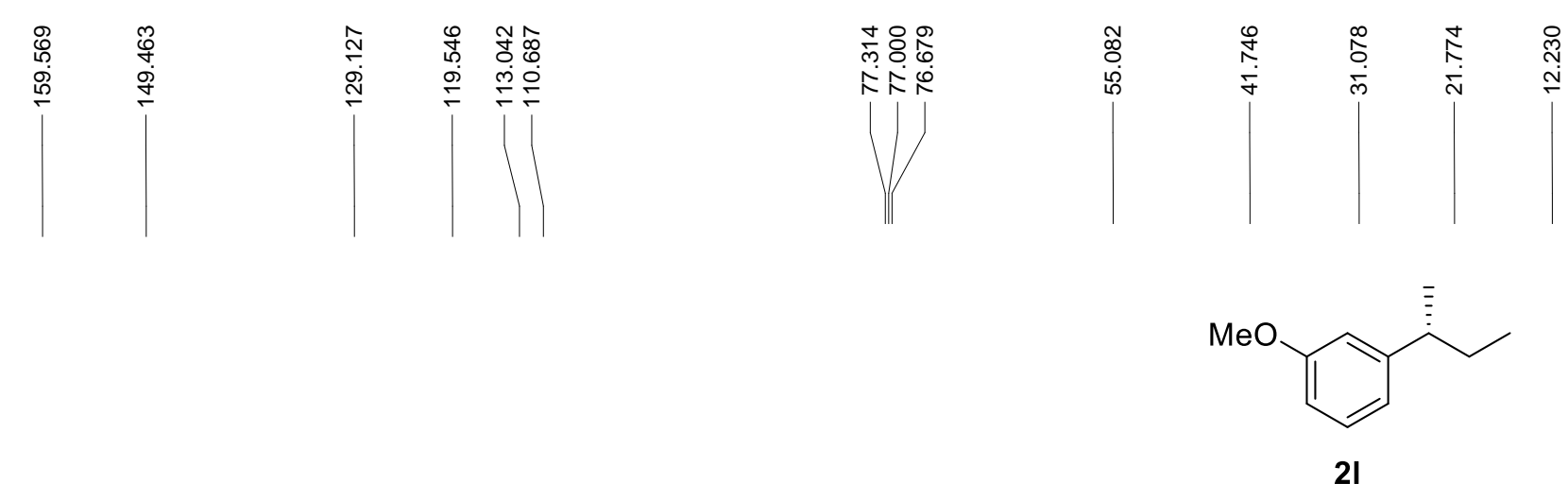

${ }^{13} \mathrm{C}$ NMR

$\mathrm{CDCl}_{3}$

$100 \mathrm{MHz}$

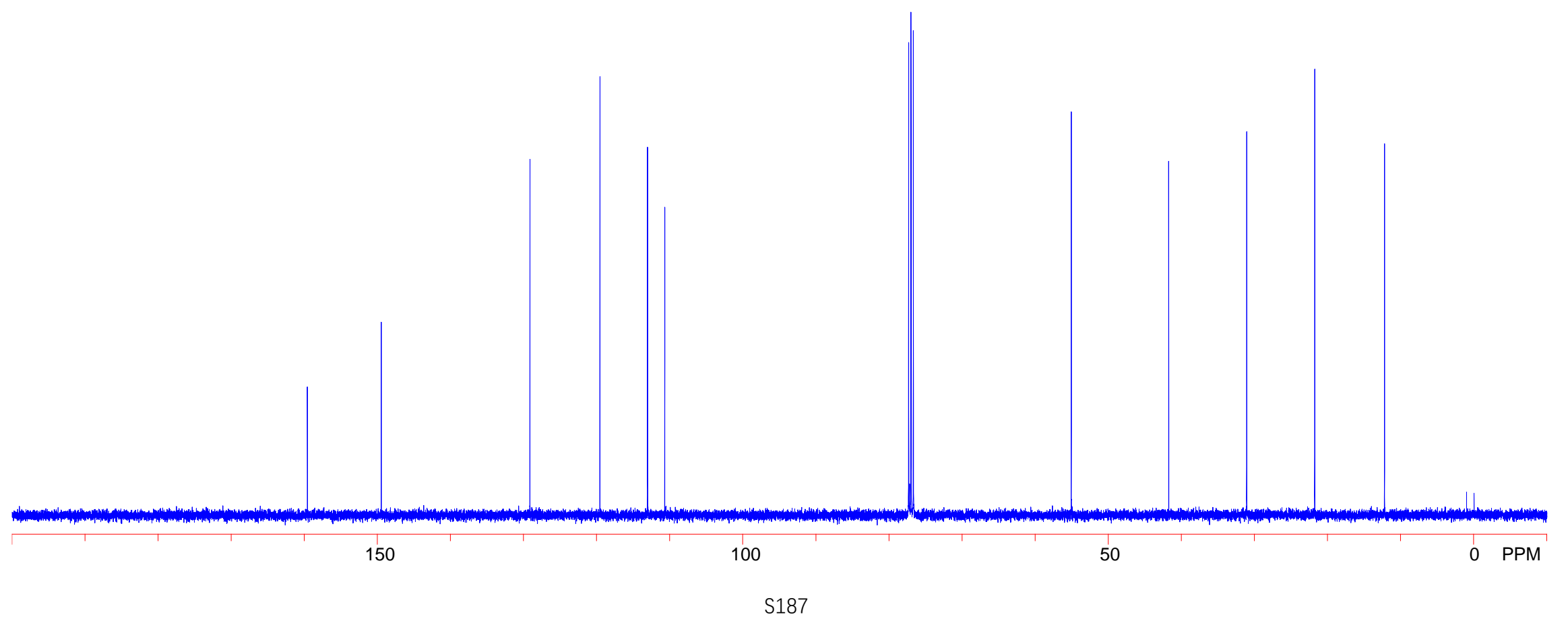




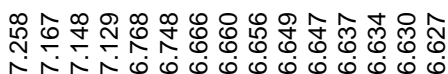

递
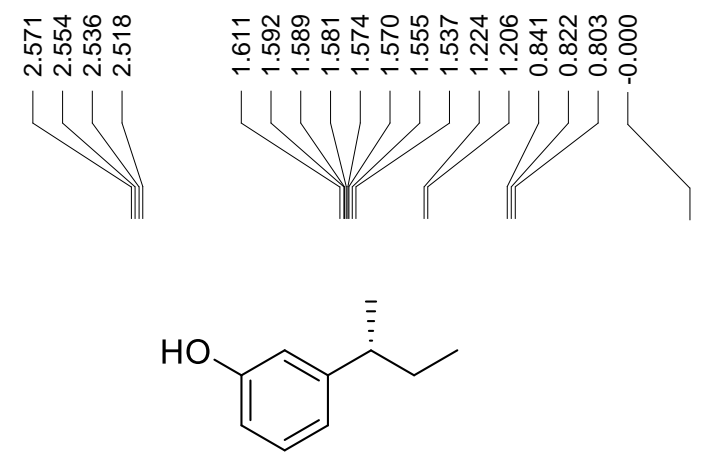

2I-1

${ }^{1} \mathrm{H}$ NMR

$\mathrm{CDCl}_{3}$

$400 \mathrm{MHz}$

2.97

1.01
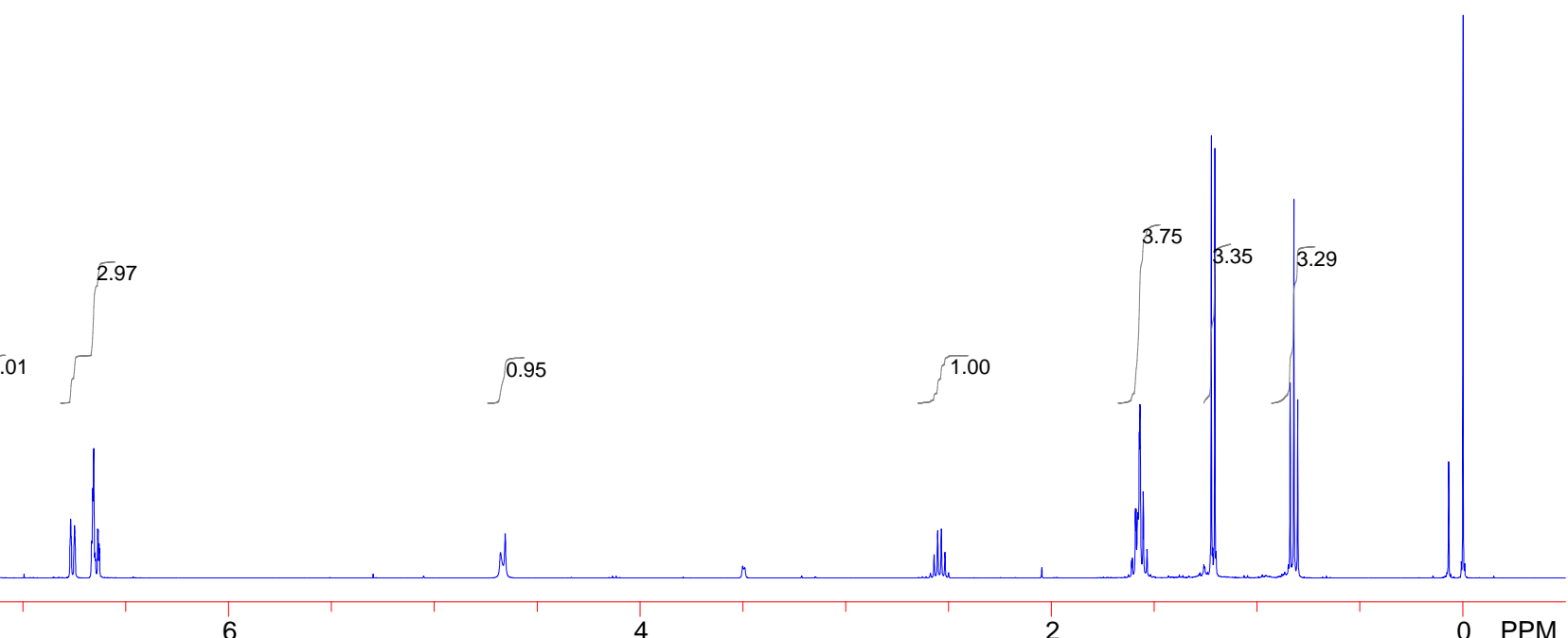


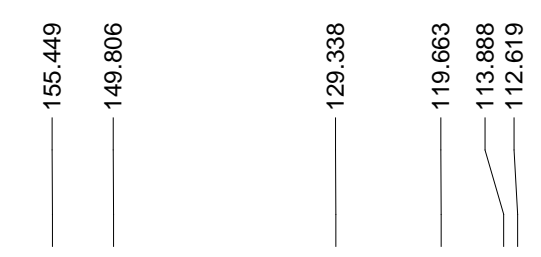

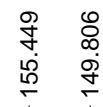

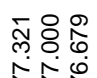

상요

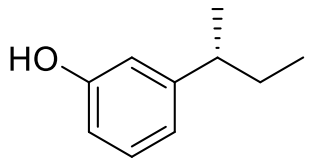

2I-1

${ }^{13} \mathrm{C}$ NMR $\mathrm{CDCl}_{3}$

$100 \mathrm{MHz}$

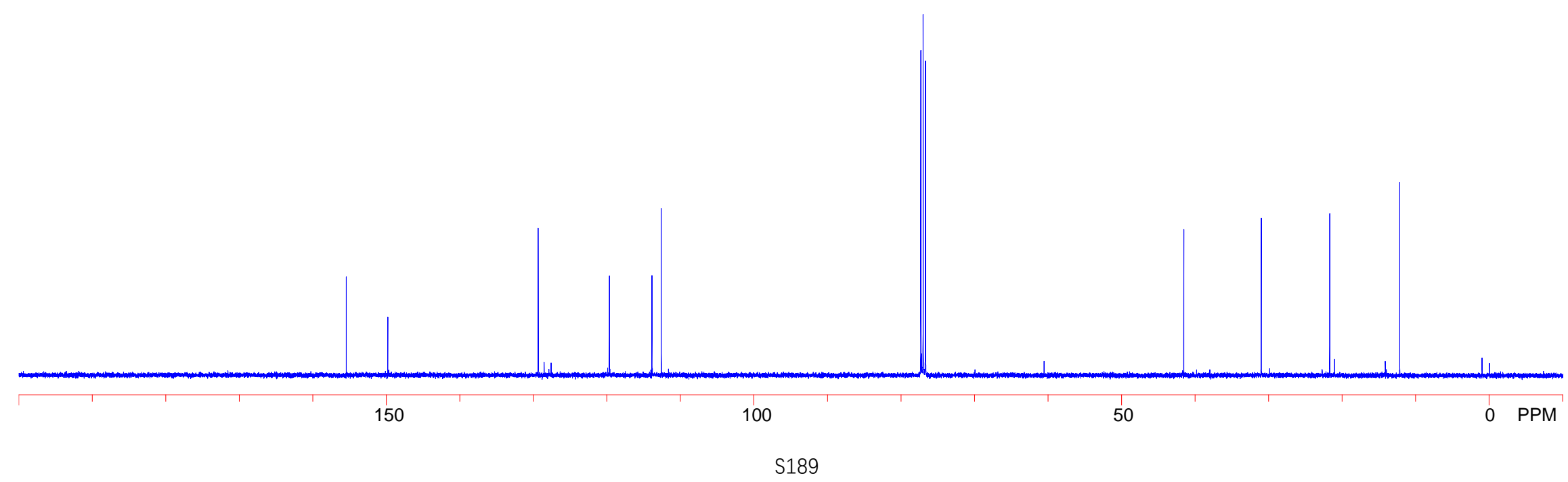




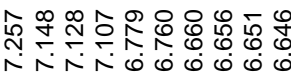

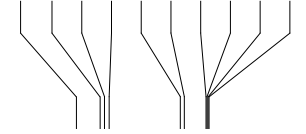

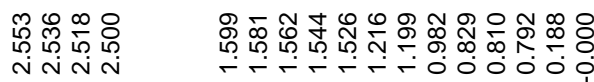

v

TBSO

${ }^{1} \mathrm{H}$ NMR

$\mathrm{CDCl}_{3}$

$400 \mathrm{MHz}$

9.98

$\begin{array}{rr} & 2.06 \\ 1.02 & 1.01\end{array}$

U. 

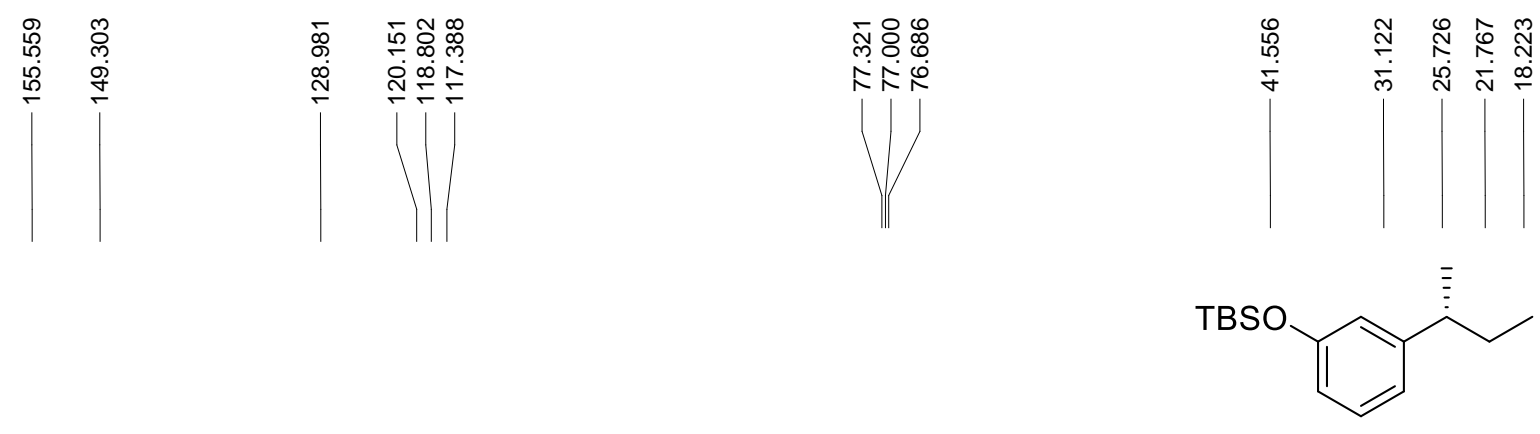

$2 \mathrm{~m}$

${ }^{13} \mathrm{H}$ NMR

$\mathrm{CDCl}_{3}$

$100 \mathrm{MHz}$

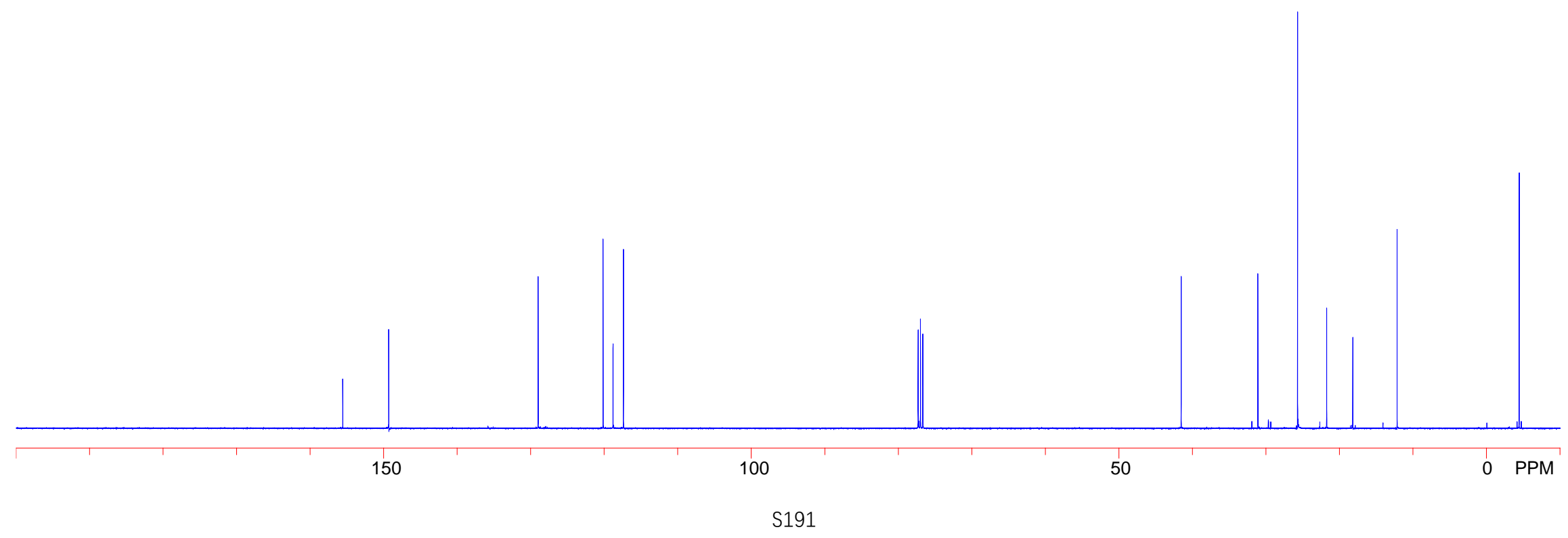




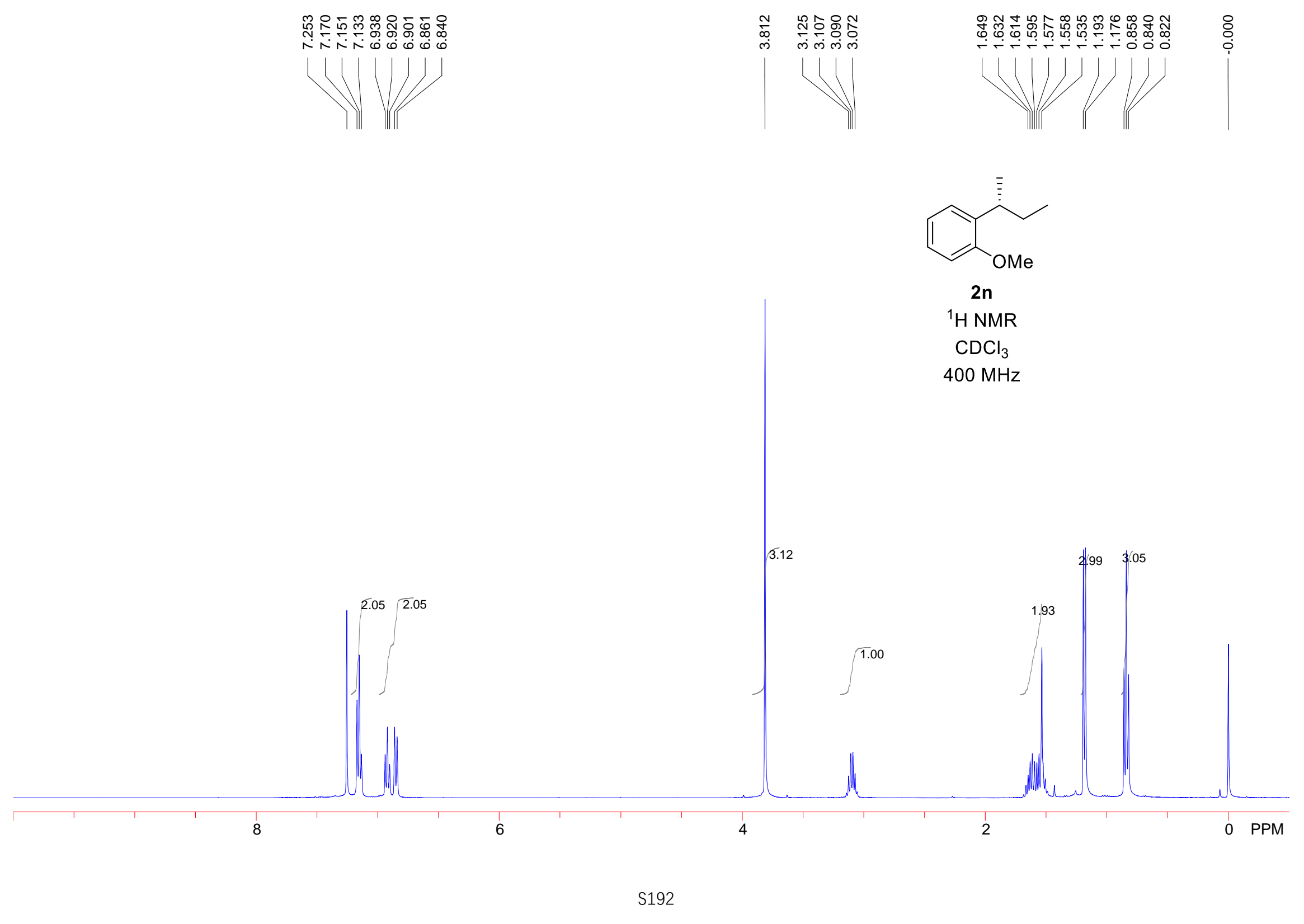




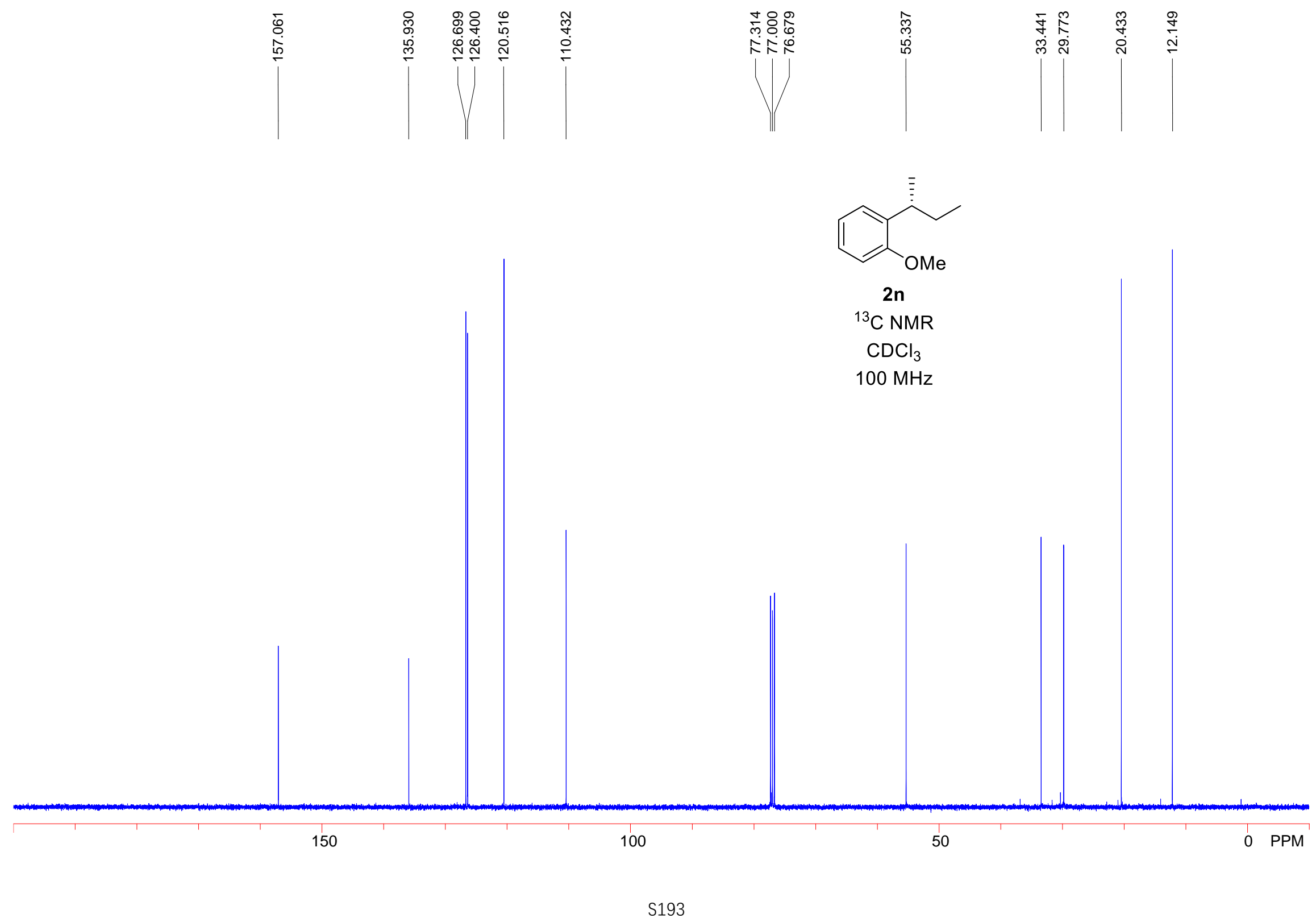




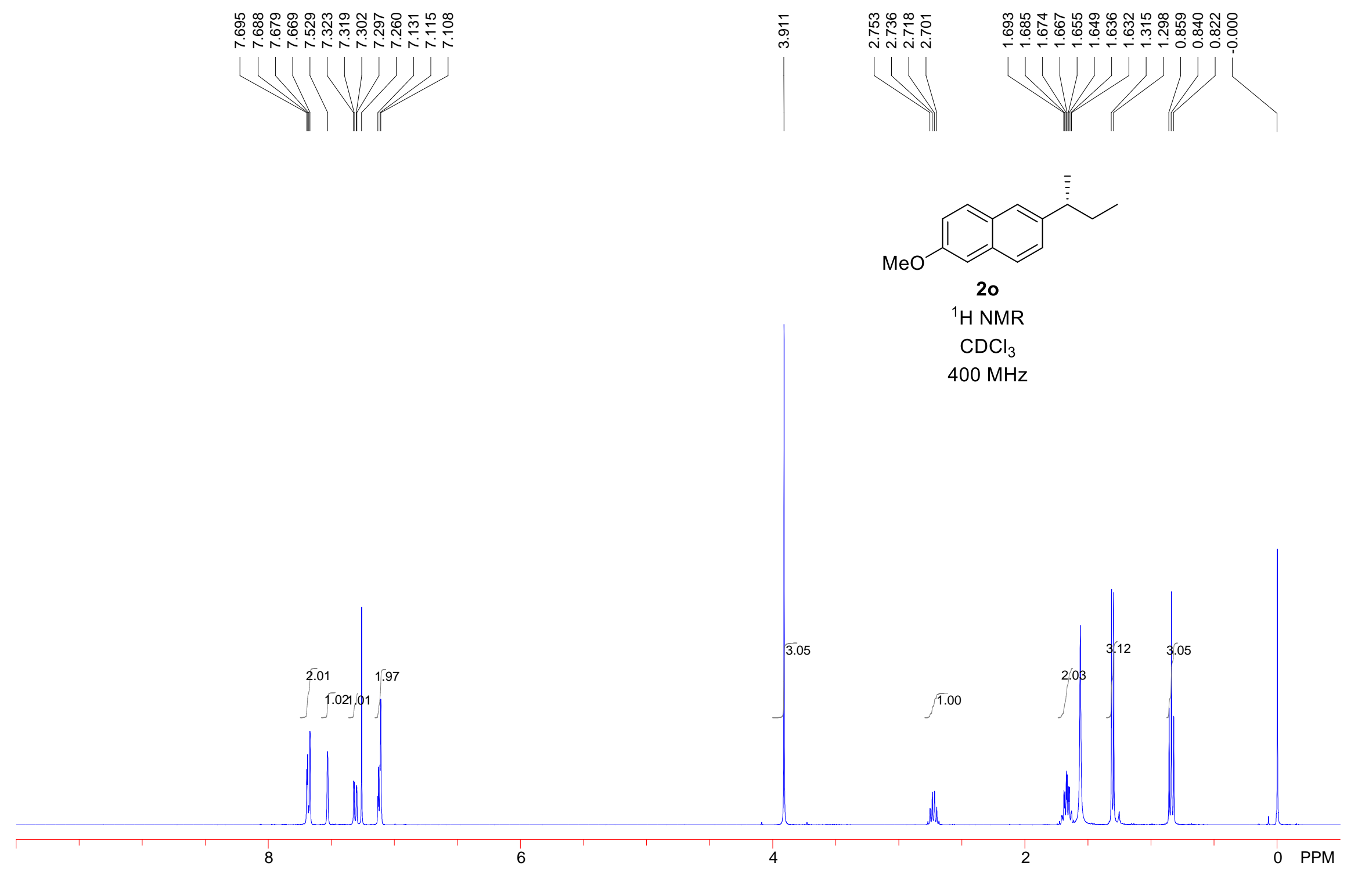




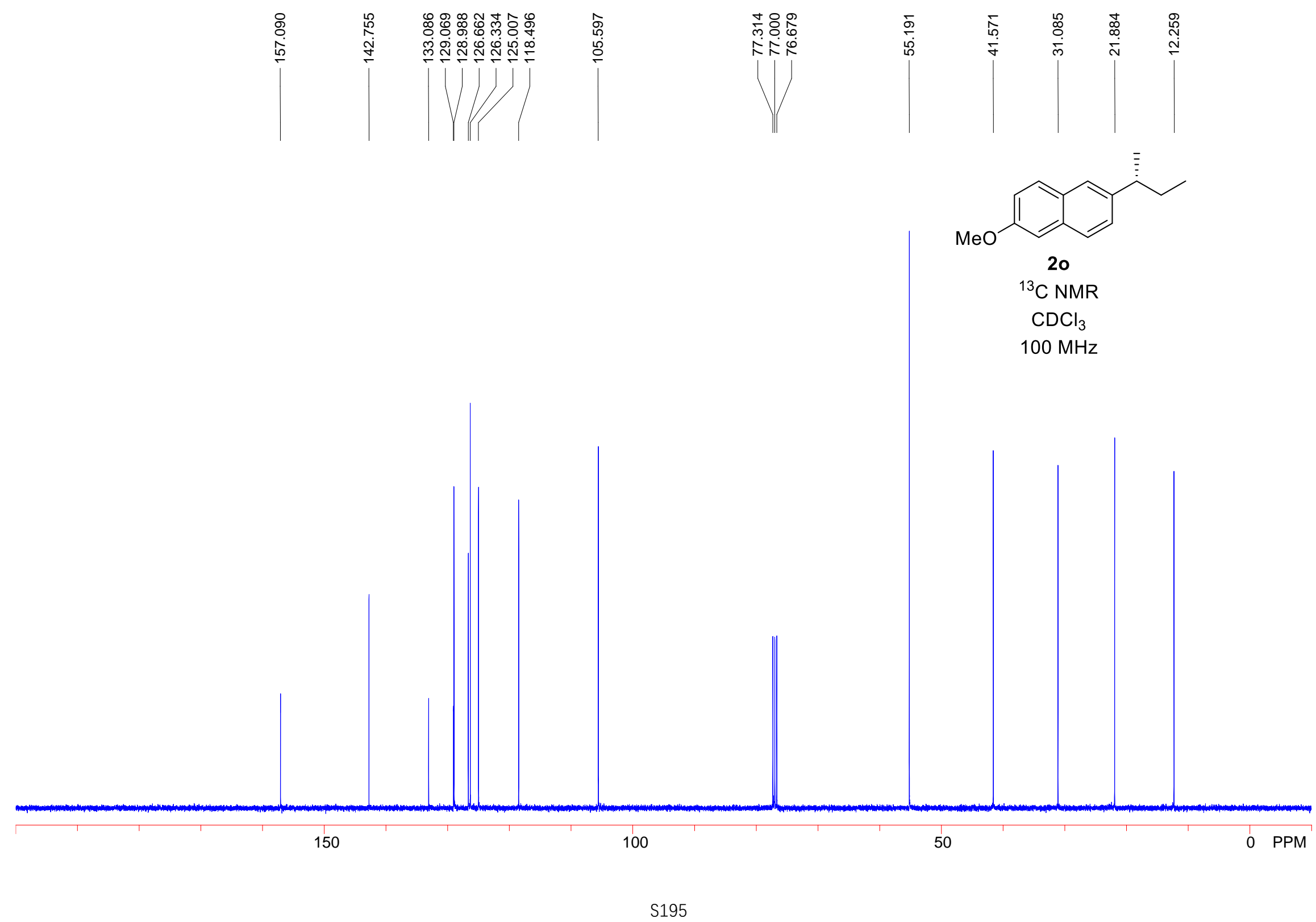




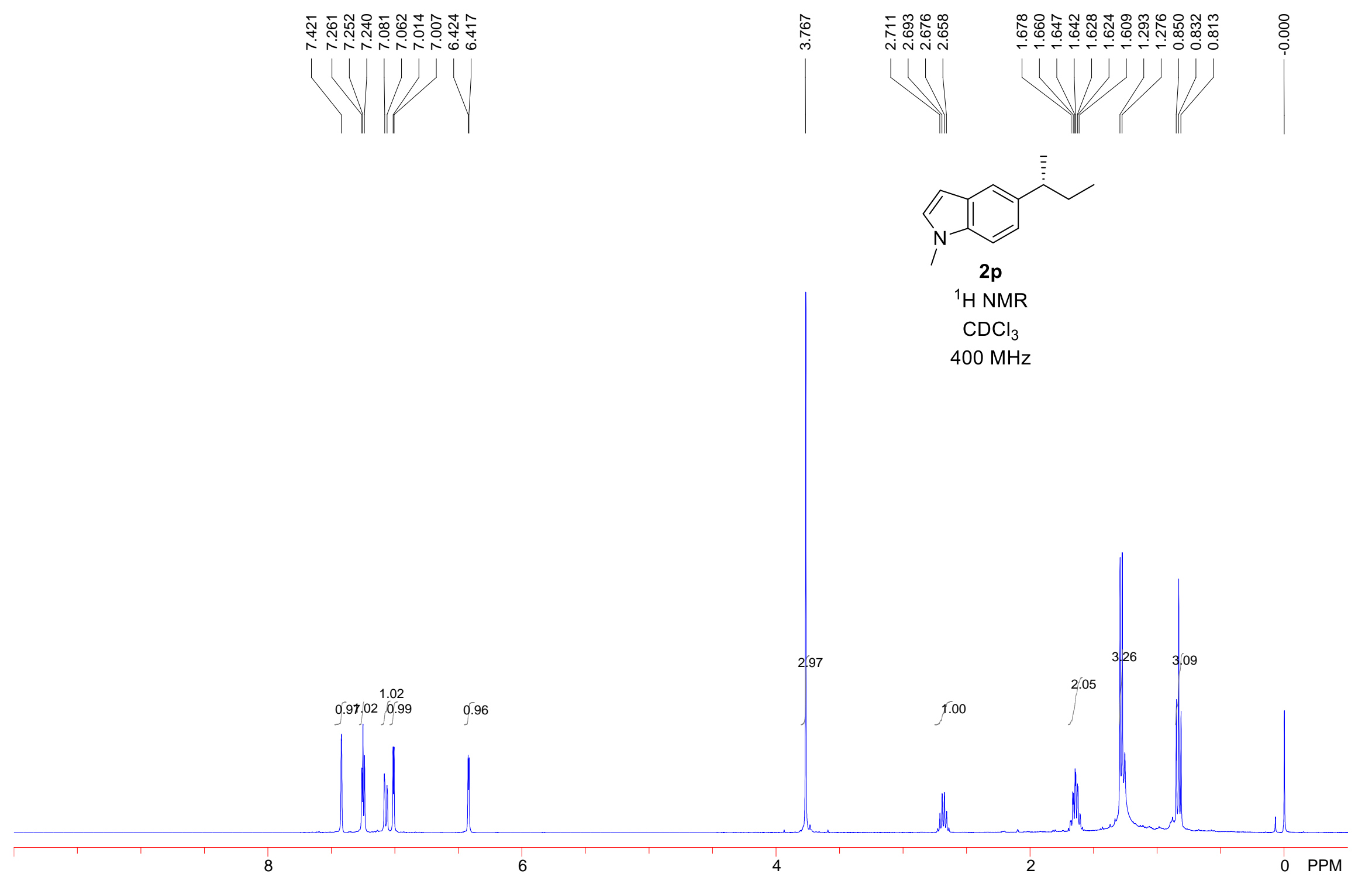




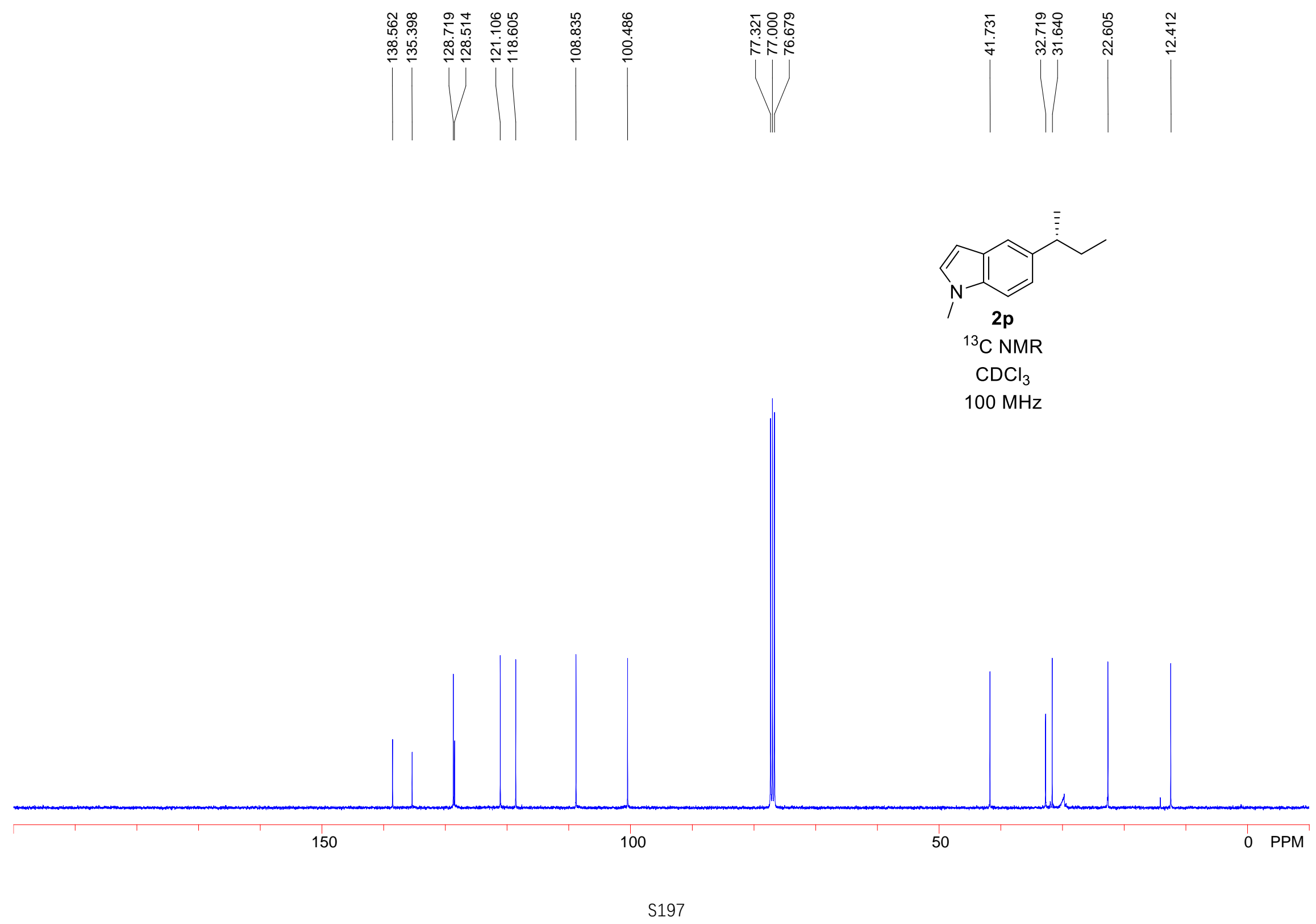



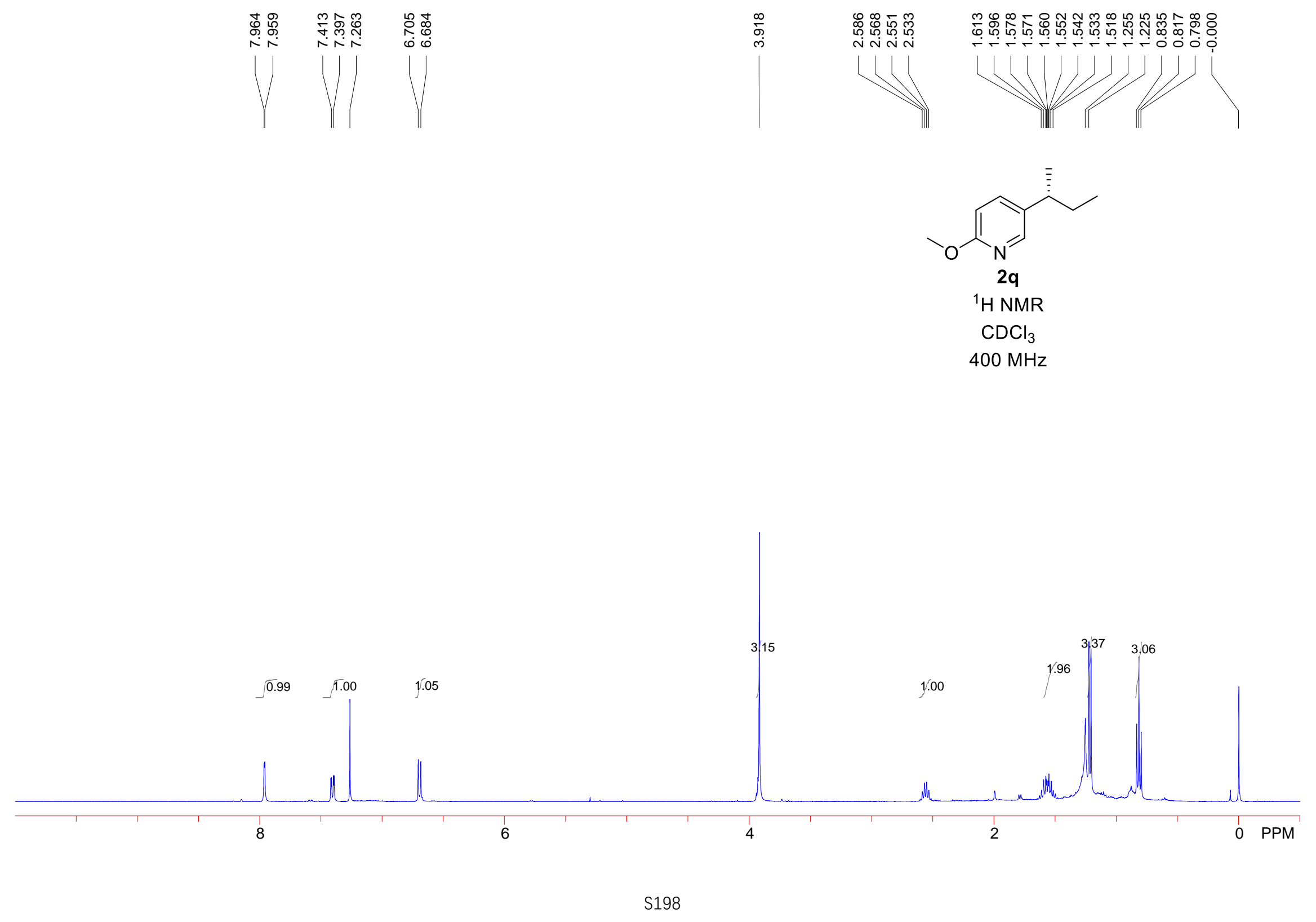


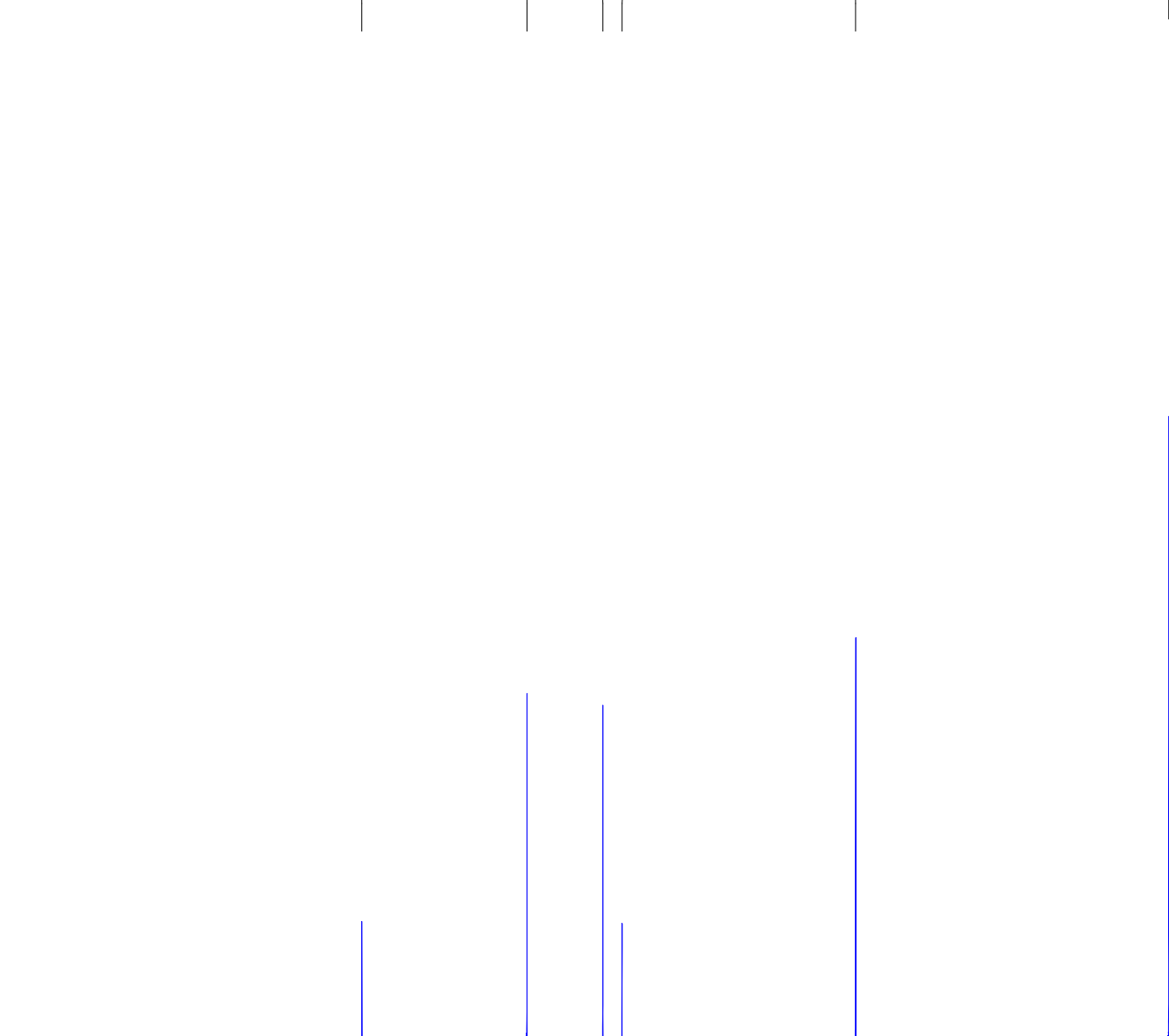




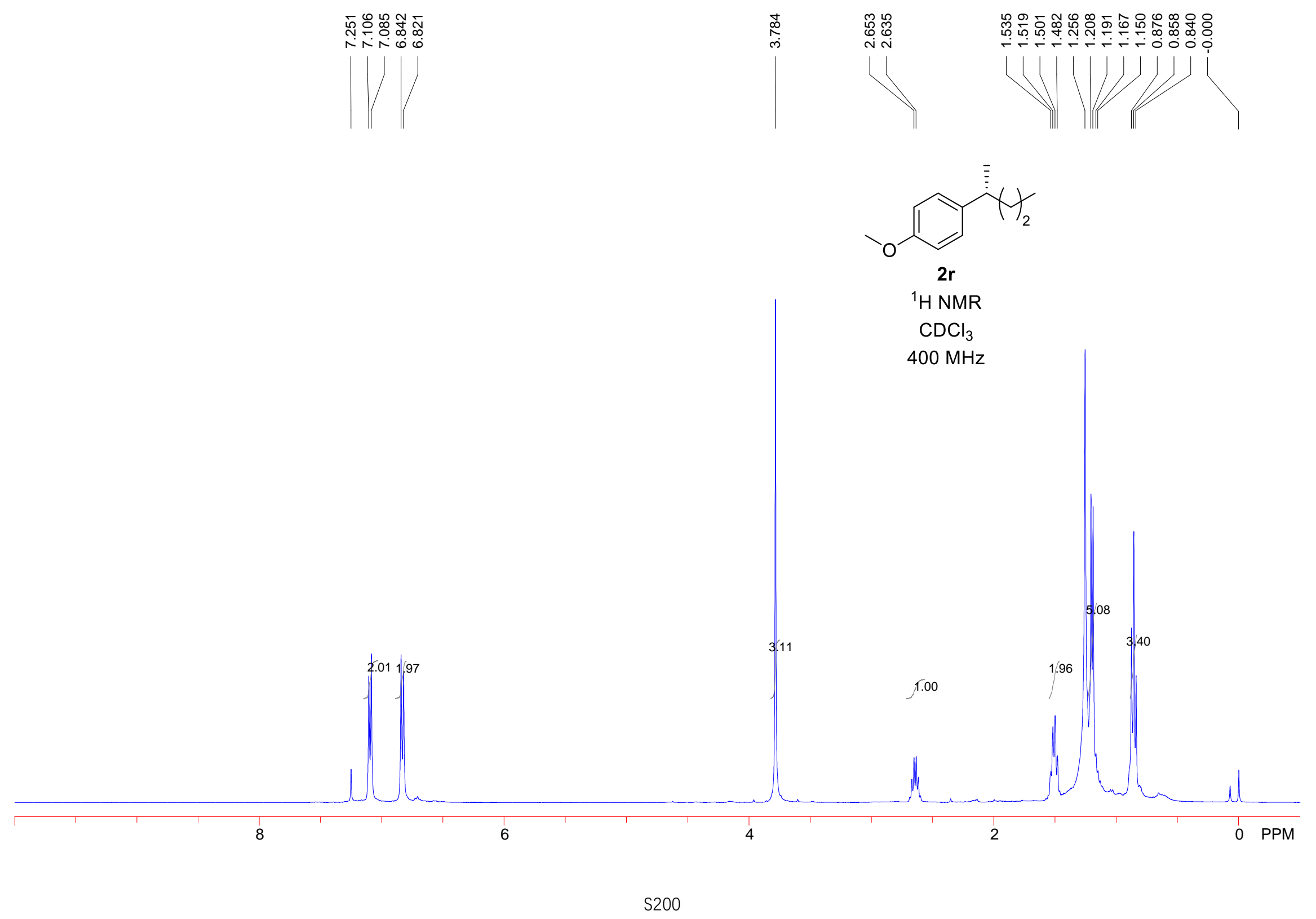



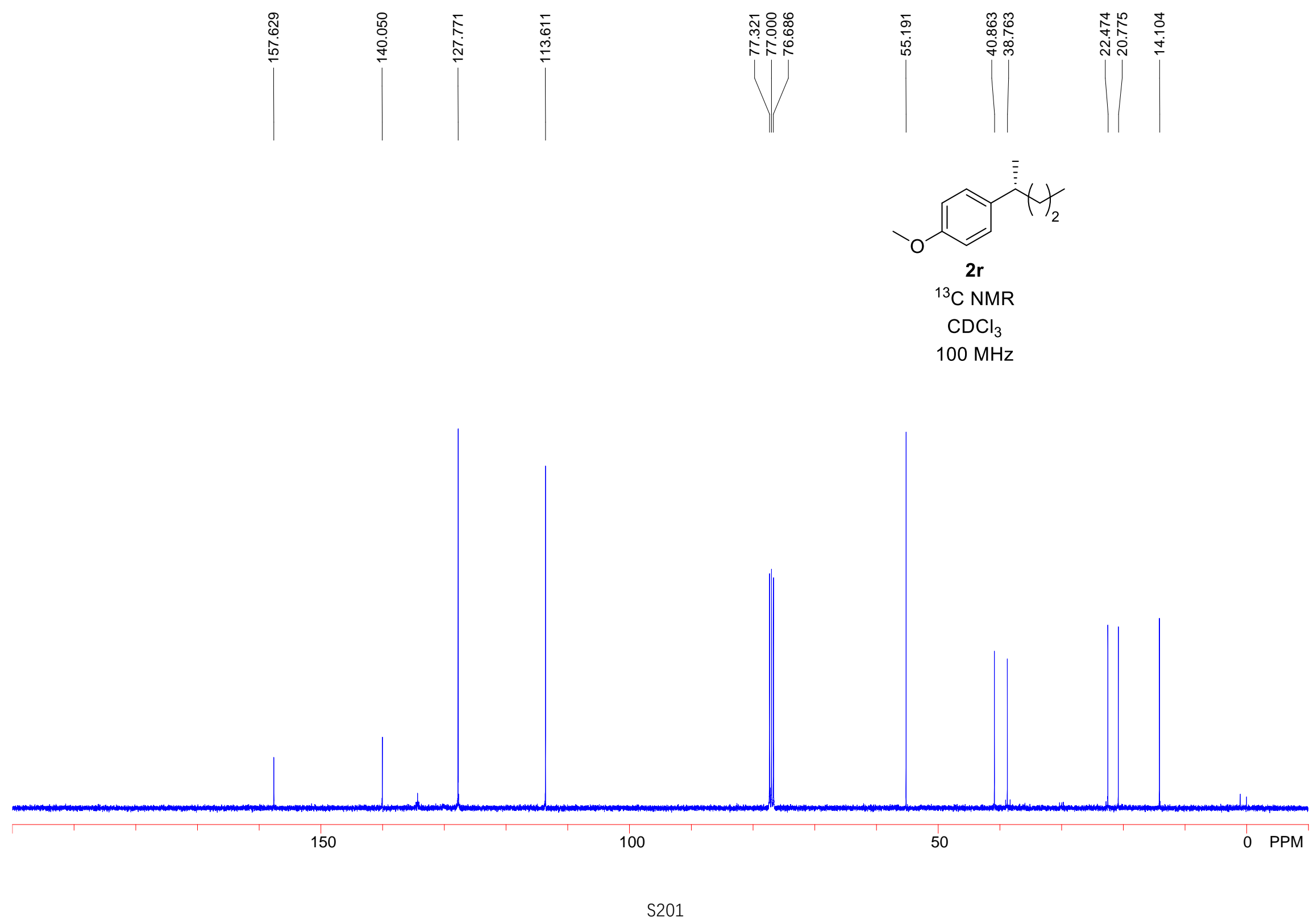


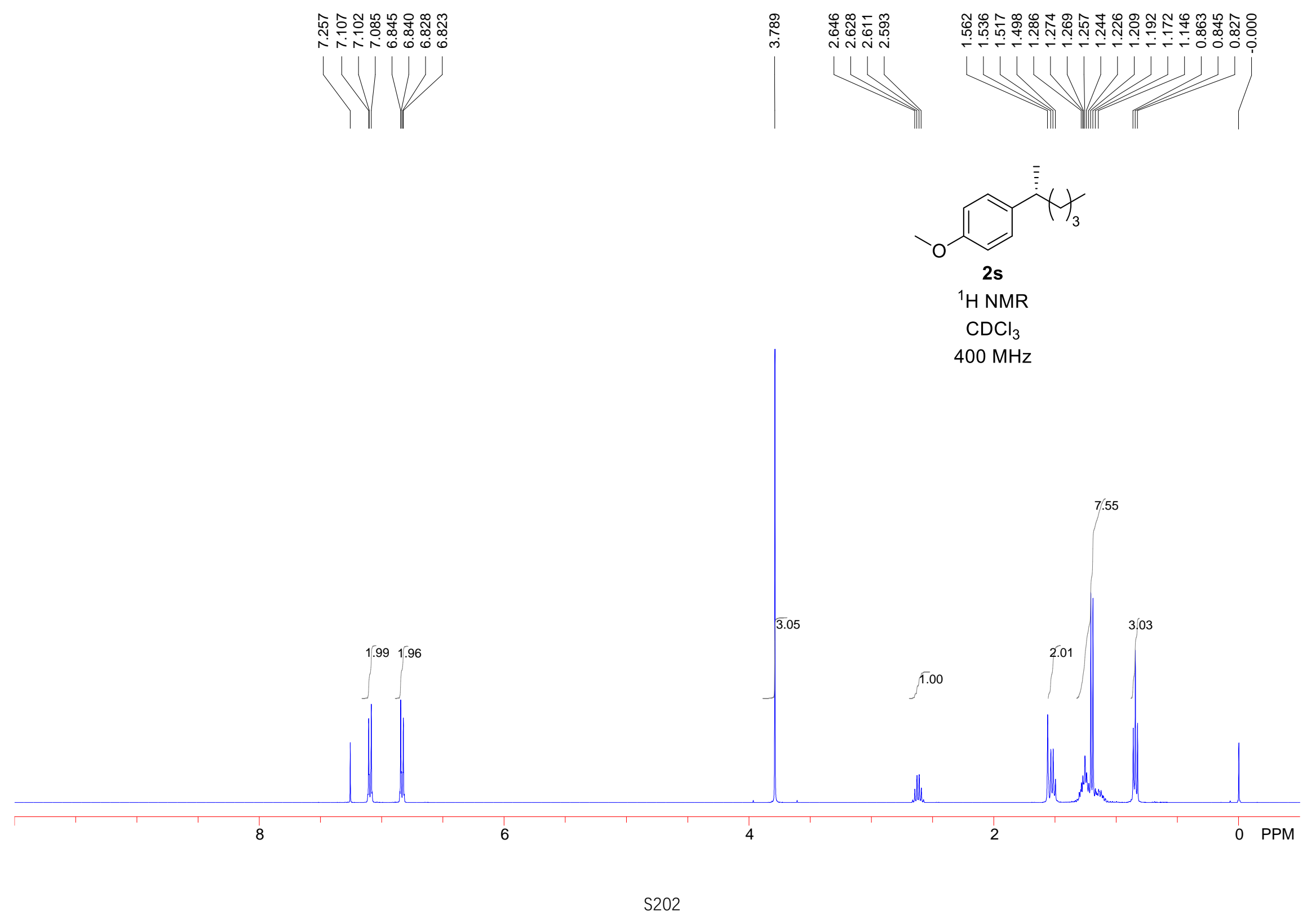



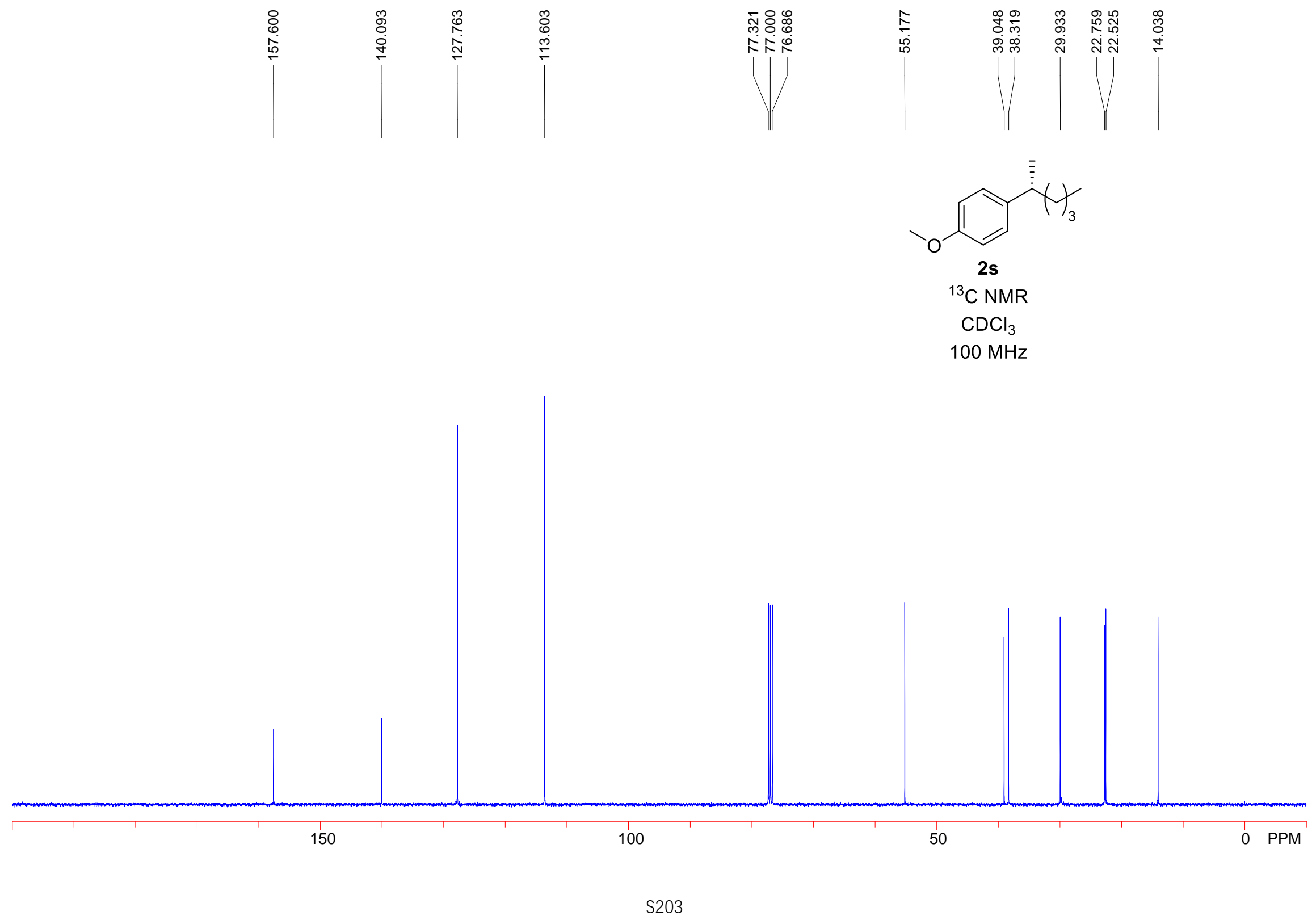


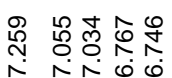

$\stackrel{\leftrightarrow}{\circ}$

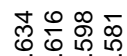

ํ่ ํํํํํำ

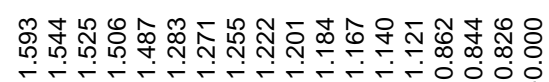

L

1.

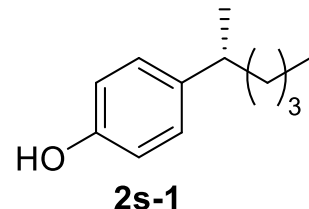

${ }^{1} \mathrm{H}$ NMR

$\mathrm{CDCl}_{3}$

$400 \mathrm{MHz}$

$2.03 \quad 2.03$

0.99 


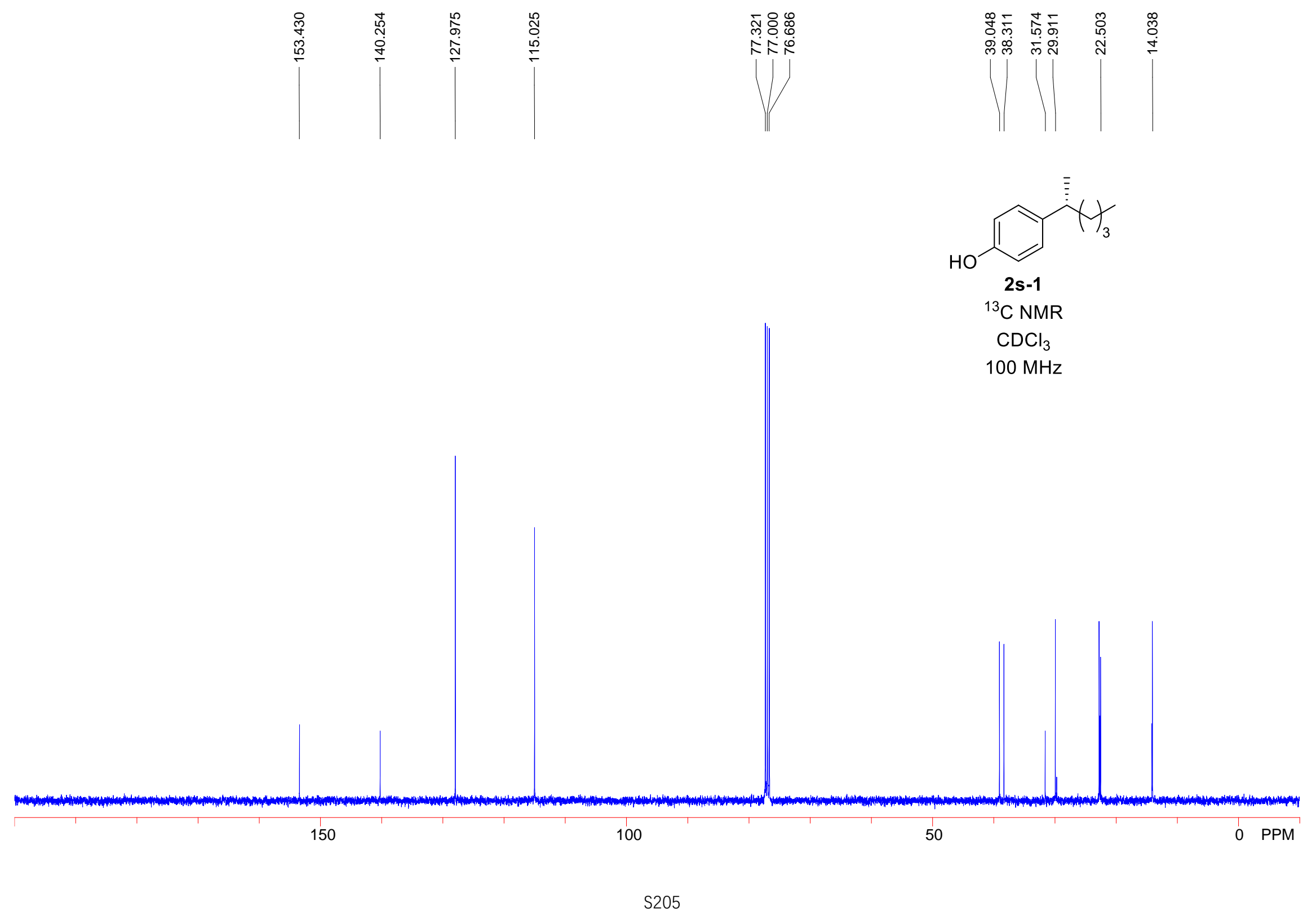




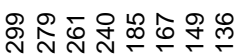
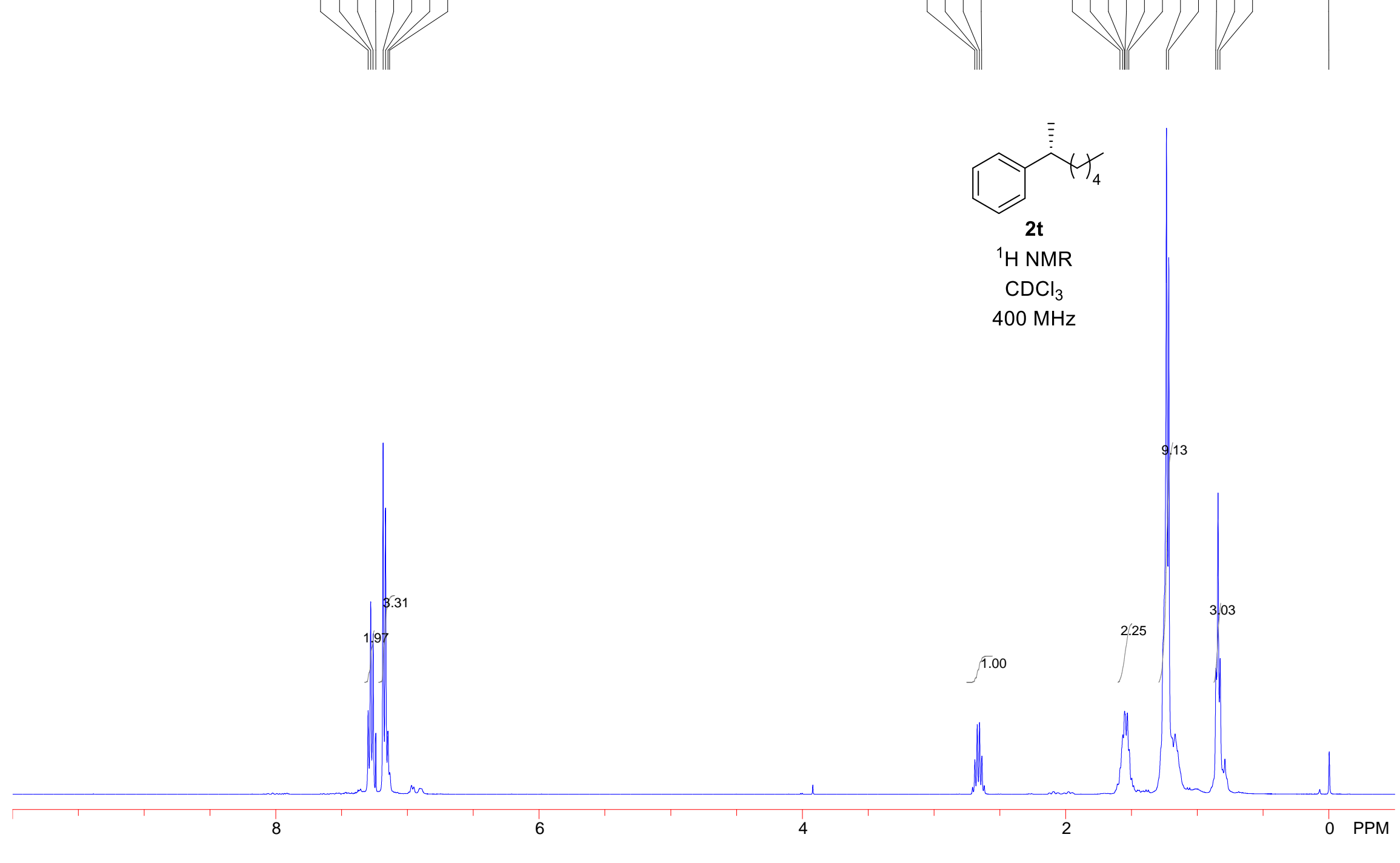


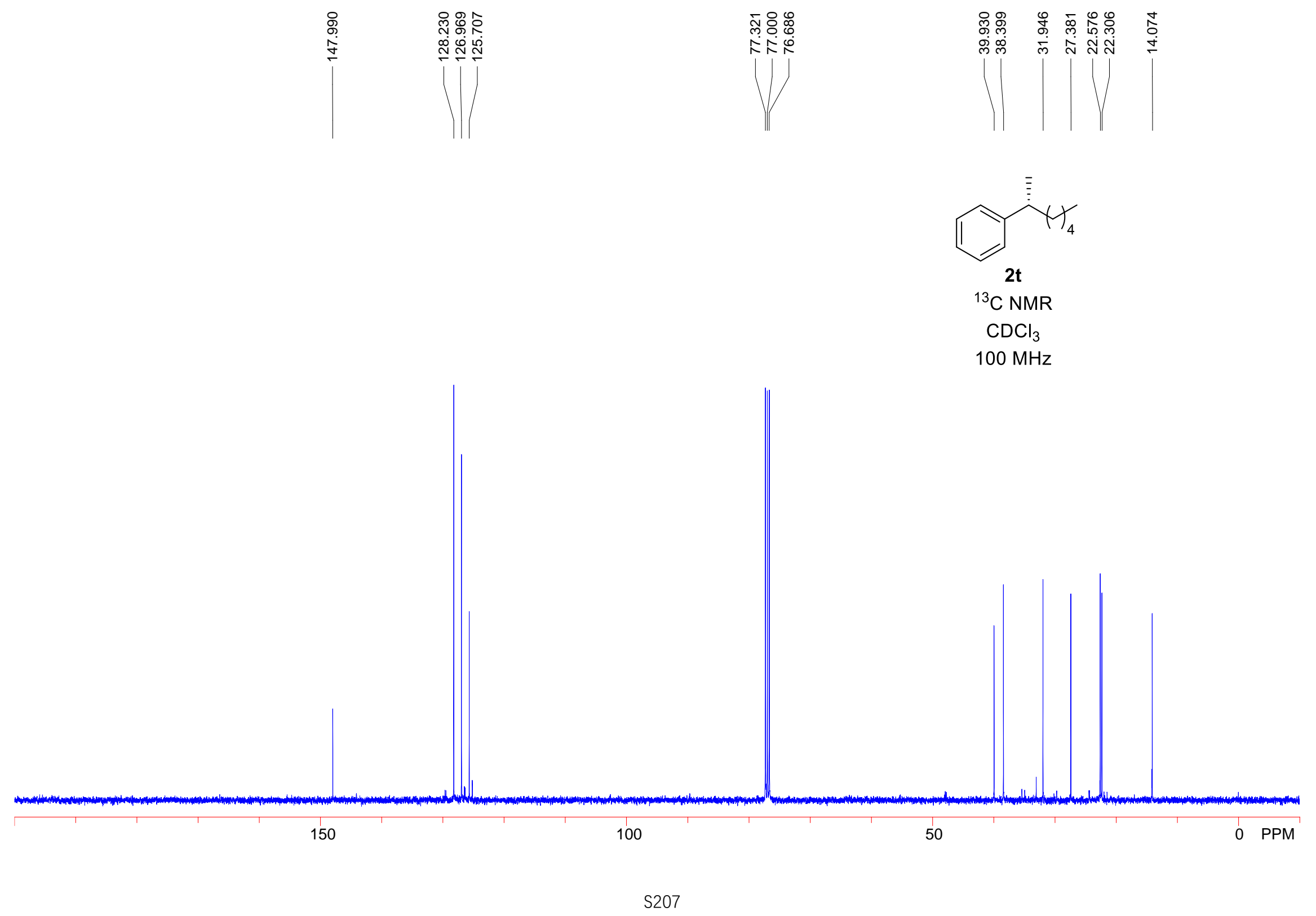




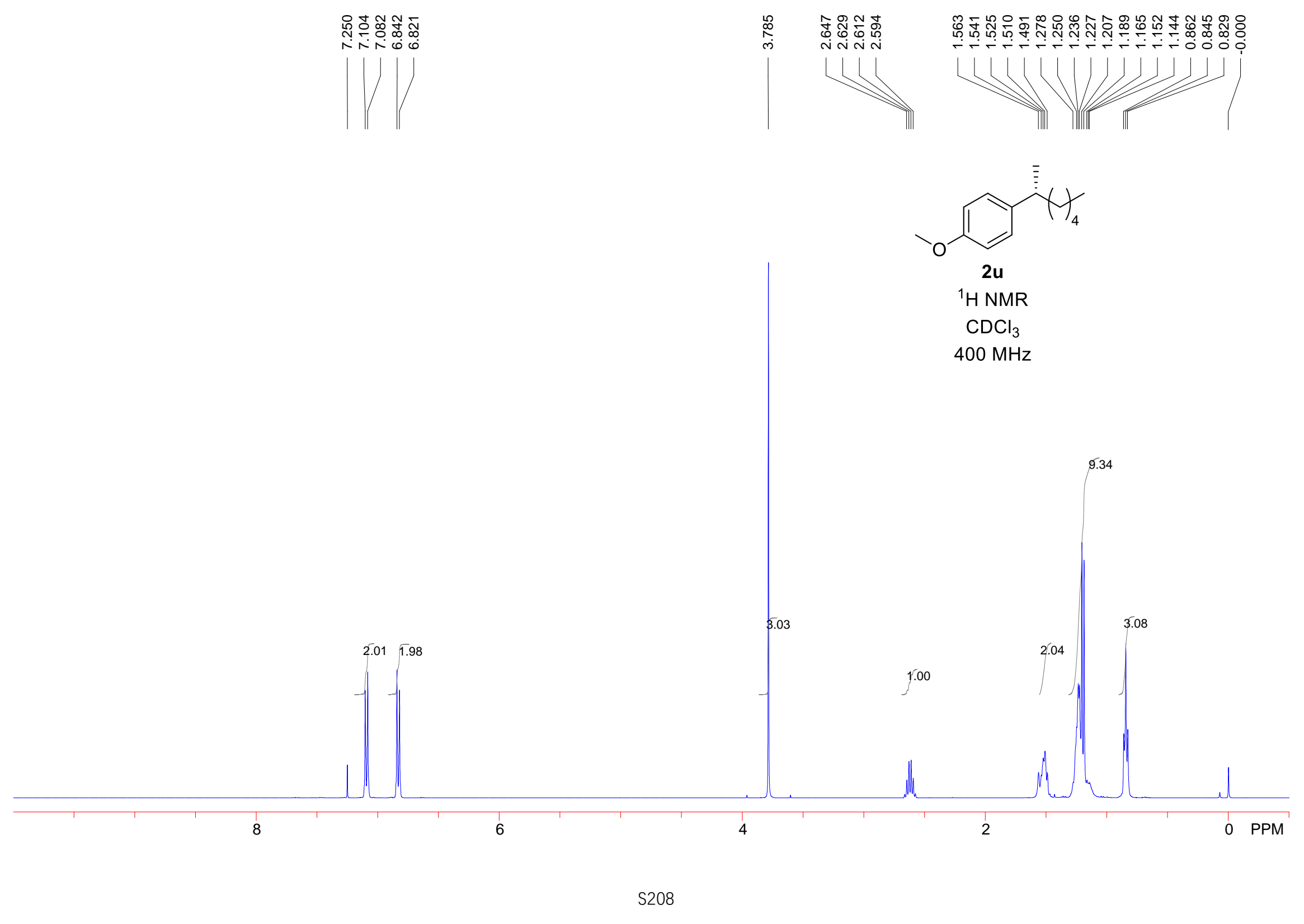




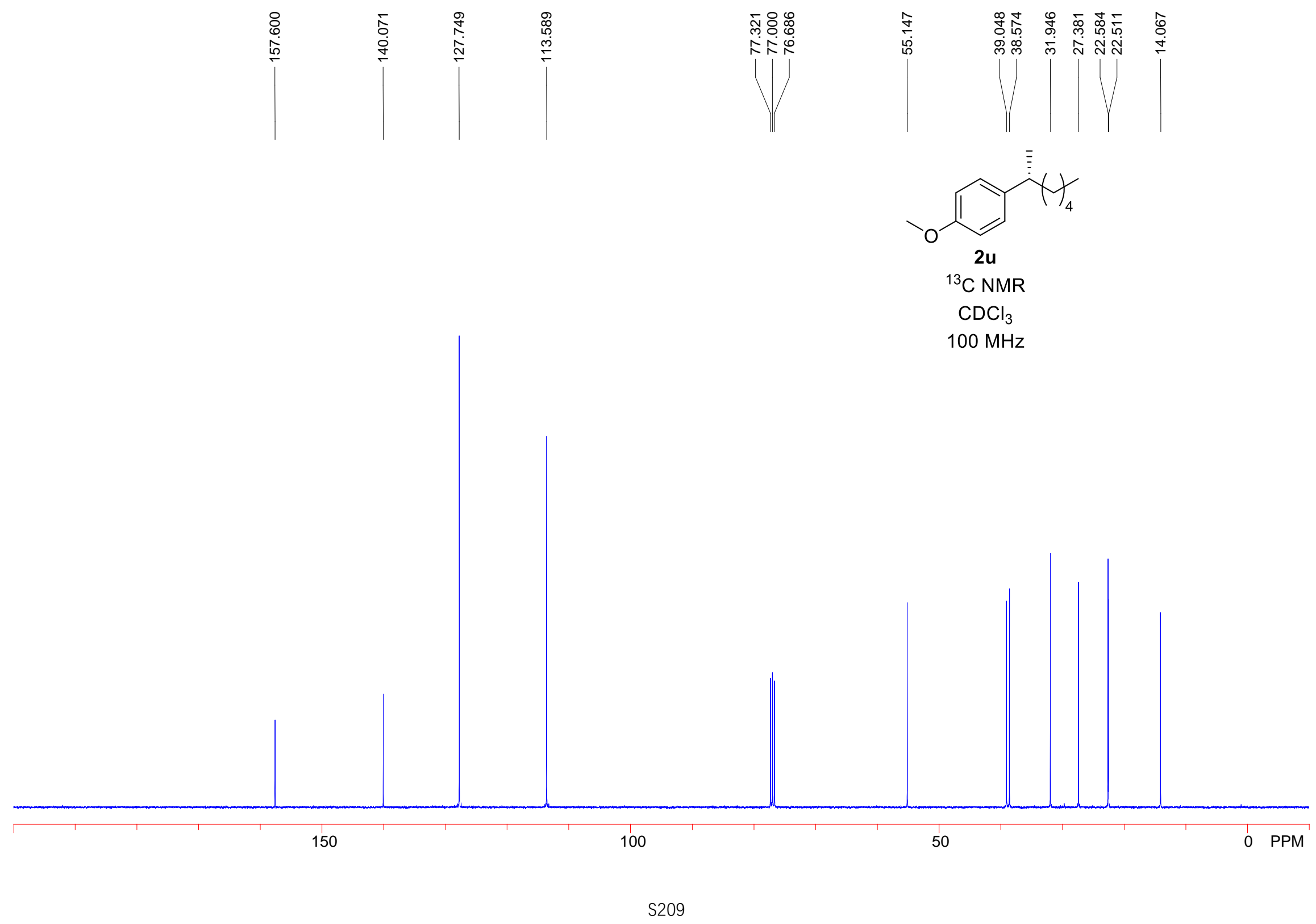




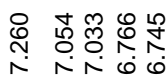

索

땡융요

ํํำ ํํำ

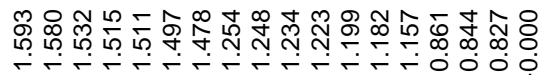

$+|+|$

ᄂ,
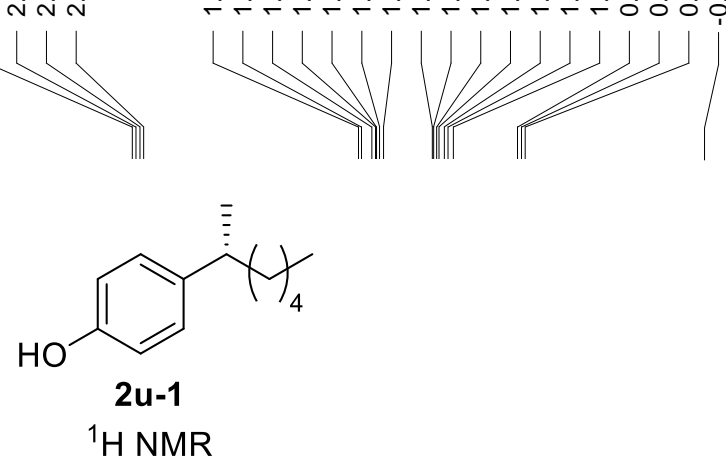

$\mathrm{CDCl}_{3}$

$400 \mathrm{MHz}$

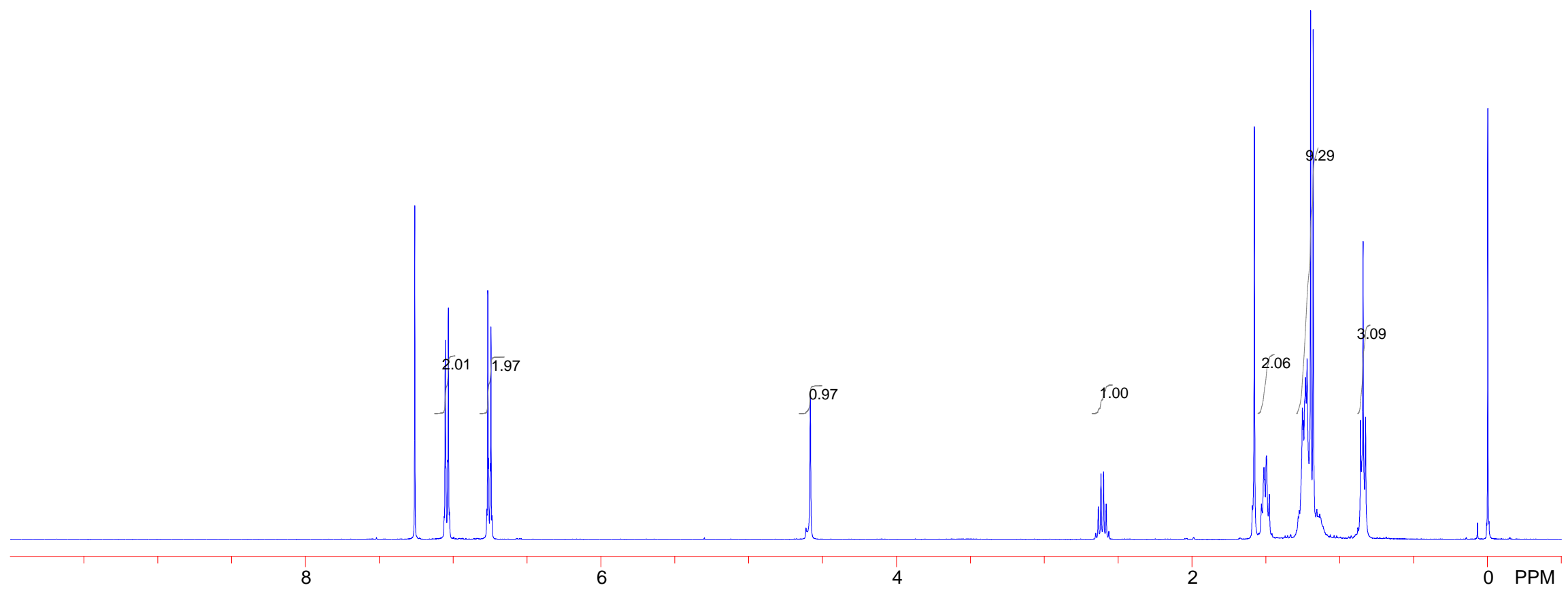




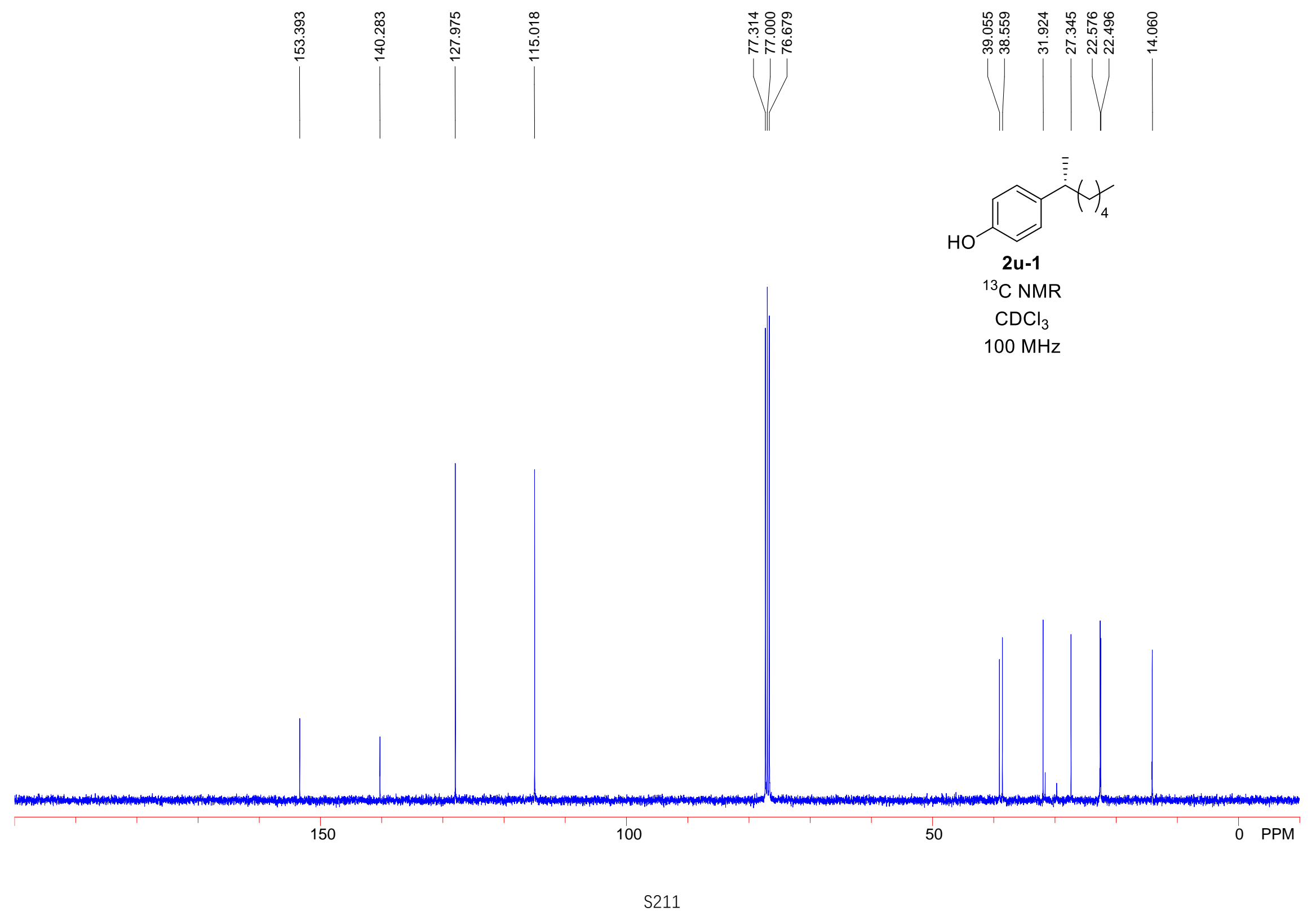




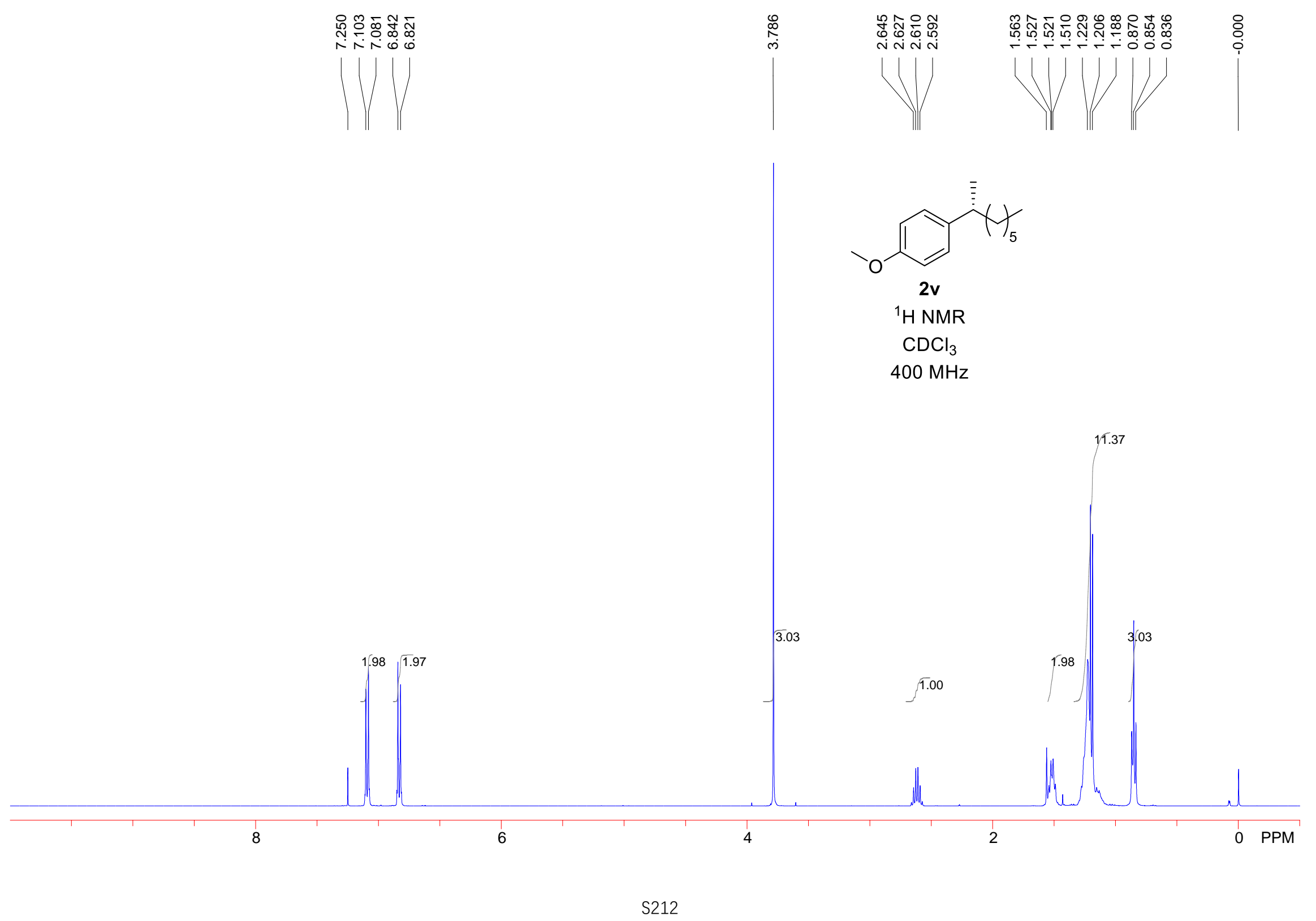




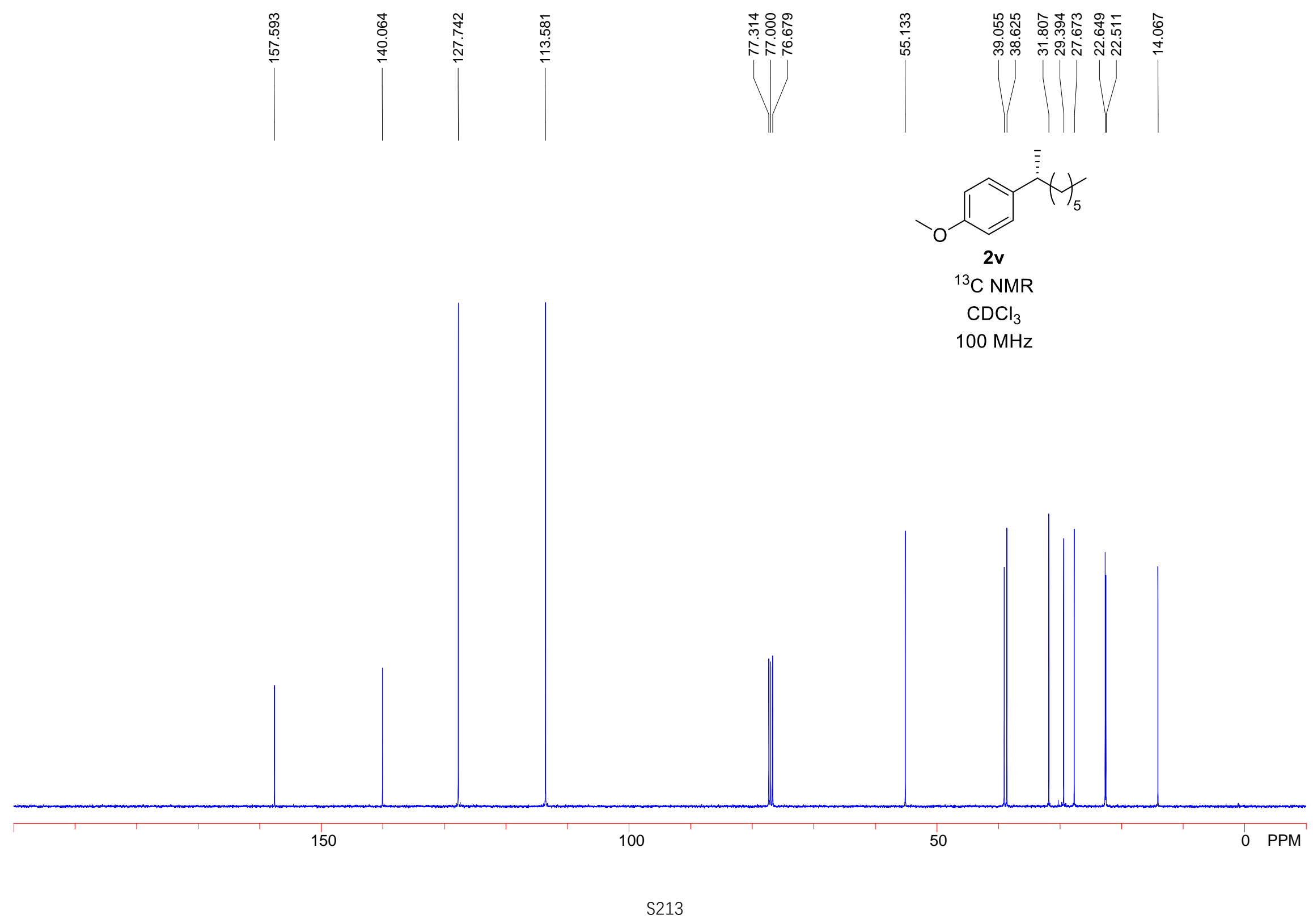




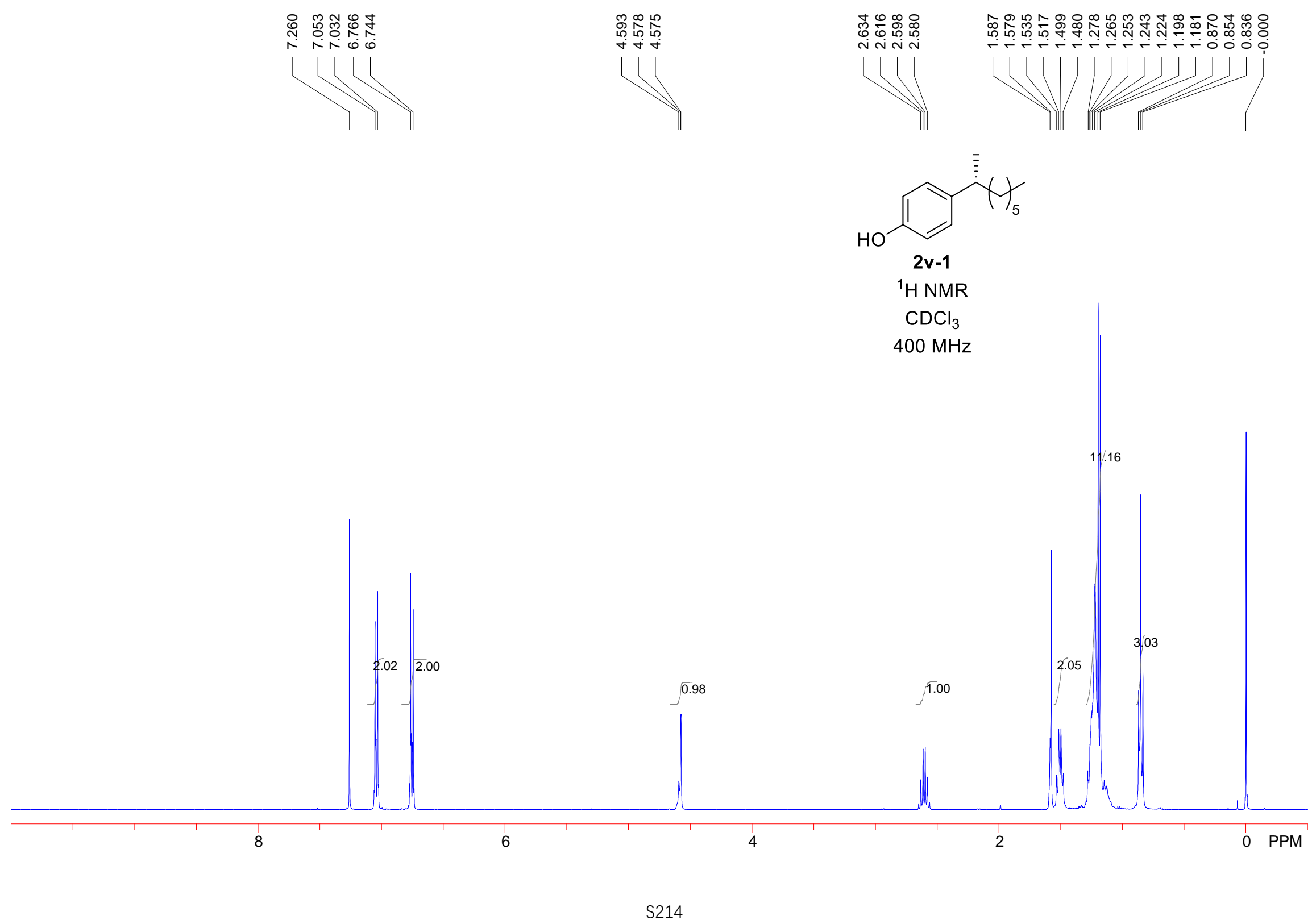




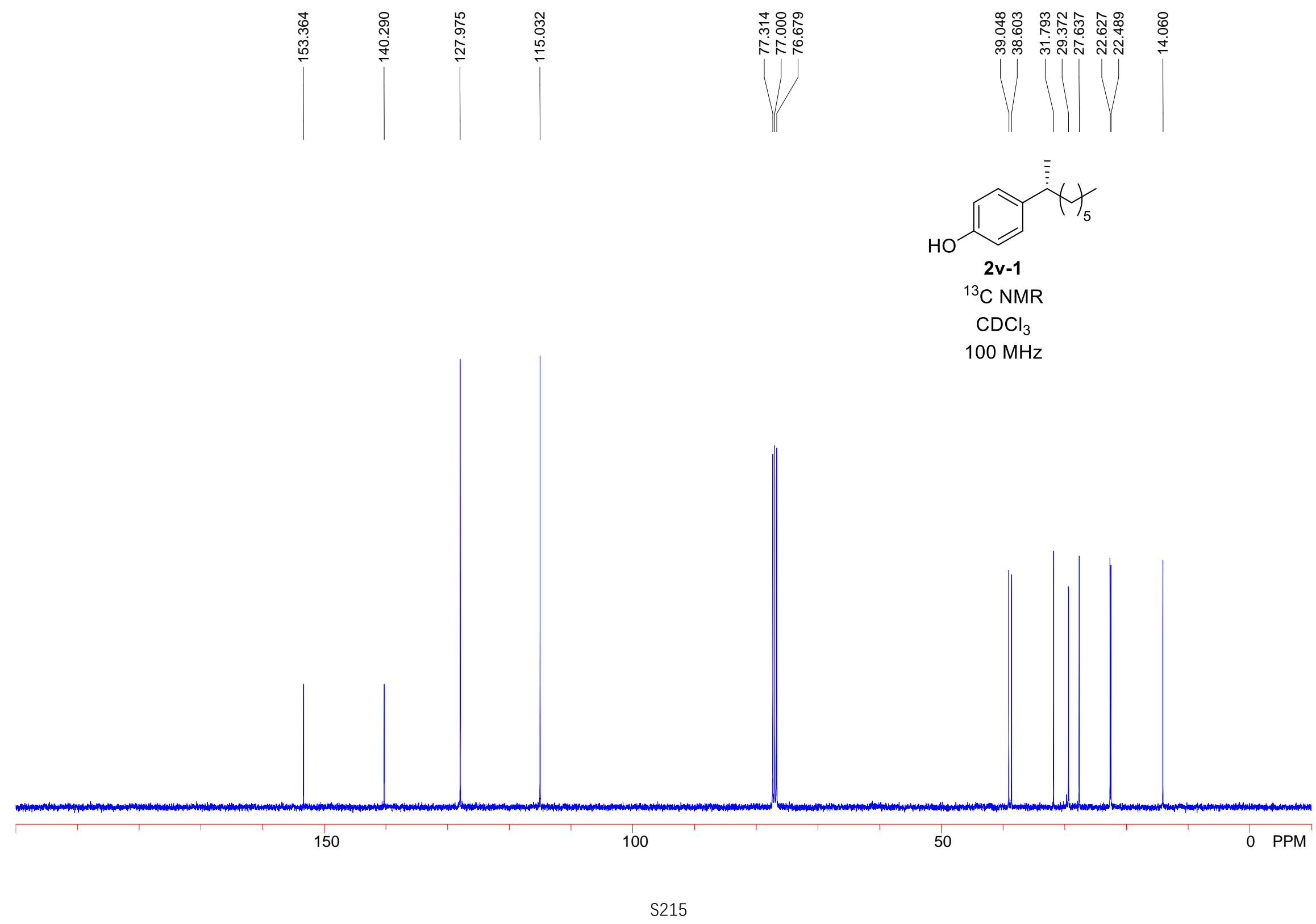




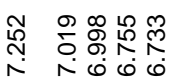

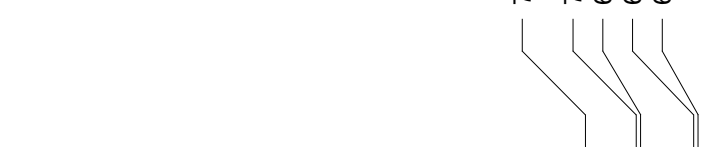

1.991 .97

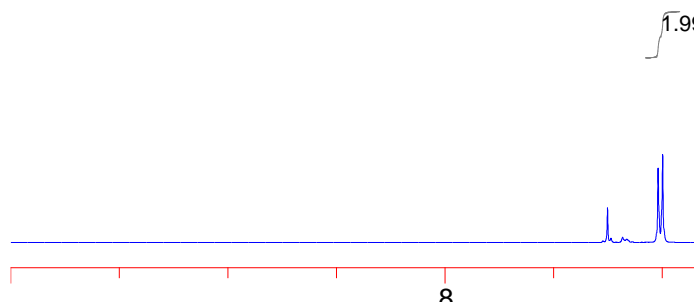

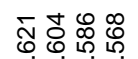

ํํํํํำ

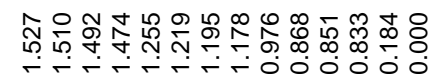

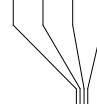

-r.

${ }^{1} \mathrm{H}$ NMR

$\mathrm{CDCl}_{3}$

$400 \mathrm{MHz}$

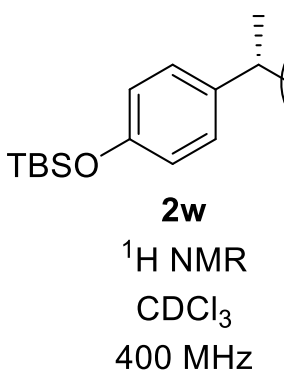

9
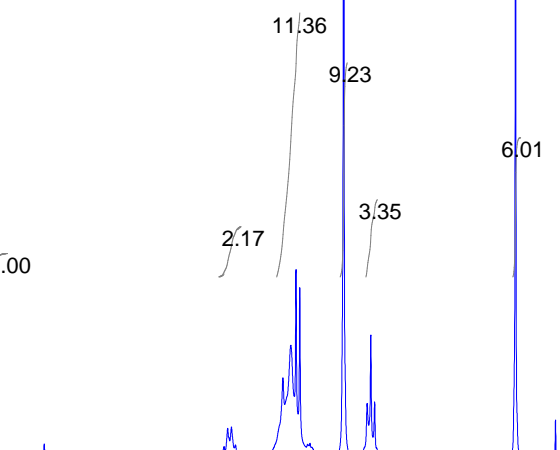

12

0 PPM 


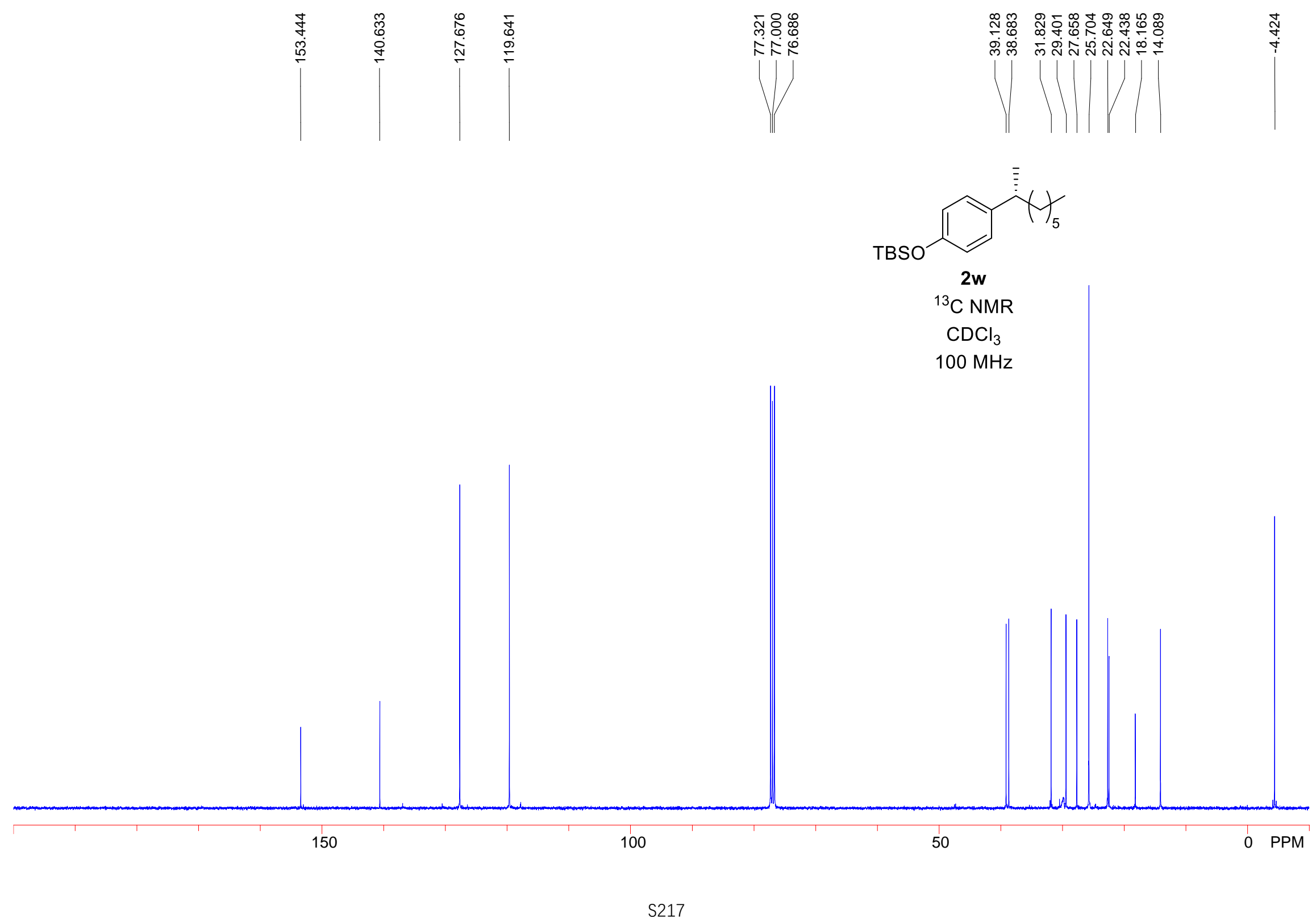




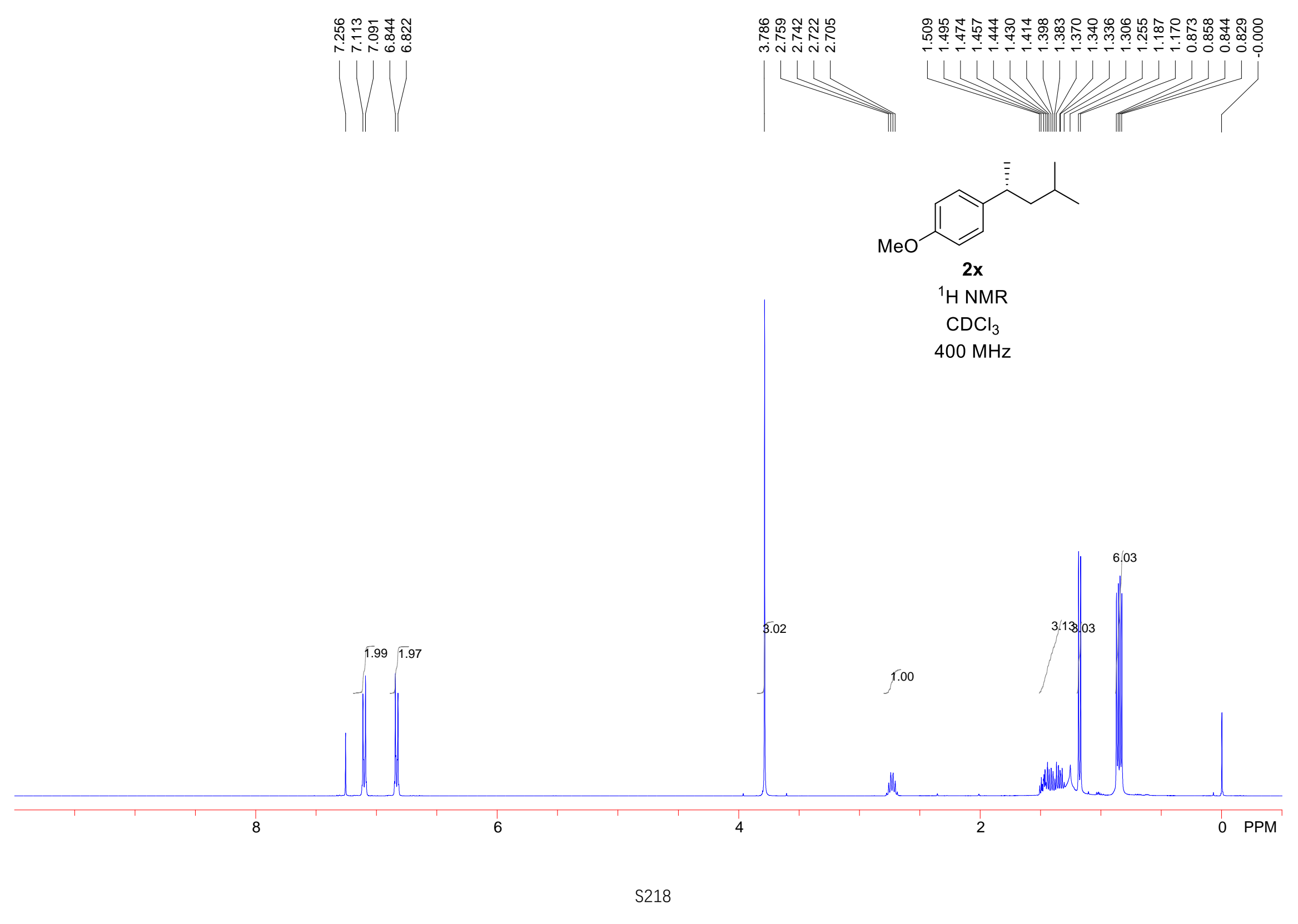




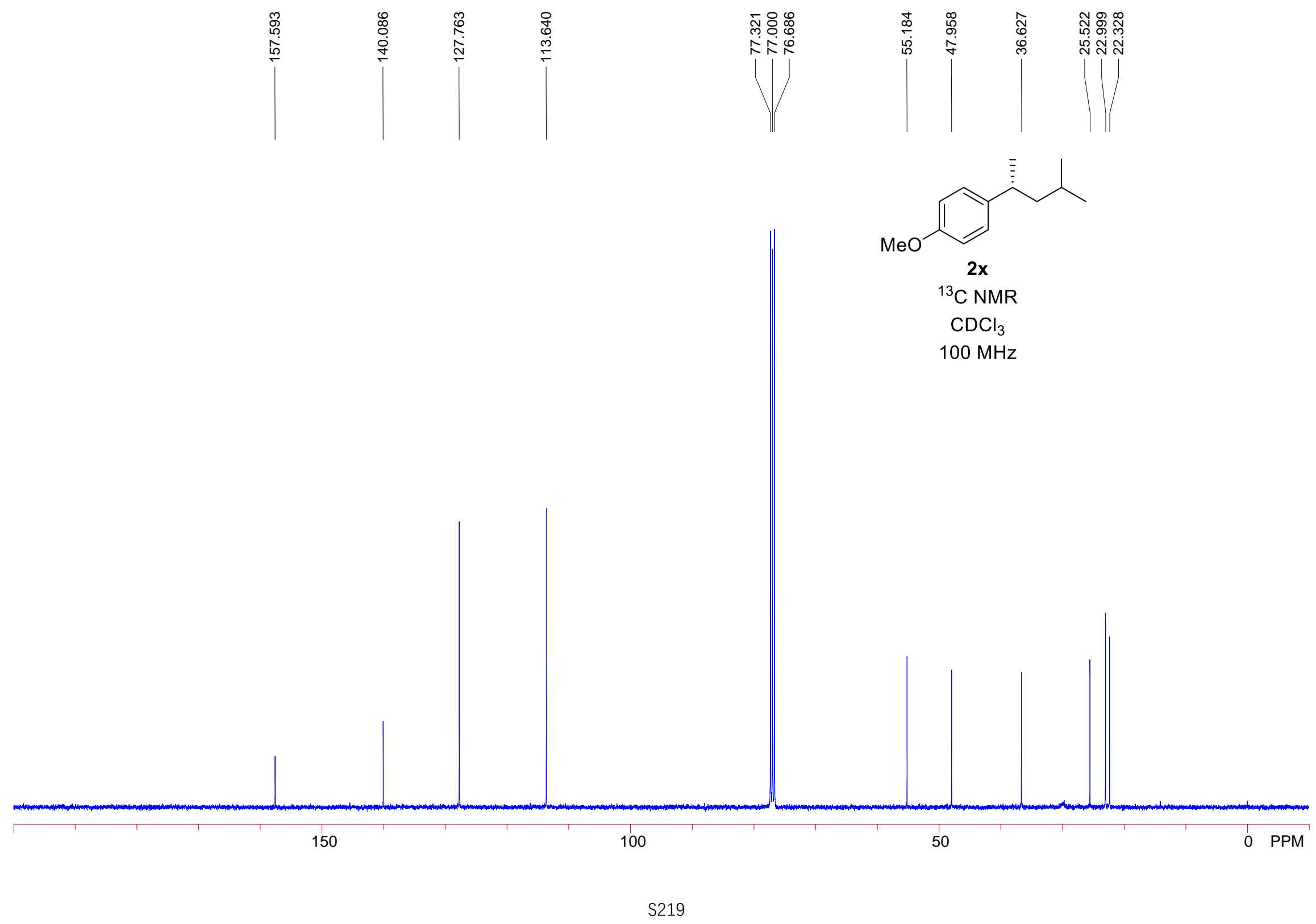




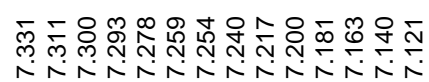

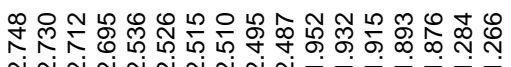

U
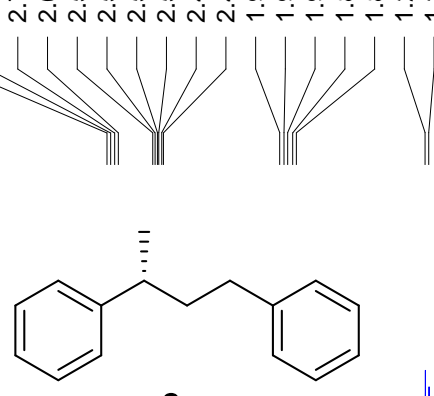

$2 y$

${ }^{1} \mathrm{H}$ NMR

$\mathrm{CDCl}_{3}$

$400 \mathrm{MHz}$

10.25

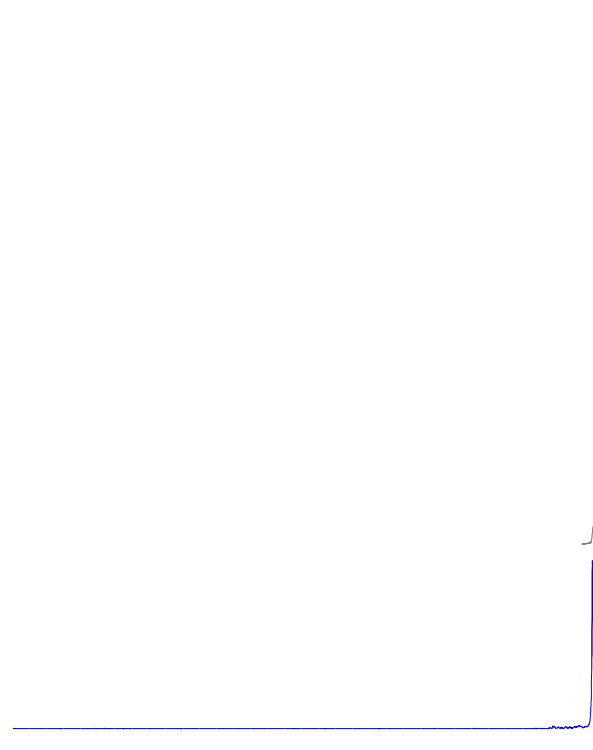




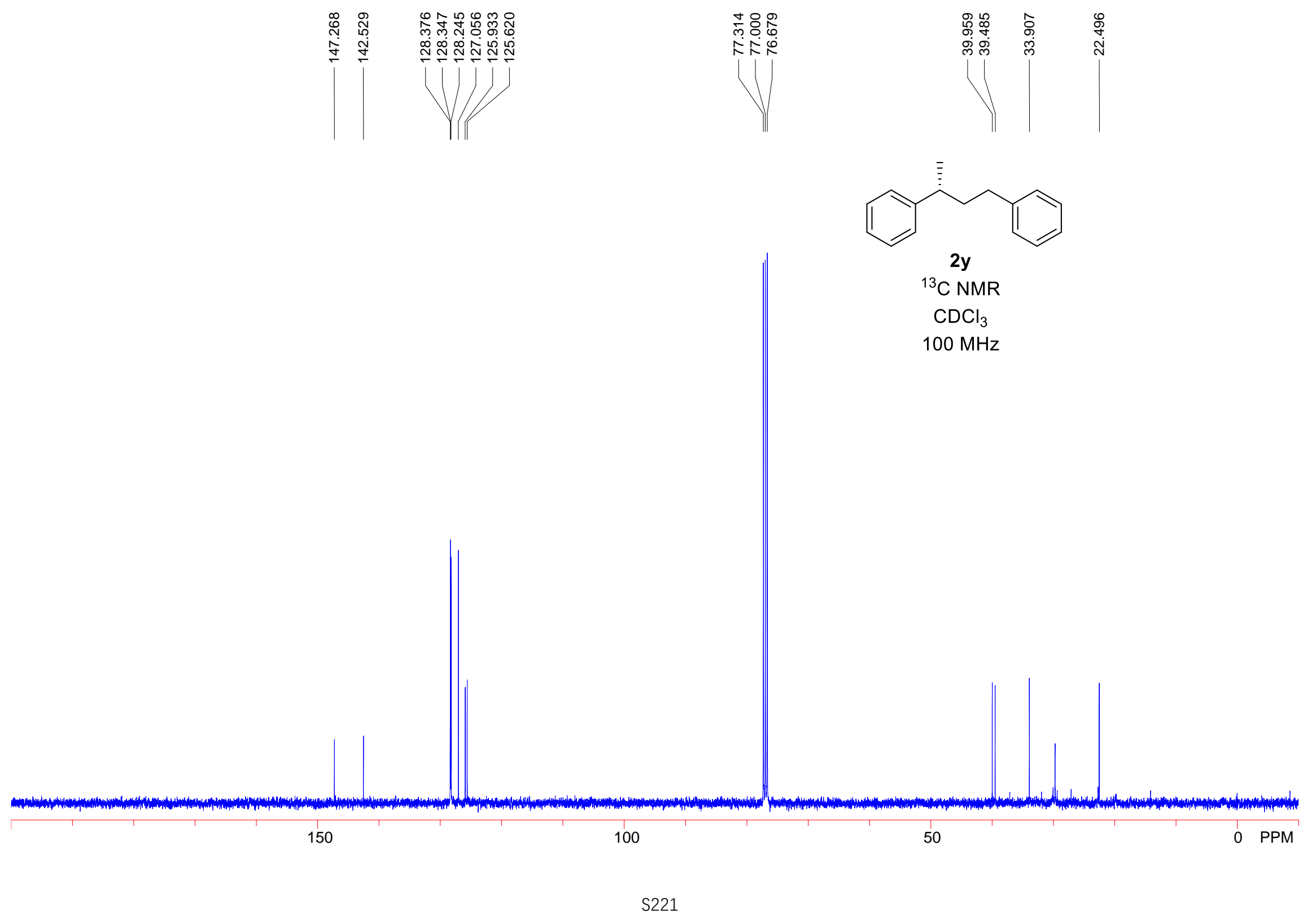




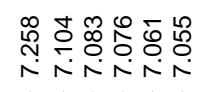

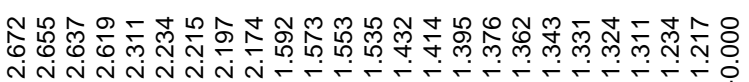

3.76
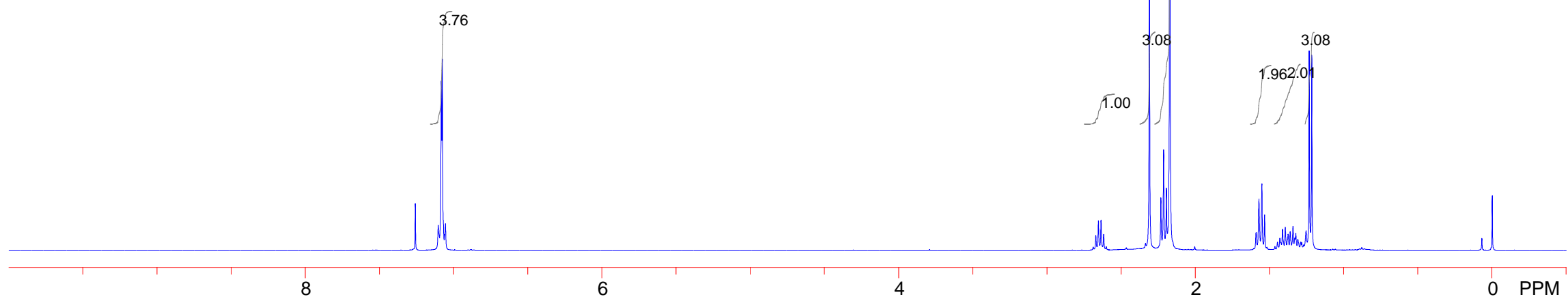

${ }^{1} \mathrm{H}$ NMR

$\mathrm{CDCl}_{3}$

$400 \mathrm{MHz}$ 


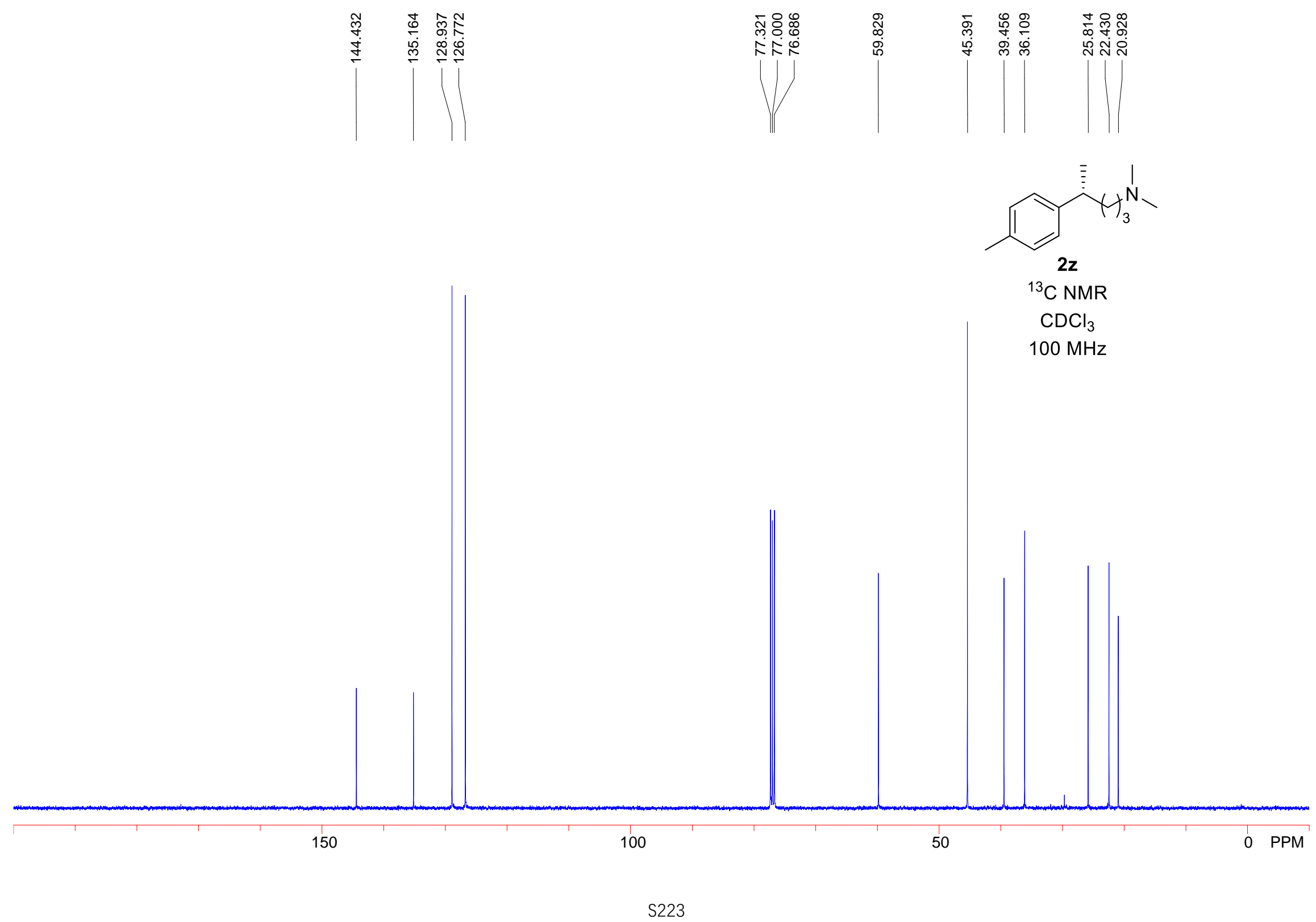




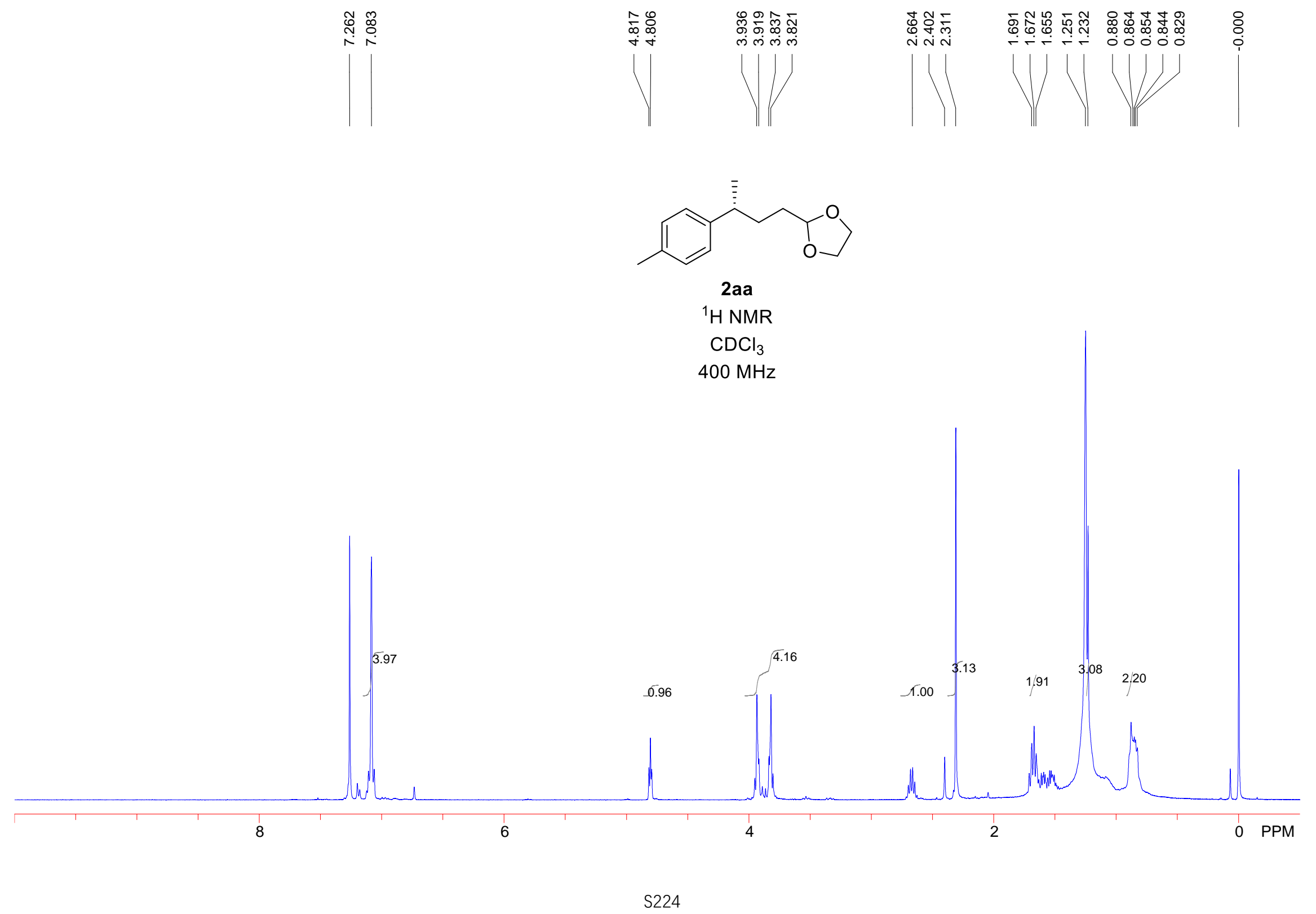




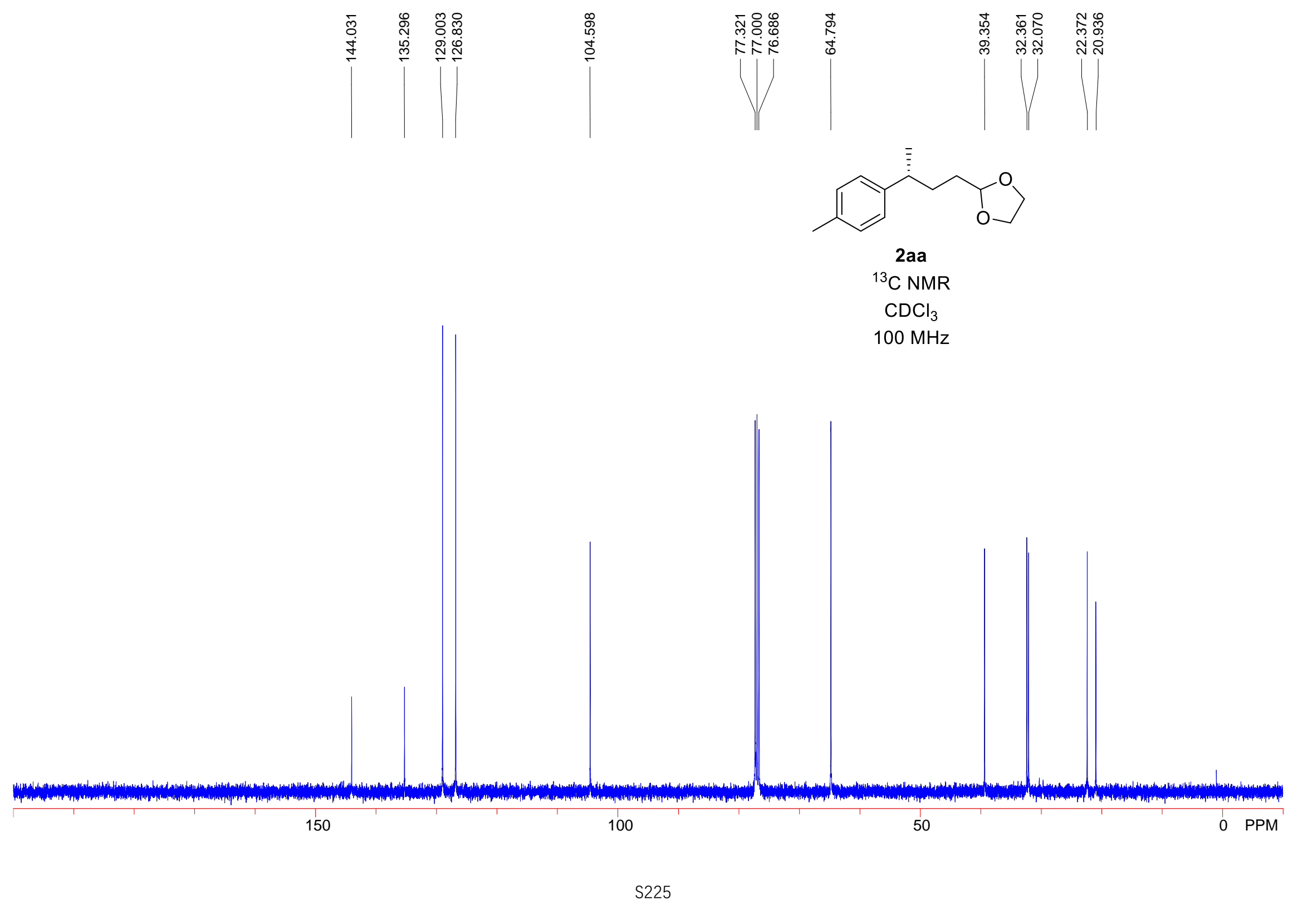




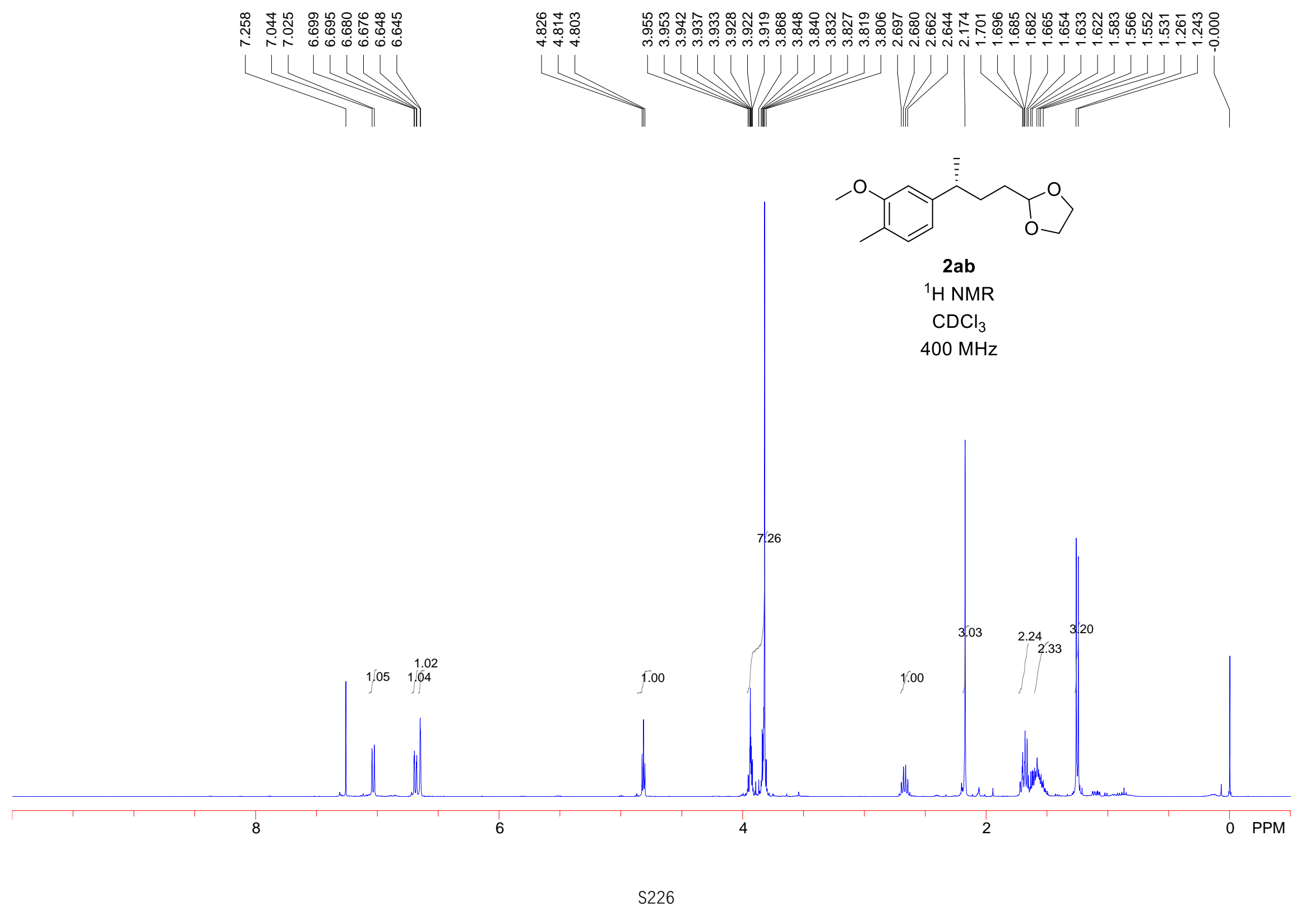




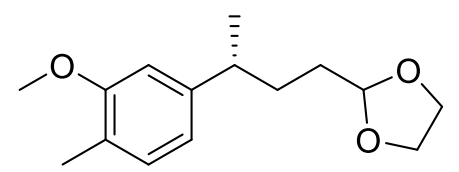

$2 a b$

${ }^{13} \mathrm{C}$ NMR

$\mathrm{CDCl}_{3}$

$100 \mathrm{MHz}$

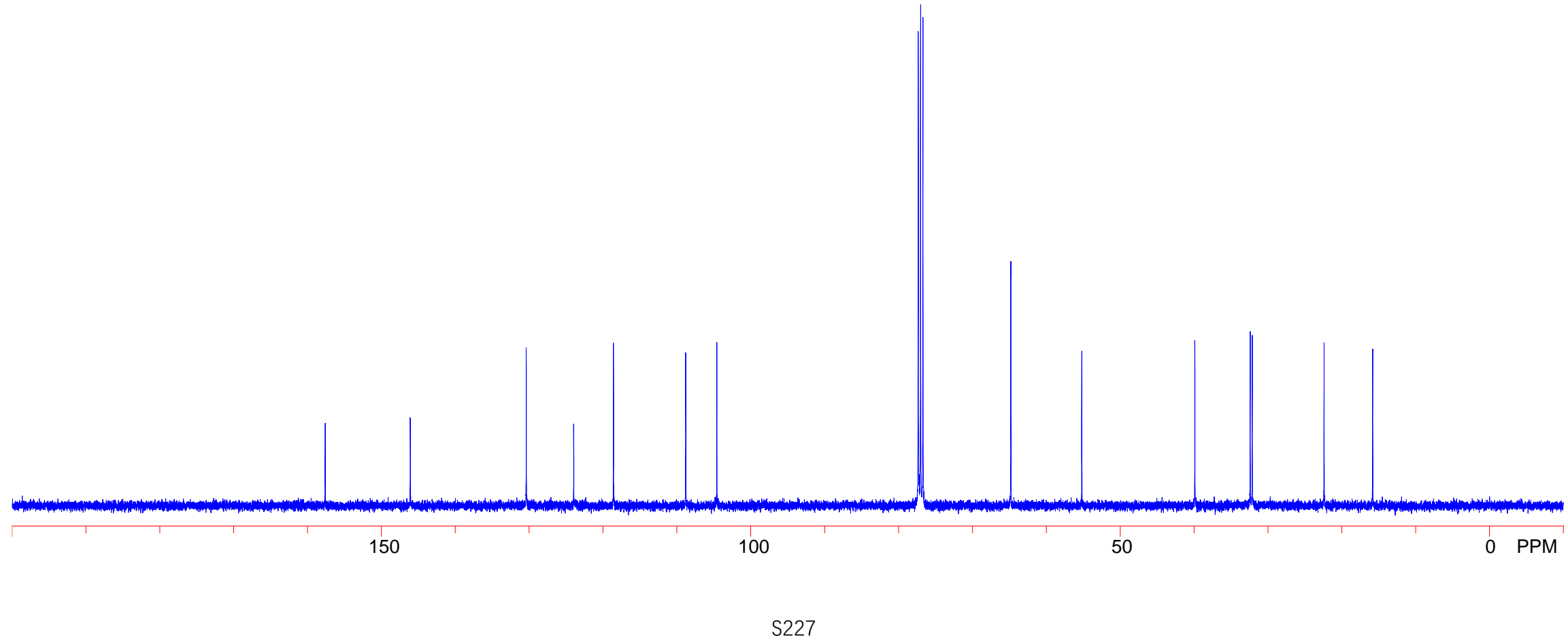




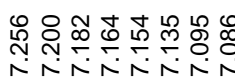

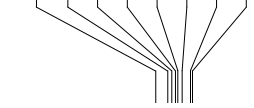

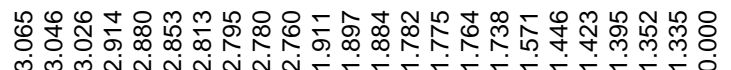

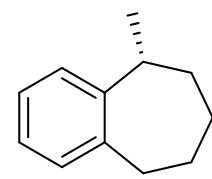

2 ac

${ }^{1} \mathrm{H}$ NMR

$\mathrm{CDCl}_{3}$

$400 \mathrm{MHz}$

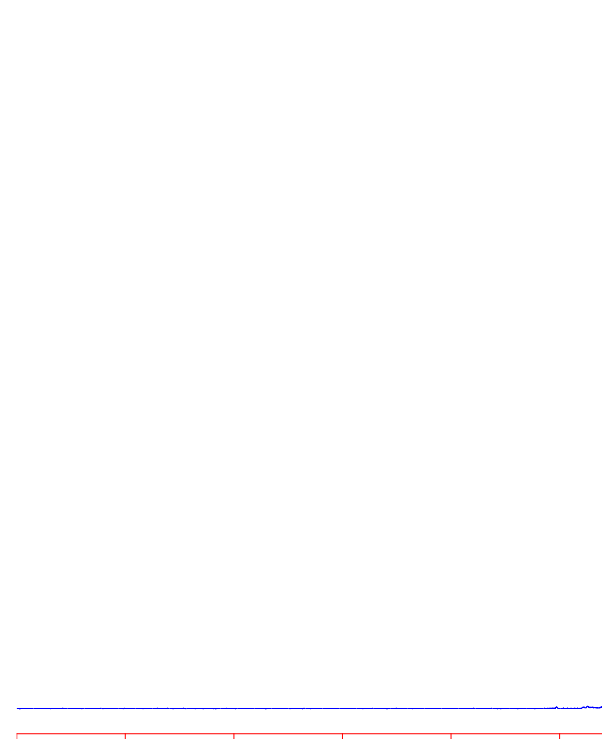

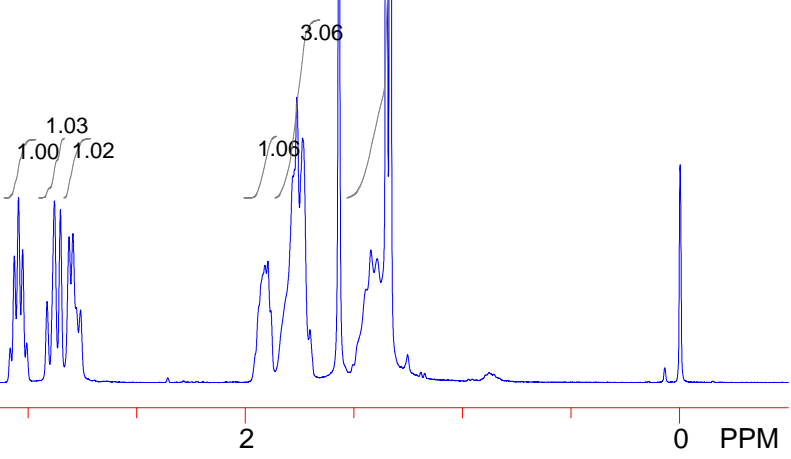




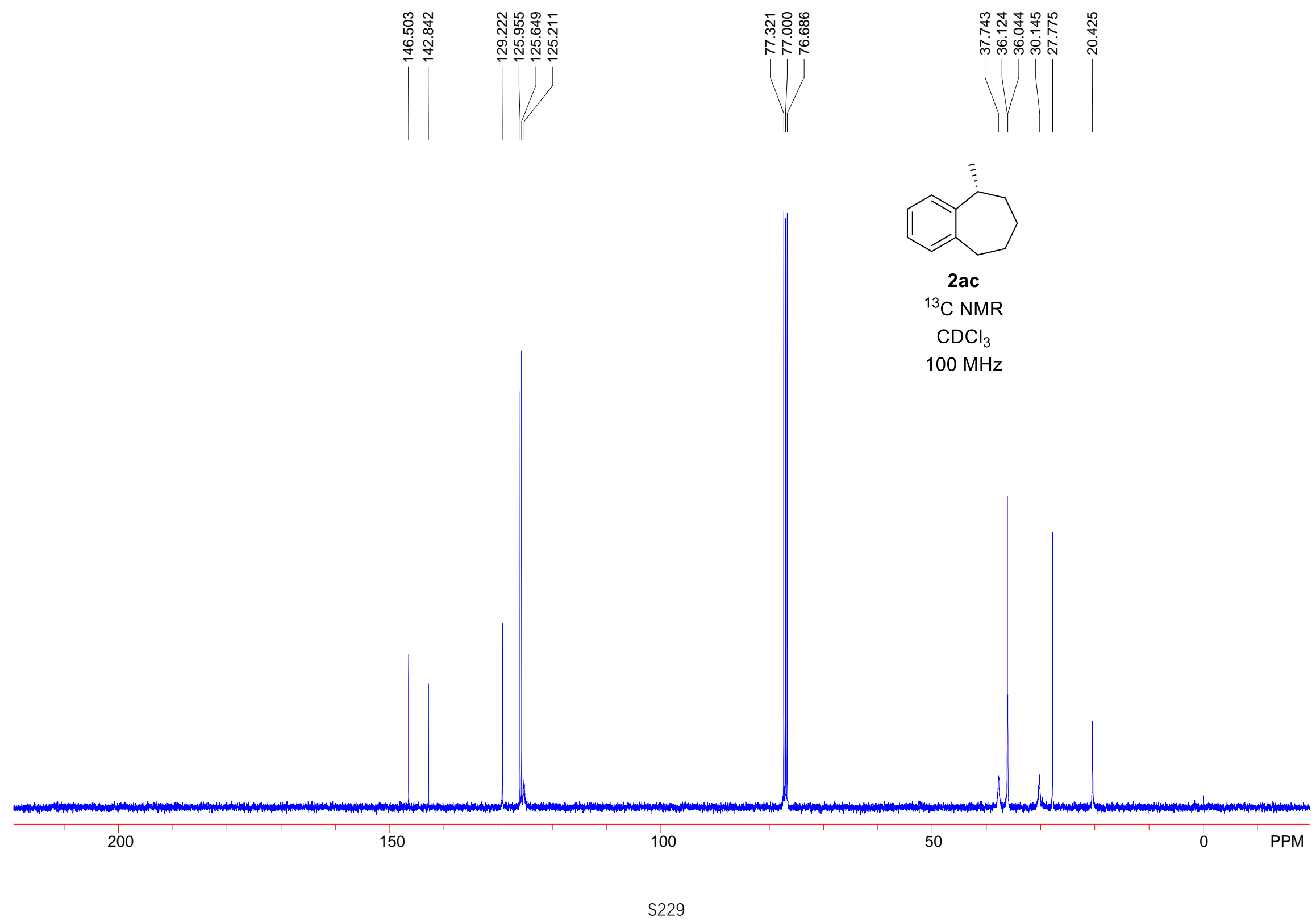




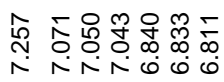

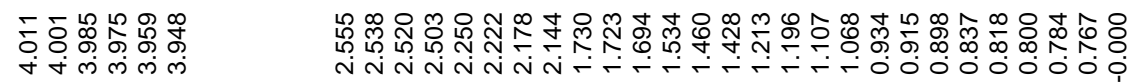

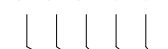
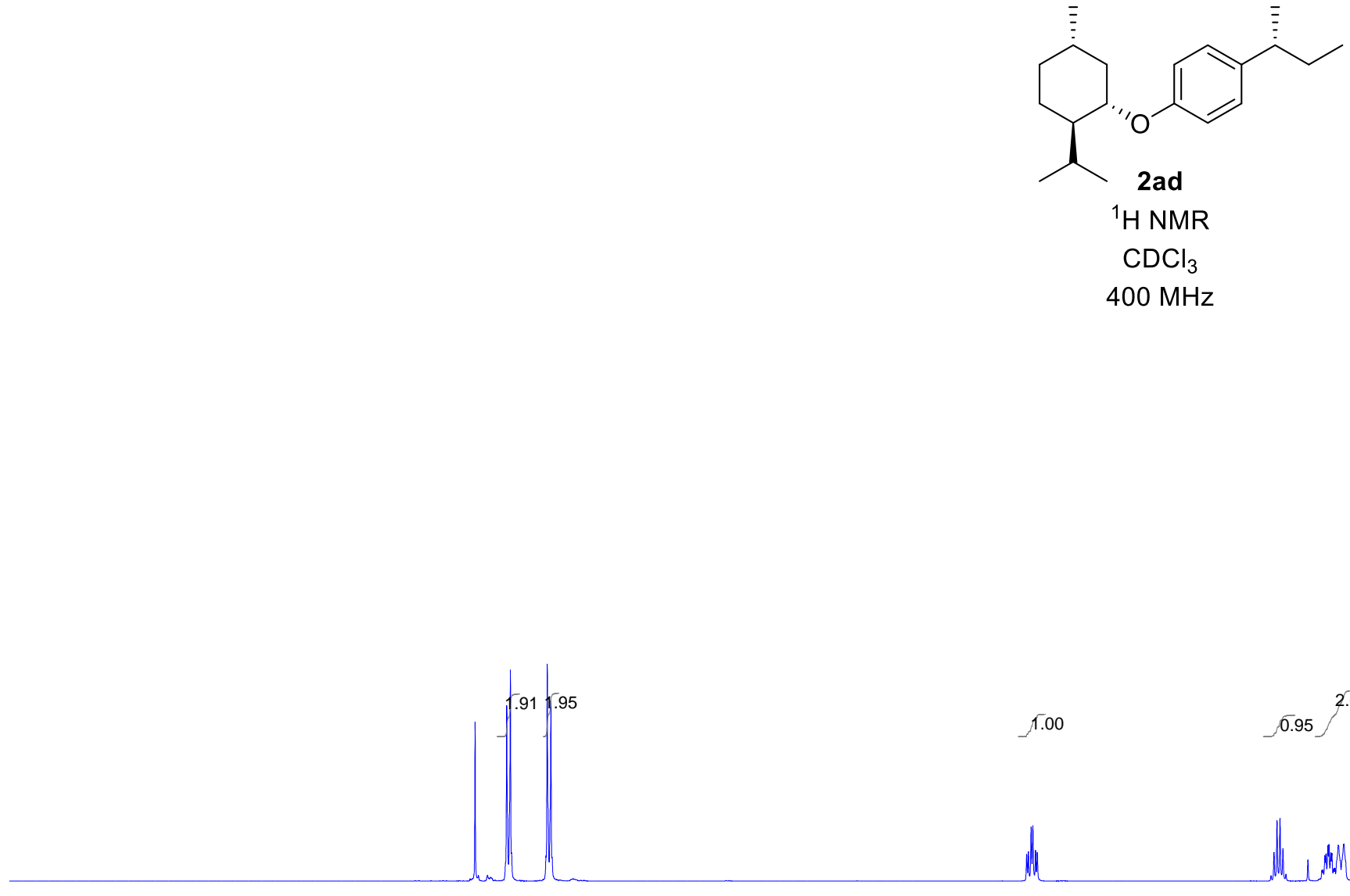

1.00

$0.95{ }^{2.05}$

2.05
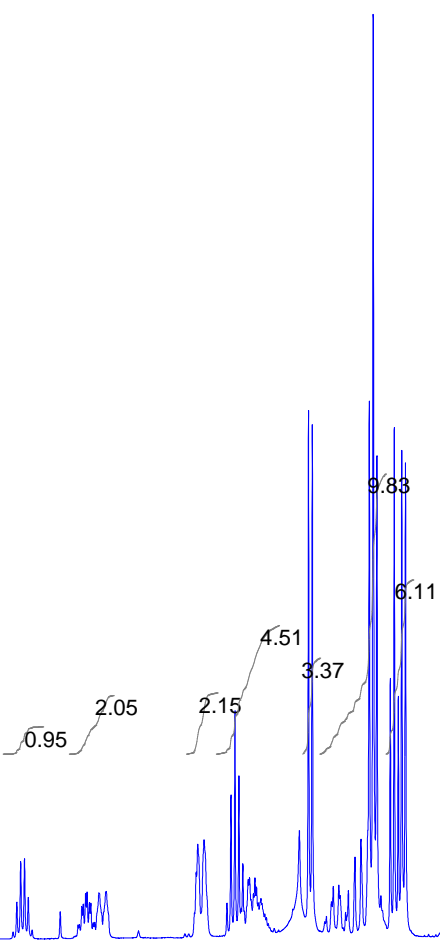

0 PPM 


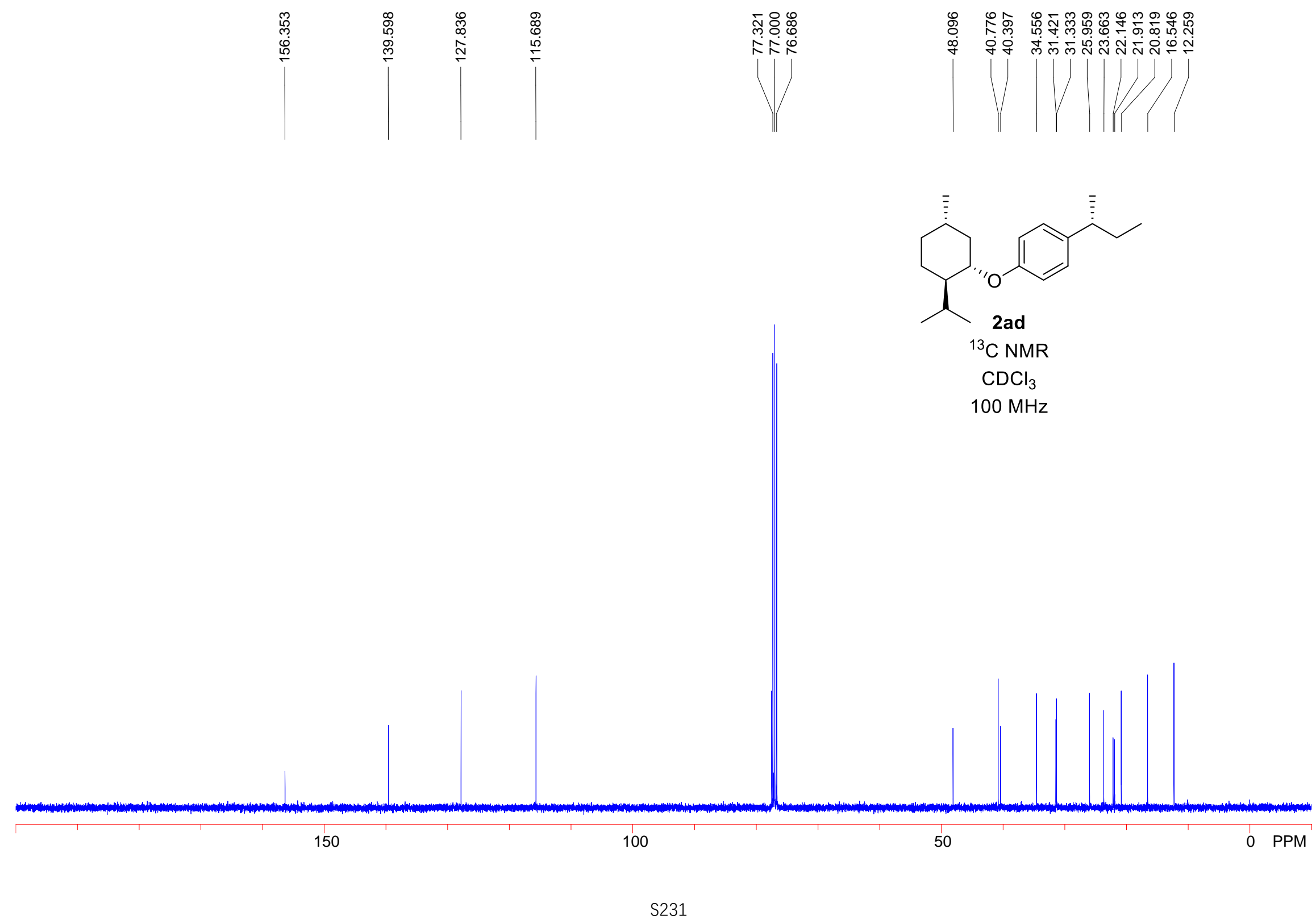




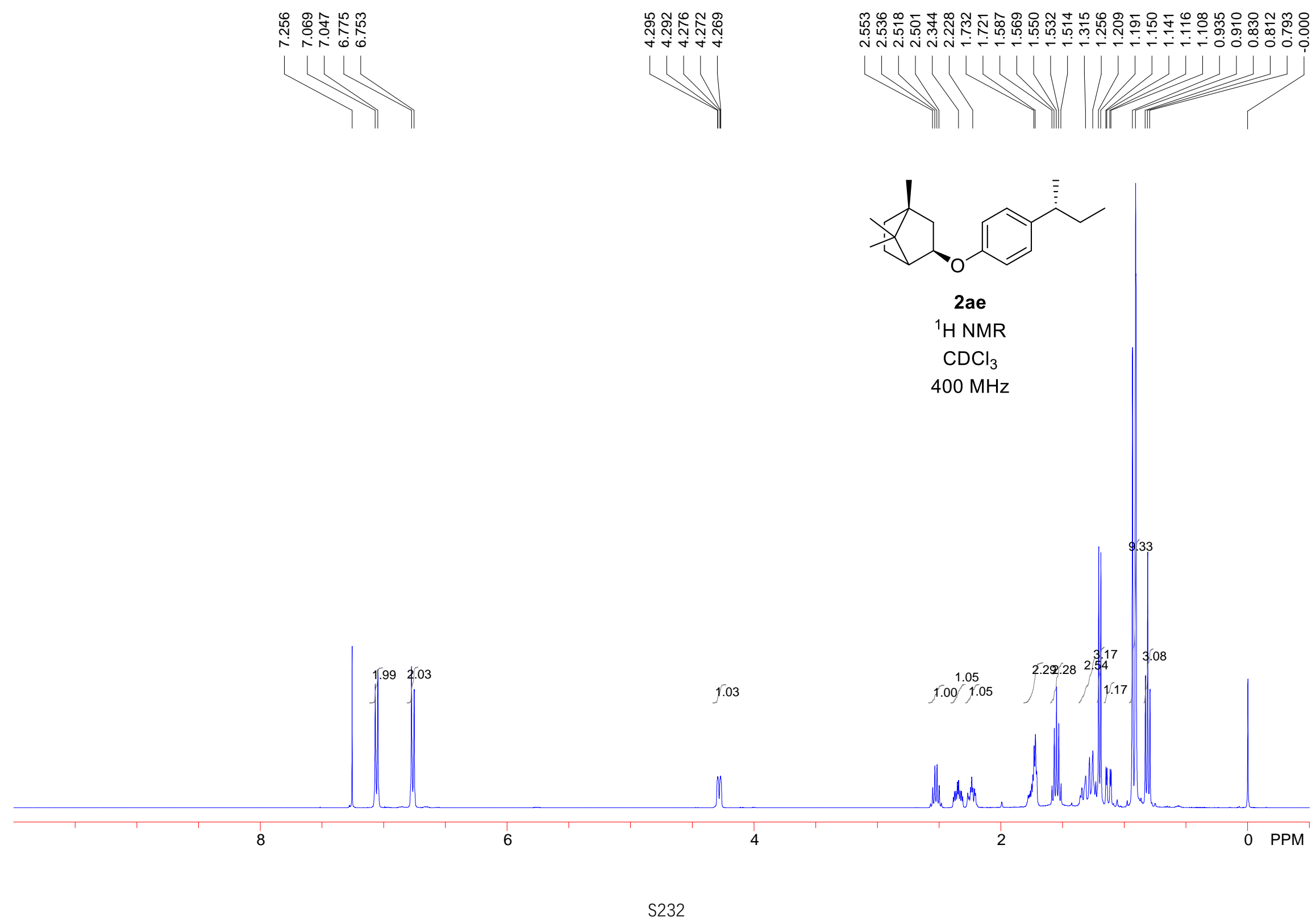




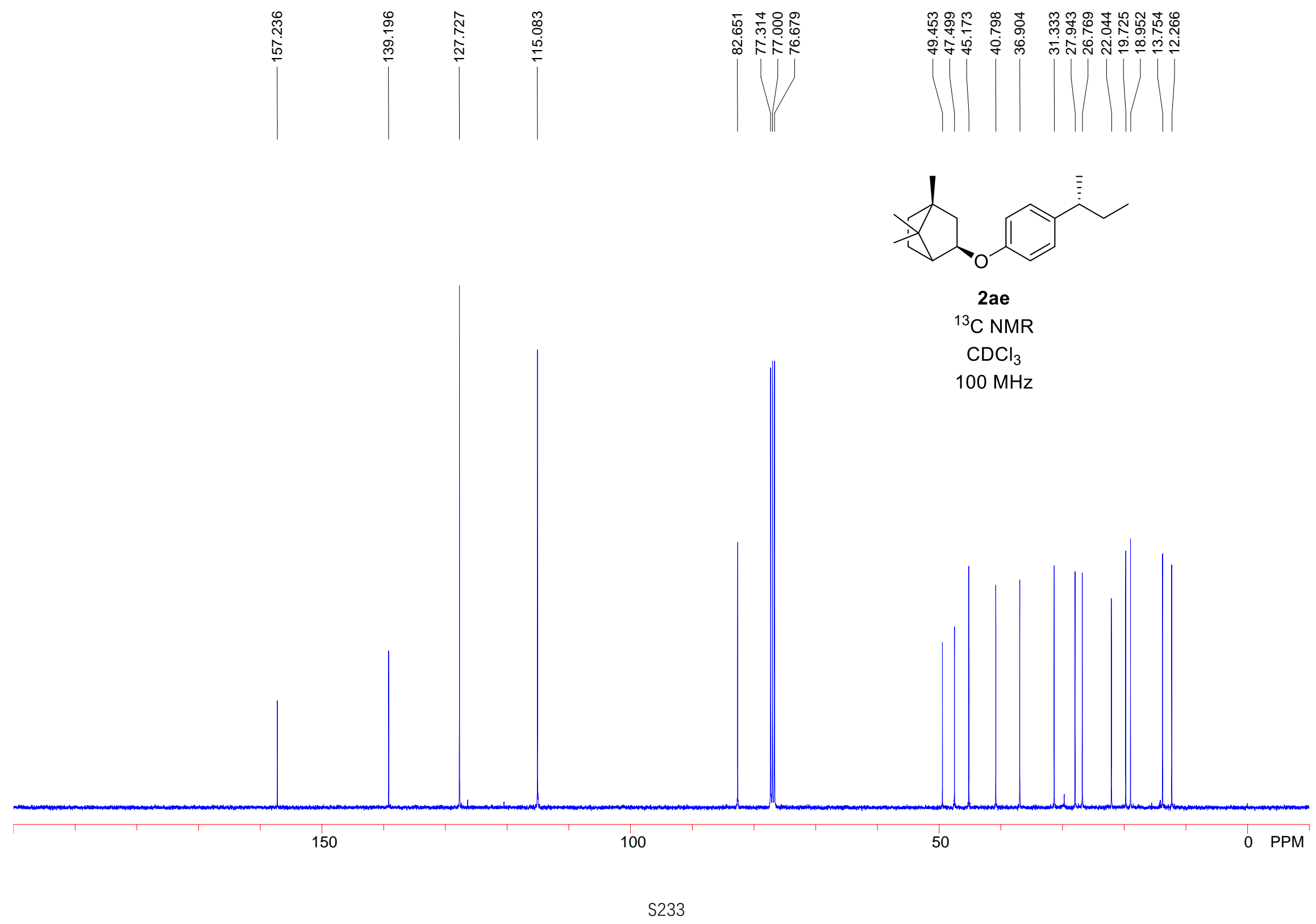




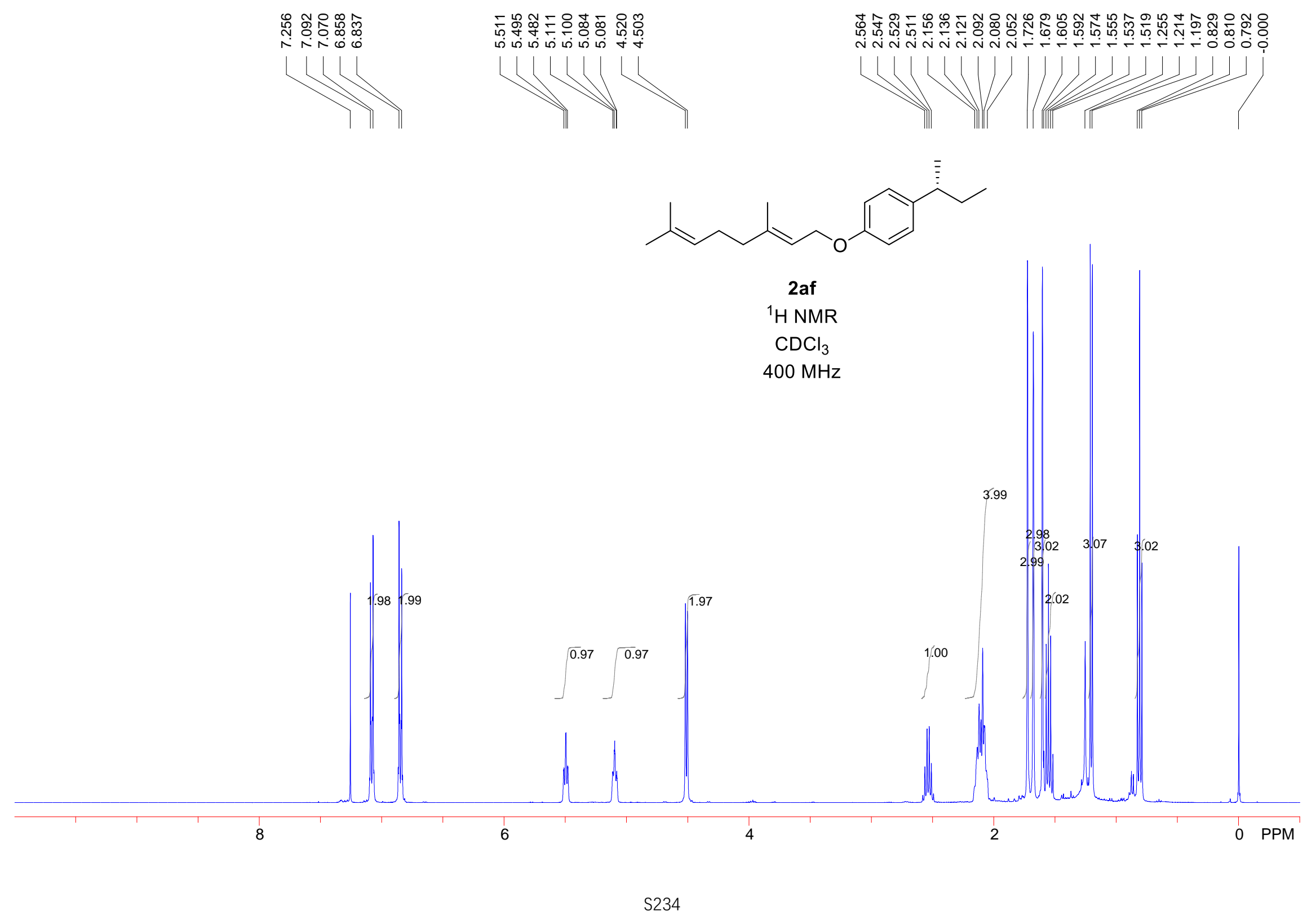




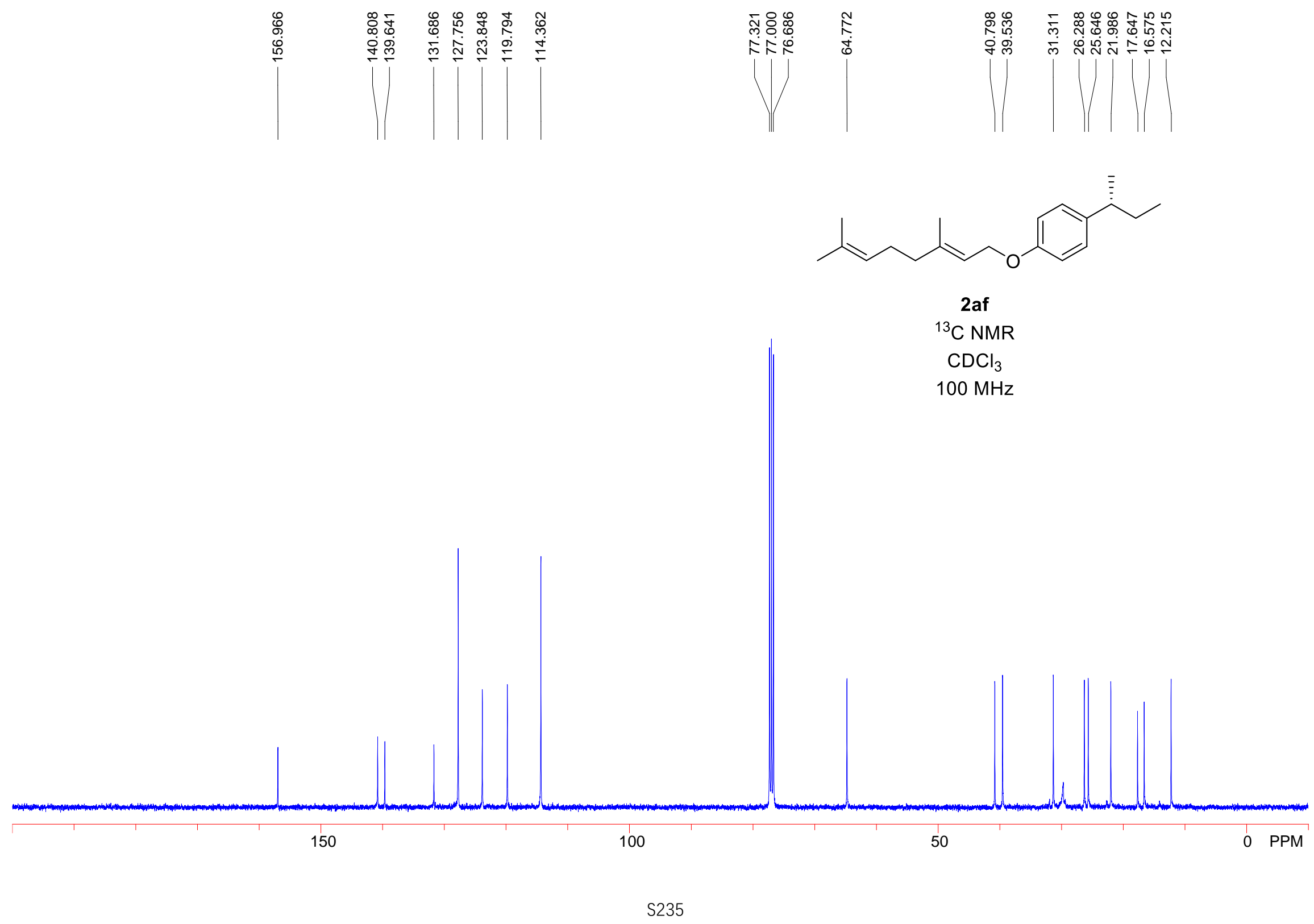




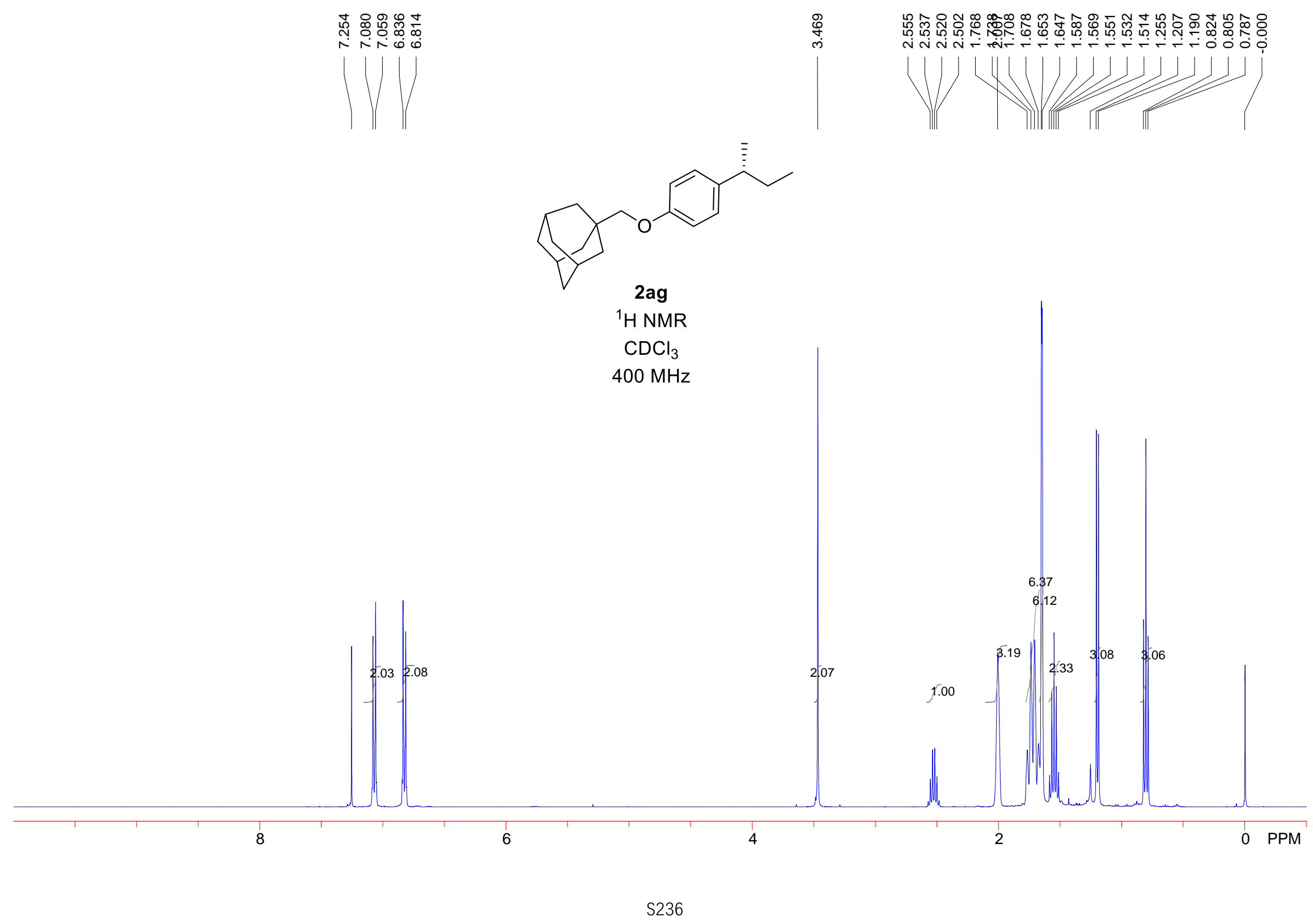




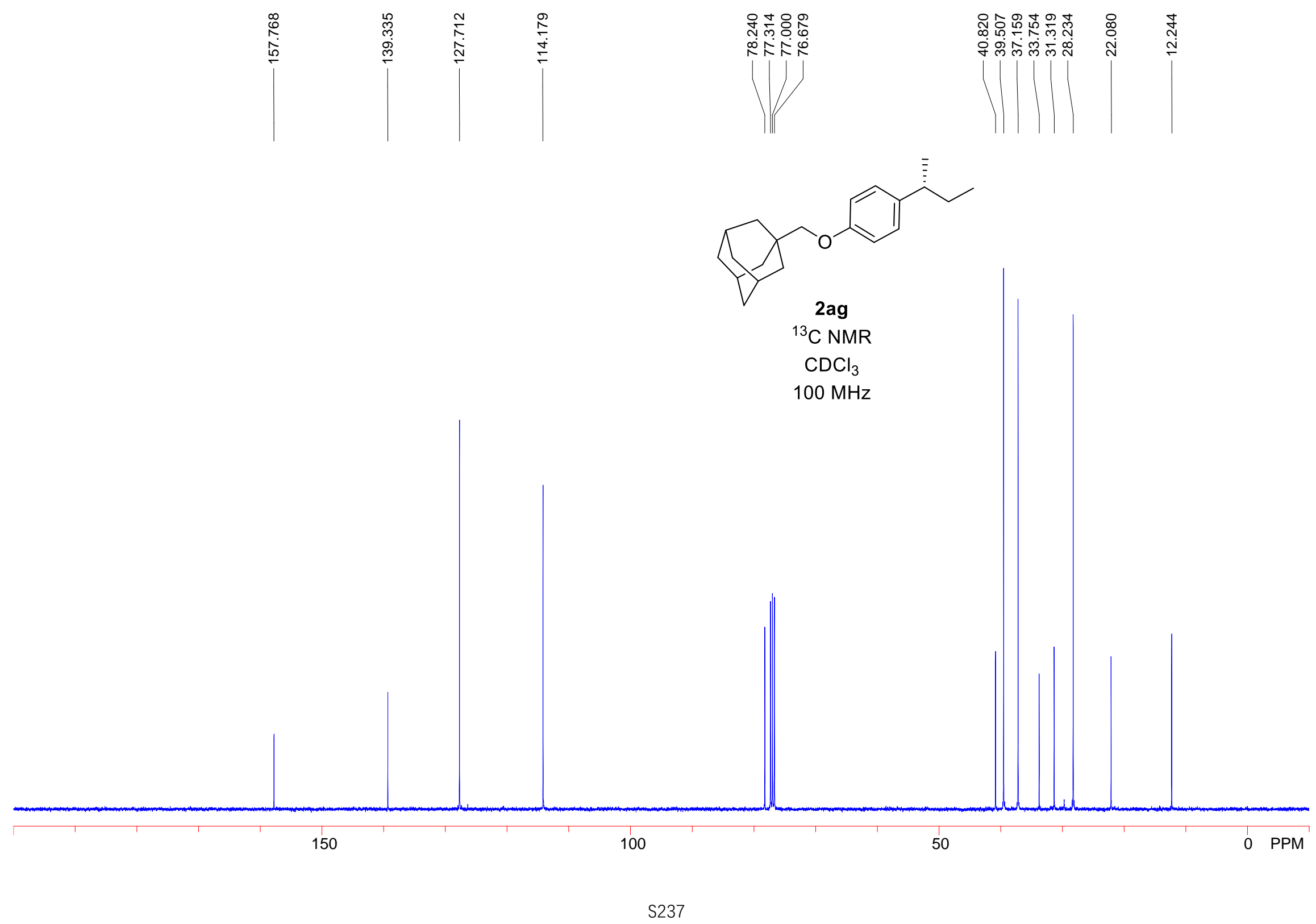


员

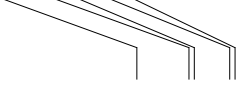

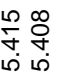

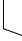

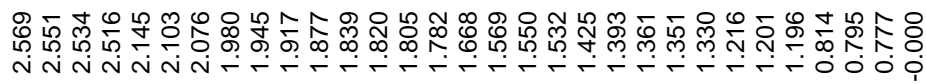
4

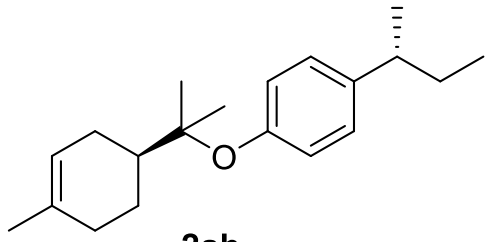

$2 a h$

${ }^{1} \mathrm{H}$ NMR

$\mathrm{CDCl}_{3}$

$400 \mathrm{MHz}$

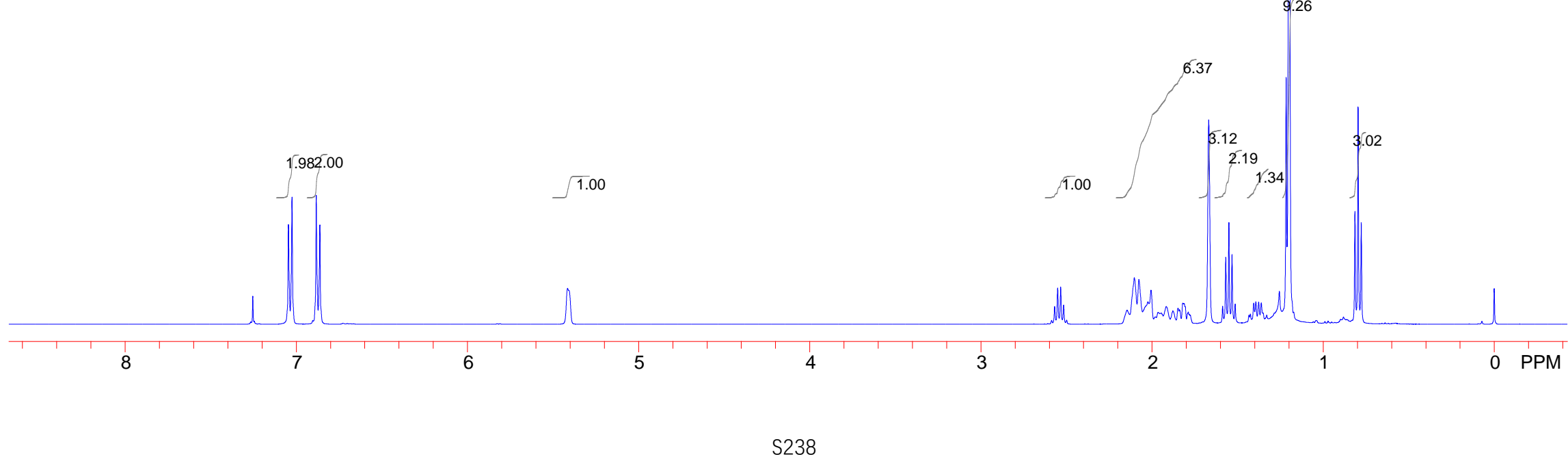




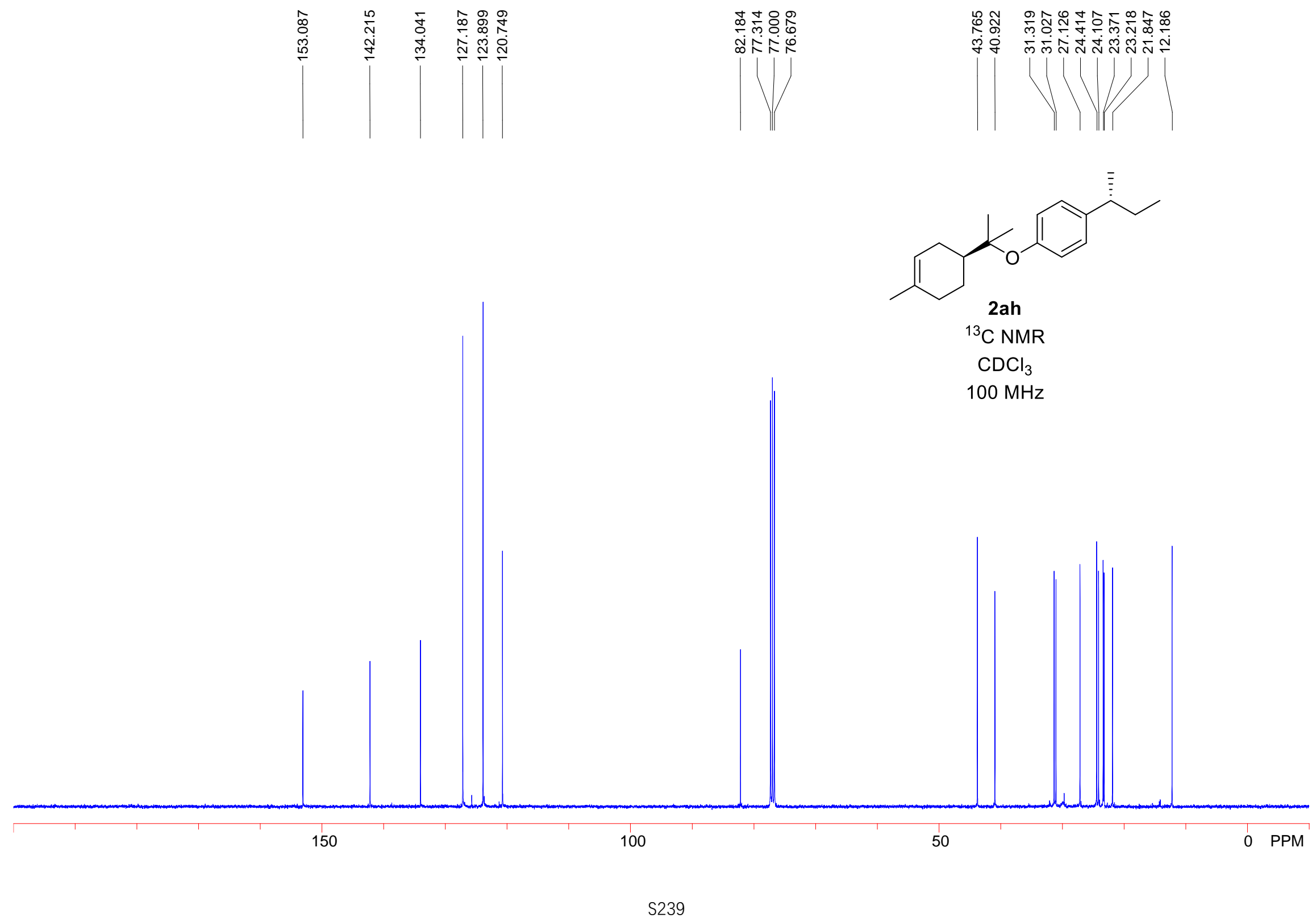




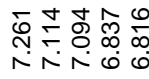

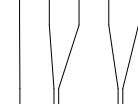

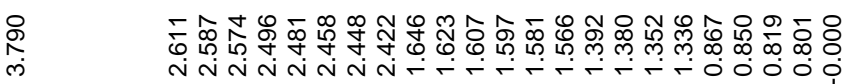

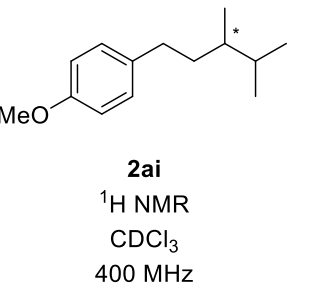

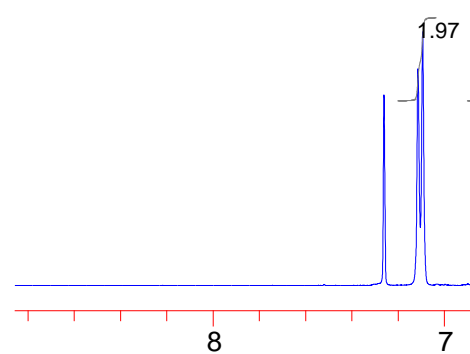

2.99

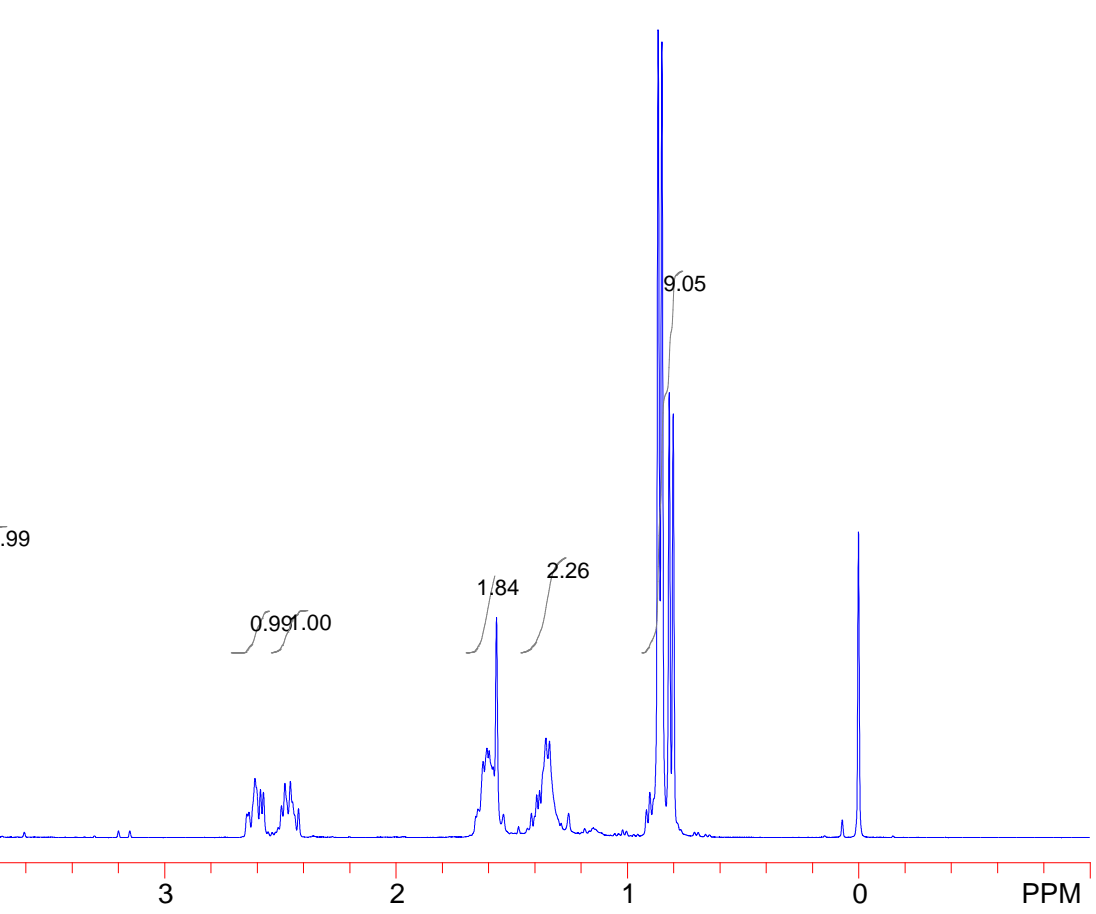




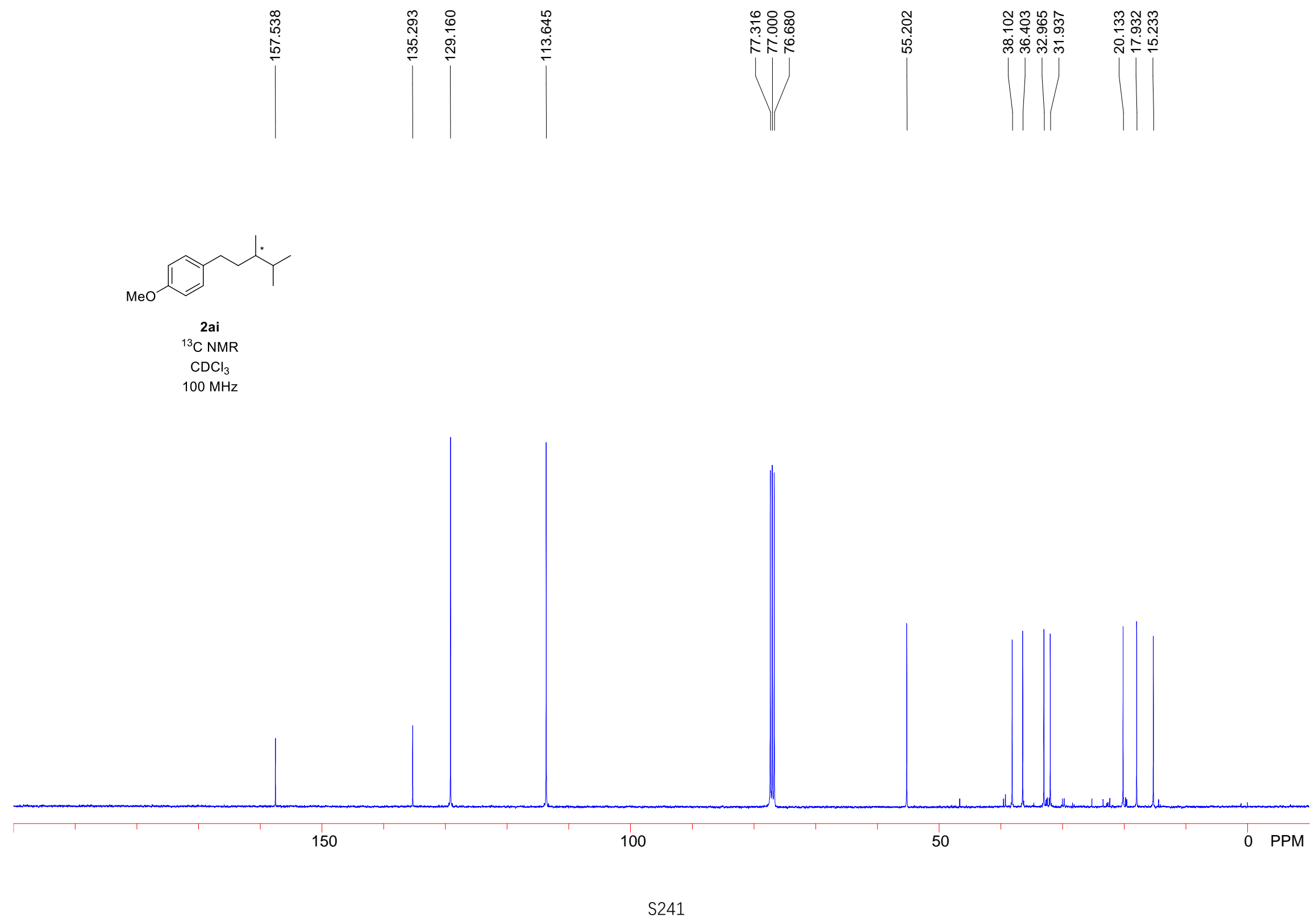




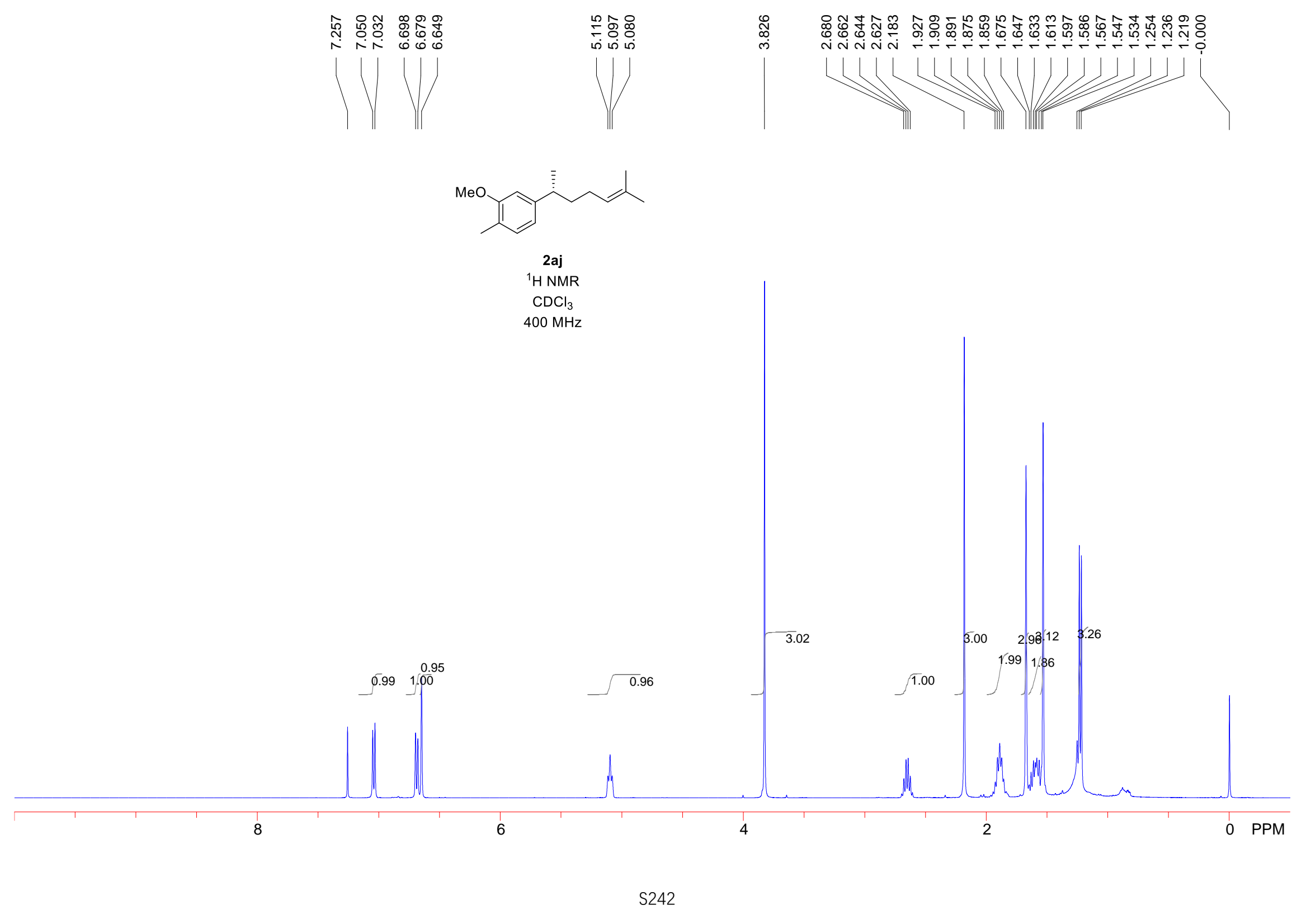




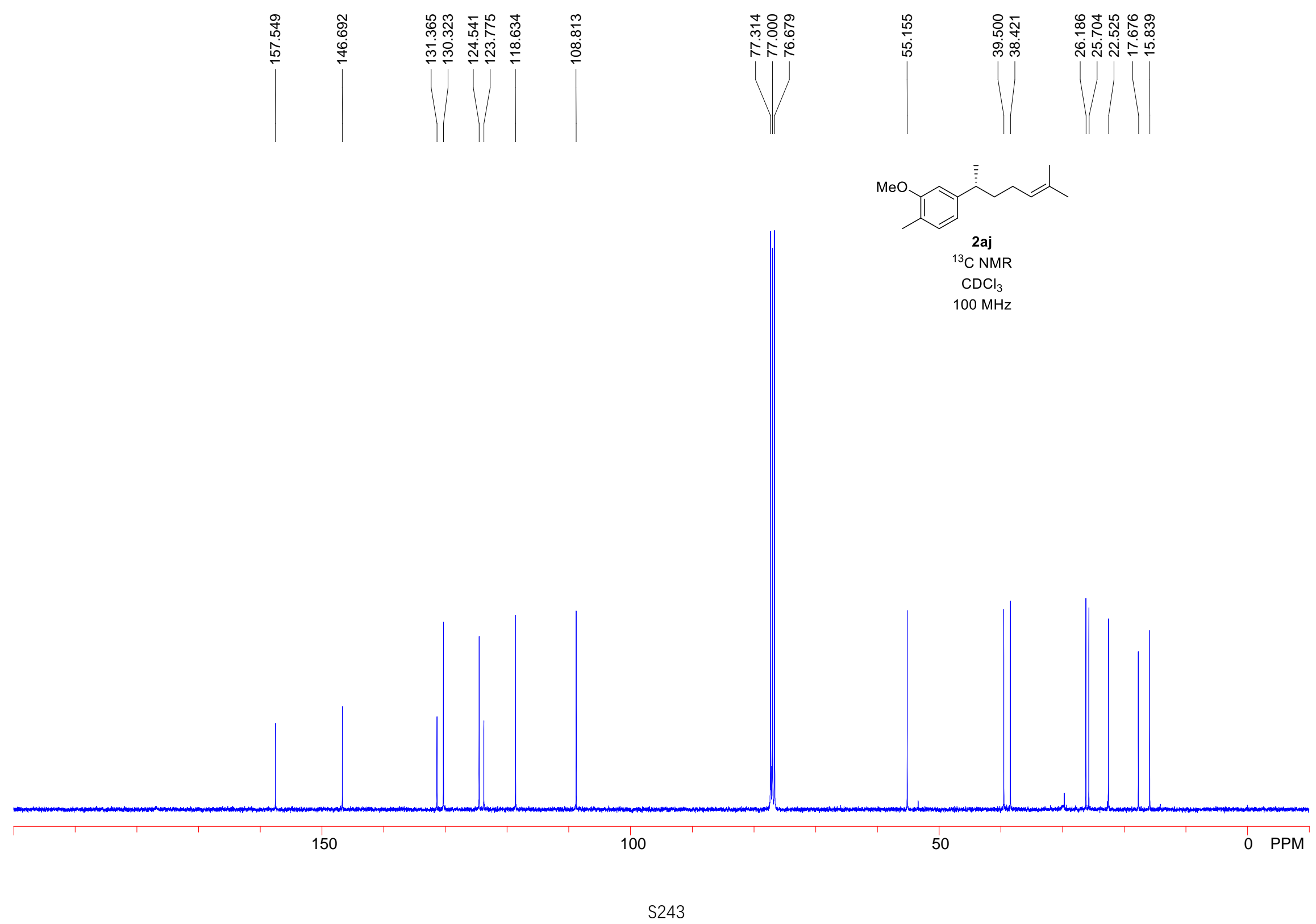




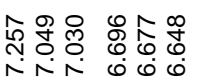

V V

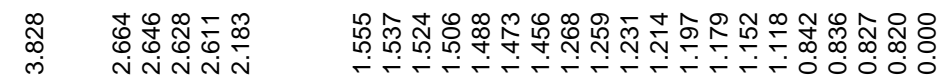

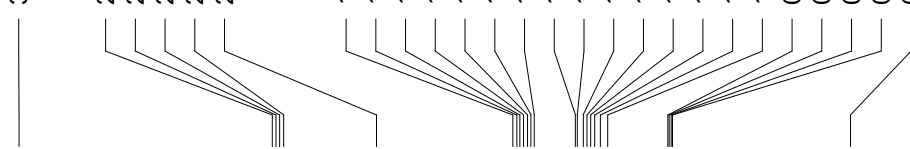

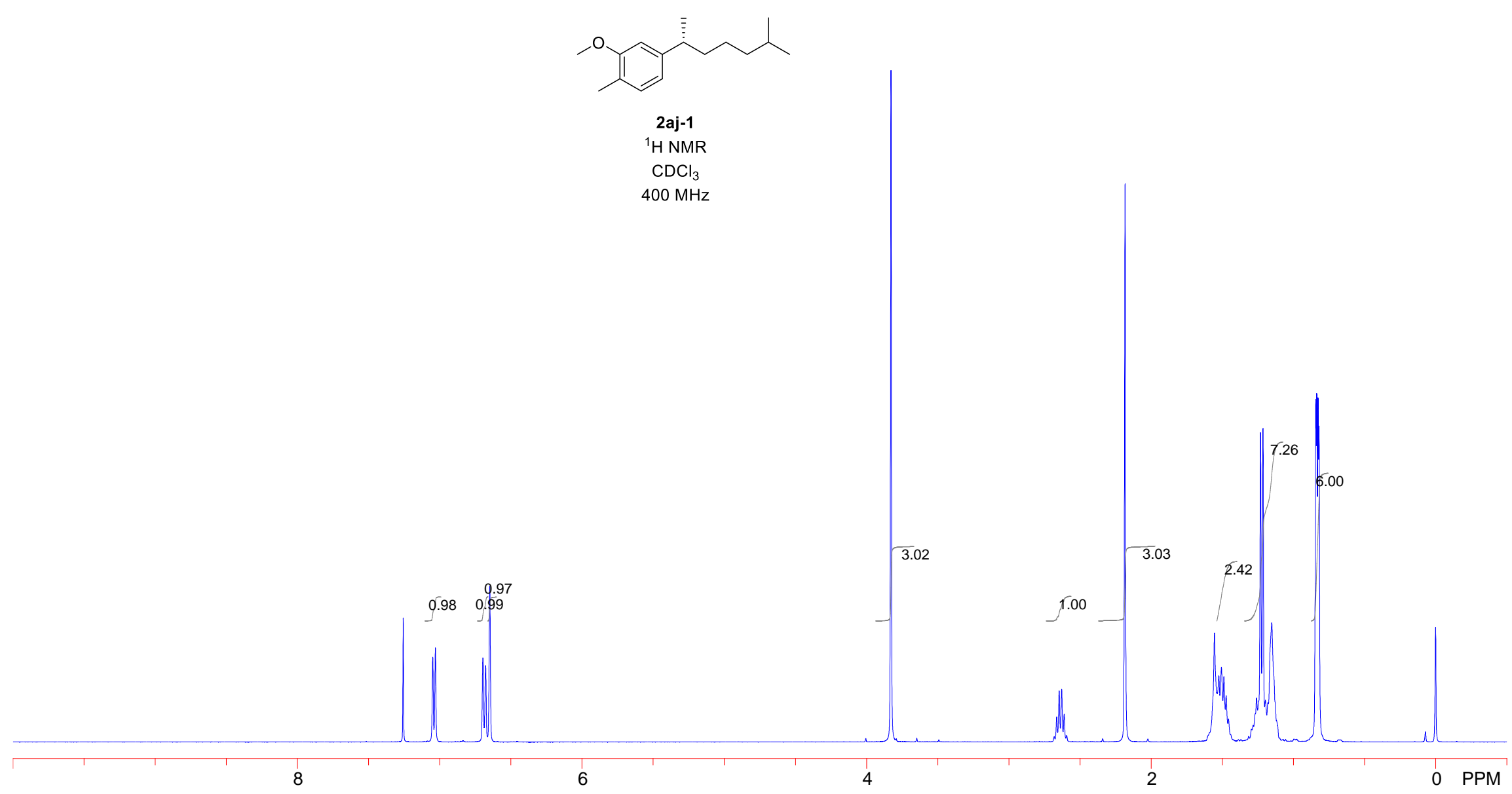




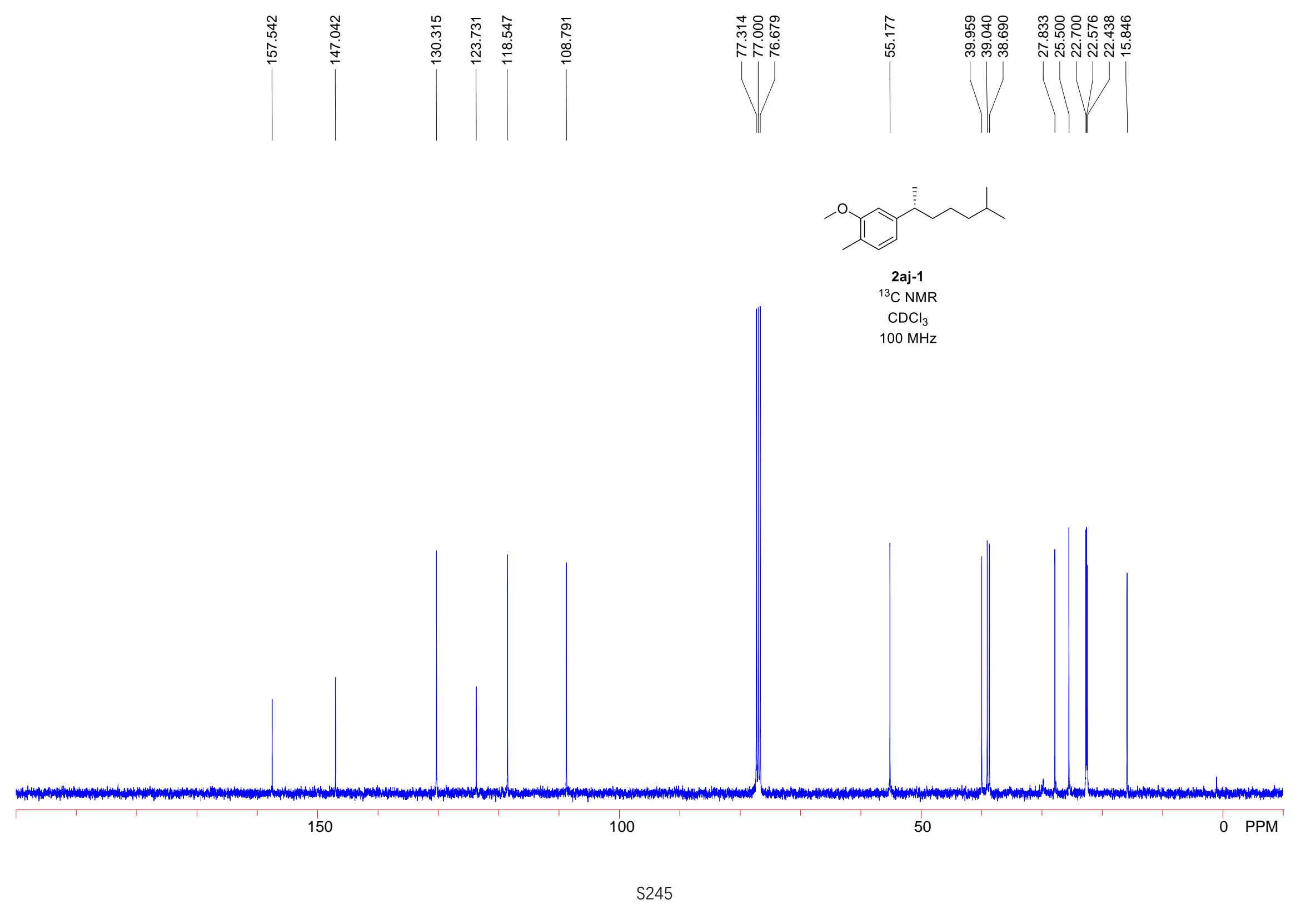




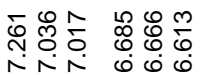

$\stackrel{\infty}{\circ}$

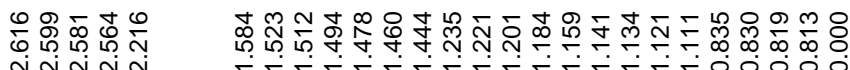

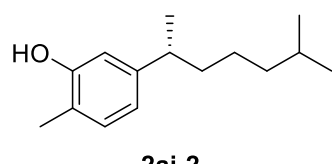

2aj-2

${ }^{1} \mathrm{H}$ NMR

$\mathrm{CDCl}_{3}$

$400 \mathrm{MHz}$

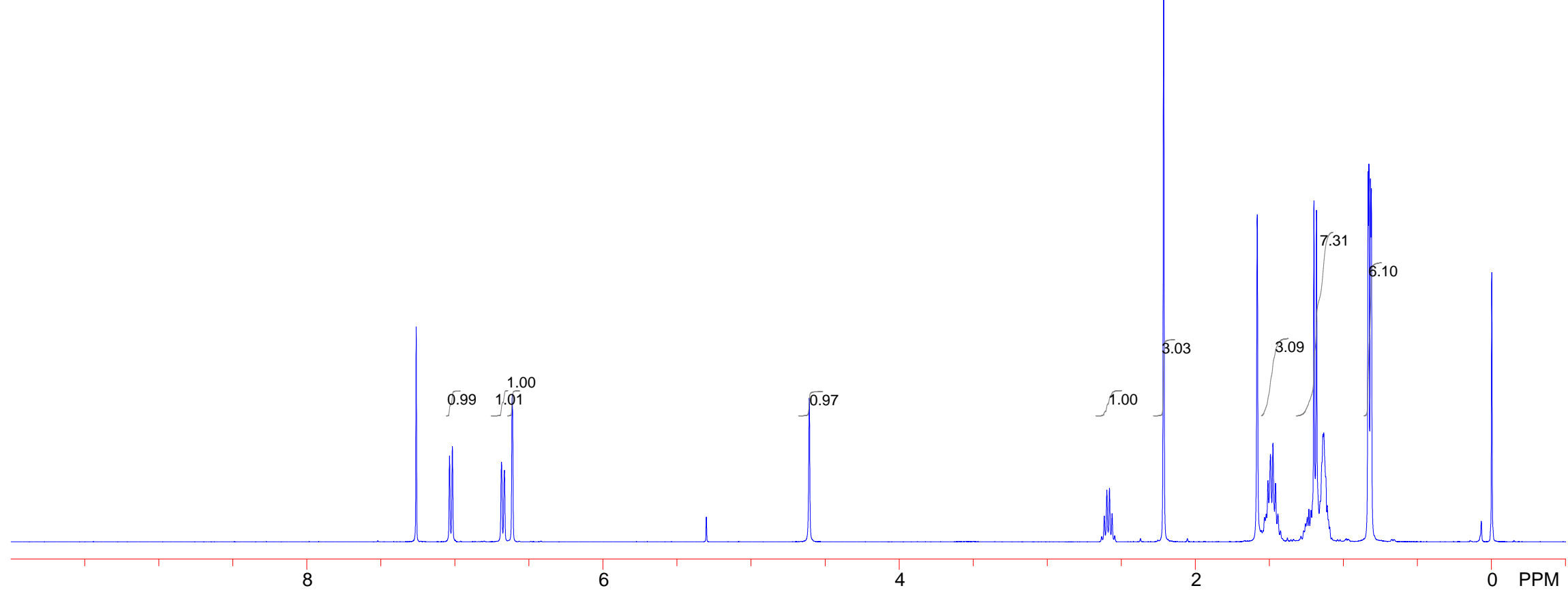




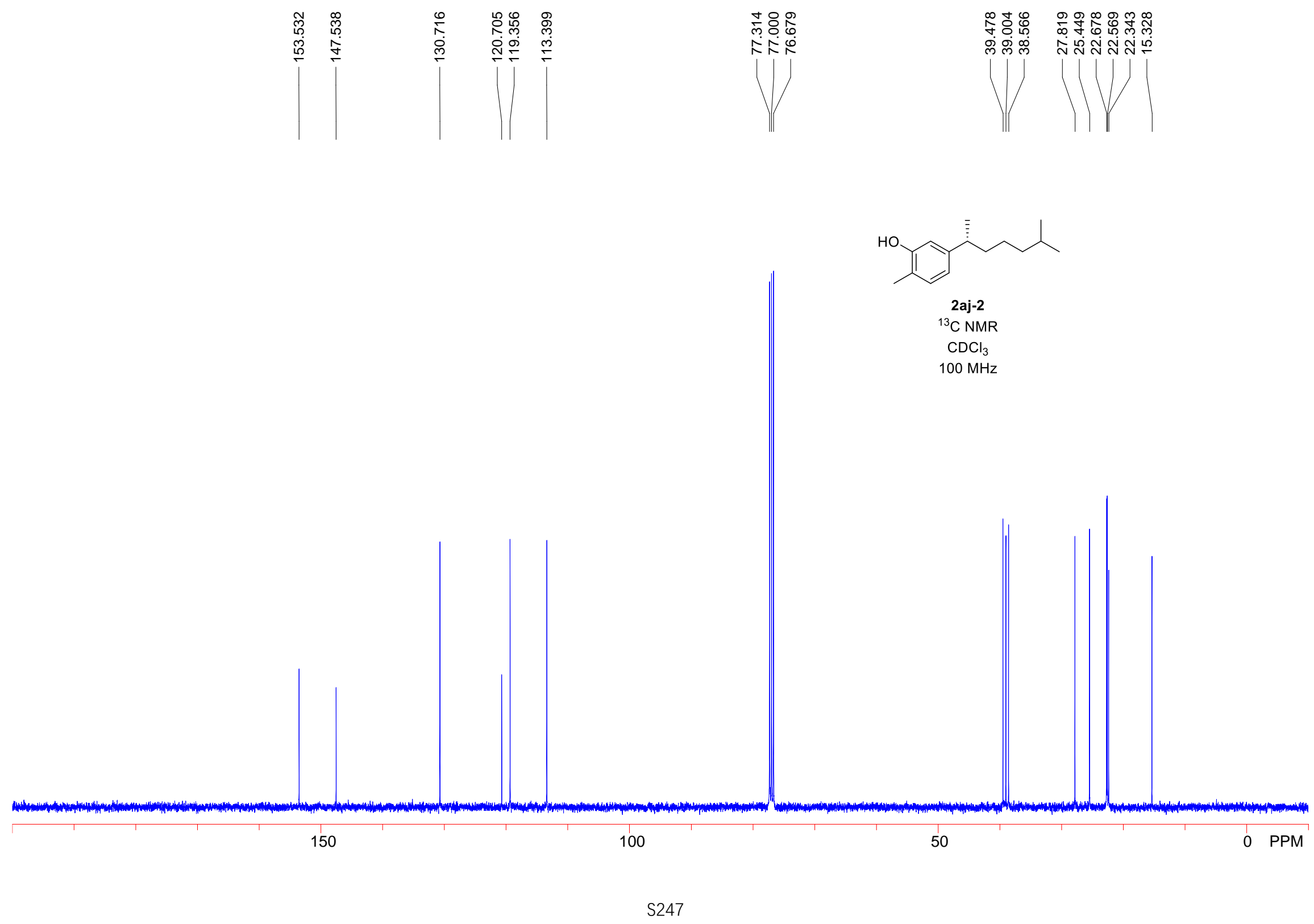




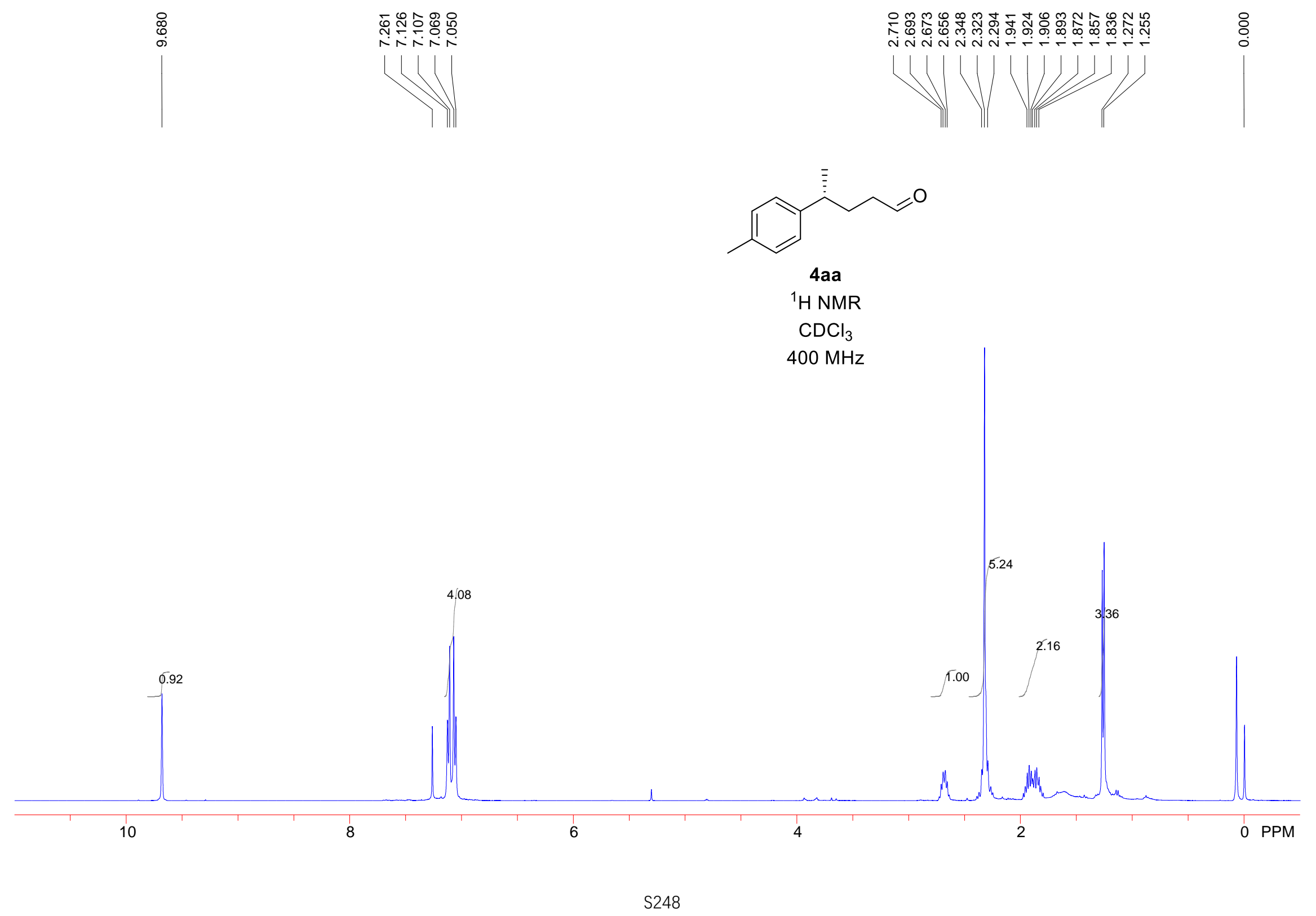




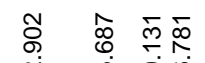

ఫ
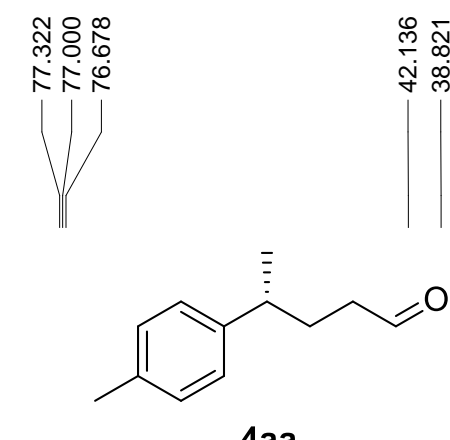

${ }^{13} \mathrm{C}$ NMR

$\mathrm{CDCl}_{3}$

$100 \mathrm{MHz}$

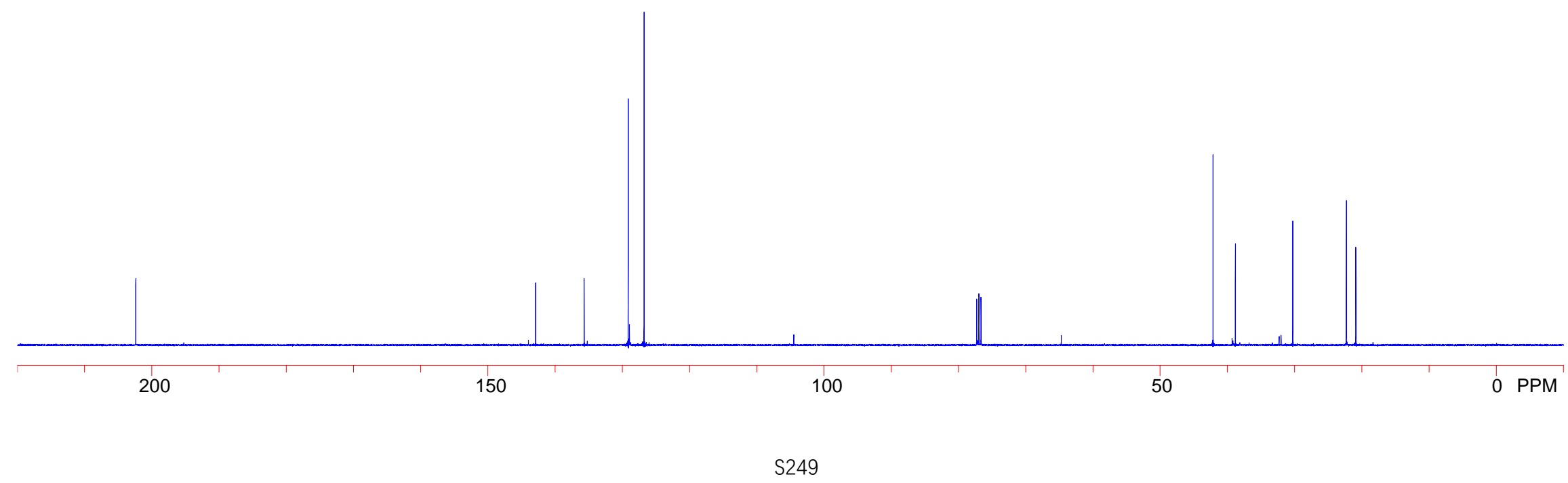




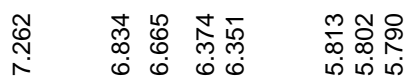

$|v| v$ $\underset{\substack{* \\ \infty}}{\substack{\infty \\ j}}$

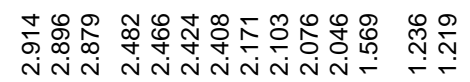

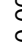

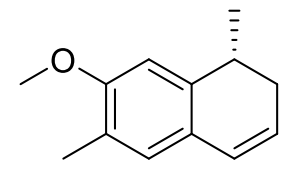

$4 a b$

${ }^{1} \mathrm{H}$ NMR

$\mathrm{CDCl}_{3}$

$400 \mathrm{MHz}$

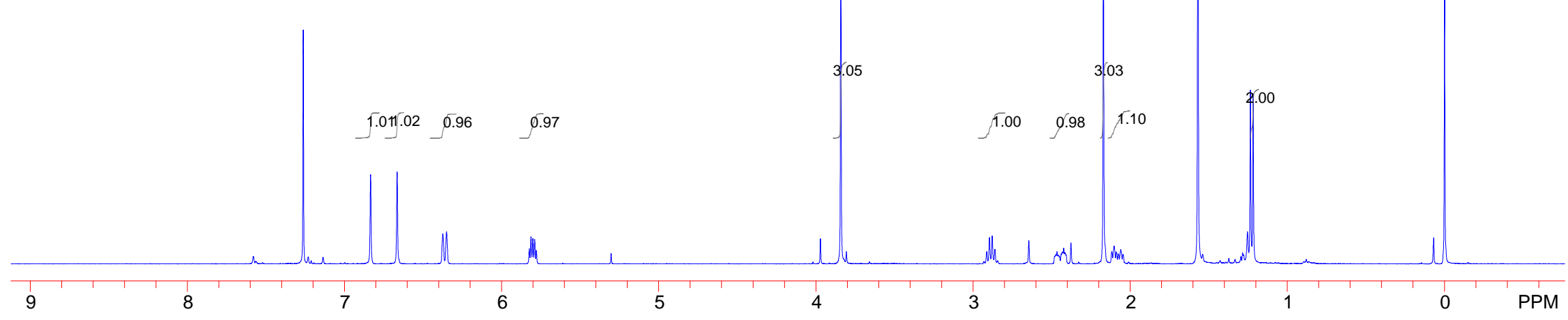




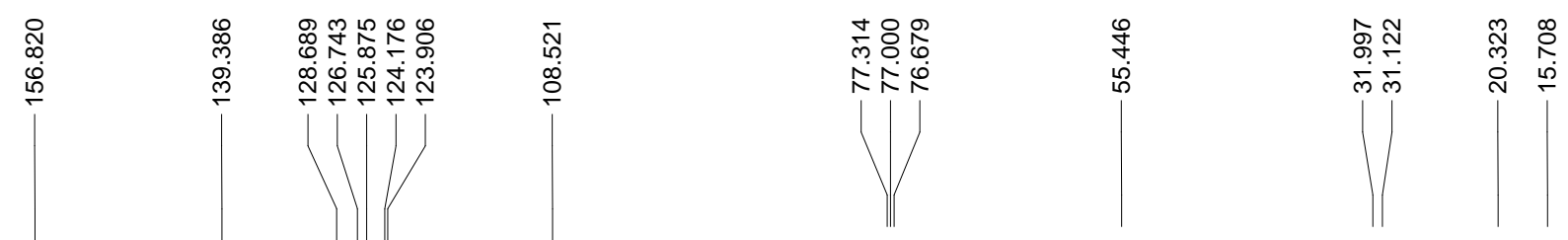

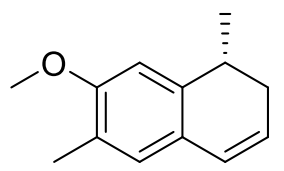

$4 a b$

${ }^{13} \mathrm{C}$ NMR

$\mathrm{CDCl}_{3}$

$100 \mathrm{MHz}$

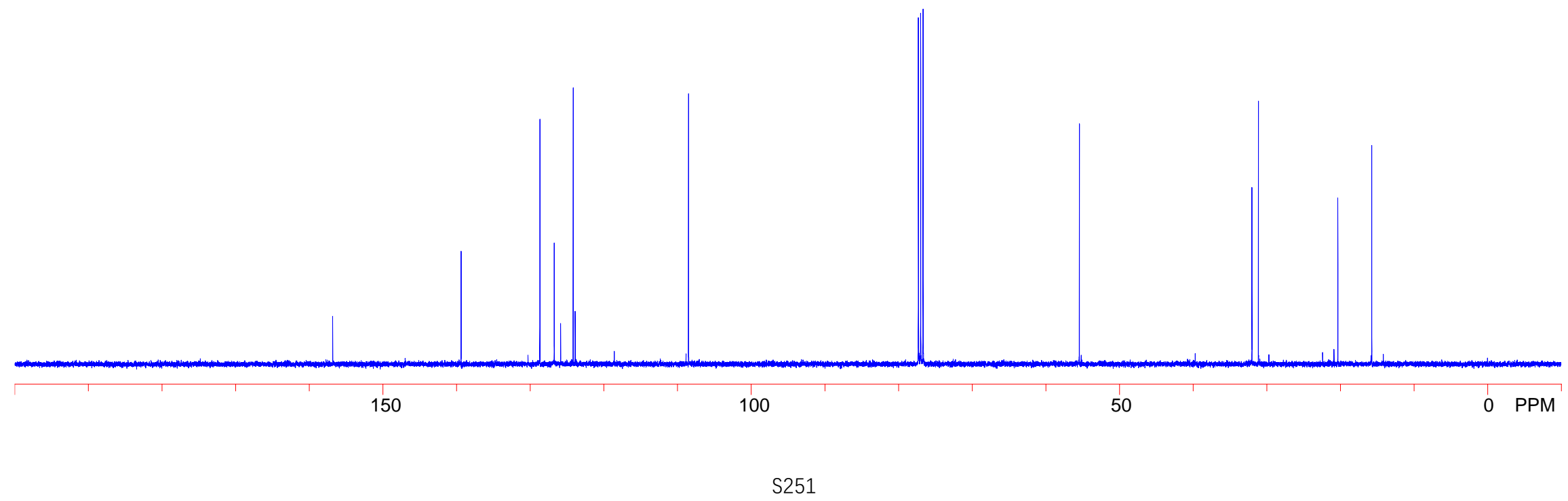




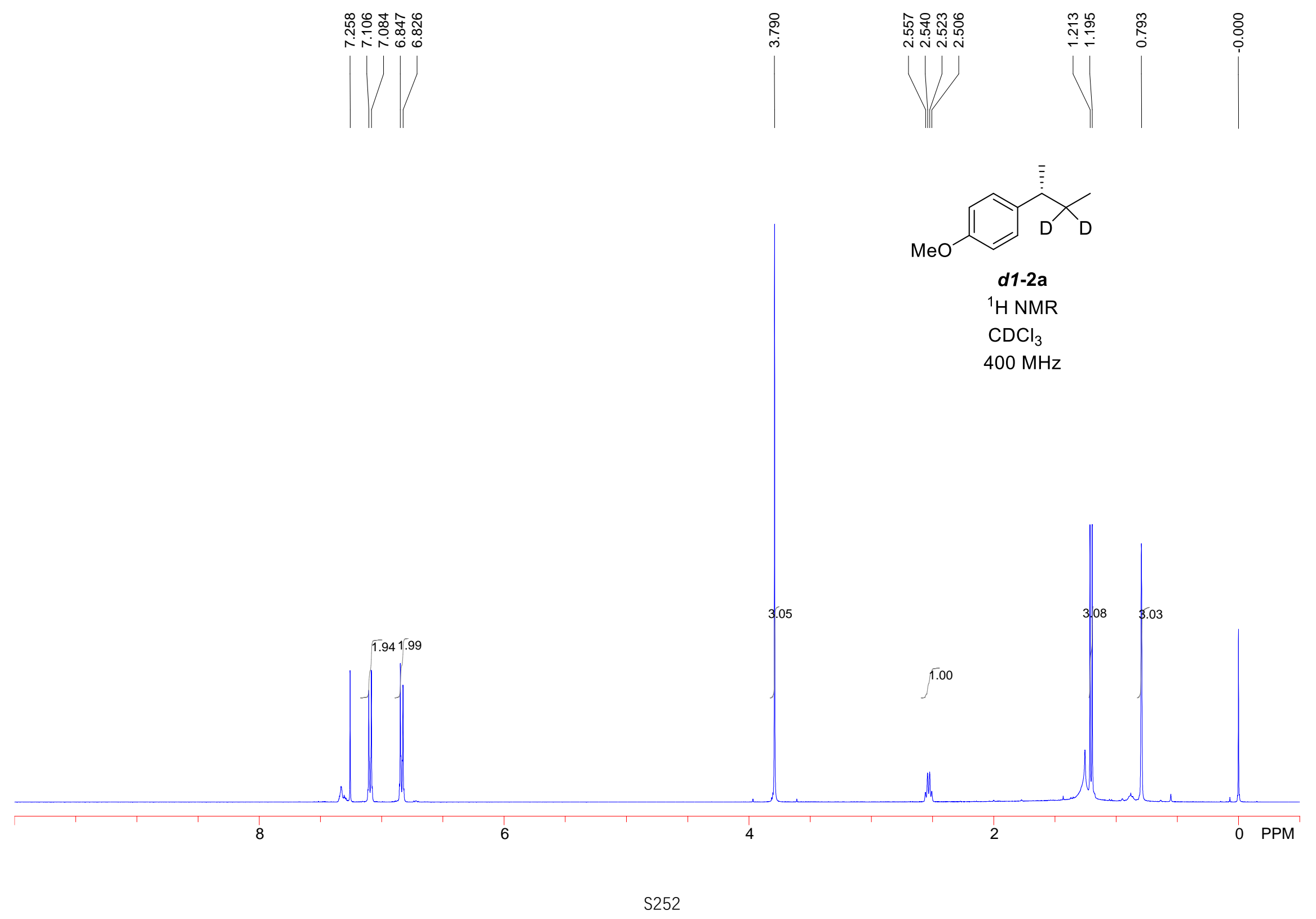




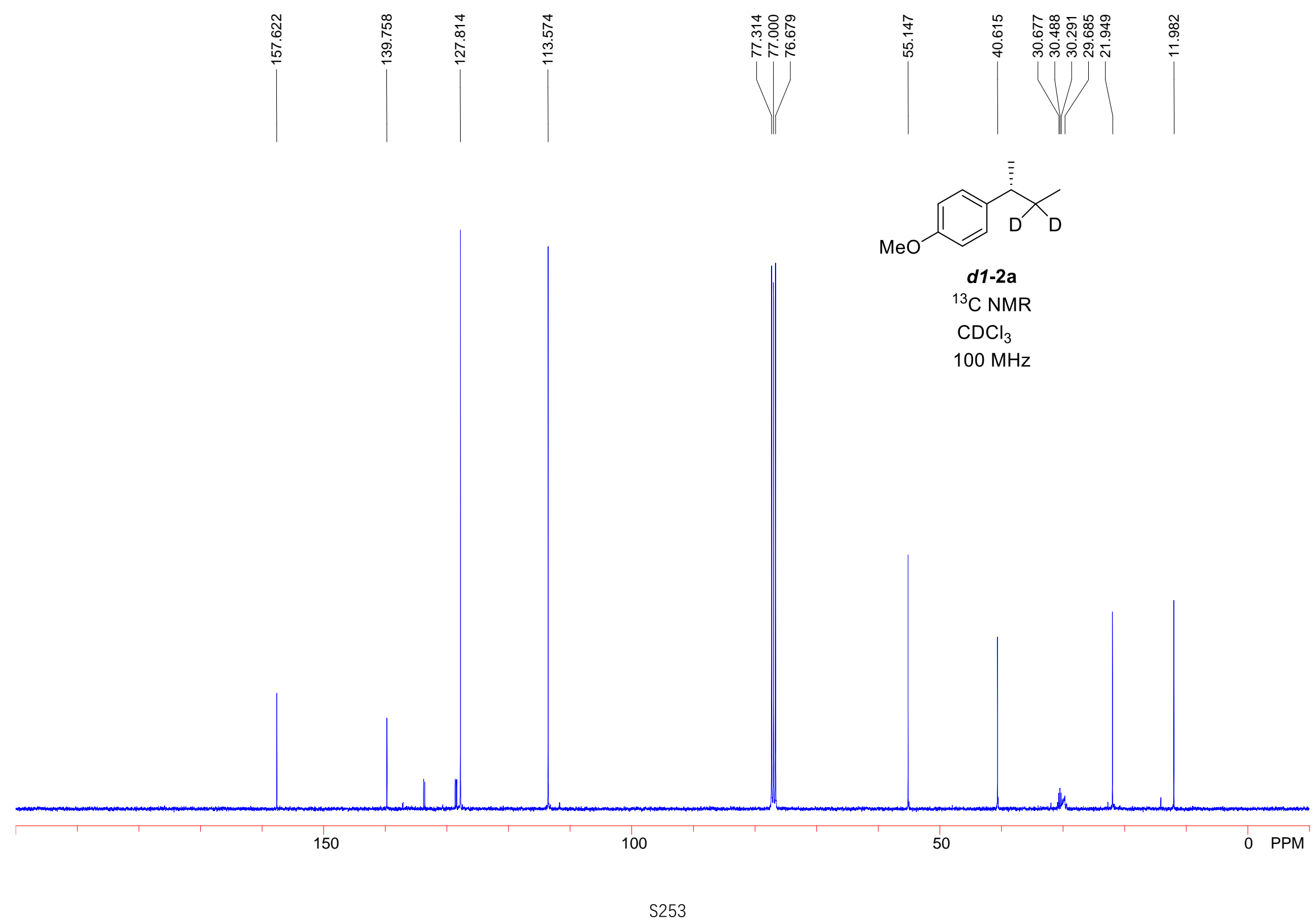




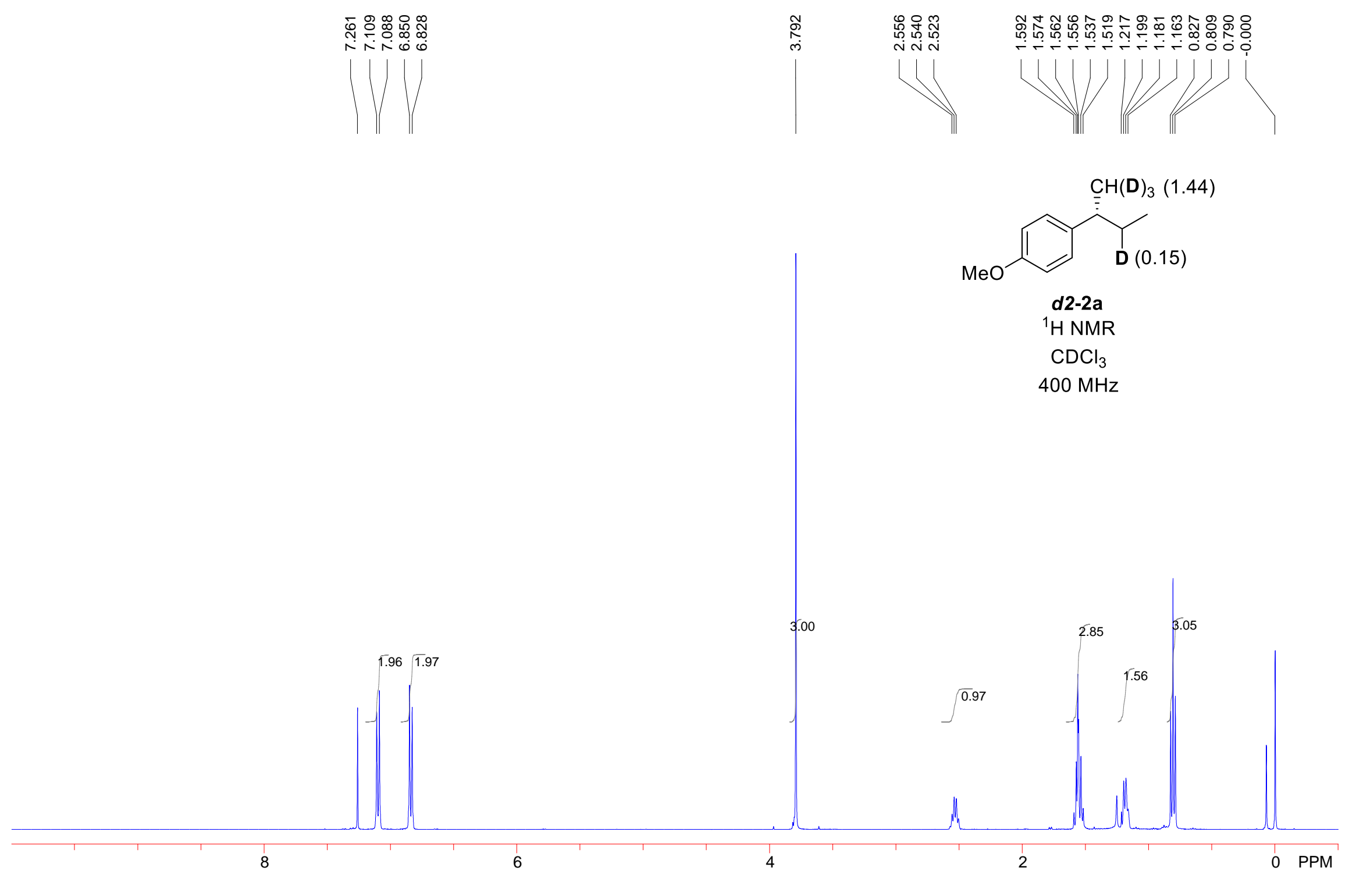




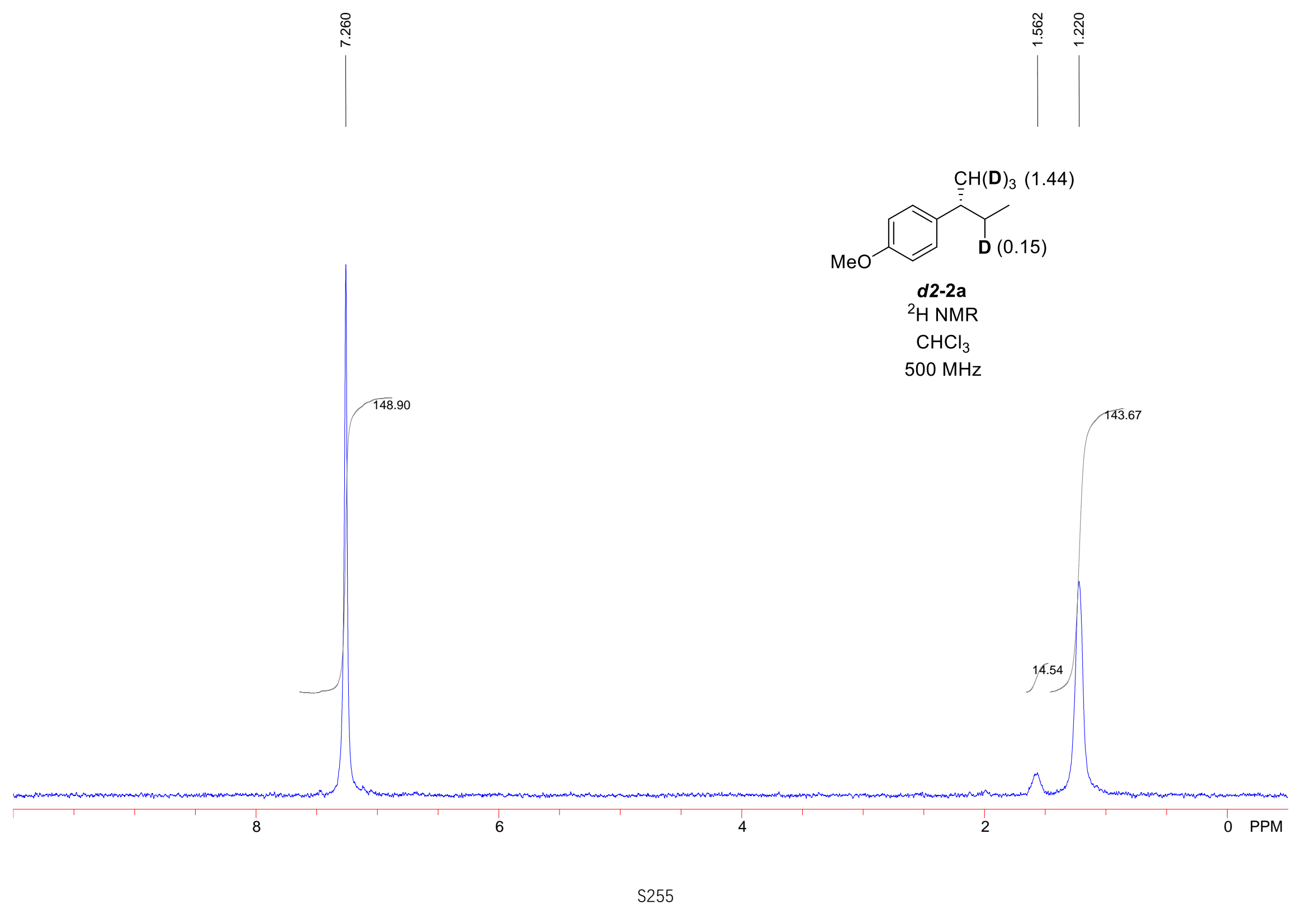




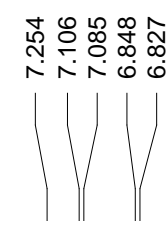

$\mathrm{Ar}=p-\mathrm{OMePh}$

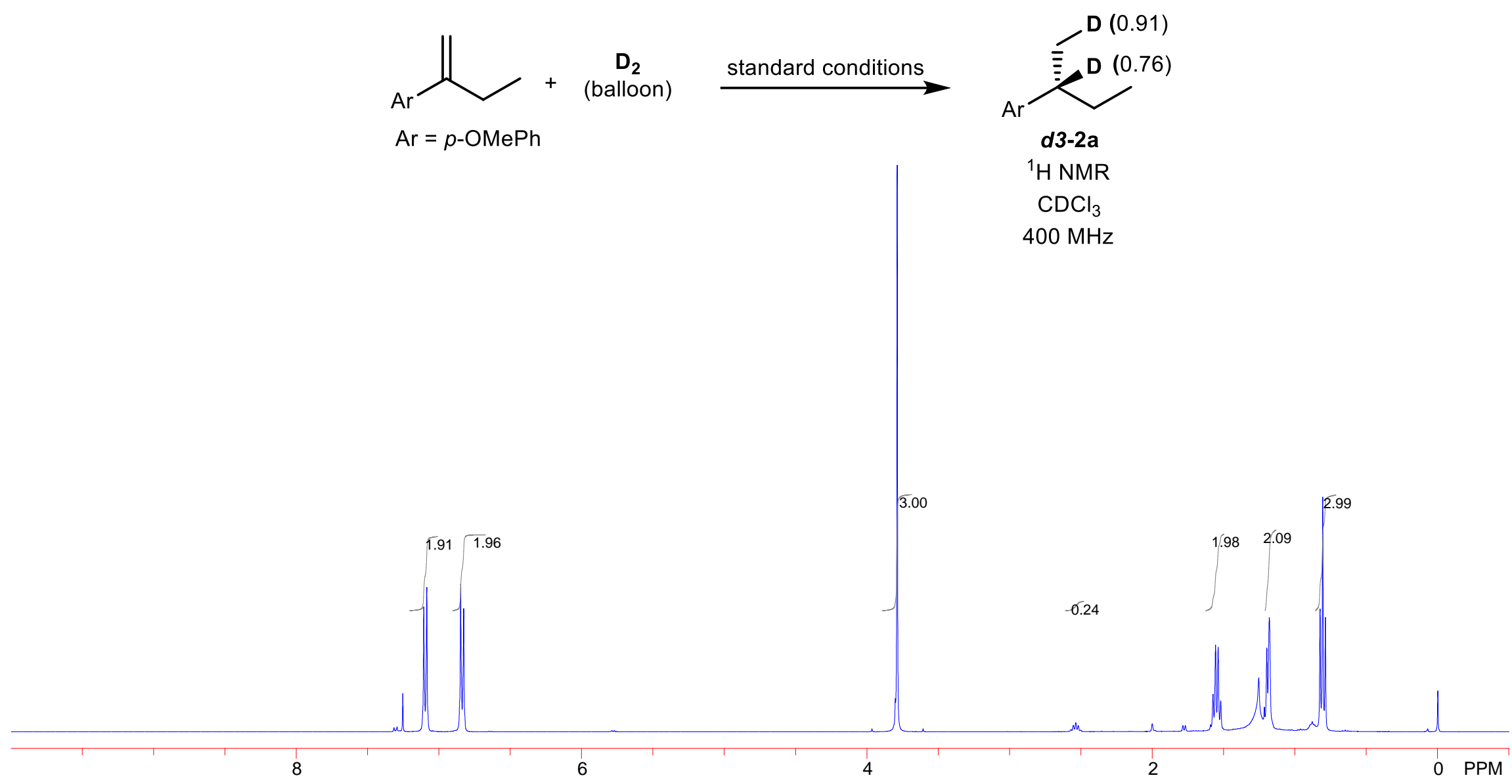




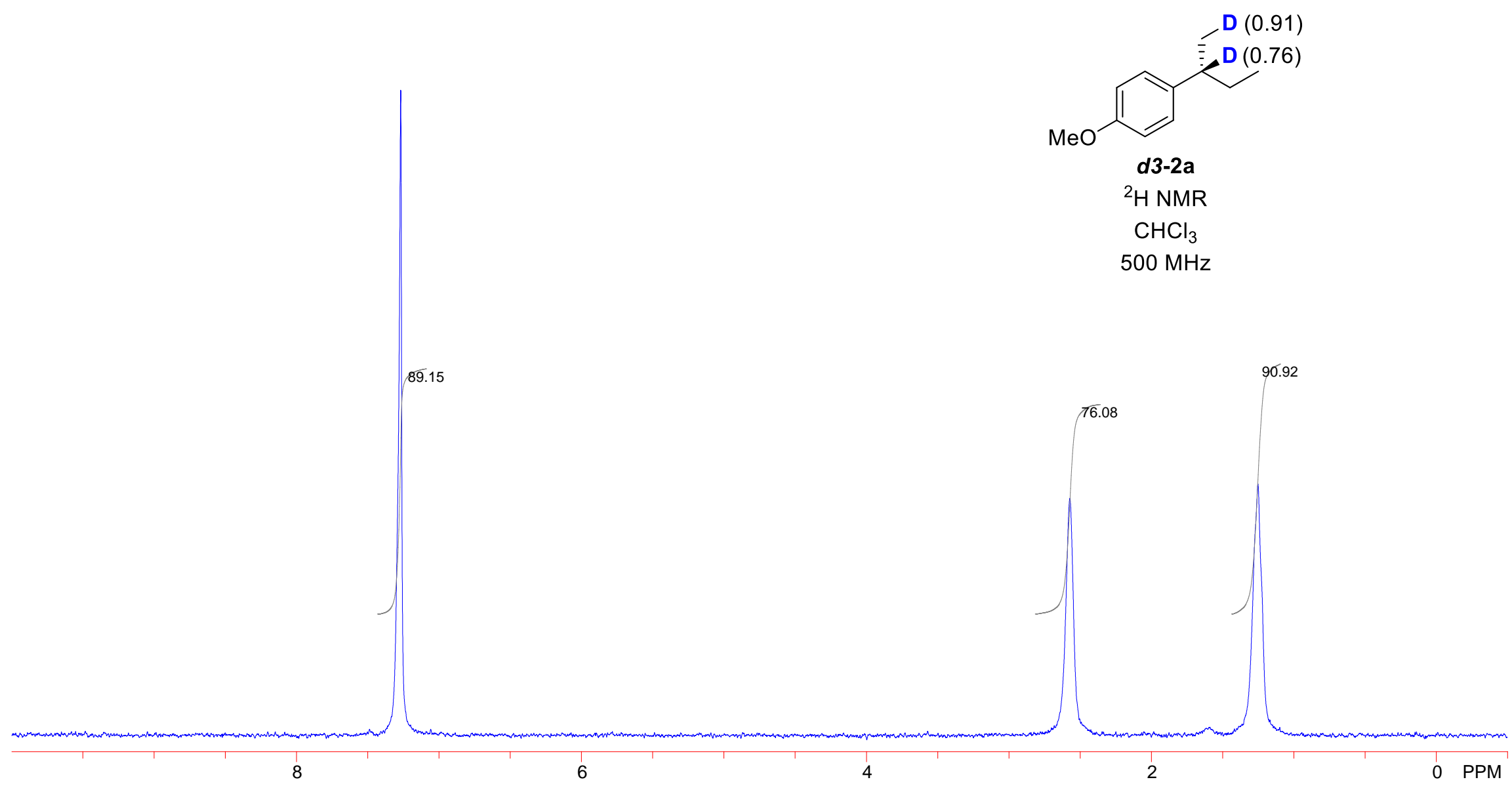




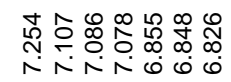

惫照品

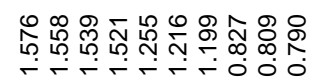

응

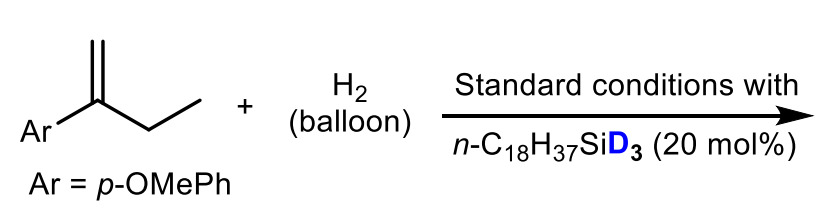

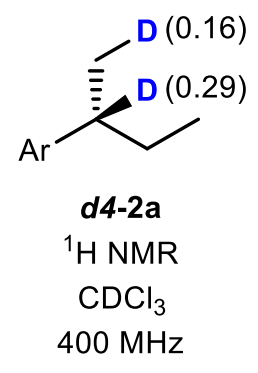

3.00

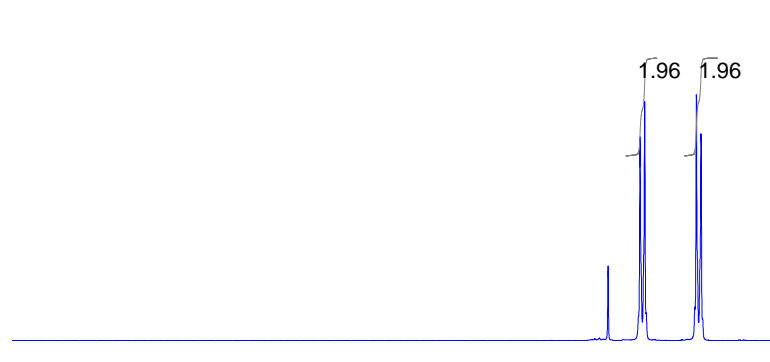




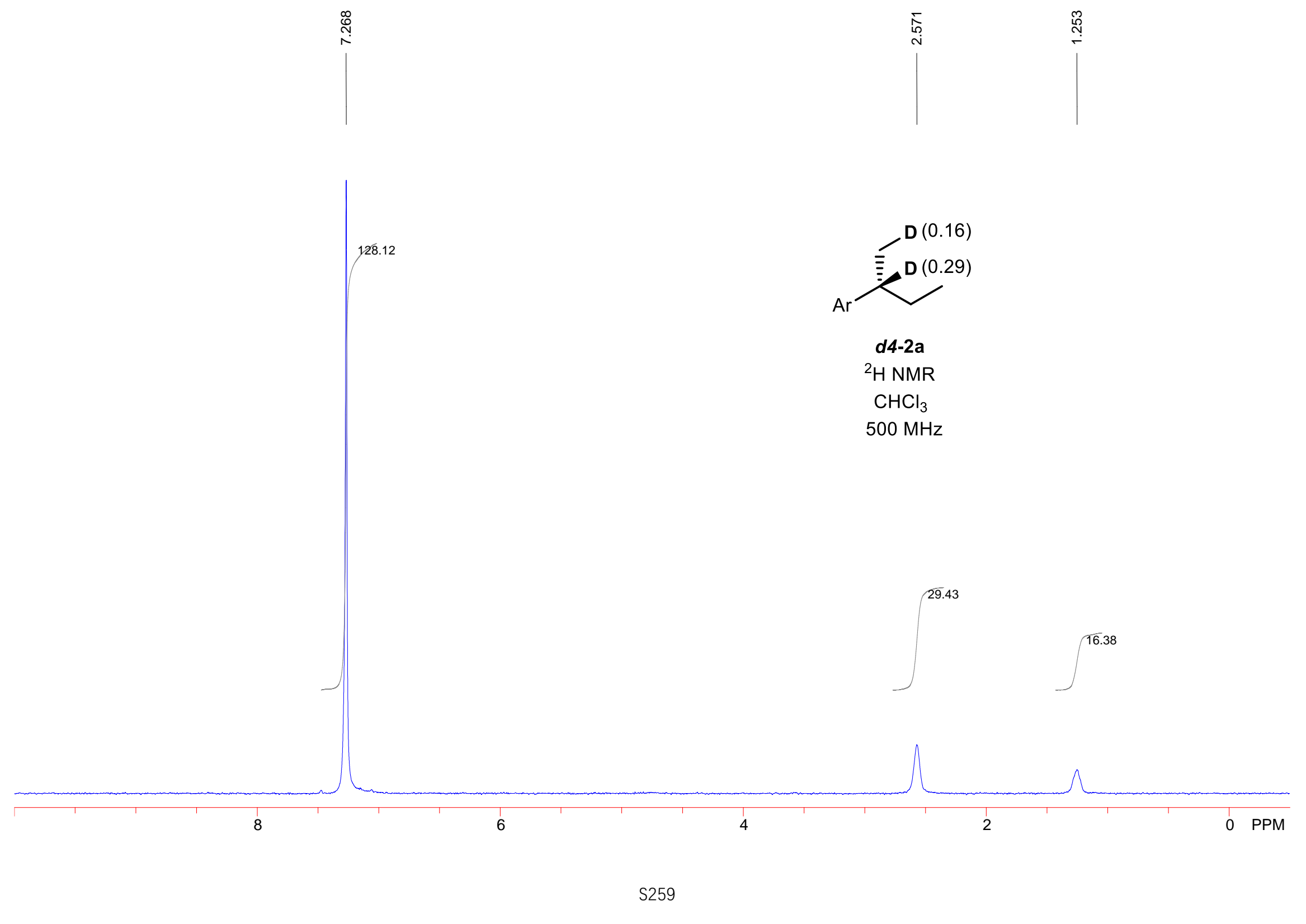




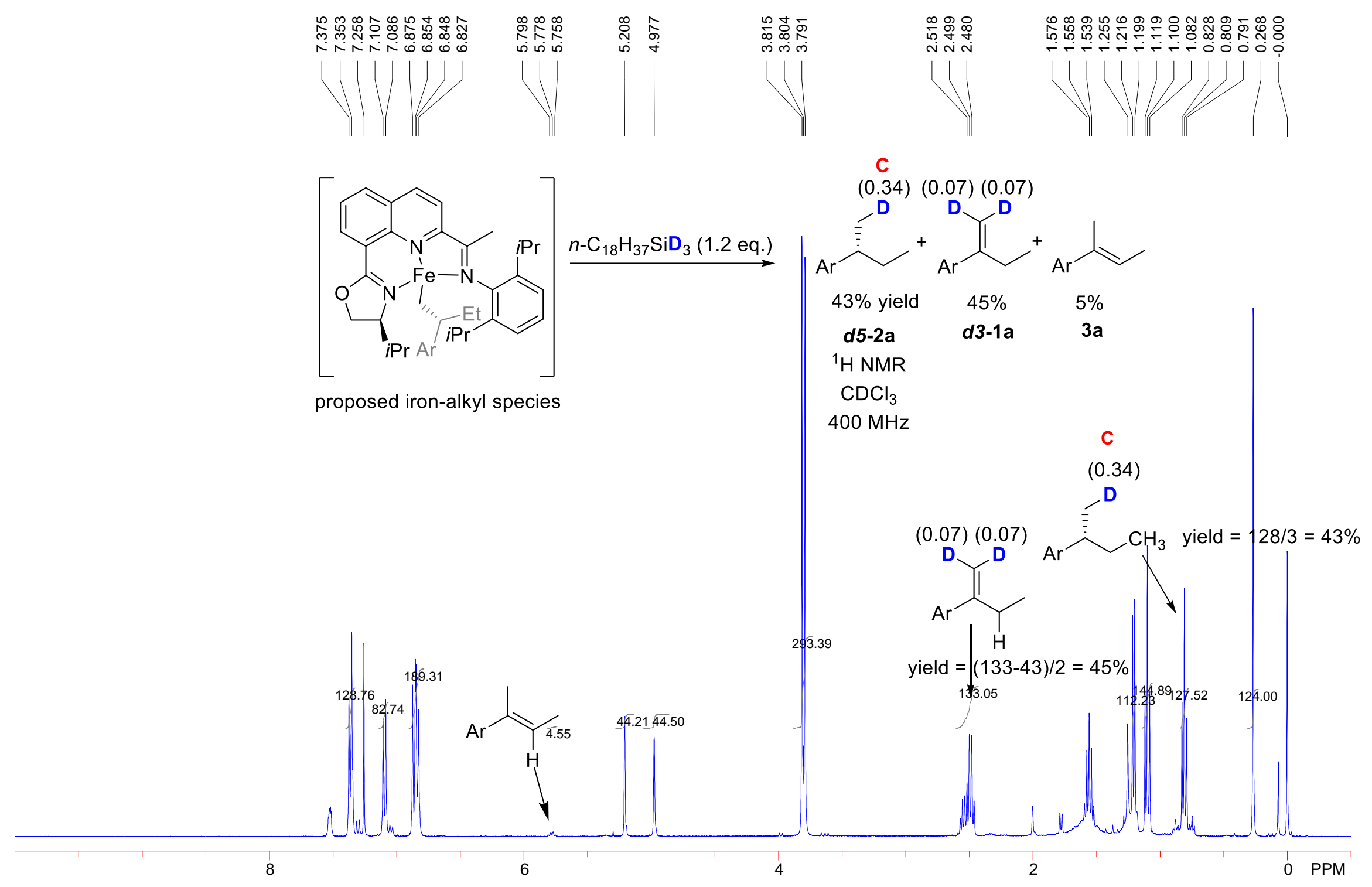




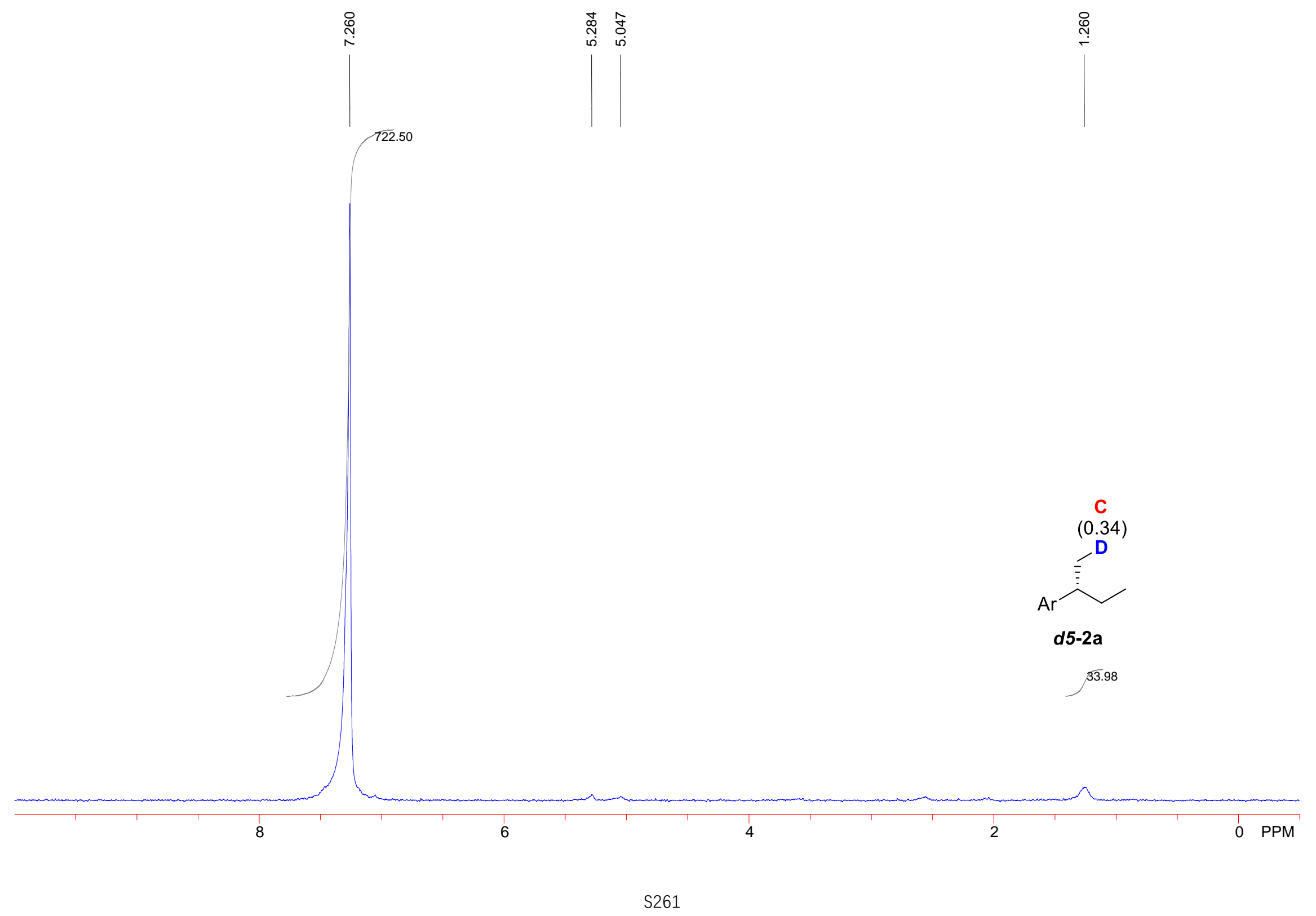




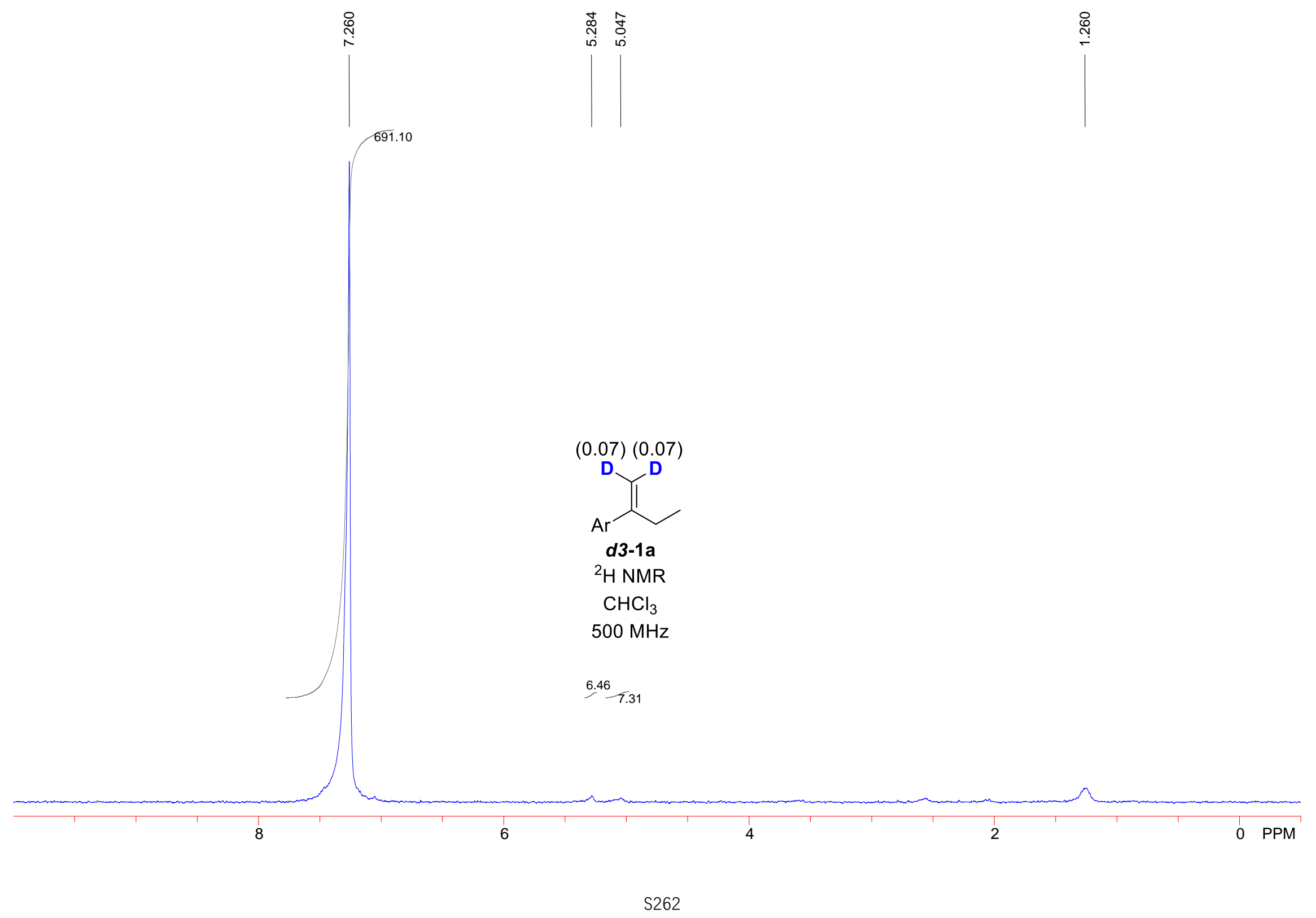




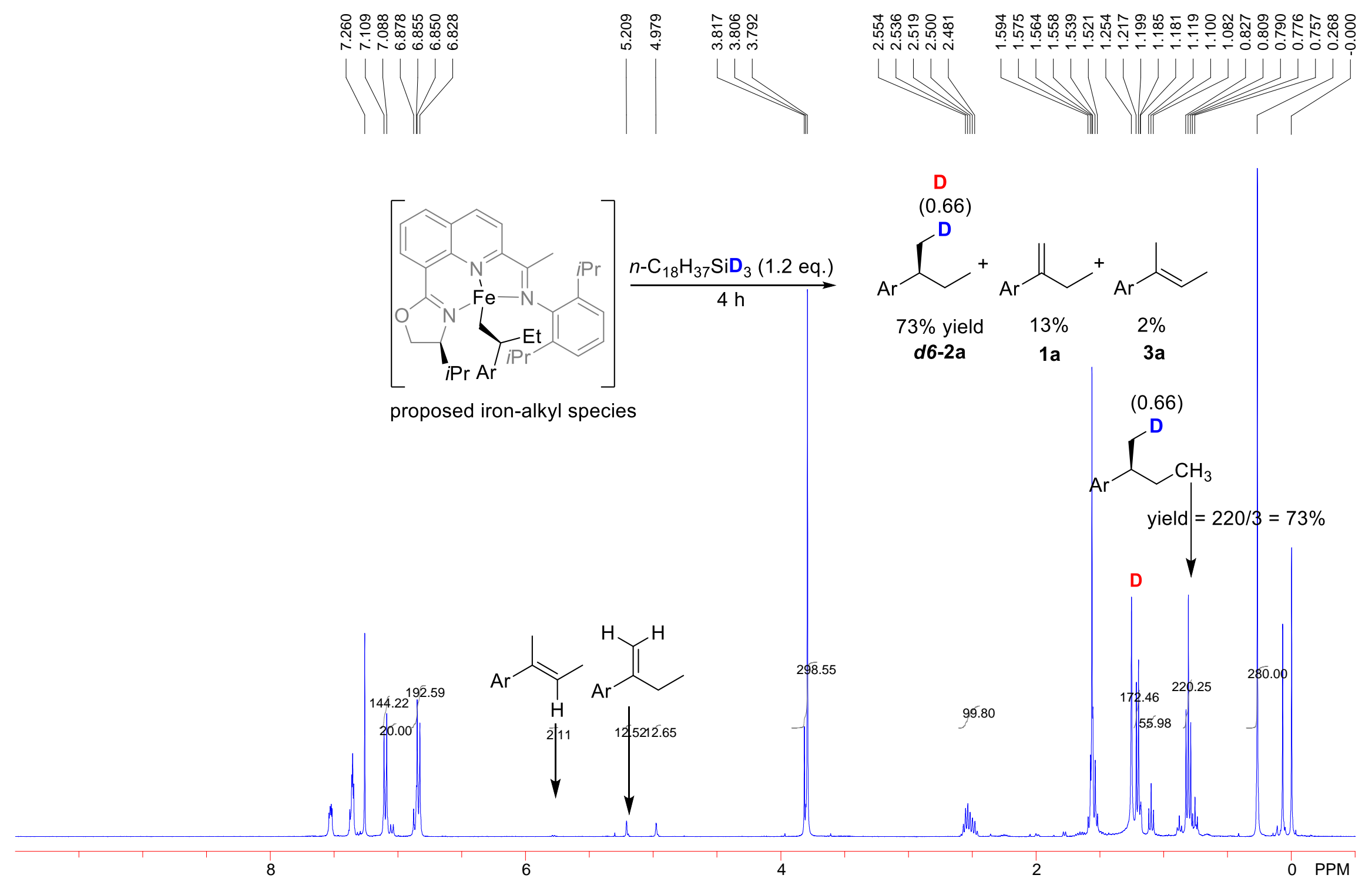


850.00

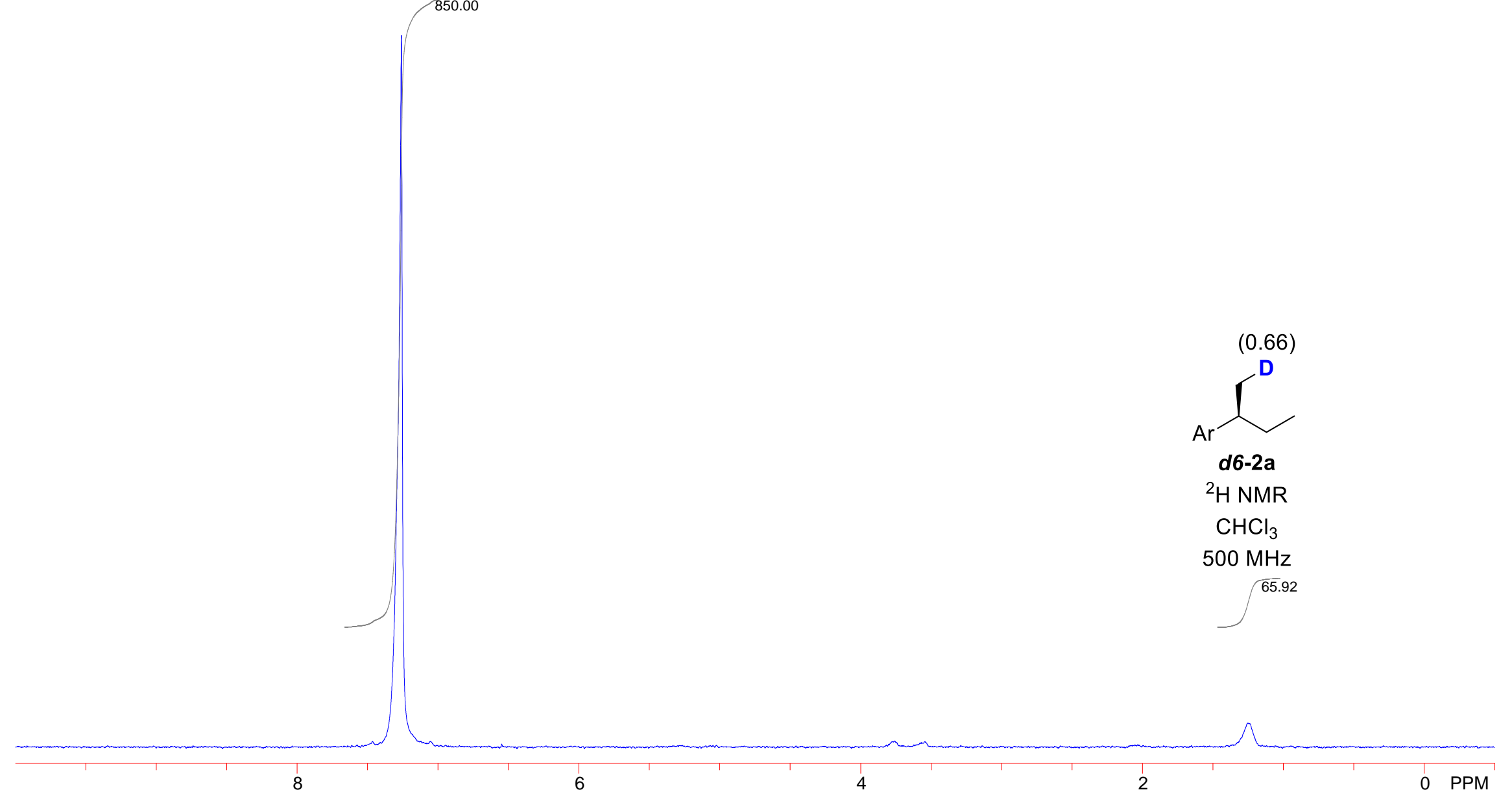




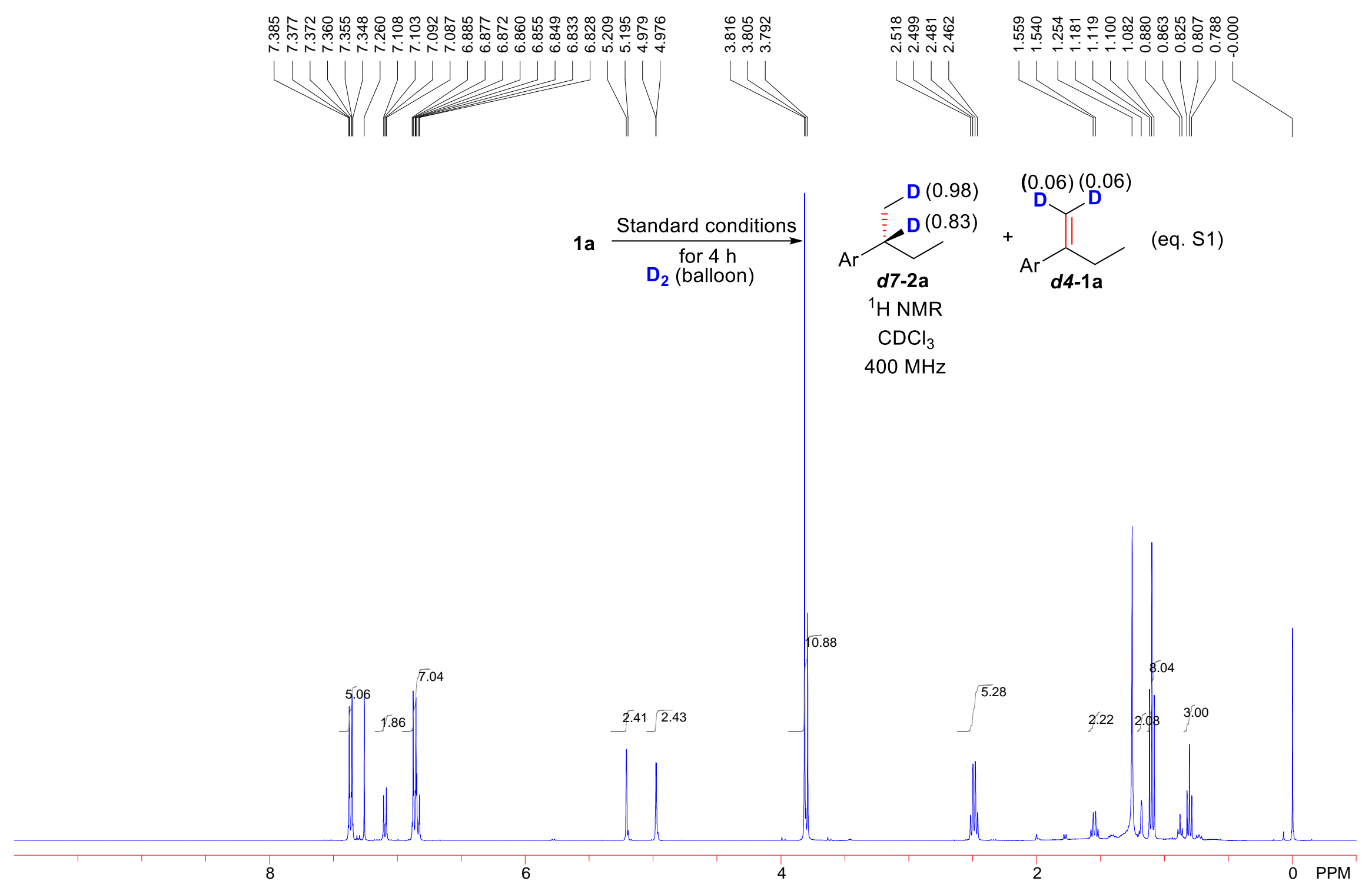




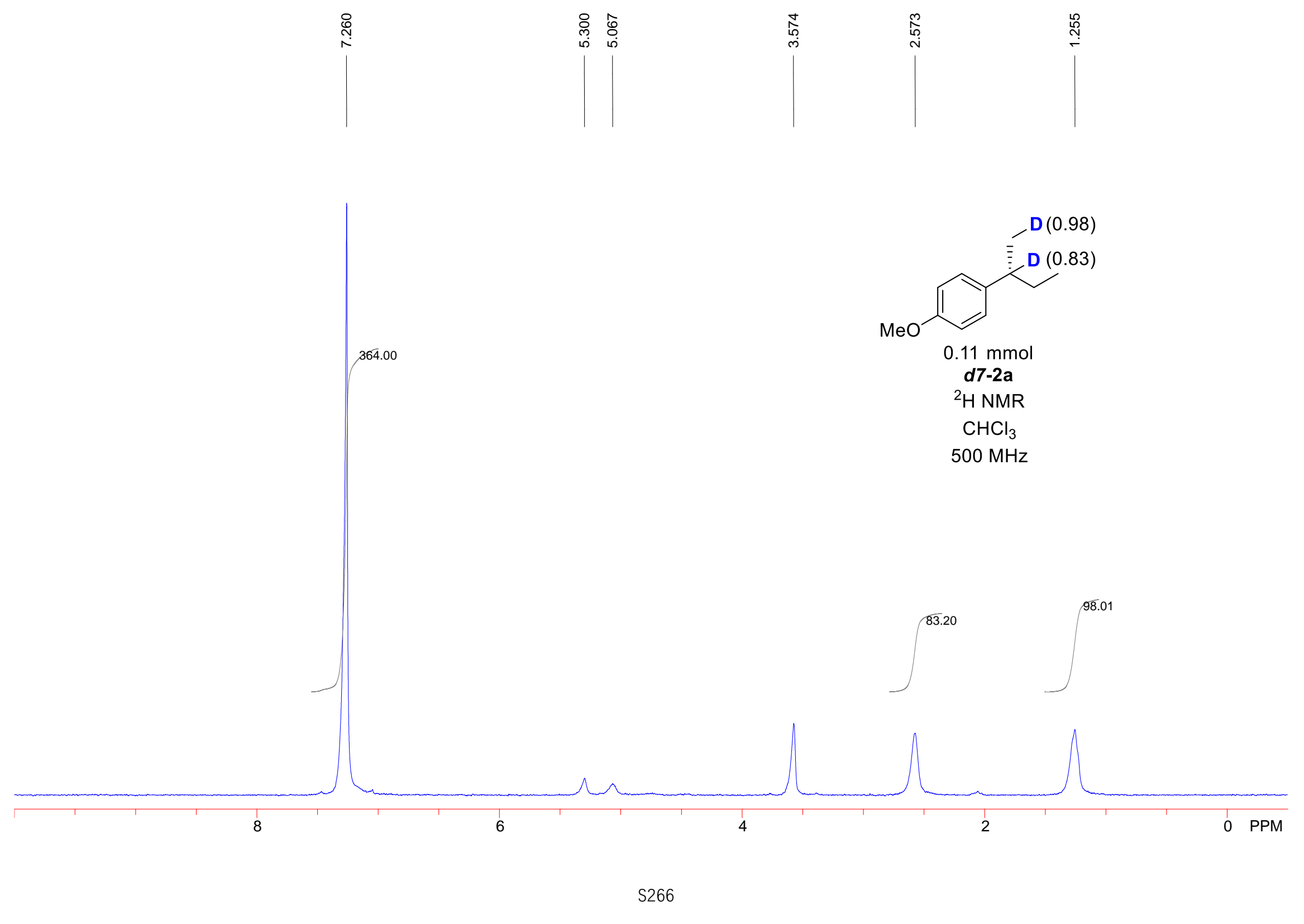




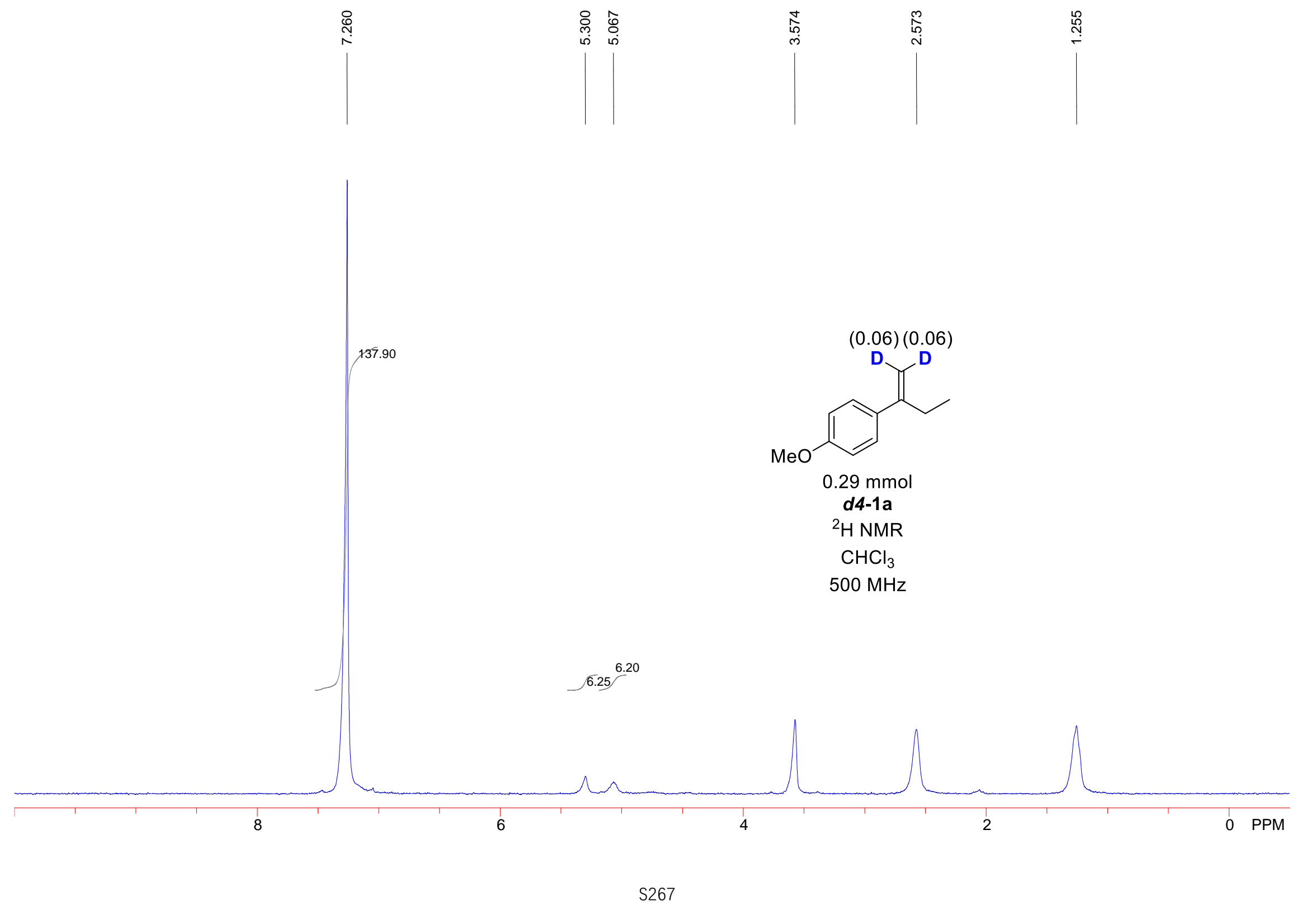




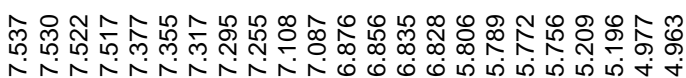

的

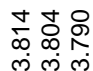

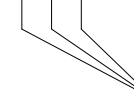

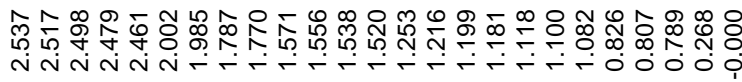

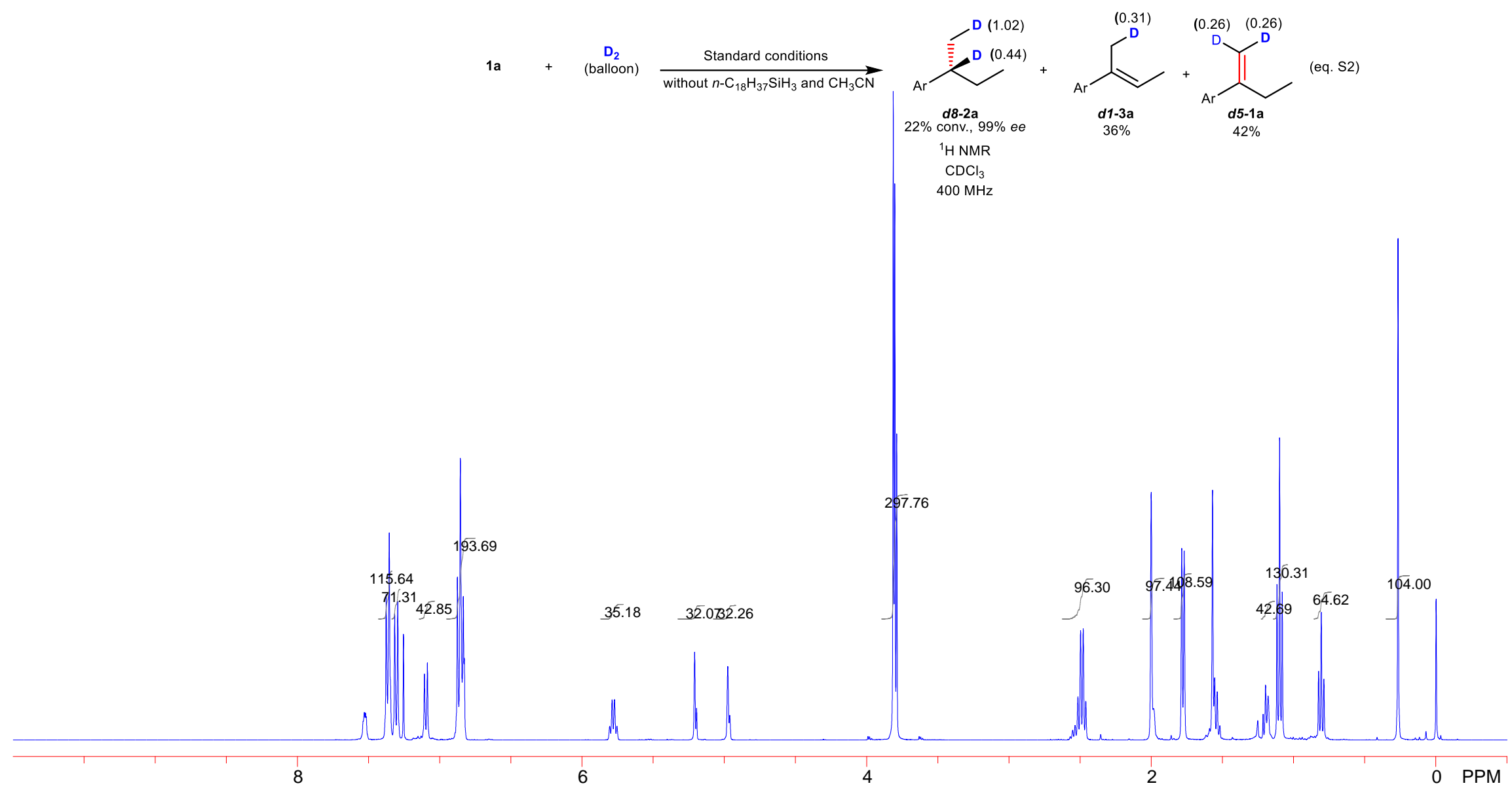




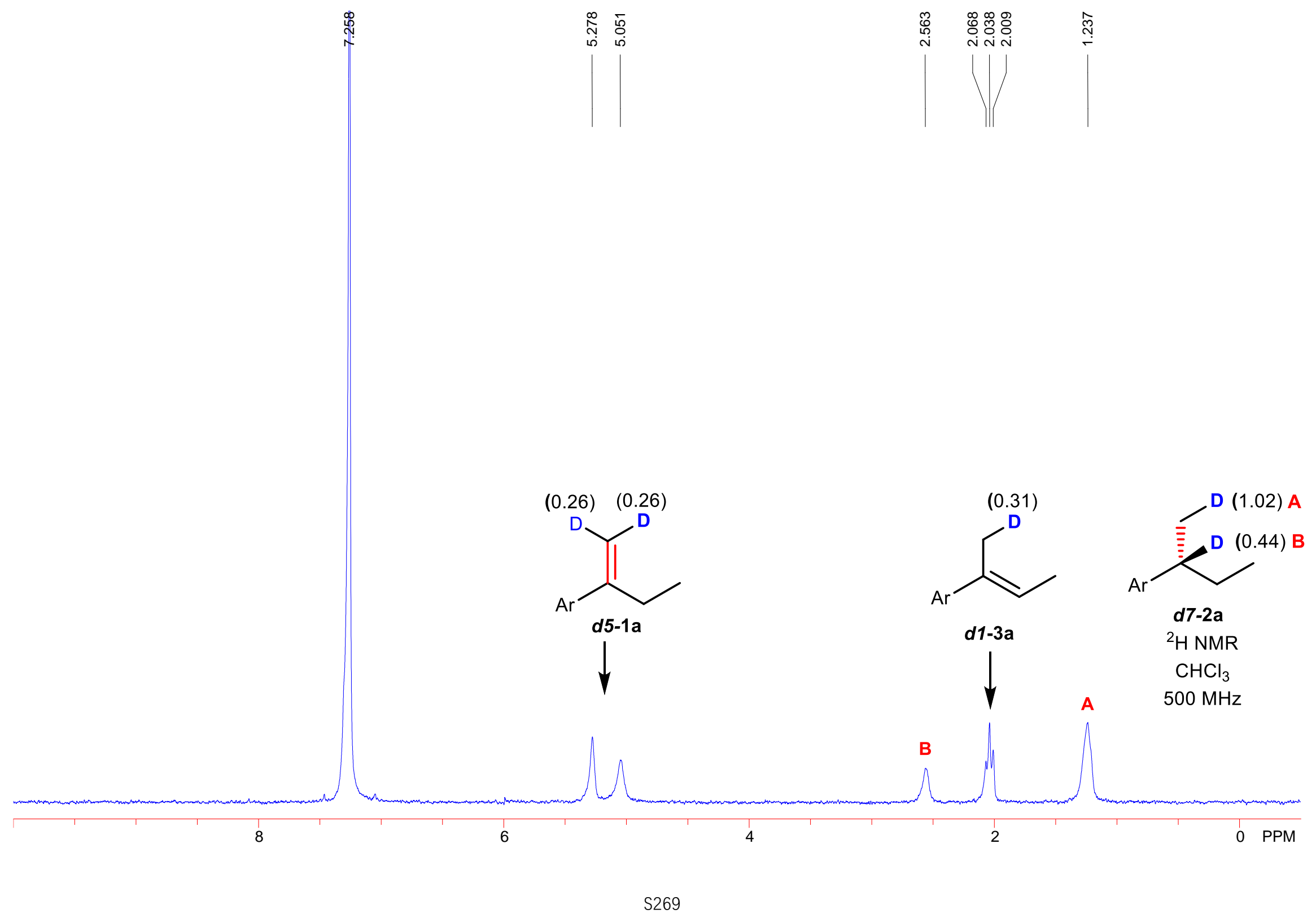


-000000000

$$
\begin{aligned}
n-\mathrm{C}_{18} \mathrm{H}_{37} \mathrm{SiD}_{3}+\underset{\text { (balloon) }}{\mathrm{H}_{2}} \stackrel{\text { standard conditions }}{\longrightarrow} n-\mathrm{C}_{18} \mathrm{H}_{37} \mathrm{SiH}(\mathrm{D})_{3} \\
\\
39 \% \text { yield of } n-\mathrm{C} 18 \mathrm{H} 37 \mathrm{SiH} 3 \\
61 \% \text { yield of } n-\mathrm{C} 18 \mathrm{H} 37 \mathrm{SiD} 3
\end{aligned}
$$




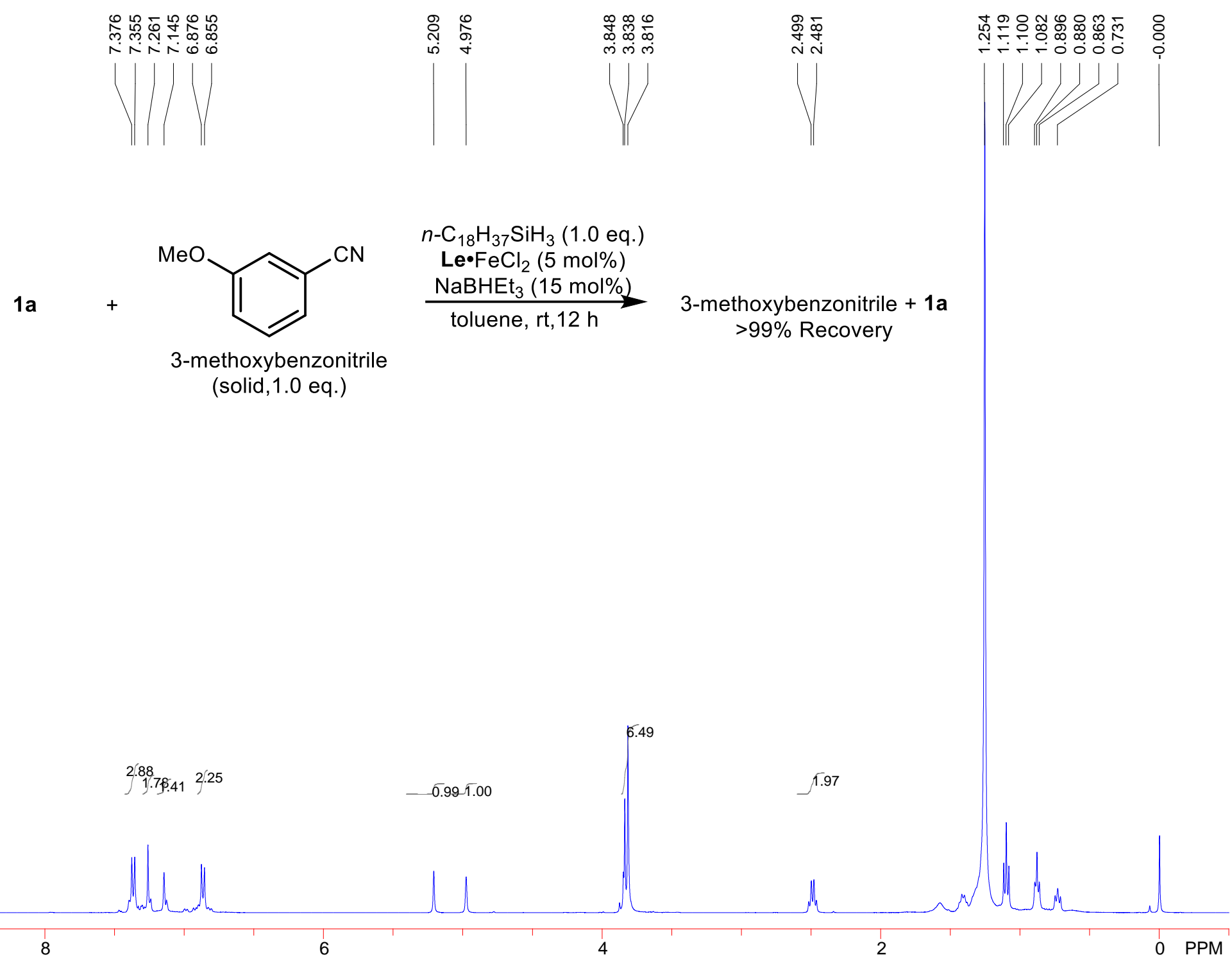




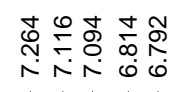

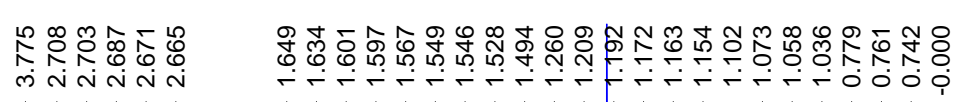

H

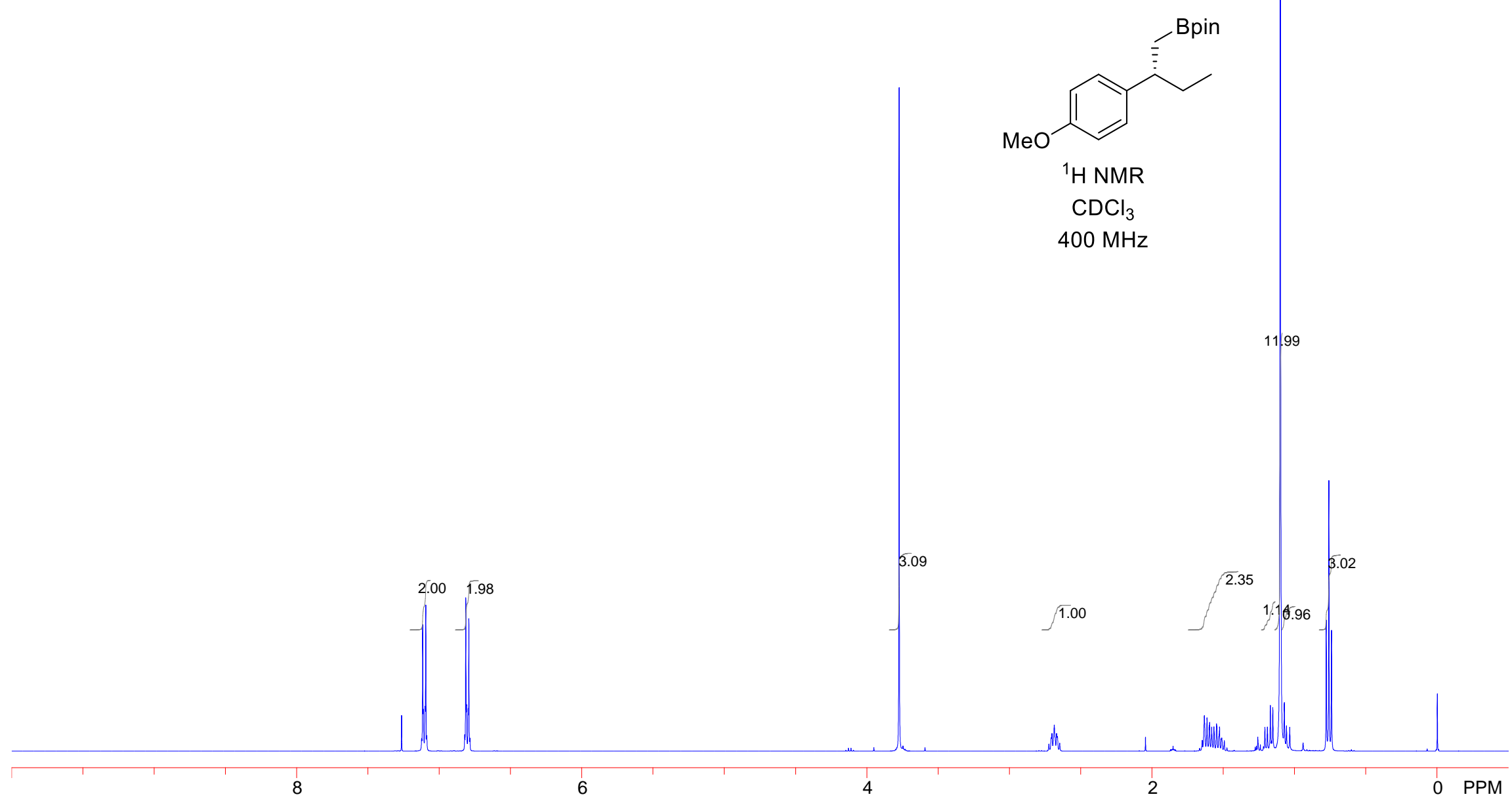




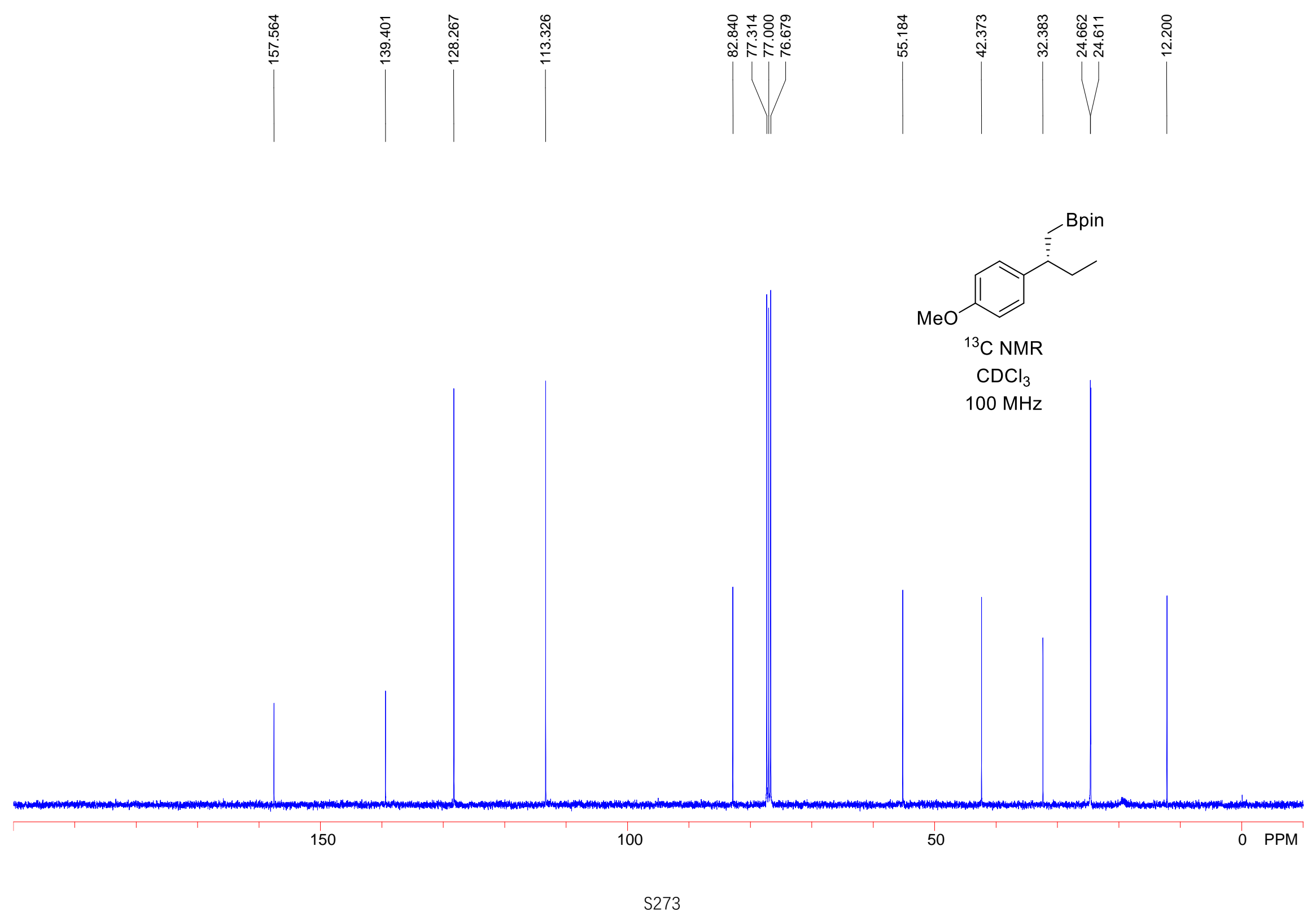




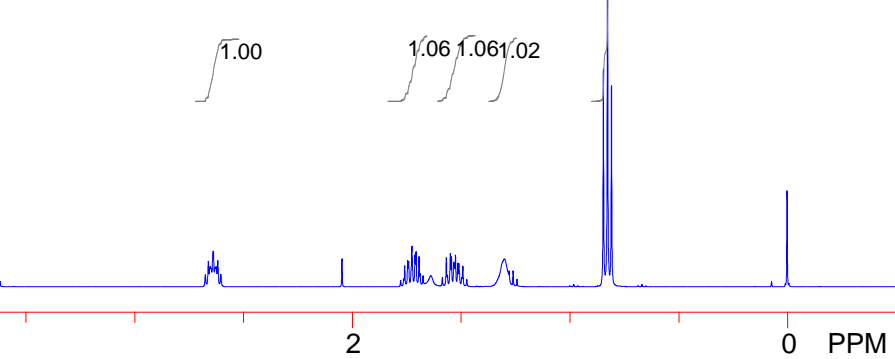




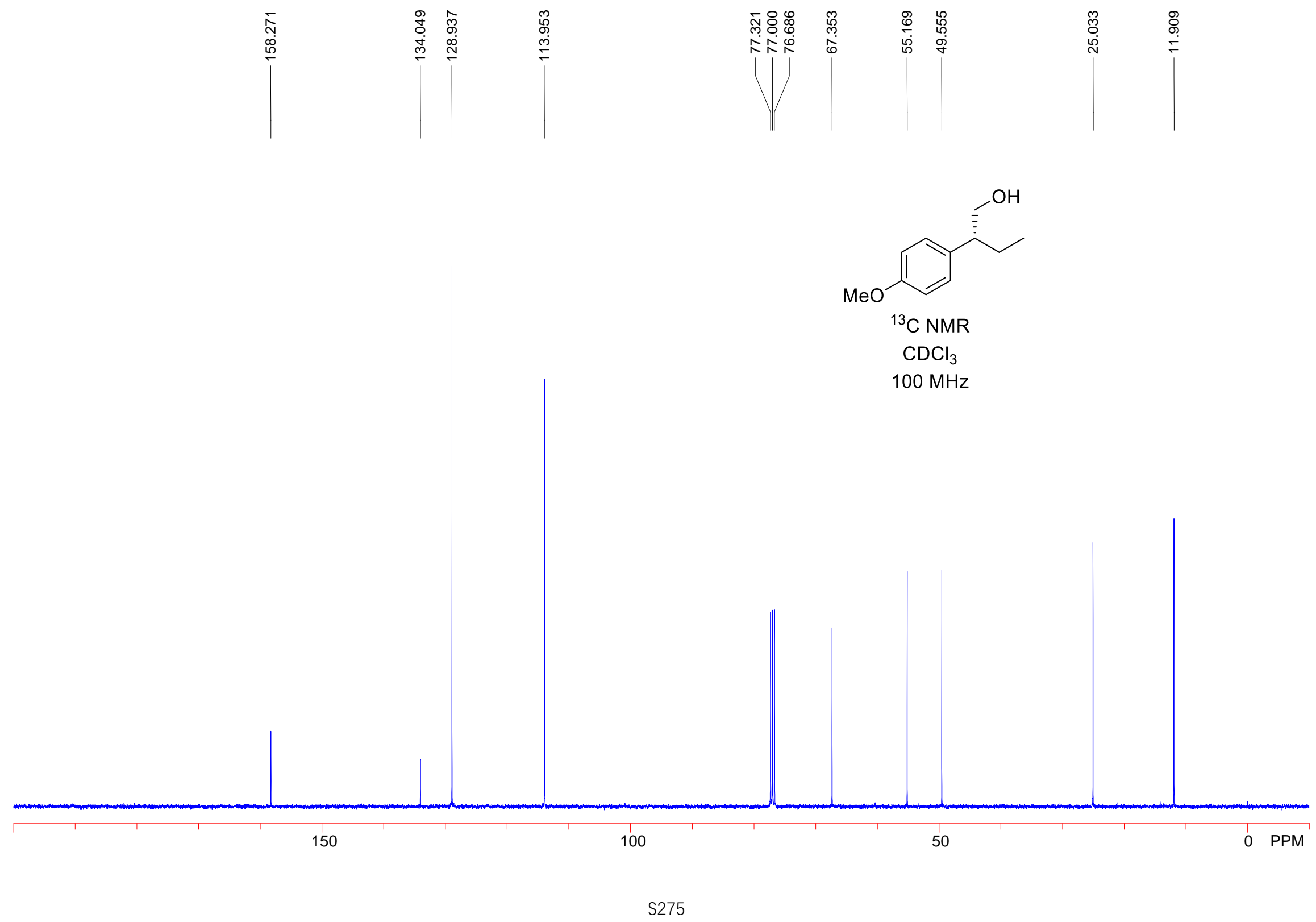




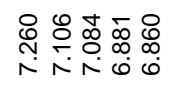

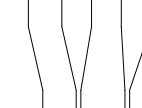

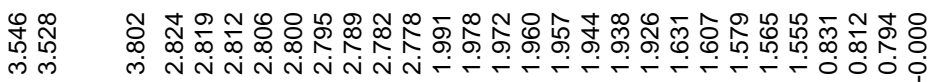

L L L L L L H H

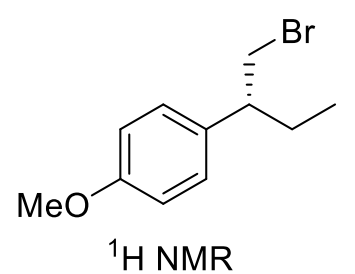

$\mathrm{CDCl}_{3}$

$400 \mathrm{MHz}$

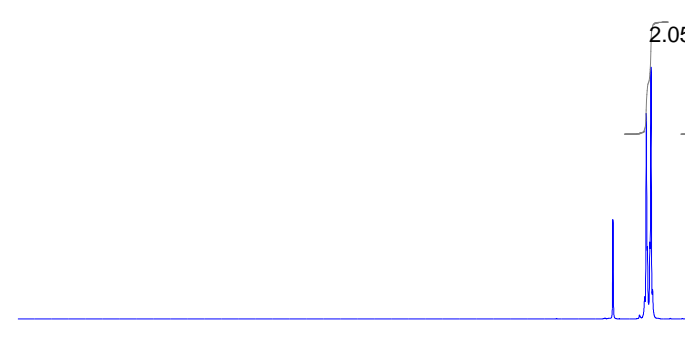




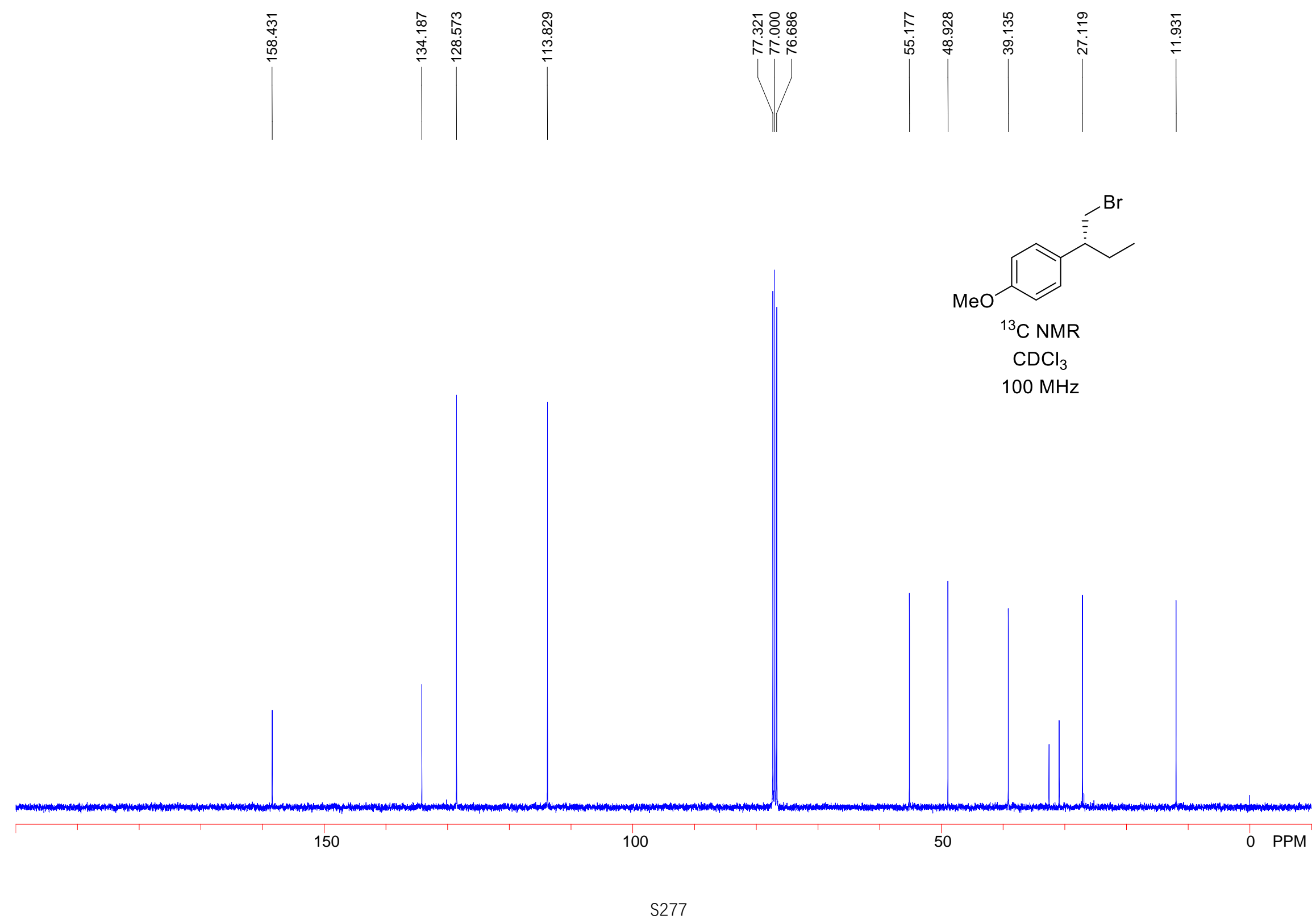




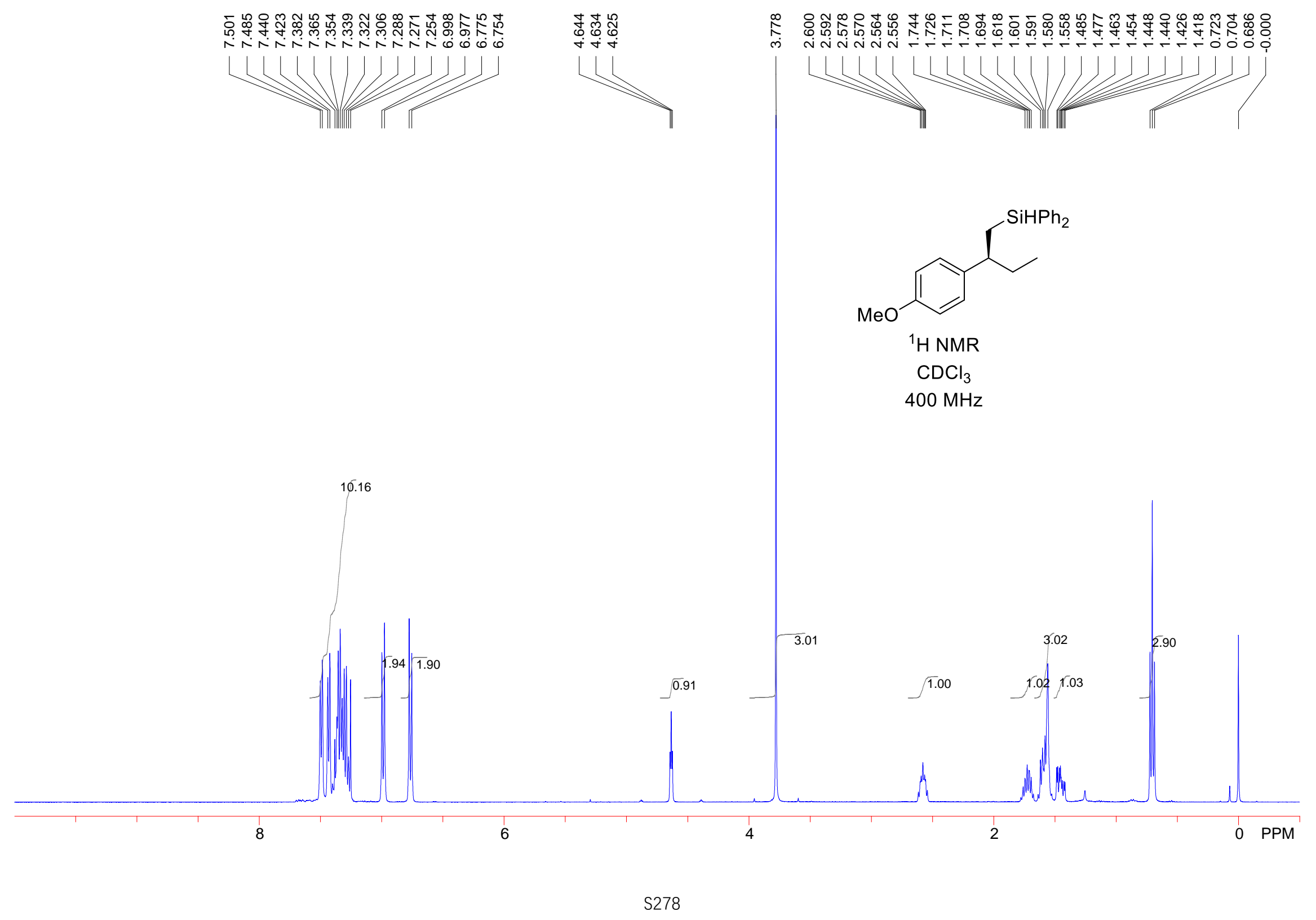




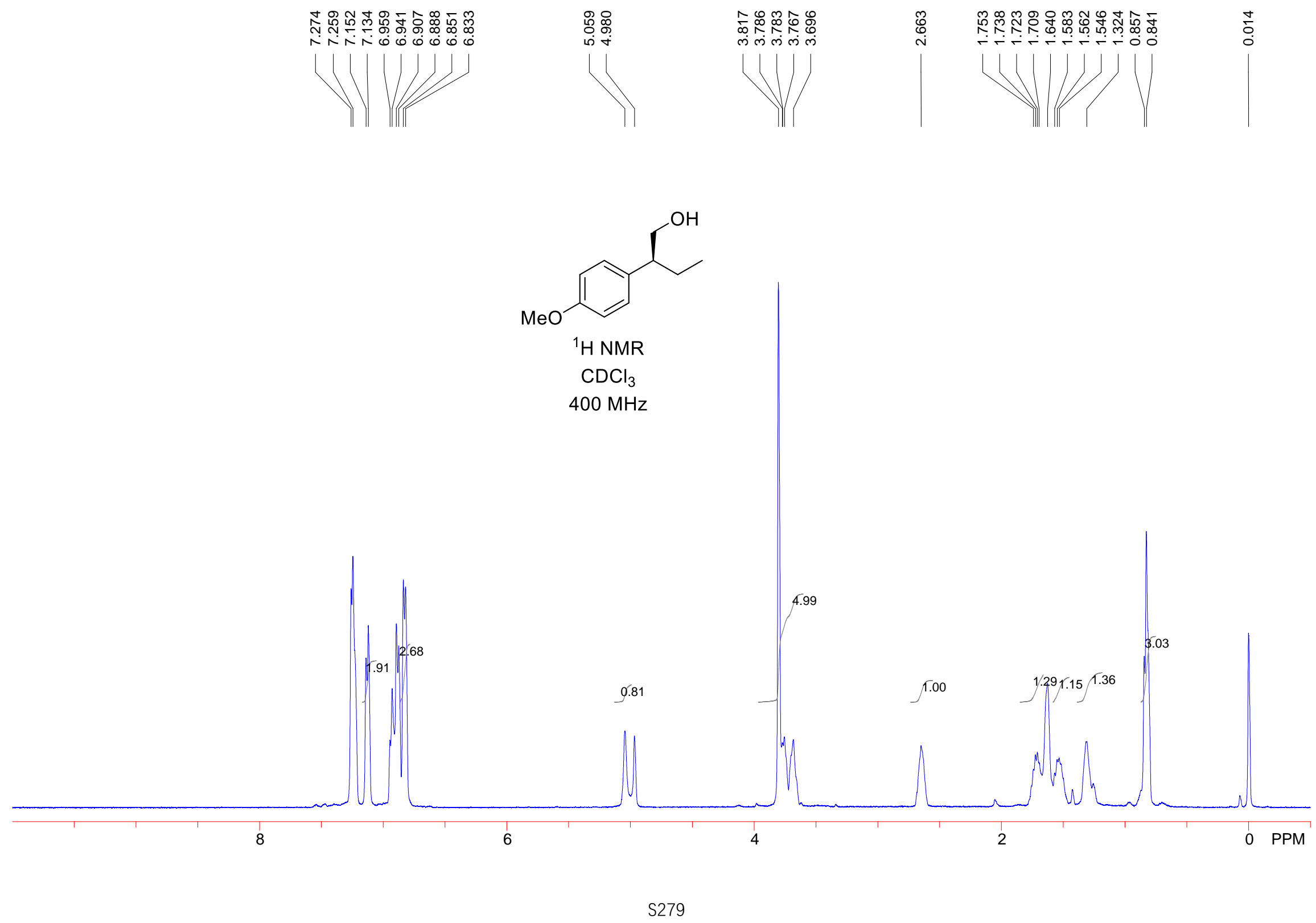




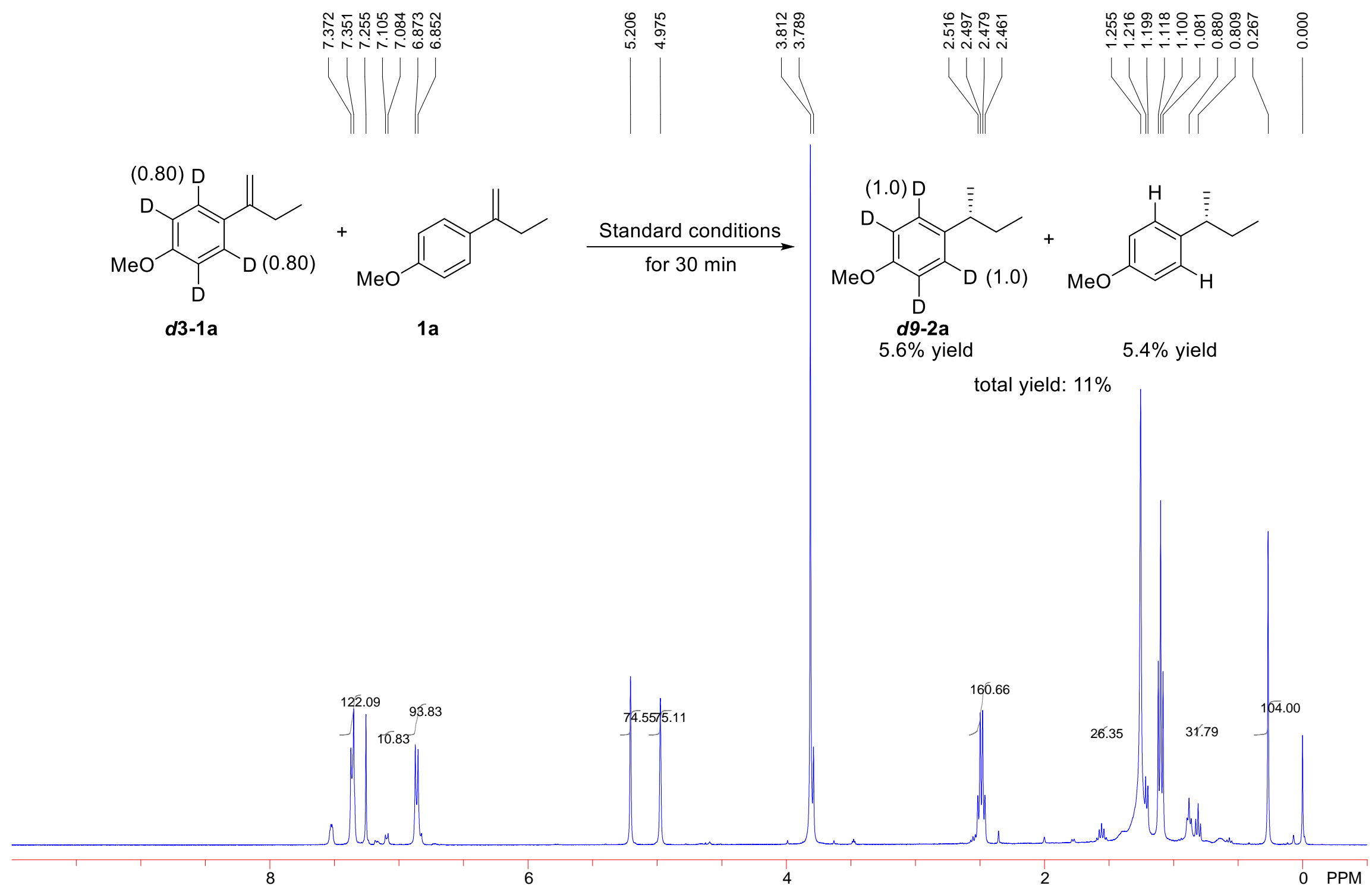




\section{HPLC and GC Spectra}

GC Conditions : Chiralcel B-DM, $100 \mathrm{kPa} \mathrm{N} 2\left(60{ }^{\circ} \mathrm{C}-30 \mathrm{~min}-1{ }^{\circ} \mathrm{C} / \mathrm{min}-180{ }^{\circ} \mathrm{C}-\right.$

\section{$5 \min )$}

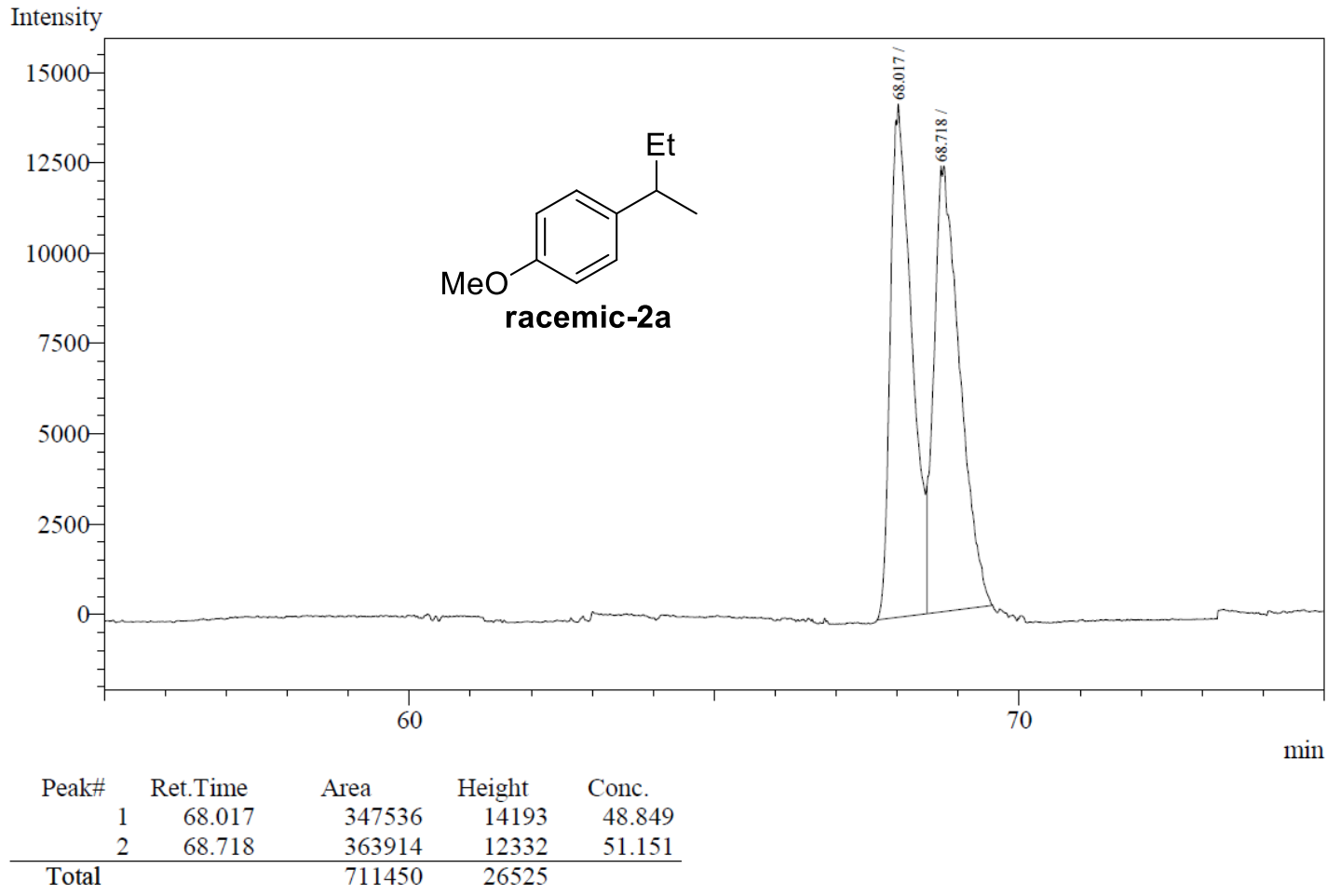

Method Name : E: $\operatorname{method~} 60$-30-1-115-5-模板-LP.gcm

Intensity

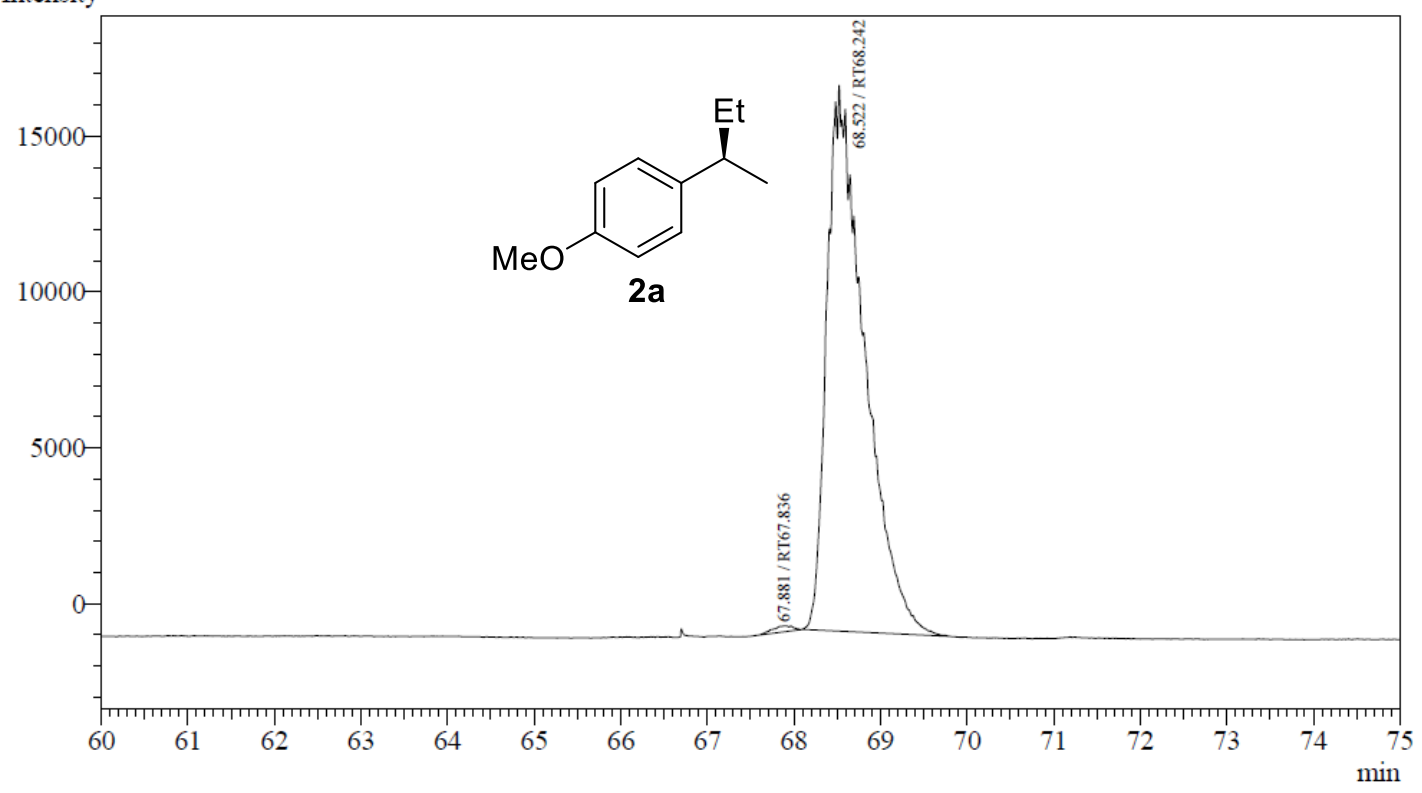

\begin{tabular}{|c|c|c|c|c|}
\hline Peak\# & Ret.Time & Area & Height & Area\% \\
\hline 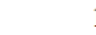 & 67.881 & 3088 & 190 & 0.5803 \\
\hline 2 & 68.522 & 529012 & 17466 & 99.4197 \\
\hline 0 & & 532100 & 17656 & 100.0000 \\
\hline
\end{tabular}


$\mathrm{mV}$

色谱图

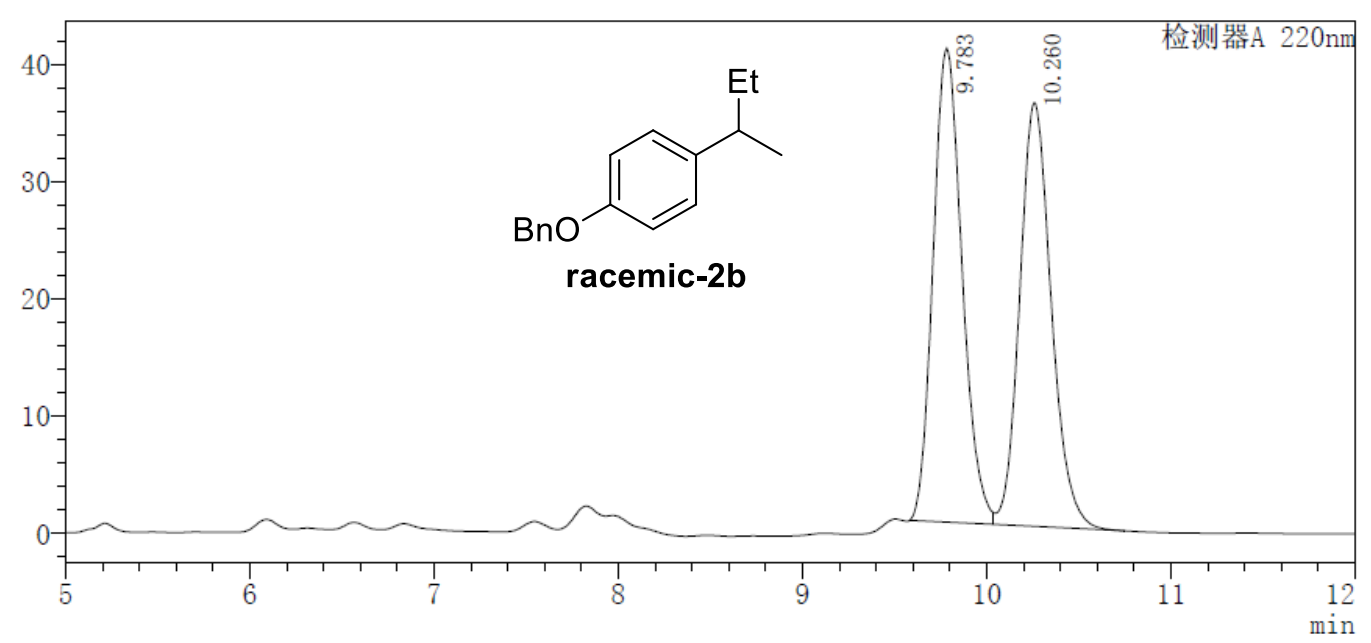

峰表

检测器A $220 \mathrm{~nm}$
\begin{tabular}{|r|r|r|r|r|r|}
\hline 峰号 & 保留时间 & 面积 & 高度 & 标记 & \multicolumn{1}{|c|}{ 面积 $\%$} \\
\hline 1 & 9.783 & 441002 & 40387 & $\mathrm{M}$ & 50.548 \\
\hline 2 & 10.260 & 431434 & 36125 & $\mathrm{~V} \mathrm{M}$ & 49.452 \\
\hline 总计 & & 872437 & 76512 & & 100.000 \\
\hline
\end{tabular}

措述

: $0 J-H, n$-hexane $/$ PrOH $=99 / 1,1.0 \mathrm{ml} / \mathrm{min}, 220 \mathrm{~nm}$

$\mathrm{mV}$

色谱图

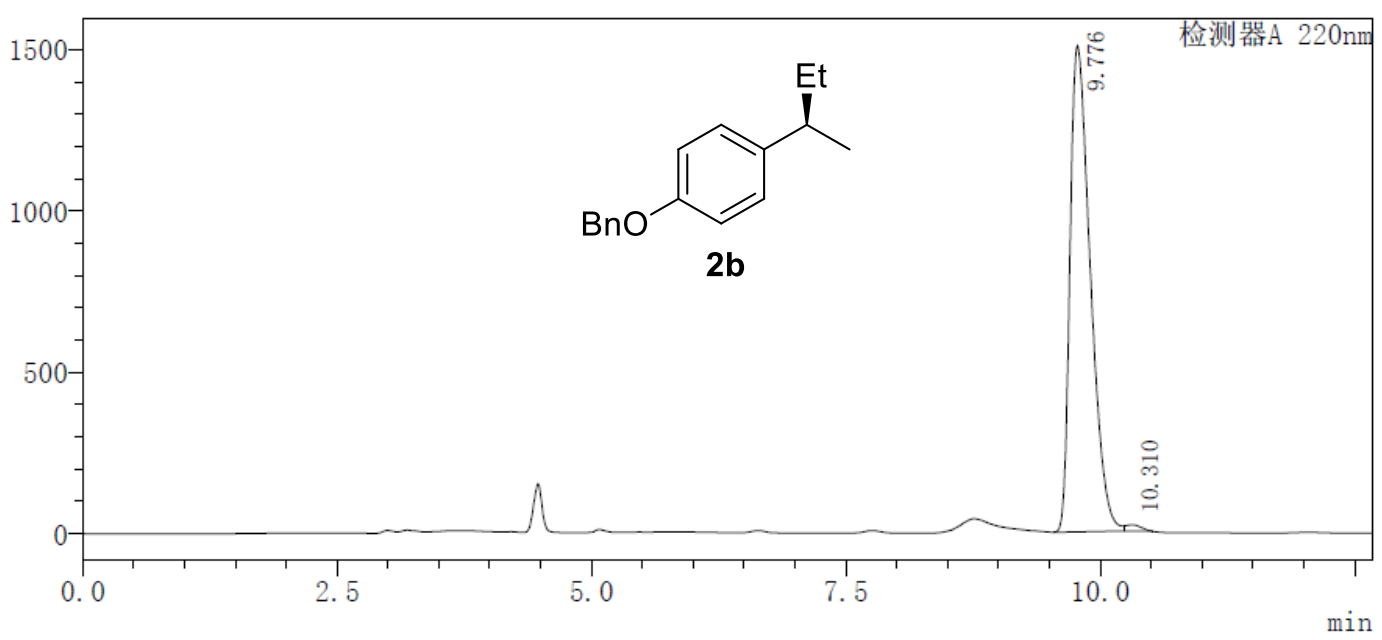

峰表

检测器A $220 \mathrm{~nm}$
\begin{tabular}{r|r|r|r|r|r|}
\hline 峰号 & 保留时间 & \multicolumn{1}{|c|}{ 面积 } & \multicolumn{1}{l|}{ 高度 } & 标记 & \multicolumn{1}{|c|}{ 面积 $\%$} \\
\hline 1 & 9.776 & 20945563 & 1506275 & $\mathrm{M}$ & 99.054 \\
\hline 2 & 10.310 & 199953 & 19464 & $\mathrm{M}$ & 0.946 \\
\hline 总计 & & 21145515 & 1525738 & & 100.000 \\
\hline
\end{tabular}


$\mathrm{mV}$

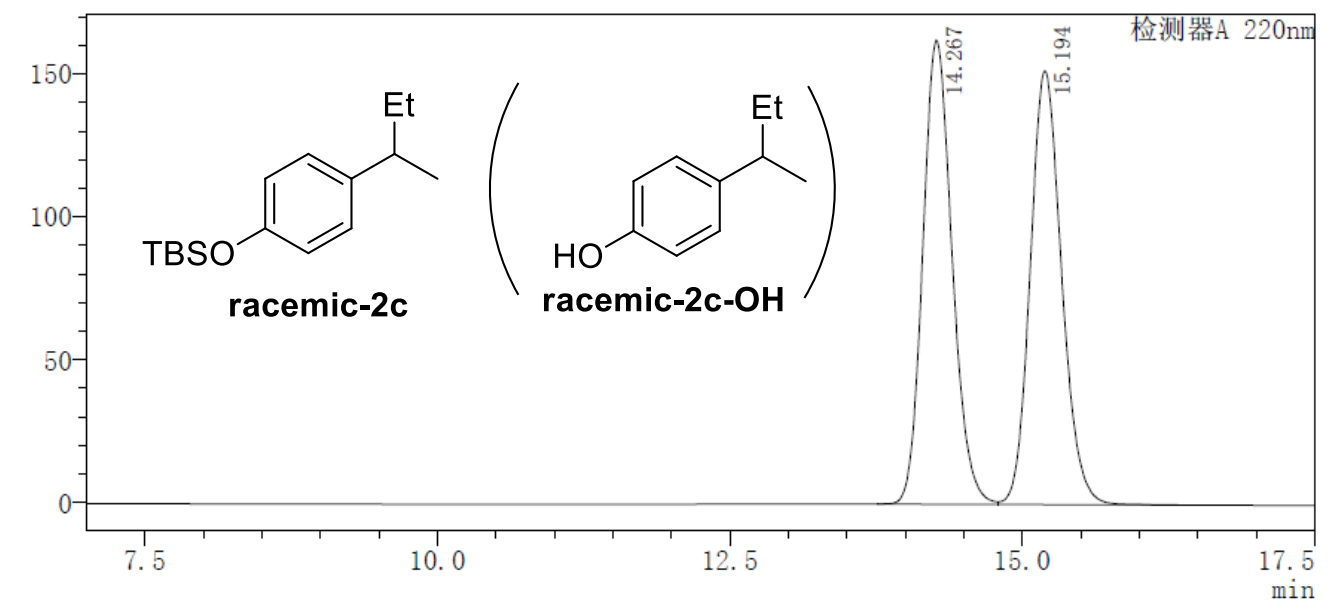

峰表

\begin{tabular}{|c|c|c|c|c|c|}
\hline & 保留 & 面和 & 高席 & 标记 & 而和 $\%$ \\
\hline 1 & 14.267 & 2826781 & 162336 & & 49.991 \\
\hline 2 & 15.194 & 2827761 & 151743 & V & 50.009 \\
\hline 总计 & & 5654542 & 314079 & & 100.000 \\
\hline
\end{tabular}

描述

: $0 J-H, n$-hexane $/$ iPrOH $=95 / 5,1.0 \mathrm{ml} / \mathrm{min}, 220 \mathrm{~nm}$

$\mathrm{mV}$

\section{色谱图}

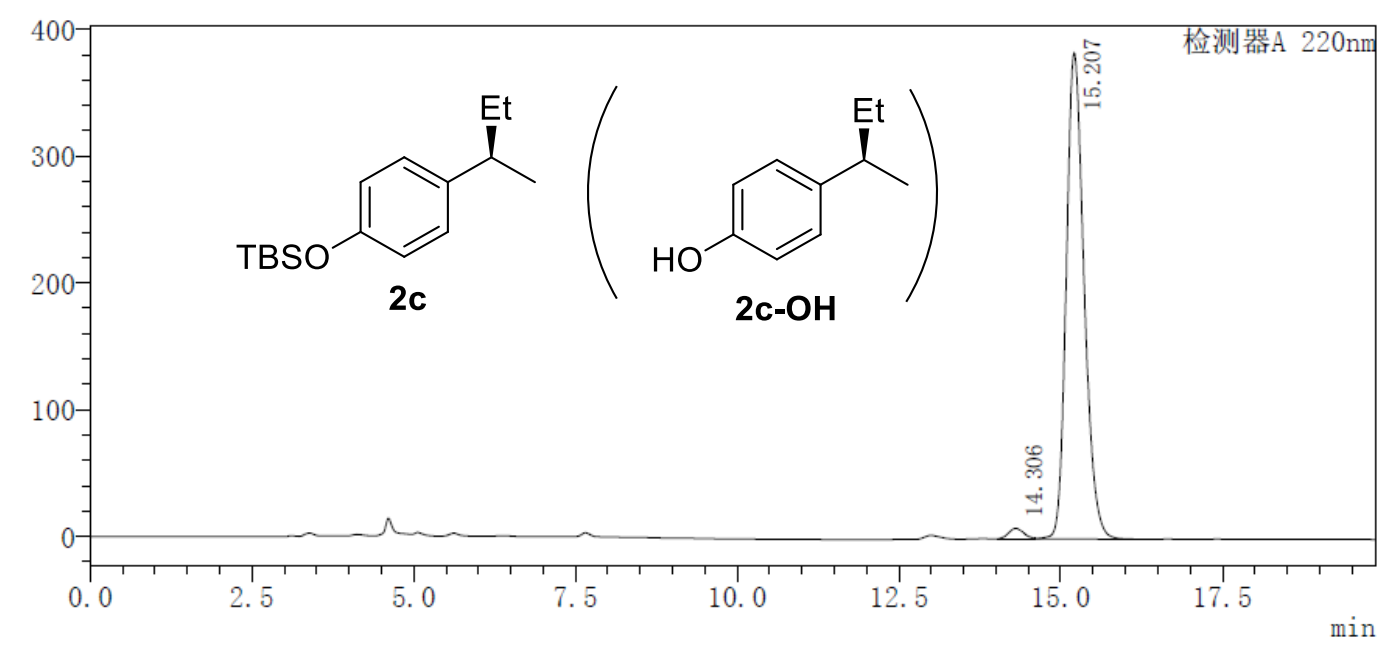

峰表

检测器A $220 \mathrm{~nm}$
\begin{tabular}{r|r|r|r|r|r|}
\hline 峰号 & 保留时间 & \multicolumn{1}{|c|}{ 面积 } & \multicolumn{1}{c|}{ 高度 } & 标记 & \multicolumn{1}{|c|}{ 面积\% } \\
\hline 1 & 14.306 & 132727 & 8183 & $\mathrm{M}$ & 1.772 \\
\hline 2 & 15.207 & 7356897 & 383292 & $\mathrm{~V} \mathrm{M}$ & 98.228 \\
\hline 总计 & & 7489624 & 391474 & & 100.000 \\
\hline
\end{tabular}


GC Conditions : Chiralcel B-DM, $100 \mathrm{kPa} \mathrm{N} 2\left(50{ }^{\circ} \mathrm{C}-5 \mathrm{~min}-1{ }^{\circ} \mathrm{C} / \mathrm{min}-160{ }^{\circ} \mathrm{C}-5\right.$ min)

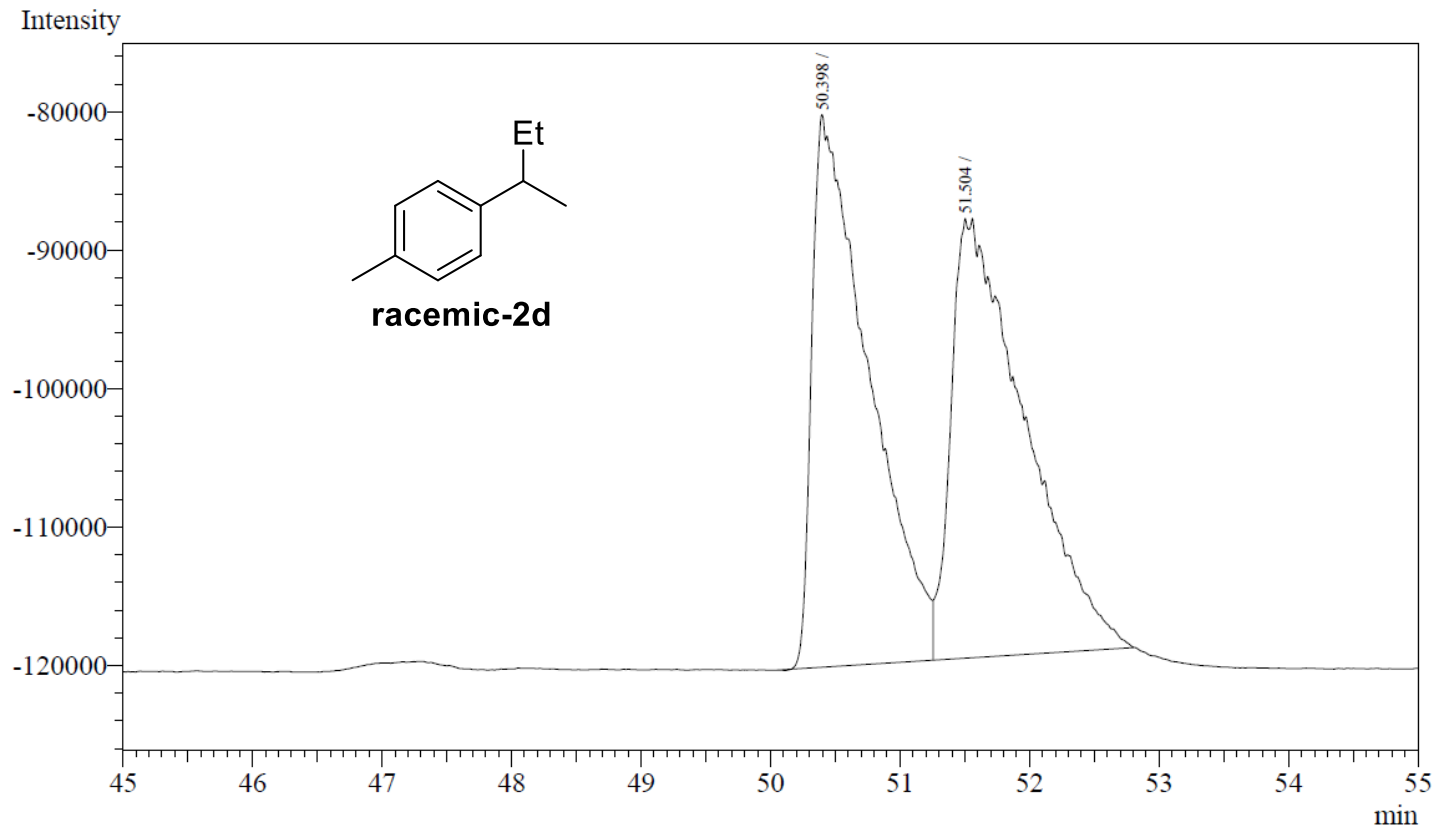

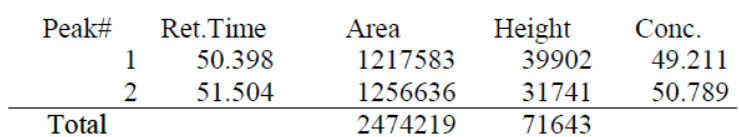

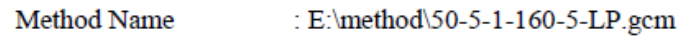

Intensity

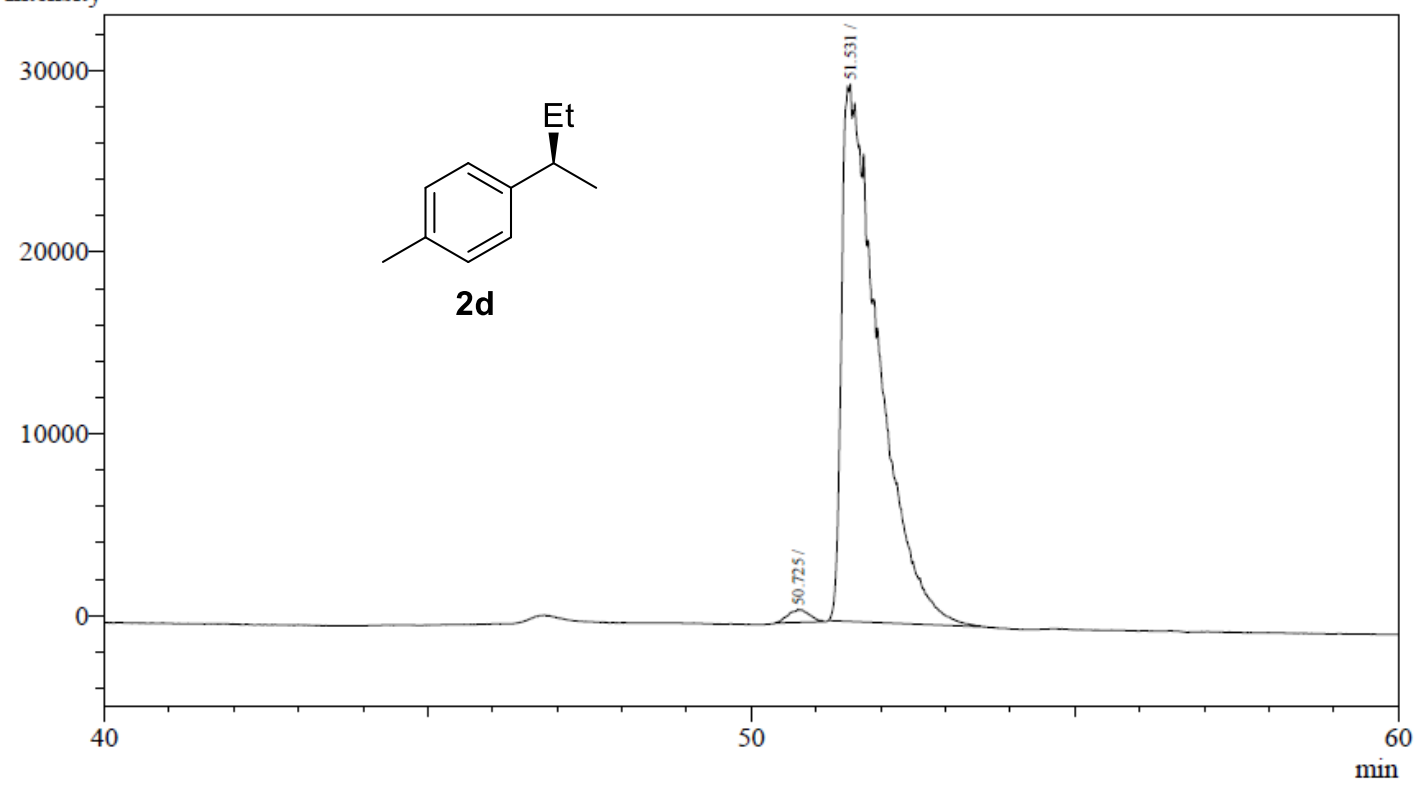

\begin{tabular}{lrrrr} 
Peak\# & Ret.Time & \multicolumn{1}{c}{ Area } & Height & \multicolumn{1}{c}{ Conc. } \\
1 & 50.725 & 16222 & 731 & 1.360 \\
2 & 51.531 & 1176753 & 29550 & 98.640 \\
\hline Total & & 1192975 & 30281 &
\end{tabular}


$\mathrm{mV}$

\section{色谱图}

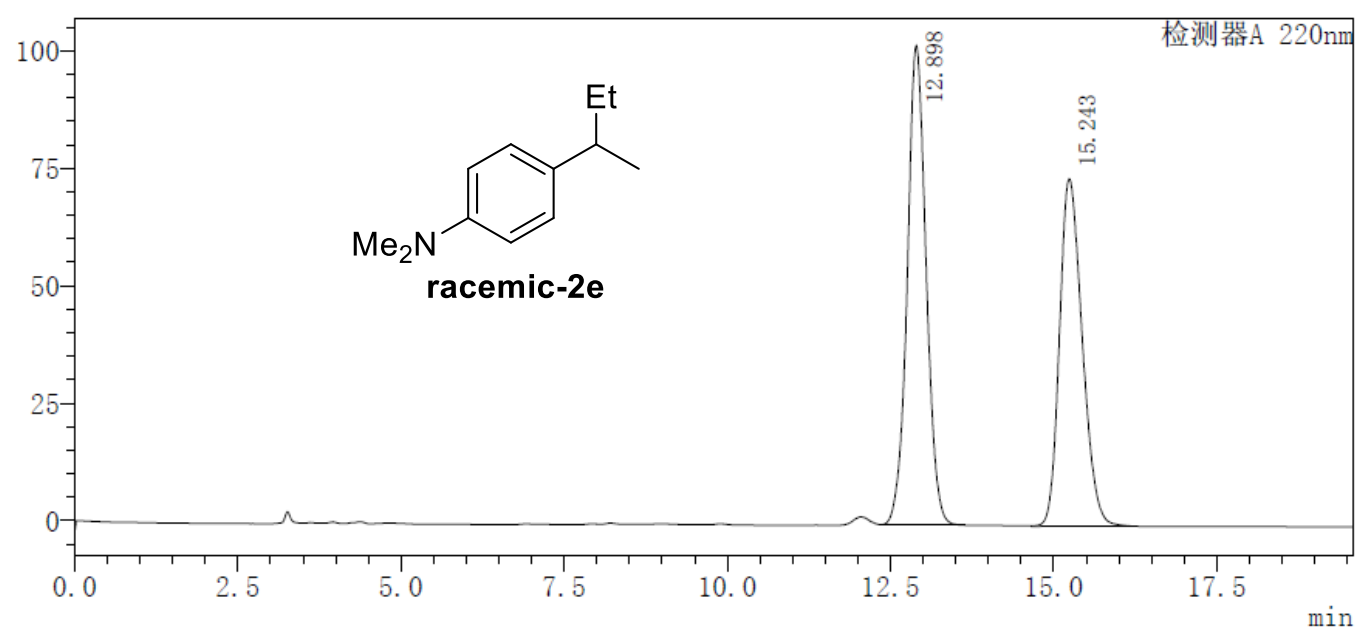

峰表

\begin{tabular}{|c|c|c|c|c|c|}
\hline 峰号 & 侣的时问 & 而和 & 高席 & 둑ㄹㄹ & 面和 \\
\hline 1 & 12.898 & 2051884 & 102114 & M & 53.373 \\
\hline 2 & 15.243 & 1792546 & 73980 & M & 46.627 \\
\hline 总计 & & 3844430 & 176095 & & 100.000 \\
\hline
\end{tabular}

描述

: $0 J-H, \quad n$-hexane $/$ PrOH $=99 / 1,1.0 \mathrm{ml} / \mathrm{min}, 220 \mathrm{~nm}$

$\mathrm{mV}$

色谱图

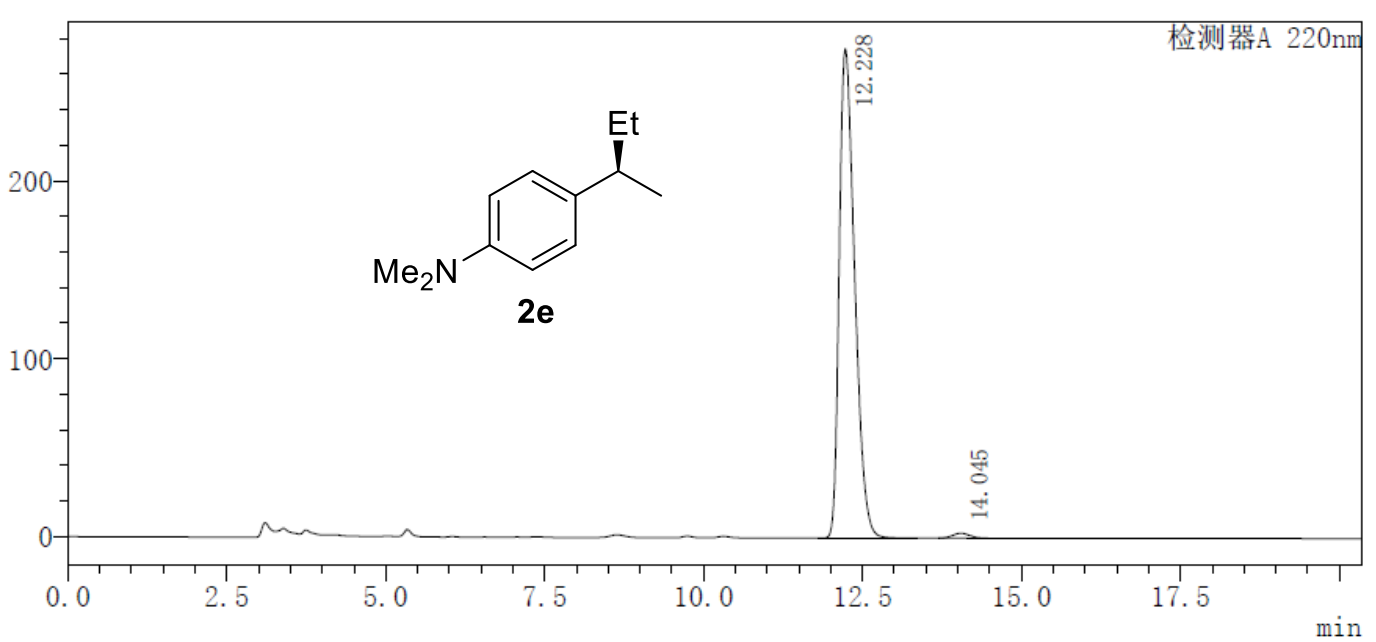

峰表

检测器 $\mathrm{A} 220 \mathrm{~nm}$

\begin{tabular}{|r|r|r|r|r|r|}
\hline 峰号 & 保留时间 & \multicolumn{1}{|c|}{ 面积 } & \multicolumn{1}{c|}{ 高度 } & 标记 & \multicolumn{1}{c|}{ 面积\% } \\
\hline 1 & 12.228 & 4643192 & 274899 & & 98.927 \\
\hline 2 & 14.045 & 50349 & 2746 & $\mathrm{M}$ & 1.073 \\
\hline 总计 & & 4693541 & 277646 & & 100.000 \\
\hline
\end{tabular}


GC Conditions : Chiralcel B-DM, $100 \mathrm{kPa} \mathrm{N} 2\left(60{ }^{\circ} \mathrm{C}-30 \mathrm{~min}-2{ }^{\circ} \mathrm{C} / \mathrm{min}-180{ }^{\circ} \mathrm{C}-5\right.$ $\min )$

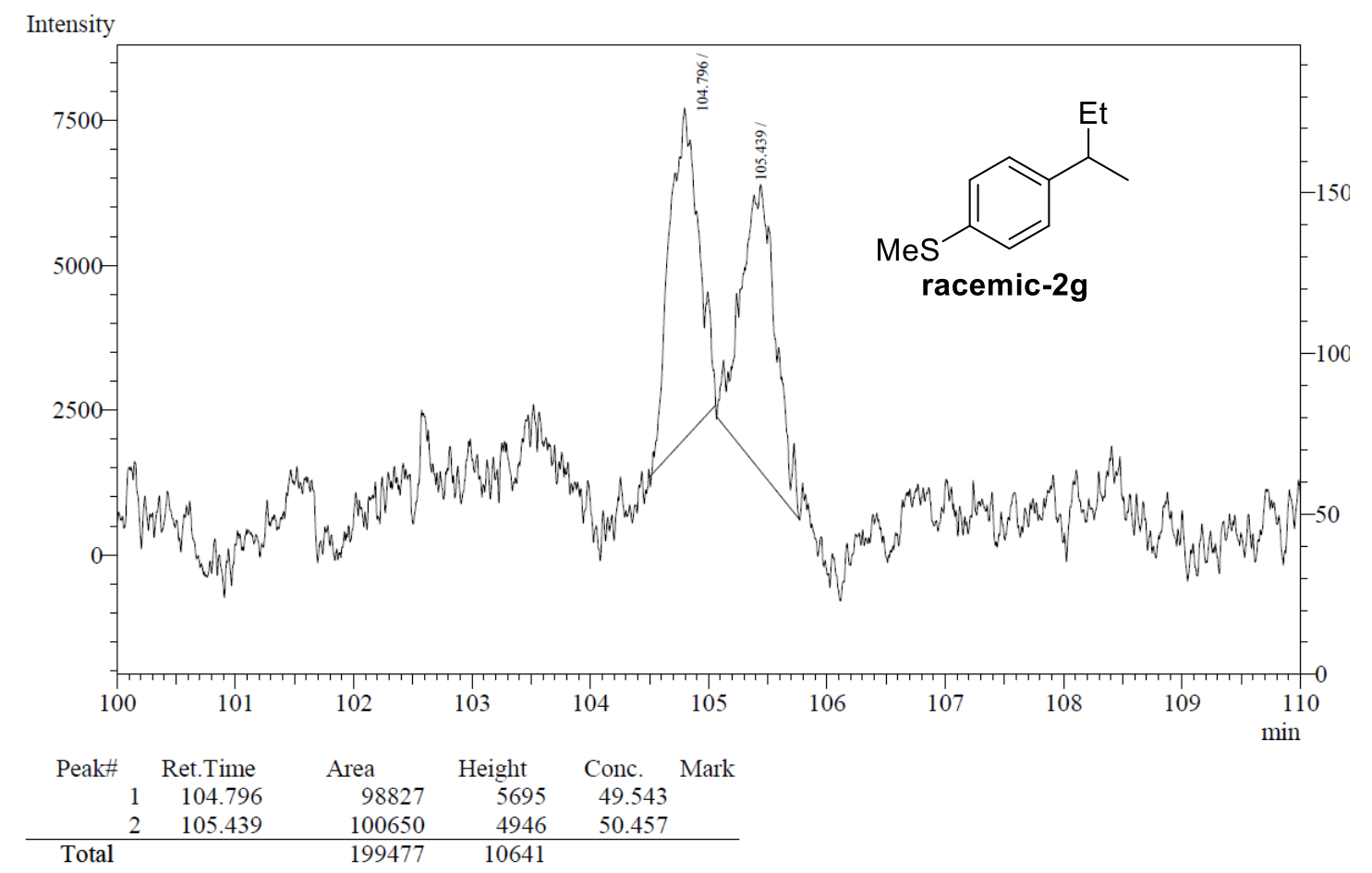

\section{Method Name $\quad$ : E: $\quad$ method $\backslash 60-40-1-175-10-4 S M e . g c m$}

Intensity

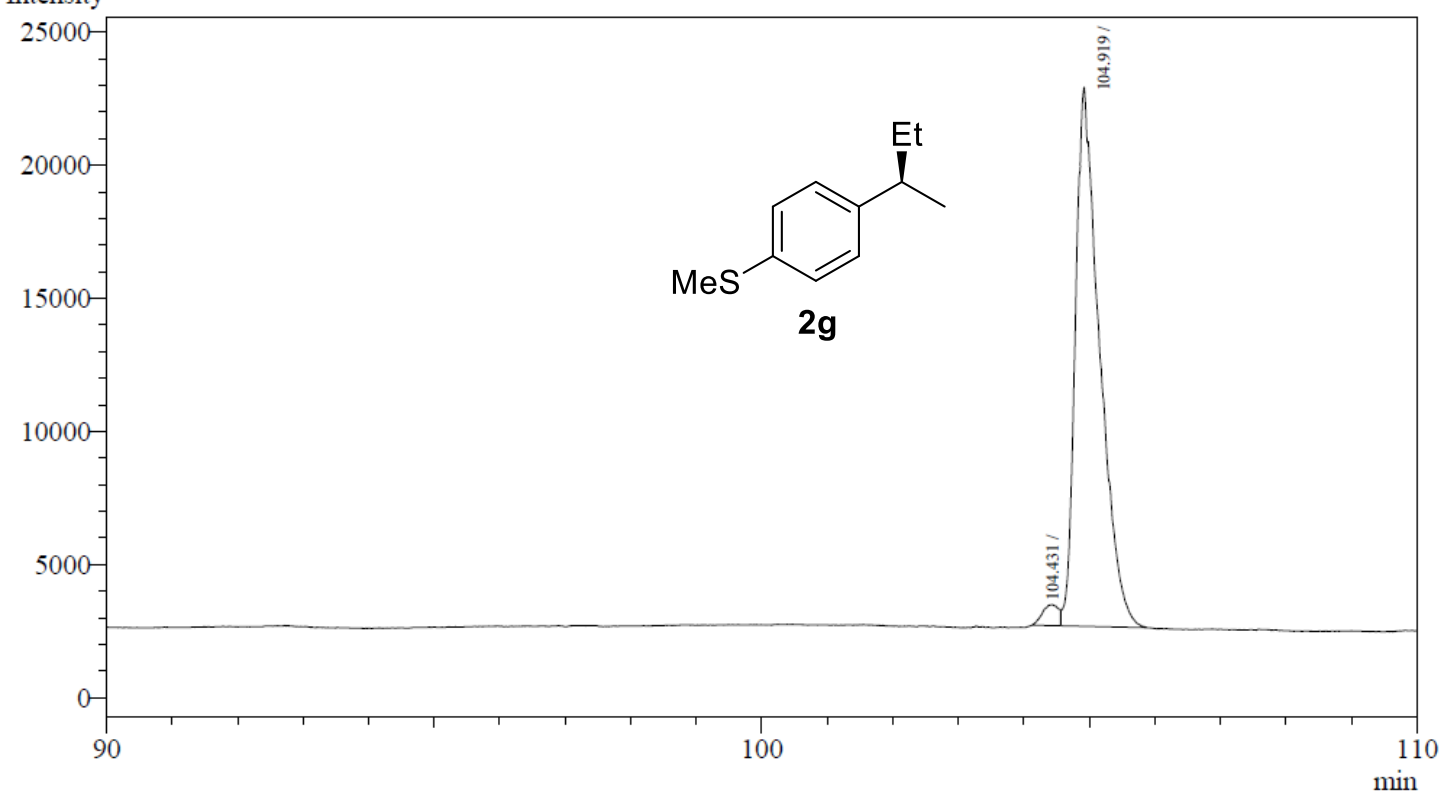

\begin{tabular}{crrrr} 
Peak\# & Ret.Time & \multicolumn{1}{c}{ Area } & Height & \multicolumn{1}{c}{ Conc. } \\
1 & 104.431 & 13208 & 789 & 2.480 \\
2 & 104.919 & 519434 & 20242 & 97.520 \\
\hline Total & & 532642 & 21031 &
\end{tabular}


GC Conditions : Chiralcel B-DM, $100 \mathrm{kPa} \mathrm{N} 2\left(110^{\circ} \mathrm{C}-200 \mathrm{~min}-1{ }^{\circ} \mathrm{C} / \mathrm{min}-180{ }^{\circ} \mathrm{C}\right.$ $-5 \mathrm{~min})$

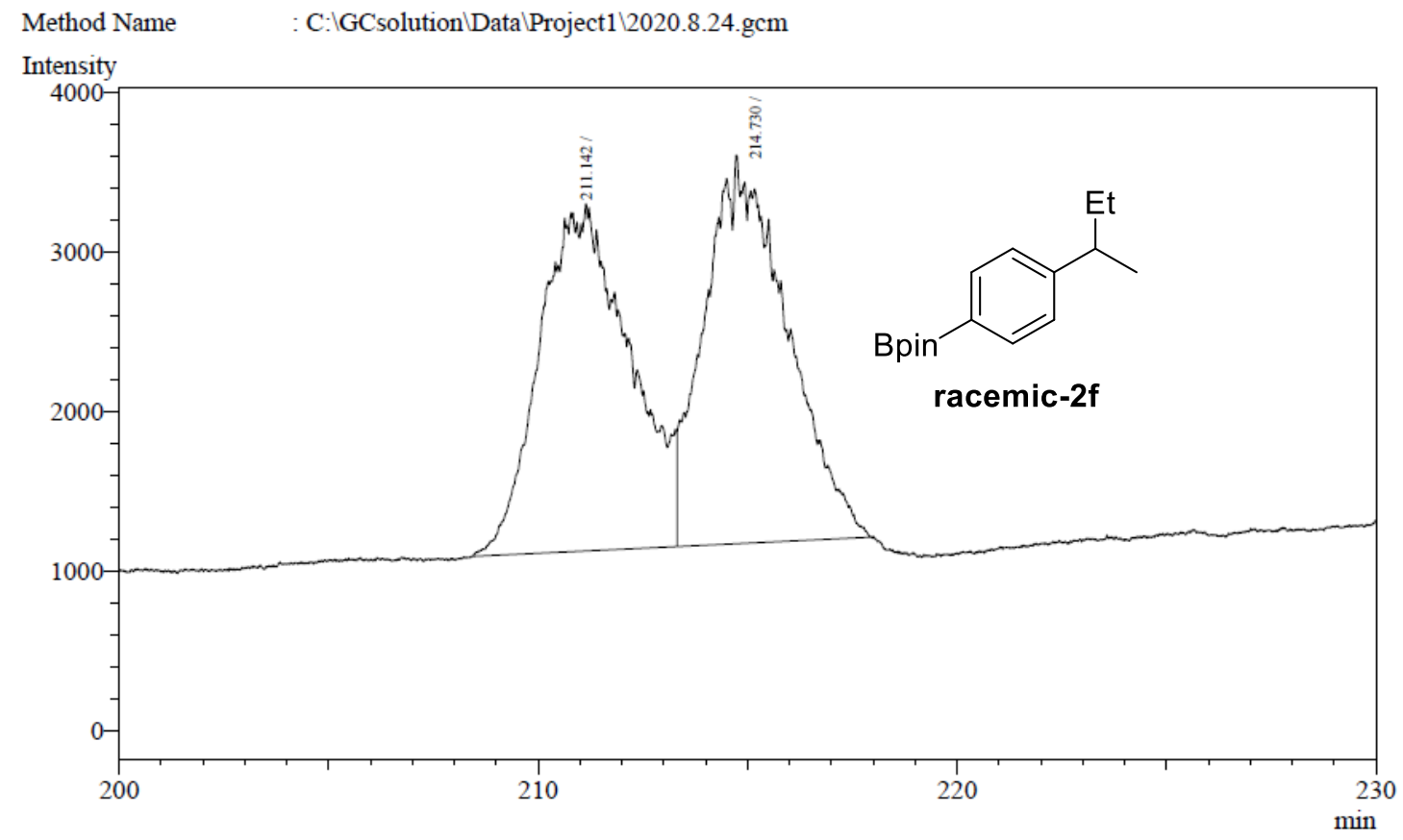

\begin{tabular}{|c|c|c|c|c|}
\hline Peak\# & Ret.Time & Area & Height & Conc. \\
\hline & 211.142 & 324239 & 2174 & 49.381 \\
\hline & 214.730 & 332368 & 2433 & 50.619 \\
\hline Total & & 656607 & 4607 & \\
\hline
\end{tabular}

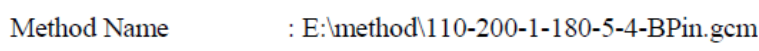

Intensity

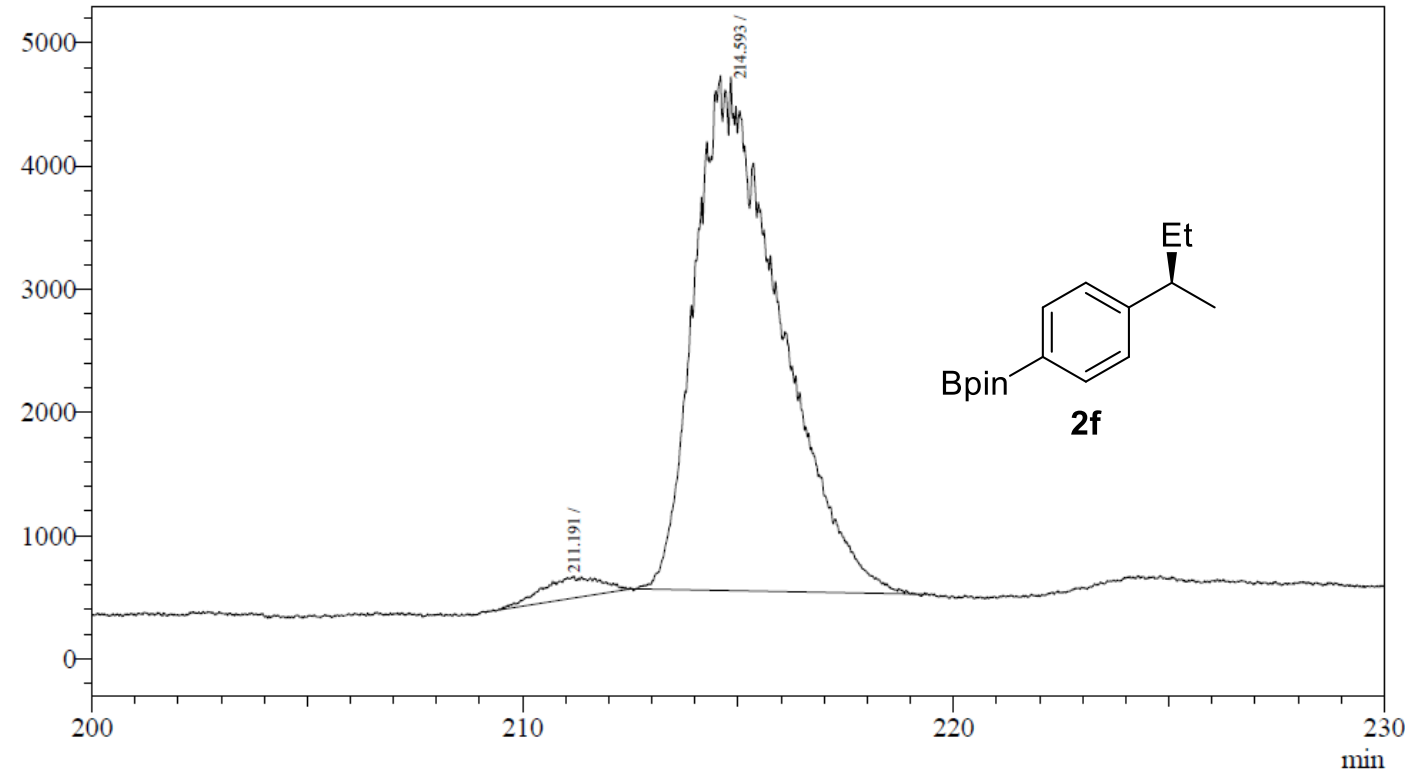

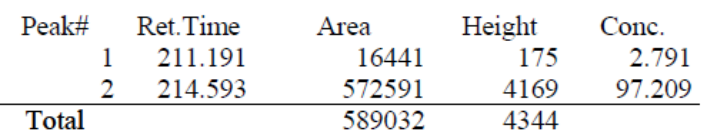


$\mathrm{mV}$

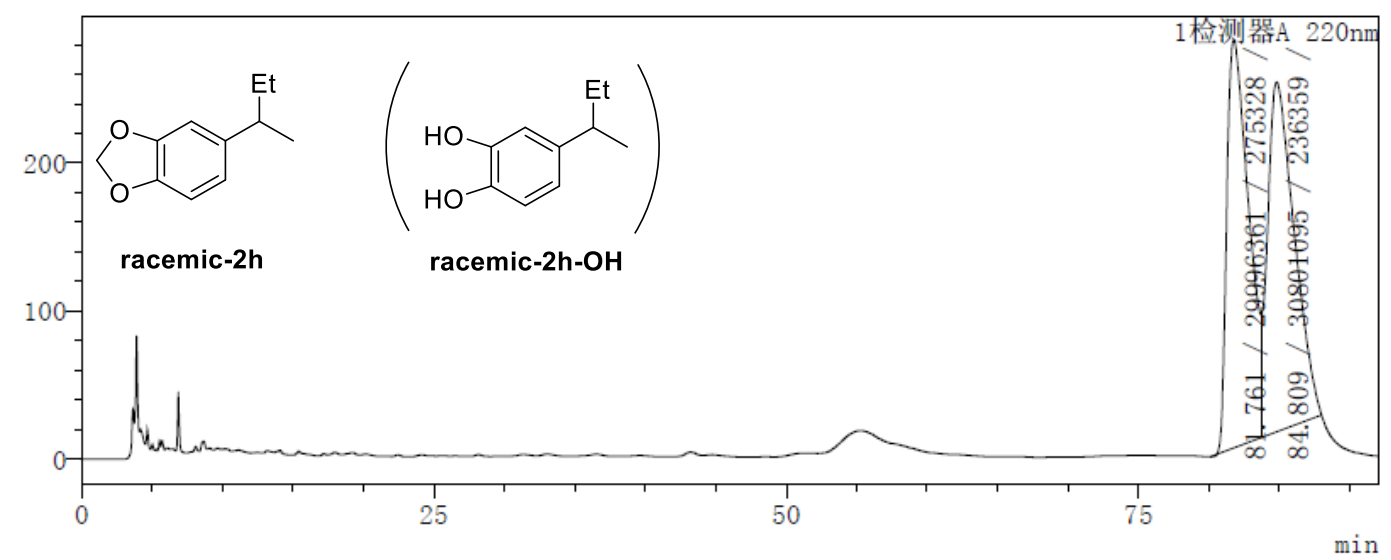

峰表

\begin{tabular}{|c|c|c|c|c|c|}
\hline 峰号 & 保留时间 & 面积 & 高度 & 标记 & 面积\% \\
\hline 1 & 81.761 & 29996361 & 275328 & M & 49.338 \\
\hline 2 & 84.809 & 30801095 & 236359 & V M & 50.662 \\
\hline 总计 & & 60797456 & 511688 & & 100.000 \\
\hline
\end{tabular}

措述

: OJ-H, n-hexane $/$ iPrOH $=98 / 2,0.8 \mathrm{ml} / \mathrm{min}, 220 \mathrm{~nm}$

$\mathrm{mV}$

\section{色谱图}

XHF1161

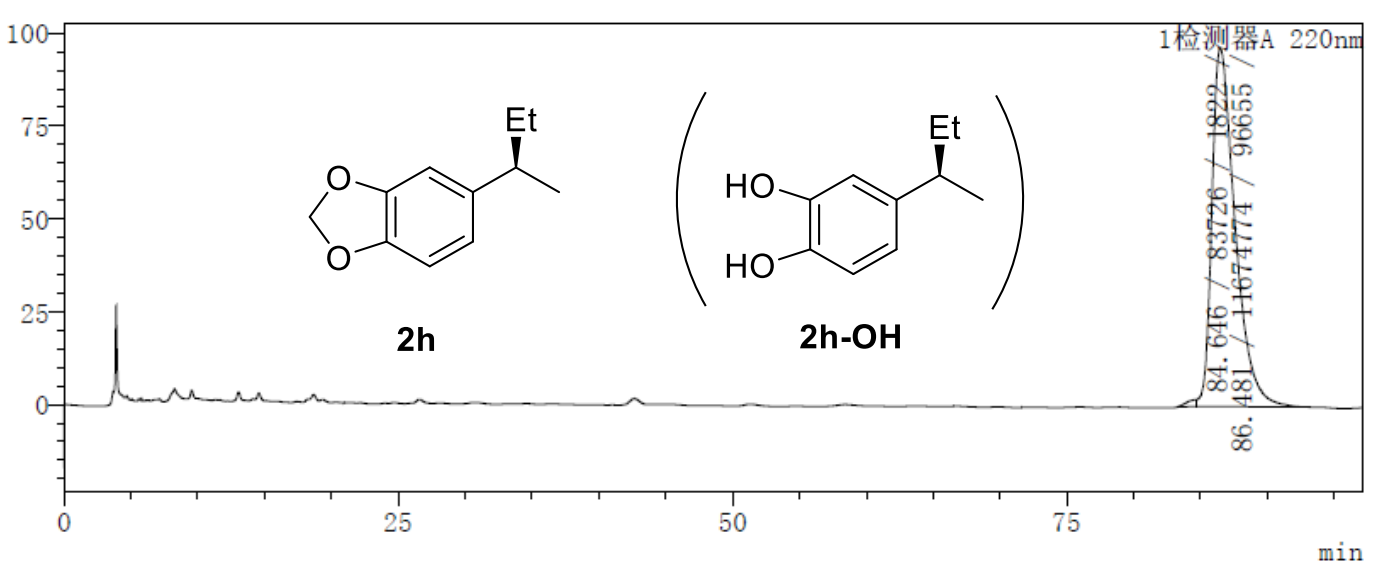

峰表

检测器 $A$. $220 \mathrm{~nm}$
\begin{tabular}{|r|r|r|r|r|r|}
\hline 峰号 & 保留时间 & \multicolumn{1}{|c|}{ 面积 } & \multicolumn{1}{c|}{ 高度 } & 标记 & \multicolumn{1}{|c|}{ 面积\% } \\
\hline 1 & 84.646 & 83726 & 1822 & $\mathrm{M}$ & 0.712 \\
\hline 2 & 86.481 & 11674774 & 96655 & $\mathrm{M}$ & 99.288 \\
\hline 总计 & & 11758499 & 98477 & & 100.000 \\
\hline
\end{tabular}


GC Conditions : Chiralcel B-DM, $100 \mathrm{kPa} \mathrm{N} 2\left(60{ }^{\circ} \mathrm{C}-30 \mathrm{~min}-2{ }^{\circ} \mathrm{C} / \mathrm{min}-180{ }^{\circ} \mathrm{C}-\right.$ $10 \min )$

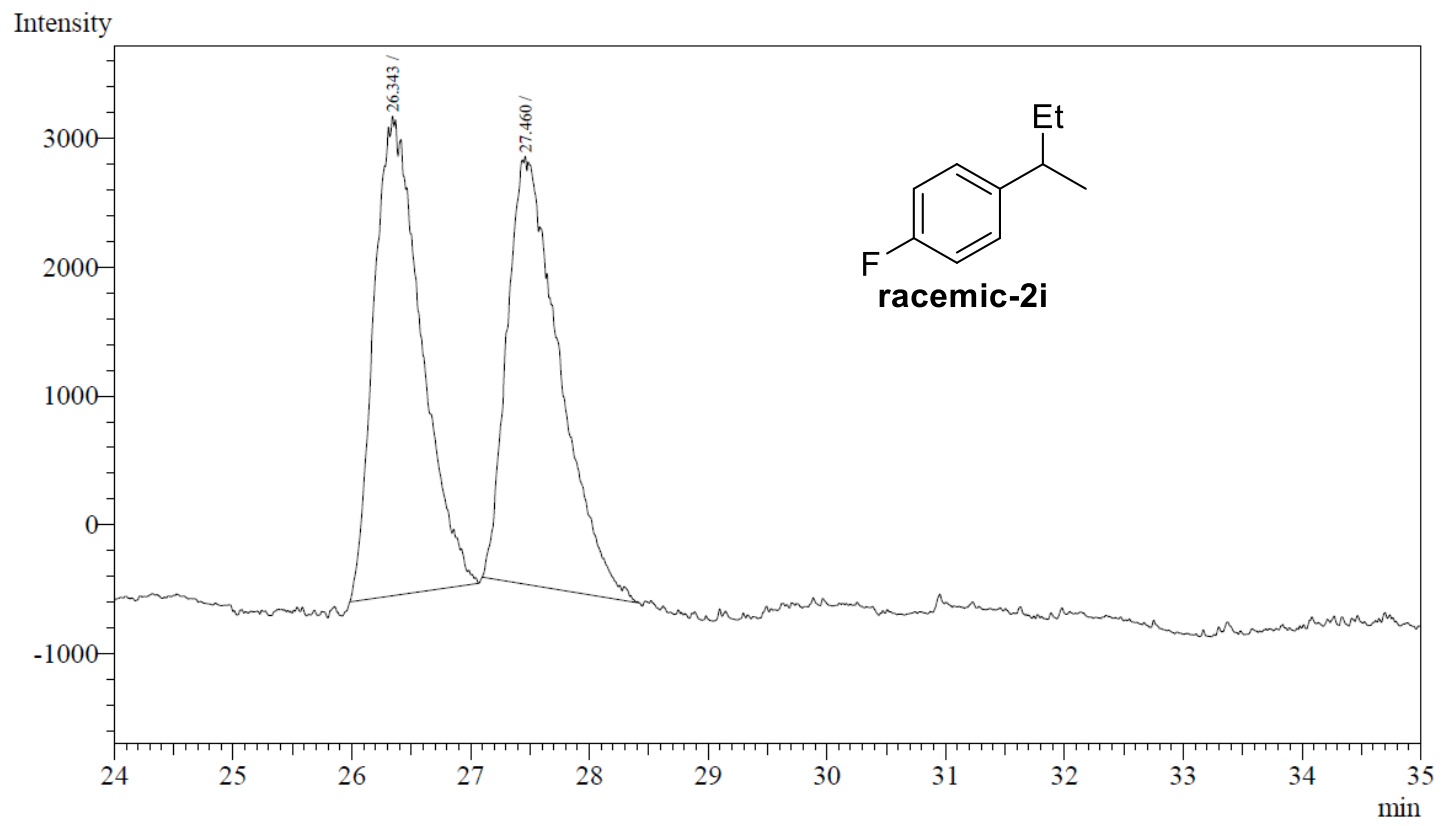

\begin{tabular}{|c|c|c|c|c|}
\hline Peak\# & Ret.Time & Area & Height & Conc. \\
\hline 1 & 26.343 & 103179 & 3718 & 50.188 \\
\hline 2 & 27.460 & 102404 & 3312 & 49.812 \\
\hline tol & & 205583 & 7030 & \\
\hline
\end{tabular}

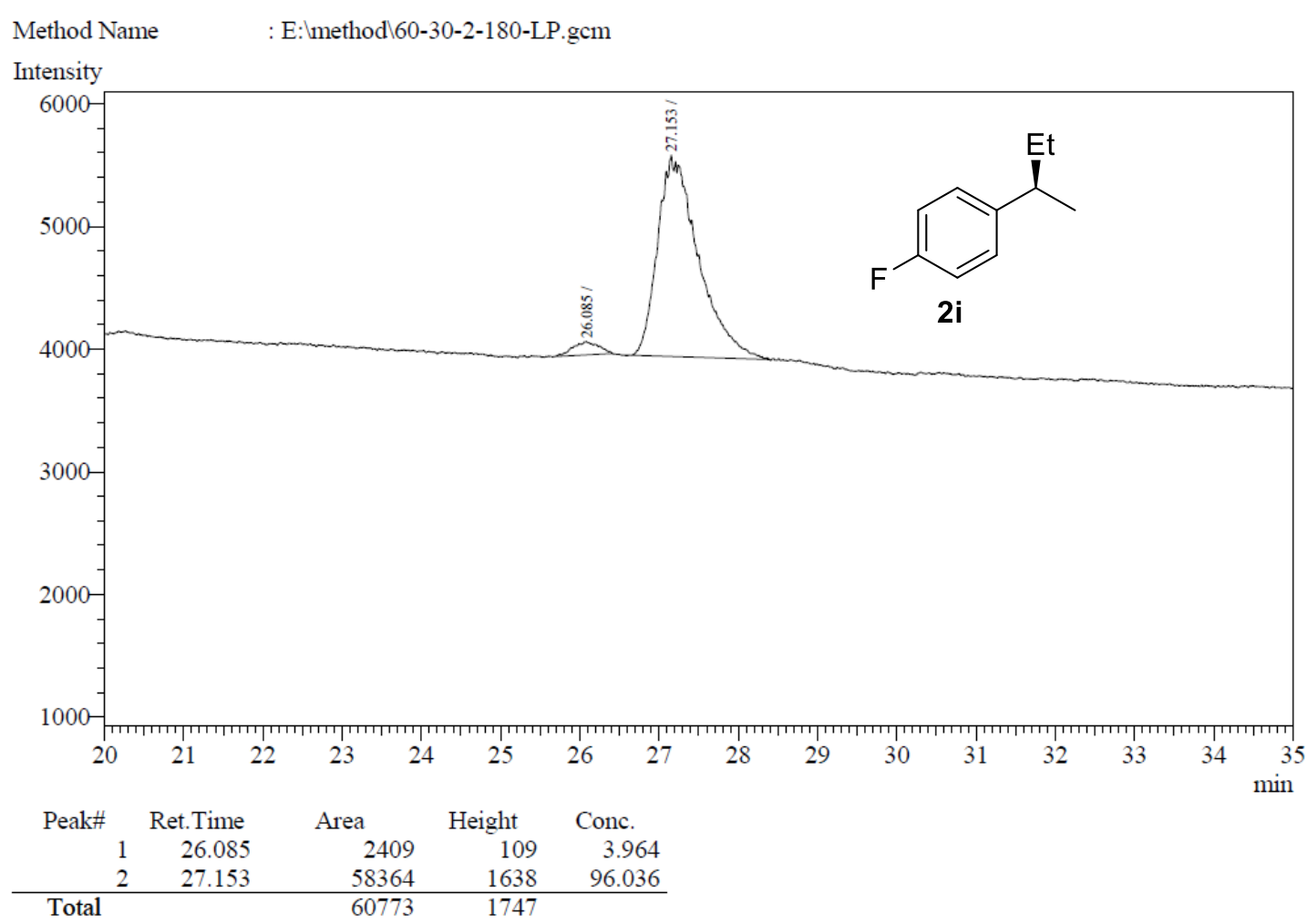


GC Conditions : Chiralcel B-DM, $100 \mathrm{kPa} \mathrm{N} 2\left(60{ }^{\circ} \mathrm{C}-90 \min -2{ }^{\circ} \mathrm{C} / \mathrm{min}-120{ }^{\circ} \mathrm{C}-\right.$ $10 \mathrm{~min}$ )

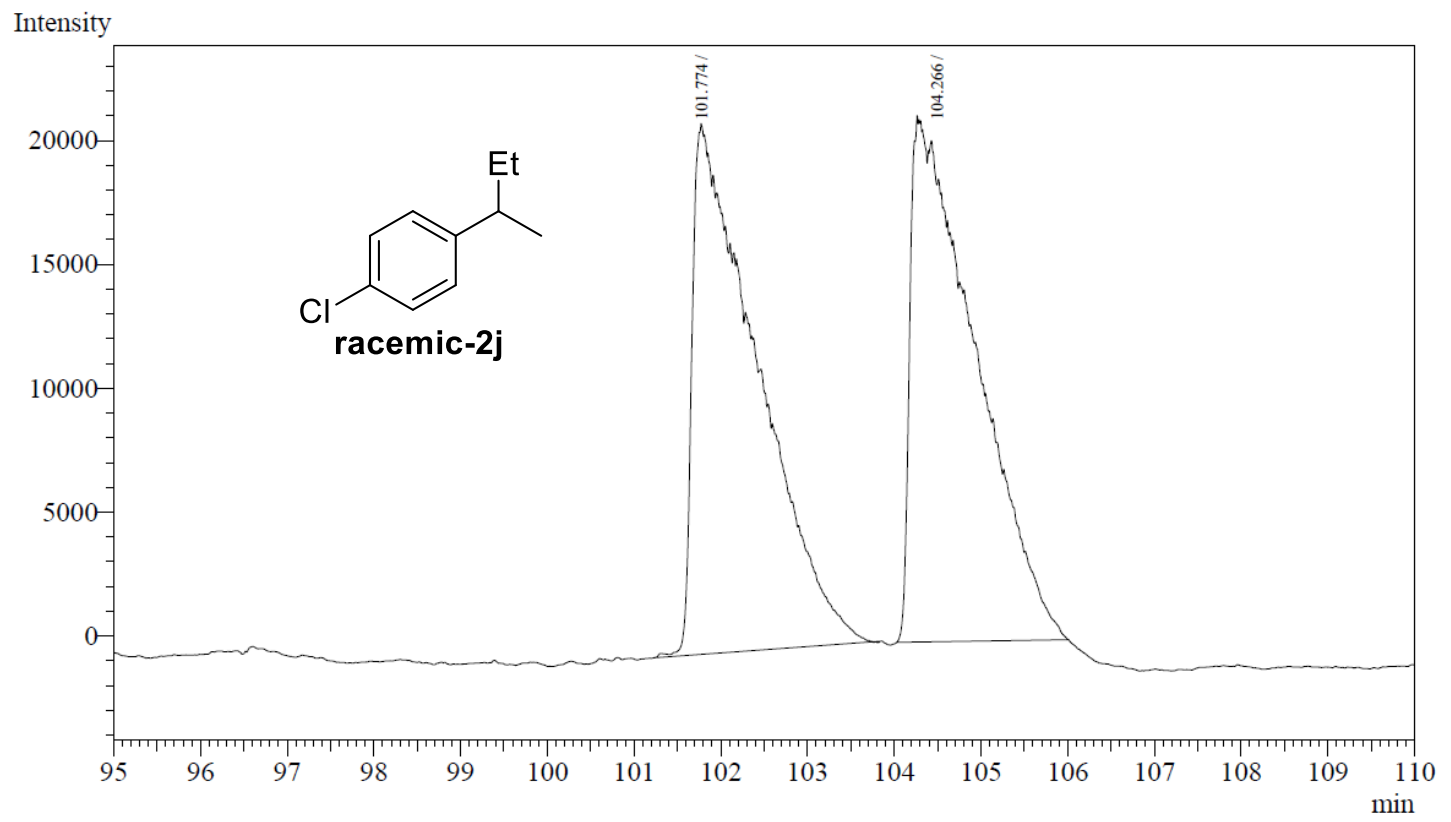

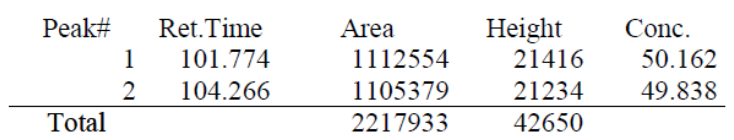

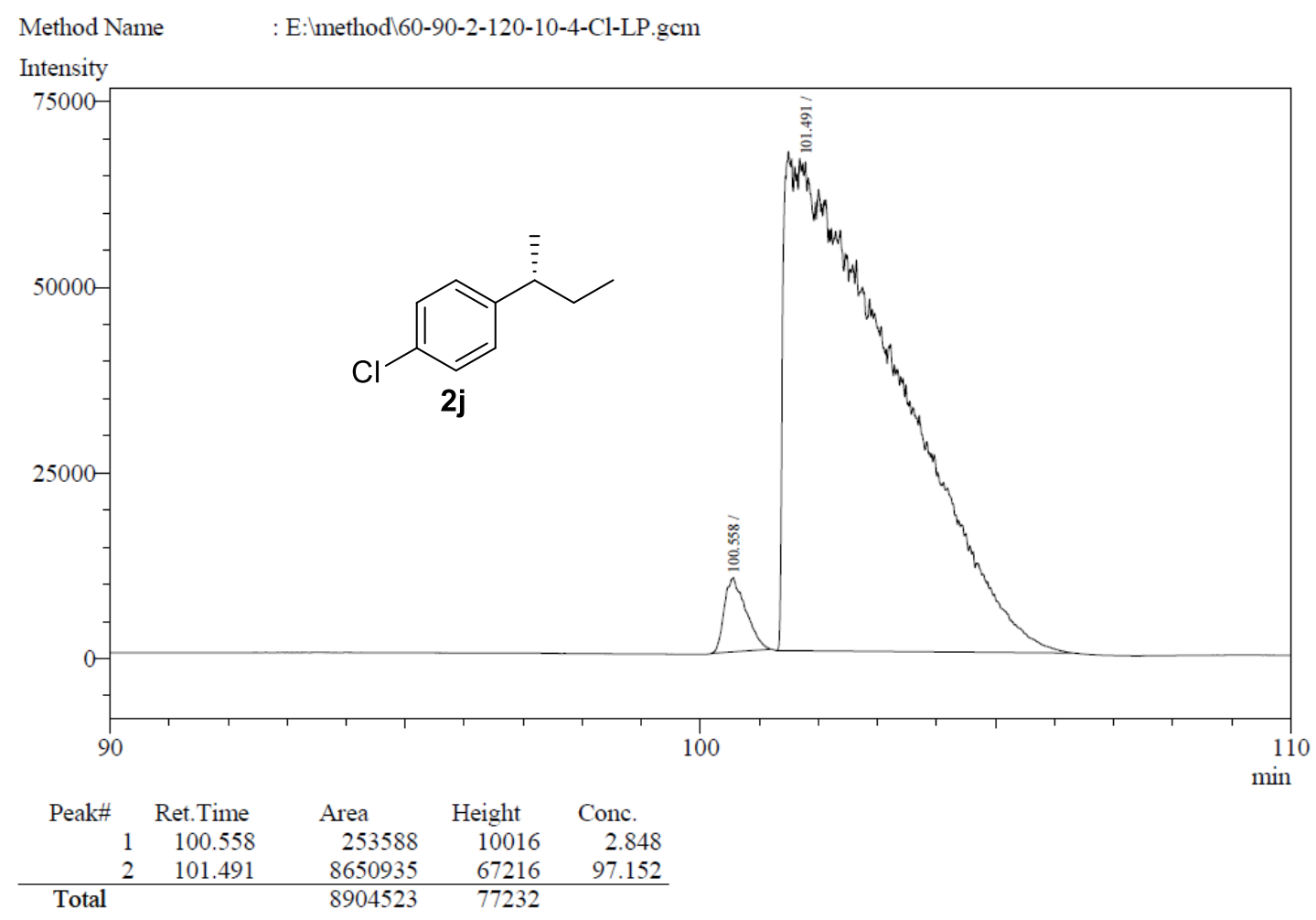


GC Conditions : Chiralcel B-DM, $100 \mathrm{kPa} \mathrm{N} 2\left(70{ }^{\circ} \mathrm{C}-90 \min -3{ }^{\circ} \mathrm{C} / \mathrm{min}-175^{\circ} \mathrm{C}-5\right.$

$\min$ )

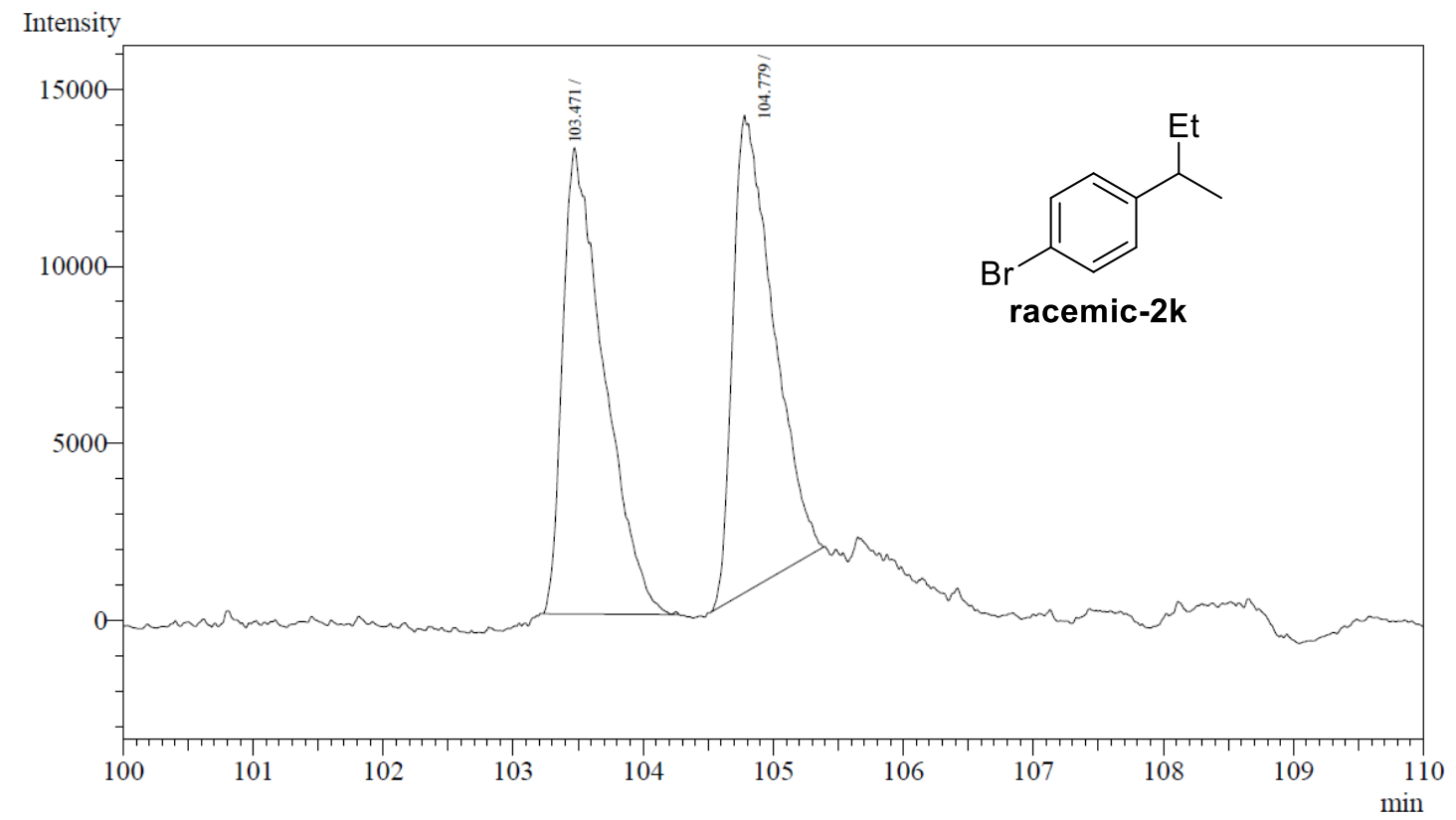

\begin{tabular}{crrrr} 
Peak\# & Ret.Time & \multicolumn{1}{c}{ Area } & Height & \multicolumn{1}{c}{ Conc. } \\
1 & 103.471 & 290639 & 13139 & 49.997 \\
2 & 104.779 & 290674 & 13469 & 50.003 \\
\hline Total & & 581313 & 26608 &
\end{tabular}

Method Name $\quad$ : E: method $170-90-3-175-5 . \mathrm{gcm}$

Intensity

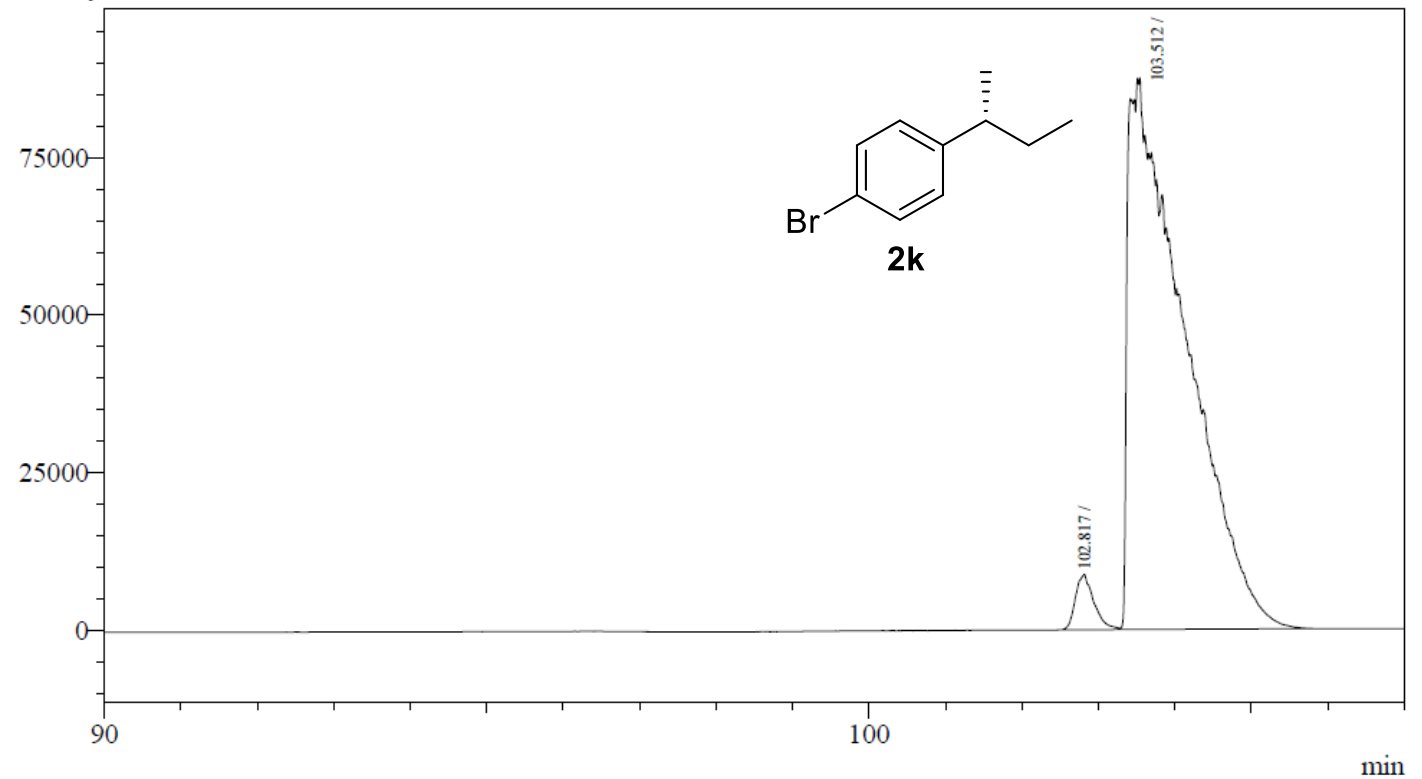

\begin{tabular}{|c|c|c|c|c|}
\hline Peak\# & Ret.Time & Area & Height & Conc. \\
\hline 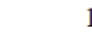 & 102.817 & 146786 & 8800 & 3.124 \\
\hline 2 & 103.512 & 4552446 & 87475 & 96.876 \\
\hline 1 & & 4699232 & 96275 & \\
\hline
\end{tabular}


$\mathrm{mV}$

\section{色谱图}

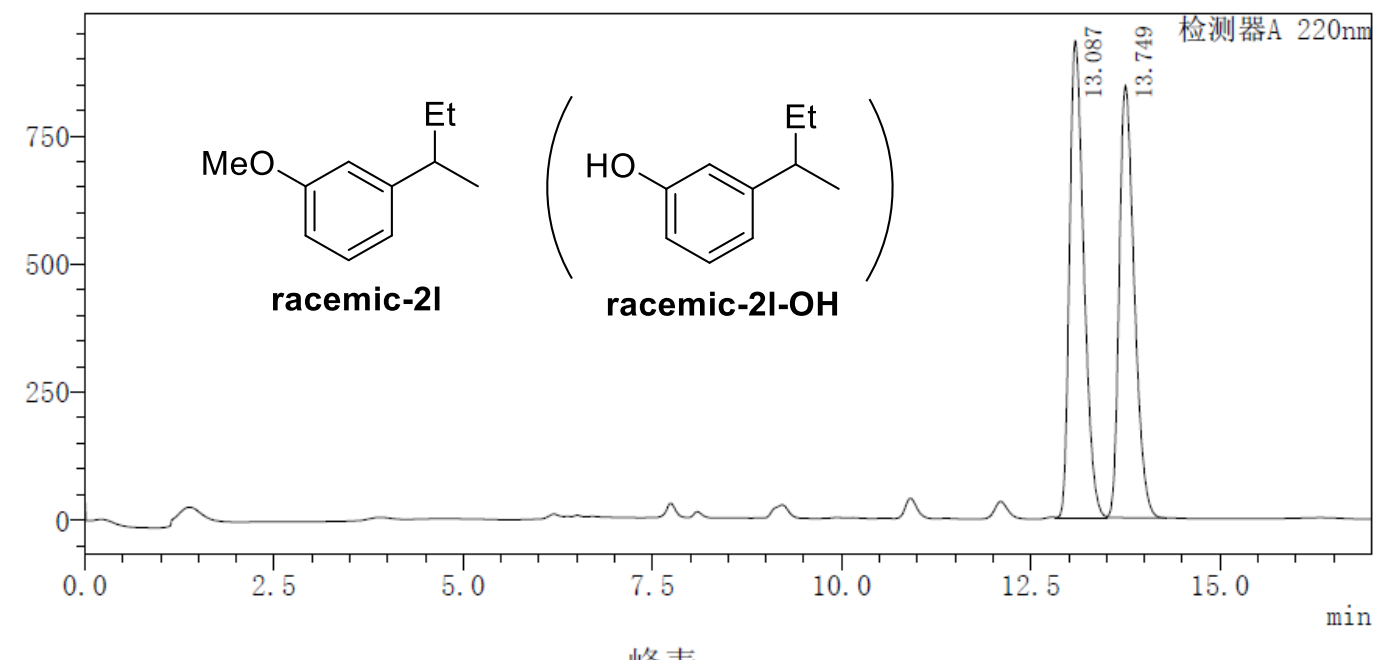

峰表

检测器A $220 \mathrm{~nm}$
\begin{tabular}{|r|r|r|r|r|r|}
\hline 峰号 & 保留时间 & 面积 & 高度 & 标记 & \multicolumn{1}{|c|}{ 面积 $\%$} \\
\hline 1 & 13.087 & 12217843 & 930055 & & 51.080 \\
\hline 2 & 13.749 & 11701172 & 843186 & $\mathrm{~V}$ & 48.920 \\
\hline 总计 & & 23919015 & 1773241 & & 100.000 \\
\hline
\end{tabular}

描述

: $0 \mathrm{~J}-\mathrm{HX} 2, \mathrm{n}$-hexane $/ \mathrm{iPrOH}=90 / 10,1.0 \mathrm{ml} / \mathrm{min}, 220 \mathrm{~nm}$

色谱图

$\mathrm{mV}$

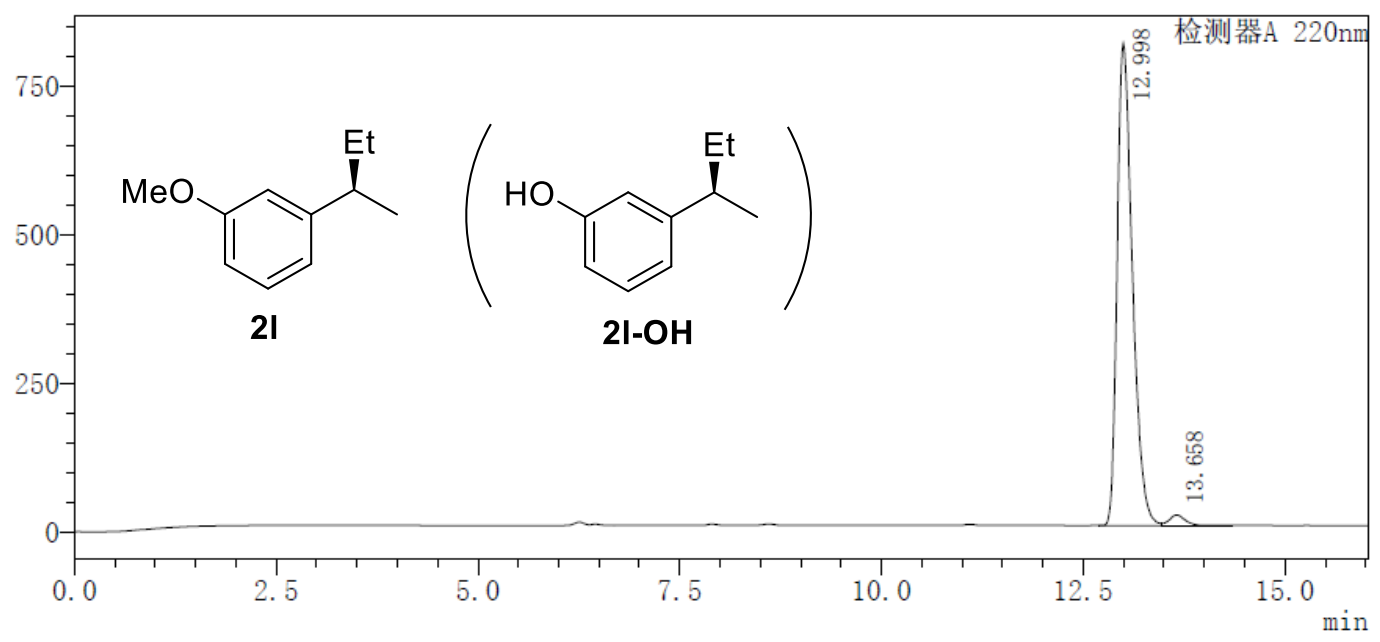

峰表

检测器A $220 \mathrm{~nm}$
\begin{tabular}{|r|r|r|r|r|r|}
\hline 峰号 & 保留时间 & \multicolumn{1}{c|}{ 面积 } & \multicolumn{1}{c|}{ 高度 } & 标记 & \multicolumn{1}{|c|}{ 面积 $\%$} \\
\hline 1 & 12.998 & 10681772 & 812546 & $\mathrm{~V}$ & 97.714 \\
\hline 2 & 13.658 & 249854 & 17854 & $\mathrm{~V}$ & 2.286 \\
\hline 总计 & & 10931626 & 830400 & & 100.000 \\
\hline
\end{tabular}


$\mathrm{mV}$

色谱图

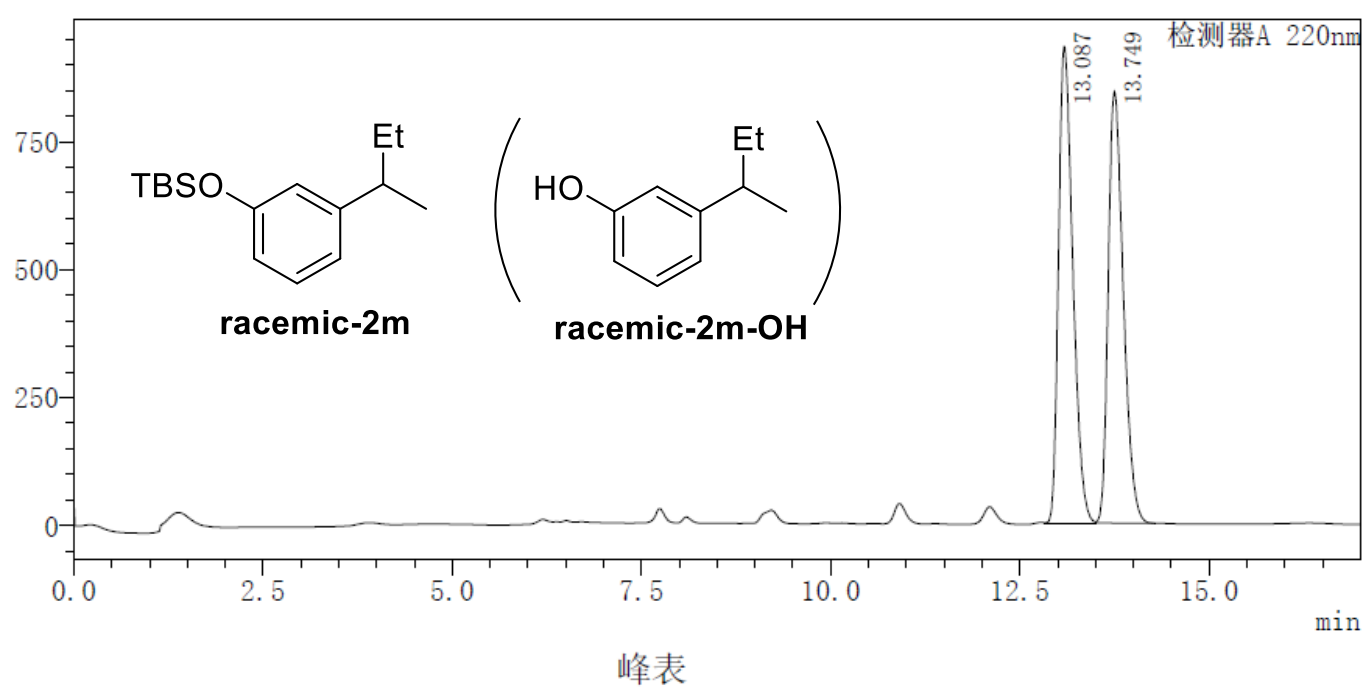

检测器A $220 \mathrm{~nm}$
\begin{tabular}{|r|r|r|r|r|r|}
\hline 峰号 & 保留时间 & 面积 & 高度 & 标记 & \multicolumn{1}{|c|}{ 面积 $\%$} \\
\hline 1 & 13.087 & 12217843 & 930055 & & 51.080 \\
\hline 2 & 13.749 & 11701172 & 843186 & $\mathrm{~V}$ & 48.920 \\
\hline 总计 & & 23919015 & 1773241 & & 100.000 \\
\hline
\end{tabular}

描述

: $0 \mathrm{~J}-\mathrm{H} * 2, \mathrm{n}$-hexane $/ \mathrm{iPrOH}=90 / 10,1.0 \mathrm{ml} / \mathrm{min}, 220 \mathrm{~nm}$

$\mathrm{mV}$

色谱图

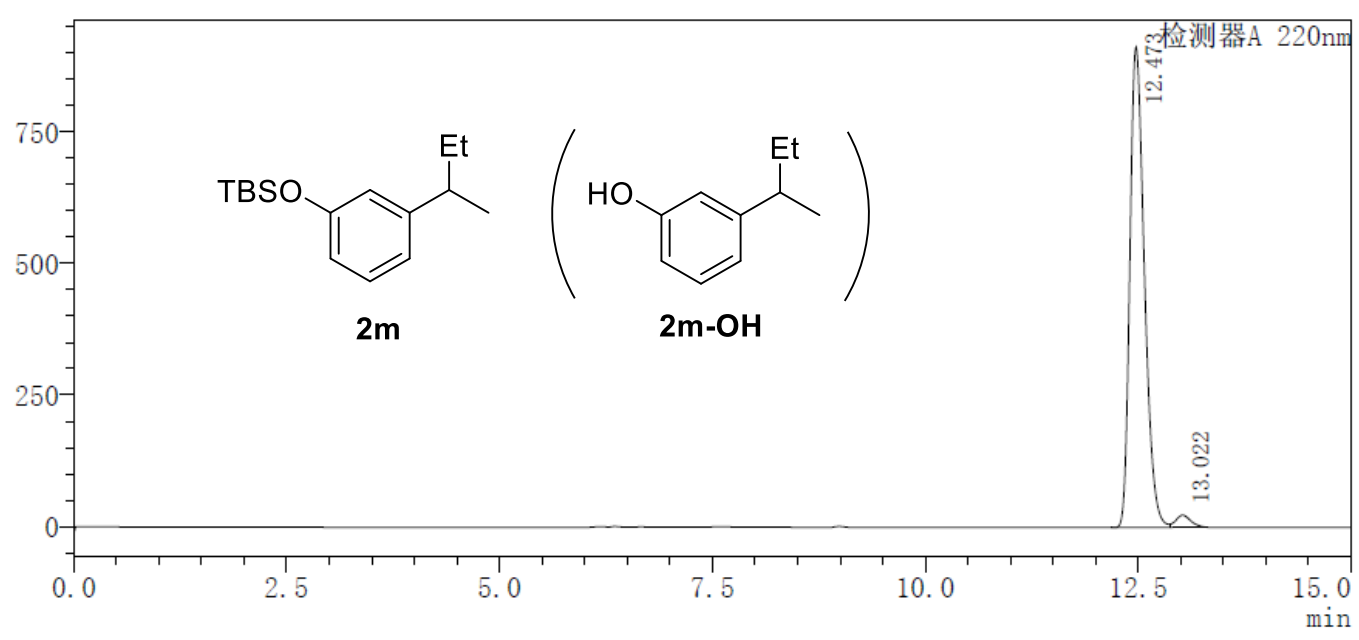

峰表

\begin{tabular}{|c|c|c|c|c|c|}
\hline 峰号 & 保留时间 & 面积 & 高度 & 标记 & 面积\% \\
\hline 1 & 12.473 & 10829407 & 911603 & & 97.550 \\
\hline 2 & 13.022 & 272023 & 22487 & V M & 2.450 \\
\hline 总计 & & 11101430 & 934090 & & 100.000 \\
\hline
\end{tabular}


IV

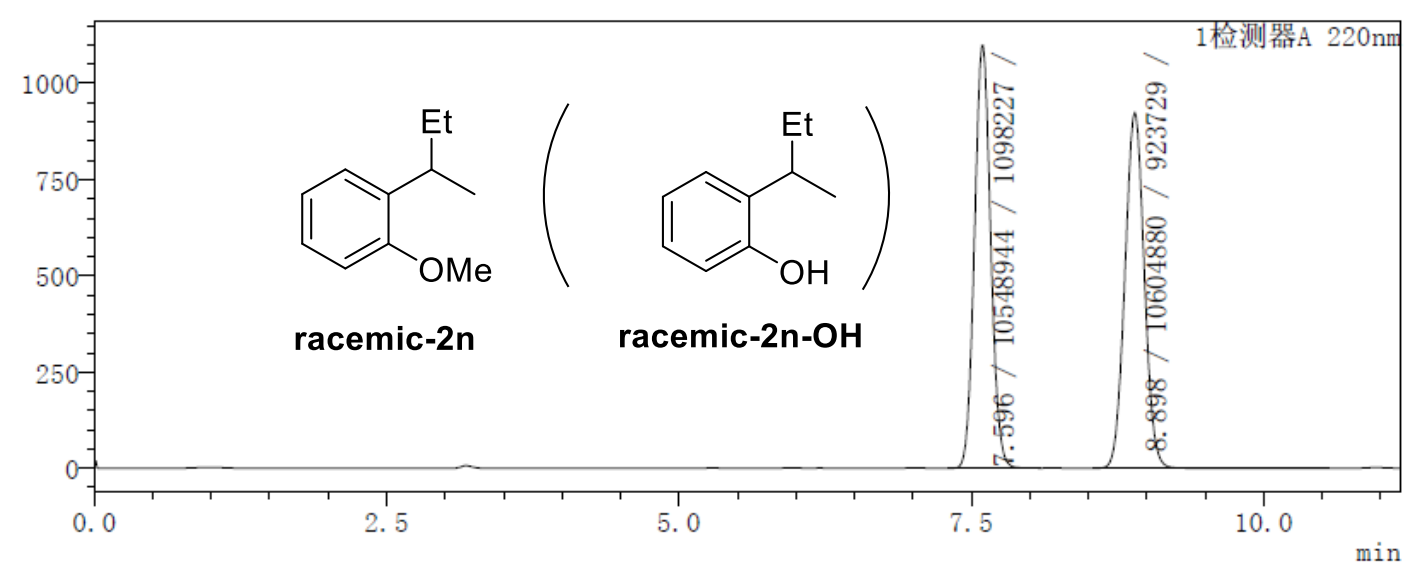

峰表

检测器A $220 \mathrm{~nm}$
\begin{tabular}{|r|r|r|r|r|r|}
\hline 峰䒓 & 保留时间 & \multicolumn{1}{|c|}{ 面积 } & \multicolumn{1}{|l}{ 高度 } & 标记 & \multicolumn{1}{c|}{ 面积\% } \\
\hline 1 & 7.596 & 10548944 & 1098227 & & 49.868 \\
\hline 2 & 8.898 & 10604880 & 923729 & & 50.132 \\
\hline 总计 & & 21153824 & 2021956 & & 100.000 \\
\hline
\end{tabular}

描述 : OJ-H, n-hexane $/ \mathrm{iPr} O \mathrm{H}=90 / 10,1.0 \mathrm{ml} / \mathrm{min}, 220 \mathrm{~nm}$

$\mathrm{mV}$

色谱图

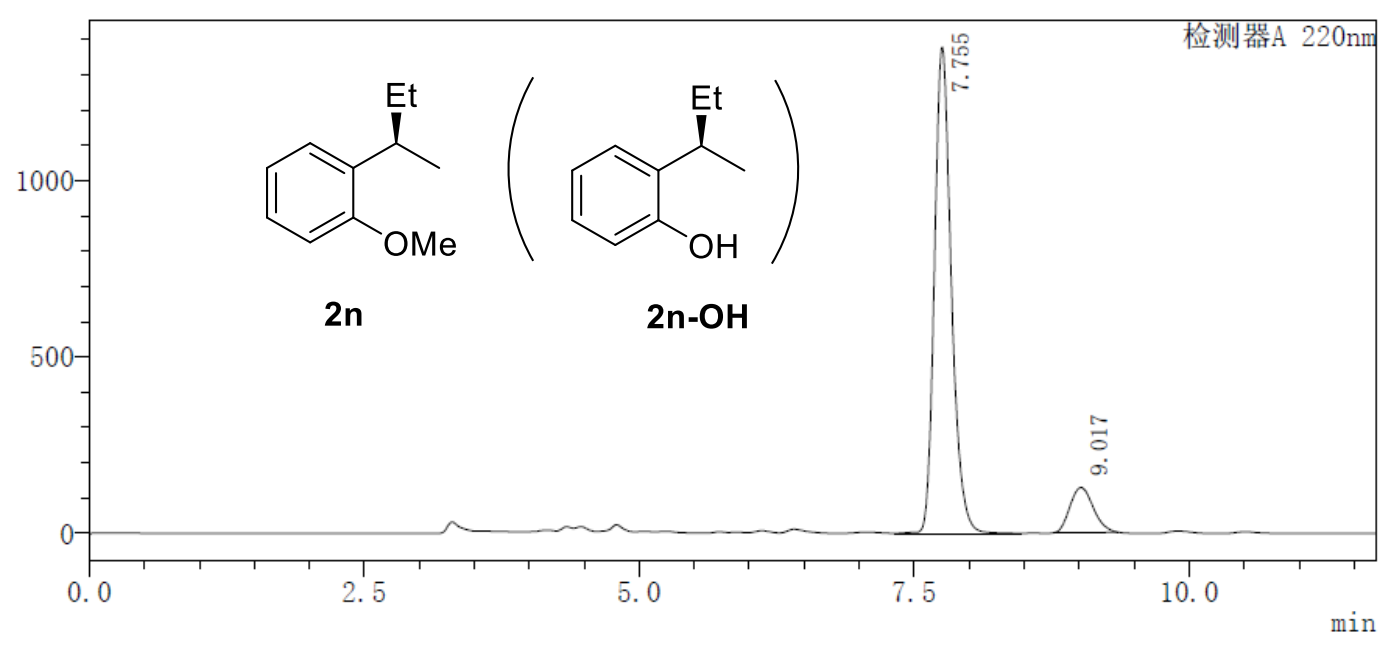

峰表

检测器A $220 \mathrm{~nm}$
\begin{tabular}{|r|r|r|r|r|r|}
\hline 峰号 & 保留时间 & \multicolumn{1}{|c|}{ 面积 } & \multicolumn{1}{c|}{ 高度 } & 标记 & \multicolumn{1}{|c|}{ 面积 $\%$} \\
\hline 1 & 7.755 & 14728124 & 1378242 & $\mathrm{M}$ & 88.678 \\
\hline 2 & 9.017 & 1880409 & 127832 & $\mathrm{M}$ & 11.322 \\
\hline 总计 & & 16608533 & 1506074 & & 100.000 \\
\hline
\end{tabular}


$\mathrm{mV}$

\section{色谱图}

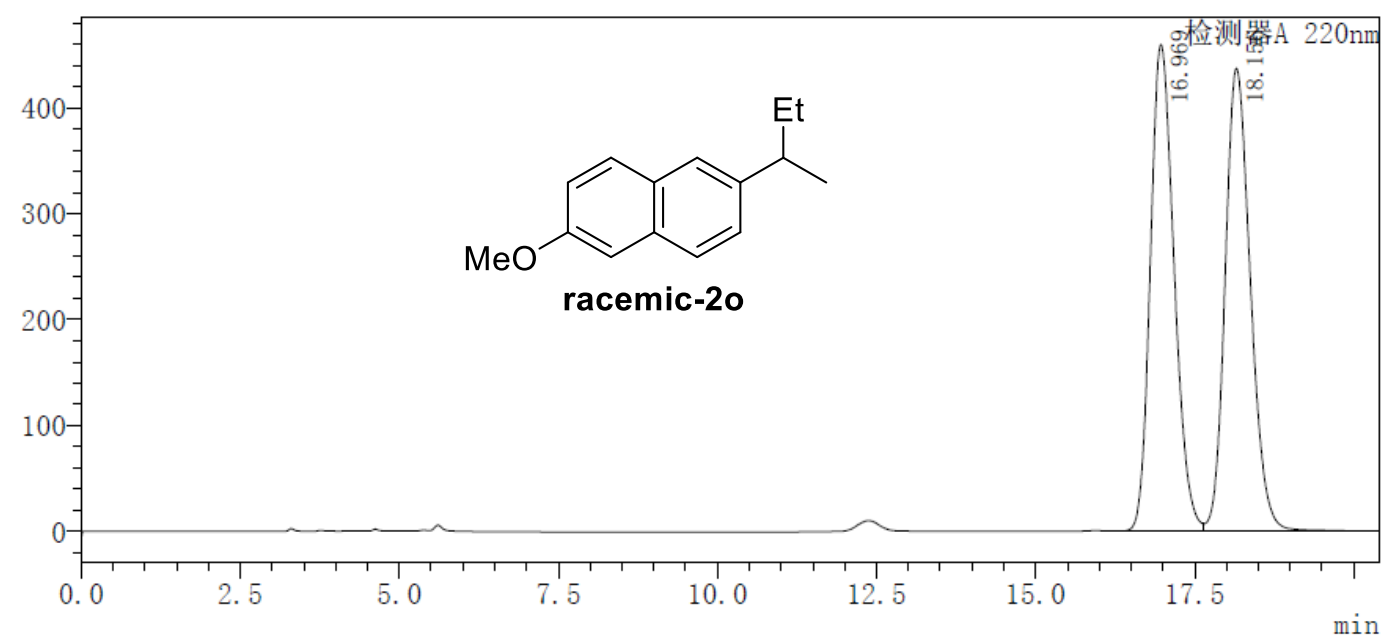

峰表

检测器A $220 \mathrm{~nm}$
\begin{tabular}{|r|r|c|c|c|r|}
\hline 峰号 & 保留时间 & 面积 & 高度 & 标记 & \multicolumn{1}{|c|}{ 面积 $\%$} \\
\hline 1 & 16.969 & 11924143 & 459445 & & 49.696 \\
\hline 2 & 18.156 & 12070103 & 437094 & V & 50.304 \\
\hline 总计 & & 23994245 & 896539 & & 100.000 \\
\hline
\end{tabular}

描述

: $0 \mathrm{~J}-\mathrm{H}, \mathrm{n}$-hexane $/ \mathrm{iPrOH}=99.5 / 0.5,1.0 \mathrm{ml} / \mathrm{min}, 220 \mathrm{~nm}$

$\mathrm{mV}$

色谱图

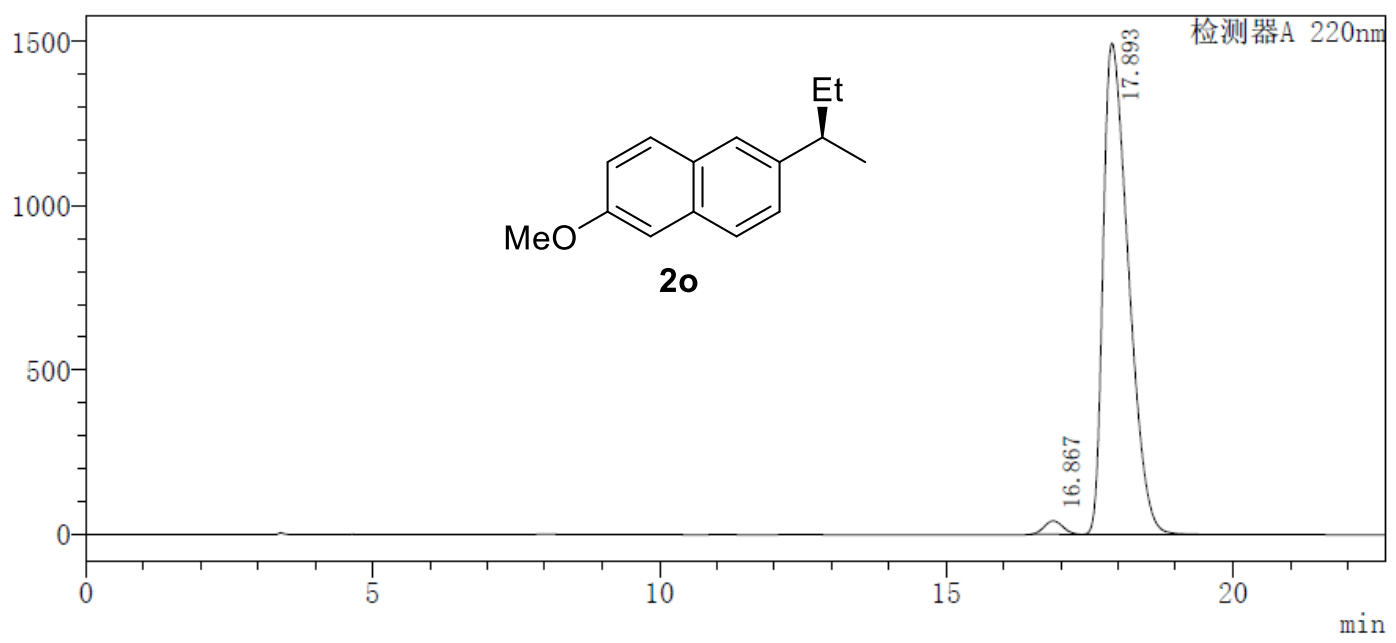

峰表

检测器 $\mathrm{A} 220 \mathrm{~nm}$

\begin{tabular}{|r|r|r|r|r|r|}
\hline 峰号 & 保留时间 & \multicolumn{1}{|c|}{ 面积 } & \multicolumn{1}{c|}{ 高度 } & 标记 & \multicolumn{1}{c|}{ 面积\% } \\
\hline 1 & 16.867 & 916337 & 40696 & $\mathrm{M}$ & 1.961 \\
\hline 2 & 17.893 & 45817396 & 1493634 & $\mathrm{M}$ & 98.039 \\
\hline 总计 & & 46733733 & 1534329 & & 100.000 \\
\hline
\end{tabular}


$\mathrm{mV}$

\section{色谱图}

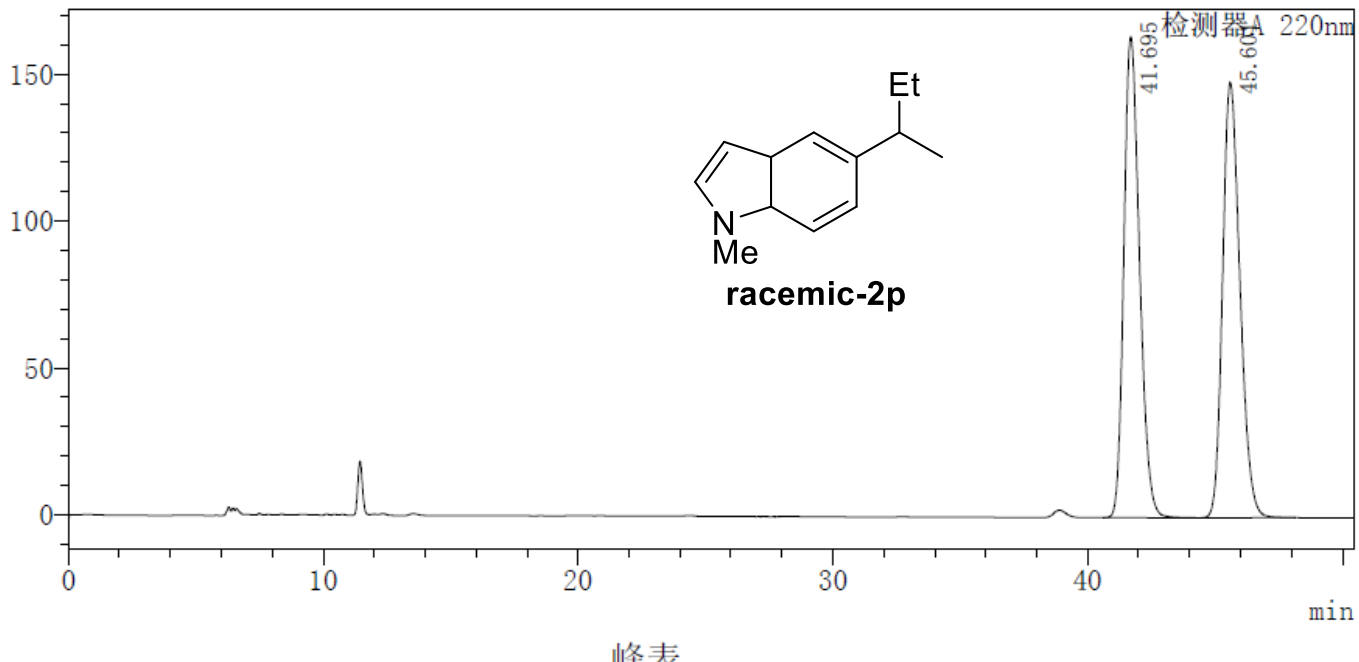

峰表

检测器A $220 \mathrm{~nm}$
\begin{tabular}{|r|r|r|c|r|r|}
\hline 峰号 & 保留时间 & \multicolumn{1}{|c|}{ 面积 } & 高度 & 标记 & \multicolumn{1}{|c|}{ 面积 $\%$} \\
\hline 1 & 41.695 & 7009920 & 163894 & & 49.920 \\
\hline 2 & 45.601 & 7032458 & 148226 & & 50.080 \\
\hline 总计 & & 14042378 & 312120 & & 100.000 \\
\hline
\end{tabular}

描述

: $0 J-H * 2, n$-hexane $/ i \operatorname{PrOH}=95 / 5,1.0 \mathrm{ml} / \mathrm{min}, 220 \mathrm{~nm}$

$\mathrm{mV}$

色谱图

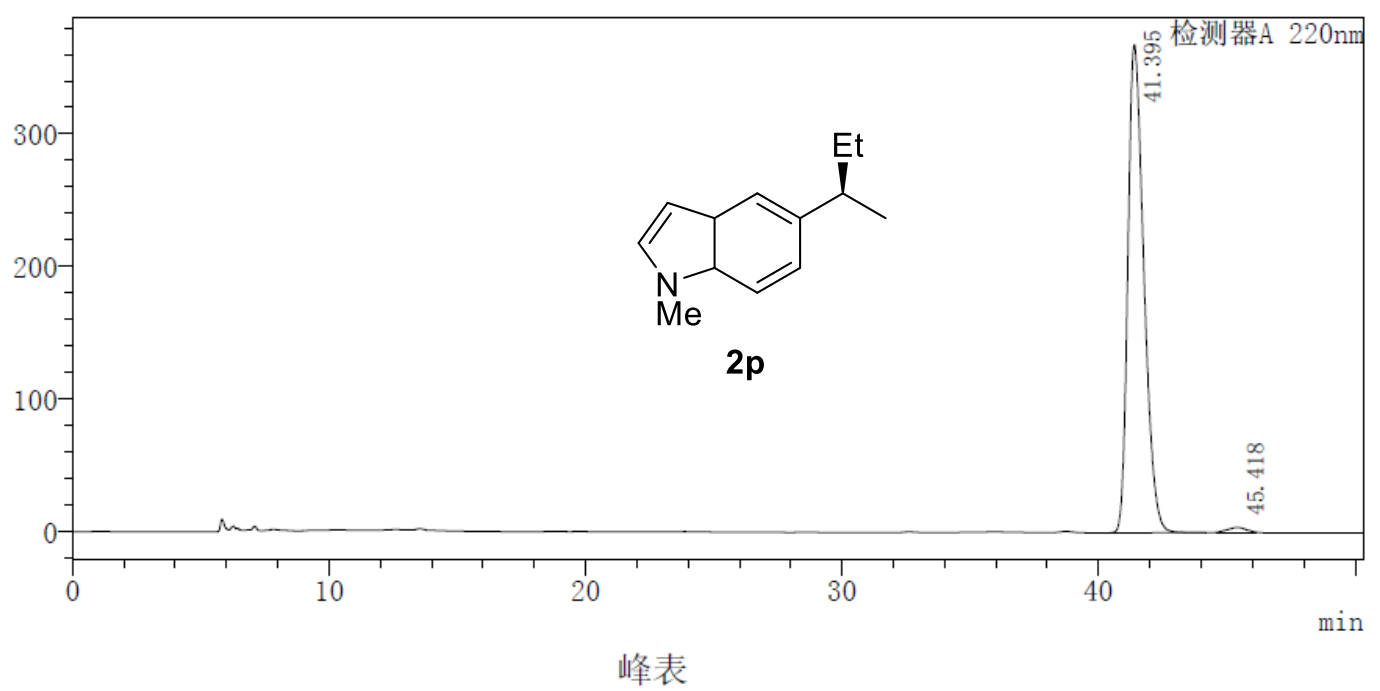

检测器A $220 \mathrm{~nm}$
\begin{tabular}{|r|r|r|r|r|r|}
\hline 峰号 & 保留时间 & \multicolumn{1}{c|}{ 面积 } & \multicolumn{1}{c|}{ 高度 } & 标记 & \multicolumn{1}{|c|}{ 面积 $\%$} \\
\hline 1 & 41.395 & 15988387 & 367947 & & 98.943 \\
\hline 2 & 45.418 & 170763 & 3563 & $\mathrm{M}$ & 1.057 \\
\hline 总计 & & 16159150 & 371510 & & 100.000 \\
\hline
\end{tabular}


GC Conditions : Chiralcel B-DM, $100 \mathrm{kPa} \mathrm{N} 2\left(60{ }^{\circ} \mathrm{C}-30 \mathrm{~min}-2{ }^{\circ} \mathrm{C} / \mathrm{min}-180{ }^{\circ} \mathrm{C}-5\right.$ $\min$ )

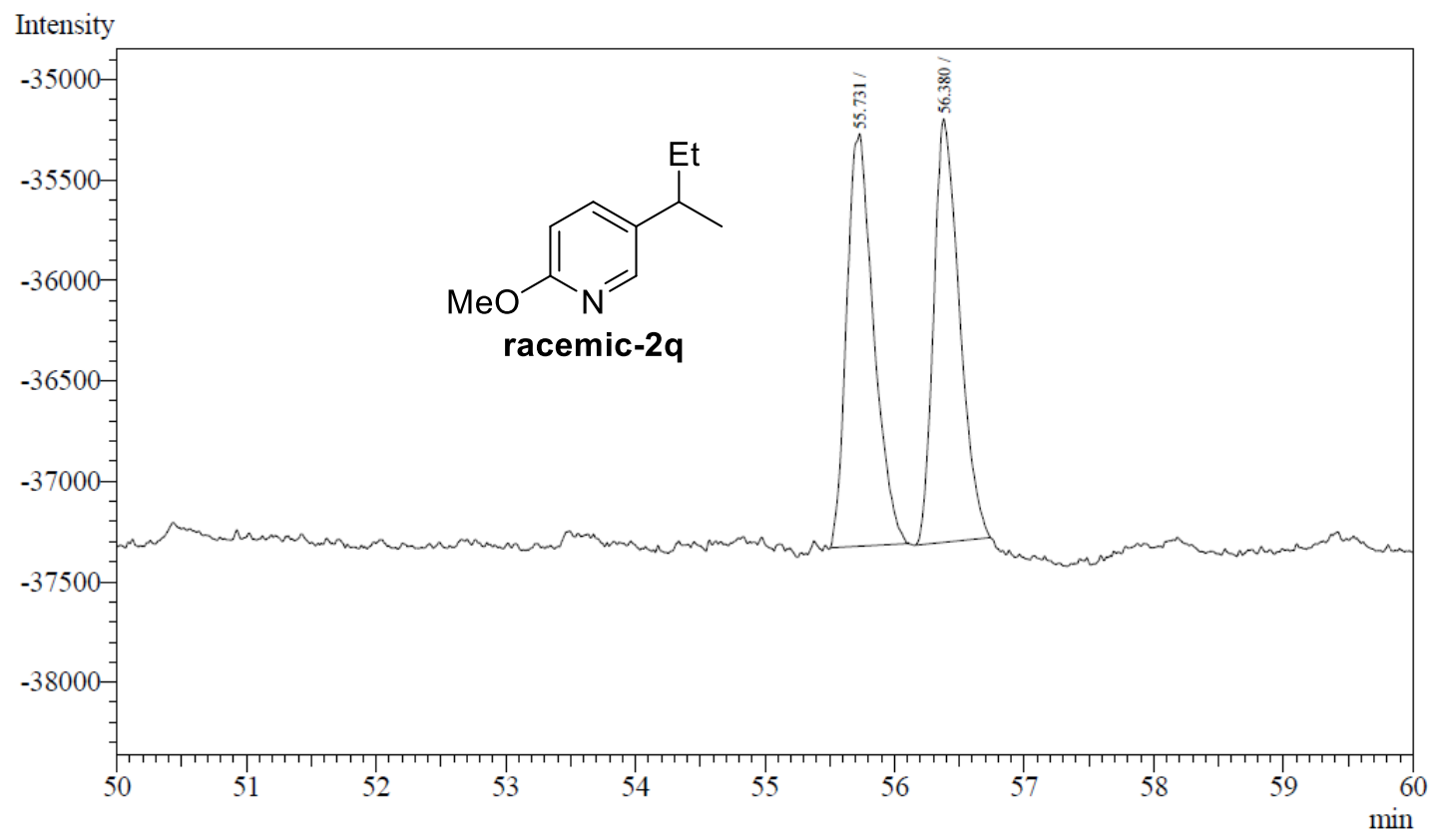

\begin{tabular}{rrrrr} 
Peak\# & Ret.Time & \multicolumn{1}{c}{ Area } & Height & \multicolumn{1}{c}{ Conc. } \\
1 & 55.731 & 29765 & 2052 & 50.450 \\
2 & 56.380 & 29234 & 2107 & 49.550 \\
\hline Total & & 58999 & 4159 &
\end{tabular}

Method Name : E: $:$ method $\backslash 2-O M e$ 吡啶.gcm

Intensity

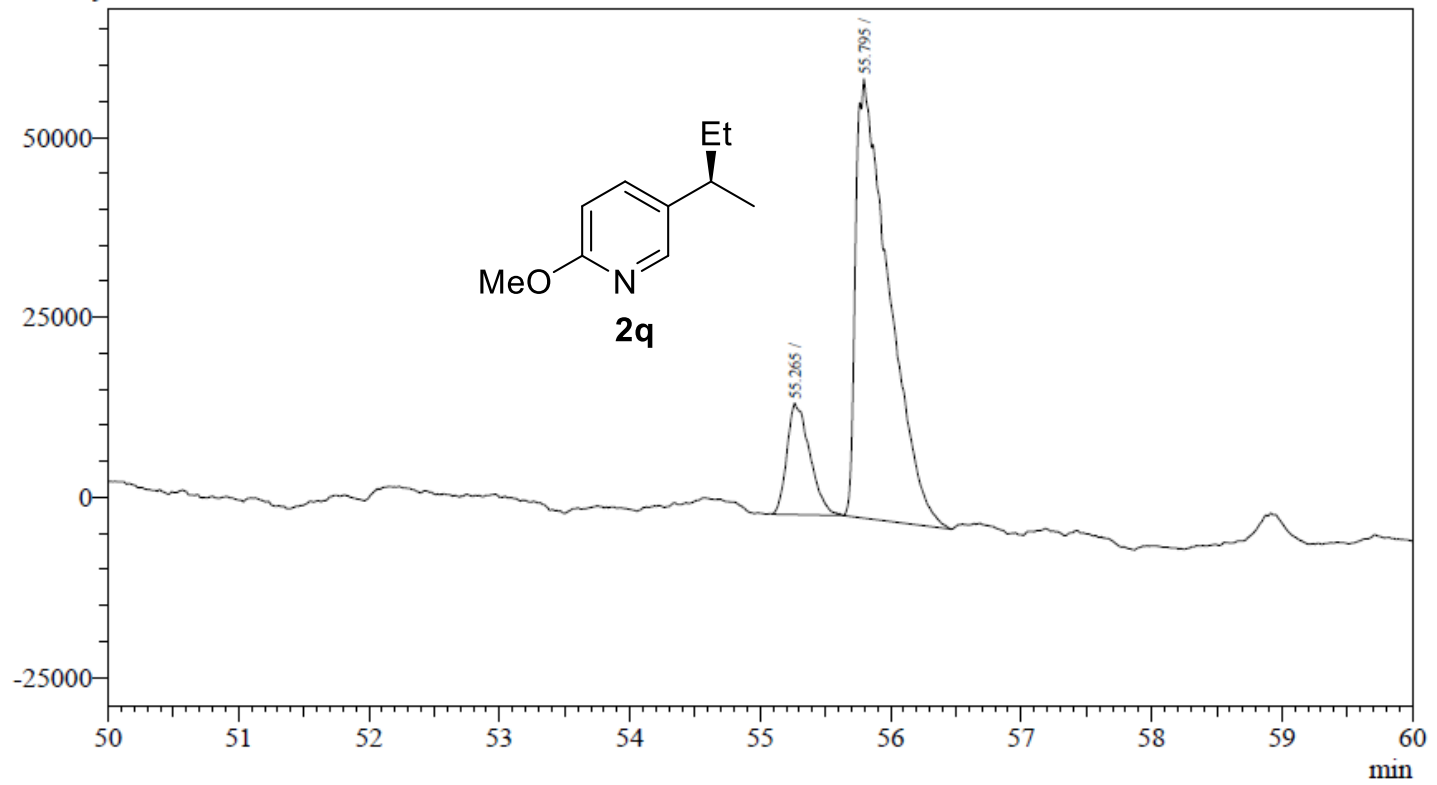

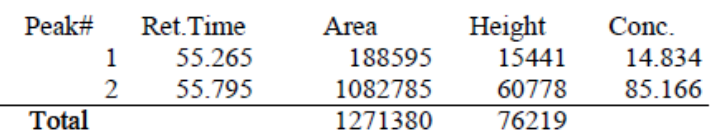


GC Conditions : Chiralcel B-DM, $100 \mathrm{kPa} \mathrm{N} 2\left(60{ }^{\circ} \mathrm{C}-30 \mathrm{~min}-2{ }^{\circ} \mathrm{C} / \mathrm{min}-180{ }^{\circ} \mathrm{C}-5\right.$ $\min )$

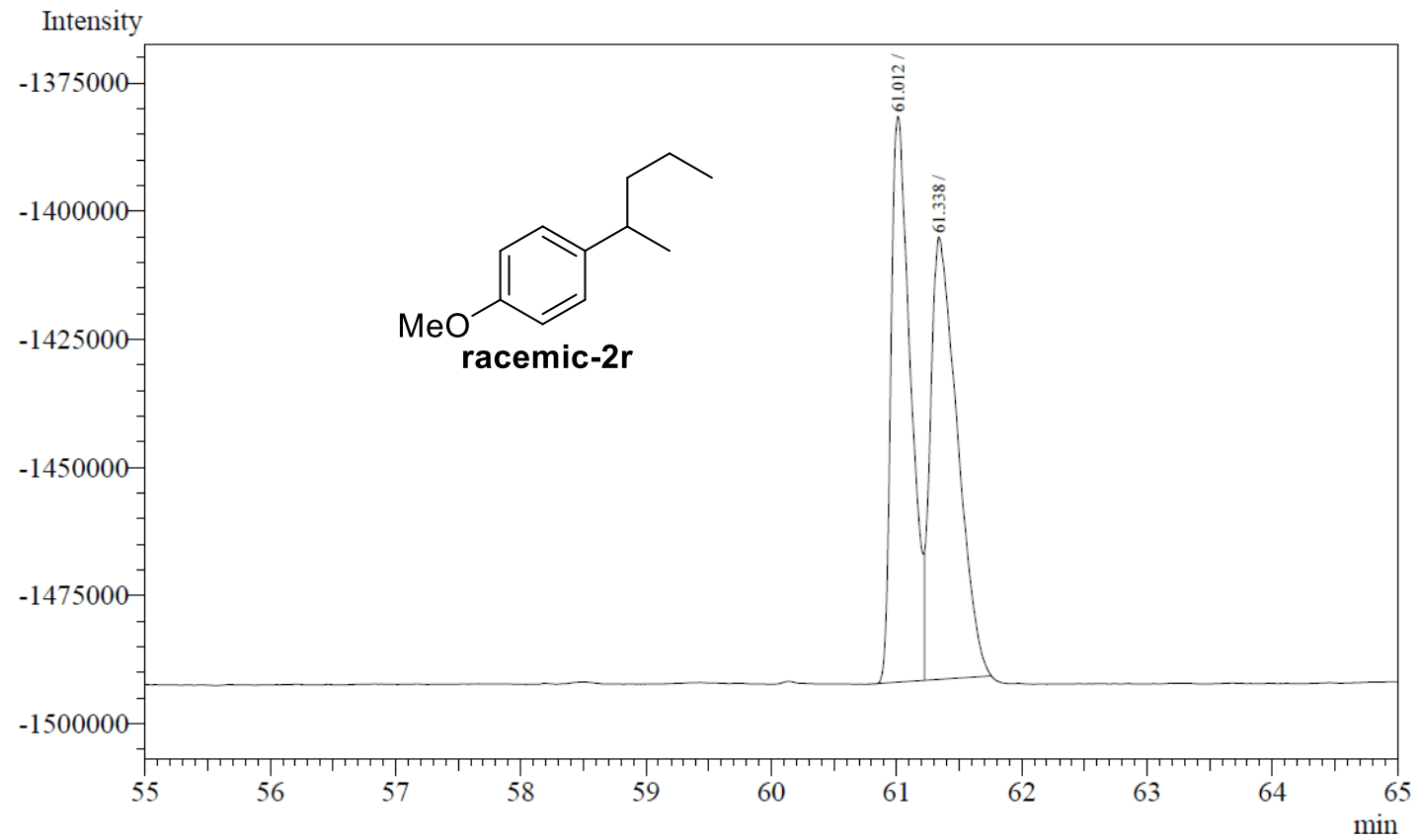

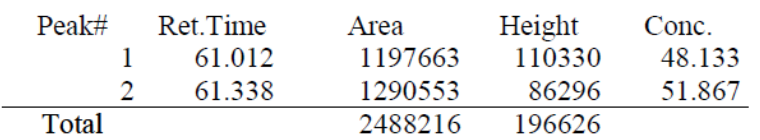

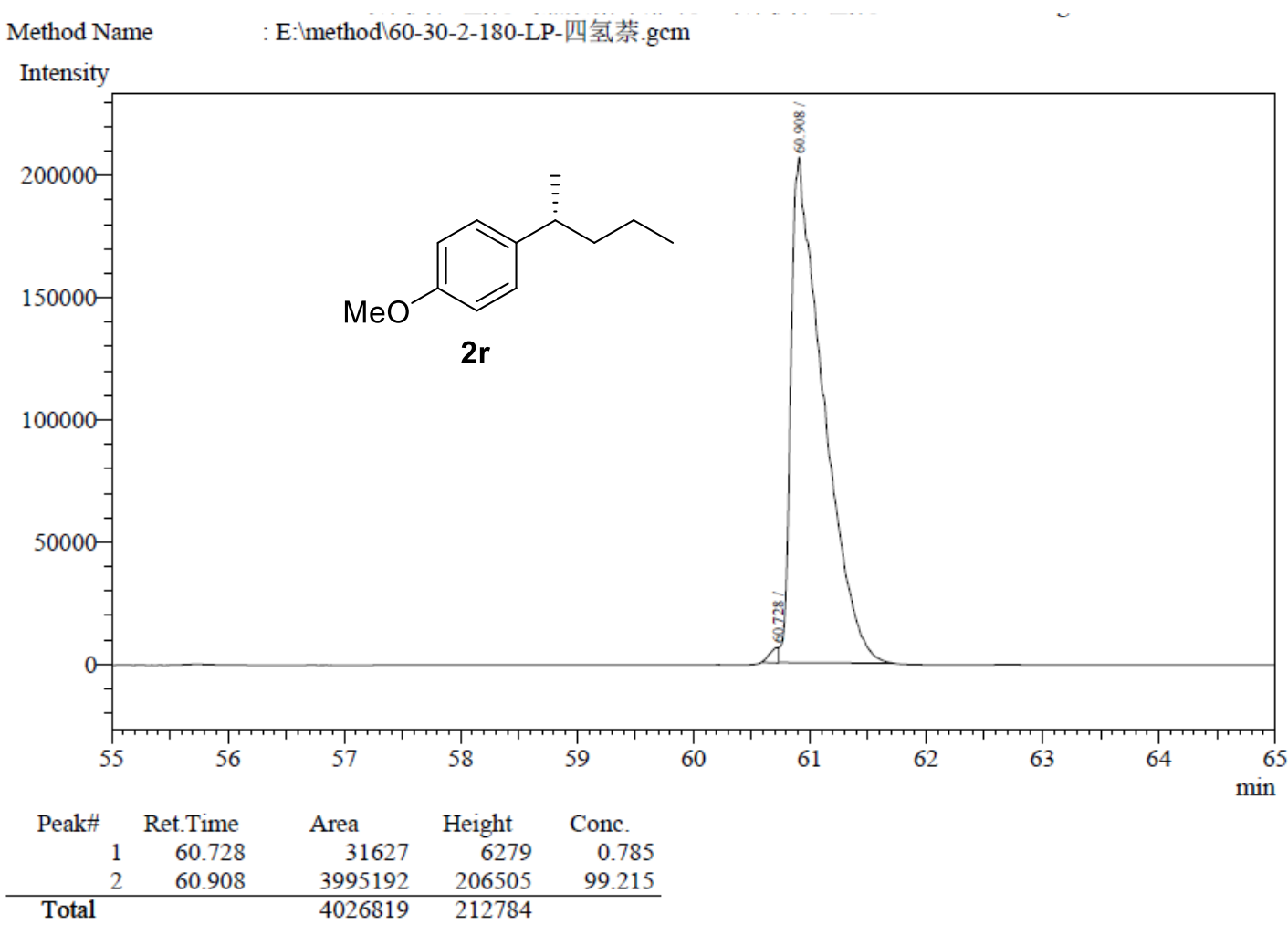


$\mathrm{mV}$

\section{色谱图}

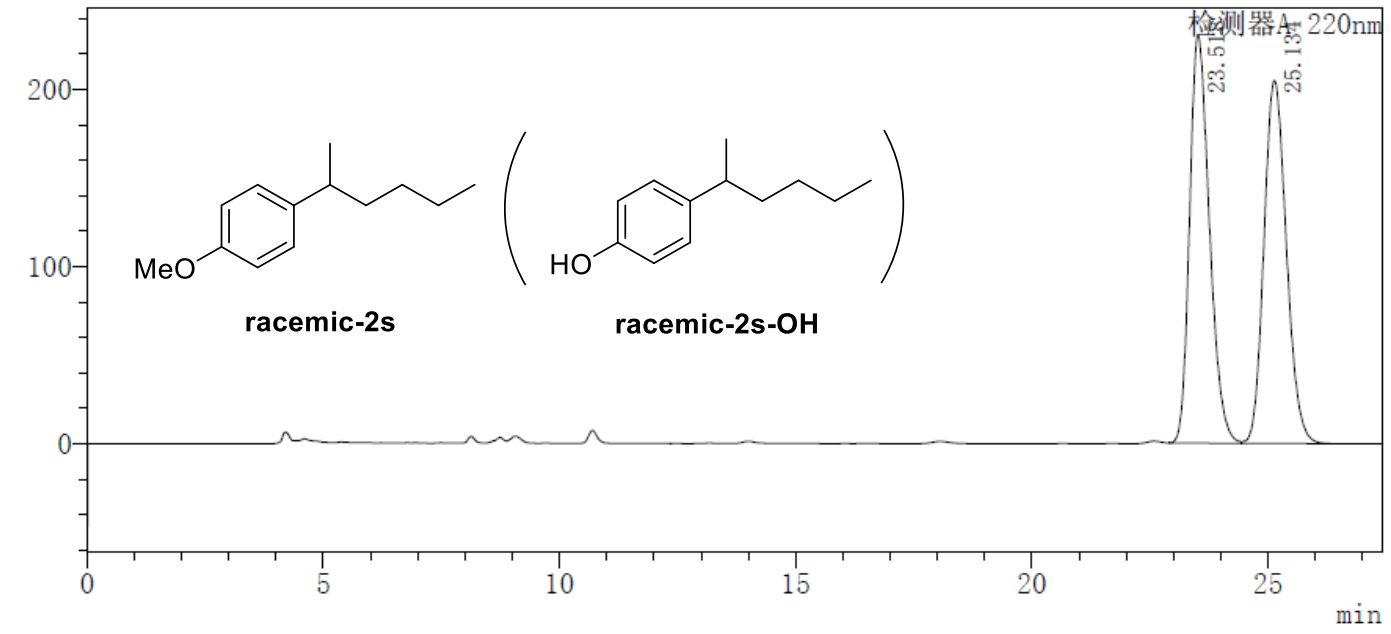

峰表

检测器A $220 \mathrm{~nm}$
\begin{tabular}{|r|r|r|r|r|r|}
\hline 峰号 & 保留时间 & \multicolumn{1}{|c|}{ 面积 } & 高度 & 标记 & \multicolumn{1}{|c|}{ 面积 $\%$} \\
\hline 1 & 23.518 & 6734132 & 230200 & & 49.896 \\
\hline 2 & 25.134 & 6762125 & 204879 & $\mathrm{~V}$ & 50.104 \\
\hline 总计 & & 13496257 & 435079 & & 100.000 \\
\hline
\end{tabular}

描述

: $0 J-H, \quad n$-hexane $/ \mathrm{iPrOH}=97 / 3,0.8 \mathrm{ml} / \mathrm{min}, 220 \mathrm{~nm}$

$\mathrm{mV}$

\section{色谱图}

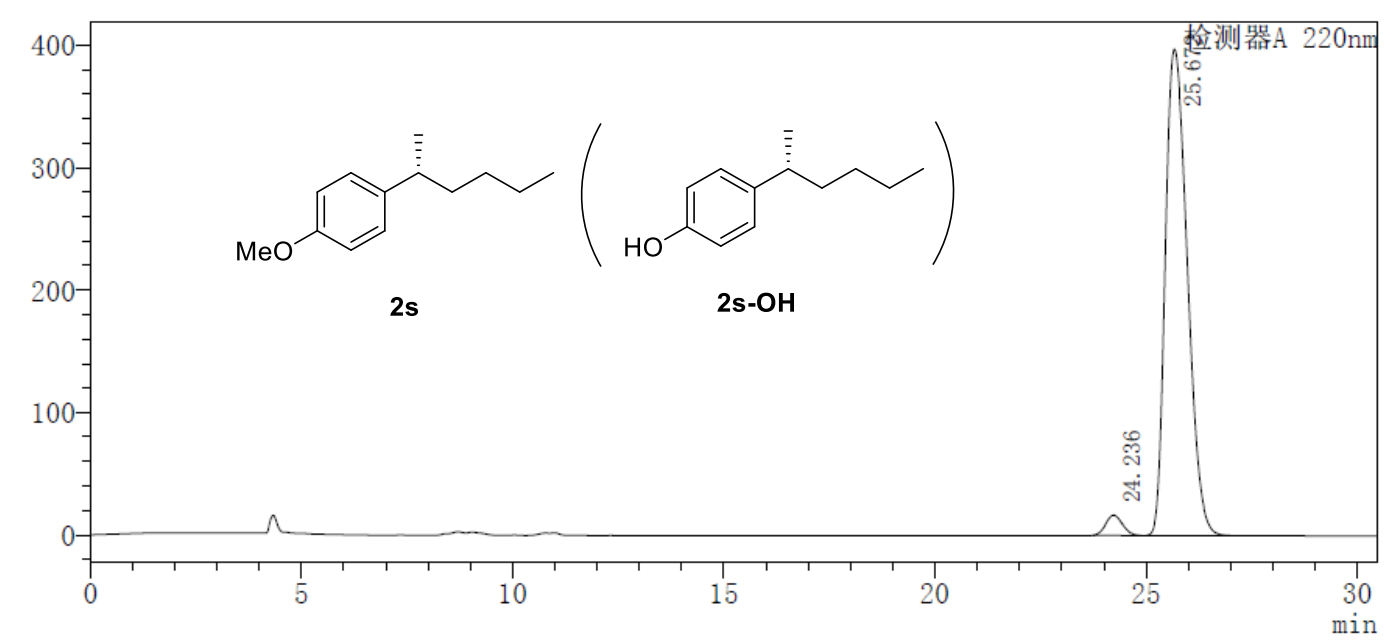

峰表

检测器A $220 \mathrm{~nm}$
\begin{tabular}{r|r|r|r|r|r|}
\hline 峰号 & 保留时间 & 面积 & 高度 & 标记 & \multicolumn{1}{|c|}{ 面积 $\%$} \\
\hline 1 & 24.236 & 452652 & 16549 & $\mathrm{M}$ & 3.051 \\
\hline 2 & 25.672 & 14384670 & 397490 & & 96.949 \\
\hline 总计 & & 14837322 & 414038 & & 100.000 \\
\hline
\end{tabular}


GC Conditions : Chiralcel B-DM, $100 \mathrm{kPa} \mathrm{N} 2\left(80{ }^{\circ} \mathrm{C}-00 \mathrm{~min}-1{ }^{\circ} \mathrm{C} / \mathrm{min}-150{ }^{\circ} \mathrm{C}-5\right.$ $\min$ )

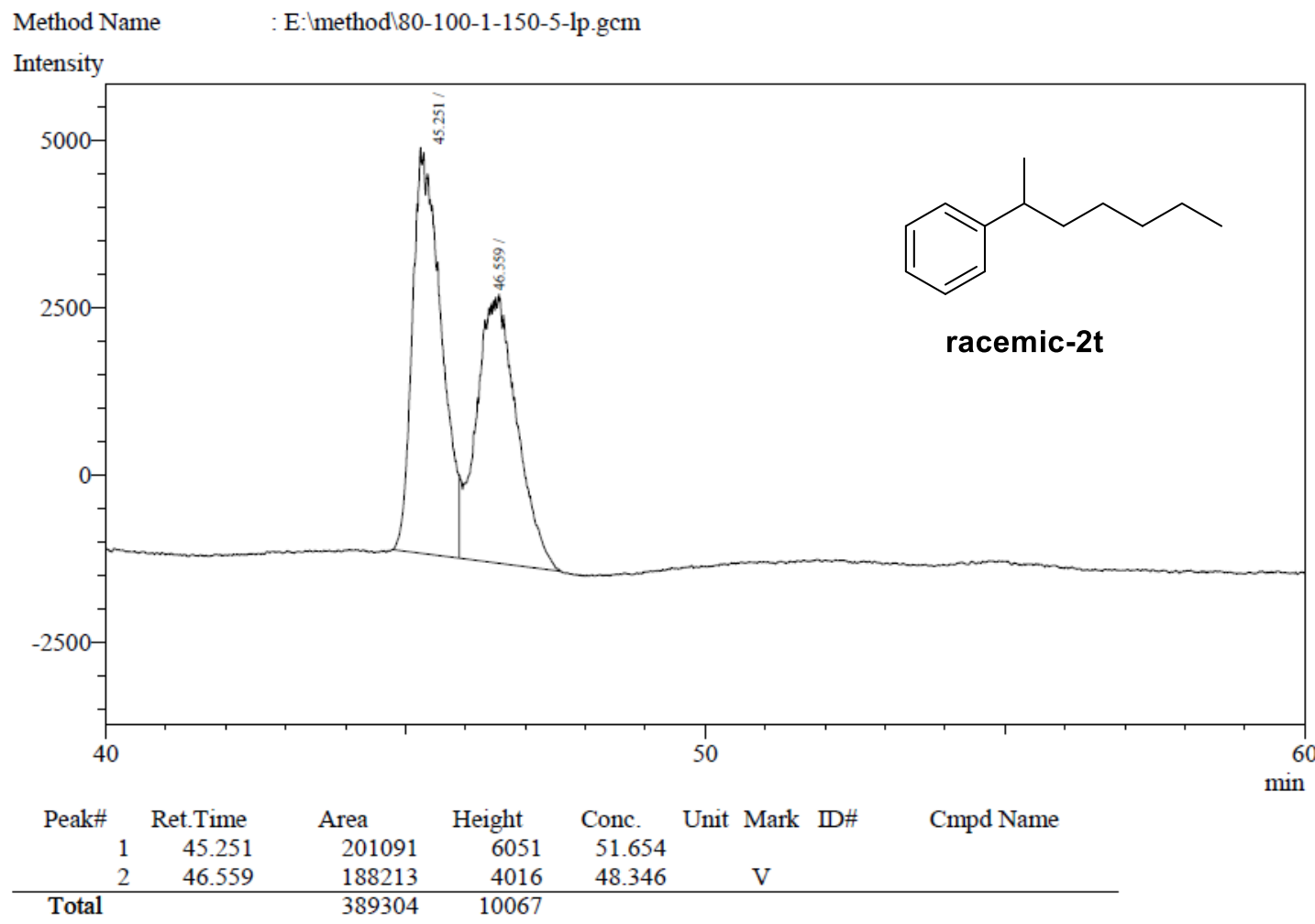

Method Name : E:Imethod $180-100-1-150-5-1 \mathrm{p} . \mathrm{gcm}$

Intensity

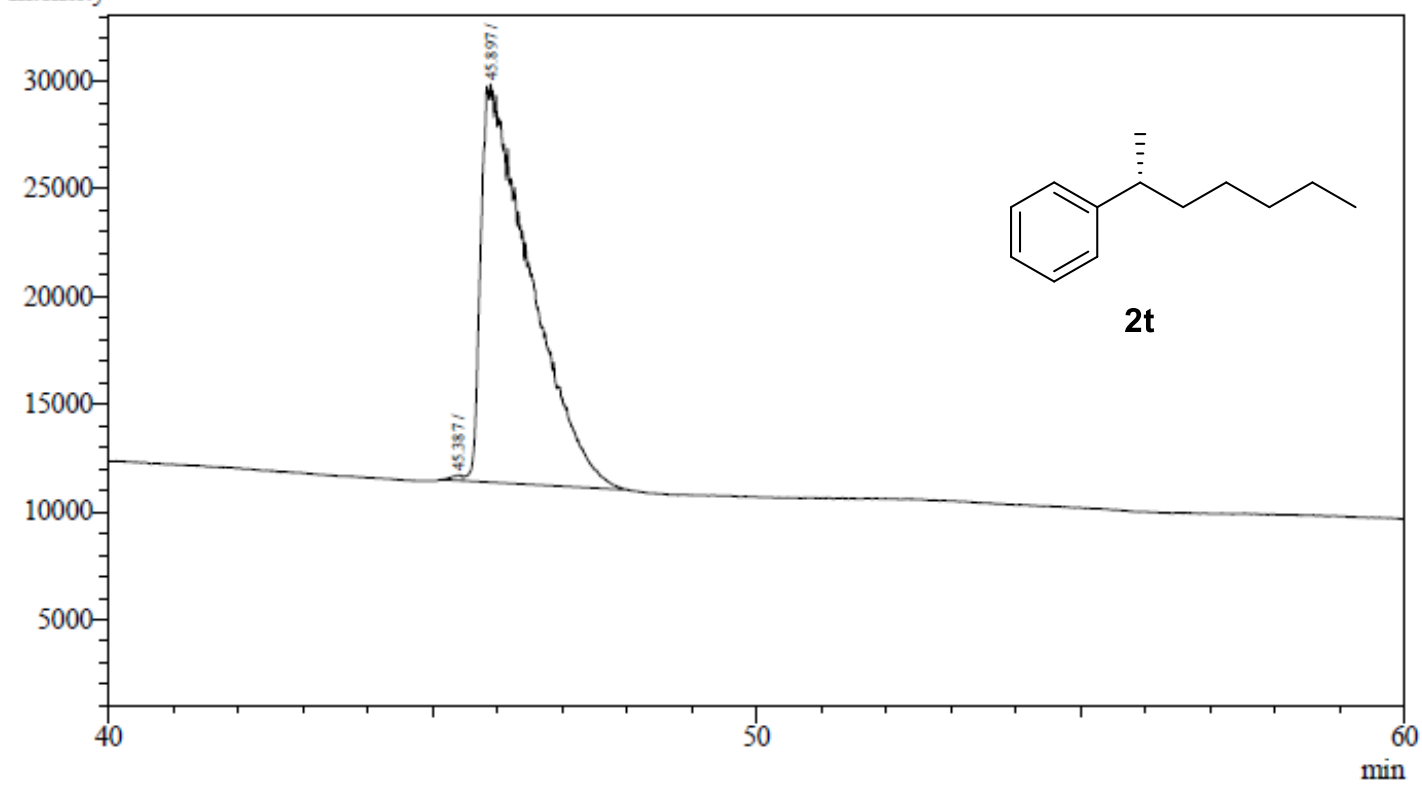

\begin{tabular}{|c|c|c|c|c|}
\hline Peak\# & $\begin{array}{r}\text { Ret.Time } \\
45.387\end{array}$ & $\begin{array}{l}\text { Area } \\
2736\end{array}$ & $\begin{array}{l}\text { Height } \\
242\end{array}$ & $\begin{array}{l}\text { Conc. } \\
0.278\end{array}$ \\
\hline 2 & 45.897 & 980984 & 18456 & 99.722 \\
\hline
\end{tabular}


$\mathrm{mV}$

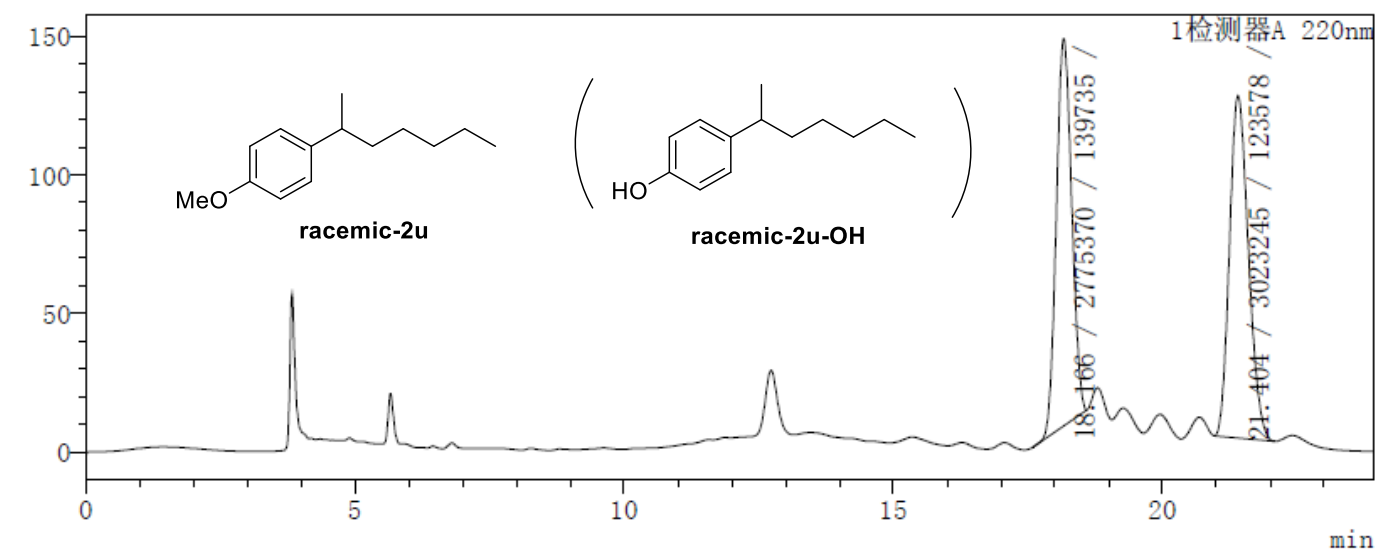

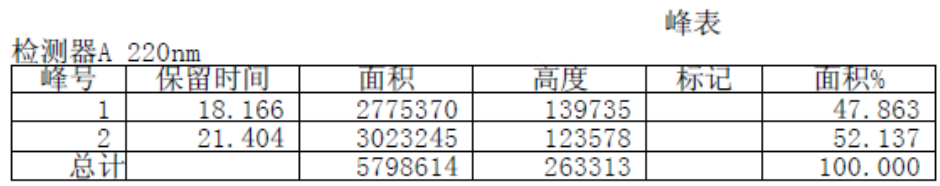

描述

: OJ-H, n-hexane $/$ iPrOH $=97 / 3,0.8 \mathrm{ml} / \mathrm{min}, 220 \mathrm{~nm}$

$\mathrm{mV}$

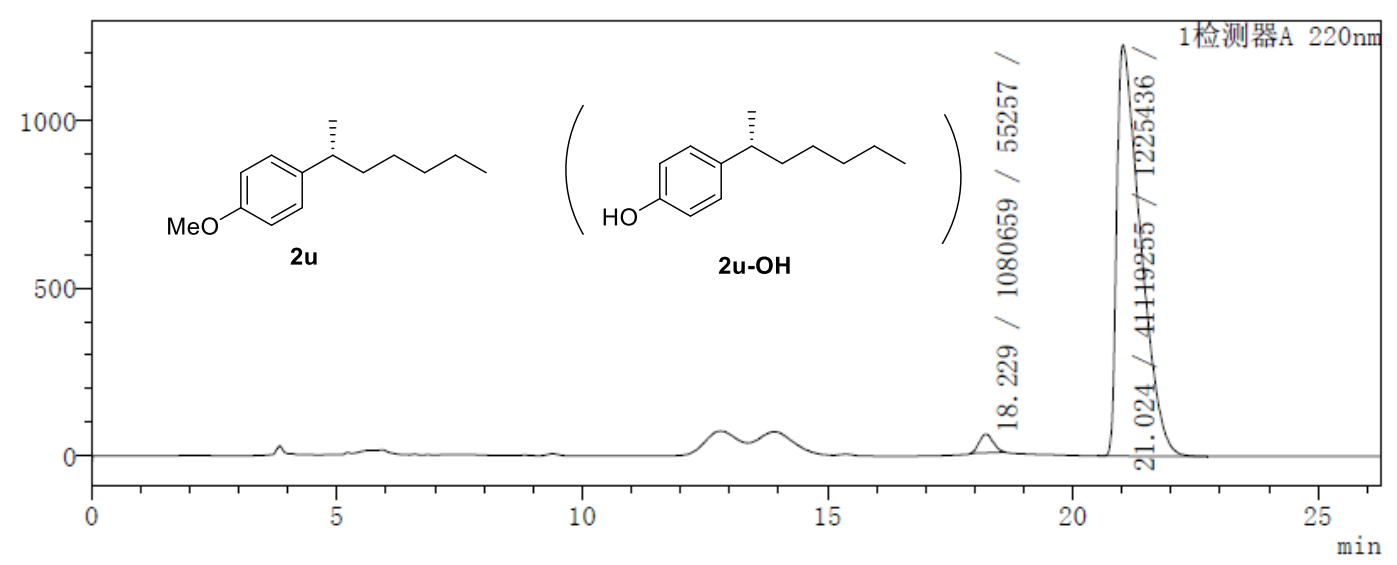

峰表

检测器A $220 \mathrm{~nm}$
\begin{tabular}{|r|r|r|r|r|r|}
\hline 峰号 & 保留时间 & \multicolumn{1}{|c|}{ 面积 } & \multicolumn{1}{c|}{ 高度 } & 标记 & \multicolumn{1}{|c|}{ 面积\% } \\
\hline 1 & 18.229 & 1080659 & 55257 & $\mathrm{M}$ & 2.561 \\
\hline 2 & 21.024 & 41119255 & 1225436 & $\mathrm{M}$ & 97.439 \\
\hline 总计 & & 42199915 & 1280693 & & 100.000 \\
\hline
\end{tabular}


$\mathrm{mV}$

\section{色谱图}

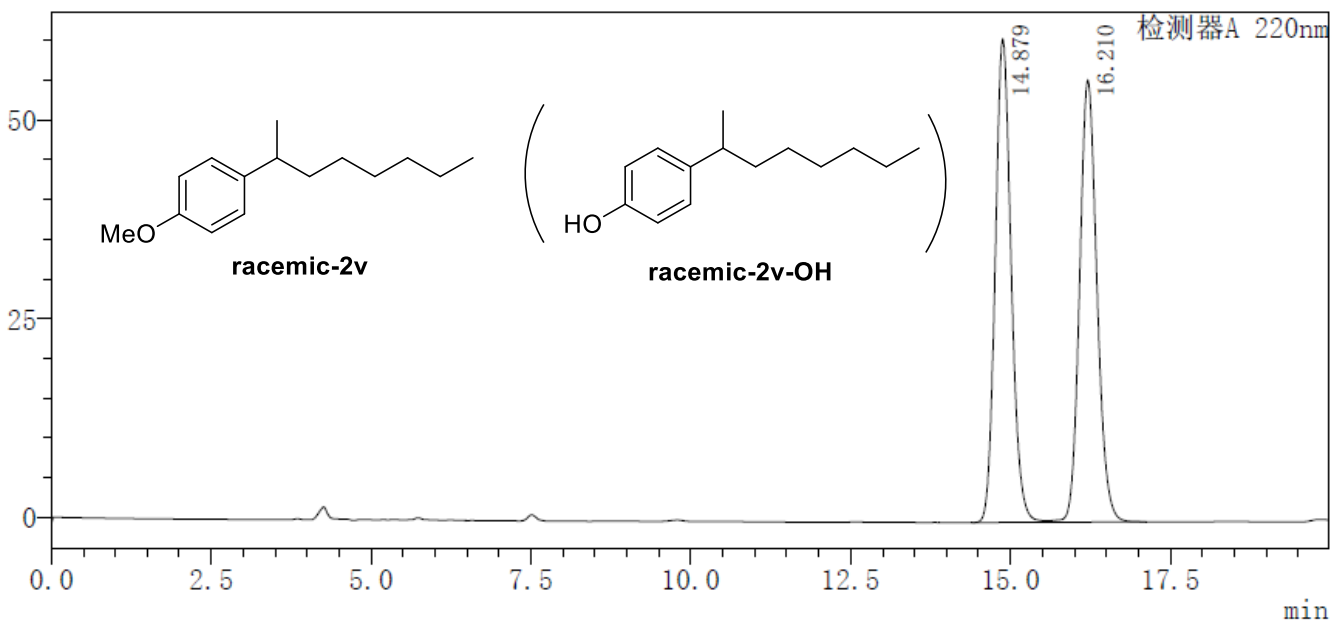

峰表

检测器A $220 \mathrm{~nm}$
\begin{tabular}{r|r|c|r|r|r|}
\hline 峰号 & 保留时间 & 面积 & 高度 & 标记 & \multicolumn{1}{|c|}{ 面积 $\%$} \\
\hline 1 & 14.879 & 1054220 & 60773 & & 49.992 \\
\hline 2 & 16.210 & 1054537 & 55571 & V & 50.008 \\
\hline 总计 & & 2108757 & 116344 & & 100.000 \\
\hline
\end{tabular}

描述

: $0 J-H, n$-hexane $/ \mathrm{iPrOH}=97 / 3,0.8 \mathrm{ml} / \mathrm{min}, 220 \mathrm{~nm}$

$\mathrm{mV}$

\section{色谱图}

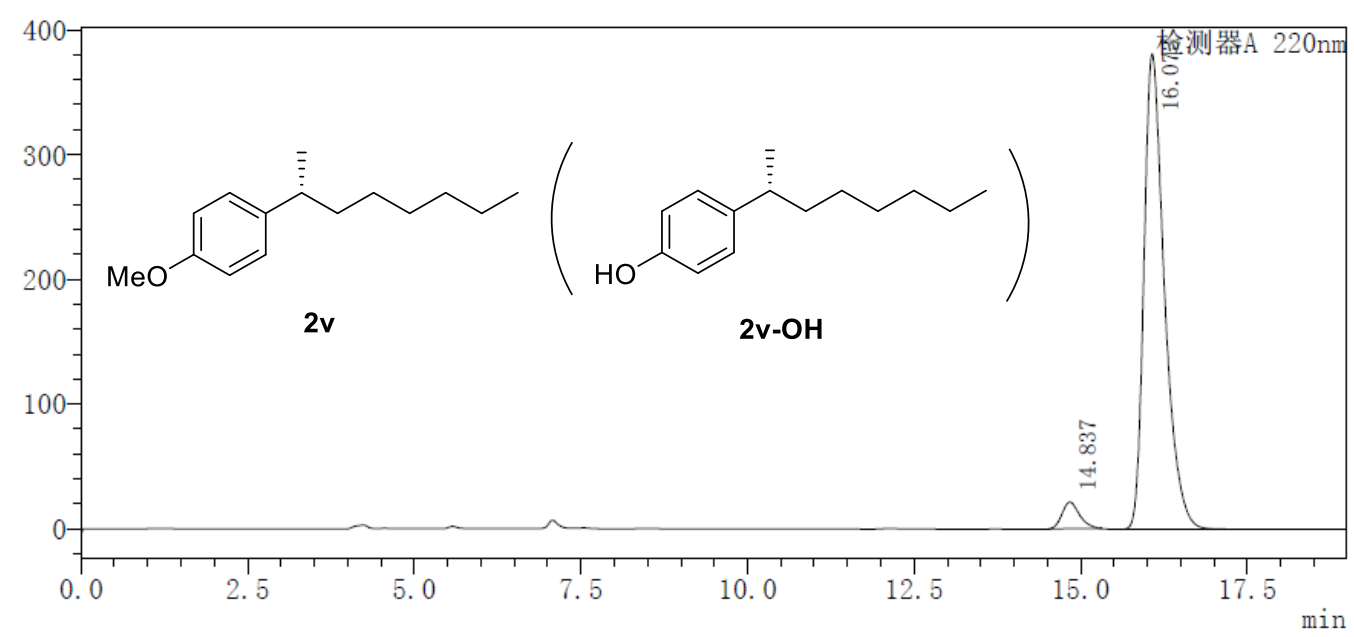

峰表

检测器A $220 \mathrm{~nm}$
\begin{tabular}{|r|r|r|r|r|r|}
\hline 峰号 & 保留时间 & \multicolumn{1}{|c|}{ 面积 } & \multicolumn{1}{c|}{ 高度 } & 标记 & \multicolumn{1}{|c|}{ 面积 $\%$} \\
\hline 1 & 14.837 & 392507 & 21409 & $\mathrm{M}$ & 4.601 \\
\hline 2 & 16.073 & 8138493 & 380702 & & 95.399 \\
\hline 总计 & & 8530999 & 402111 & & 100.000 \\
\hline
\end{tabular}


$\mathrm{mV}$

\section{色谱图}

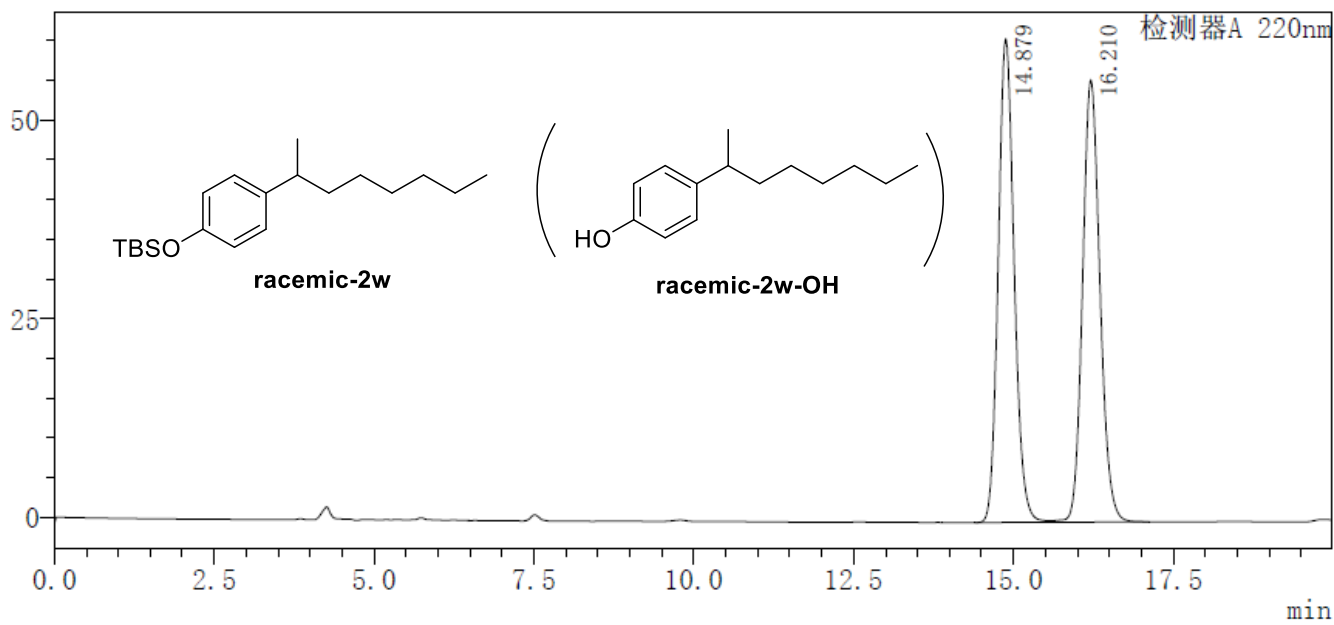

峰表

检测器A $220 \mathrm{~nm}$
\begin{tabular}{r|r|c|r|r|r|}
\hline 峰号 & 保留时间 & 面积 & 高度 & 标记 & \multicolumn{1}{|c|}{ 面积 $\%$} \\
\hline 1 & 14.879 & 1054220 & 60773 & & 49.992 \\
\hline 2 & 16.210 & 1054537 & 55571 & V & 50.008 \\
\hline 总计 & & 2108757 & 116344 & & 100.000 \\
\hline
\end{tabular}

描述 : OD-H, n-hexane $/ \mathrm{iPrOH}=97 / 3,0.8 \mathrm{ml} / \mathrm{min}, 220 \mathrm{~nm}$

$\mathrm{mV}$

色谱图

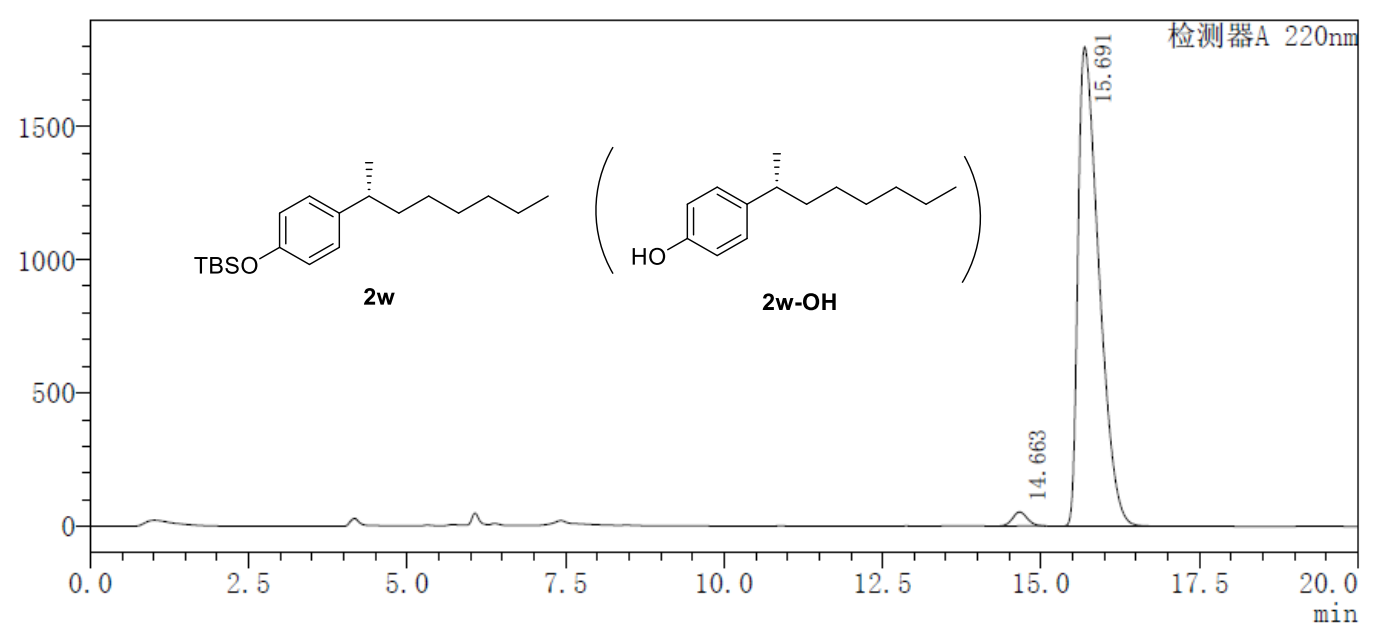

峰表

检测器A $220 \mathrm{~nm}$
\begin{tabular}{|r|r|r|r|r|r|}
\hline 峰号 & 保留时间 & \multicolumn{1}{|c|}{ 面积 } & \multicolumn{1}{c|}{ 高度 } & 标记 & \multicolumn{1}{|c|}{ 面积 $\%$} \\
\hline 1 & 14.663 & 856955 & 52515 & M & 2.034 \\
\hline 2 & 15.691 & 41268501 & 1797822 & M & 97.966 \\
\hline 总计 & & 42125456 & 1850337 & & 100.000 \\
\hline
\end{tabular}


GC Conditions : Chiralcel B-DM, $100 \mathrm{kPa} \mathrm{N} 2\left(60{ }^{\circ} \mathrm{C}-30 \mathrm{~min}-1{ }^{\circ} \mathrm{C} / \mathrm{min}-130{ }^{\circ} \mathrm{C}-5\right.$ min)

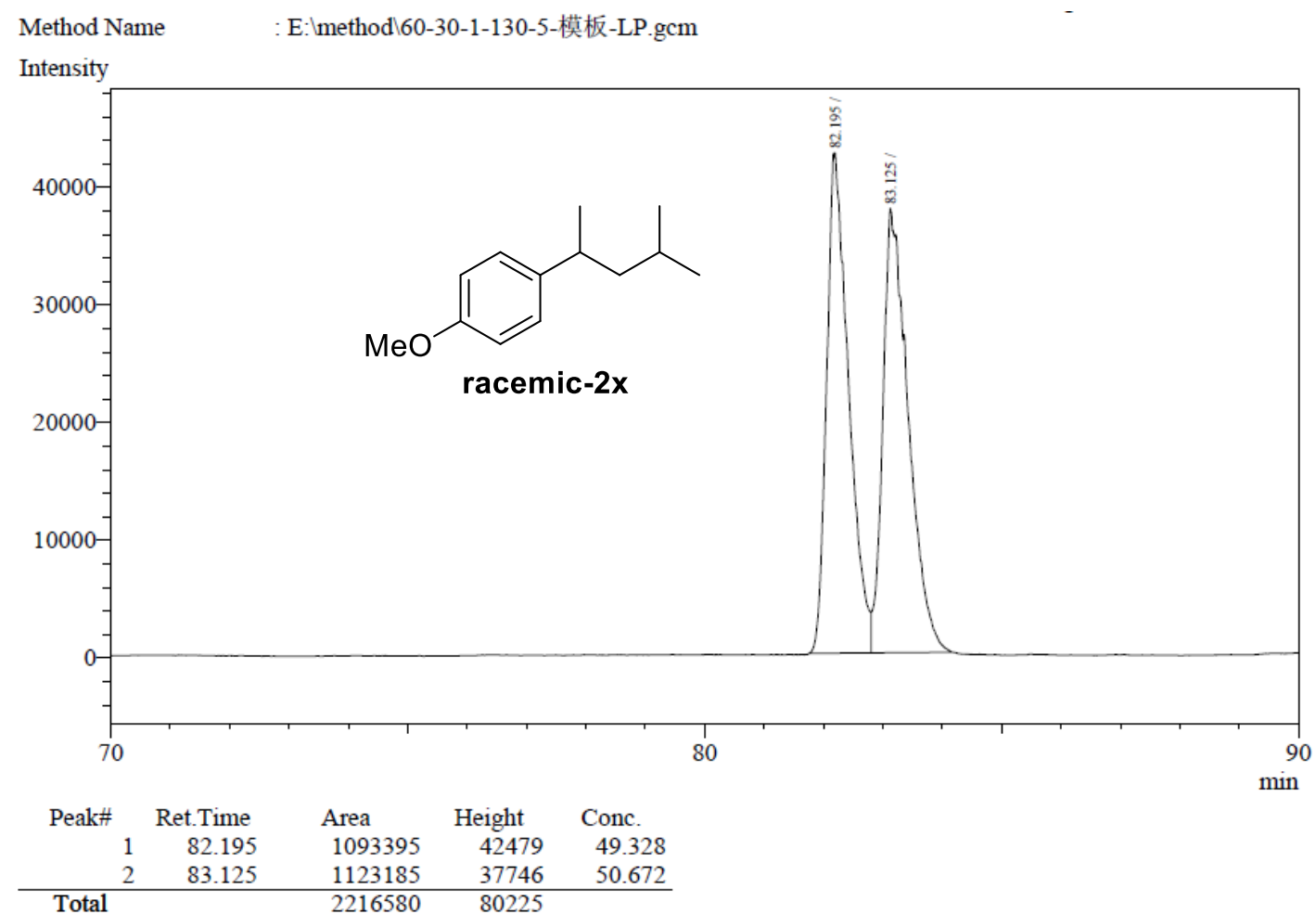

Method Name : E: $\quad$ method $60-30-1-130-5-$ 模板-LP.gcm

Intensity

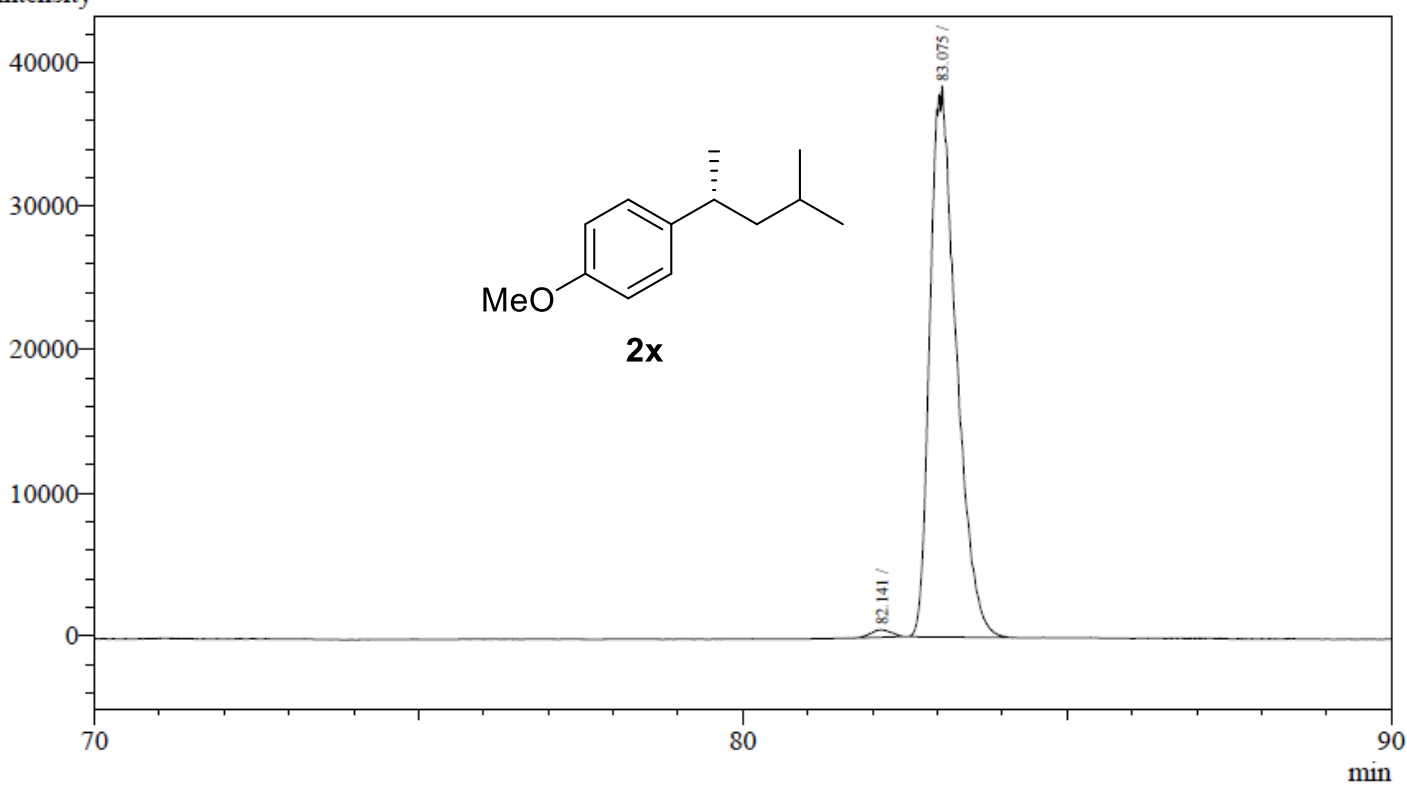

\begin{tabular}{|c|c|c|c|c|}
\hline Peak\# & Ret.Time & Area & Height & Conc. \\
\hline 1 & 82.141 & 11675 & 523 & 1.063 \\
\hline 2 & 83.075 & 1086283 & 38454 & 98.937 \\
\hline Total & & 1097958 & 38977 & \\
\hline
\end{tabular}


$\mathrm{mV}$

\section{色谱图}

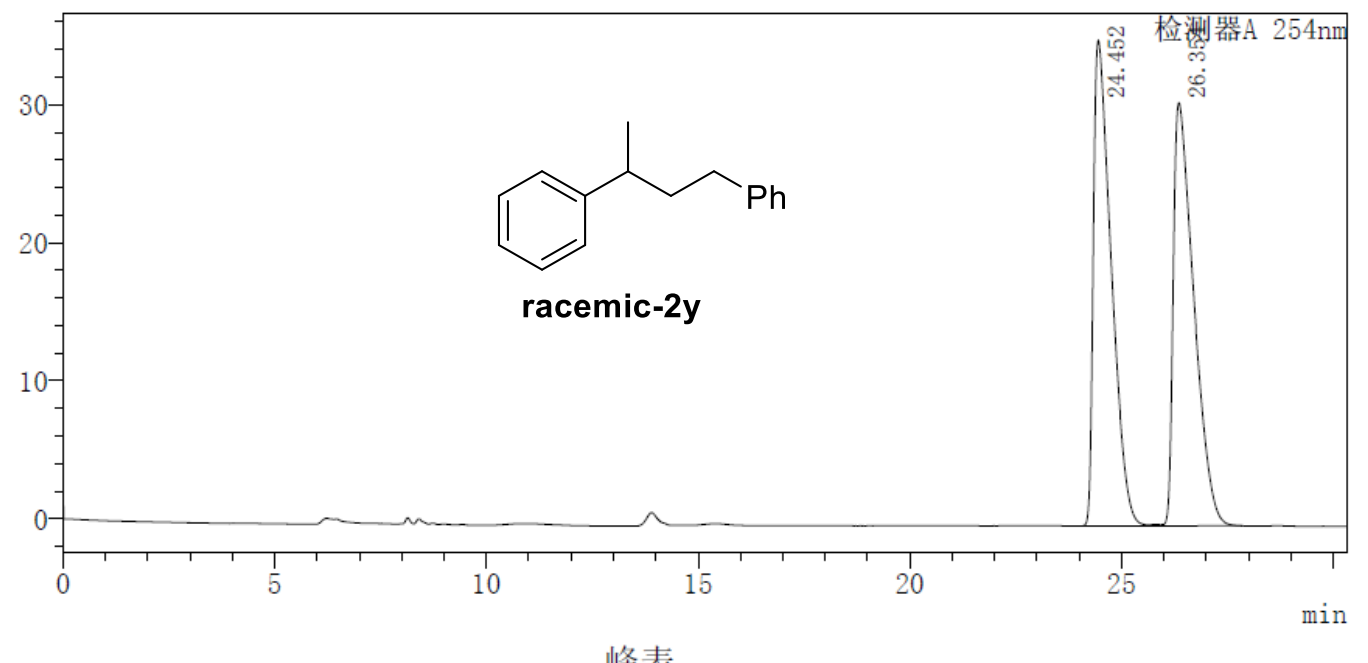

检测器A $254 \mathrm{~nm}$
\begin{tabular}{|r|r|l|l|l|r|}
\hline 峰号 & 保留时间 & 面积 & 高度 & 标记 & 面积 $\%$ \\
\hline 1 & 24.452 & 1028752 & 35174 & $\mathrm{~S}$ & 50.014 \\
\hline 2 & 26.354 & 1028174 & 30662 & $\mathrm{~V}$ & 49.986 \\
\hline 总计 & & 2056927 & 65836 & & 100.000 \\
\hline
\end{tabular}

描述

: $0 J-H X 2, \mathrm{n}$-hexane $/ \mathrm{i} \operatorname{PrOH}=99.5 / 0.5,1.0 \mathrm{ml} / \mathrm{min}, 254 \mathrm{~nm}$

$\mathrm{mV}$

色谱图

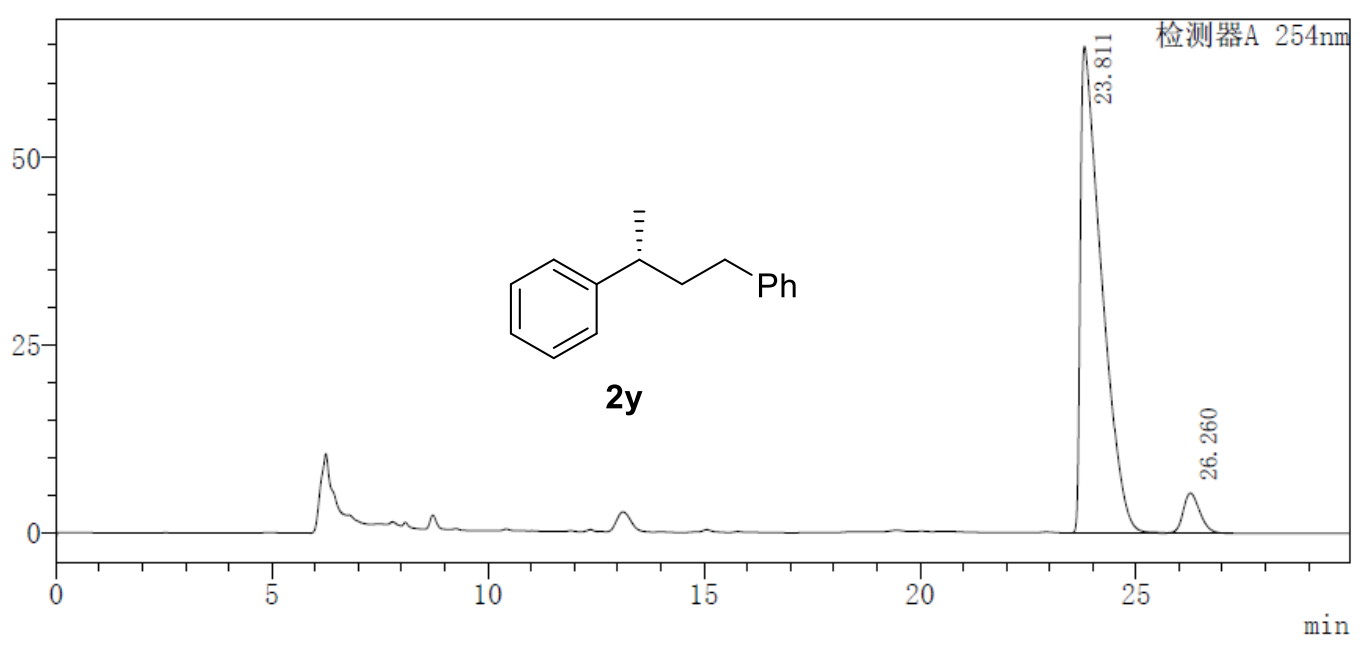

峰表

检测器A $254 \mathrm{~nm}$
\begin{tabular}{|r|r|r|r|r|r|}
\hline 峰号 & 保留时间 & \multicolumn{1}{|c|}{ 面积 } & \multicolumn{1}{c|}{ 高度 } & 标记 & \multicolumn{1}{|c|}{ 面积 $\%$} \\
\hline 1 & 23.811 & 2207438 & 64855 & & 93.927 \\
\hline 2 & 26.260 & 142729 & 5341 & V & 6.073 \\
\hline 总计 & & 2350167 & 70196 & & 100.000 \\
\hline
\end{tabular}


$\mathrm{mV}$

色谱图

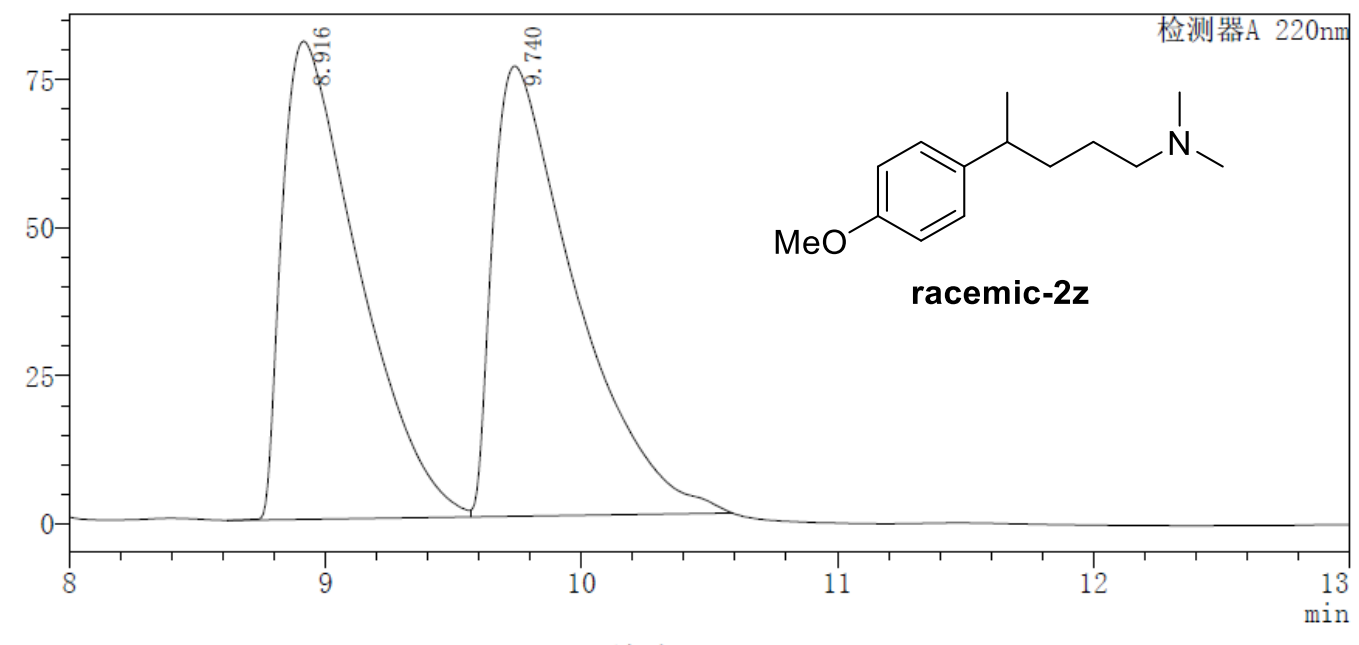

峰表

检测器A $220 \mathrm{~nm}$
\begin{tabular}{|r|r|r|r|r|r|}
\hline 峰号 & 保留时间 & 面积 & \multicolumn{1}{c|}{ 高度 } & 标记 & \multicolumn{1}{|c|}{ 面积 $\%$} \\
\hline 1 & 8.916 & 1675423 & 80765 & & 49.430 \\
\hline 2 & 9.740 & 1714031 & 76055 & V M & 50.570 \\
\hline 总计 & & 3389454 & 156819 & & 100.000 \\
\hline
\end{tabular}

描述 : OJ-HX2, n-hexane $/ \mathrm{iPrOH}=95 / 5,1.0 \mathrm{ml} / \mathrm{min}, 220 \mathrm{~nm}$

$\mathrm{mV}$

色谱图

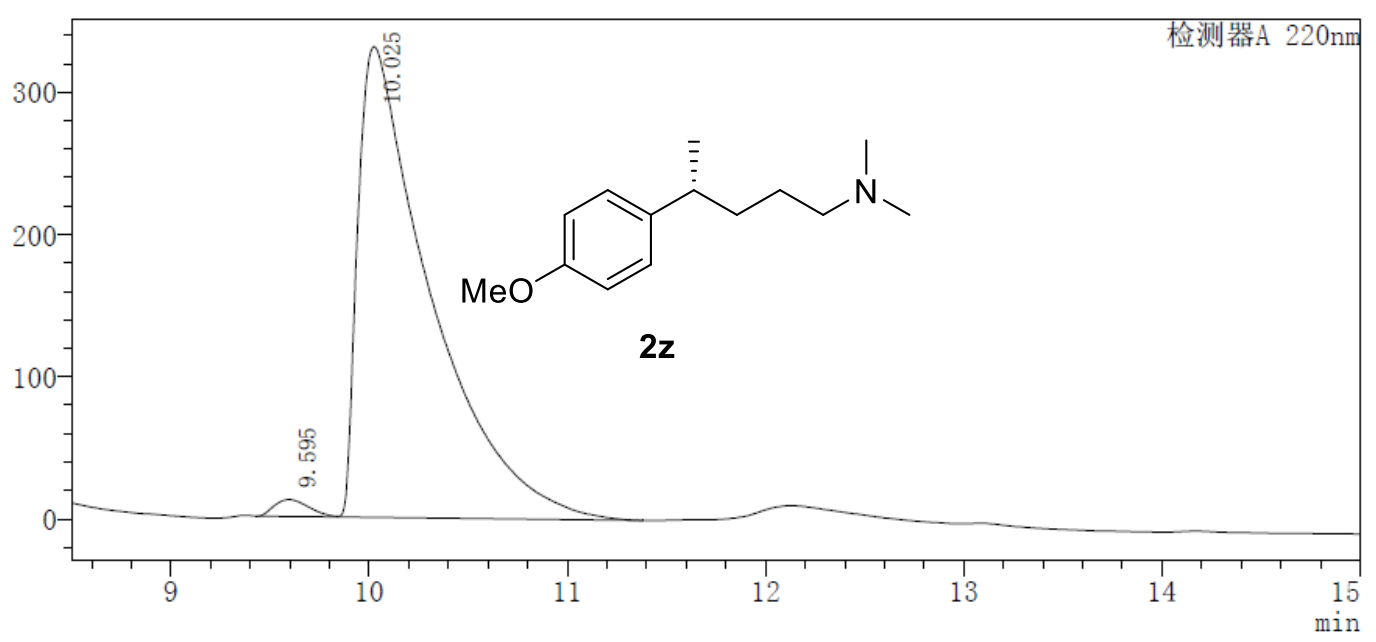

峰表

检测器A $220 \mathrm{~nm}$
\begin{tabular}{|r|r|r|r|r|r|}
\hline 峰号 & 保留时间 & \multicolumn{1}{|c|}{ 面积 } & 高度 & 标记 & \multicolumn{1}{|c|}{ 面积 $\%$} \\
\hline 1 & 9.595 & 144672 & 11898 & & 1.684 \\
\hline 2 & 10.025 & 8444867 & 331140 & $\mathrm{~V}$ & 98.316 \\
\hline 总计 & & 8589539 & 343039 & & 100.000 \\
\hline
\end{tabular}


$\mathrm{mV}$

\section{色谱图}

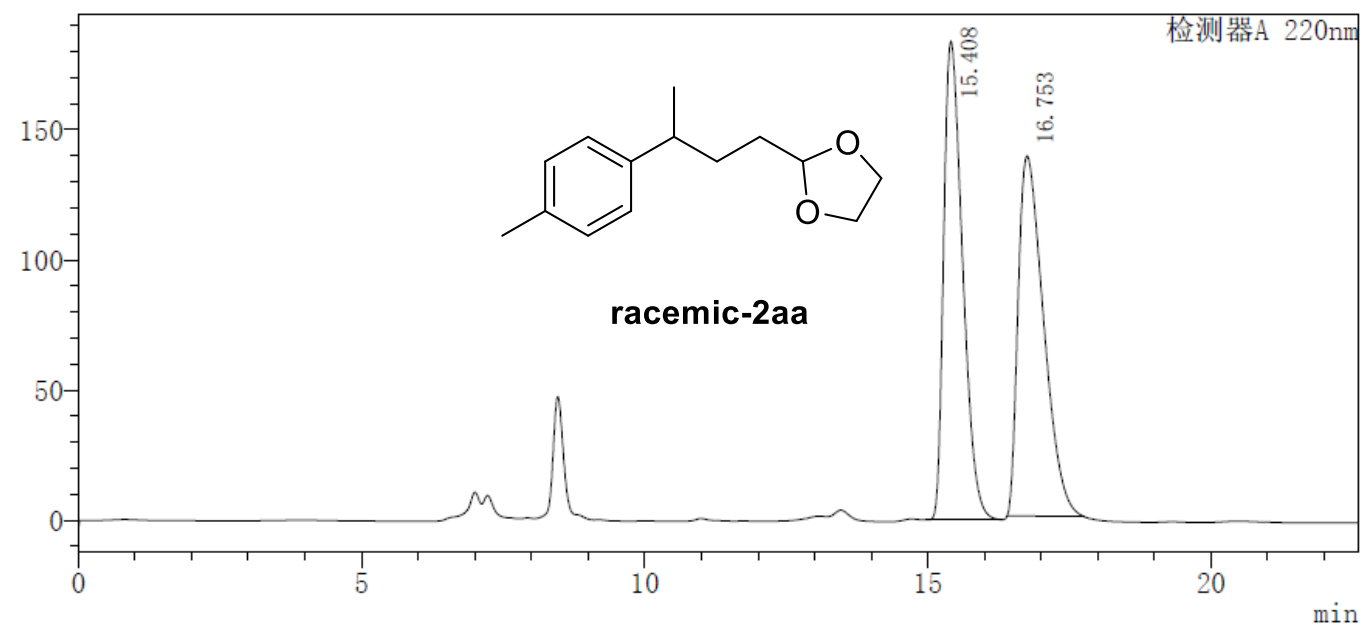

峰表

\begin{tabular}{|c|c|c|c|c|c|}
\hline 峰㕺 & 保留时间 & 面积 & 高度 & 标记 & 面积\% \\
\hline 1 & 15.408 & 4255641 & 183771 & & 49.636 \\
\hline 2 & 16.753 & 4318030 & 138225 & M & 50.364 \\
\hline 总计 & & 8573671 & 321996 & & 100.000 \\
\hline
\end{tabular}

描述 $\cdots$ AD-H, n-hexane/iPrOH $=99.5 / 0.5,0.5 \mathrm{ml} / \mathrm{min}, 220 \mathrm{~nm}$

$\mathrm{mV}$

色谱图

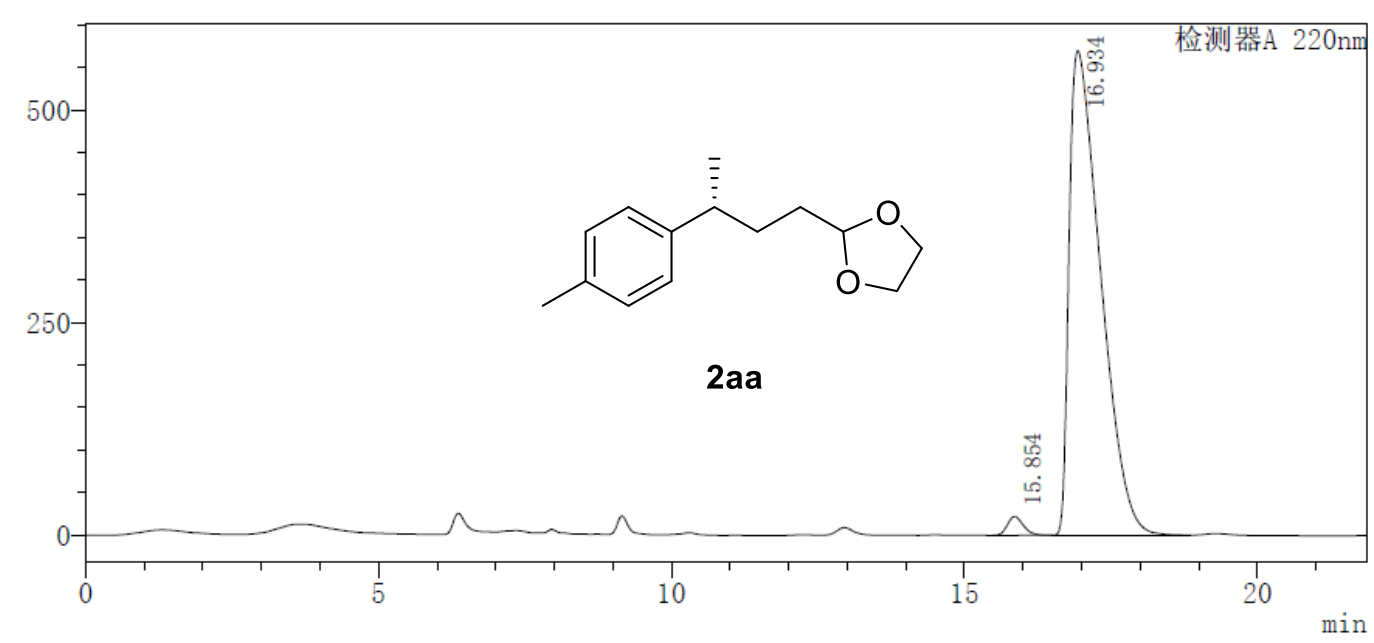

峰表

检测器A $220 \mathrm{~nm}$
\begin{tabular}{|r|r|r|r|r|r|}
\hline 峰号 & 保留时间 & \multicolumn{1}{|c|}{ 面积 } & \multicolumn{1}{c|}{ 高度 } & 标记 & \multicolumn{1}{|c|}{ 面积 $\%$} \\
\hline 1 & 15.854 & 419390 & 22039 & & 1.936 \\
\hline 2 & 16.934 & 21247579 & 569537 & V & 98.064 \\
\hline 总计 & & 21666969 & 591576 & & 100.000 \\
\hline
\end{tabular}


$\mathrm{mV}$

色谱图

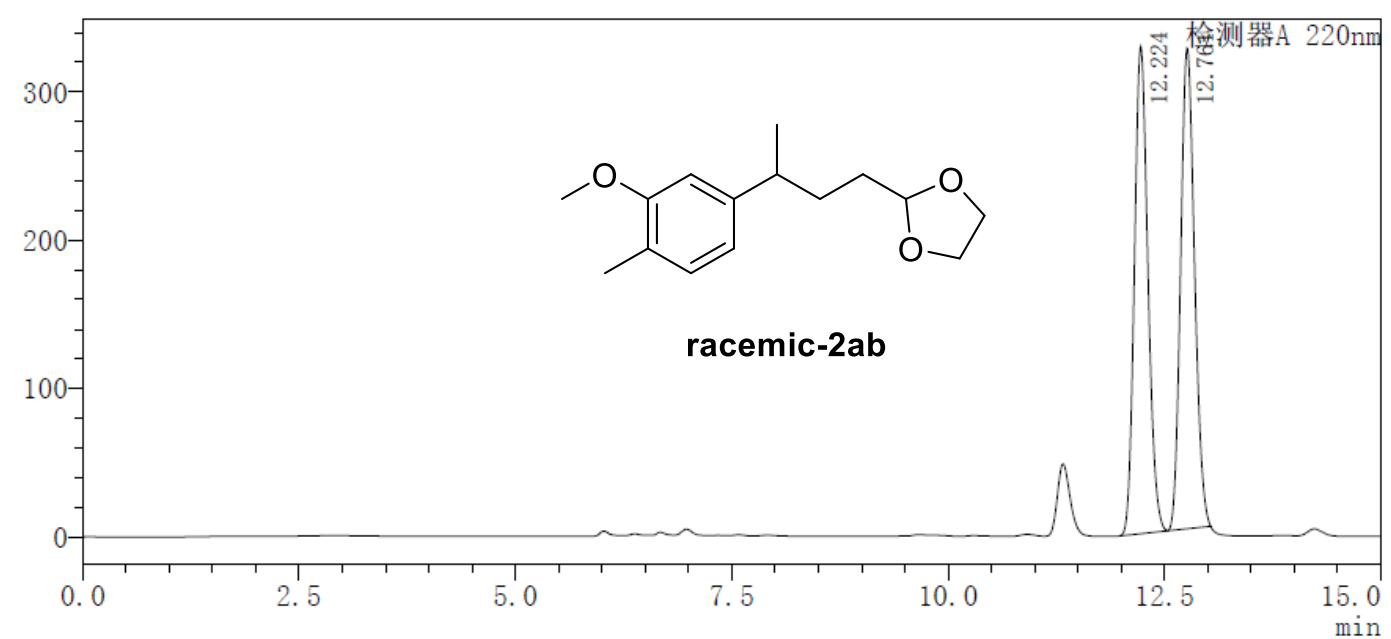

峰表

检测器A $220 \mathrm{~nm}$
\begin{tabular}{|r|r|r|r|r|r|}
\hline 峰号 & 保留时间 & 面积 & 高度 & 标记 & \multicolumn{1}{|c|}{ 面积 $\%$} \\
\hline 1 & 12.224 & 3574838 & 328501 & M & 49.620 \\
\hline 2 & 12.761 & 3629531 & 324127 & V M & 50.380 \\
\hline 总计 & & 7204368 & 652628 & & 100.000 \\
\hline
\end{tabular}

描述

: AD-HX2, n-hexane $/ \mathrm{iPrOH}=99 / 1,1.0 \mathrm{ml} / \mathrm{min}, 220 \mathrm{~nm}$

$\mathrm{mV}$

色谱图

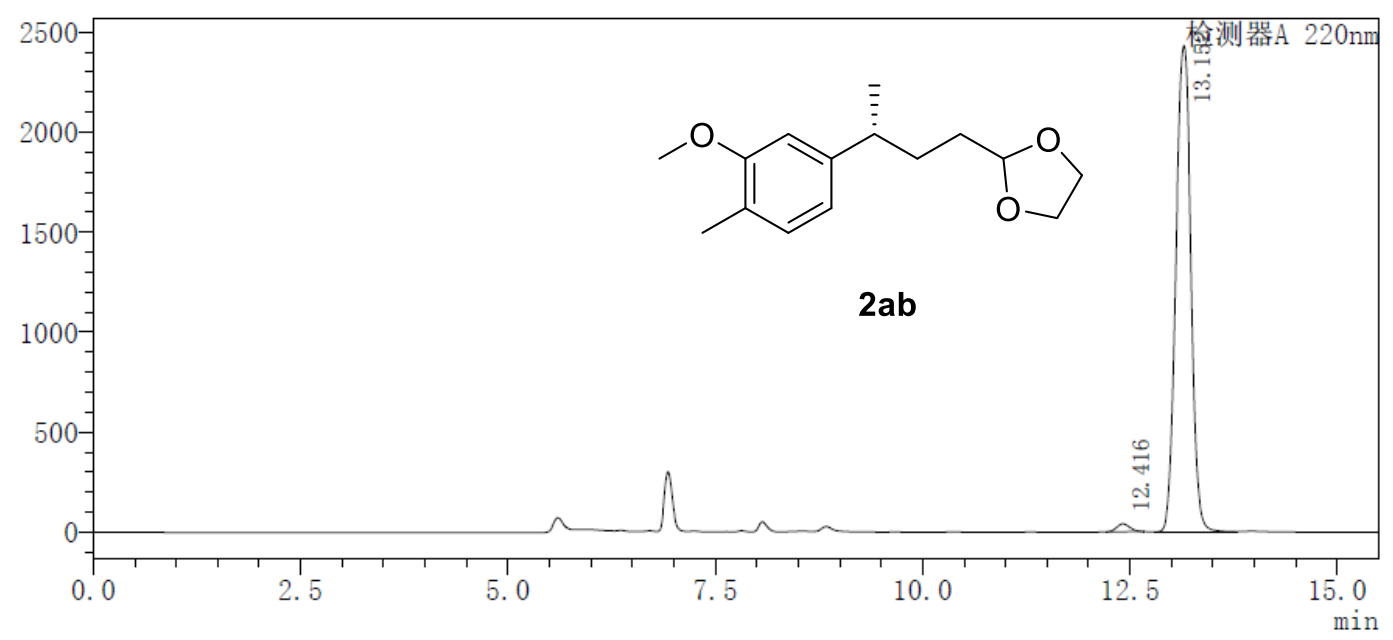

峰表

检测器A $220 \mathrm{~nm}$
\begin{tabular}{|r|r|r|r|r|r|}
\hline 峰号 & 保留时间 & \multicolumn{1}{|c|}{ 面积 } & 高度 & 标记 & \multicolumn{1}{|c|}{ 面积 $\%$} \\
\hline 1 & 12.416 & 430084 & 39925 & $\mathrm{M}$ & 1.388 \\
\hline 2 & 13.152 & 30547273 & 2428819 & $\mathrm{M}$ & 98.612 \\
\hline 总计 & & 30977357 & 2468744 & & 100.000 \\
\hline
\end{tabular}




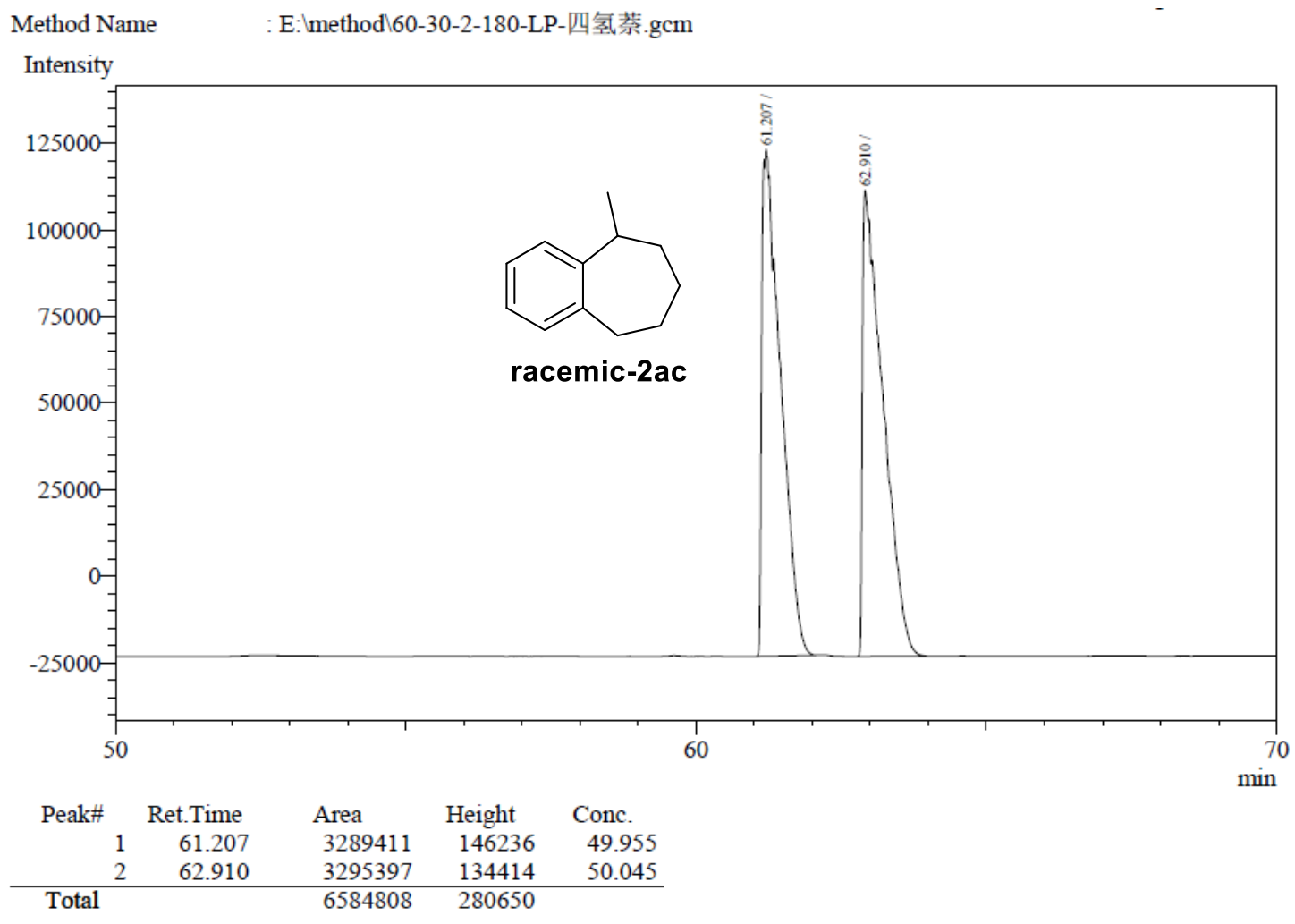

Method Name : E: method $60-30-2-180-L P$-四氢䒺.gcm

Intensity

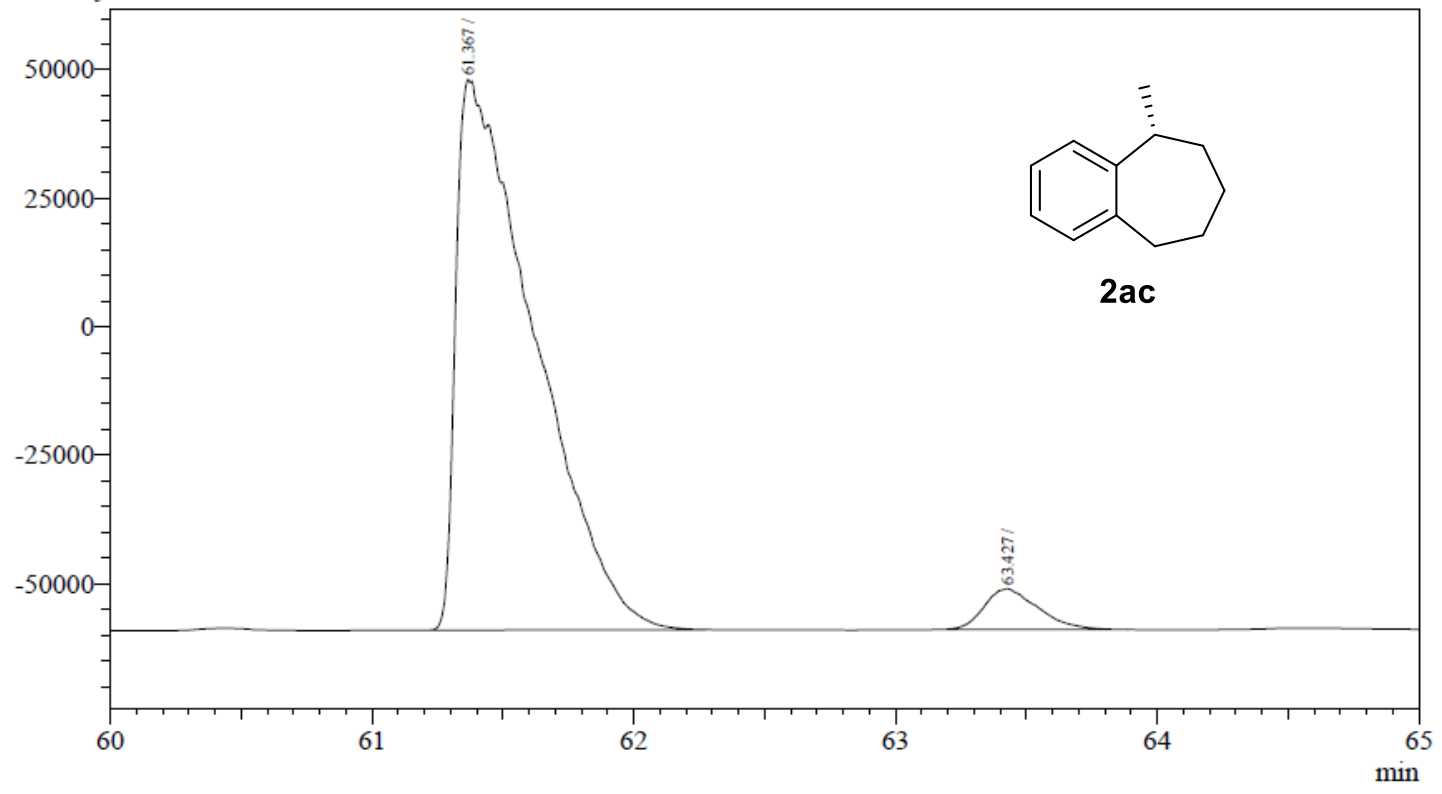

Peak\# Ret.Time Area Height Conc. Unit Mark ID\# Cmpd Name

$\begin{array}{rrrrr}1 & 61.367 & 2227112 & 107078 & 95.212 \\ 2 & 63.427 & 112006 & 7898 & 4.788\end{array}$

$\begin{array}{rrrr}2 & 63.427 & 112006 & 7898\end{array}$ 
$\mathrm{mV}$

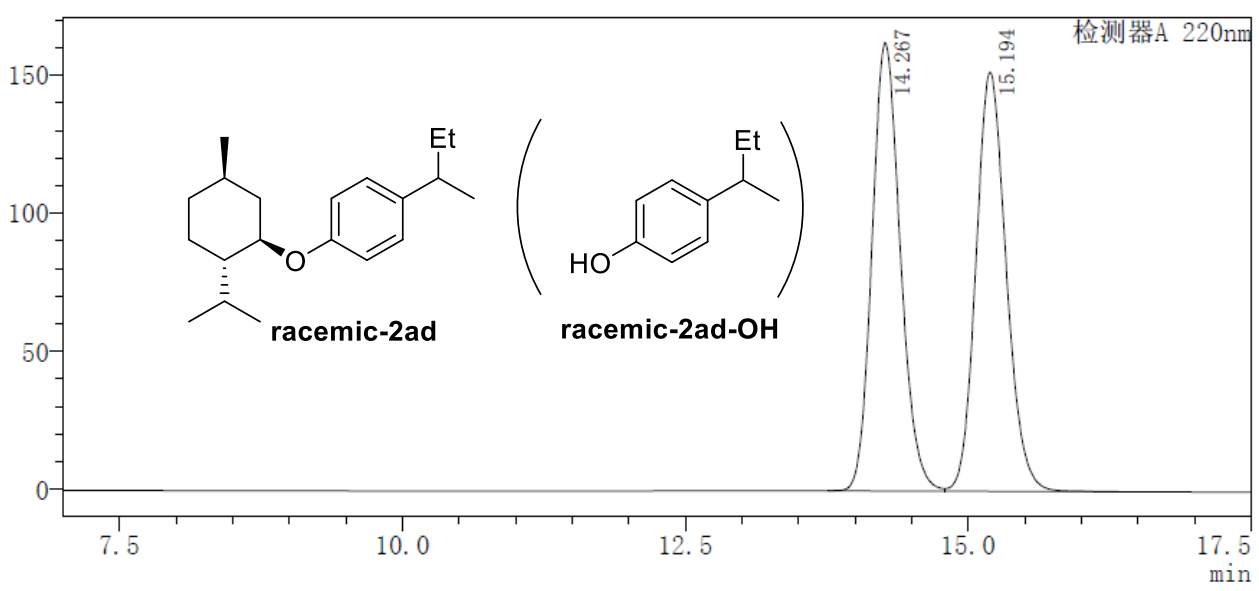

峰表

检测器A $220 \mathrm{~nm}$
\begin{tabular}{|r|r|l|l|l|r|}
\hline 峰号 & 保留时间 & 面积 & 高度 & 标记 & \multicolumn{1}{|c|}{ 面积 $\%$} \\
\hline 1 & 14.267 & 2826781 & 162336 & & 49.991 \\
\hline 2 & 15.194 & 2827761 & 151743 & V & 50.009 \\
\hline 总计 & & 5654542 & 314079 & & 100.000 \\
\hline
\end{tabular}

描述

: $0 J-H, n$-hexane $/ \mathrm{iPr} 0 \mathrm{H}=95 / 5,1.0 \mathrm{ml} / \mathrm{min}, 220 \mathrm{~nm}$

$\mathrm{mV}$

\section{色谱图}

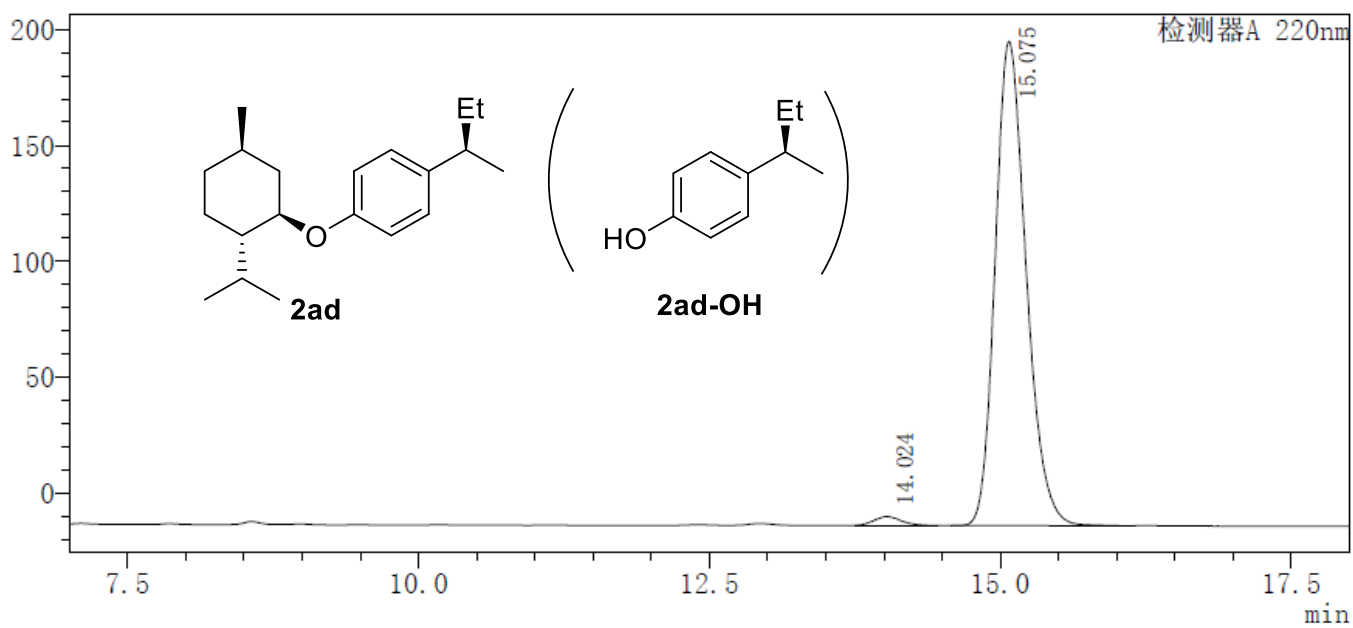

峰表

检测器A $220 \mathrm{~nm}$
\begin{tabular}{|r|r|r|r|r|r|}
\hline 峰号 & 保留时间 & 面积 & \multicolumn{1}{|c|}{ 高度 } & 标记 & 面积 $\%$ \\
\hline 1 & 14.024 & 61848 & 3792 & M & 1.573 \\
\hline 2 & 15.075 & 3868838 & 208877 & & 98.427 \\
\hline 总计 & & 3930686 & 212670 & & 100.000 \\
\hline
\end{tabular}


$\mathrm{mV}$

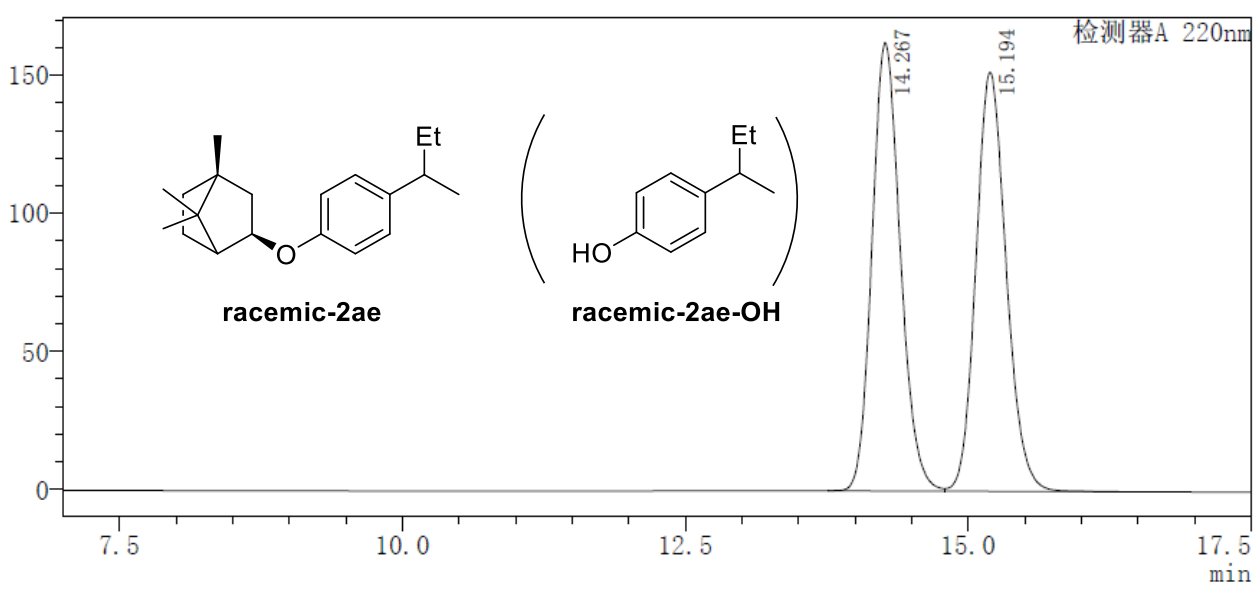

峰表

检测器A $220 \mathrm{~nm}$
\begin{tabular}{|r|r|l|l|l|r|}
\hline 峰号 & 保留时间 & 面积 & 高度 & 标记 & \multicolumn{1}{|c|}{ 面积 $\%$} \\
\hline 1 & 14.267 & 2826781 & 162336 & & 49.991 \\
\hline 2 & 15.194 & 2827761 & 151743 & V & 50.009 \\
\hline 总计 & & 5654542 & 314079 & & 100.000 \\
\hline
\end{tabular}

描述

: $0 J-H, n$-hexane $/$ PrOH $=95 / 5,1.0 \mathrm{ml} / \mathrm{min}, 220 \mathrm{~nm}$

$\mathrm{mV}$

\section{色谱图}

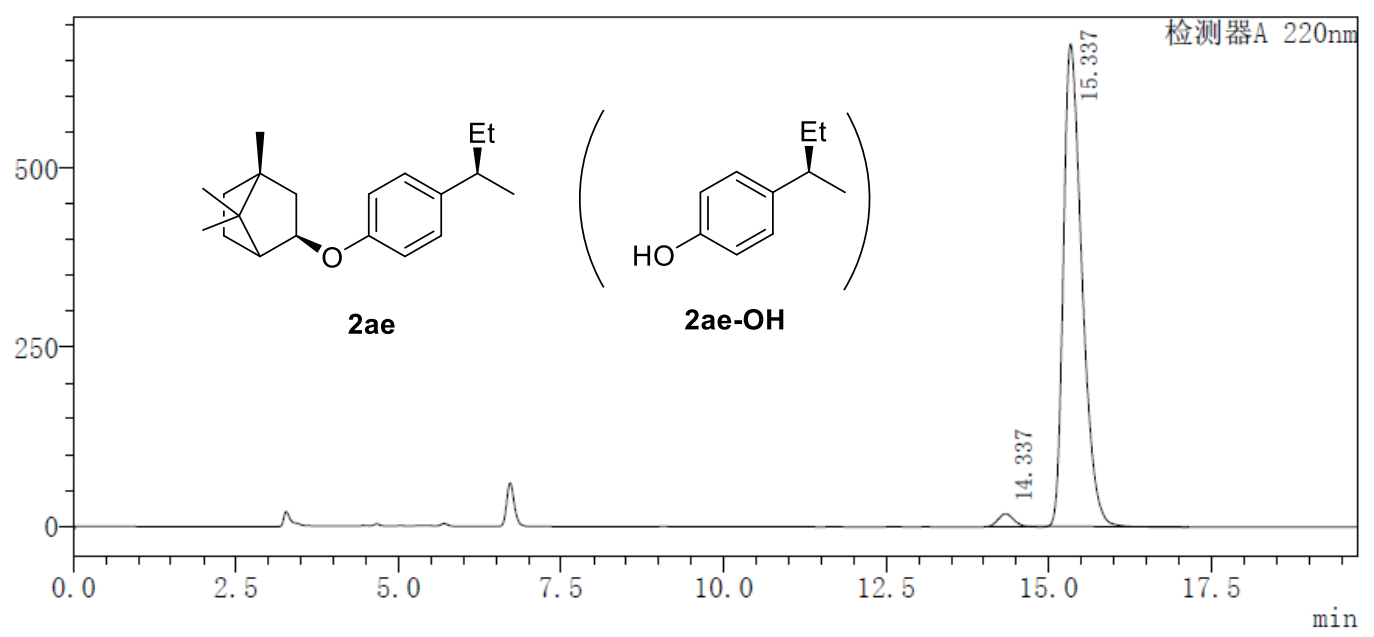

峰表

检测器A $220 \mathrm{~nm}$
\begin{tabular}{r|r|r|r|r|r|}
\hline 峰号 & 保留时间 & 面积 & 高度 & 标记 & \multicolumn{1}{|c|}{ 面积 $\%$} \\
\hline 1 & 14.337 & 283009 & 17722 & $\mathrm{M}$ & 2.076 \\
\hline 2 & 15.337 & 13349801 & 672948 & $\mathrm{~V} \mathrm{M}$ & 97.924 \\
\hline 总计 & & 13632810 & 690670 & & 100.000 \\
\hline
\end{tabular}


$\mathrm{mV}$

\section{色谱图}

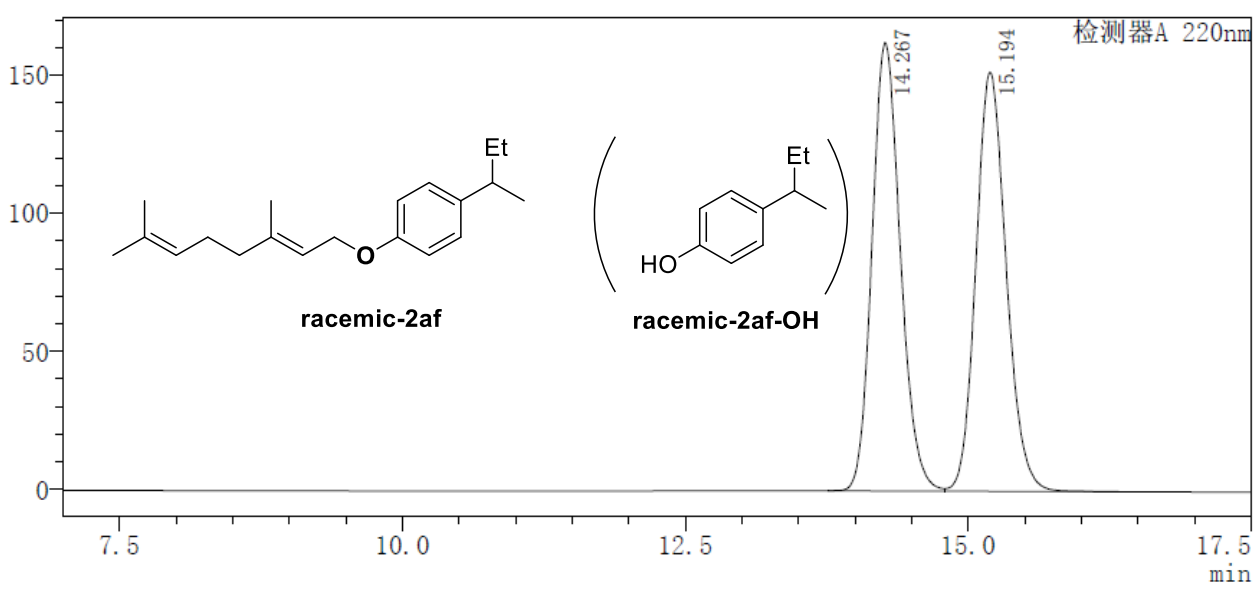

峰表

\begin{tabular}{|r|r|l|l|l|r|}
\hline 峰号 & 保留时间 & 面积 & 高度 & 标记 & \multicolumn{1}{|c|}{ 面积\% } \\
\hline 1 & 14.267 & 2826781 & 162336 & & 49.991 \\
\hline 2 & 15.194 & 2827761 & 151743 & V & 50.009 \\
\hline 总计 & & 5654542 & 314079 & & 100.000 \\
\hline
\end{tabular}

描述 $\cdots$ O J

$\mathrm{mV}$

\section{色谱图}

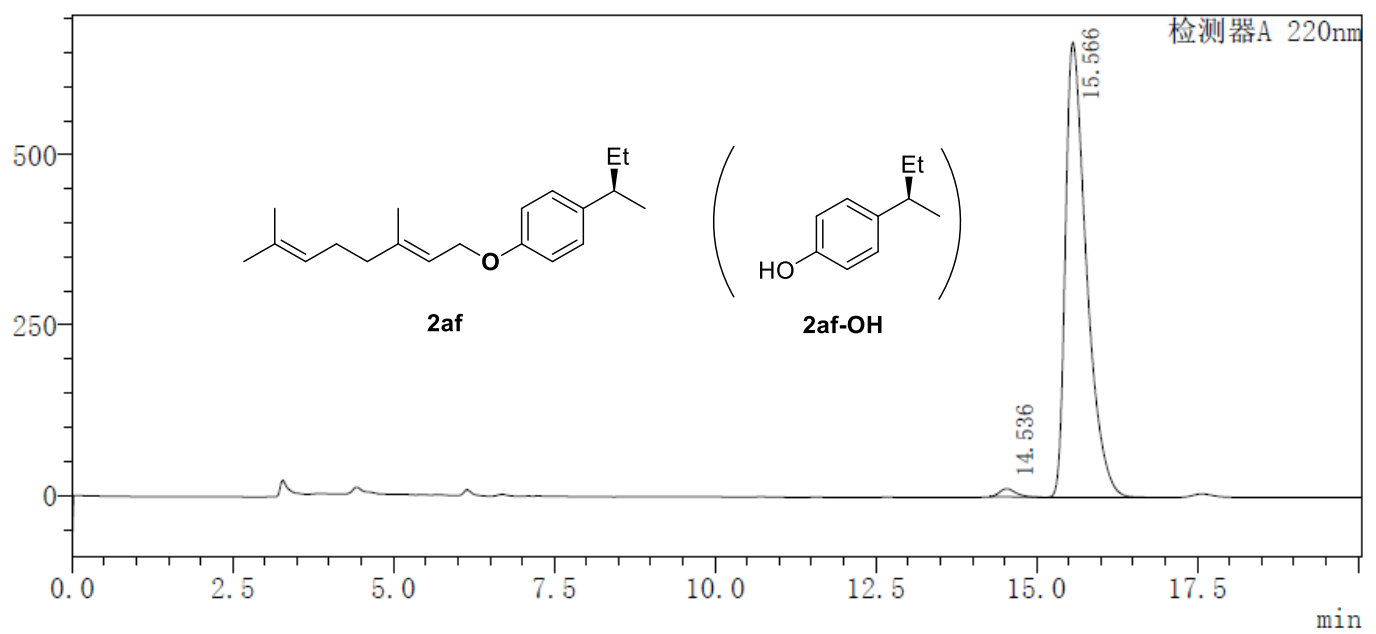

峰表

检测器A $220 \mathrm{~nm}$
\begin{tabular}{r|r|r|r|r|r|}
\hline 峰号 & 保留时间 & 面积 & 高度 & 标记 & \multicolumn{1}{|c|}{ 面积 $\%$} \\
\hline 1 & 14.536 & 213392 & 11916 & $\mathrm{M}$ & 1.388 \\
\hline 2 & 15.566 & 15160543 & 667417 & & 98.612 \\
\hline 总计 & & 15373935 & 679333 & & 100.000 \\
\hline
\end{tabular}


$\mathrm{mV}$

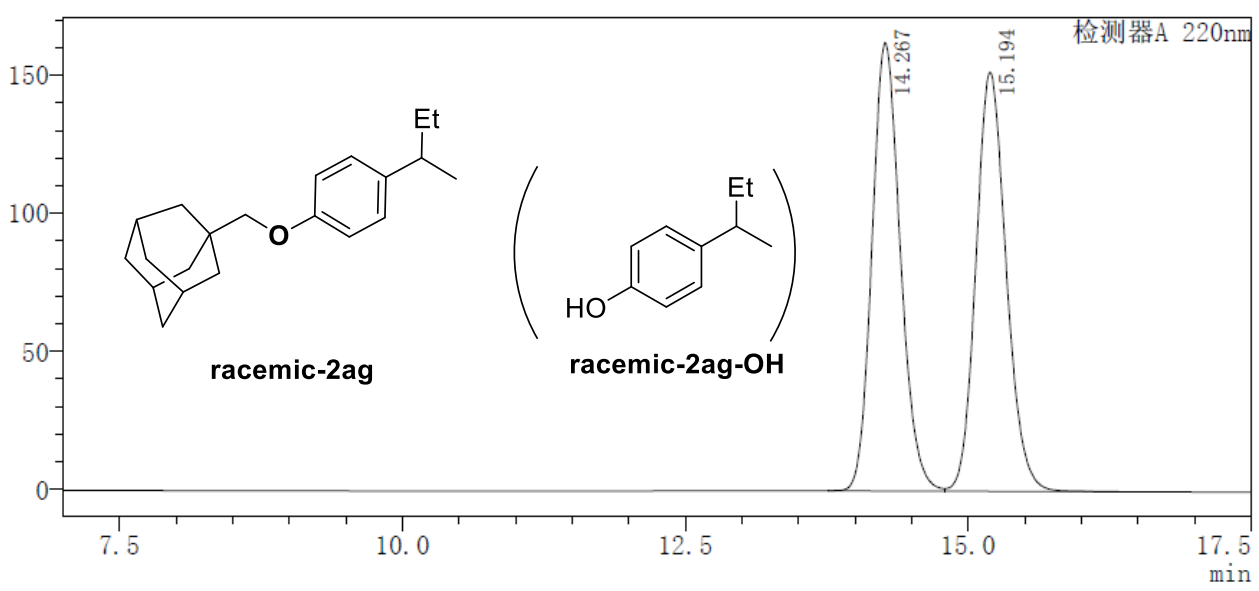

峰表

检测器A $220 \mathrm{~nm}$
\begin{tabular}{|r|r|l|l|l|r|}
\hline 峰号 & 保留时间 & 面积 & 高度 & 标记 & \multicolumn{1}{|c|}{ 面积 $\%$} \\
\hline 1 & 14.267 & 2826781 & 162336 & & 49.991 \\
\hline 2 & 15.194 & 2827761 & 151743 & V & 50.009 \\
\hline 总计 & & 5654542 & 314079 & & 100.000 \\
\hline
\end{tabular}

描述

: $0 \mathrm{~J}-\mathrm{H}, \mathrm{n}$-hexane $/ \mathrm{iPrOH}=95 / 5,1.0 \mathrm{ml} / \mathrm{min}, 220 \mathrm{~nm}$

$\mathrm{mV}$

\section{色谱图}

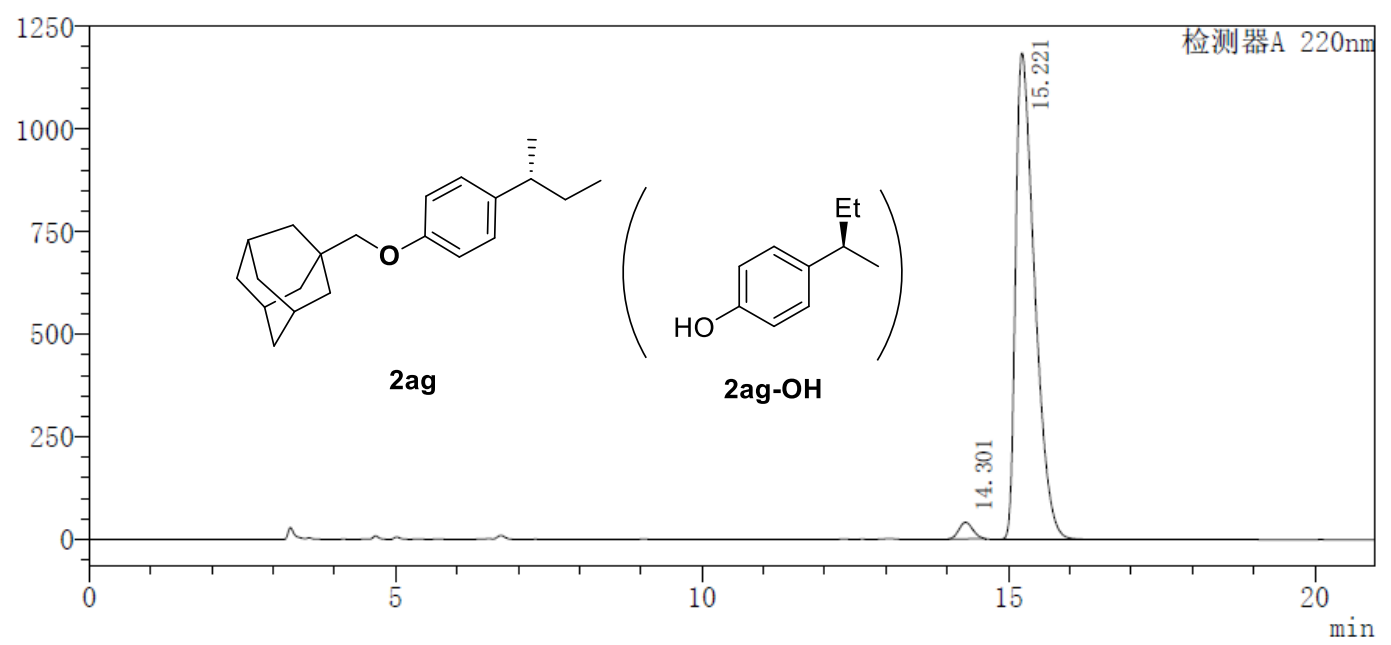

峰表

检测器A $220 \mathrm{~nm}$
\begin{tabular}{|r|r|r|r|r|r|}
\hline 峰号 & 保留时间 & \multicolumn{1}{|c|}{ 面积 } & 高度 & 标记 & \multicolumn{1}{|c|}{ 面积 $\%$} \\
\hline 1 & 14.301 & 638309 & 40481 & $\mathrm{M}$ & 2.437 \\
\hline 2 & 15.221 & 25552332 & 1184591 & $\mathrm{M}$ & 97.563 \\
\hline 总计 & & 26190641 & 1225071 & & 100.000 \\
\hline
\end{tabular}


$\mathrm{mV}$

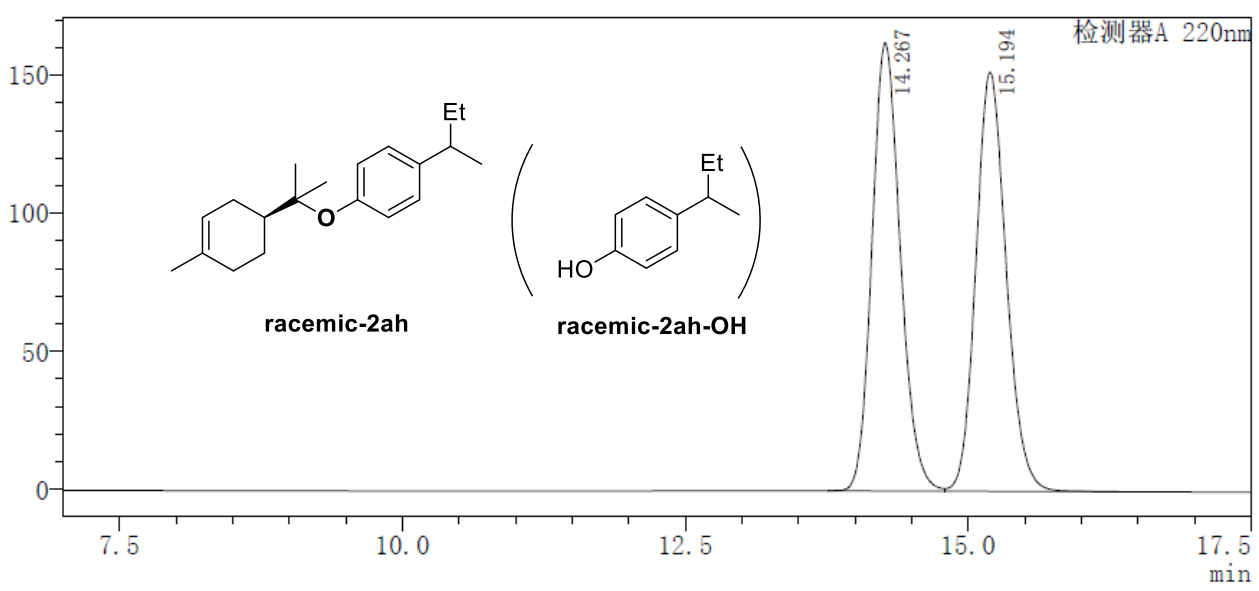

峰表

检测器A $220 \mathrm{~nm}$
\begin{tabular}{|r|r|l|l|l|r|}
\hline 峰号 & 保留时间 & 面积 & 高度 & 标记 & \multicolumn{1}{|c|}{ 面积 $\%$} \\
\hline 1 & 14.267 & 2826781 & 162336 & & 49.991 \\
\hline 2 & 15.194 & 2827761 & 151743 & V & 50.009 \\
\hline 总计 & & 5654542 & 314079 & & 100.000 \\
\hline
\end{tabular}

描述

: $0 J-H, n$-hexane $/ i \operatorname{PrOH}=95 / 5,1.0 \mathrm{ml} / \mathrm{min}, 220 \mathrm{~nm}$

$\mathrm{mV}$

\section{色谱图}

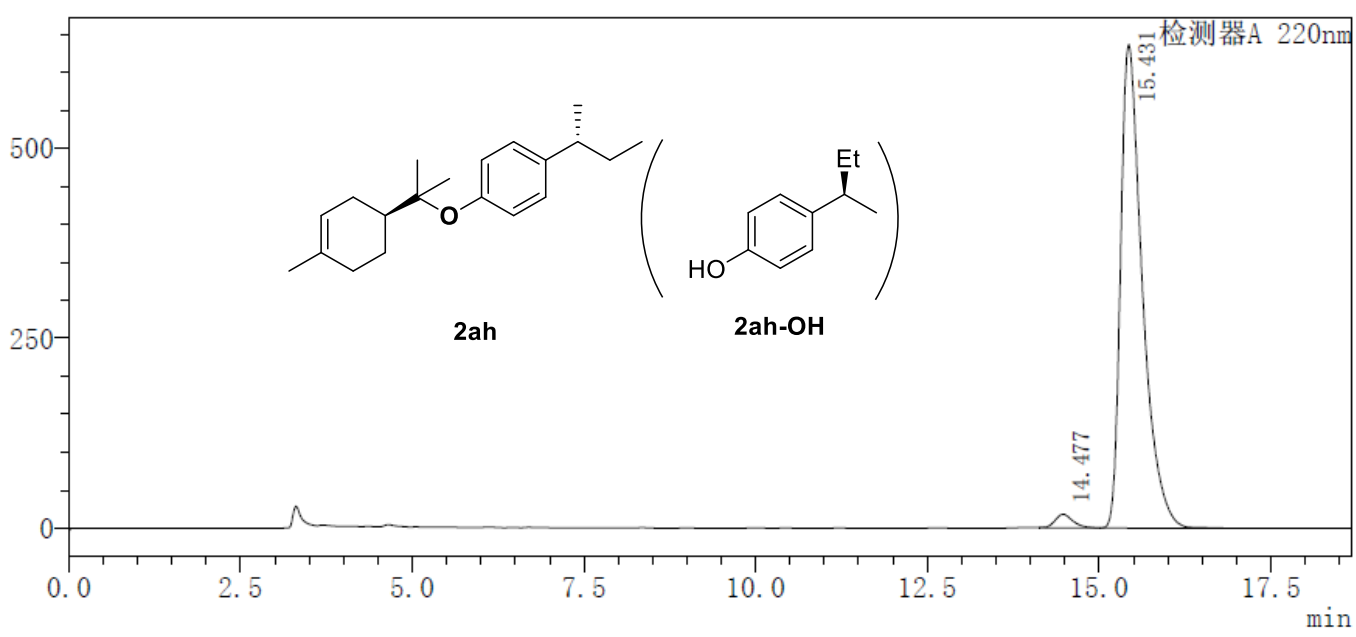

峰表

检测器A $220 \mathrm{~nm}$
\begin{tabular}{r|r|r|r|r|r|}
\hline 峰号 & 保留时间 & \multicolumn{1}{|c|}{ 面积 } & 高度 & 标记 & \multicolumn{1}{|c|}{ 面积 $\%$} \\
\hline 1 & 14.477 & 321456 & 17753 & & 2.236 \\
\hline 2 & 15.431 & 14052456 & 635754 & & 97.764 \\
\hline 总计 & & 14373912 & 653507 & & 100.000 \\
\hline
\end{tabular}


GC Conditions : Chiralcel B-DM, $100 \mathrm{kPa} \mathrm{N} 2\left(60{ }^{\circ} \mathrm{C}-30 \mathrm{~min}-2{ }^{\circ} \mathrm{C} / \mathrm{min}-180{ }^{\circ} \mathrm{C}-\right.$ $5 \mathrm{~min})$
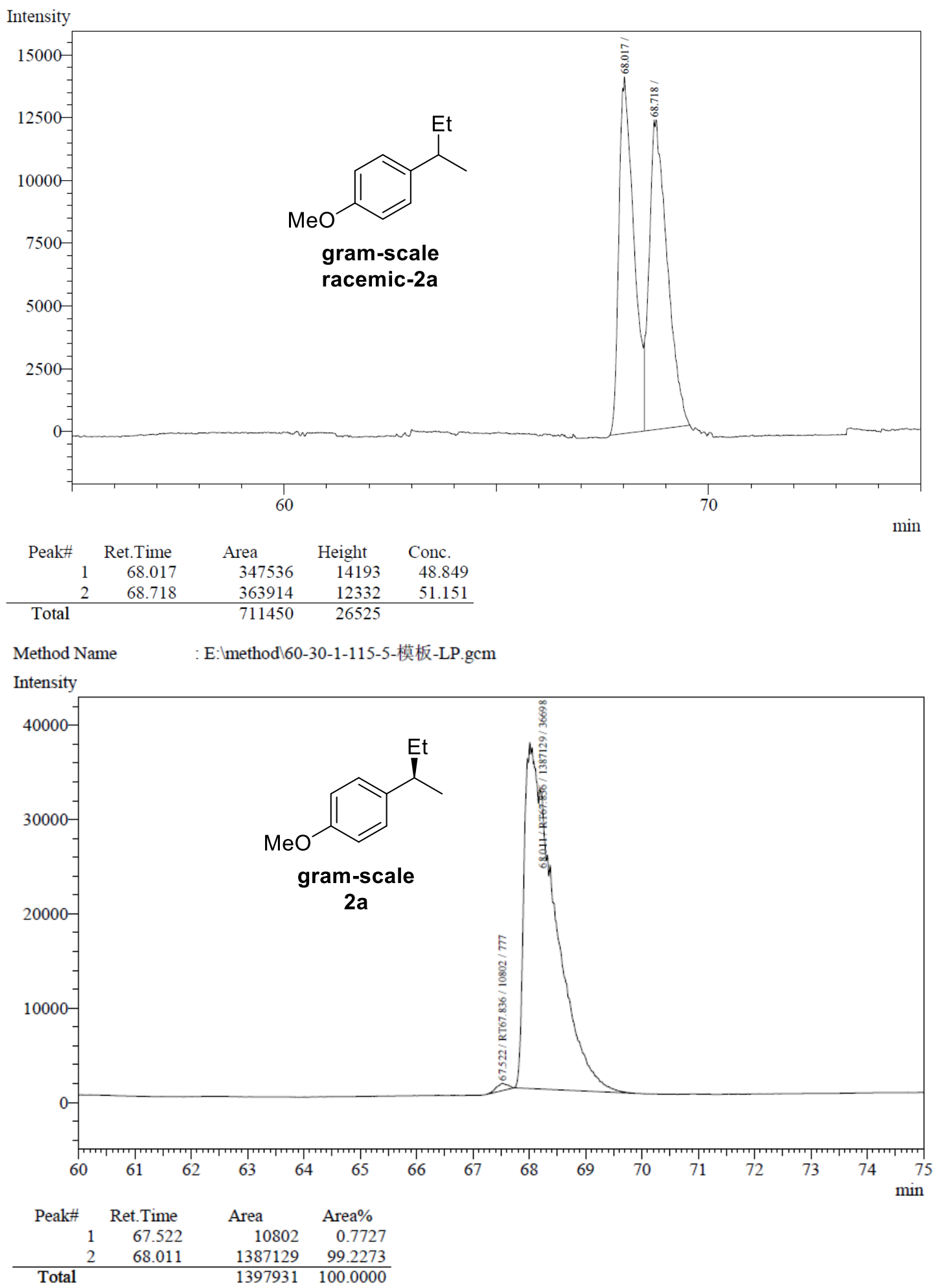
$\mathrm{mV}$

色谱图

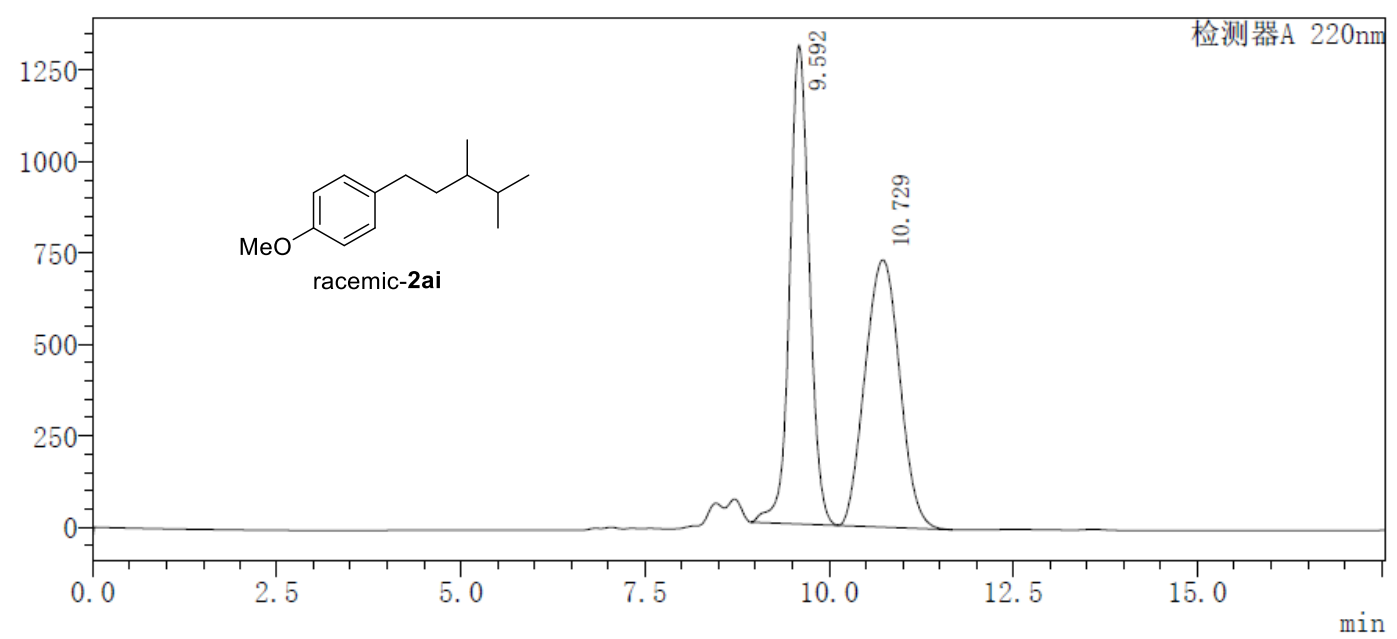

峰表

\begin{tabular}{|c|c|c|c|c|c|}
\hline \\
\hline 峰号 & 保留时间 & 面积 & 高度 & 标记 & 面积 \% \\
\hline 1 & 9.592 & 24396116 & 1309523 & & 50.417 \\
\hline 2 & 10.729 & 23992909 & 731004 & & 49.583 \\
\hline 总计 & & 48389025 & 2040527 & & 100.000 \\
\hline
\end{tabular}

描述

: OB-H, n-hexane $/ \mathrm{PrOH}=99.5 / 0.5,0.5 \mathrm{ml} / \mathrm{min}, 220 \mathrm{~nm}$

$\mathrm{mV}$

色谱图

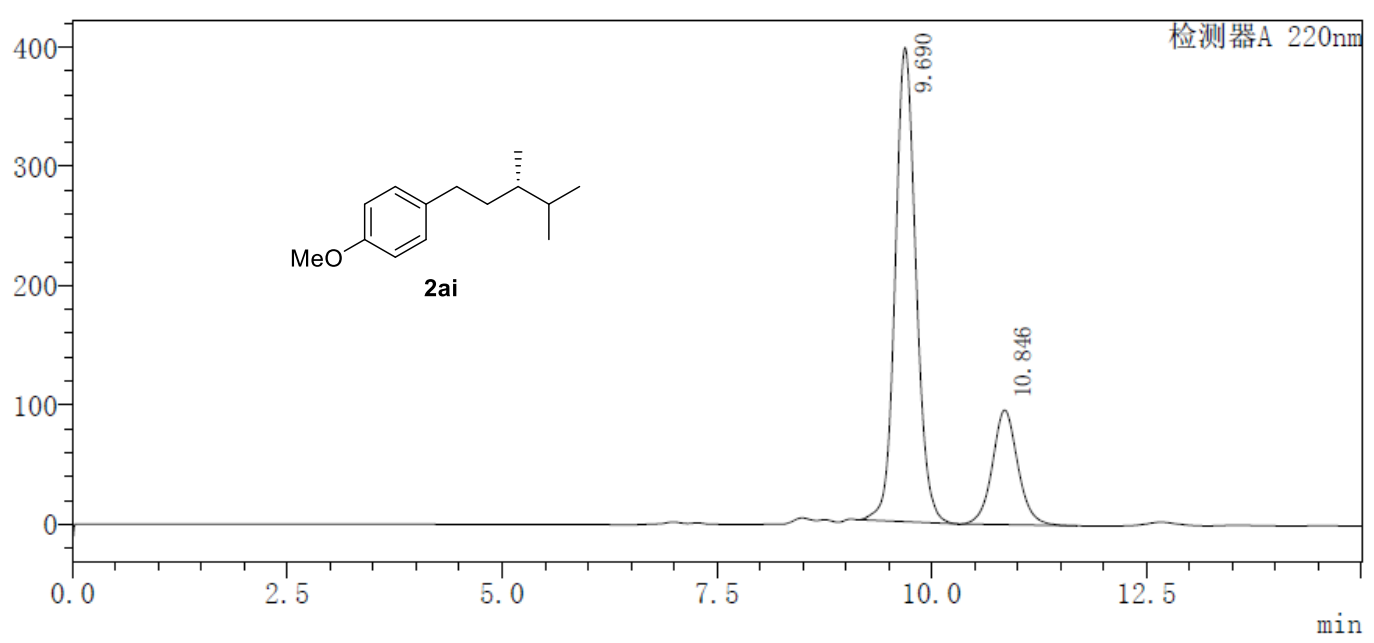

峰表

检测器A $220 \mathrm{~nm}$
\begin{tabular}{|r|r|r|r|r|r|}
\hline 峰号 & 保留时间 & 面积 & \multicolumn{1}{|c|}{ 高度 } & 标记 & \multicolumn{1}{c|}{ 面积\% } \\
\hline 1 & 9.690 & 6681295 & 397295 & & 77.082 \\
\hline 2 & 10.846 & 1986515 & 96027 & & 22.918 \\
\hline 总计 & & 8667809 & 493322 & & 100.000 \\
\hline
\end{tabular}


$\mathrm{mV}$

色谱图

LP17143-1

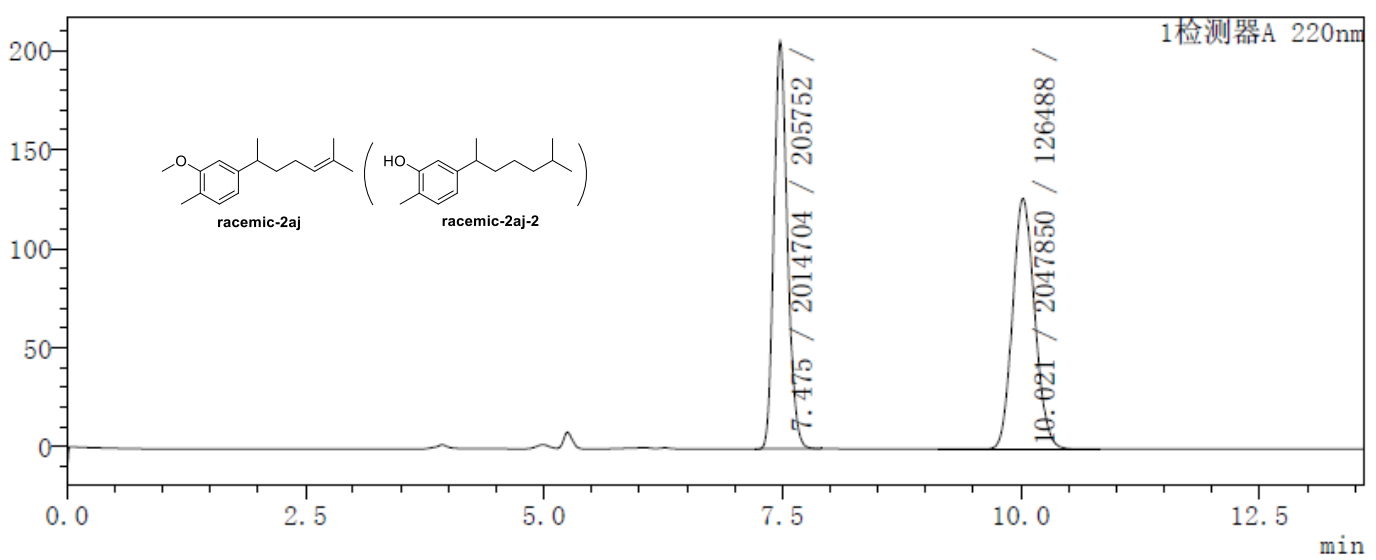

峰表

检测器 $\mathrm{A} 220 \mathrm{~nm}$
\begin{tabular}{|r|r|r|r|r|r|}
\hline 峰䒓 & 保留时间 & \multicolumn{1}{|c|}{ 面积 } & 高度 & 标记 & 面积 $\%$ \\
\hline 1 & 7.475 & 2014704 & 205752 & & 49.592 \\
\hline 2 & 10.021 & 2047850 & 126488 & & 50.408 \\
\hline 总计 & & 4062554 & 332240 & & 100.000 \\
\hline
\end{tabular}

描述

: OJ-H, n-hexane $/$ iPrOH $=95 / 5,0.8 \mathrm{ml} / \mathrm{min}, 220 \mathrm{~nm}$

$\mathrm{mV}$

色谱图

LP17147

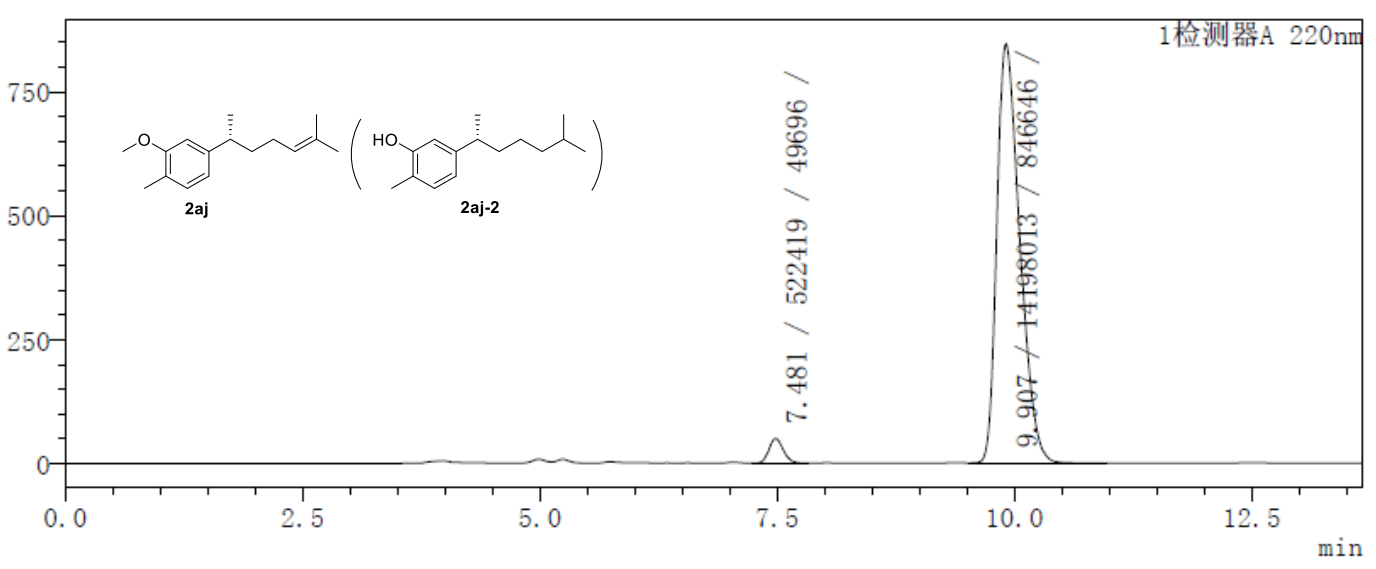

峰表

检测器A $220 \mathrm{~nm}$
\begin{tabular}{|r|r|r|r|r|r|}
\hline 峰号 & \multicolumn{1}{|c|}{ 保留时间 } & \multicolumn{1}{|c|}{ 面积 } & \multicolumn{1}{c|}{ 高度 } & 标记 & \multicolumn{1}{|c|}{ 面积 $\%$} \\
\hline 1 & 7.481 & 522419 & 49696 & & 3.549 \\
\hline 2 & 9.907 & 14198013 & 846646 & & 96.451 \\
\hline 总计 & & 14720432 & 896343 & & 100.000 \\
\hline
\end{tabular}


$\mathrm{mV}$

色谱图

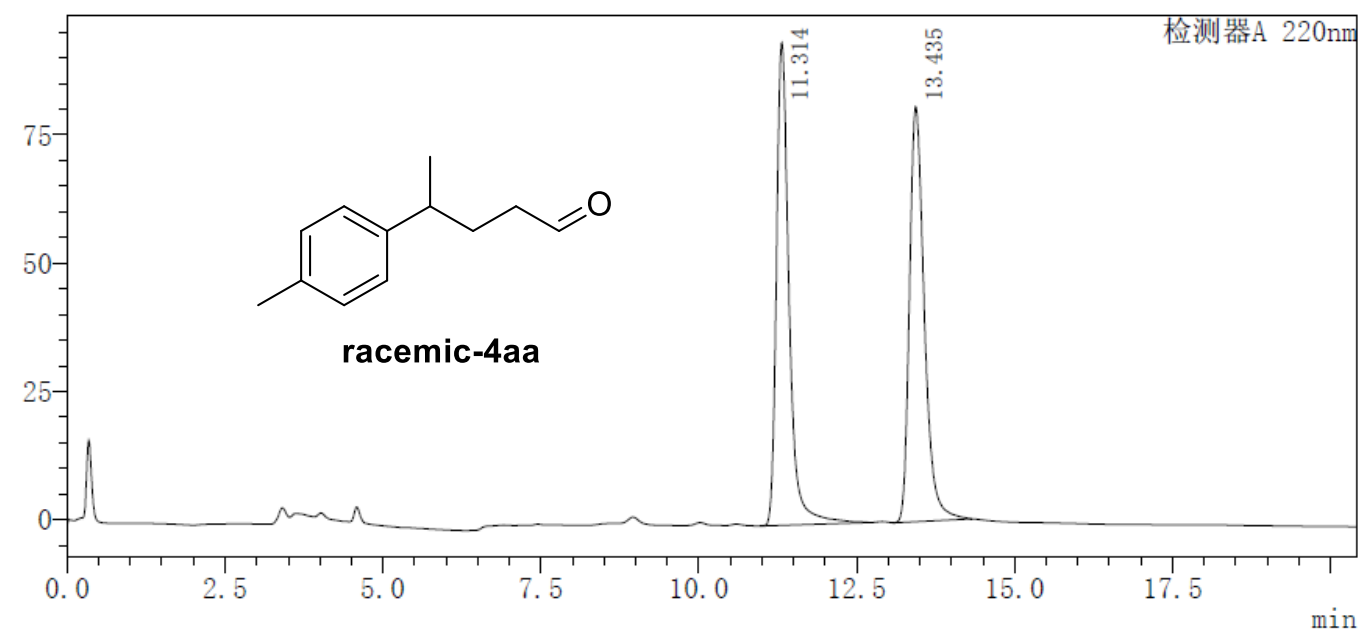

峰表

检测器A $220 \mathrm{~nm}$
\begin{tabular}{|r|r|r|r|r|r|}
\hline 峰号 & 保留时间 & 面积 & \multicolumn{1}{c|}{ 高度 } & 标记 & \multicolumn{1}{|c|}{ 面积 $\%$} \\
\hline 1 & 11.314 & 1313944 & 93993 & & 49.857 \\
\hline 2 & 13.435 & 1321496 & 80872 & $\mathrm{M}$ & 50.143 \\
\hline 总计 & & 2635440 & 174865 & & 100.000 \\
\hline
\end{tabular}

描述

: OJ-H, n-hexane $/ \mathrm{iPrOH}=99 / 1,1.0 \mathrm{ml} / \mathrm{min}, 220 \mathrm{~nm}$

$\mathrm{mV}$

色谱图

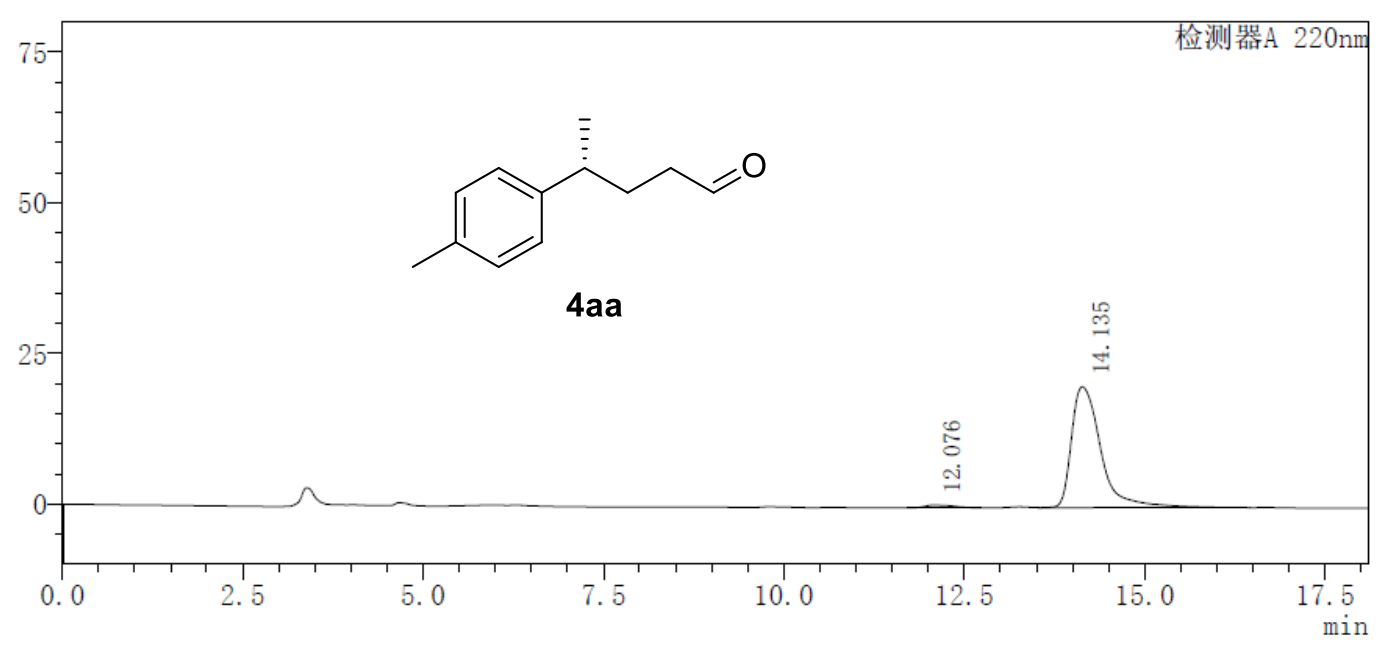

峰表

检测器A $220 \mathrm{~nm}$
\begin{tabular}{|r|r|r|r|r|r|}
\hline 峰号 & 保留时间 & 面积 & \multicolumn{1}{l|}{ 高度 } & 标记 & \multicolumn{1}{|c|}{ 面积 $\%$} \\
\hline 1 & 12.076 & 11660 & 434 & & 2.017 \\
\hline 2 & 14.135 & 566381 & 20037 & & 97.983 \\
\hline 总计 & & 578041 & 20471 & & 100.000 \\
\hline
\end{tabular}


GC Conditions : Chiralcel B-DM, $100 \mathrm{kPa} \mathrm{N} 2\left(40{ }^{\circ} \mathrm{C}-90 \min -1{ }^{\circ} \mathrm{C} / \mathrm{min}-150{ }^{\circ} \mathrm{C}-\right.$ $10 \min )$
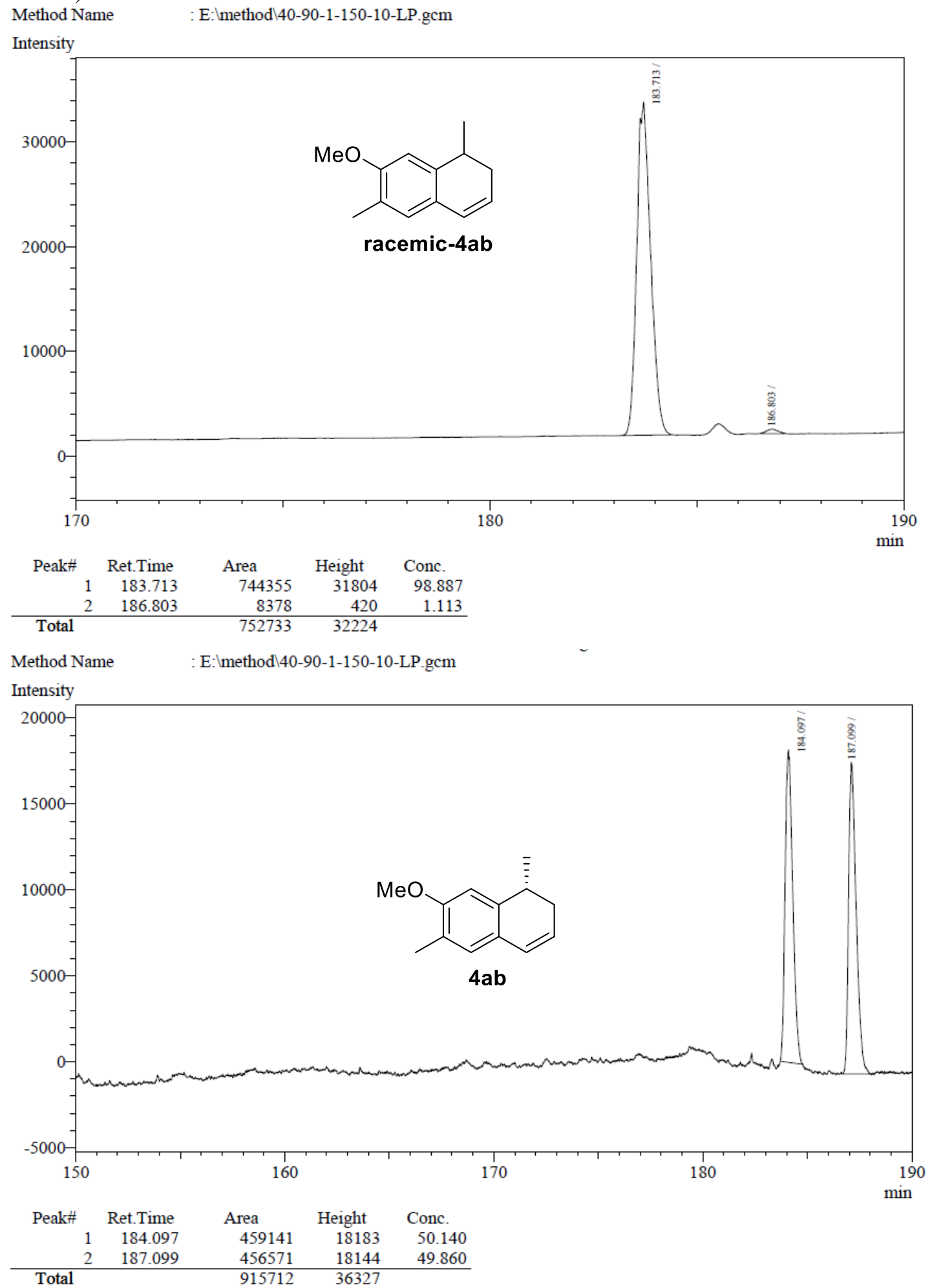
GC Conditions : Chiralcel B-DM, $100 \mathrm{kPa} \mathrm{N} 2\left(60{ }^{\circ} \mathrm{C}-30 \mathrm{~min}-2{ }^{\circ} \mathrm{C} / \mathrm{min}-180{ }^{\circ} \mathrm{C}-\right.$ $5 \mathrm{~min})$
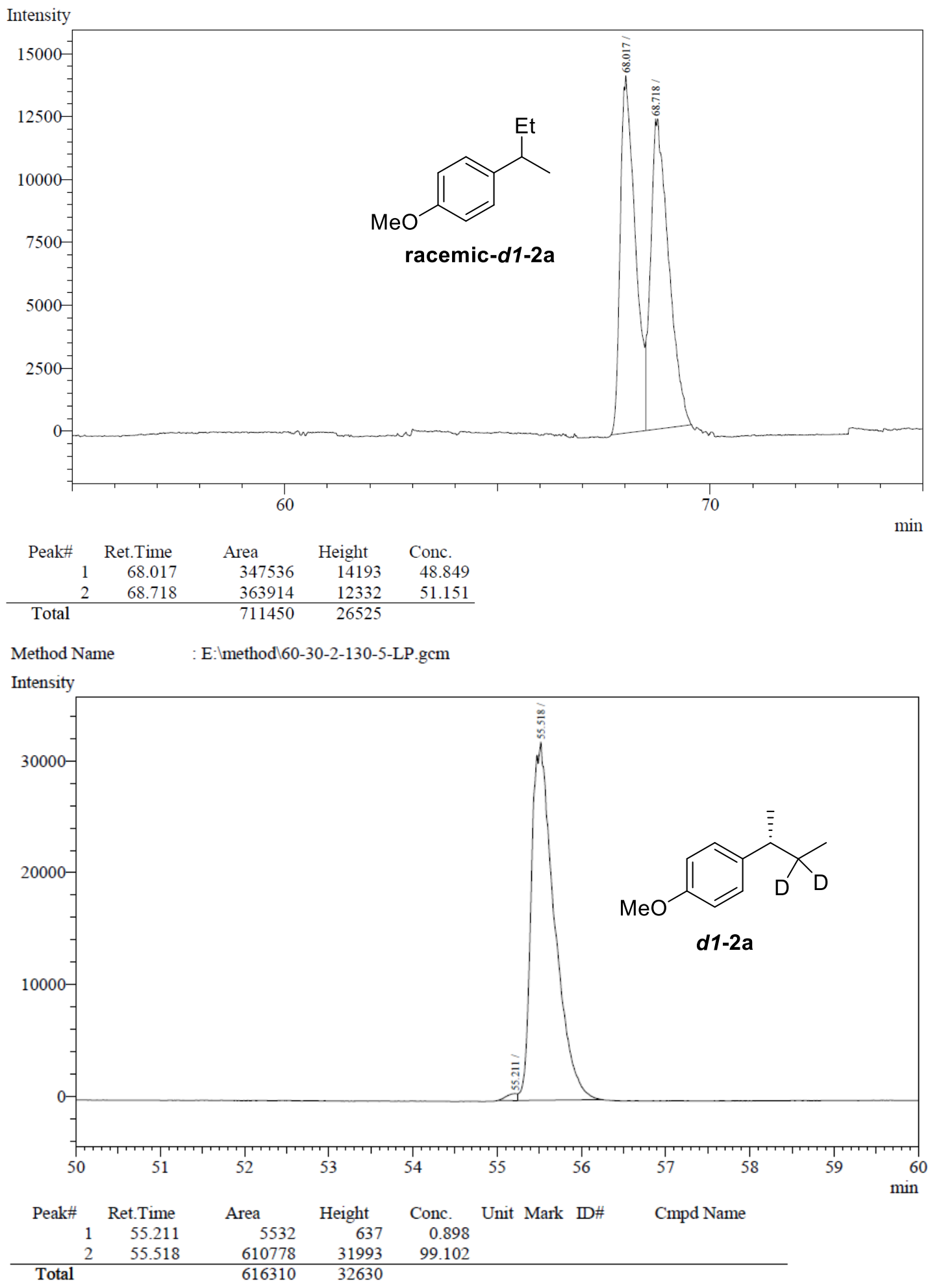
GC Conditions : Chiralcel B-DM, $100 \mathrm{kPa} \mathrm{N} 2\left(60{ }^{\circ} \mathrm{C}-30 \mathrm{~min}-2{ }^{\circ} \mathrm{C} / \mathrm{min}-180{ }^{\circ} \mathrm{C}-\right.$ $5 \mathrm{~min})$

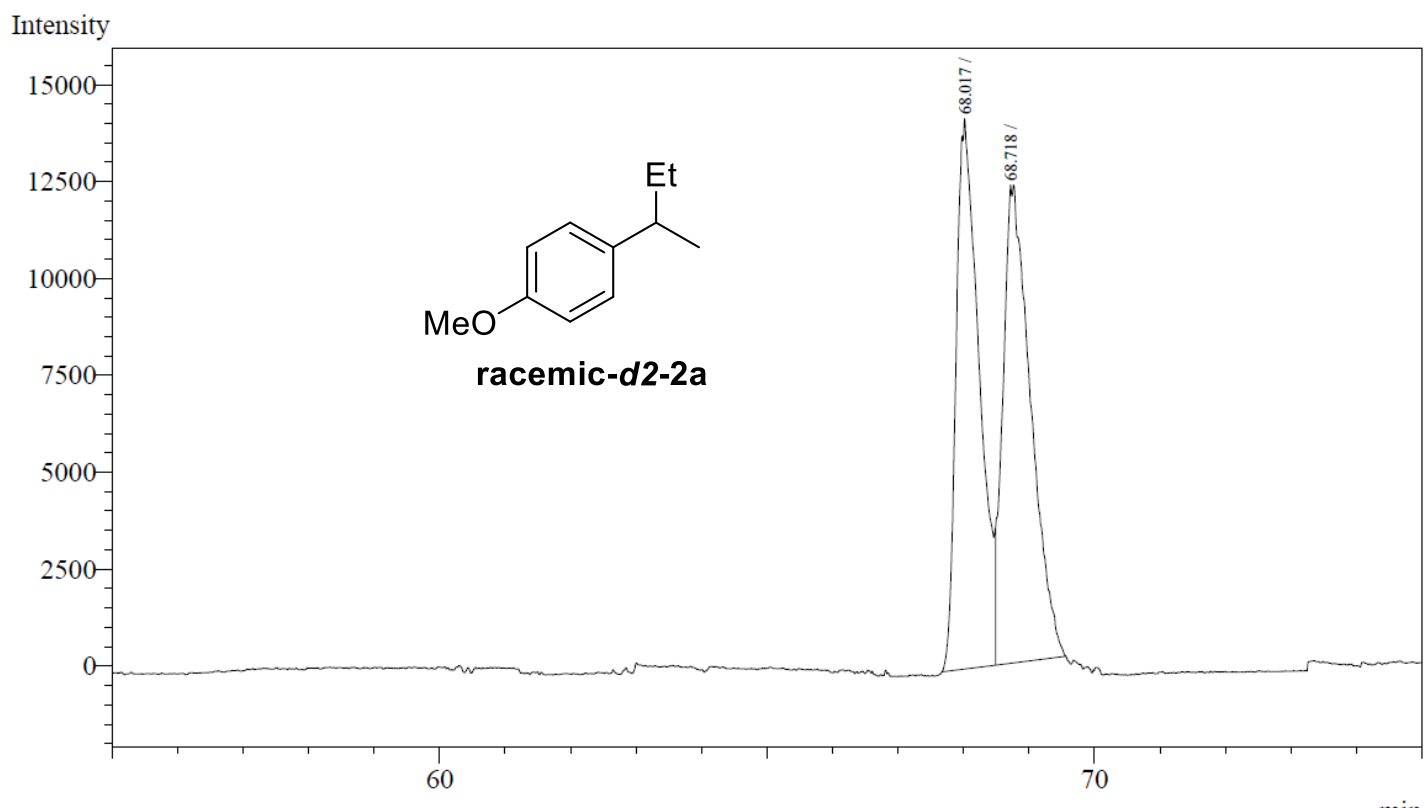

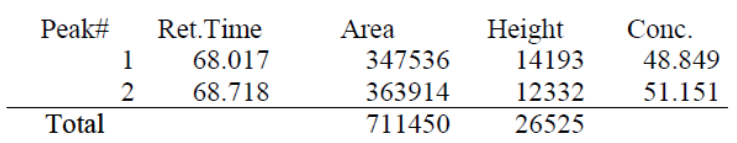

Method Name : E: :method $160-30-1-130-5$-模板-LP.gcm

Intensity

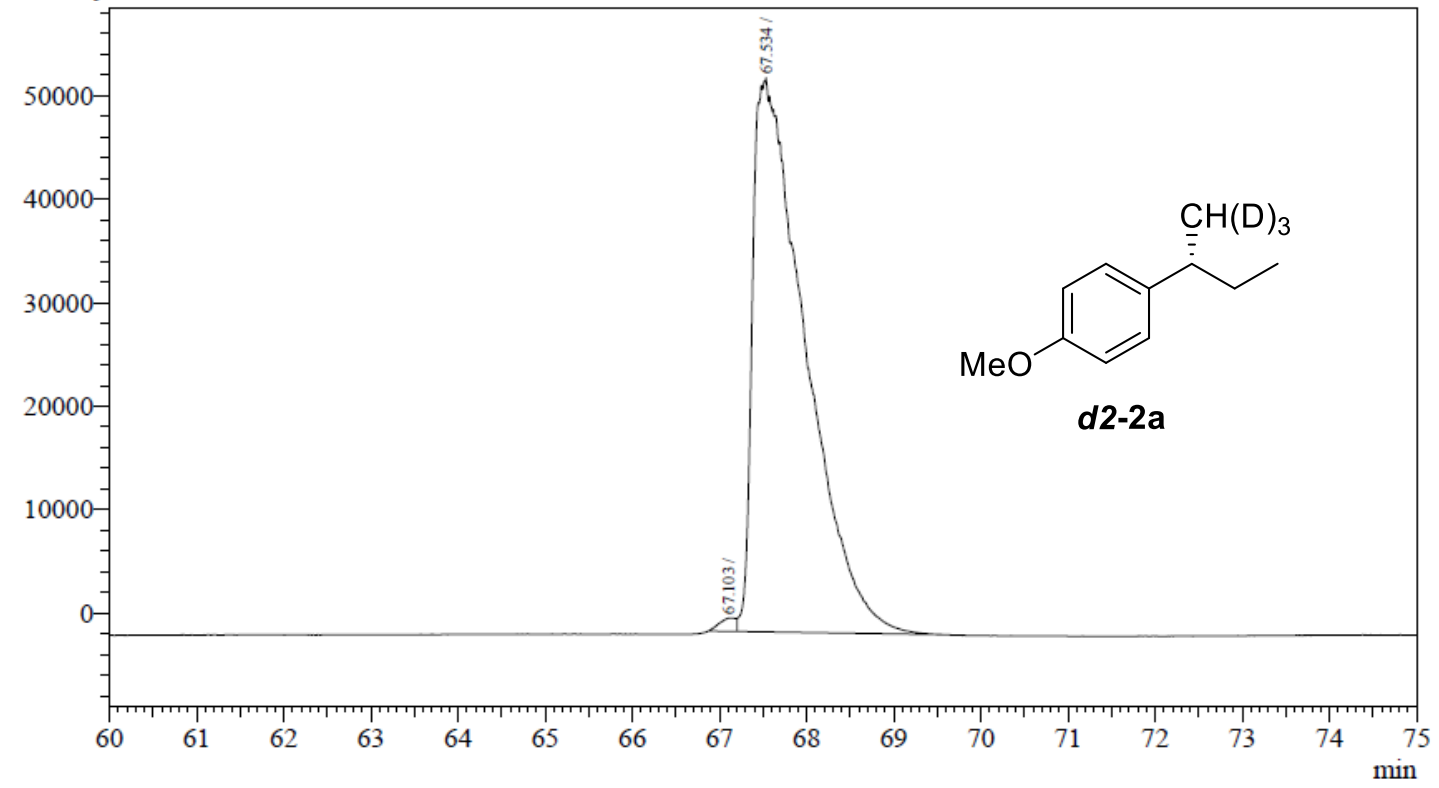

Peak\# Ret.Time Area Height Conc.

\begin{tabular}{rrrrr}
1 & 67.103 & 17190 & 1273 & 0.760 \\
2 & 67.534 & 2243818 & 53502 & 99.240 \\
\hline Total & & 2261008 & 54775 &
\end{tabular}


GC Conditions : Chiralcel B-DM, $100 \mathrm{kPa} \mathrm{N} 2\left(60{ }^{\circ} \mathrm{C}-30 \mathrm{~min}-2{ }^{\circ} \mathrm{C} / \mathrm{min}-180{ }^{\circ} \mathrm{C}-\right.$ $5 \mathrm{~min})$
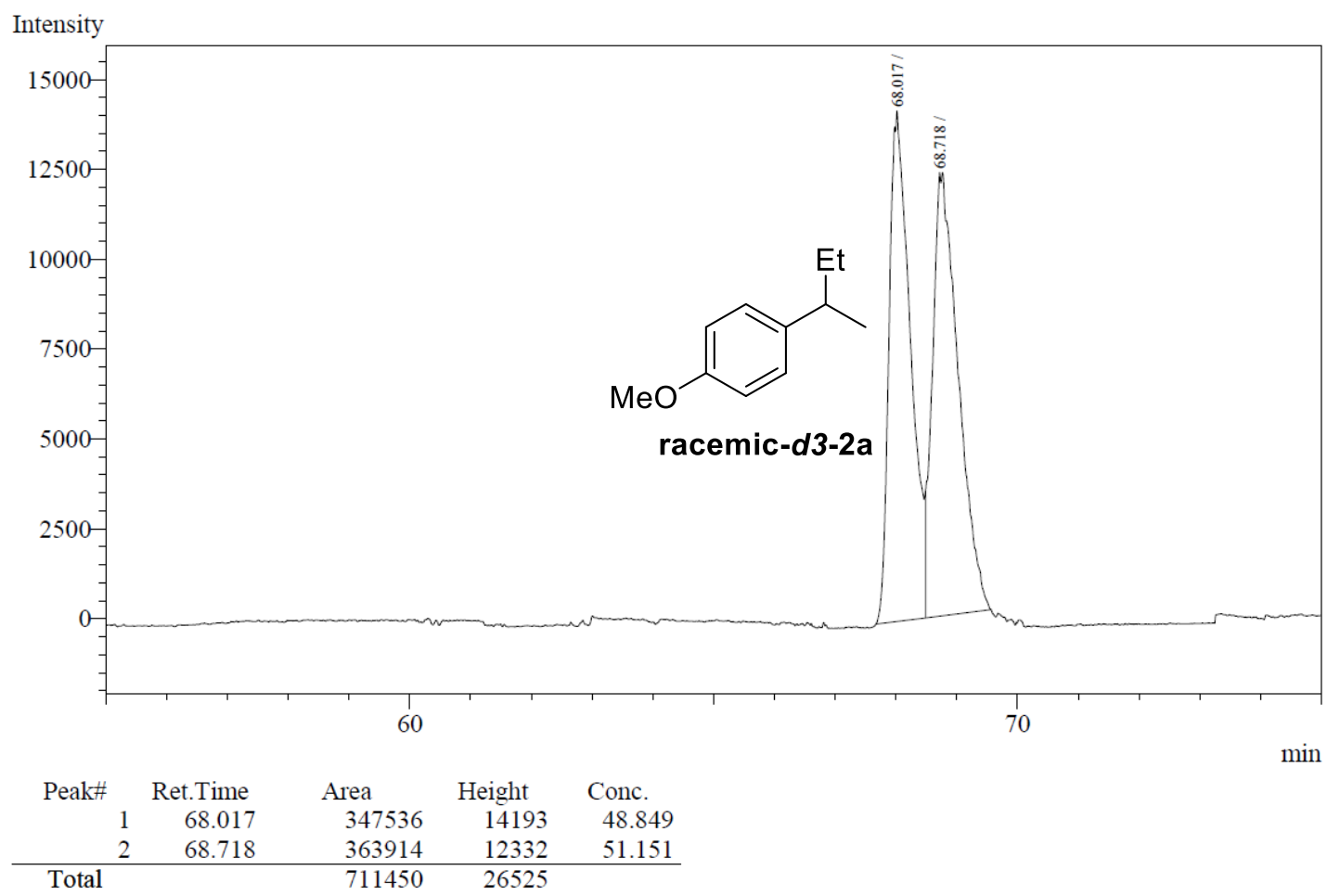

Method Name : E: method 60 -30-1-115-5-模板-LP.gcm

Intensity

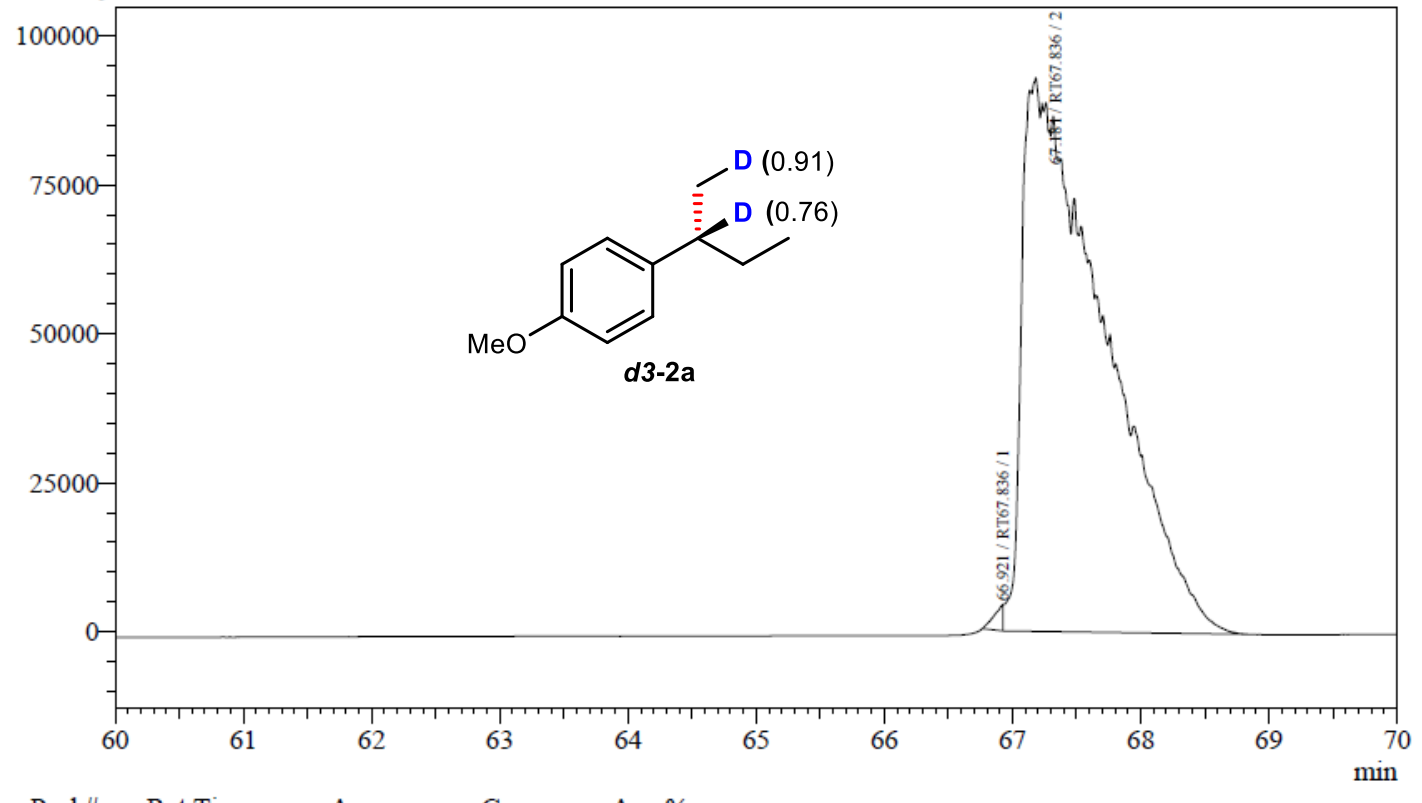

Peak\# Ret.Time Area Conc. Area\%

\begin{tabular}{rrrrr}
1 & 66.921 & 20407 & 0.000 & 0.4890 \\
2 & 67.181 & 4152553 & 0.000 & 99.5110 \\
\hline Total & & 4172960 & & 100.0000
\end{tabular}


GC Conditions : Chiralcel B-DM, $100 \mathrm{kPa} \mathrm{N} 2\left(60{ }^{\circ} \mathrm{C}-30 \mathrm{~min}-2{ }^{\circ} \mathrm{C} / \mathrm{min}-180{ }^{\circ} \mathrm{C}-\right.$ $5 \mathrm{~min})$
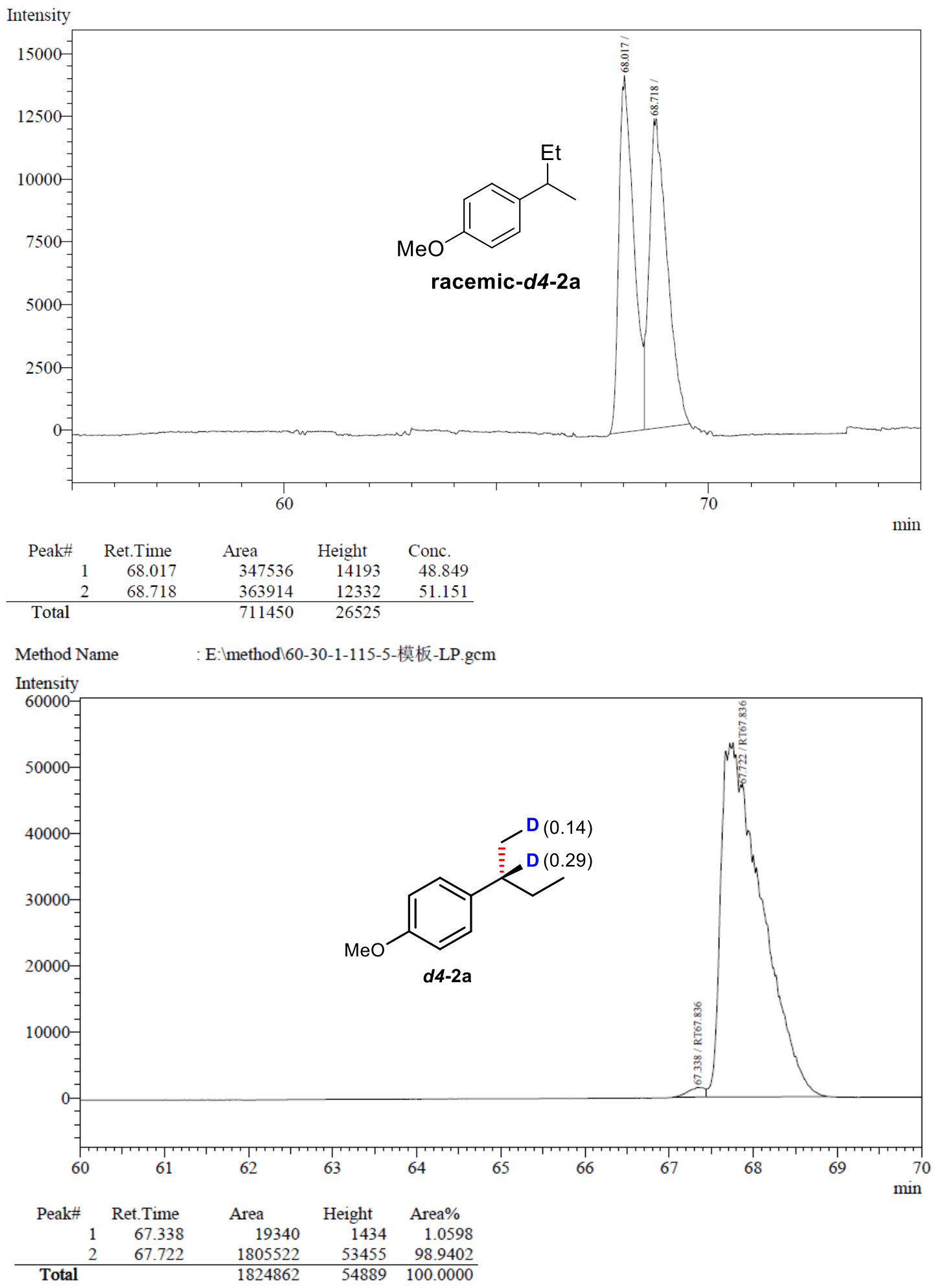
GC Conditions : Chiralcel B-DM, $100 \mathrm{kPa} \mathrm{N} 2\left(60{ }^{\circ} \mathrm{C}-30 \mathrm{~min}-1{ }^{\circ} \mathrm{C} / \mathrm{min}-180{ }^{\circ} \mathrm{C}-\right.$ $5 \mathrm{~min})$

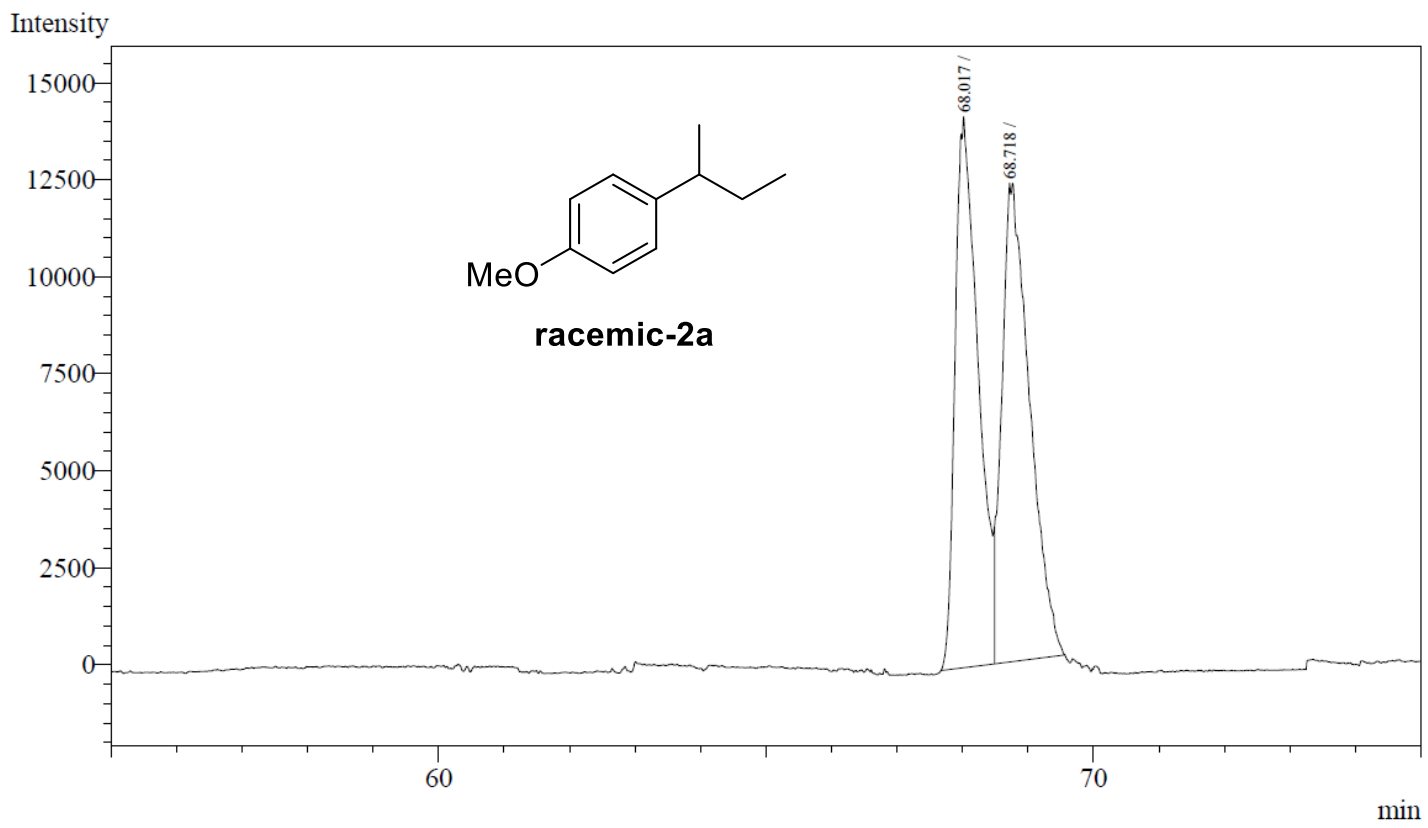

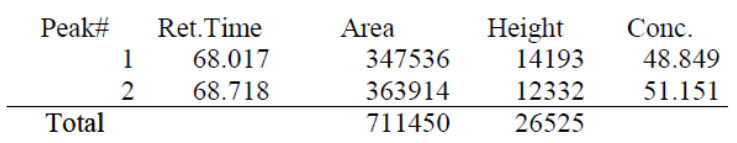

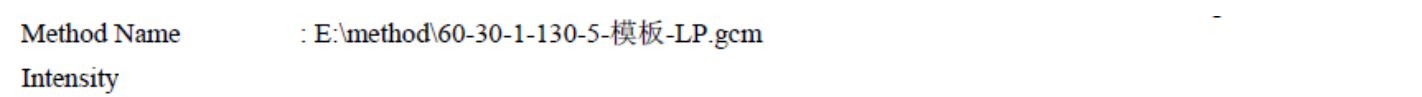

Intensity

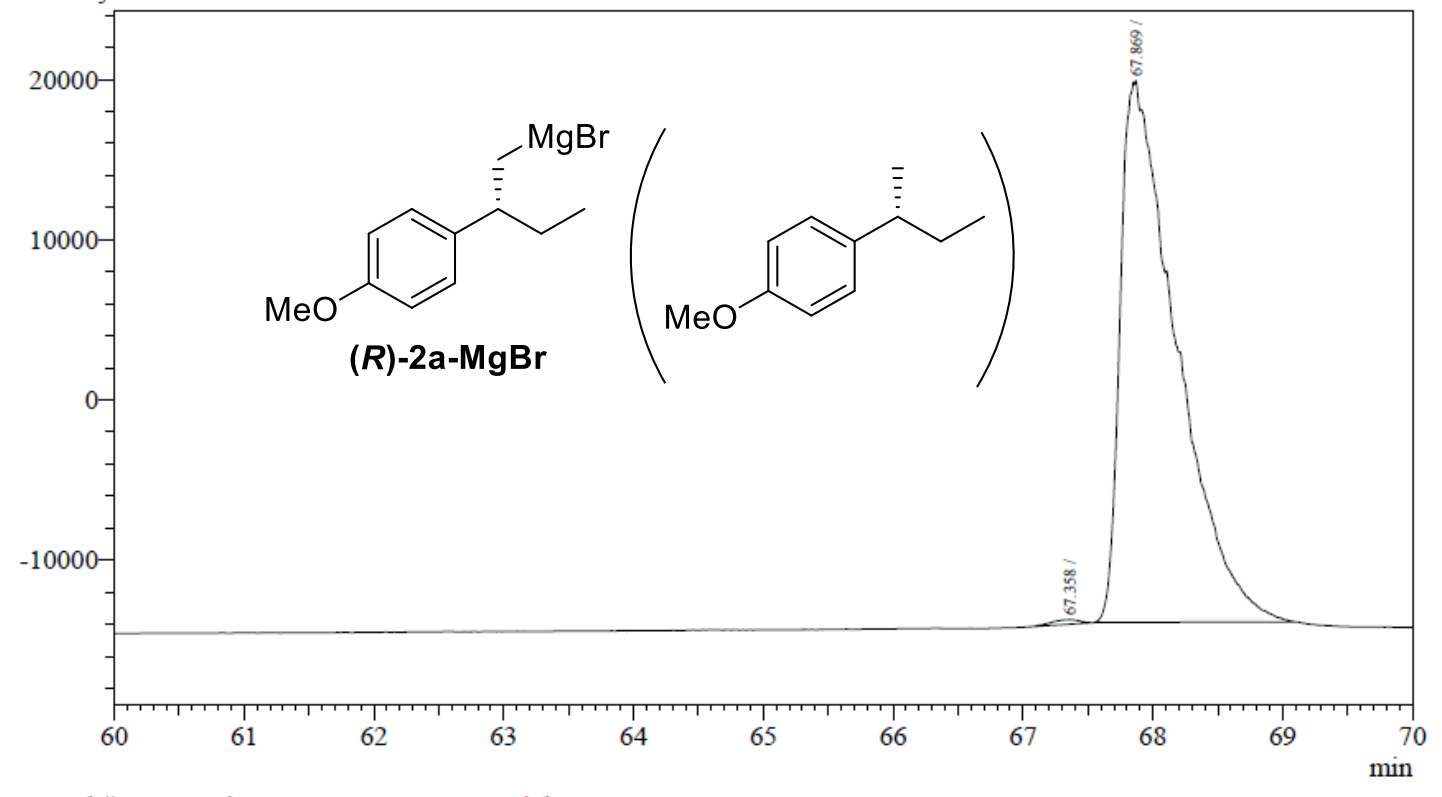

\begin{tabular}{lrrrr} 
Peak\# & Ret.Time & \multicolumn{1}{c}{ Area } & Height & \multicolumn{1}{c}{ Conc. } \\
1 & 67.358 & 4444 & 289 & 0.436 \\
2 & 67.869 & 1014942 & 33779 & 99.564 \\
\hline Total & & 1019386 & 34068 &
\end{tabular}


GC Conditions : Chiralcel B-DM, $100 \mathrm{kPa} \mathrm{N} 2\left(60{ }^{\circ} \mathrm{C}-30 \mathrm{~min}-1{ }^{\circ} \mathrm{C} / \mathrm{min}-180{ }^{\circ} \mathrm{C}-\right.$ 5 min)

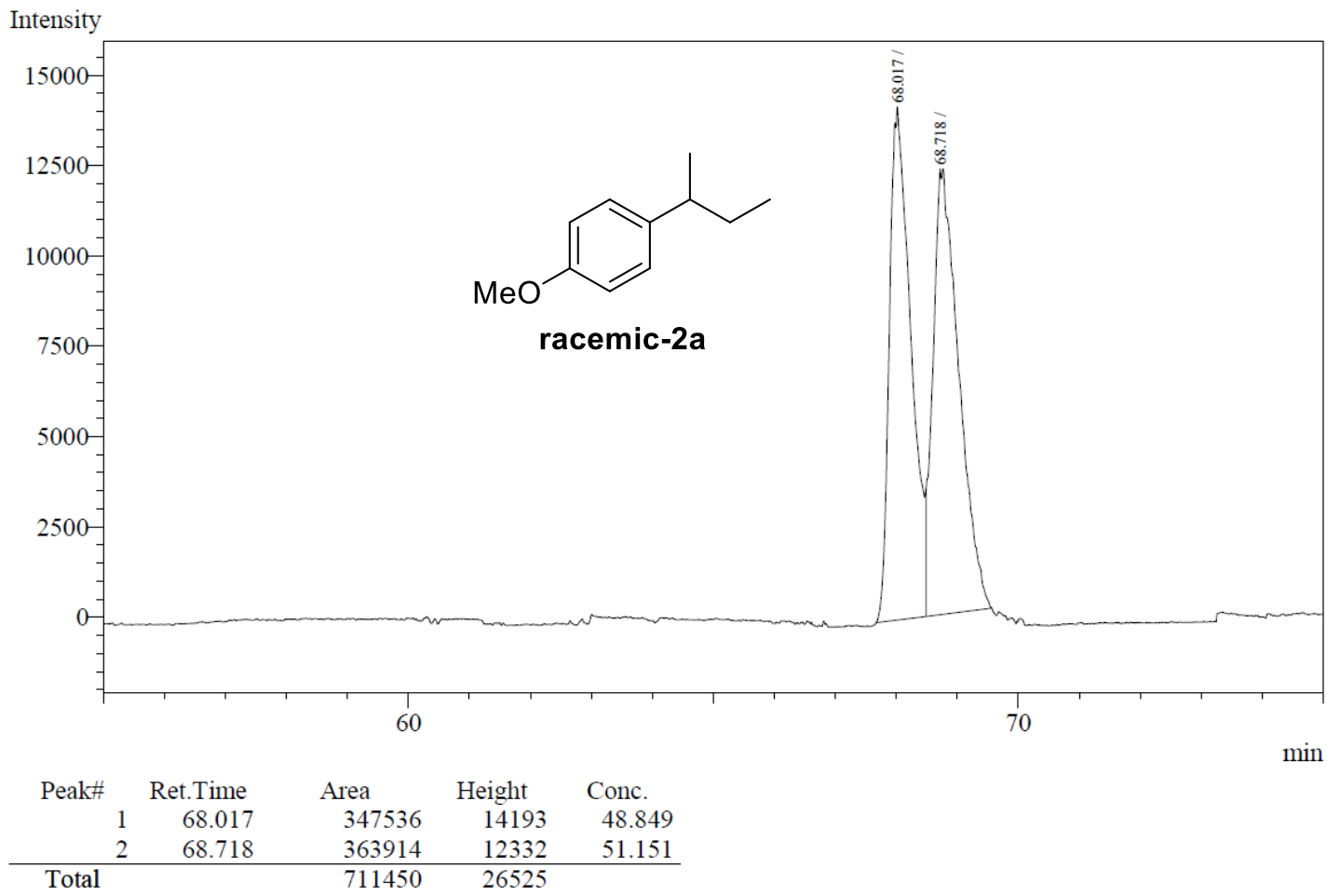

Method Name : E: Imethod 60 -30-1-115-5-模板-LP.gcm

Intensity

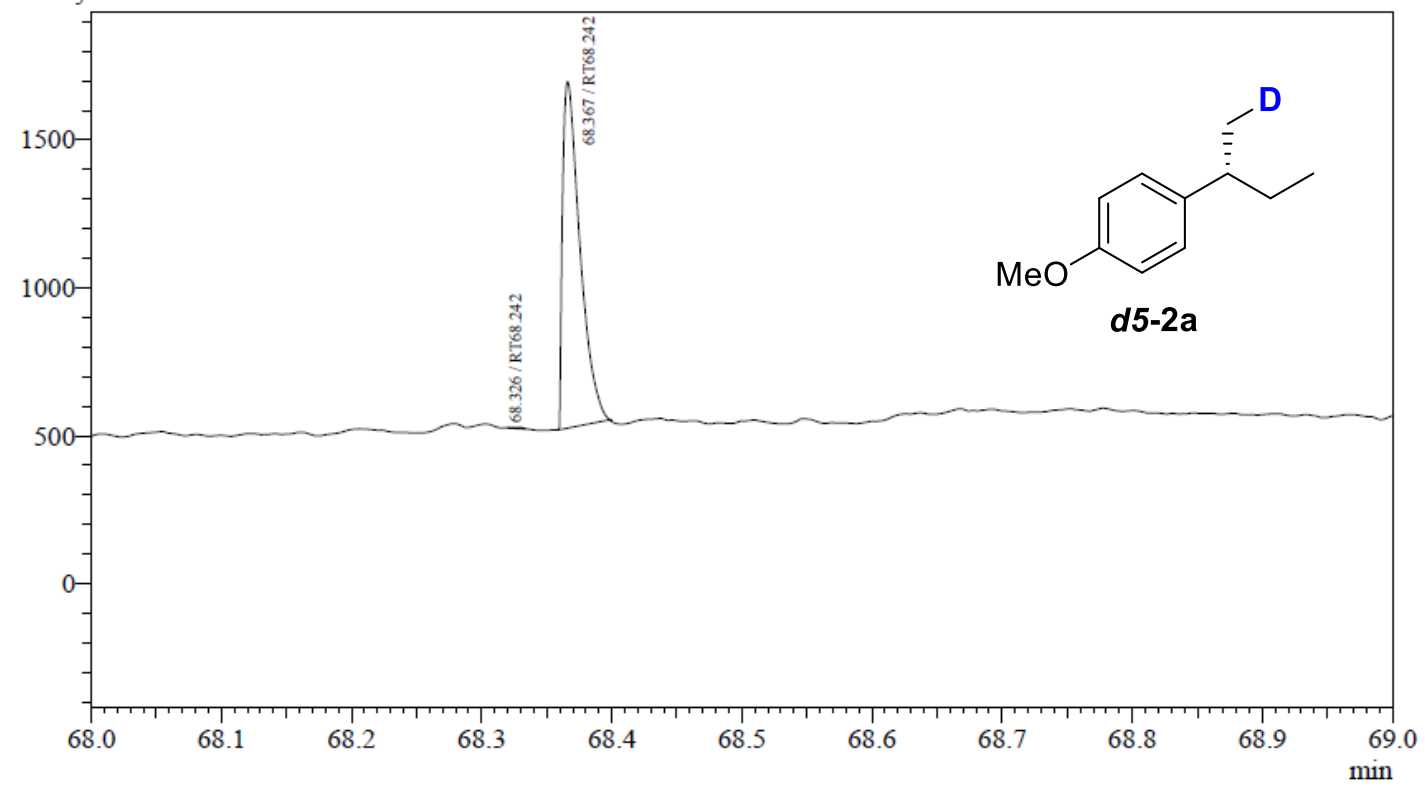

\begin{tabular}{|c|c|c|c|c|}
\hline Peak\# & Ret.Time & Area & Height & Area $\%$ \\
\hline & 68.326 & 2 & 3 & 0.2138 \\
\hline & 68.367 & 1088 & 1114 & 99.7862 \\
\hline Total & & 1090 & 1117 & 100.0000 \\
\hline
\end{tabular}


$\mathrm{mV}$

色谱图

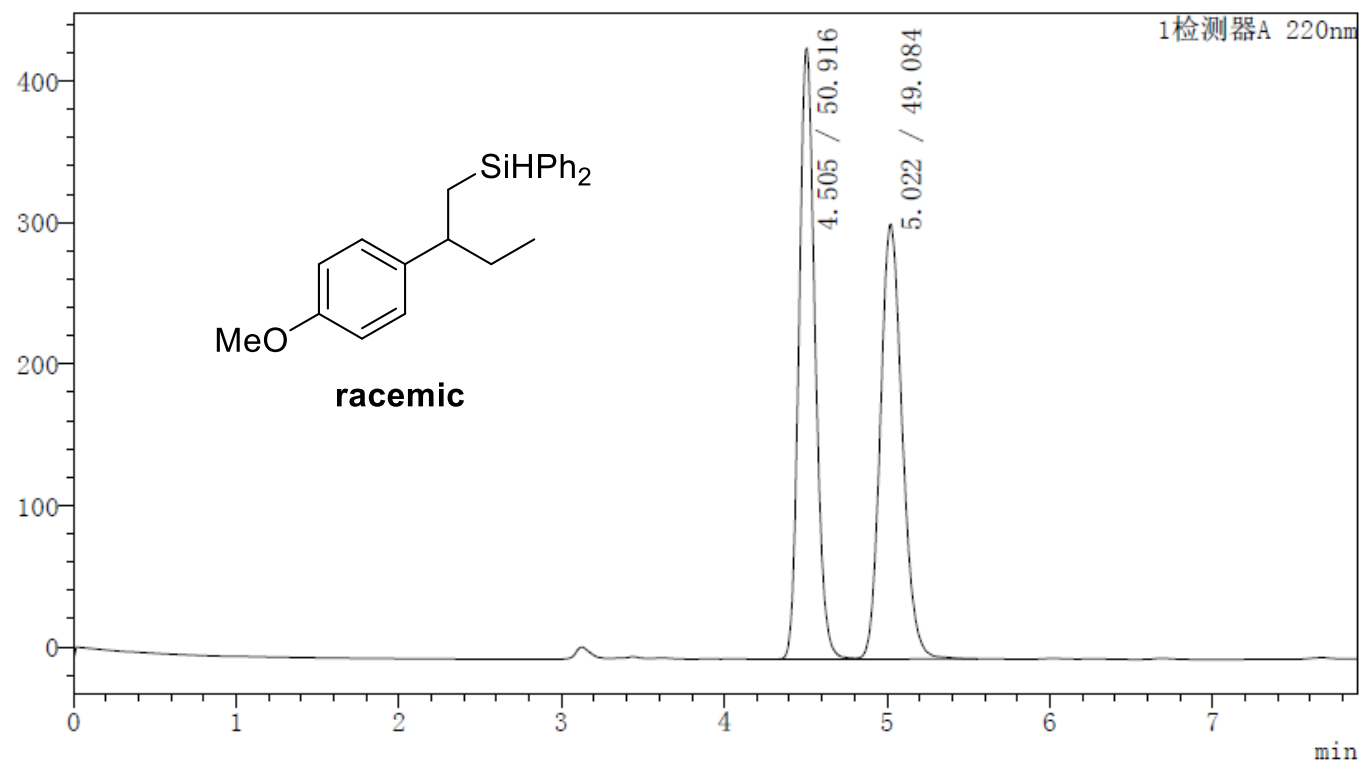

峰表

检测器A $220 \mathrm{~nm}$
\begin{tabular}{|r|r|r|r|r|r|}
\hline \multicolumn{1}{|c|}{ 峰号 } & 保留时间 & 面积 & 高度 & 标记 & 面积 $\%$ \\
\hline 1 & 4.505 & 2975109 & 431722 & & 50.916 \\
\hline 2 & 5.022 & 2868100 & 307053 & V & 49.084 \\
\hline 总计 & & 5843210 & 738775 & & 100.000 \\
\hline
\end{tabular}

描述

: $0 J-H, \quad n$-hexane $/ \mathrm{iPr}=95 / 5,1 \mathrm{ml} / \mathrm{min}, 220 \mathrm{~nm}$

$\mathrm{mV}$

色谱图

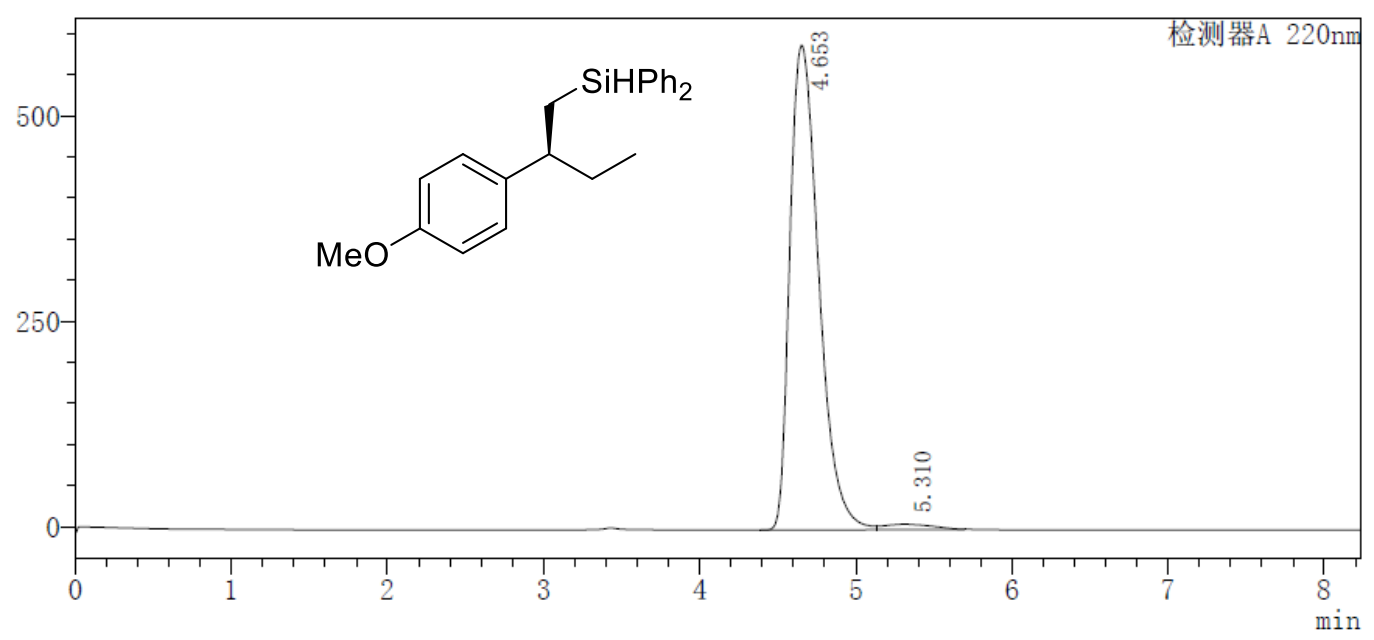

峰表

检测器A $220 \mathrm{~nm}$
\begin{tabular}{|r|r|r|r|r|r|}
\hline 峰号 & 保留时间 & \multicolumn{1}{|c|}{ 面积 } & \multicolumn{1}{|c|}{ 高度 } & 标记 & \multicolumn{1}{c|}{ 面积\% } \\
\hline 1 & 4.653 & 7580208 & 589844 & & 98.223 \\
\hline 2 & 5.310 & 137104 & 6651 & V M & 1.777 \\
\hline 总计 & & 7717312 & 596495 & & 100.000 \\
\hline
\end{tabular}


$\mathrm{mV}$

\section{色谱图}

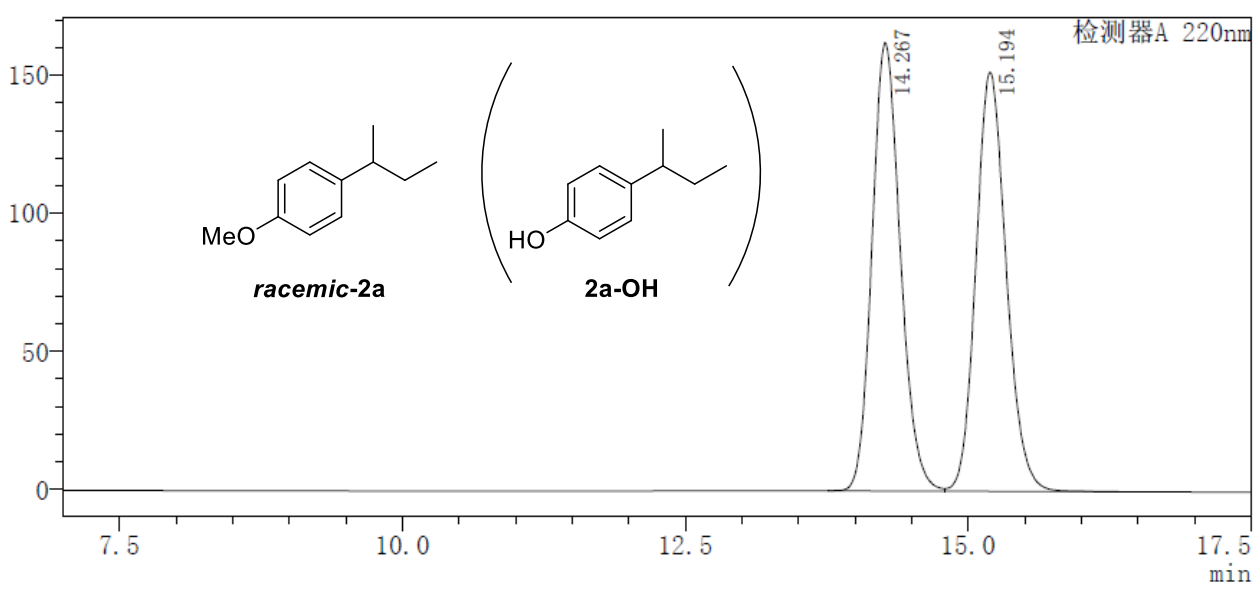

峰表

检测器A $220 \mathrm{~nm}$
\begin{tabular}{|r|r|l|l|l|r|}
\hline 峰号 & 保留时间 & 面积 & 高度 & 标记 & \multicolumn{1}{|c|}{ 面积 $\%$} \\
\hline 1 & 14.267 & 2826781 & 162336 & & 49.991 \\
\hline 2 & 15.194 & 2827761 & 151743 & V & 50.009 \\
\hline 总计 & & 5654542 & 314079 & & 100.000 \\
\hline
\end{tabular}

描述

: $0 \mathrm{~J}-\mathrm{H}, \mathrm{n}$-hexane $/ \mathrm{iPr}=95 / 5,1 \mathrm{ml} / \mathrm{min}, 220 \mathrm{~nm}$

$\mathrm{mV}$

\section{色谱图}

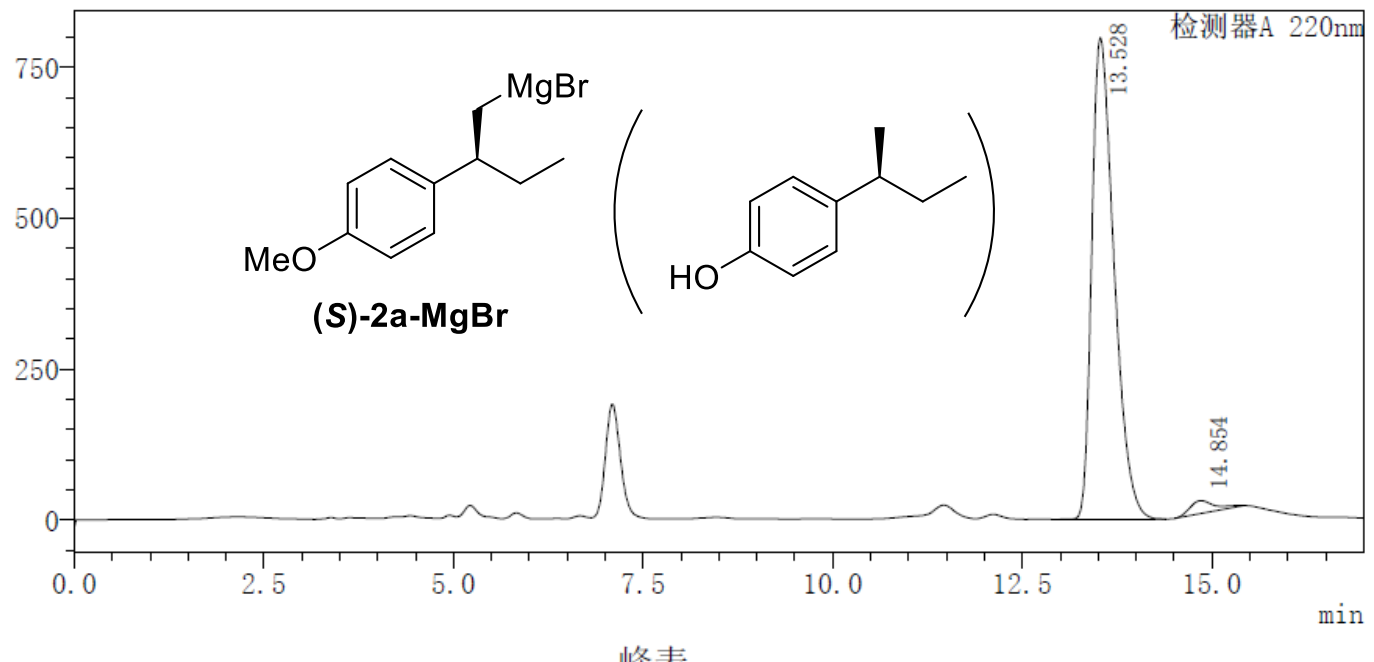

峰表

检测器A $220 \mathrm{~nm}$
\begin{tabular}{|r|r|r|r|r|r|}
\hline 峰号 & 保留时间 & \multicolumn{1}{|c|}{ 面积 } & \multicolumn{1}{c|}{ 高度 } & 标记 & \multicolumn{1}{|c|}{ 面积 $\%$} \\
\hline 1 & 13.528 & 16335873 & 797932 & & 97.078 \\
\hline 2 & 14.854 & 491675 & 21612 & $\mathrm{M}$ & 2.922 \\
\hline 总计 & & 16827548 & 819544 & & 100.000 \\
\hline
\end{tabular}


$\mathrm{mV}$

\section{色谱图}

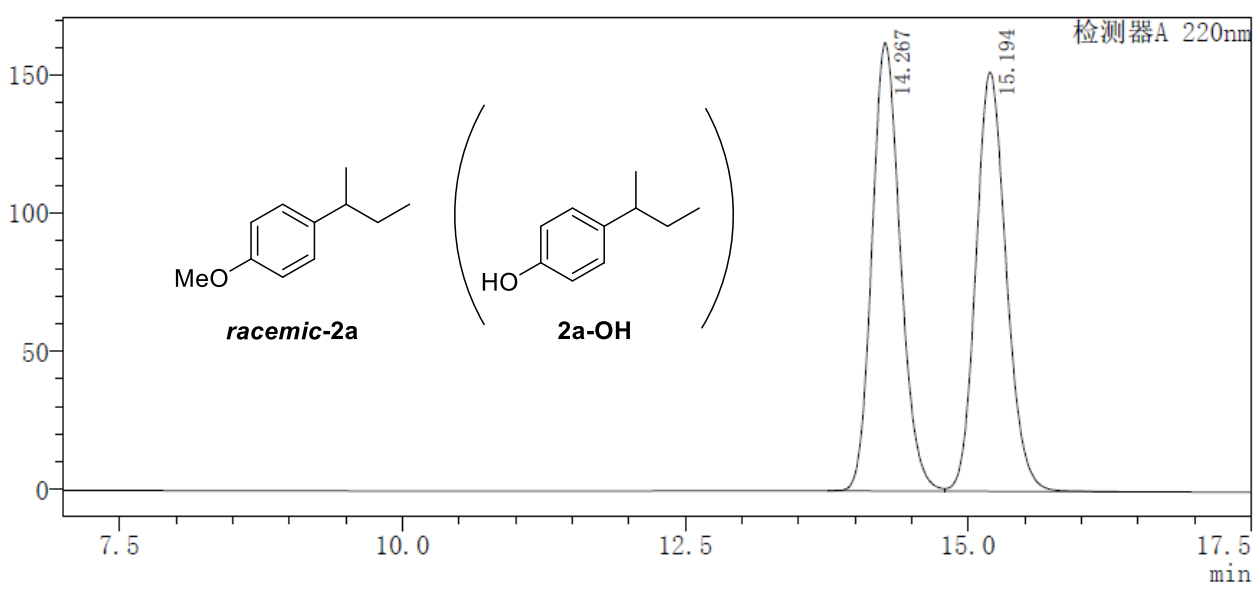

峰表

\begin{tabular}{|c|c|c|c|c|c|}
\hline 峰号 & 保留时间 & 面积 & 高度 & 标记 & 面积\% \\
\hline 1 & 14.267 & 2826781 & 162336 & & 49.991 \\
\hline 2 & 15.194 & 2827761 & 151743 & $\mathrm{~V}$ & 50.009 \\
\hline 总计 & & 5654542 & 314079 & & 100.000 \\
\hline
\end{tabular}

描述

$\mathrm{mV}$

\section{色谱图}

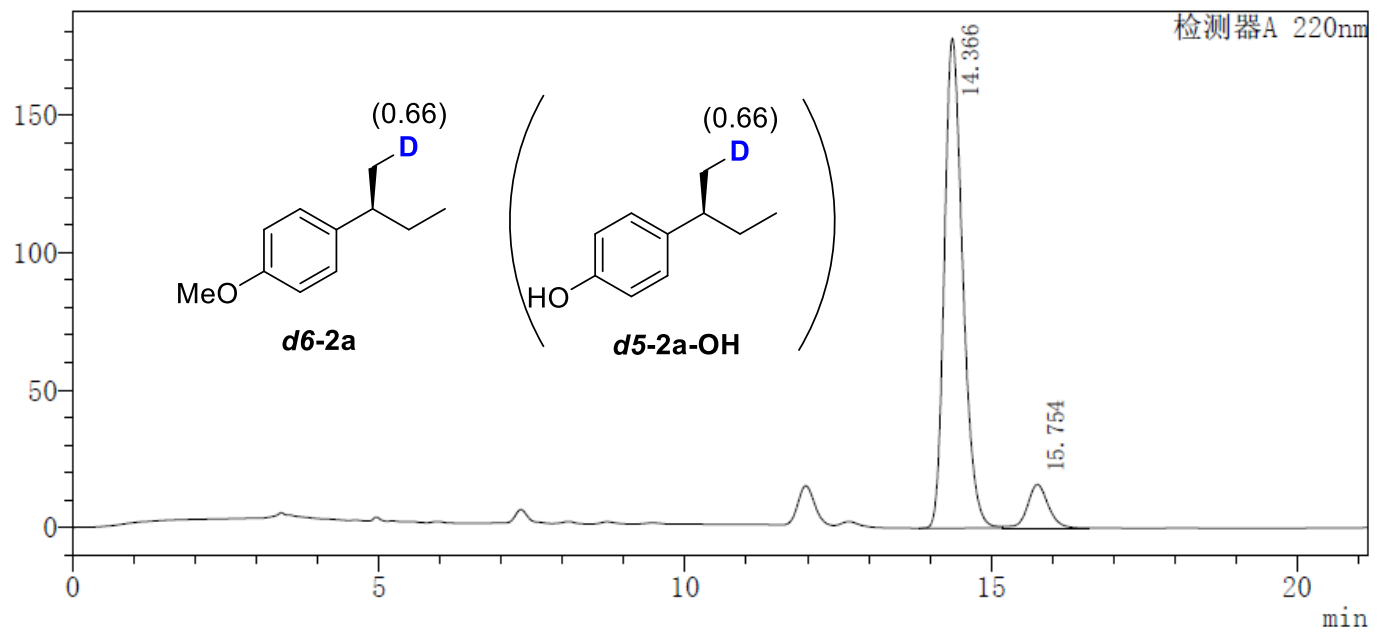

峰表

检测器A $220 \mathrm{~nm}$
\begin{tabular}{|r|r|r|r|r|r|}
\hline 峰号 & 保留时间 & \multicolumn{1}{|c|}{ 面积 } & \multicolumn{1}{c|}{ 高度 } & 标记 & \multicolumn{1}{|c|}{ 面积 $\%$} \\
\hline 1 & 14.366 & 3723352 & 177935 & & 90.902 \\
\hline 2 & 15.754 & 372633 & 15831 & V & 9.098 \\
\hline 总计 & & 4095985 & 193767 & & 100.000 \\
\hline
\end{tabular}


GC Conditions : Chiralcel B-DM, $100 \mathrm{kPa} \mathrm{N} 2\left(60{ }^{\circ} \mathrm{C}-30 \mathrm{~min}-2{ }^{\circ} \mathrm{C} / \mathrm{min}-180{ }^{\circ} \mathrm{C}-\right.$ $5 \mathrm{~min})$
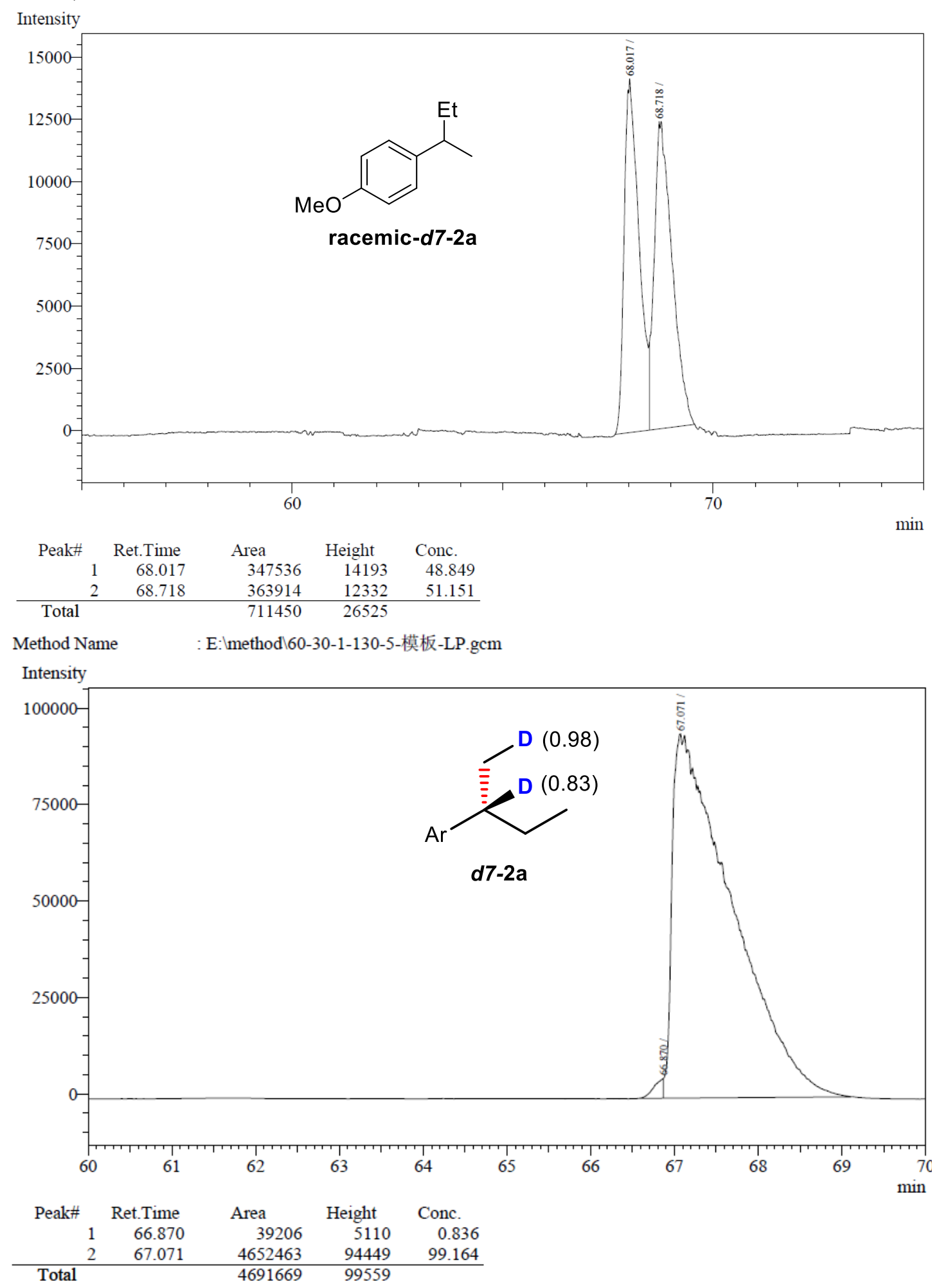
GC Conditions : Chiralcel B-DM, $100 \mathrm{kPa} \mathrm{N} 2\left(60{ }^{\circ} \mathrm{C}-30 \mathrm{~min}-2{ }^{\circ} \mathrm{C} / \mathrm{min}-180{ }^{\circ} \mathrm{C}-\right.$ $5 \mathrm{~min})$
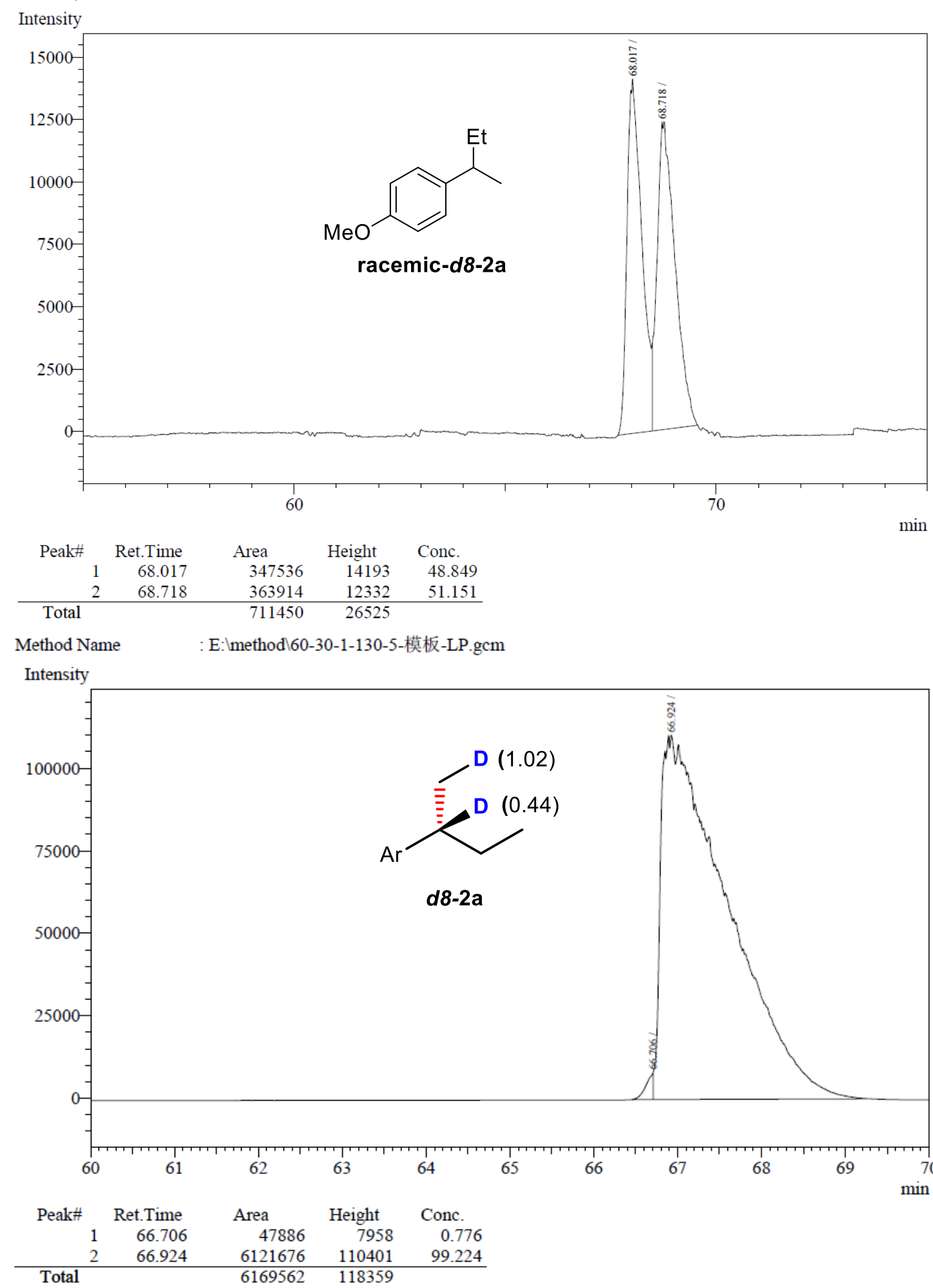